
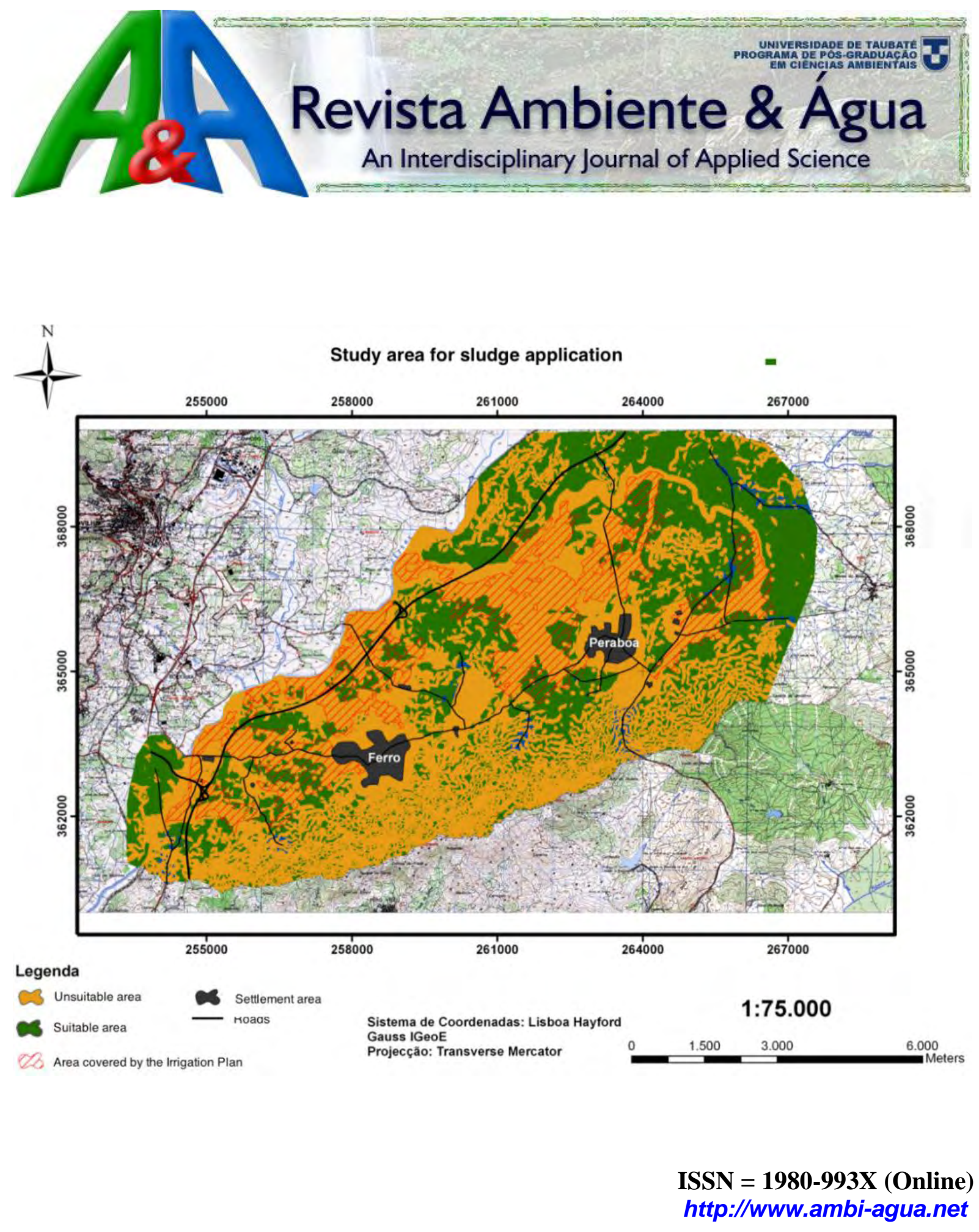

$28^{\text {th }}$ Edition of Revista Ambiente \& Água - An Interdisciplinary Journal of Applied Science, Taubaté, v. 10, n. 1, p. 1-233, Jan./Mar. 2015. (doi:10.4136/ambi-agua.v10.n1) 


\section{EDITORIAL BOARD}

\section{Editor}

\section{Getulio Teixeira Batista}

Instituto de Pesquisas Ambientais em Bacias Hidrográficas (IPABHi), SP, BR

\section{Associate Editors}

Amaury Paulo de Souza

Ana Aparecida da Silva Almeida

Andrea Giuseppe Capodaglio

Antonio Evaldo Klar

Antonio Teixeira de Matos

Carlos Eduardo de M. Bicudo

Cláudia S. de C. M. dos S. Cordovil

Dar Roberts

Delly Oliveira Filho

Ehsan Noroozinejad Farsangi

Gabriel Constantino Blain

Giordano Urbini

Gustaf Olsson

Hélio Nobile Diniz

János Fehér

João Vianei Soares

José Carlos Mierzwa

Julio Cesar Pascale Palhares

Luis Antonio Merino

Marcelo dos Santos Targa

Massimo Raboni

Nelson Wellausen Dias

Petr Hlavínek

Richarde Marques da Silva

Sergio Francisco de Aquino

Silvio Jorge Coelho Simões

Stefan Stanko

Teresa Maria Reyna

Yosio Edemir Shimabukuro

Zhongliang Liu Beijing
Universidade Federal de Viçosa (UFV), BR

Universidade de Taubaté (UNITAU), BR

University of Pavia, Italy

Universidade Est. Paulista Júlio de Mesquita Filho (UNESP), BR

Universidade Federal de Viçosa (UFV), BR

Instituto de Botânica, IBT, BR

Centro de estudos de Engenharia Rural (CEER), Lisboa, Portugal

University of California, Santa Barbara, United States

Universidade Federal de Viçosa (UFV), BR

International Institute of Earthquake Engineering and

Seismology(IIEES), Farmanieh, Tehran, Iran

Instituto Agronômico de Campinas, IAC, Brasil.

University of Insubria, Varese, Italy

Lund University, Lund, Sweden

Inst. Geológico, Sec. do Meio Amb. do Est. de SP (IG/SMA),BR

Debrecen University, Hungary

Instituto Nacional de Pesquisas Espaciais (INPE), BR

Universidade de São Paulo, USP, BR

Embrapa Pecuária Sudeste, CPPSE, São Carlos, SP, BR

Institute of Regional Medicine, National University of the Northeast,

Corrientes, Argentina

Universidade de Taubaté, (UNITAU), BR

Polytechnic University of Milan, Milan, Italy

Inst. Bras. de Geogr. e Estatística (IBGE). Aracaju, SE, BR

Brno University of Technology República Tcheca

Universidade Federal da Paraíba, UFPB, BR

Universidade Federal de Ouro Preto, UFOP, BR

Univ. Est. Paulista Júlio de Mesquita Filho, UNESP, BR

Slovak Technical University in Bratislava Slovak, Eslováquia

Universidad Nacional de Córdoba, Argentina

Instituto Nacional de Pesquisas Espaciais (INPE), BR

University of Technology, China

\section{Editorial Section}

Text (EN and ES) Editors

Reference Editor

Layout Editors

Technical Support

\author{
Nelson Wellausen Dias, PPGCA, UNITAU, IBGE, BR \\ Theodore D'Alessio, FL, USA \& Getulio T. Batista, UNITAU, BR \\ Liliane Castro, Bibliotecária - CRB/8-6748, Taubaté, BR \\ Vera L. F. Batista, IPABHi \& Getulio T. Batista, UNITAU, BR \\ Tiago dos Santos Agostinho, LAGEO, UNITAU, BR
}

Library catalog entry by SIBi - Sistema Integrado de Bibliotecas / UNITAU

Revista Ambiente \& Água - An Interdisciplinary Journal of Applied Science / Instituto de Pesquisas Ambientais em Bacias Hidrográficas. Taubaté. v.10, n. 1 (2006)- Taubaté: IPABHi, 2015

Quadrimestral

ISSN 1980-993X

1. Ciências ambientais

2. Recursos hídricos

I. Instituto de Pesquisas Ambientais em Bacias Hidrográficas

CDD - 333.705

CDU - (03)556.18 


\section{TABLE OF CONTENTS}

\section{COVER:}

Suitable agricultural area for paper mill sludge application in Covilhã, Portugal $\left(40^{\circ} 15^{\prime} 00^{\prime \prime} \mathrm{N}, 7^{\circ} 2^{\prime}\right.$ 00" W).

Source: ALBUQUERQUE, A. J. C. et al. Multi-criteria analysis for site selection for the reuse of reclaimed water and biosolids. Rev. Ambient. Água, Taubaté, vol. 10 n. 1, p. 23-34, 2015 http://dx.doi.org/10.4136/ambi-agua. 1550

\section{EDITORIAL}

Internationalization, education and technological innovation: three key factors to improve the quality of the environment and public health

doi:10.4136/ambi-agua.1574

Giordano Urbini

\section{ARTICLES}

\footnotetext{
A comprehensive analysis of the current and future role of biofuels for transport in the European

01 Union (EU)

01 doi:10.4136/ambi-agua.1492

Massimo Raboni; Paolo Viotti; Andrea G. Capodaglio

Multi-criteria analysis for site selection for the reuse of reclaimed water and biosolids

02 doi: 10.4136/ambi-agua.1550

António Albuquerque; Paulo Sérgio Scalize; Nilson Clementino Ferreira; Flora Silva

Hydrogeochemical attributes and ground water quality of Ngbo Community in Ohaukwu Area Council, Ebonyi State, Nigeria
}

03 doi: 10.4136/ambi-agua.1453

Omaka Ndukaku Omaka; Ifeanyi Francis Offor; Ikechukwu Moses Onwe

Induction of cytotoxic and genotoxic effects of Guandu River waters in the Allium cepa system doi: 10.4136/ambi-agua.1487

Jennifer Vieira Gomes; Jéssica Tamara dos Santos Teixeira; Viviane Moreira de Lima; Helcio Resende Borba

Remoción de plomo en soluciones acuosas empleando nanoaluminofosfatos amorfos doi: 10.4136/ambi-agua.1437

Wendy Rondón; Ángela B. Sifontes; David Freire; Manuelita Quintal; Edgar Cañizales; Franklin J. Méndez1; Ligia Llovera; Yraida Diaz; Joaquin L. Brito

Construção e emprego de diagramas de coagulação como ferramenta para o monitoramento contínuo da floculação em águas de abastecimento

doi: 10.4136/ambi-agua.1239

Daniel Bartiko; Marcelo De Julio

Análise de tendência em séries históricas de vazão e precipitação: uso de teste estatístico não paramétrico

07 doi: 10.4136/ambi-agua.1427

Eduardo Morgan Uliana; Demetrius David da Silva; Estevão Morgan Uliana; Beatriz Silveira Rodrigues; Lucas de Paula Corrêdo

Modelagem neural e análise estatística do processo de degradação de chorume por foto-Fenton solar

08 doi: 10.4136/ambi-agua.1341

Alessandro Sampaio Cavalcanti; Hélcio José Izário Filho; Oswaldo Luiz Cobra Guimarães; Adriano Francisco Siqueira 
Tratamento de lixiviados de aterros de resíduos sólidos utilizando Processos Fenton e Foto-Fenton Solar

Fábio Moraes da Costa; Juacyara Carbonelli Campos; Fabiana Valéria da Fonseca; Daniele Maia Bila

Uso de biomarcadores para monitoramento das águas do Córrego Arara no município de Rio Brilhante, MS, Brasil

Elisangela Bortoluci Maceda; Alexéia Barufatti Grisolia; Jussara Oliveira Vaini; Liliam Silvia Candido

Citotoxicidade e genotoxicidade da água do rio Subaé (Humildes, Bahia, Brasil) usando Allium cepa L. como bioindicador

Jacqueline Ramos Machado Braga; Diêgo Menezes Lopes

Vulnerabilidade à contaminação das águas subterrâneas do município de Nova Palma, Rio Grande do Sul, Brasil

Carlos Alberto Löbler; José Luiz Silvério da Silva

Manganês, zinco, cádmio, chumbo, mercúrio e crômio no chorume de aterro sanitário em Dourados, MS, Brasil

Priscilla Fracalossi Riguetti; Cláudia Andréa Lima Cardoso; Alberto Adriano Cavalheiro; Ervim Lenzi; Antonio Rogério Fiorucci; Margarete Soares da Silva

\section{Avaliação de sistemas de gestão ambiental em granjas de suínos}

14 doi: 10.4136/ambi-agua.1504

Bruno Vieira de Carvalho; Angelita Pereira Melo e Sousa; Francisco Rafael Martins Soto

Análise probabilística da distribuição de dados diários de chuva no estado do Paraná

Airton Kist; Jorim Sousa das Virgens Filho

Eventos extremos de precipitação em Belém-PA: uma revisão de notícias históricas de jornais doi: 10.4136/ambi-agua.1433

Thamiris Luisa de Oliveira Brandão Campos; Maria Aurora Santos da Mota; Sergio Rodrigo Quadros dos Santos

Influência das características morfométricas da bacia hidrográfica do rio Benevente nas enchentes no município de Alfredo Chaves-ES

17 doi: 10.4136/ambi-agua.1475

Alexandre Simões Lorenzon; Micael de Souza Fraga; Amanda Rodrigues Moreira; Eduardo Morgan Uliana; Demetrius David da Silva; Carlos Antonio Alvares Soares Ribeiro; Alisson Carraro Borges

Caracterização de ambientes aquáticos no rio de Ondas, cerrado baiano

Elis Regina Rodrigues de Souza Castro; Michel Castro Moreira; Luís Gomes Carvalho; Demetrius David da Silva

\section{Adubação com NPK e irrigação do girassol em Luvissolo: Comportamento vegetativo}




Ambiente \& Água - An Interdisciplinary Journal of Applied Science
ISSN 1980-993X - doi:10.4136/1980-993X
www.ambi-agua.net
E-mail: ambi-agua@agro.unitau.br

\title{
Internationalization, education and technological innovation: three key factors to improve the quality of the environment and public health
}

\author{
doi:10.4136/ambi-agua.1574
}

\author{
Giordano Urbini
}

\author{
Member of the Editorial Committee of Ambiente \& Agua Journal \\ Università degli Studi dell'Insubria, Varese, Italy \\ Department of Biotechnologies and Life Sciences \\ e-mail: giordano.urbini@uninsubria.it
}

\begin{abstract}
The environment and the health of mankind are subject to strong pressures from pollutants. The ambition of a better quality of life is often frustrated by the increase in emissions as a result of the increasing consumption of resources and energy to satisfy the growth of the world's population and its expectations for socioeconomic development. At times, problems take on a planetary dimension, the best known example being greenhouse gas emission, with the resulting effects of climate change. Pollution highlights our failure to provide adequate education, which is essential if we are to become fully aware of problems and actively seek sustainable solutions. To this end, it is essential to set up an extended environmental and health education program in all communities that will accompany younger generations from the beginning of their education in order to make them conscious players in protecting the environment and their own health. The size of the problems requires that a joint effort be made at an international level in the political realm and in the scientific-cultural realm in order to share experiences and sensitivity and to seek joint solutions. It is necessary to assign an increasingly important role to scientific and technological innovation which, in the last century, from Marconi to Einstein to Fleming, to the most recent innovations in the fields of genomics and microchips, has allowed humanity to achieve a rate of growth of wellbeing never previously seen in its thousands of years of history. This innovation must rationally be entrusted with our expectations to provide a practical solution to the problems of pollution that threaten our everyday lives and the survival of the planet. This text discusses the types of progress most eagerly awaited in the fields of water supply, wastewater treatment, atmospheric pollution and waste disposal.
\end{abstract}

Keywords: environmental education, pollution control, water resource.

\section{Internacionalização, educação e inovação tecnológica: três fatores-chave para melhorar a qualidade do meio ambiente e saúde pública}

\section{RESUMO}

O meio ambiente e a saúde humana estão sob forte pressão de poluentes. A ambição de uma qualidade de vida melhor é muitas vezes frustrada pelo crescimento das emissões, devido 
ao aumento do consumo de recursos e energia para atender à crescente população mundial e às expectativas de desenvolvimento socioeconômico. Às vezes os problemas assumem uma dimensão global, da qual o exemplo mais conhecido é constituído pela emissão de gases de efeito estufa e os consequentes efeitos nas alterações climáticas. Poluição tem as suas raízes na falta de educação adequada, como base imprescindível para uma tomada de consciência das questões e participação na busca de soluções sustentáveis. Neste sentido, é essencial começar, em todas as comunidades, um extenso programa de educação ambiental e de saúde que possa acompanhar a geração mais jovem desde as fases iniciais do estudo, para torná-los protagonistas conscientes do meio ambiente e da sua própria saúde. A dimensão do problema exige um esforço conjunto a nível internacional pela política e pela ordem científico-cultural para transferir experiências e sentimentos e também buscar soluções compartilhadas. Um papel sempre mais importante deve ter a inovação científica e tecnológica, que no século passado, a partir de Marconi a Einstein e a Fleming até as últimas inovações nas áreas de genômica e microchips, permitiu à humanidade alcançar uma taxa de crescimento do bemestar nunca conseguida antes, em milhares de anos de história. Esta inovação está atrelada às nossas expectativas de fornecer uma solução prática para os problemas de poluição que ameaçam a nossa vida diária e a sobrevivência do planeta. No texto, são citados os progressos mais esperados nas áreas de abastecimento de água e tratamento de esgotos, poluição do ar e disposição dos resíduos sólidos.

Palavras-chave: controle da poluição, educação ambiental, recursos hídricos.

\section{DISCUSSION}

\subsection{The strong need for internationalization and environmental education}

Today, the word "internationalization" is one of the most used and abused. Articles and books are even written to explain its meaning, also giving it philological and philosophical interpretations. Apart from these literary ruses, the practical meaning of the term should refer to any of man's activities that have international involvement. Internationalization produces exchange at various levels, and expands knowledge to a broad audience; as such, it is an instrument for personal gratification, for professional fulfillment and, without being rhetorical, of social progress. Over time, the concept of "internationalization" and its actual practice have been extended greatly, favored by very rapid instruments and means of communication. We know of the internationalization of markets and economies, of universities, of the sciences and of culture, of sport, of businesses, banking systems and commerce. It is essential also to include the internationalization of thought and of ideas, where the world's best minds come together. The main objective is to enhance excellence in individual countries by expanding and intensifying cooperation with the best researchers in other countries, fields and institutions.

A lot is expected of internationalization: progress in living conditions, greater fairness in the use and distribution of resources, better protection of the environment and of public health, peace and widespread well-being. The large organizations that govern world politics are being called upon to give substance to these hopes, by promoting joint efforts on the big themes concerning the life of man and of the planet. The greatest challenges this century faces inevitably relate to climate change, the planet's nutrition, the safety and sustainability of energy supplies, the fight against poverty and infectious diseases, as well as the construction of basic health and sanitation infrastructure. It is worth remembering that for a large part of the world's population, the scarcity of water and the absence of such infrastructure constitute a grave danger to health and are a limiting factor in socioeconomic development. The UN data is shocking: approximately $40 \%$ of the world's population suffers these shortages, and the trend is upward, to $65 \%$ by 2025 (UN-Human Rights et al., 2010). In this situation, no 
fewer than 1,500,000 children die every year in the world because of contaminated water or the scarcity of water (UN Water, 2013).

Water is a limited resource; but at the same time, it is vital for all of man's activities, so much so that any scarcity will stunt a society's development. Thus, the development trend taking place in many of the world's countries requires ever greater consumption of water, exploiting the natural water resources. However, there are many situations in which this resource is shared by more than one nation, and unilateral exploitation leads to dangerous tensions and competition. The UN has indicated that this danger concerns no fewer than 180 areas of the planet.

A great number of impediments to improving the environment and the sustainable exploitation of resources stem from extremely poor knowledge on the part of a very large proportion of the world's population. We need to be aware that, only with extended and effective environmental education of new generations, starting as early as primary school, will it be possible to promote and sustain the proper actions, both locally and globally. Unfortunately, we must acknowledge that this path must still be constructed entirely, because the young generations are currently very detached from the nature surrounding them, of which they themselves are a vital and integral part. The objective we must set is to educate young students to respect nature as a universal value and to assess its benefits for the life of man itself. The education process should make students genuine "environmental stewards", bringing them back into direct contact with animal and vegetable life, allowing them to rediscover the importance of things that are only apparently small, like the planting of a sapling which they can see grow over time, giving them the important understanding of the extraordinary value of life. Schools need encouragement and time to dedicate to environmental education; they also need funds with which to train teachers adequately. The students thus educated will be able to turn their knowledge into positive environmental actions in the community.

\subsection{Science and technology in defense of the environment and of the health of man}

In the last few years, mankind's need to live in a less polluted environment has driven science and industry to introduce a multitude of innovations: from recycling materials, to biodegradable plastics, to using eco-friendly propellants for domestic and health sprays; to replacing the phosphorus in detergents, to using biodegradable dyes in industry, to exploiting the waste biomass to produce biofuels, animal feed and energy, and many others.

In the field of wastewater treatment, it is known that the processes most applied are biological ones that are about 100 years old (Tchobanoglous et al., 2003). However, the original plant configuration remained all but unaltered until the 1980s-1990s, when an impressive development of more refined, efficient and innovative ideas and technologies began. I am referring to biological, submerged bed systems, to UASB (Upflow Anaerobic Sludge Blanket) reactors, to SBBR (Sequencing Batch Biological Reactor) systems, to MBBR (Mobile Bed Biological Reactor) systems, and, above all, to the recent introduction of MBR (Membrane Biological Reactor) systems, which can be more compact, and can give better purification yields (Farabegoli et al., 2003, Raboni et al., 2013a; 2013b, Torretta et al., 2013a; Raboni et al., 2014a; 2014b). Many new plants are designed with these innovative technologies and, in particular, with biofiltration processes and advanced MBR processes (Torretta et al., 2013a). In the near future, it is expected that these processes will be implemented with technologies aimed at removing the emerging micro-pollutants (particularly active ingredients of medicines and abused drugs), the accumulation of which in the aquatic ecosystems is a worrisome danger for them and for public health.

In the field of water, the great, future challenge for mankind will be to distribute fresh water to satisfy the demands of the whole of the world's population. This will be a very 
difficult task, because it will only be possible by desalinating water from the oceans, a process that is already done today, but at a cost that makes it only accessible to the richest nations. While waiting for this, mankind should immediately focus on reusing water. Indeed, the growing consumption for civil, industrial and, above all, agricultural purposes (Figure 1) has brought many areas of the world to conditions of genuine water stress (water drawn at a rate of $20 \%$ above actual availability). These areas even include a number of countries in the European Union, such as Cyprus, Spain, Bulgaria, Belgium, Malta and Italy.

The UN estimates that 700 million people currently live in areas where there is a scarcity of water (insufficient for essential needs) and that, by 2025, this number will rise to 1800 million; furthermore, by this date two thirds of the world's population will be living under conditions of water stress (Figure 2).

Today, many countries already resort to reusing treated sewage, primarily for irrigation purposes (e.g. the USA reuse of 7-8\%, Australia 8\%, Saudi Arabia 16\%; Singapore 30\%; Israel has the record for recovery, of $70 \%$; in Europe, the two countries that stand out are Italy, with 4-5\%, and Spain, with 6-7\%). In the future, these percentages will inevitably increase, and the help that technological innovation can give will be essential.

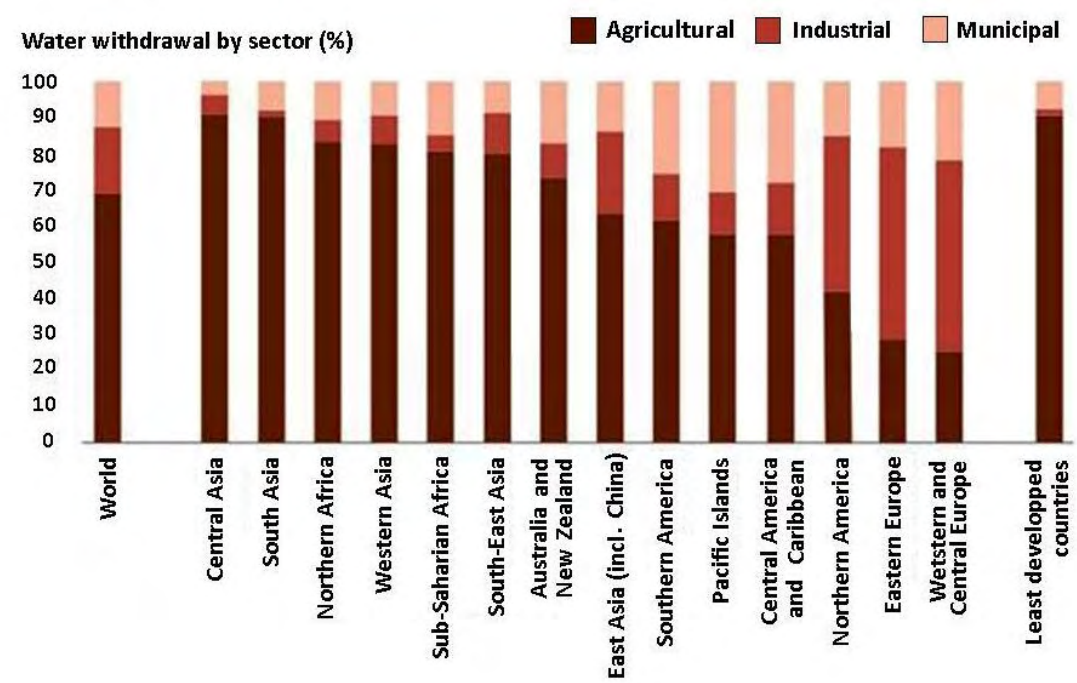

Figure 1. Water withdrawal by sector and by region (UN World Water, 2012).

It is worth remembering that among the forms of reuse that currently exist, there is also reuse as drinking water; the areas affected are obviously those that are climatically very disadvantaged. Of the plants for direct reuse as drinking water, particular mention should go to the service in the city of Windhoek $\left(21,000 \mathrm{~m}^{3} / \mathrm{d}\right)$, the capital of Namibia, as it was the first of its kind in the world. Also in the USA, there are cases of obtaining drinking water from civil sewage, albeit indirectly. The most typical example is the plant in Orange County, California $\left(265,000 \mathrm{~m}^{3} / \mathrm{d}\right)$, which improves the quality of the effluent from the mechanical-biological plant, with a process based on using membranes (microfiltration and hyperfiltration) and advanced oxidative treatments. The water produced in this way is used to recharge the water table destined for the production of drinking water.

The waste sector has much more complex consequences and, in particular, the disposal choices are often greatly affected by public opposition. At the moment, the most advanced countries base their choices on the so-called "integrated solution" (Rada et al., 2014a; Torretta et al., 2014), which consists of a mixture of organizational and technological systems that include a very intense, initial phase of separated waste collection, with recycling of the materials recovered, and the residual fraction is then sent for incineration to recover energy. 
The landfill is only used for disposal of the incineration residues. Within this scheme, where the proportion of waste collected separately can be as high as $50 \%$, incineration has a better degree of acceptance, given also that it is technologically very advanced (Mancini et al., 2013).

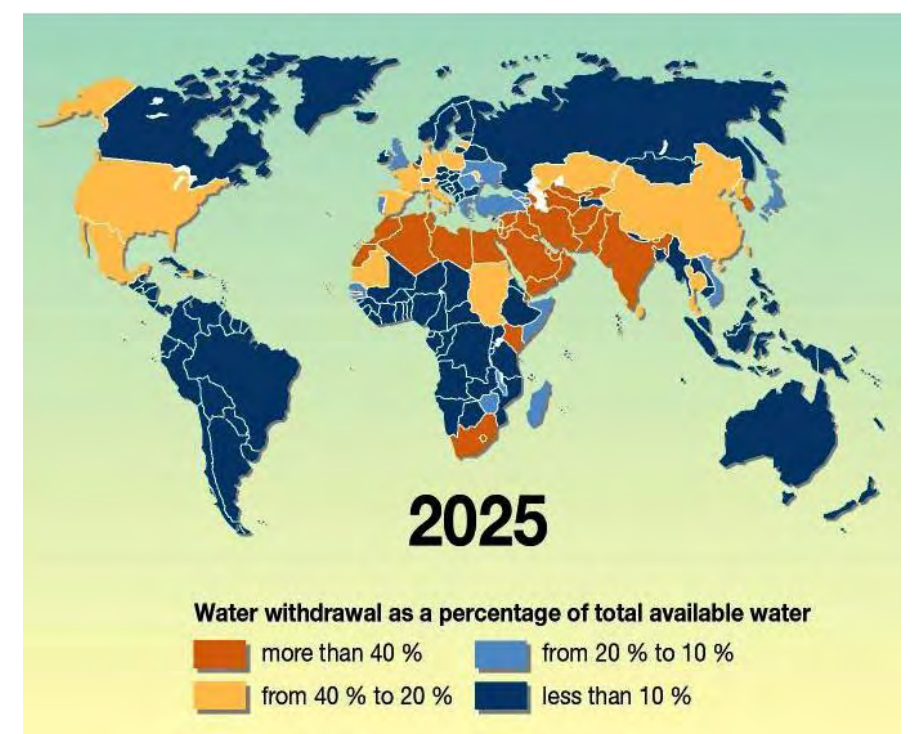

Figure 2. Water withdrawal as a percentage of total available water. Values of $20 \%$ above water availability represent water stress conditions; of $40 \%$ above water availability, water scarcity (UN World Water, 2012).

Technological innovation is currently making a considerable effort to limit the atmospheric emission of pollutants that have a direct impact on the health of mankind. Urban areas have a high presence of fine particulates, of polycyclic aromatic hydrocarbons (PAHs) and of volatile organic compounds (VOCs), the toxicity, cancerogenicity and mutagenicity characteristics of which are well-known (Copelli et al., 2012; Chiavola et al., 2010; Torretta et al., 2013b; 2013c; Rada et al., 2014b; Raboni et al., 2014c). The sector most under accusation is transport, which is responsible also for significant $\mathrm{CO}_{2}$ emissions. The European Union is looking at this issue with great interest, and three years ago they adopted an extensive strategy to reduce carbon dioxide emissions in the transport sector by $60 \%$ by the year 2050 (European Commission, 2011). From now until 2050, the ambitious targets will require several measures, including the limitation of oil-derived fuels in favor of renewable fuels. In this field, Brazil is the best example in the world, because it had the farsightedness back in the 1970s to implement an extraordinary policy of developing bioethanol and of producing hydroelectric energy, and for a number of years has been working on a program of biodiesel production (Torres et al., 2013). Europe does not have the natural resources for a similar policy, so in the long term interest in using hydrogen might re-emerge, while in the short to medium term a significant development of electric cars is expected. In the current phase, there are many opportunities for using biomethane and biodiesel (Raboni e Urbini, 2014). In fact, all of the European production of biodiesel is already currently used as an additive for mineral diesel. What is really new is biomethane, the use of which in transport is particularly popular in Germany, the Netherlands, Finland, Austria, and especially in Sweden. In this country, several thousands of light-duty vehicles as well as several fleets of vehicles for public transport fueled by biomethane (or biomethane mixed with natural gas) are currently in circulation. In many Swedish towns, the use of biomethane for transport is encouraged with different types of incentives which create an excellent level of acceptance for this kind of biofuel. Sweden's ambition is to have a fossil fuel-free vehicle fleet by 2030 . 


\section{CONCLUSIONS}

Over time, the concept of "internationalization" has extended a great deal to a multitude of mankind's activities. For progress in the quality of the environment and in health, it is essential that we have internationalization, particularly in science and culture; that is, internationalization of thought, of ideas and of research, by bringing together the world's best minds. The main objective is to enhance excellence in individual countries by expanding and intensifying cooperation with the best researchers in other countries, fields and institutions. Mankind expects much from the development of international relations: progress in living conditions, greater fairness in the use and distribution of resources, better protection of the environment and public health, peace and widespread well-being. The greatest challenges of this century concern climate change, nutrition of the planet, the safety and sustainability of energy supplies, the fight against poverty and infectious diseases, as well as the construction of basic health and sanitation infrastructure. It is worth remembering that, for a large part of the world's population, the scarcity of water and of this infrastructure constitutes a grave danger to health and represents a limiting factor in socioeconomic development. The UN data is shocking: approximately $40 \%$ of the world's population suffers these shortages, and the trend is upward, to $65 \%$ by 2025 . In this situation, no fewer than $1,500,000$ children die every year because of contaminated water or water scarcity. A worldwide policy for water seems urgent, so that we may define fair access to resources by everyone, and prevent dangerous tensions and competition between nations, which already exist. However, it is necessary quickly also to start an extended education process, beginning in primary schools, to teach young students to respect nature as a universal value, in order to make them genuine "environmental stewards".

Science and technological innovation will play a decisive role in improving the quality of the environment and of health. The expected progress concerns, as a priority, actions to limit greenhouse gas emissions, which are responsible for the current climate changes, limiting emissions into the urban atmosphere of the contaminants that most affect health (PAHs, VOCs, fine particulates), controlling the release of emerging micro-pollutants (particularly medicines and abuse drugs) into surface waters and, last but not least, actions to fairly satisfy the water needs of the world's entire population in order to reverse the dangerous trend towards growing situations of water stress and scarcity.

\section{REFERENCES}

CHIAVOLA, A.; BACIOCCHI, R.; GAVASCI, R. Biological treatment of PAHscontaminated sediments in a Sequencing Batch Reactor. Journal of Hazardous Materials, v. 184, n. 1-3, 2010.

COPELli, S.; TORRETTA, V.; RABONI, M.; VIOTTI, P.; LUCIANO, A.; MANCINI, G. Et al. Improving biotreatment efficiency of hot waste air streams: experimental upgrade of a full plant. Chemical Engineering Transactions, v. 30, p. 49-54, 2012. http://dx.doi.org/10.3303/CET1230009.

EUROPEAN COMMISSION. White paper 2011 - Roadmap to a Single European Transport Area - Towards a competitive and resource efficient transport system. Report COM(2011) 144 final. Brussels, 28 Mar. 2011.

FARABEGOLI, G.; GAVASCI, R.; LOMBARDI, F.; ROMANI, F. Denitrification in tertiary filtration: Application of an up-flow filter. Journal of Environmental Science and Health - Part A Toxic/Hazardous Substances and Environmental Engineering, v. 38, n. 10, p. 2169-2177, 2003. http://dx.doi.org/10.1081/ESE-120023349 
MANCINI, G.; VIOTTI, P.; LUCIANO, A.; RABONI, M.; FINO, D. Full scale treatment of ASR wastes in a modified rotary kiln. Waste management, v. 33, p. 2670-2674, 2013. http://dx.doi.org/10.1016/j.wasman.2014.06.028.

RABONI, M.; TORRETTA, V.; URBINI, G. Influence of strong diurnal variations in sewage quality on the performance of biological denitrification in small community wastewater treatment plants (WWTPs). Sustainability, v. 5, n. 9, p. 3679-3689, $2013 \mathrm{a}$. http://dx.doi.org/10.3390/su5093679

RABONI, M.; TORRETTA, V.; VIOTTI, P.; URBINI, G. Experimental plant for the physical-chemical treatment of groundwater polluted by municipal solid waste (MSW) leachate, with ammonia recovery. Revista Ambiente e Agua, v. 8, n. 3, p. 22-32, 2013b. http://dx.doi.org/10.4136/ambi-agua. 1250

RABONI, M.; GAVASCI, R.; URBINI, G. UASB followed by sub-surface horizontal flow phytodepuration for the treatment of the sewage generated by a small rural community. Sustainability, v. 6, n. 10, p. 6998-7012, 2014a. http://dx.doi.org/10.3390/su6106998

RABONI, M.; TORRETTA, V.; VIOTTI, P.; URBINI, G. Pilot experimentation with complete mixing anoxic reactors to improve sewage denitrification in treatment plants in small communities. Sustainability, v. 6, n. 1, p. 112-122, $2014 \mathrm{~b}$. http://dx.doi.org/10.3390/su6010112

RABONI, M.; TORRETTA, V.; URBINI, G.; VIOTTI, P. Automotive shredded residue: a survey of the hazardous organic micro-pollutants spectrum in landfill biogas. Waste Management and Research, 2014c. Published online before print November 26, 2014. http://dx.doi.org/10.1177/0734242X14559300

RABONI, M.; URBINI, G. Production and use of biogas in Europe: a survey of current status and perspectives. Revista Ambiente e Água, v. 9, n. 2, Apr./Jun. 2014. http://dx.doi.org/10.4136/ambi-agua. 1324

RADA, E. C.; RAGAZZI, M.; IONESCU, G.; MERLER, G.; MOEDINGER, F.; RABONI, M. Municipal Solid Waste treatment by integrated solutions: Energy and environmental balances. Energy Procedia, v. 50, p. 1037-1044, 2014a.

RADA, E. C.; RABONI, M.; TORRETTA, V.; COPELLI, S.; RAGAZZI, M., CARUSON, P. Removal of benzene from oil refinery wastewater treatment plant exchausted gases with a multi-stage biofiltration pilot plant. Revista de Chimie, v. 65, n. 1, p. 68-70, 2014 b.

TCHOBANOGLOUS, G.; FRANKLIN, R. B.; STENSEL, H. D. Wastewater engineeringtreatment and reuse. $4^{\text {th }}$ Edition. New York: Mc Graw Hill, 2003.

TORRES, E. A.; CERQUEIRA, G. S.; FERRER, T. M.; QUINTELLA, C. M.; RABONI, M.; TORRETTA, V. Recovery of different waste vegetable oils for biodiesel production: a pilot experience in Bahia State, Brazil. Waste management, v. 33, p. 2670-2674, 2013. http://dx.doi.org/10.1016/j.wasman.2013.07.030.

TORRETTA, V.; URBINI, G.; RABONI, M.; COPELLI, S.; VIOTTI, P.; LUCIANO, A. Effect of powdered activated carbon to reduce fouling in membrane bioreactors: A sustainable solution. Case study. Sustainability, v. 5, n. 4, p. 1501-1509, 2013a. http://dx.doi.org/10.3390/su5041501 
TORRETTA, V.; RABONI, M.; COPELLI, S.; CARUSON, P. Application of multi-stage biofilter pilot plants to remove odor and VOCs from industrial activities air emissions. WIT Transactions on Ecology and the Environment, v. 176, p. 225-233, $2013 \mathrm{~b}$. http://dx.doi.org/10.2495/ESUS130191

TORRETTA, V.; RABONI, M.; COPELLI, S.; RADA, E. C.; RAGAZZI, M,; IONESCU, G. Application of strategies for particulate matter reduction in urban areas: an Italian case. UPB Scientific Bulletin, Series D: Mechanical Engineering, v. 75, n. 4, p. 221-228, $2013 \mathrm{c}$.

TORRETTA, V.; IONESCU, G.; RABONI, M.; MERLER, G. The mass and energy balance of an integrated solution for municipal solid waste treatment. WIT Transactions on

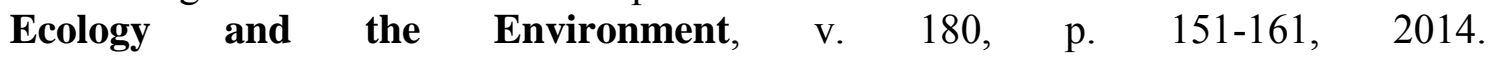
http://dx.doi.org/10.2495/WM140131

UNITED NATIONS WORLD WATER. Managing water under uncertainty and risk. Development Report 4, v. 1, 2012.

UNITED NATIONS HUMAN RIGHTS; UNITED NATIONS HABITAT; WORLD HEALTH ORGANIZATION. The Right to Water. Geneva, 2010. (Fact Sheet, 35)

UNITED NATIONS WATER. World Water Day 2013. Fact Sheet Sanitation. Geneva, 2013. 


Ambiente \& Água - An Interdisciplinary Journal of Applied Science
ISSN 1980-993X - doi:10.4136/1980-993X
www.ambi-agua.net
E-mail: ambi-agua@agro.unitau.br

\title{
A comprehensive analysis of the current and future role of biofuels for transport in the European Union (EU)
}

\author{
doi: 10.4136/ambi-agua.1492 \\ Received: 25 Aug. 2014; Accepted: 27 Nov. 2014

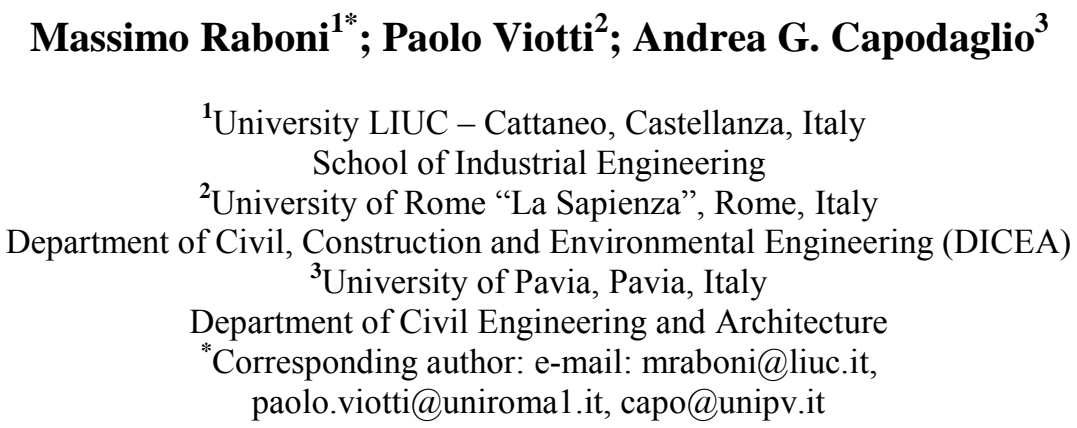

ABSTRACT

The production of biofuels is strongly supported all over the world as a renewable energy source for reducing dependence on the unstable oil market. Bioethanol, the main biofuel produced in the world, is widely used to power vehicles in both the USA and Brazil, but concerns exist in both places regarding its sustainability. In Brazil, it is produced from a by-product of the sugar cane industry, while in the USA it is manufactured from food crops. The production of biogas and biodiesel is growing rapidly, but neither has outpaced the production of bioethanol. The European Union (EU) is greatly interested in this issue, and in 2011 adopted an extensive strategy to reduce carbon dioxide emissions related to transport by $60 \%$ by the year 2050 . In order to achieve this result, the current European transportation system must be transformed. This ambitious goal will require the implementation of complex measures including the reduction of fossil fuels in favor of renewable fuels. This program has various options regarding the development of biofuels (e. g., biogas, bioethanol and biodiesel) and their related technologies, which are still on trial (mainly regarding the bioethanol production), and must also analyze their sustainability from a social and economic standpoint. The paper discusses the use of biofuels for transport in the European setting, and shows that their sustainability may result in relevant negative social effects due mainly to the use of land for energy crops (e.g., change of food price and world food shortage).

Keywords: biodiesel, bioethanol, European policy, sustainable mobility.

\section{Análise geral do papel atual e futuro dos biocombustíveis nos transportes na União Europeia (UE)}

\section{RESUMO}

A produção de biocombustíveis é fortemente reconhecida em todo o mundo como uma fonte de energia renovável para reduzir a dependência do mercado instável do petróleo. Bioetanol, o principal biocombustível produzido no mundo, é largamente utilizado para a 
mobilidade no Brasil, e também nos EUA, mas com diferenças de sustentabilidade, porque no Brasil ele é produzido a partir de um subproduto da indústria de cana-de-açúcar, enquanto nos EUA é fabricado com culturas alimentares. Biogás e biodiesel estão crescendo rapidamente, mesmo que em um nível muito menor do que o bioetanol. A União Europeia (UE) observa este assunto com grande interesse e, há dois anos, adoptou uma ampla estratégia para reduzir em $60 \%$ as emissões de dióxido de carbono no setor de transporte, até o ano 2050. A fim de alcançar esse resultado, será necessária a transformação do atual sistema europeu de transportes. A ambiciosa meta implicará em medidas complexas, incluindo a limitação do uso de combustíveis fósseis em favor de combustíveis renováveis. Este programa abre várias possibilidades de desenvolvimento dos biocombustíveis (ou seja, biogás, bioetanol e biodiesel). Para concretizar este avanço, é preciso se desenvolver tecnologias de nova geração em fase de experimentação (principalmente para a produção de bioetanol a partir de resíduos florestais), bem como a análise da sustentabilidade econômica e social desta nova fonte de combustível. Este artigo trata da utilização de biocombustíveis nos transportes no cenário europeu e mostra que a produção de biocombustíveis pode aumentar efeitos sociais negativos relevantes, principalmente ligados ao uso da terra para culturas energéticas em vez de alimentos com efeitos sobre o preço de mercado e da escassez mundial de alimentos.

Palavras-chave: biodiesel, bioetanol, políticas europeias, mobilidade sustentável.

\section{INTRODUCTION}

\subsection{The world policy toward biofuels}

The term "biofuels" includes the following products derived from biomasses or sub-derived products: biogas, biodiesel, bioethanol, bio-methanol, bio-ethers (Bio DME - DiMethylEhter; bio-ETBE - EthylTerButylEther; bio-MTBE - MethylTerButylEther), synthetic biofuels, bio-hydrogen and vegetable oils. Considering the level of production, market interests are primarily focused on the first three (Morosini et al., 2012; Raboni et al., 2013).

As renewable energy sources, biofuels have several advantages:

1. Significant benefits in reducing $\mathrm{CO}_{2}$, micro- and macro-pollutants (e.g., particulate matter, PM) and emissions (FAO, 2008; Torretta et al., 2013);

2. Helping countries to increase their energy security creating a more stable energy market;

3. Development of a new economy associated with both energy crop cultivation and biofuels production.

For these reasons many countries promoted programs in favour of biofuels. The consequence has been a rapid growth of biofuel production in the last decade (US-EIA, 2014), even though since 2010 a decline due to the global economic crisis has appreciably reduced the energy demand. Also, social sustainability of biofuels influenced the trend because land exploitation for energy crops instead of food production threatens food availability.

The transport sector is the main biofuels consumer. Figure 1 shows biofuels' share of overall fuel consumption for road transport (WEC, 2014; EurObserv'ER, 2014; US-EIA, 2014).

Biofuels represent $3.3 \%$ of the total fuel used in road transport. This amount of biofuel is produced by less than $3 \%$ of all cultivated land on earth $\left(4.7 \times 10^{5} \mathrm{~km}^{2}\right)$. Asia and Pacific area have low shares of biofuel (below 1\%), while good performances are demonstrated by Brazil, which stands out for its effective policy towards renewable energies. Since the early 70's, Brazil has had a vast program in place for ethanol production from sugar cane, primarily for 
motor vehicles. In 2013, 52\% of the Brazilian cars were equipped with "flex" engines fueled with gasoline, ethanol or mixtures of both (Sindipeças and Abipeças, 2014).

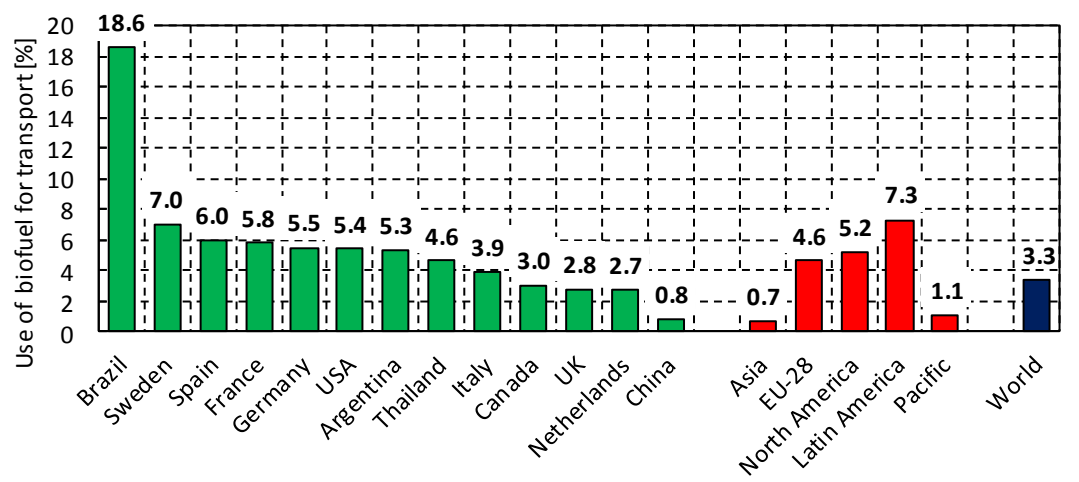

Figure 1. Share of biofuels for road transport (2011 data).

Brazilian energy policy has also included biodiesel. The production of biodiesel from vegetable oils (mainly from palm, castor and Jathropha curcas seeds) is actually under development, while great interest is focused on Used Cooking Oil - UCO (Torres et al., 2013).

Since 2000, the European Union (EU) has devoted great effort to promote biofuels, issuing several programmatic actions, such as (Raboni et al., 2013):

1. The "Green Paper" (European Commission, 2000), which introduced the target of replacing $20 \%$ of energy derived from fossil fuels with renewable energies by 2020 ;

2. The Directive 2003/30/EC (European Parliament, 2003), which stated targets for biofuels use: $2 \%$ by 2005 and $5.75 \%$ by 2010 ;

3. The package of proposals which established a common European energy policy (European Commission, 2007). It states that by 2020 biofuels will replace at least $10 \%$ of fossil fuels;

4. The Directive 2009/28/CE regarding the promotion of energy from renewable sources (European Parliament, 2009), which defines mandatory targets for the Member States in order to allow the sector to grow more efficiently. The Renewable Energy Directive (RED) aims at achieving 20\% of renewable energy consumption in the whole EU by 2020 . Concerning the transport sector, the share of renewable energy sources should be at least $10 \%$;

5. The "Transport 2050" plan (European Commission, 2011), aiming at creating a European common space able to improve the mobility of people and goods, as well as reducing $60 \%$ of $\mathrm{CO}_{2}$ emissions in transport by 2050. In order to achieve this target, the plan provides for a deep transformation of the current European transportation system through a complex plan based on the following main points:

5.1 Urban transport: halving the number of cars that relies upon traditional fuels (diesel and gasoline) in cities by 2030 and excluding them by 2050;

5.2 Intercity travels: by 2050 , the majority of passengers' mid-range transport (over $300 \mathrm{~km}$ ) will take place by train. Thirty percent and $50 \%$ of freight transport by road will switch to other methods of transport such as rail and inland waterways by 2030 and 2050, respectively; 
5.3 Long range trips and intercontinental freight transport: the use of low $\mathrm{CO}_{2}$ emission fuels in aviation sector will reach $40 \%$ of total fuel consumption by 2050. By the same date, $40 \%$ of the maritime navigation $\mathrm{CO}_{2}$ emission will be cut.

The EU strongly needs to reduce its oil dependence, which nowadays amounts to more

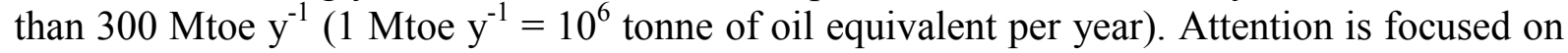
the transport system, traditionally based on oil and responsible for more than $21 \%$ of overall greenhouse gas (GHG) emissions, with an increasing trend higher than other economic sectors. Actually, the EU policy is fundamentally focused on biofuels. The EU estimated a $14 \%$ share of biofuels in 2020 , which could reduce GHGs emissions $(101 \div 103$ Mtonne $\left.\mathrm{CO}_{2} \mathrm{e}^{-1}\right)$.

The Directives 2001/77/EC (European Parliament, 2001) and 2003/30/EC established different national targets for renewable energy production and biofuel for transport consumption in order to reach a share of $21 \%$ and $5.75 \%$, respectively, by 2010 . Neither of the goals was reached (European Commission, 2011b). Therefore, in this decade the EU will work to double the production of renewable energies dedicated to transport in order to achieve the target of $10 \%$, as demonstrated by the member states' plans (Figure 2).

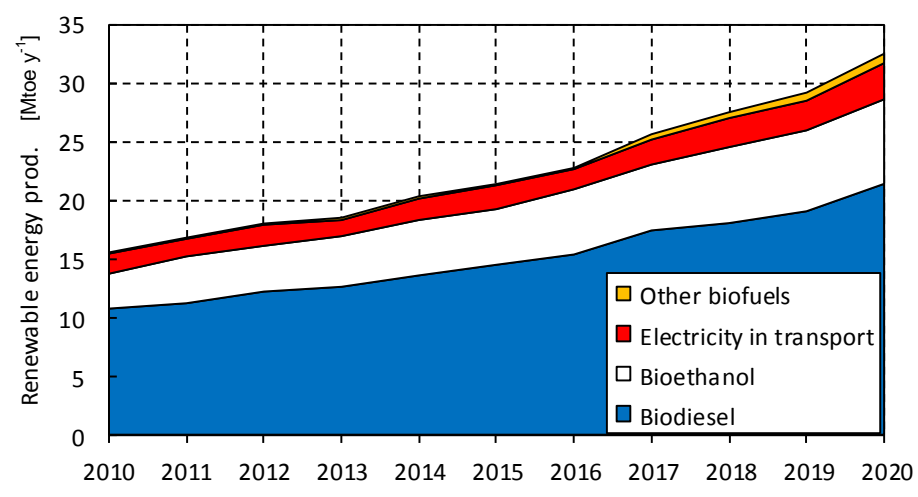

Figure 2. Growth of renewable energies for transport in the EU (EC, 2011b).

Biodiesel is and will be the main biofuel source ( $66 \%$ of the total renewable energy for transport in 2020), followed by bioethanol. The other biofuels (e.g. bio-hydrogen) have and will have almost no contribution. The EU will have to invest in the field of advanced technologies related to renewable energies in order to reach the 2020 targets both in terms of cost-effectiveness and environmental sustainability.

This paper analyzes the role of biofuel (biogas, biodiesel and bioethanol, in particular) in the EU transport sector, comparing the results with global production and considering the social and environmental sustainability of the policies.

\section{MATERIALS AND METHODS}

The research methodology was as follows:

1. Bibliographic research on the production and use of biogas, bioethanol and biodiesel in the European member states and comparison with world production;

2. Collection of documents concerning legislation and strategies issued by the EU and its member states aimed at encouraging and promoting biofuel production, conversion into energy and use in transport; 
3. Collection and analysis of technical and economic data regarding 285 European plants (biogas: 225; biodiesel: 55; bioethanol: 5);

4. Collection of documents regarding environmental sustainability of biofuels.

The results presented in this paper are a concise part of the more complex work produced by the research team.

\section{RESULTS AND DISCUSSION}

\subsection{Biofuels production}

\subsubsection{Biogas and biomethane}

Biogas production comes mainly from Municipal Solid Waste (MSW) landfills, sewage sludge anaerobic digestion, MSW Organic Fraction (MSWOF) and the agro-zootechnical waste biogasification plants (Urbini and Torretta, 2008; Martinez et al., 2014). Figure 3 shows global biogas production in 2012 and its trend till 2022.

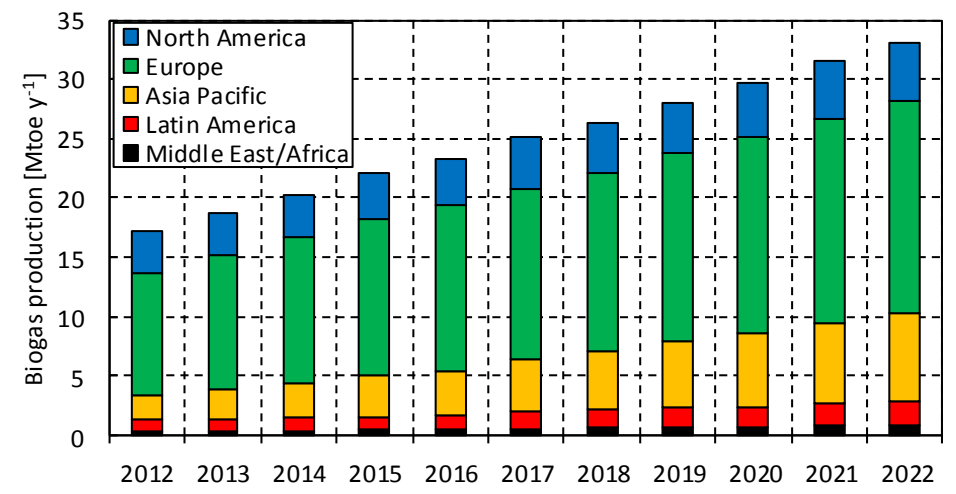

Figure 3. Global biogas production in 2012 and its trend to 2022 (Raboni and Urbini, 2014).

Europe is the major producing continent (about 12 Mtoe $\mathrm{y}^{-1}$ ): $53 \%$ is supplied by Germany. United Kingdom (1.8 $\mathrm{Mtoe}^{-1}$ ) is the second producer, followed by France $\left(1.2\right.$ Mtoe $\left.^{-1}\right)$ and Italy $\left(0.4\right.$ Mtoe $\left.^{-1}\right)$. Nevertheless, the biomethane contribution to European biofuels market is limited to $0.3 \%$. In Italy, France and even more in the UK, biogas production is strongly bound to old landfills. Germany proves to be the only country that promoted a confident and effective policy for the promotion of this renewable energy through the construction of numerous biogasification plants based on organic matrices (including energy crops). It should be noted that in 2012 this kind of biogas amounts to about $66 \%$ of all EU production. According to EU plans, the biogas production target in 2020 will raise to $15 \mathrm{Mtoe}^{-1}$, with a corresponding electricity production of $56,400 \mathrm{GWh} \mathrm{y}^{-1}$.

In the EU, the conversion of biogas to energy is devoted almost entirely to electricity production, while the use in vehicles is still very limited. In fact, in only a few cases the biogas produced is converted into pure methane (Biomethane with a $\mathrm{CH}_{4}$ content higher than 95\%) and used as biofuel. The greatest use as Biomethane for transport is a concern of Central and Northern European countries (Switzerland and Sweden, in particular). In Sweden, the new biodigesters fed by organic matrices are equipped with systems able to upgrade the biogas into Biomethane for vehicles and the natural gas network. In this country, 4,000 light-duty vehicles as well as several fleets of vehicles for public transport fed by Biomethane or natural gas are currently circulating. In many Swedish cities, the use of methane for transport is favored by different forms of incentives (e.g., free parking, detaxation of 
Biomethane purchase, toll exemption, dedicated lanes for Biomethane taxies, financial support for the purchase of Biomethane fed vehicles) which created an excellent level of acceptance for such a biofuel (Van Foreest, 2012).

In the EU, these kind of solutions are still rather isolated; even in Italy, the majority of the biogas is converted into electricity in cogeneration plants, by virtue of relevant incentives allowed by the Italian Financial Act 2008, known as "Green certificates" (GSE, 2014).

\subsubsection{Liquid biofuels (biodiesel and bioethanol)}

Due to the marginal role of biomethane, it is possible to assert that the world biofuels market is currently dominated by biodiesel and, above all, bioethanol. Figure 4 shows the world and EU production of biodiesel and bioethanol during the period 2000-2012 (EurObserv'ER, 2014; Ren21, 2014; US-EIA, 2014).
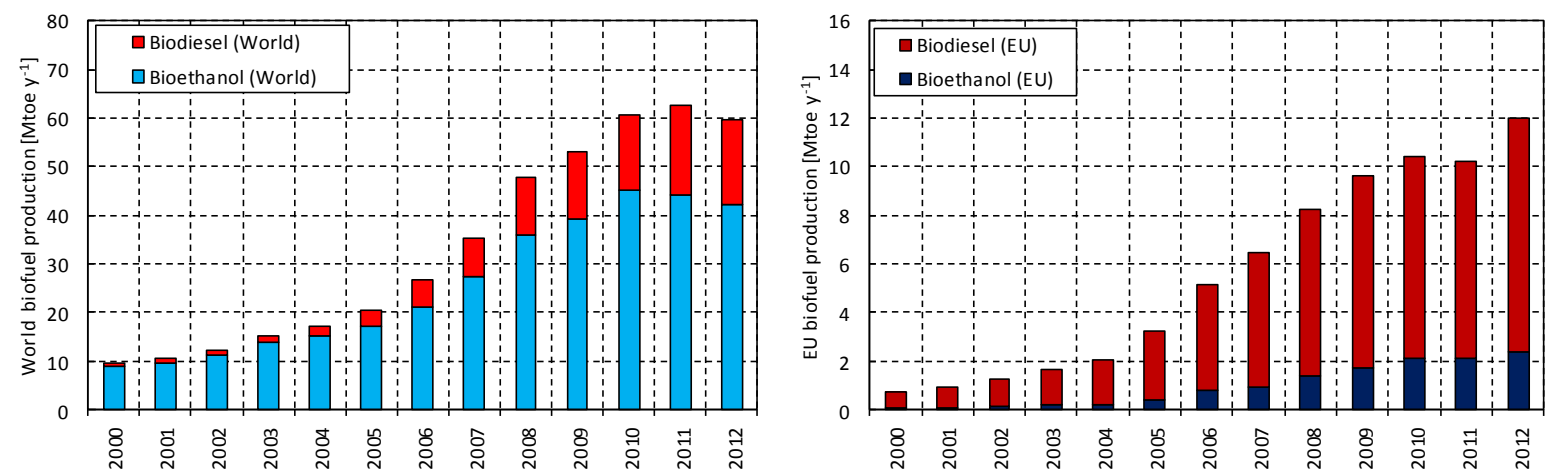

Figure 4. Biodiesel and bioethanol production trend in the World (left) and in the EU (right).

The 2012 global biodiesel and bioethanol production was about $60 \mathrm{Mtoe}^{-1}$; its growth in the last 9 years is notable even if in 2012 it suffered a slight decline (-4\%), mainly due to

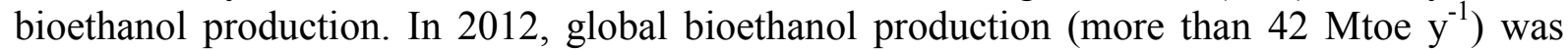
mainly concentrated in North and South America: Brazil and USA are responsible for about $90 \%$ of overall production, while the European share is around $6 \%$. Regarding biodiesel, the

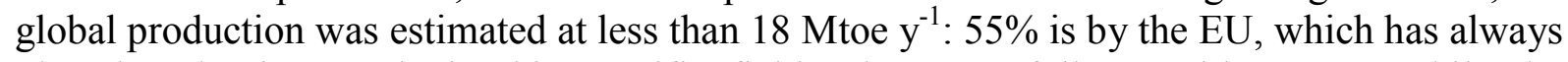
played a dominant role in this specific field. The USA follows with $16.7 \%$, while the remainder is distributed throughout several emerging countries (Brazil and Argentina, particularly). Table 1 lists detailed data concerning the most important European producers and consumers for transport (EurObserv'er, 2014).

Table 1. EU biofuel production and use for transport in $2012\left(\mathrm{ktoe}^{-1}\right)$.

\begin{tabular}{lccccc}
\hline Country & \multicolumn{2}{c}{ Bioethanol } & & \multicolumn{2}{c}{ Biodiesel } \\
\cline { 1 - 2 } \cline { 5 - 6 } & Production & $\begin{array}{c}\text { Use for } \\
\text { transport }\end{array}$ & & Production & $\begin{array}{c}\text { Use for } \\
\text { transport }\end{array}$ \\
\hline Germany & 387 & 403 & & 2,861 & 2,191 \\
France & 600 & 209 & & 1,910 & 2,268 \\
Spain & 191 & 101 & & 925 & 1,899 \\
Italy & 75 & 40 & & 706 & 1,264 \\
Poland & 106 & 77 & & 370 & 669 \\
Austria & 108 & 34 & & 289 & 390 \\
Other member states & 952 & 538 & & 2,509 & 2,980 \\
\hline Total & $\mathbf{2 , 4 1 8}$ & $\mathbf{1 , 4 0 1}$ & & $\mathbf{9 , 5 7 0}$ & $\mathbf{1 1 , 6 6 1}$ \\
\hline
\end{tabular}


EU bioethanol production was about $2.4 \mathrm{Mtoe}^{-1}$, with a remarkable contribution from France $(25 \%)$ and Germany $(16 \%)$ while imported bioethanol is less than 1 Mtoe $\mathrm{y}^{-1}$ (EurObserv'ER, 2014). The major biodiesel-producing countries are Germany (30\%), France $(20 \%)$ and Spain (10\%).

The reasons why Europe has favored biodiesel production in the past are both historical and economic. The most-used fuel in the EU is diesel. Moreover, the EU is also a net importer of mineral diesel and a gasoline exporter. Therefore the priority for the EU policy was the search for a valid substitute for mineral diesel. From the economic point of view, European bioethanol production relies on uncompetitive or unsuitable crops respective to the Brazilian one, which is based on sugar cane which has an energy productivity from four to seven times higher than wheat, corn and sugar beet. Moreover, Brazilian bioethanol production has great advantages in terms of sustainability because it is based mainly on molasses, the by-product of the sugar cane industry. Bioethanol offers a great support to the Brazilian program for energy sustainability, together with hydro-electricity. It is important to consider that a federal decree forbids the sale of gasoline unless it is mixed with $25 \%$ bioethanol (E25). The growing success of "flex" vehicles allowed bioethanol to overtake gasoline consumption in 2008 .

In 2012, the EU biofuels consumption reached 14.6 Mtoe $\mathrm{y}^{-1}$, including the modest contribution offered by biogas and straight vegetable oils, which are $0.9 \%$ of overall biofuels consumption. This value represents $4 \%$ of total energy consumption in the EU. Therefore, the $5.75 \%$ target for 2010, defined by Directive 2003/30/CE, still remains difficult to achieve.

Both biodiesel and bioethanol produced in the EU are almost completely used in blends with oil-derived fuels (gasoline and diesel); their development perspectives are sensitively bound with the increase of mixing quotas allowed in the different member states. In Germany, the overall incorporation rate (biodiesel and bioethanol) has been $6.25 \%$ since 2009. Italy and the UK are still far from the 2010 EU target (4.5\% and 3.9\%, respectively). France is the most progressive of all: $7.57 \%$ compared to $5.15 \%$ (the EU average target).

The European Commission, looking at 2020 target, believes that the real target for biofuels growth should be placed around 14\%. This means that the production of biofuels should increase to 43.1 Mtoe $^{-1}$ (27 Mtoe $\mathrm{y}^{-1}$ composed of biodiesel). This growth should be achieved respecting the principles of social and environmental sustainability.

\subsection{Biofuels social sustainability}

The social sustainability of biofuels production is strictly bound to the competition of arable lands for energy crops rather than food production. The EU acknowledges not having enough cropland to achieve the 2020 goal: the most realistic strategy takes into account the importation of $50 \%$ the amount needed from third countries (Commission of EC, 2007).

The analysis of data regarding the 2000-2010 food market showed that biofuel policies alone were responsible for the increase in the price of cereals and vegetable oils by $1-2 \%$ and $4 \%$, respectively (Ecofys et al., 2012). Other studies confirm the growth trend in the future (up to $5-7 \%$ and $15-16 \%$ in 2018 for wheat and vegetable oils, respectively). Such food-price growth will have a large impact mainly in low-income countries and could create social instability. Moreover, the need for large amounts of cropland to produce energy crops is increasing land-grabbing in developing countries, with negative socio-economic impacts on local communities (e.g. loss of access to land, water and other natural resources; Ecofys et al., 2012).

The EU is very sensible to such issues and reinforced RED with an agreement among its member states regarding the Indirect Land Use Change (ILUC). The new agreement will generate a clear and net GHGs saving without negatively impacting on biodiversity and land use because it strongly promotes the development and use of the $2^{\text {nd }}-3^{\text {rd }}$ generation biofuels, such as the bioethanol produced by lignocellulosic matrices and waste (Council of the EU, 
2014). In fact, the EU internal production of biofuels will come from (i) $7.6 \%$ of the total arable lands ( $2 \%$ in 2012) and (ii) new generation biofuel processes (7.5 Mtoe $\mathrm{y}^{-1}$ ). Pilot plants implementing such technologies are already working with mixtures of MSWOF and organic waste from orange industry (Valencia, Spain), straw (Salamanca, Spain), straw and ditch reed (Alessandria, Italy). Even in the USA, there are currently about ten plants running or under construction operating on various agricultural, forestry and MSW. Several other experiments are reported in Japan, Canada and other countries.

\subsection{Biofuels environmental sustainability}

Biofuels environmental sustainability can be examined considering several aspects. One of the most significant is net carbon emission, assessed by the means of the Life Cycle Analysis (LCA) technique. Biofuels aim to be carbon neutral, that is to say the carbon released during fuel use (e.g. combustion in power transport or electricity generation) is absorbed and balanced by new plant growth. Carbon neutral fuels do not increase atmospheric $\mathrm{CO}_{2}$ levels, helping to reduce the greenhouse effect. Actually, every biofuel has a net carbon emission considering the contribution of both the production (e.g. feedstock production; process technology) and the combustion processes. In the last years, a relevant improvement in biofuel production technologies decreased carbon emissions (UK-DfT, 2014a). Figure 5 shows the current biofuels carbon intensity statistics resulting from different feedstock and technology, compared to traditional fossil fuels (UK-DfT, 2014a).

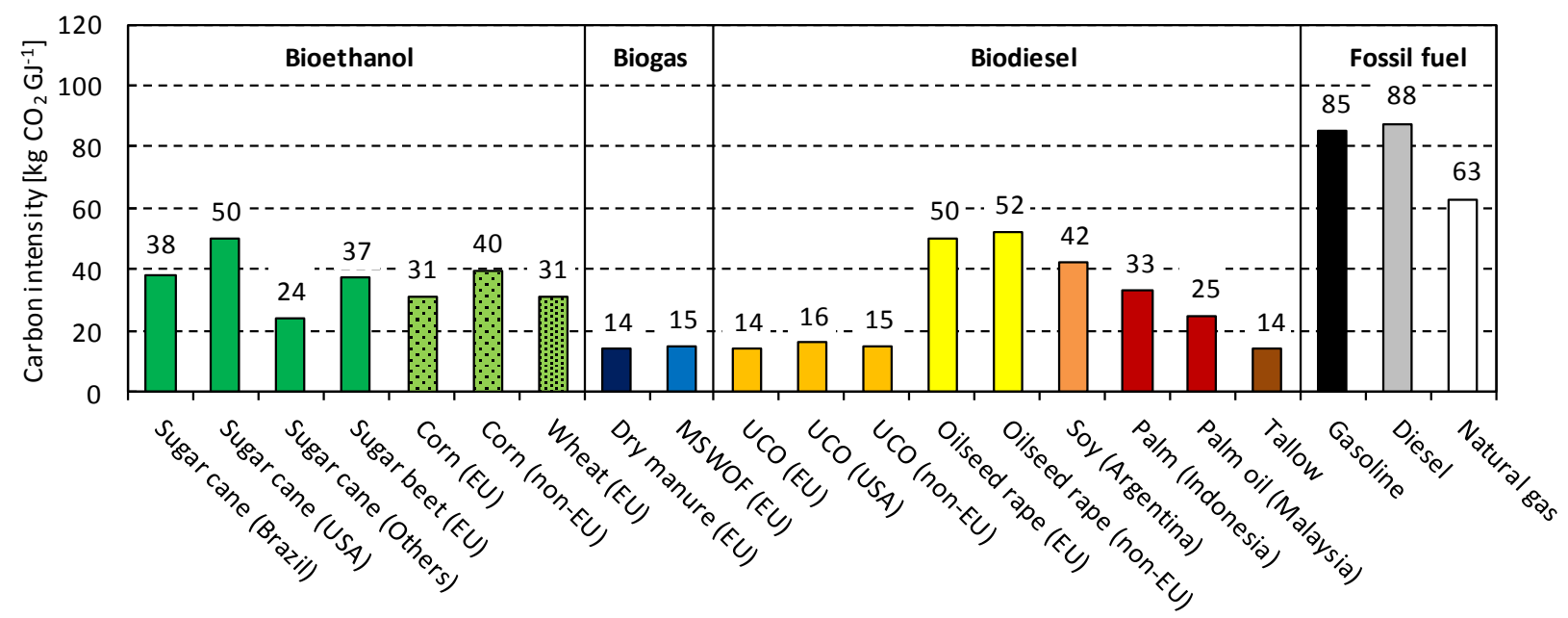

Figure 5. Carbon intensity of biofuels resulting from different feedstock and technology, compared to traditional fossil fuels.

Central American sugar cane, EU corn and wheat prove to be the most attractive source for the bioethanol production. On the contrary, it is noted that many other sources (e.g. USA sugar cane and corn; South Africa and Pakistan sugar cane; Ukraine wheat) do not allow substantial advantages respect to gasoline.

Considering biodiesel and biomethane, particularly interesting are the use of waste (e.g. UCO, tallow, MSWOF) and manure. On the other hand, biodiesel from oilseed rape and soy has a very high carbon intensity.

Another aspect of particular interest is represented by the emissions of pollutants derived from motor-vehicle combustion. Figure 6 shows the trend of emissions of truck fleets in relation to rising biodiesel-diesel blends. 


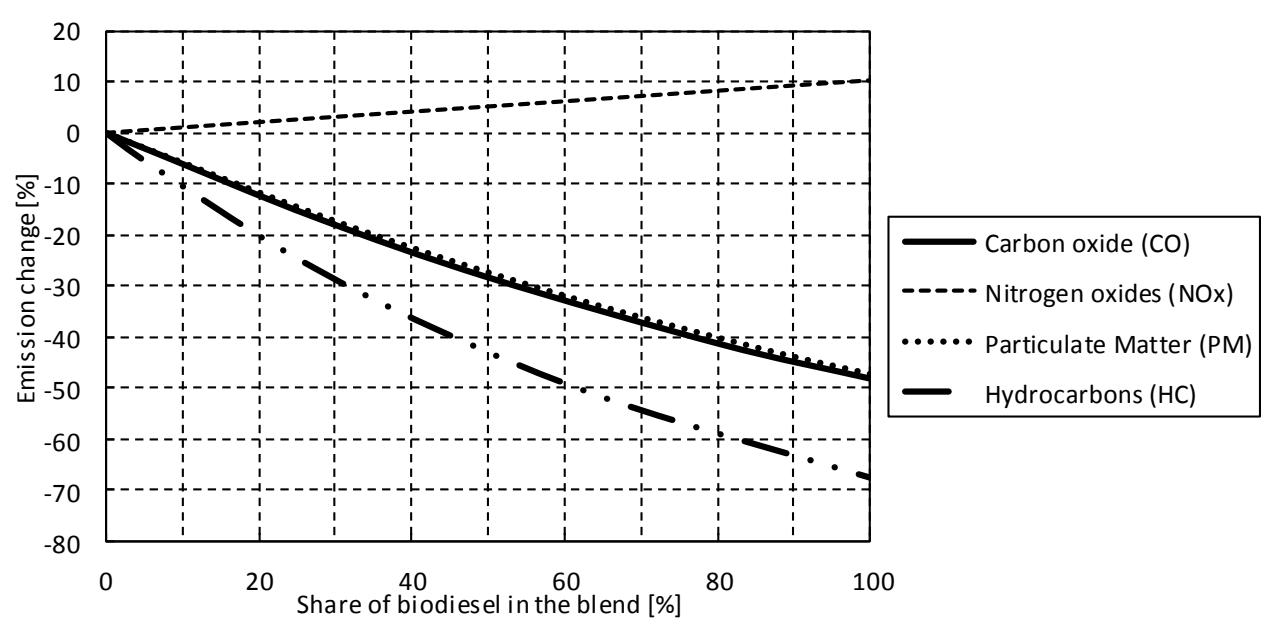

Figure 6. Macro-pollutants average emissions changes from the exhaust pipes of trucks fueled by rising rates of biodiesel (US-EPA, 2002).

The graph of Figure 6 clearly illustrates the following:

1. The complex of macro-pollutants emissions is reduced progressively moving from mineral diesel toward biodiesel blends, up to pure biodiesel (B100). PM-CO and hydrocarbon $(\mathrm{HC})$ reductions reach values of about $50 \%$ and $70 \%$, respectively;

2. A slight NOx increase occurs, up to values of about $10 \%$ for B100.

No substantial variations have been highlighted in relation to engine age or type. PM, HC and $\mathrm{CO}$ reductions are certainly correlated to the better combustion of the methyl-ester compared to traditional diesel due to both the presence of oxygen in the molecule and the high Cetane Number $(\mathrm{CN})$. The NOx increase $(5 \div 20 \%$ depending on the authors) has not been unequivocally justified by scientists: there is a tendency to correlate the phenomenon with (i) the presence of oxygen in the molecule and (ii) the combustion conditions (e.g.: pressure, temperature, low injection delay). Another hypothesis is the greater viscosity of biodiesel with respect to mineral diesel. In terms of macro-pollutant emissions, biodiesel benefits SOx reduction due to the near absence of sulfur, while significant concentrations of this compound characterize mineral diesel. A US-EPA study involving a total of 11 organic compounds (e.g. Acetaldehyde, Acrolein, Benzene, 1,3-Butadiene, Ethylbenzene, Formaldehyde, n-Hexane, Naphthalene, Styrene, Toluene, Xylene) confirms that pure biodiesel reduces macro-pollutant emissions by about 16\% (US-EPA, 2002).

Regarding bioethanol and gasoline, most of the experiments were carried out with ethanol/gasoline mixtures and the results are often contradictory. However, most significant data show a NOx, $\mathrm{CO}$ and $\mathrm{HC}$ reduction when the ethanol quota is increased. In an experiment in which 85\% bioethanol (E85) was compared with pure gasoline, emissions were reduced by $6 \%, 27 \%$ and $49 \%$ for NOx, CO and $\mathrm{HC}$, respectively (Avella, 2009).

An advantage stated for bioethanol is that the unburned emissions contribute significantly less to the formation of ozone, compared to organic compounds present in the gasoline exhaust emissions. In addition, the very low sulfur content of bioethanol produces virtually no sulfur dioxide $\left(\mathrm{SO}_{2}\right)$ emissions and improves the efficiency of catalytic emission control (UK-DfT, 2014b). However, while some of the most toxic pollutants (e.g. benzene, 1, 3-butadiene, toluene, xylene) decrease when using bioethanol, others (e.g. formaldehyde, acetaldehyde, peroxyacetyl nitrate) increase. For this aspect alone, there is a strong need for an in-depth human health and risk exposure assessment (Urbini and Stella, 2007; UK-DfT, 2014b). 


\section{CONCLUSIONS}

The biofuels future production and use in transport will be much diversified in different regions of the world. Countries which made important progress in the field of bioethanol (e.g. Brazil) have relevant margins for further growth because they have vast uncultivated areas and enjoy favourable climatic conditions for energy crops. Similar developments could affect other regions, including parts of Eastern Asia as well as Central and Southern Africa. Unfortunately, Europe does not enjoy the same favorable conditions. Nevertheless, the EU demonstrated a high sensibility to health and environmental protection upon issuing the "Transport 2050" plan, which aims at very ambitious targets in terms of sustainable mobility to be achieved by 2050. Consequently, biofuels (biodiesel, bioethanol and its derivatives, in particular) will play a significant role in energy policy. The contribution of biomethane will continue to be very marginal, except in few localities (e.g. Sweden).

The estimated growth of biodiesel and the bioethanol will present two fundamental issues:

1. The limited willingness of the EU to produce bioethanol for transport;

2. The environmental and, moreover, social sustainability due to land use for energy crops rather than food.

In order to overcome these obstacles, it will be necessary to import significant amounts of bioethanol and vegetable oils suitable for biodiesel production. In addition, the EU plans to produce bioethanol from lignocellulosic residues. Only the results of the current experiments in this field will confirm the effectiveness and the economic convenience of such processes.

In order to achieve the biofuels growth targets, it is expected that incentives through concrete legislative support (e.g. adoption of incentives such as tax exemption; increase of blending quote with fossil fuels; benefits for production and purchase of dedicated/hybrid vehicles; facilitation in road circulation and parking) will be necessary. Therefore, in the near future biofuels could play a significant role in transportation and could effectively contribute, together with electric vehicles which are already increasing in medium to large cities, to the achievement of the EU "Transport 2050" plan targets, with a hopeful eye toward the promising development of hydrogen cars.

\section{ACKNOWLEDGEMENTS}

The results presented are part of: (i) a two-year research program funded by Regione Lombardia (Italy) concerning "Biodiesel project for the sustainable development - Production of energy and economic self-sufficiency of a small community - Bahia State, Brazil " carried out in collaboration with the Laboratory of energy and gas of UFBA - Federal University of Bahia, Brazil and FEP - Foundation of Polytechnic School of UFBA; (ii) a two-year research program funded by the Italian Government in the framework of the PRIN projects (Research Programme of Projects with a National Relevant Interest) concerning the status of biogas production and use in the European countries.

\section{REFERENCES}

AVELLA, F. Bioethanol as fuel for transport - Behaviour in engines and influence on pollutant emissions (in Italian). Technical report 200904183. Combustibles Experimental Station. Milan: [s.n.], 2009. 
COMMISSION OF THE EUROPEAN COMMUNITIES. Biofuels progress report - Report on the progress made in the use of biofuels and other renewable fuels in the Member States of the European Union. Brussels, 2007.

COUNCIL OF THE EUROPEAN UNION. Proposal on indirect land-use change: Council reaches agreement. Luxembourg, $13^{\text {th }}$ June 2014.

ECOFYS; FRAUNHOFER INSTITUTE; BECKER BÜTTNER HELD - BBH; ENERGY ECONOMICS GROUP - EEG; WINROCK INTERNATIONAL. Renewable energy progress and biofuels sustainability. Report for the EC ENER/Ci/463-2011-Lot2. Utrecht, The Netherlands, 2012.

EUROBSERV'ER. l'Observatorie des energies renouvelables database. Available in: http://www.eurobserv-er.org/. Access in August 2014.

EUROPEAN COMMISSION. A European strategy for sustainable, competitive and secure energy. Green paper of the European Commission. Brussels, Belgium, 2000.

EUROPEAN COMMISSION. An energy policy for Europe. Brussels, Belgium, 2007.

EUROPEAN COMMISSION. White Paper 2011 - Roadmap to a Single European Transport Area - Towards a competitive and resource efficient transport system. Brussels, Belgium, 2011a.

EUROPEAN COMMISSION. Recent progress in developing renewable energy sources and technical evaluation of the use of biofuels and other renewable fuels in transport in accordance with Article 3 of Directive 2001/77/EC and Article 4(2) of Directive 2003/30/EC. Brussels: Commission Staff Working Document, 2011b.

EUROPEAN PARLIAMENT. Directive 2001/77/EC on the promotion of electricity produced from renewable energy sources in the internal electricity market. Brussels, 2001.

EUROPEAN PARLIAMENT. Directive 2003/30/EC of the European Parliament and of the Council of 8 May 2003 on the promotion of the use of biofuels or other renewable fuels for transport. Brussels, 2003.

EUROPEAN PARLIAMENT. Directive 2009/28/EC on the promotion of the use of energy from renewable sources and amending and subsequently repealing Directives 2001/77/EC and 2003/30/EC. Brussels, 2009.

FOOD AND AGRICULTURE ORGANIZATION (FAO). The state of food and agriculture. Rome, 2008. 128 p.

GESTORE SERVIZI ENERGETICI - GSE. Green Certificates (GCs). Available in: www.gse.it/en/qualificationandcertificates/GreenCertificates/Pages/default.aspx. Access in August 2014.

MARTINEZ, S.; TORRETTA, V.; MINGUELA, J.; SIÑERIZ, F.; RABONI, M.; COPELLI, S. et al. Treatment of slaughterhouse wastewaters using anaerobic filters. Environ. Technol., v. 35, n. 3, p. 322-332, 2014. http://dx.doi.org/10.1080/09593330.2013.827729 
MOROSINI, C.; STELLA, S.; URBINI, G. Biofuels for the sustainable mobility: current and future role in Italy and in the EU. In: INTERNATIONAL SYMPOSIUM OF SANITARY AND ENVIRONMENTAL ENGINEERING SIDISA, 9., 26-29 June 2012, Milan, Italy. Proceedings... Milan, 2012.

RABONI, M.; TORRETTA, V.; URBINI, G. The future of biofuels for a sustainable mobility. In: WORLD SUSTAIN. FORUM, 3.; SCIFORUM ELECTRONIC CONFERENCE SERIES, 3., 1-30 November, 2013. Proceedings... http://dx.doi.org/doi:10.3390/wsf3-f009

RABONI, M.; URBINI, G. Production and use of biogas in Europe: a survey of current status and perspectives. Rev. Ambient. Água, Taubaté, v. 9, n. 2, p. 191-202, 2014. http://dx.doi.org/10.4136/ambi-agua. 1324

REN21 STEERING COMMITTEE. Renewables 2014 Global Status Report. Paris, 2014.

SINDICATO NACIONAL DAS INDUSTRIA DE COMPONENTES PARA VEÍCULOS AUTOOMOTORES - SINDIPEÇAS; ASSOCIAÇÃO BRASILEIRA DAS INDÚSTRIAS DE AUTOPEÇAS - ABIPEÇAS. Relatório da frota circulante de 2014. São Paulo, Available at:

http://automotivebusiness.anankecdn.net.br/pdf/pdf_171.pdf.

TORRES, E. A.; CERQUEIRA, G. S.; FERRER, T. M., QUINTELLA, C. M.; RABONI, M.; TORRETTA, V. et al. Recovery of different waste vegetable oils for biodiesel production: A pilot experience in Bahia State, Brazil. Waste Management, v. 33, n. 12, p. 2670-2674, 2013. http://dx.doi.org/10.1016/j.wasman.2013.07.030.

TORRETTA, V.; RABONI, M.; COPELLI, S.; RADA, E.C.; RAGAZZI, M.; IONESCU, G. et al. Application of strategies for particulate matter reduction in urban areas: an Italian case. UPB Sci. Bull. Series D, v. 75, n. 4, p. 221-228, 2013.

UNITED KIGDOM. Department for Transport (UK-DfT). Biofuel statistics. London, 2014a. Available in: https://www.gov.uk/government/collections/biofuels-statistics. Access in August 2014.

UNITED KIGDOM. Department for Transport (UK-DfT). Renewable Transport Fuels Obligation (RTFO) guidance. London, 2014b.

URBINI, G.; STELLA, S. Development perspectives for biodiesel: new EU strategies, technological trends, environmental effects (in Italian). Ingegneria Ambientale, v. 10/11, 2007.

URBINI, G.; TORRETTA, V. Anaerobic digestion and biogas recovery from MSWOF and other organic matrices (in Italian). PRIN-2006 final report. [S.1.]: [s.n.], November 2008.

UNITED STATES. Energy Information Administration (US-EIA). International energy statistics database. Available in: http://www.eia.gov/countries/data.cfm. Access in August 2014.

UNITED STATES. Environmental Protection Agency (US-EPA). A Comprehensive analysis of Biodiesel impacts on exhaust emissions. Report EPA420-P-02-001, 2002. Washington, DC, 2002.

VAN FOREEST, F. Perspectives for biogas in Europe. Report NG 70. Oxford: The Oxford Institute for Energy Studies, 2012. 54 p. 
WORLD ENERGY COUNCIL. ENERDATA. Energy efficiency indicators database. Available in: http://www.wec-indicators.enerdata.eu/. Access in August 2014. 


Ambiente \& Água - An Interdisciplinary Journal of Applied Science
ISSN 1980-993X - doi:10.4136/1980-993X
www.ambi-agua.net
E-mail: ambi-agua@agro.unitau.br

\title{
Multi-criteria analysis for site selection for the reuse of reclaimed water and biosolids
}

\author{
doi: 10.4136/ambi-agua.1550
}

Received: 23 Oct. 2014; Accepted: 19 Nov. 2014

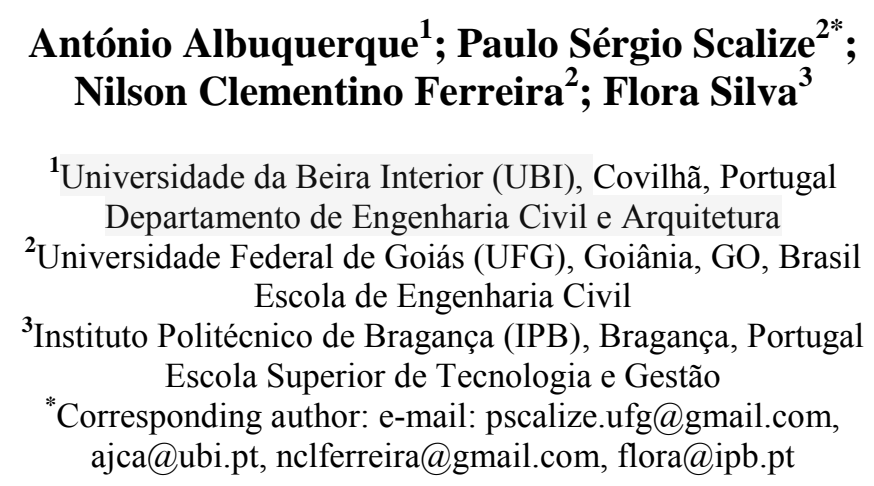

ABSTRACT

Low $\mathrm{pH}$ soils with insufficient organic matter can benefit from the application of reclaimed water (RW) and biosolids. The presence of nutrients also aids plant growth. This paper presents the results of two integrated research studies, both carried out in the Beira Interior Region (Covilhã, Portugal); one used RW for irrigation, the other applied paper mill sludge to agricultural land. In both cases, multiple criteria based on GIS tools were used for site selection. In the first study, the characteristics of RW analyzed over 2 years were found suitable for crop irrigation. The RW had moderate organic content, low electrical conductivity (CE), high nutrient content $(\mathrm{N}, \mathrm{P})$, and low concentrations of nitrate, metals and phytotoxic elements (Al, B, Cl and $\mathrm{Na}$ ). The multi-criteria analysis was carried out taking into account environmental, technical and economic criteria and a suitable area of 30.5 ha was found for RW irrigation. In the second work, the paper mill sludge was considered suitable for application to agricultural land. Its concentrations of $\mathrm{N}, \mathrm{P}$ and heavy metals did not a present risk for soil contamination and were suitable for soil improvement and crop production. A multi-criteria analysis based on similar criteria was conducted and a suitable area of 253 ha was found for sludge application.

Keywords: reutilization, spatial analysis, topographic location.

\section{Análise multicritério para a seleção de locais para o reuso de águas residuais tratadas e biosólidos}

\section{RESUMO}

Os solos com baixo teor em matéria orgânica e com baixo $\mathrm{pH}$ podem se beneficiar da aplicação da água residual tratada e de biosólidos. A presença de nutrientes é também útil para o crescimento de plantas. Este artigo apresenta os resultados de dois trabalhos de pesquisa, ambos realizados na região da Beira Interior (Covilhã, Portugal), um com água 
residuária tratada (RW) utilizada para irrigação agrícola, o outro com lodo resultante do tratamento de efluentes da pasta de papel para aplicação em solo agrícolas. Em ambos os casos, uma análise multicritério com utilização de SIG foi utilizada para localização de sítios mais adequados para a sua aplicação. No primeiro trabalho, as características de uma RW analisada durante 2 anos foram consideradas adequadas para a irrigação de culturas, uma vez que apresentavam teores de matéria orgânica moderada, baixa condutividade elétrica (CE), alto teor de nutrientes ( $\mathrm{N}$ e $\mathrm{P}$ ) e baixas concentrações de nitrato, metais e elementos fitotóxicos (Al, B, Cl e Na). A análise multicritério foi realizada levando-se em consideração critérios ambientais, técnicos e econômicos, e resultou na seleção de uma área de 30,5 ha para irrigação. No segundo trabalho, o lodo foi considerado adequado para aplicação em solo agrícola, uma vez que seu teor de $\mathrm{N}, \mathrm{P}$ e metais pesados não apresentavam risco de contaminação do solo e foram adequados para a melhoria da sua qualidade e da produção agrícola. A análise multicritério com base em critérios semelhantes permitiu selecionar uma área de 253 ha para a aplicação do lodo.

Palavras-chave: Análise espacial, localização topográfica, reaproveitamento.

\section{INTRODUCTION}

In regions with large population growth, the demand for water for human, industrial and agricultural activities is expected to grow, and the imbalance between available water supply and demand is expected to increase, especially in regions with initial deficits of water. Due to growing water stress, it is necessary to look at efficient and alternative uses of water resources in order to narrow the gap between supply and demand. On the other hand, industrialization also requires wastewater treatment and the consequent production of sludge (biosolids), the treatment and disposal of which is costly for users. Therefore, the integrated reuse of treated wastewaters (reclaimed water) and biosolids seems an attractive option for agricultural activities. It allows for the control of the pollution potential of these residues and for their application to irrigation, aquifer recharge, land recovery, crop production and urban uses.

Both reclaimed water (RW) and biosolids include organic matter and nutrients that can be useful for soil conservation or crop production. The organic matter aids in the recovery of degraded or eroded soils, while the presence of nutrients makes the residue suitable as fertilizer (USEPA, 2012). Since they normally have a $\mathrm{pH}$ over 7, they can also be useful for the correction of $\mathrm{pH}$ in acid soils. However, they can also include harmful compounds (e.g. heavy metals, PCBs and PAHs), dissolved salts (e.g. Al, B, $\mathrm{Cl}$ and $\mathrm{Na}$ ) that can cause soil salinity, and pathogens. Therefore, the quality of both residues should be properly controlled before being applied to agricultural lands, which may cause additional operational costs for users.

RW results from wastewater treatment and is subject to strict regulations prior to discharge into streams or soils. The residuals present in RW (namely organic matter, N, P and other micronutrients) may be useful for soil correction and crop irrigation. However, the presence of pathogens may require a polishing treatment as observed in Marecos do Monte and Albuquerque $(2010 \mathrm{a}, \mathrm{b})$. The use of RW for irrigation is recognized internationally as a practice for integrated water resource management (Asano et al., 2007; Locussol et al., 2009; UNESCO, 2012; Jimenez et al., 2010). Brazilian and European legislation (EP, 1991; Brasil, 2005) has pointed out the need for increasing the use of RW in agricultural activities.

Paper mill sludge compost (PMSC) is produced during the treatment of wastewater from the paper industry. In the last decades, PMSC has been mainly burned or disposed of at landfills (Glenn, 1998). PMSC is high in cellulose and has suitable organic matter and nutrients (e.g. Ca, K, N, Na and $\mathrm{P}$ ) for poor soil recovery and crop production (Foley and Cooperband, 2002; Jordan and Rodriguez, 2004; Camberato et al., 2006). The content of toxic 
elements is considered low (Phillips et al., 1997) and its organic matter, carbonate and silicate can even reduce harmful mobile metals in soils since they can be adsorbed into the soil particle surface (Battaglia et al., 2007). Current waste management strategies developed by international institutions such as the World Bank, the United Nations and the European Union point out the need of increasing the reuse of sludge (EP, 2008; EC, 2012; Hoornweg and Bhada-Tata, 2012). This practice, besides improving soil fertility and waste reuse, also reduces treatment and disposal costs. Brazilian and European legislation classifies the biosolids as solid waste that is not inert (EC, 2000; ABNT, 2004), suggesting that such wastes should be reused or valorised rather than disposed of (Brasil, 2010; EC, 2012). In Brazil, there are studies of WWTP irrigation agriculture in different cultures. Dantas et al. (2014), using WWTP of the city of São Cristóvão-SE to irrigate radishes, found that the resulting radishes were within the standards established by law. Deon et al. (2010) and Freitas et al. (2013) showed gains in productivity of cane sugar with drip irrigation of effluent. Varallo et al. (2010), working with red-yellow latosol reported that the use of reclaimed water for agronomic purposes should be applied in a rational manner, especially by monitoring the elevation of sodium.

Therefore, the integrated reuse of RW and PMSC in soils can increase its organic matter and nutrient content, improve soil's physical properties (e.g. lower bulk density, greater soil aggregation and water holding capacity), and increase its $\mathrm{pH}$ (Camberato et al., 2006; Asano et al., 2007), and may therefore be viewed as suitable soil amendments and plant nutrient sources.

Due to the large amount of complex information that must be accessed, integrated and analyzed (e.g. characteristics of the RW and biosolids, soil characteristics, type of crop production, water demand and nutrients for crop production, land use, environmental and legal restrictions, accessibility) geographical information systems (GIS) multicriteria analysis can be useful for data georeferencing, manipulation, conversion, analysis and map construction (Gemitzia et al., 2007; Kallali et al., 2007).

This paper presents the results of the application of GIS-based multicriteria for site selection of suitable areas for the application of RW and biosolids for agriculture.

\section{MATERIAL AND METHODS}

\subsection{Characterization of the reclaimed water and the area for irrigation}

The first study was conducted in a peri-urban region of Covilhã (Portugal) near an agricultural area with small, but with well-defined agricultural parcels $\left(40^{\circ} 15^{\prime} 00^{\prime \prime} \mathrm{N}, 72^{\circ} 29^{\prime} 00^{\prime \prime} \mathrm{W}\right)$. The RW produced at the local wastewater treatment plant (an infrastructure sized for the equivalent of 4000 inhabitants, consisting of bar racks, grit chambers, a primary settling tank and biological aerated filters) was monitored between January and December of 2007 including the daily flow-rate measurement and the collection of bi-monthly samples (22 samples in total) of the raw influent and the RW. The samples were analyzed to evaluate the following parameters: $\mathrm{pH}$, temperature, electrical conductivity (EC), biochemical oxygen demand $\left(\mathrm{BOD}_{5}\right)$, chemical oxygen demand (COD), total suspended solids (TSS), total nitrogen $(\mathrm{TN})$, ammonia $\left(\mathrm{NH}_{4}\right)$, nitrite $\left(\mathrm{NO}_{2}\right)$, nitrate $\left(\mathrm{NO}_{3}\right)$, total phosphorus $(\mathrm{TP})$, sodium $(\mathrm{Na})$, magnesium $(\mathrm{Mg})$, calcium $(\mathrm{Ca})$, potassium $(\mathrm{K})$, chloride $(\mathrm{Cl})$, boron $(\mathrm{B})$, arsenic $(\mathrm{As})$, cadmium $(\mathrm{Cd})$, chromium $(\mathrm{Cr})$, copper $(\mathrm{Cu})$, mercury $(\mathrm{Hg})$, nickel $(\mathrm{Ni})$, lead $(\mathrm{Pb})$, zinc $(\mathrm{Zn})$, total coliforms $(\mathrm{TC})$, faecal coliforms $(\mathrm{FC})$, E. coli and helminth eggs (HE). All of the analyses were conducted using the standard methods presented in APHA et al. (1999).

An area of 410 ha of agricultural parcels were demarcated near the village of Boidobra (Covilhã, Portugal) as shown in Figure 1, using extracts of Military Map No. 235 
$(1 / 25,000$ scale), altimetry data $(1 / 25,000$ scale) and orthophotomaps (photogrammetric flight of 2004, 1/5,000 scale) and other local georeferenced elements (e.g. location of the WWTP and the agricultural parcels).

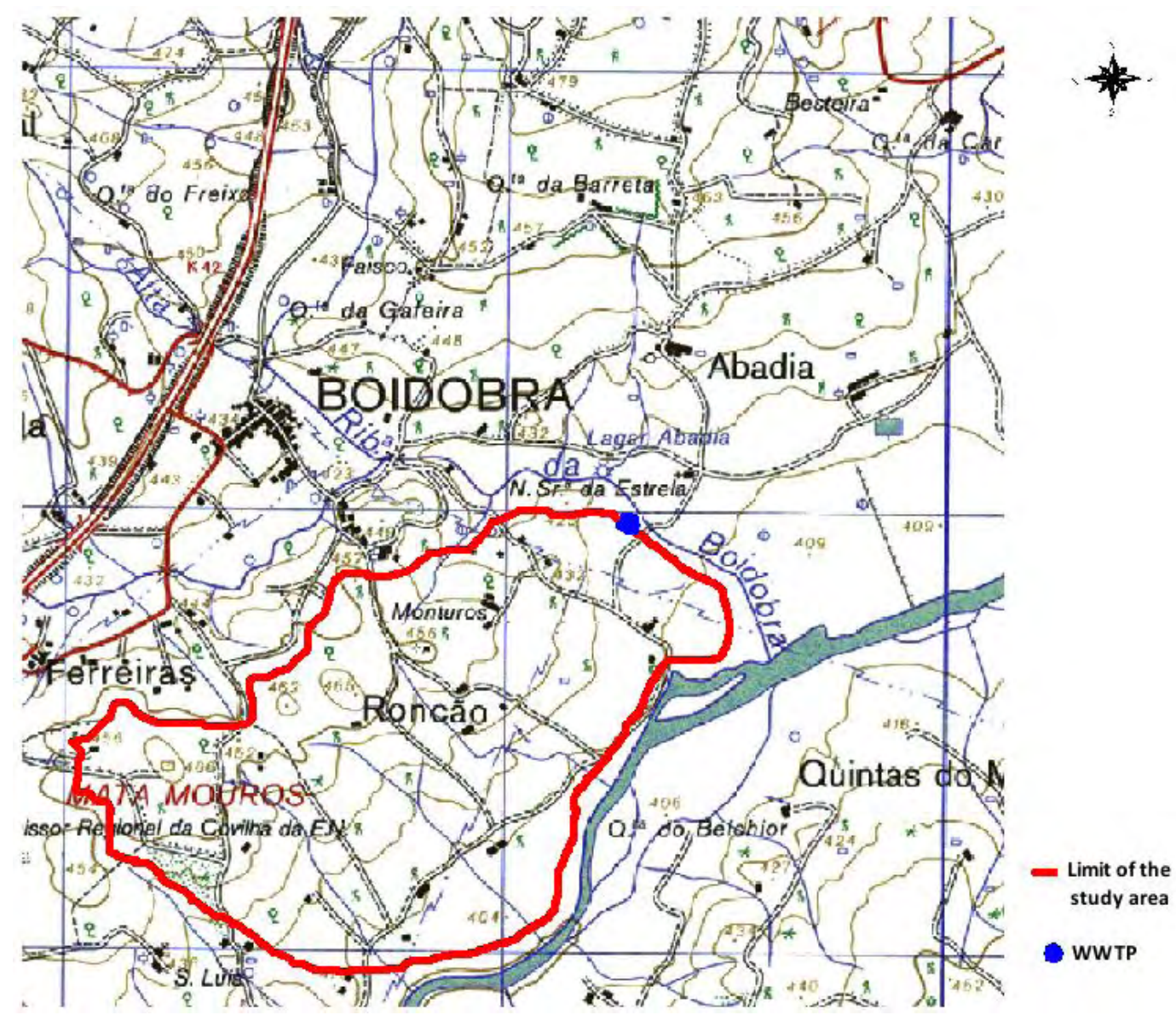

Figure 1. Selected area for reclaimed water irrigation in Covilhã, Portugal $\left(40^{\circ} 15^{\prime} 00^{\prime \prime} \mathrm{N}, 72^{\circ} 29^{\prime} 00^{\prime \prime} \mathrm{W}\right)$.

The selection of the agricultural parcels for irrigation was based on environmental, technical and economic criteria according to the suggestions of Asano et al. (2007), Marecos do Monte and Albuquerque (2010b) and USEPA (2012) as follows:

a) Environmental criteria

- Land use (the Corine Land Cover map was used to evaluate the potential land use of the studied area);

- A safety distance of $50 \mathrm{~m}$ from natural water resources, $100 \mathrm{~m}$ from water supply sources and water reservoirs used for human consumption; and $200 \mathrm{~m}$ from urban residential areas.

b) Technical criteria

- Annually available RW volume and its physical, chemical and microbiological characteristics;

- Water demand for crop production in the agricultural parcels;

- Nutrient demand for crop production;

- Tolerance of crops to toxicity and salinity;

- Slopes (irrigation should be preferably applied to areas with slope up to $12 \%$, since higher slope increases runoff, soil erosion and thus soil instability, which risks basin safety and increases refilling costs); 
- Groundwater table (aquifers should be sufficiently deep and transmissive to prevent excessive rises of the groundwater table due to infiltration; the minimum static level was setup in $5 \mathrm{~m}$ in order to prevent groundwater contamination).

c) Economic criteria

- A maximum distance of $4 \mathrm{~km}$ from the RW production point was defined as economically viable for reuse (this criterion included water transfer costs from the WWTP to the agricultural parcels).

\subsection{Characterization of the paper mill sludge and the area for application}

The second work was developed in a larger agricultural area close to the first study $\left(40^{\circ} 15^{\prime} 00^{\prime \prime} \mathrm{N}, 72^{\circ} 29^{\prime} 00^{\prime \prime} \mathrm{W}\right)$ and within an area covered by an irrigation plan, using paper mill sludge produced at a paper industry WWTP (Figure 2), which produces around 17 ton day $^{-1}$ of sludge. The data were collected from a sludge characterization report supplied by the industry, which included information on the following parameters: $\mathrm{pH}$, organic matter content, TN, $\mathrm{NH}_{4}-\mathrm{N}, \mathrm{TP}, \mathrm{Cd}, \mathrm{Cr}, \mathrm{Cu}, \mathrm{Ni}, \mathrm{Pb}$ and $\mathrm{Zn}$. All the analyses were conducted using standard methods presented in APHA et al. (1999).

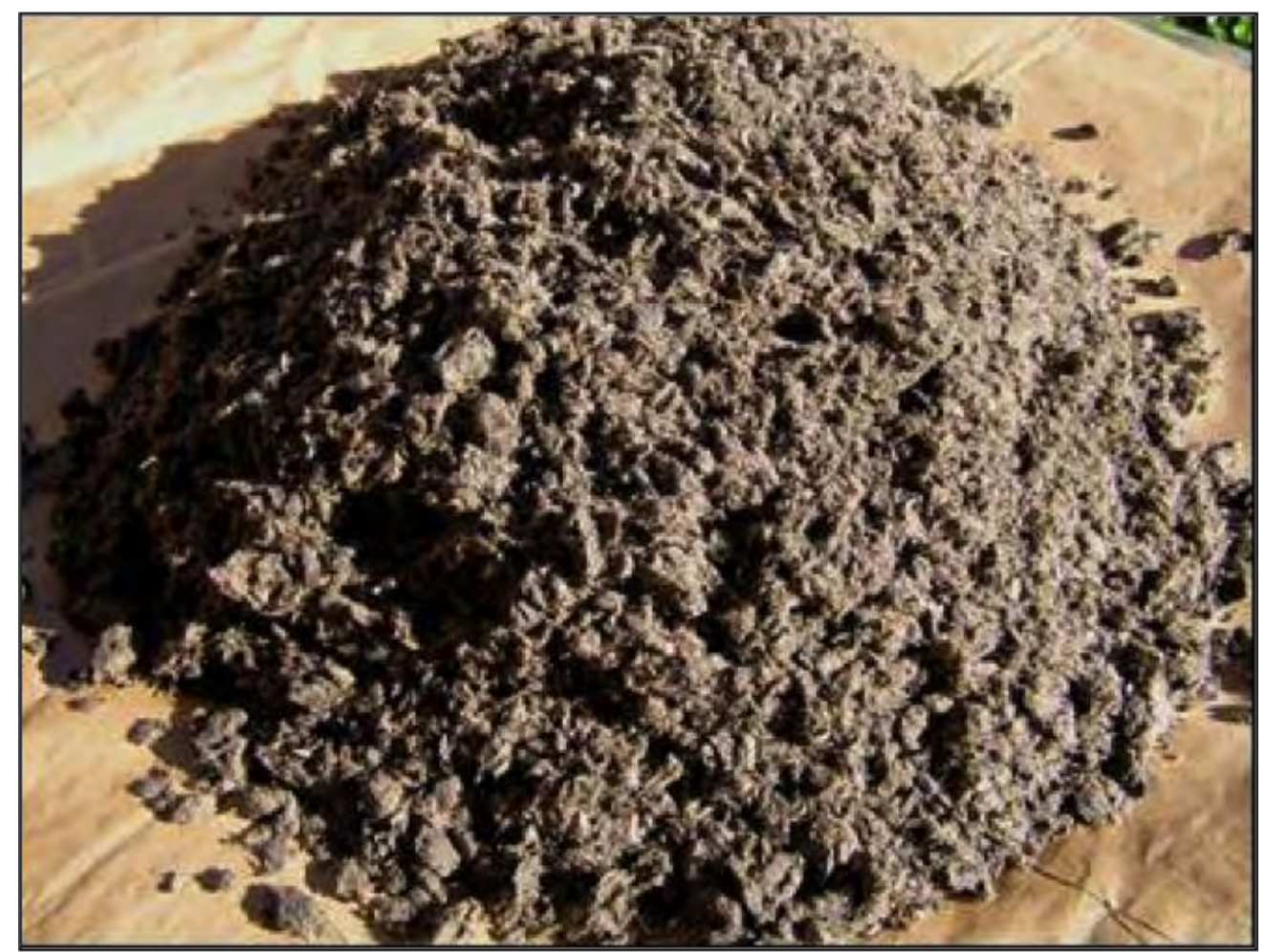

Figure 2. Sample of pulp mill sludge.

An agricultural area of 1600 ha was selected in the peri-urban area of Covilhã (Portugal) for potential sludge application as shown in Figure 3, using extracts of Military Map No. 235 and $236(1 / 25,000$ scale), altimetry data $(1 / 25,000$ scale), ecological protected areas and agricultural protected areas $(1 / 25,000$ scale), a map of the Perimeter Irrigation Block of Covilhã-Fundão (1/25000, scale) orthophotomaps (photogrammetric flight of 2004, $1 / 5,000$ scale) and other local georeferenced elements (e.g. location of the industry and the agricultural parcels). 


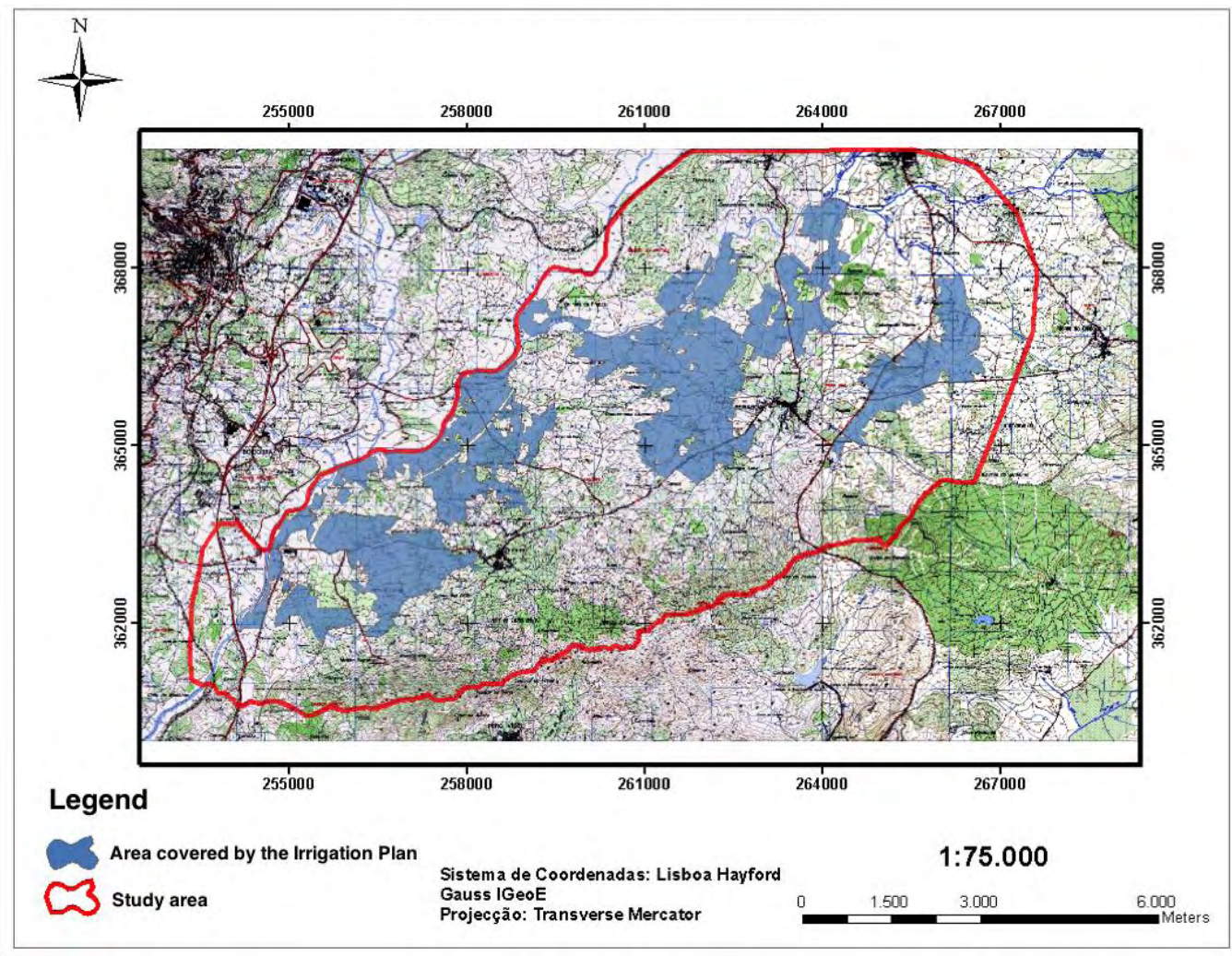

Figure 3. Selected area for sludge application in Covilhã, Portugal $\left(40^{\circ} 15^{\prime} 00^{\prime \prime} \mathrm{N}\right.$, $\left.72^{\circ} 29^{\prime} 00^{\prime \prime} \mathrm{W}\right)$.

The selection of the agricultural parcels for irrigation was based on environmental, technical and economic criteria according to the suggestions of MADRP (Portugal, 1997), USEPA (1997), ESD (Alberta, 1999) and DELG (Ireland, 2008), as follows:

a) Environmental criteria

- Areas with deep soils and classified as classes A and B according to the World Reference Base for soil resources (Deckers et al., 1998);

- A safety distance of $50 \mathrm{~m}$ from natural water resources, $100 \mathrm{~m}$ from water supply sources and water reservoirs used for human consumption, $200 \mathrm{~m}$ from urban residential areas, $30 \mathrm{~m}$ from borders of navigable waters and $10 \mathrm{~m}$ away from borders of non-navigable waters;

- Land uses (the Corine Land Cover map was used to evaluate the potential land use of the studied area). Areas for biological agriculture were skipped since sludge application is not allowed in soils with such activity.

b) Technical criteria

- Dry sludge application rate of 6 ton $\mathrm{ha}^{-1}$ year $^{-1}$;

- Annually available sludge volume and its physical and chemical characteristics;

- Nutrient demand for crop production;

- Tolerance of crops to toxicity and salinity;

- Slopes (sludge application should be preferably applied in agricultural parcels with slopes up to $12 \%$ );

- Groundwater table lower than $5 \mathrm{~m}$. 
c) Economic criteria

- A maximum distance of $8 \mathrm{~km}$ from the sludge production point was defined as economically viable for reuse (this criterion included sludge drying and transfer costs from the Celtejo industry to the agricultural parcels).

For each work (application of RW or biosolids), a spatial analysis was conducted over thematic layers (thematic map). The thematic layers were developed from geographical data obtained from official sources, georeferenced data and data generated using satellite images and orthophotos. Each point (cells representing $10 \mathrm{~m} \times 10 \mathrm{~m}$ ) in each layer took a value ( 0 or 1$)$ according to exclusion and inclusion criteria. Map algebra was used to make a boolean operation between grid cells of different thematic maps in order to generate a final map with the agricultural parcels for the application of RW. All the operations were conducted using the software ArcGIS 9.1 (ESRI, USA) and the ArcCatalog and ArcMap applications.

All the physical, chemical and microbiological parameters were determined according to standard methods (APHA et al., 1999).

In both studies, taking into account the environmental, technical and economic criteria, the exclusion areas were coded as 0 and the inclusion areas as 1. A GIS in raster format was used to create a map with the location of the agricultural parcels and the results were processed according to the algebra of maps (maps overlapping for the different variables, operated in $10 \mathrm{~m} \times 10 \mathrm{~m}$ sized cells).

\section{RESULTS AND DISCUSSION}

\subsection{Site location for reclaimed water reuse}

The characteristics of the RW are presented in the Table 1. The annual net volume of RW produced at the WWTP is approximately $170,000 \mathrm{~m}^{3}$, based on the average flow-rate.

Table 1. Reclaimed water characteristics

\begin{tabular}{|c|c|c|c|}
\hline Parameter & Value & Parameter & Value \\
\hline Flow rate $\left(\mathrm{m}^{3} \mathrm{~d}^{-1}\right)$ & 461 & $\mathrm{~K}\left(\mathrm{mg} \mathrm{L}^{-1}\right)$ & 56.7 \\
\hline Temperature $\left({ }^{\circ} \mathrm{C}\right)$ & $16.4-2.5$ & $\mathrm{Na}\left(\mathrm{mg} \mathrm{L}^{-1}\right)$ & 102.2 \\
\hline $\mathrm{pH}$ & $6.6-7.5$ & $\operatorname{Mg}\left(\mathrm{mg} \mathrm{L}^{-1}\right)$ & 12.2 \\
\hline $\mathrm{EC}\left(\mathrm{dS} \mathrm{m} \mathrm{m}^{-1}\right)$ & 0.38 & As $\left(\mathrm{mg} \mathrm{L}^{-1}\right)$ & 0.01 \\
\hline $\mathrm{BOD}_{5}\left(\mathrm{mg} \mathrm{L}^{-1}\right)$ & 32.1 & $\mathrm{Cd}\left(\mathrm{mg} \mathrm{L}^{-1}\right)$ & 0.02 \\
\hline $\mathrm{DQO}\left(\mathrm{mg} \mathrm{L}^{-1}\right)$ & 96.1 & $\mathrm{Cr}\left(\mathrm{mg} \mathrm{L}^{-1}\right)$ & 0.15 \\
\hline $\mathrm{TSS}\left(\mathrm{mg} \mathrm{L}^{-1}\right)$ & 25.2 & $\mathrm{Co}\left(\mathrm{mg} \mathrm{L}^{-1}\right)$ & 0.01 \\
\hline $\mathrm{NH}_{4}-\mathrm{N}\left(\mathrm{mg} \mathrm{L}^{-1}\right)$ & 33.4 & $\mathrm{Hg}\left(\mathrm{mg} \mathrm{L}^{-1}\right)$ & 0.01 \\
\hline $\mathrm{NO}_{3}-\mathrm{N}\left(\mathrm{mg} \mathrm{L}^{-1}\right)$ & 3.4 & $\mathrm{Ni}\left(\mathrm{mg} \mathrm{L}^{-1}\right)$ & 0.11 \\
\hline $\mathrm{TN}\left(\mathrm{mg} \mathrm{L}^{-1}\right)$ & 44.2 & $\mathrm{~Pb}\left(\mathrm{mg} \mathrm{L}^{-1}\right)$ & 0.08 \\
\hline $\mathrm{TP}\left(\mathrm{mg} \mathrm{L}^{-1}\right)$ & 8.2 & $\mathrm{Zn}\left(\mathrm{mg} \mathrm{L}^{-1}\right)$ & 0.21 \\
\hline $\mathrm{Al}\left(\mathrm{mg} \mathrm{L}^{-1}\right)$ & 1.13 & TC (NMP $100 \mathrm{~mL}^{-1}$ ) & $1.1 \times 10^{5}$ \\
\hline $\mathrm{B}\left(\mathrm{mg} \mathrm{L}^{-1}\right)$ & 0.18 & FC (NMP $\left.100 \mathrm{~mL}^{-1}\right)$ & $2.1 \times 10^{4}$ \\
\hline $\mathrm{Ca}\left(\mathrm{mg} \mathrm{L}^{-1}\right)$ & 44.2 & E. coli $\left(\mathrm{NMP} 100 \mathrm{~mL}^{-1}\right)$ & $3.4 \times 10^{4}$ \\
\hline $\mathrm{Cl}\left(\mathrm{mg} \mathrm{L}^{-1}\right)$ & 31.3 & $\mathrm{HE}\left(\operatorname{eggs} 10 \mathrm{~L}^{-1}\right)$ & ND \\
\hline
\end{tabular}

Note: average values for 22 samples, ND: not detected. 
The physicochemical characteristics of the RW suggest that it is suitable for crop irrigation, namely for the irrigation of fruit trees and vinery (Asano et al., 2007; Dantas et al., 2014). It has moderate organic content, high nutrient content (which is useful for crop production), low nitrate concentrations (it does not present a risk for groundwater contamination), low heavy metal concentrations, low EC (only values above $0.7 \mathrm{dS} \mathrm{m}^{-1}$ may be considered a risk for soil salinity) and is not phytotoxic for those crops (the concentration of $\mathrm{Al}, \mathrm{B}, \mathrm{Cl}$ and $\mathrm{Na}$ are below the recommended values for crop irrigation set up in USEPA (2012), Asano et al. (2007). However, the pathogenic content (TC, FC and E. coli) is not suitable for irrigation (WHO, 2006; Marecos do Monte e Albuquerque, 2010b; USEPA, 2012) and must be removed before the use of RW.

By overlapping all the criteria associated with each variable to the parcels with dominant crops (mainly fruit trees, corn, olive trees and vine) the agricultural parcels most suitable for irrigation were obtained as presented in Table 2 and Figure 4. A total suitable area of 30.5 ha was thereby found for irrigation, located within $1.8 \mathrm{~km}$ from the WWTP, which is the economical criterion allowed for the study.

Table 2. Areas for crop irrigation.

\begin{tabular}{|c|c|c|c|c|}
\hline \multirow{2}{*}{$\begin{array}{l}\text { Type of } \\
\text { crop }\end{array}$} & \multirow{2}{*}{$\begin{array}{l}\text { Needs of water } \\
\left(\mathrm{m}^{3} \mathbf{h a}^{-1} \mathbf{a n o}^{-1}\right)\end{array}$} & \multirow{2}{*}{$\begin{array}{l}\text { Volume of reclaimed water } \\
\text { to be used }\left(\mathrm{m}^{3} \mathrm{ha}^{-1} \text { year }^{-1}\right)\end{array}$} & \multicolumn{2}{|c|}{ Area for irrigation } \\
\hline & & & (ha) & $(\%)$ \\
\hline Fruit trees & 7500 & 85200 & 11.36 & 37.2 \\
\hline Corn & 2500 & 11300 & 4.52 & 14.8 \\
\hline Olive trees & 5300 & 46100 & 8.70 & 28.5 \\
\hline Vine & 4600 & 27400 & 5.96 & 19.5 \\
\hline Total & & 170000 & $\mathbf{3 0 . 5 3}$ & 100 \\
\hline
\end{tabular}

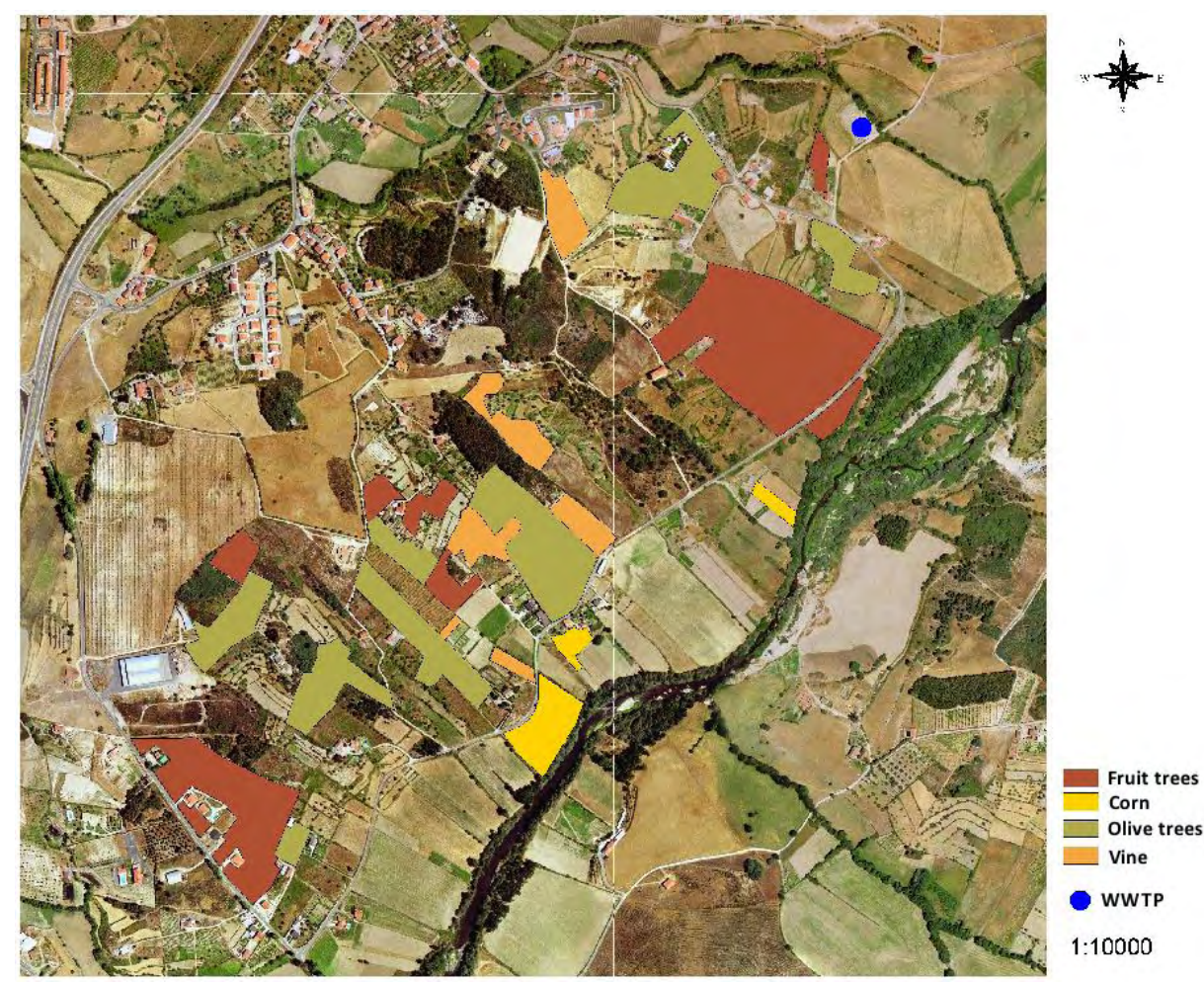

Figure 4. Agricultural parcels selected for irrigation with reclaimed water, Covilhã, Portugal $\left(40^{\circ} 15^{\prime} 00^{\prime \prime} \mathrm{N}, 72^{\circ} 29^{\prime} 00^{\prime \prime} \mathrm{W}\right)$. 
Taking into account the average concentrations of nutrients $(\mathrm{N}$ and $\mathrm{P})$ and heavy metals, the yearly available volume of RW and the total area for crop irrigation, the yearly load of each compound to be added to the soil was computed. With respect to metals, the results show an higher load of $1.7 \mathrm{~kg} \mathrm{ha}^{-1}$ year ${ }^{-1}$ for $\mathrm{Zn}$ and values under $1 \mathrm{~kg} \mathrm{ha}^{-1}$ year $^{-1}$ for other metals, which are all much lower than the limits defined by WHO (2006), Asano et al. (2007) and Marecos do Monte e Albuquerque (2010b).

Regarding the content of $\mathrm{N}$ and $\mathrm{P}$, the maximum loads that would be added to the soil yearly would be $143 \mathrm{~kg} \mathrm{TN} \mathrm{ha}^{-1}$ year $^{-1}$ and $34 \mathrm{~kg} \mathrm{TP} \mathrm{ha}^{-1}$ year $^{-1}$, respectively, which are below the limits defined by several guides for good agricultural practices (USDA, 1994; Portugal, 1997; Ireland, 2008).

\subsection{Site location for the resuse of biosolids}

Primary sludge (produced at the primary settling tank) presents both higher organic matter and higher dry matter than secondary sludge (a biological sludge produced at the secondary settling tank), because it is less mineralized (Table 3). It also has a higher content of heavy metals, but less concentration of $\mathrm{N}$ and $\mathrm{P}$ as also observed by Camberato et al. (2006). However, the concentrations of heavy metals in both types of sludge are below the limits recommended by the same guides and high concentrations are not expected in soils after the incorporation of sludge. Both sludges present a good $\mathrm{pH}$ for land application since the limit stated in several manuals and codes of good practice is 5 (USEPA, 1997; Alberta, 1999; Portugal, 1997; Ireland, 2008).

Table 3. Pulp mill sludge characteristics.

\begin{tabular}{|c|c|c|c|c|c|}
\hline Parameter & Primary sludge & Secondary sludge & Parameter & Primary sludge & Secondary sludge \\
\hline $\begin{array}{l}\text { Dry matter } \\
(\mathrm{DM}, \%)\end{array}$ & 25 & 10 & $\mathrm{Cd}\left(\mathrm{mg} \mathrm{kg}^{-1}\right)$ & 1.4 & 0.34 \\
\hline $\begin{array}{l}\text { Organic matter } \\
(\mathrm{OM}, \%)\end{array}$ & 47 & 11 & $\mathrm{Cu}\left(\mathrm{mg} \mathrm{kg}^{-1}\right)$ & 13 & 2.8 \\
\hline $\mathrm{pH}$ & 7.2 & 7.8 & $\mathrm{Cr}\left(\mathrm{mg} \mathrm{kg}^{-1}\right)$ & 19 & 1.9 \\
\hline $\mathrm{TN}\left(\mathrm{mg} \mathrm{kg}^{-\mathbf{1}}\right)$ & 38 & 2560 & $\mathrm{Ni}\left(\mathrm{mg} \mathrm{kg}^{-\mathbf{1}}\right)$ & 10.5 & 1.44 \\
\hline $\mathrm{NH}_{4}-\mathrm{N}\left(\mathrm{mg} \mathrm{kg}^{-1}\right)$ & 4 & 1090 & $\mathrm{~Pb}\left(\mathrm{mg} \mathrm{kg}^{-\mathbf{1}}\right)$ & 13.2 & 1.1 \\
\hline $\mathrm{TP}\left(\mathrm{mg} \mathrm{kg}^{-1}\right)$ & 167 & 370 & $\mathrm{Zn}\left(\mathrm{mg} \mathrm{kg}^{-1}\right)$ & 83 & 12.9 \\
\hline
\end{tabular}

Results also show that the amount of $\mathrm{N}$ to be introduced in the soils of the study area is much lower than the limits recommended by several guides (Portugal, 1997; Ireland, 2008) even for areas sensitive to nitrate leaching. Therefore, it seems reasonable to assume that sludge application rates higher than 6 ton $\mathrm{ha}^{-1}$ year $^{-1}$ can be applied if the amount of $\mathrm{N}$ in soil does not exceed $210 \mathrm{~kg} \mathrm{~N} \mathrm{ha}^{-1}$ year $^{-1}$ and if both the quality of water sources and the risk of nitrate percolation in the soil were properly assessed.

The characteristics and types of crops can have different susceptibility to pulp mill sludge. Therefore, taking into account the restrictions set forth in Directive 86/278/EEC, agricultural parcels with the following crops were not considered: grassland or forage crops that are used for cattle feeding; vegetable crops and fruit crops with direct contact during the growing period; soils for vegetable or fruit cultures with direct contact for a period of 10 months before harvest.

A multi-criteria analysis was carried out over all the thematic maps and restrictions, resulting a suitable area of 253 ha for sludge application as shown in Figure 5 (green areas), which is enough for the application of all the sludge produced at the paper industry (approximately 6,386 ton year $^{-1}$ ), considering a rate of application of 6 ton ha $^{-1}$ year ${ }^{-1}$. 


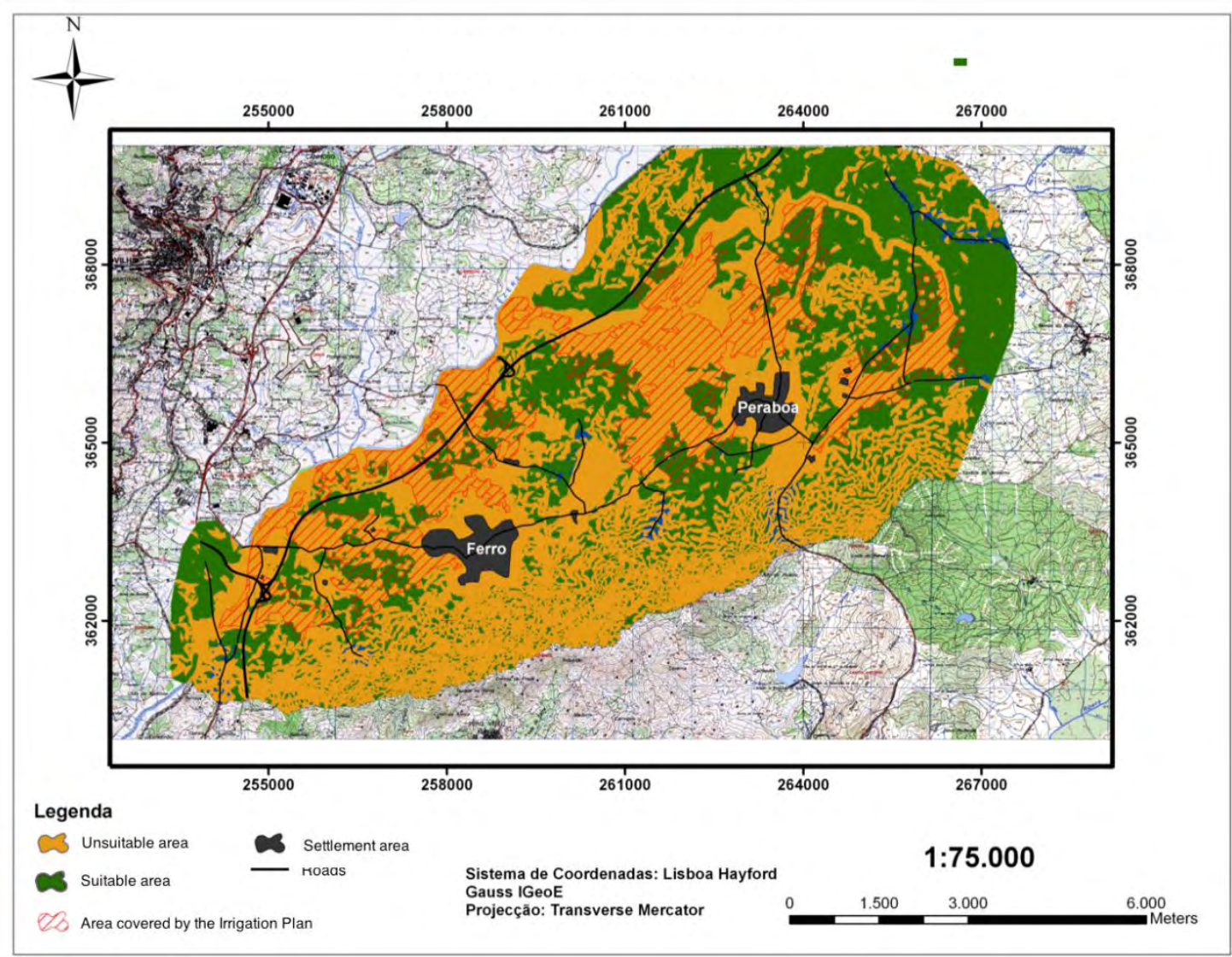

Figure 5. Suitable agricultural area for paper mill sludge application in Covilhã, Portugal $\left(40^{\circ} 15^{\prime} 00^{\prime \prime} \mathrm{N}, 72^{\circ} 29^{\prime} 00^{\prime \prime} \mathrm{W}\right)$.

\section{CONCLUSIONS}

GIS multi-criteria analysis proved to be a very useful tool for selecting suitable agricultural parcels for the application of RW and biosolids. This tool allows georeferencing, storing, processing and manipulating complex information, as well as the use of map algebra operations over environmental, technical and economic criteria in order to create suitability maps. The use of reclaimed water represents an important water source for irrigation in peri-urban regions with water shortages, with important environmental and economic benefits. Pulp mill sludge is suitable for application to agricultural land either for organic correction of soils or as fertilizer for crops.

\section{REFERENCES}

ALBERTA. Environmental Sciences Division. Standards and guidelines for the land application of mechanical pulp mill sludge to agricultural land. Alberta, 1999. $44 \mathrm{p}$.

ASSOCIAÇÃO BRASILEIRA DE NORMAS TÉCNICAS - ABNT. NBR 10004 - Resíduos sólidos - classificação. Rio de Janeiro, 2004.

AMERICAN PUBLIC HEALTH ASSOCIATION - APHA; AMERICAN WATER WORKS ASSOCIATION - AWWA; WATER ENVIRONMENT FEDERATION - WEF. Standard methods for the examination of water and wastewater. $20^{\text {th }}$ edition. Washington DC, 1999. 
ASANO, T.; BURTON, F.; LEVERENZ, H.; TSUCHIHASHI, R.; TCHOBANOGLOUS, G. Water Reuse. 1st ed. New York: McGraw Hill, 2007.

BATTAGliA, A.; CALACE, N.; NARDI, E.; PETRONIO, B.; PIETROLETTI, M. Reduction of $\mathrm{Pb}$ and $\mathrm{Zn}$ bioavailable forms in metal polluted soils due to paper mill sludge addition. Effects on $\mathrm{Pb}$ and $\mathrm{Zn}$ transferability to barley. Biores. Techn. v. 98, n. 16, p. 2993-2999, 2007. http://dx.doi.org/10.1016/j.biortech.2006.10.007

BRASIL. Conselho Nacional do Meio Ambiente. Resolução nr. 54, de 28 de novembro de 2005. Estabelece modalidades, diretrizes e critérios gerais para a prática de reuso direto não potável de água, e dá outras providências. Brasília, 2005.

BRASIL. Ministério do Meio Ambiente. Lei no 12.305, de 2 de Agosto de 2010. Institui a Política Nacional de Resíduos Sólidos; altera a Lei nº 9.605, de 12 de fevereiro de 1998; e dá outras providências. Brasília, 2010.

CAMBERATO, J.; GAGNON, B.; ANGERS, D.; CHANTIGNY, M.; PAN, W. Pulp and paper mill by-products as soil amendments and plant nutrient sources. Can. J. of Soil Sci., v. 86, p. 641-653, 2006. http://dx.doi.org/10.4141/S05-120

DANTAS, I. L. A. et al . Viability of using treated wastewater for the irrigation of radish (Raphanus sativus L.). Rev. Ambient. Água, Taubaté, v. 9, n. 1, Mar. 2014. http://dx.doi.org/10.4136/ambi-agua.1220.

DECKERS, J.; NACHTERGAELE, F.; SPAARGAREN, O. World reference base for soil resources. Rome: ISSS; ISRIC; FAO, 1998. 172p. (World Soil Resources Report, n. 84)

DEON, M. D.; GOMES, T. M.; MELFI, A. J.; MONTES, C. R.; SILVA, E. Sugarcane yield and quality under irrigation with sewage treatment plant effluent. Pesq. agropec. bras., Brasília, v. 45 , n. 10, Oct. 2010. http://dx.doi.org/10.1590/S0100$204 X 2010001000014$.

EUROPEAN COMMISSION - EC. Use of economic instruments and waste management performances. Final report. Paris, 2012.

EUROPEAN COMMISSION - EC. European waste catalogue 20305. European Commission Decision 2000/532/EC of 2000.09.06. Brussels, 2000.

EUROPEAN PARLIAMENT - EP. Waste and repealing certain directives. European Directive 2008/98/EC of 2008.11.19. Brussels, 2008.

EUROPEAN PARLIAMENT - EP. Urban waste water treatment. European Directive 1991/271/EEC of 1991.05.21. Brussels, 1991.

FOLEY, B.; COOPERBAND, L. Paper mill residuals and compost effects on soil carbon and physical properties. J. of Environ. Quality, v. 31, n. 6, p. 2086-2095, 2002. http://dx.doi.org/10.2134/jeq2002.2086

FREITAS, C. A. S. et al. Reuse of treated domestic sewage effluent as an alternative water source for the production of sugarcane. Rev. bras. eng. agríc. ambient., Campina Grande, v. 17, n. 7, July 2013. http://dx.doi.org/10.1590/S1415-43662013000700006.

GEMITZIA, A.; TSIHRINTZIS, V. A.; CHRISTOUC, O.; PETALAS, C. Use of GIS in siting stabilization pond facilities for domestic wastewater treatment. J. Environ Manag, v. 82, p. 155-166, 2007. http://dx.doi.org/10.1016/j.jenvman.2005.12.022 
GLENN, J. Paper mill sludge: feedstock for tomorrow. BioCycle. v. 38, n. 11, p. 30-36, 1998.

HOORNWEG, D.; BHADA-TATA, P. What a waste: a global review of solid waste management. Washington: The World Bank, 2012. 116p. (Urban Development Series. Knowledge Papers, No. 15).

IRELAND. Dep. of Agriculture and Rural Development. The code of good agricultural practice for the prevention of pollution of water, air and soil. Dublin, 2008. $165 \mathrm{p}$.

JIMENEZ, B.; DRECHSEL, P.; KONE, D.; BAHRI, A.; RASCHID-SALLY, L.; QADIR, M. Wastewater, sludge and excreta use in developing countries: an overview. In: DRECHSEL, P.; SCOTT, C.; RASCHID-SALLY, L.; REDWOOD, M.; BAHRI, A. (Eds.). Wastewater irrigation and health: assessing and mitigating risk in low-income countries. London: Earthscan; IDRC-IWMI, 2010. p. 3-27.

JORDAN, M.; RODRIGUEZ, E. Effect of solid residues from the cellulose industry on plant growth. J. of Plant. Nutr. Soil. Scienc., v. 167, n. 3, p. 351-356, 2004. http://dx.doi.org/10.1002/jpln.200321183

KALLALI, H.; ANANE, M.; JELLALI, S.; TARHOUNI, J. GIS-based multi-criteria analysis for potential wastewater aquifer recharge sites. Desalination, v. 215, p. 111-119, 2007. http://dx.doi.org/10.1016/j.desal.2006.11.016

LOCUSSOL, A.; FALL, M.; DICKSON, E. Guiding principles for successful reforms of urban water supply and sanitation sectors. Washington, DC: The World Bank, 2009. (Water Working Notes, n. 19).

MARECOS DO MONTE H.; ALBUQUERQUE, A. Analysis of constructed wetland performance for irrigation reuse. Wat. Sc. and Tech., v. 61, n. 7, p. 1699-1705, 2010a. http://dx.doi.org/10.2166/wst.2010.063.

MARECOS DO MONTE H.; ALBUQUERQUE, A. Wastewater reuse. Lisboa: ERSAR, 2010b. 319 p. (Technical guide, n. 14).

PHILLIPS, V.; KIRKPATRICK, N.; SCOTFORD, L.; WHITE, R.; BURTON, R. The use of paper-mill sludges on agricultural land. Bior. Tech., v. 60, n. 1, p. 73-80, 1997. http://dx.doi.org/10.1016/S0960-8524(97)00006-0

PORTUGAL. Ministério da Agricultura, do Desenvolvimento Rural e das Pescas. Guide for good agriculture practices: protection of water streams against the nitrate pollution. Lisbon, 1997. 53 p.

UNITED NATIONS EDUCATIONAL, SCIENTIFIC AND CULTURAL ORGANIZATION - UNESCO. Managing water under uncertainty and risk. Paris, 2012. (The UN World Water Development Report 4, v. 1).

UNITED STATES. Department of Agriculture - USDA. Phosphorus assessment tool. Forth Worth, 1994. (Technical note, Series No. 1901, South National Technical Center).

UNITED STATES. Environmental Protection Agency - USEPA. Guidelines for water reuse. Report EPA/600/R-12/618. Washington, 2012. 643 p.

UNITED STATES. Environmental Protection Agency - USEPA. Land application of biosolids: process design manual. 1st edition. New York: CRC, 1997. 310p. 
VARAllo, A. C. T.; CARVAlHO, L.; SANTORO, B. L.; SOUZA, C. F. Alterations in attributes of a Red-yellow Latosol irrigated with reuse water. Rev. bras. eng. agríc. ambient., Campina Grande, v. 14, n. 4, Apr. 2010. http://dx.doi.org/10.1590/S141543662010000400005.

WORLD HEALTH ORGANIZATION - WHO. Guidelines for the safe use of wastewater, excreta and greywater. Vol. 2: wastewater use in agriculture. Geneva, 2006. 


Ambiente \& Água - An Interdisciplinary Journal of Applied Science
ISSN 1980-993X - doi:10.4136/1980-993X
www.ambi-agua.net
E-mail: ambi-agua@agro.unitau.br

\title{
Hydrogeochemical attributes and ground water quality of Ngbo Community in Ohaukwu Area Council, Ebonyi State, Nigeria
}

\author{
doi: 10.4136/ambi-agua.1453 \\ Received: 03 Jul. 2014; Accepted: 29 Oct. 2014

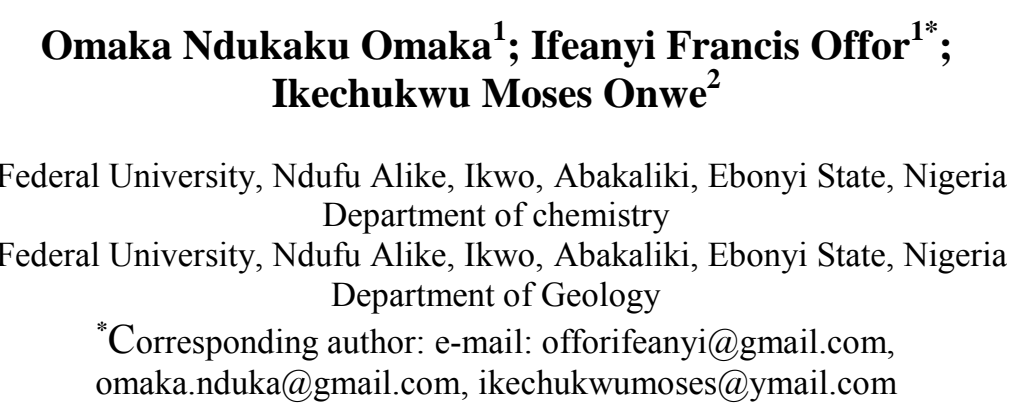

\section{ABSTRACT}

This study evaluated the hydrogeochemical attributes and quality of groundwater resources in Ngbo, Ohaukwu Area Council of Ebonyi State, Nigeria in order to determine whether boreholes in the area were suitable for potable uses. Eleven groundwater samples were collected from hand-dug boreholes between February and March, 2013. The physiochemical parameters of the samples were then analyzed to determine electrical conductivity, total dissolved solids, total alkalinity, major cations and anions, and trace metals. The quality of these characteristics was evaluated by comparing them to the Nigerian Institute of Standards, the Bureau of Indian Standards and the World Health Organization standards for drinking water quality. Mass abundance of the major ions was in the order of $\mathrm{Mg}^{2+}>\mathrm{Ca}^{2+}$ for cations, $\mathrm{Cl}^{-}>\mathrm{SO}_{4}{ }^{2-}>\mathrm{NO}_{3}{ }^{-}>\mathrm{PO}_{4}{ }^{3^{-}}$for anions and $\mathrm{Fe}>\mathrm{As}>\mathrm{Mn}>\mathrm{Cu}>\mathrm{Zn}$ $>\mathrm{Cr}>\mathrm{Ni}>\mathrm{Pb}>\mathrm{Cd}$ for trace metals. Correlation analysis revealed both positive and negative correlations among the parameters. Also, one-way ANOVA tests revealed that no significant differences existed between physiochemical parameters $(F=1.004<$ Fcrit $=1.977)$, major cations and anions $(\mathrm{F}=0.547<\mathrm{Fcrit}=2.008)$ and trace metals $(\mathrm{F}=0.772<\mathrm{Fcrit}=1.940)$ regardless of the sampling location. Groundwater in the area was generally hard, alkaline and highly mineralized, making it unsuitable for drinking in some places due to high total hardness and TDS; but it was generally suitable for irrigation purposes. It is recommended that boreholes be flushed regularly to aid in the removal of mineralized deposits, and that regular hydrogeochemical studies be conducted in order to detect future deterioration of water quality.

Keywords: borehole, hydrogeochemistry, water quality.

\section{Atributos hydrogeoquímicos e qualidade das águas subterrâneas da Comunidade Ngbo de Ohaukwu, Estado de Ebonyi, Nigéria}

\section{RESUMO}

As investigações sobre os atributos hydrogeoquímicos e a qualidade das águas subterrâneas da Comunidade Ngbo no Conselho de Área Ohaukwu, Estado de Ebonyi, 
Nigéria foram realizadas para avaliar a adequação dos poços para água potável. O estudo envolveu onze amostras de água subterrânea coletadas em poços cavados a mão entre Fevereiro e Março de 2013. Parâmetros físico-químicos incluindo condutividade elétrica, sólidos dissolvidos totais, alcalinidade total, os principais cátions e ânions superóxidos e metais foram analisados em amostras de água subterrânea e suas características de qualidade avaliadas e comparadas com o padrão do Nigerian Institute of Standards, do Bureau of Indian Standards e com o World Health Organization Standards para o consumo de água. A ocorrência dos principais íons apresentou a seguinte ordem: $\mathrm{Mg}^{2+}>\mathrm{Ca}^{2+}$ para os cátions, $\mathrm{Cl}^{-}$ $>\mathrm{SO}_{4}{ }^{2-}>\mathrm{NO}_{3}{ }^{-}>\mathrm{PO}_{4}{ }^{3-}$ para os ânions e $\mathrm{Fe}>\mathrm{Mn}>\mathrm{Cu}>\mathrm{Zn}>\mathrm{Cr}>\mathrm{Ni}>\mathrm{Pb}>\mathrm{Cd}$ para os metais traços. A análise correlações positivas e negativas entre os parâmetros. Além disso, testes de ANOVA revelaram que não houve diferença significativa $(\mathrm{F}=1,004<\mathrm{Fcrit}=$ 1.977) entre os parâmetros físico-químicos, e entre os principais cátions e ânions $(\mathrm{F}=0,547<$ Fcrit = 2.008), assim como entre os elementos traços $(\mathrm{F}=0,772<$ Fcrit $=1.940)$ independentemente do local de amostragem. As águas subterrâneas na área eram de modo geral duras, alcalinas e altamente mineralizadas, portanto, impróprias para consumo em alguns lugares devido à alta dureza total e TDS, mas geralmente adequadas para irrigação. Lavagens regulares dos poços para auxiliar a remoção de minerais depositados e estudos hidrogeoquímicos regulares são importantes para a detecção de deterioração da qualidade da água no futuro.

Palavras-chave: hidrogeoquímica, poço artesiano, qualidade da água.

\section{INTRODUCTION}

Water is vital to man's existence and without it there would be no life on earth. It has numerous applications ranging from domestic applications such as drinking, cooking, washing and bathing to agricultural and industrial applications such as irrigation, power generation and industrial production. The earth holds approximately $1.4 \times 10^{9}$ cubic kilometers of water in the form of oceans, seas, rivers, lakes, ice, etc.; but only $3 \%$ of the total available water resources are in the form of fresh water found in rivers, lakes and groundwater. The fresh water that is needed for a clean water supply is limited, and demand far exceeds the available supply due to increasing population and industrialization (APHA et al., 2012).

Recently, the need to determine the quality of public water supply has been intensified as a result of an increase in water pollution on a global scale caused by increasing population, urbanization and industrialization (Achi, 2003; Olatunji et al., 2005; Nwankwoala et al., 2007; Amadi et al., 2012).

A clean water supply is one of the key indicators for development in any country; however, the situation of most African countries is not encouraging. For instance, eighteen African countries are expected to experience a water shortage by 2025, and there are currently more than 300 million people in Africa living in water scarce environments (Abam, 2001). In Sub-Saharan Africa, the water requirements for major domestic and industrial purposes are usually not met. The available water resources continue to dwindle, and the amount of freshwater available for each person in Africa is about one-quarter of what it was in 1950 (Abam, 2001).

Nigeria as a nation is blessed with abundant surface and groundwater resources, especially in south-southern, south-eastern and south-western regions of the country (Obatoyinbo and Oyedotun, 2011).

According to Rijswijk (1981), groundwater found between a $0-50 \mathrm{~m}$ depth in Nigeria is estimated to cover about $6 \times 10^{6} \mathrm{~km}^{3}$, while using the eight mega regional aquifiers in 
Nigeria to estimate the country's total ground water yielded 50 million trillion liters per year (Akujieze et al., 2003). On the other hand, surface water resources in Nigeria are estimated at 224 trillion liters per year (Hanidu, 1990).

As far back as the 1980s, when the international drinking water supply and sanitation decade (1980-1990) was declared, Nigeria had already launched a national borehole program which proposed water for all by the year 2000. About 760 boreholes were earmarked for the program; but only 228 were actually installed, thus leading to a failure of the project (Akujieze, 2003; NPC, 2006).

Due to increasing cases of water pollution associated with surface waters, more attention has been shifted to groundwater sources, leading to an increase in well and borehole digging in both rural and urban centers in Nigeria (Oyedotun and Obatoyinbo, 2012). Several factors, such as lithology and prevailing conditions during formation, water availability in the aquifer and its rate of circulation, the activity of microorganisms, and temperature and pressure affect both the physical and chemical characteristics of groundwater (Ramanathan, 2004).

Groundwater usually contains dissolved mineral ions which can affect the water's usage depending on the type and concentrations of the ions involved (Okagbue, 1988; Boyle, 1988). Major cations and anions found in groundwater include $\mathrm{Ca}^{2+}, \mathrm{Mg}^{2+}, \mathrm{Na}^{+}, \mathrm{K}^{+}, \mathrm{HCO}_{3}{ }^{-}, \mathrm{SO}_{4}{ }^{2-}$ and $\mathrm{Cl}^{-}$. Non-ionic constituents such as oxides, phenols, synthetic detergents, dissolved $\mathrm{O}_{2}$ and $\mathrm{CO}_{2}$ are also found in groundwater (Tijani, 1994). These constituents determine the quality of ground water; and, if they are present in excessive amounts, the water may need to be treated before use (Tijani, 1994).

The inhabitants of Ngbo and the surrounding area are mostly farmers, traders and skilled craftsmen. These economic activities attract people to the area, and thus further increase the demand for potable water even as clean sources decline due to water pollution.

The major aim of this research is to investigate the infiltration of mineral ions into the groundwater of Ngbo community in Ohaukwu Area Council of Ebonyi State and thereafter compare results obtained with the standards set for water quality by the Nigerian Institute of Standards, the Bureau of Indian Standards and the World Health Organization.

\subsection{Description of study area}

Ohaukwu Area Council is one of the 13 Area Councils in Ebonyi State, South-East, Nigeria and it has three main communities namely Izhia, Ngbo and Effium. According to the 2006 population figures, Ohaukwu Council Area has an estimated population of 196,000 (NPC, 2006). The area lies within latitudes $6^{\circ} 3^{\prime} \mathrm{N}$ to $6^{0} 50^{\prime} \mathrm{N}$ and longitudes $7^{\circ} 80^{\prime} \mathrm{E}$ to $8^{0} 00^{\prime} \mathrm{E}$ with climatic conditions such as rainy season (March-October) and dry season (October-February). Two distinct vegetative regions exist in the study area: the Savannah in the Northern part of the study area, and tropical rainforests in the southern parts. More than $70 \%$ of the inhabitants of Ngbo engage in economic activities such as subsistent agriculture, petty trading, hunting and fishing (A.C. Umeji, Personal Communication, 2005).

Figure 1 shows a map of the study area with the sample locations identified.

\subsection{Geology of study site}

The bedrock in the study area is the Abakaliki Shale Formation, which is part of the Albian Asu River Group (99.6 - 112Ma). The area is mainly characterized by bedded shale with occasional sandy, splintery metamorphosed mudstone; lenses of sandstone and sandy limestone are highly jointed and fractured. (A.C. Umeji, Personal communication, 2005). The influence of tectonic activities introduced the discordant dip between the Asu River Group and the overlying Turonian Eze-Aku formation (Afikpo basin). Younger intrusive bodies, in combination with numerous faults and joint systems, have created secondary porosity in the shale. (A.C. Umeji, Personal communication, 2005). The fracture system spread across the Abakaliki anticlinorium and the Afikpo syncline in the Benue rift during the deformational 
episode originated from the vertical movement associated with the rising and cooling of magma, which intruded into the sediments in the sanitonian stage (A. C. Umeji, Personal communication, 2005). Sedimentary rock is folded and fractured, particularly south of Abakaliki, and the fold axes and dominant rock fractures are oriented northwest-southeast. The aquifer in the study area is formed by weathered rocks, alluvium and fractured zones, some of which are isolated.

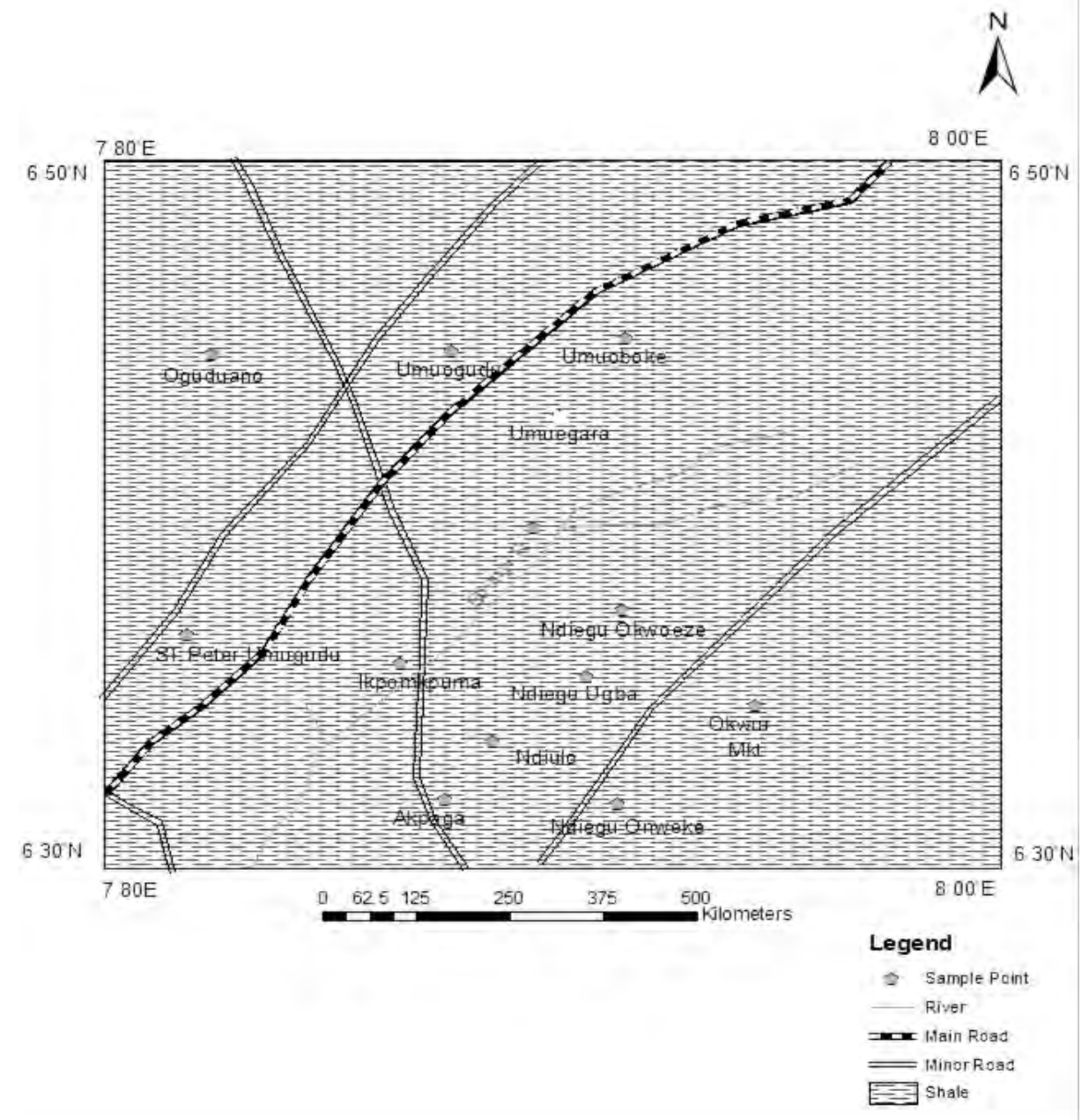

Figure 1. Map of the study area showing sampling locations.

\section{MATERIALS AND METHODS}

\subsection{Sampling}

Groundwater samples were collected at various locations within the study area from 11 boreholes during the dry season (February-March, 2013) at depths less than 40m (<132 ft). Sampling was done in the dry season under good weather conditions in order to avoid rain water contamination which would have affected the quality of the rain water samples. Sample bottles were pre-cleaned before sampling by soaking them in 1:1 dilute $\mathrm{HCl}$ solution for 24 hours, and were later rinsed about 3 times with distilled water and further rinsed with the borehole water to be sampled in each case (Omaka, 2010). Sampling was done using two sets of pre-labelled one-liter polyethylene bottles for cation and anion analysis. Each pair of water samples was collected near the well head of each of the 11 sample boreholes by filling the sample bottle to the brim before corking it to ensure that dissolved $\mathrm{CO}_{2}$ was retained and also to minimize $\mathrm{O}_{2}$ contamination (Hem, 1985). Water samples for the determination of cations were stabilized by adding a few drops of dil. $\mathrm{HCl}$ after collection. To maintain the integrity of 
the water samples, physiochemical parameters sensitive to environmental changes such as $\mathrm{pH}$, conductivity and temperature were measured and recorded in-situ using portable digital meters. The samples were later transported to the laboratory in an ice chest for further analysis.

\subsection{Laboratory analyses}

The collected samples were analyzed for physicochemical properties $[\mathrm{pH}$, electrical conductivity (EC), turbidity, total dissolved solids (TDS), total hardness, chemical oxygen demand], cations $\left(\mathrm{Ca}^{2+}, \mathrm{Mg}^{2+}\right)$, anions $\left(\mathrm{SO}_{4}{ }^{2-}, \mathrm{Cl}^{-}, \mathrm{NO}_{3}{ }^{-}, \mathrm{PO}_{4}{ }^{3-}\right)$ and trace metals $(\mathrm{As}, \mathrm{Cu}, \mathrm{Ni}$, $\mathrm{Fe}, \mathrm{Pb}, \mathrm{Zn}, \mathrm{Cr}, \mathrm{Cd}, \mathrm{Mn}$ ) using the American Public Health Association (APHA et al., 2012) and American Standard for Testing Materials (ASTM, 2012) standard procedures (Table 1).

Table 1. Summary of analytical methods used for the analyses of groundwater samples.

\begin{tabular}{ll}
\hline Parameter measured & Standard Analytical Method used \\
\hline $\mathrm{pH}$ & ASTM D1293-12 \\
Temperature & APHA 2550 \\
Conductivity & APHA 2510B \\
$\mathrm{Ca}^{2+}, \mathrm{Mg}^{2+}$ & ASTM D511-09A \\
$\mathrm{As}^{2+}$ & D2972-08B \\
$\mathrm{Cu}^{2+}, \mathrm{Ni}^{2+}, \mathrm{Fe}^{2+}, \mathrm{Pb}^{2+}, \mathrm{Zn}^{2+}$, & ASTM D1688-07A, APHA 3113B, \\
$\mathrm{Cr}^{2+}, \mathrm{Cd}^{2+}, \mathrm{Mn}^{2+}$ & APHA, 3120B, ASTM D3559-08D, \\
$\mathrm{Total} \mathrm{dissolved} \mathrm{solids} \mathrm{(TDS)}^{2+}$ & APHA 3111B \\
$\mathrm{Total}^{2+}$ hardness & APHA 2540C \\
$\mathrm{SO}_{4}^{2-}$ & - \\
$\mathrm{Cl}^{-}$ & ASTM D4327-03 \\
$\mathrm{NO}_{3}^{-}$ & ASTM D4327-03 \\
$\mathrm{PO}_{4}{ }^{3-}$ & ASTM D3867-90A \\
\hline
\end{tabular}

\subsection{Statistical analyses}

Relevant statistical packages (SPSS Version 17, Microsoft Excel 2009 Statistical Tool Pack) were used to analyze the data obtained and their levels of significance. Descriptive statistics, correlation analysis and one-way analysis of variance (ANOVA) were some of the analyses carried out in this study.

\section{RESULTS AND DISCUSSION}

A summary of the results of water chemistry (physico-chemical properties, cation and anion content and trace metal content) of the groundwater samples is presented in Tables 2 and 3. 
Table 2. Hydrochemical parameters of water samples from various sampling points within Ngbo, Ebonyi State, Nigeria.

\begin{tabular}{|c|c|c|c|c|c|c|c|c|c|c|c|c|c|c|c|}
\hline $\mathbf{S} / \mathbf{N}$ & Sample location & Code & Temp & pH & DO & TDS & EC & TA & TH & $\mathbf{C a}$ & Mg & $\mathbf{C l}$ & $\mathrm{SO}_{4}$ & $\mathrm{NO}_{3}$ & $\mathrm{PO}_{4}$ \\
\hline 1 & Ikpomkpuma & BH1 & 29 & 8.5 & 9.5 & 786 & 1754 & 640 & 172 & 31 & 141 & 167 & 48 & 0.62 & 0.10 \\
\hline 2 & Oguduanoo & BH2 & 29 & 8.2 & 8.6 & 188 & 432 & 164 & 89 & 35 & 55 & 82 & 27 & 0.74 & 0.19 \\
\hline 3 & Umuoboke & $\mathrm{BH} 3$ & 29 & 8.0 & 9.0 & 10 & 10 & 380 & 271 & 50 & 222 & 105 & 26 & 0.75 & 0.12 \\
\hline 4 & Akpegu & $3 \mathrm{H} 4$ & 29 & 8.2 & 9.2 & 388 & 753 & 310 & 202 & 21 & 181 & 112 & 21 & 0.76 & 0.10 \\
\hline 5 & Ndiegu Okwoeze & BH5 & 28 & 8.5 & 9.5 & 415 & 754 & 384 & 36 & 8 & 28 & 131 & 54 & 0.74 & 0.20 \\
\hline 6 & Ndiegu Ugba & BH6 & 29 & 8.3 & 9.4 & 213 & 495 & 190 & 177 & 31 & 147 & 150 & 42 & 0.74 & 0.23 \\
\hline 7 & St Peters Umuogudu & BH7 & 27 & 8.8 & 8.5 & 654 & 1240 & 476 & 41 & 25 & 16 & 136 & 36 & 0.50 & 0.14 \\
\hline 8 & Ndiulo & $3 \mathrm{H} 8$ & 29 & 8.4 & 9.5 & 397 & 857 & 327 & 76 & 25 & 51 & 70 & 63 & 0.47 & 0.15 \\
\hline 9 & Ndiegu Onweke & BH9 & 29 & 8.3 & 9.5 & 282 & 644 & 274 & 164 & 56 & 108 & 160 & 50 & 0.62 & 0.22 \\
\hline 10 & Okwor Market & BH10 & 27 & 8.7 & 9.5 & 338 & 922 & 296 & 140 & 30 & 110 & 147 & 63 & 0.73 & 0.23 \\
\hline 11 & Ebenyi River & BH11 & 28 & 7.8 & 4.0 & 35 & 42 & 15 & 14 & 35 & 10 & 42 & 21 & 0.62 & 0.24 \\
\hline
\end{tabular}

Note: DO-Dissolved oxygen, EC-Electrical conductivity, TDS-Total dissolved solids, TA-Total alkalinity, TH-Total hardness. All parameters have been expressed as mg/L except $\mathbf{p H}$ and $\mathbf{E C}$. The unit of $\mathbf{E C}$ is $\mu \mathrm{S} / \mathrm{cm}$.

Table 3. Trace metal levels in groundwater samples from various locations within Ngbo, Ebonyi State.

\begin{tabular}{|c|c|c|c|c|c|c|c|c|c|c|}
\hline $\mathbf{S} / \mathbf{N}$ & Code & As & $\mathrm{Cu}$ & $\mathbf{N i}$ & $\mathrm{Fe}$ & $\mathbf{P b}$ & $\mathbf{Z n}$ & $\mathrm{Cr}$ & Cd & Mn \\
\hline 1 & BH1 & 0.44 & 0.024 & 0.014 & 0.424 & 0.058 & 0.004 & BDL & BDL & 0.125 \\
\hline 2 & $\mathrm{BH} 2$ & 0.90 & 0.056 & 0.020 & 2.890 & $\mathrm{BDL}$ & 0.173 & BDL & BDL & 0.330 \\
\hline 3 & $\mathrm{BH} 3$ & 0.90 & 0.100 & 0.015 & 0.452 & $\mathrm{BDL}$ & 0.012 & 0.040 & BDL & 0.167 \\
\hline 4 & $\mathrm{BH} 4$ & 0.41 & 0.130 & 0.009 & 0.362 & BDL & 0.007 & 0.088 & 0.002 & 0.030 \\
\hline 5 & BH5 & 0.95 & 0.024 & 0.008 & 0.438 & BDL & 0.003 & 0.064 & BDL & BDL \\
\hline 6 & BH6 & 0.61 & 0.016 & 0.012 & 0.435 & $\mathrm{BDL}$ & 0.004 & BDL & BDL & 0.294 \\
\hline 7 & $\mathrm{BH} 7$ & 0.41 & 0.033 & 0,012 & 0.455 & BDL & 0.004 & BDL & BDL & $\mathrm{BDL}$ \\
\hline 8 & BH8 & 0.47 & 0.023 & 0.011 & 0.543 & BDL & 0.003 & 0.024 & BDL & 0.027 \\
\hline 9 & BH9 & 0.73 & 0.158 & 0.011 & 1.772 & $\mathrm{BDL}$ & 0.256 & $\mathrm{BDL}$ & BDL & 0.122 \\
\hline 10 & BH10 & 1.20 & 0.026 & 0.010 & 0.545 & BDL & 0.004 & BDL & BDL & 0.221 \\
\hline 11 & BH11 & 0.35 & 0.030 & 0.010 & 0.400 & 0.030 & 0.020 & BDL & BDL & 0.100 \\
\hline
\end{tabular}

Note: BDL-Below detection limit. Concentration of trace metals are given in parts per million (ppm). 
Table 4 gives the descriptive statistics of the data generated showing the minimum, maximum, mean, standard deviation and variance values of various parameters investigated in this study compared with national and global drinking water standards such as the Nigerian Institute of Standards (NIS) (SON, 2007), the Bureau of Indian Standards (BIS) (India, 2006) and the World Health Organization (WHO) water standards for drinking and public health purposes.

The temperature of the water samples ranged from $27-29^{\circ} \mathrm{C}$ with a mean temperature of $28.45^{\circ} \mathrm{C}$, which compares favorably with mean atmospheric temperature $\left(28^{\mathrm{O}} \mathrm{C}\right)$, thus suggesting the influence of local climatic conditions. For the $\mathrm{pH}$, values ranged from 7.8-8.8 with a mean of 8.34, which is within the WHO-stipulated tolerable range of 6.5-.8.5 for potable water (WHO, 2011). This shows that majority of the groundwater samples were mildly alkaline, which can be traced to the nature of weathered rocks and aquifers found within the Ngbo area of Ebonyi State. However, the observed $\mathrm{pH}$ range (7.8-8.8) in this study was higher than the $\mathrm{pH}$ ranges of 3.84-7.72 and 5.8-6.9 recorded for groundwater in parts of the eastern Niger Delta (Nwankwoala and Udom, 2011) and parts of Bayelsa State (Okiongbo and Douglas, 2013), respectively. On the other hand, the recorded $\mathrm{pH}$ range (7.8-8.8) of groundwater samples in this study is lower than that (8.3-9.6) obtained for groundwater in Ekiti State by Talabi and Tijani in 2010.

The mean EC and TDS values of the groundwater samples were $718.45 \mu \mathrm{S} / \mathrm{cm}$ and $336.91 \mathrm{mg} / \mathrm{L}$ as compared to the WHO maximum standards of $1250 \mu \mathrm{S} / \mathrm{cm}$ and $1200 \mathrm{mg} / \mathrm{L}$, respectively, for potable water (WHO, 2011). The relatively high EC value suggests the presence of highly mineralized freshwater in Ngbo area of Ebonyi State. Electrical conductivity is a measure of salinity, which can affect the taste of water and hence its suitability as drinking water source (Langenegger, 1990). The maximum allowable EC limit for groundwater is $1400 \mu \mathrm{S} / \mathrm{cm}$ which, if exceeded, renders such water unsuitable for irrigation (Ragunath, 1987). Except for the borehole at Ikpomkpuma (BH1), with an EC value of $1754 \mu \mathrm{S} / \mathrm{cm}$ which exceeded the $1400 \mu \mathrm{S} / \mathrm{cm}$ limit, all other boreholes investigated produced water suitable for irrigation.

The total alkalinity (TA) of the groundwater samples ranged from $15 \mathrm{mg} / \mathrm{L}$ to $640 \mathrm{mg} / \mathrm{L}$ with a mean value of $314.18 \mathrm{mg} / \mathrm{L}$, showing that all the TA values were within the maximum allowable limit $(600 \mathrm{mg} / \mathrm{L})$ set by ISI, except for BH1 $(640 \mathrm{mg} / \mathrm{L})$. Thus, except for BH1, all other boreholes investigated contain water that is suitable for drinking or domestic use. Excessive amounts of calcium in the form of calcium carbonates form temporary hardness in water, while the presence of sulphates, nitrates and chlorides tend to form permanent hardness in water (Twort et al., 2000). The groundwater samples can be classified into different water categories based on their total hardness $(\mathrm{TH})$ values as follows: 3 boreholes (BH5, BH7, BH11) fall into the soft water category $(<75 \mathrm{mg} / \mathrm{L} \mathrm{TH}), 2$ boreholes $(\mathrm{BH} 2, \mathrm{BH} 8)$ fall into the moderate water category $(75-150 \mathrm{mg} / \mathrm{L})$ while 6 boreholes $(\mathrm{BH} 1, \mathrm{BH} 3, \mathrm{BH} 4, \mathrm{BH} 6, \mathrm{BH} 9$, BH10) fall into the hard water category $(150-300 \mathrm{mg} / \mathrm{L} \mathrm{TH})$.

The mean Ca content $(28.68 \mathrm{mg} / \mathrm{L})$ of water samples was lower than the WHO-stipulated standard $(75 \mathrm{mg} / \mathrm{L})$ for $\mathrm{Ca}$ in potable water (WHO, 2011). Similarly, the mean $\mathrm{Mg}$ content $(97.18 \mathrm{mg} / \mathrm{L})$ of water samples was also higher than the WHO stipulated standard $(50 \mathrm{mg} / \mathrm{L})$ for $\mathrm{Mg}$ in potable water (WHO, 2011). Chloride concentrations in the groundwater samples ranged from $42-167 \mathrm{mg} / \mathrm{L}$, with a mean value of $118.36 \mathrm{mg} / \mathrm{L}$, which is below the WHO standard $(250 \mathrm{mg} / \mathrm{L})$ for $\mathrm{Cl}^{-}$in potable water (WHO, 2011). Sulphate concentrations in the groundwater samples ranged from $21-63 \mathrm{mg} / \mathrm{L}$, with a mean value of $41 \mathrm{mg} / \mathrm{L}$, which is below the WHO maximum limit $(500 \mathrm{mg} / \mathrm{L})$ for $\mathrm{SO}_{4}{ }^{2-}$ in potable water (WHO, 2011). The low values recorded for $\mathrm{SO}_{4}{ }^{2-}$ in these water samples could be due to its removal from the water by bacteria (Freeze and Cherry, 1979). Nitrate concentrations in the water samples ranged from $0.47-0.76 \mathrm{mg} / \mathrm{L}$, with a mean value of $0.66 \mathrm{mg} / \mathrm{L}$, which is well below the WHO 
standard $(50 \mathrm{mg} / \mathrm{L})$ for $\mathrm{NO}_{3}{ }^{-}$in domestic/public water supply (WHO, 2011). The major percentage of nitrogen found in groundwater is gotten from the biosphere (Saleh et al., 1999). The cycle for formation of nitrate in nature begins with nitrogen fixation in the atmosphere with mineralization into ammonia by soil bacteria, followed by the conversion of nitrogen into nitrate by nitrifying bacteria under aerobic conditions (Tindall et al., 1995). Toxic effects of high concentrations of nitrate in drinking water include blue baby disease in children and gastric carcinomas (Gilly et al., 1984).

Trace metal levels in the groundwater samples were in minute quantities when compared to major cations and anions. Cadmium had the least mean concentration $(0.000182 \mathrm{mg} / \mathrm{L})$ while Fe had the highest mean concentration $(0.792 \mathrm{mg} / \mathrm{L})$. The noticeably high concentration of $\mathrm{Fe}$ in groundwater samples throughout the study area can be attributed to factors influencing iron solubility and concentration in ground water (Okiongbo and Douglas, 2013). The relative abundance of the trace metals arranged in increasing order of magnitude is as follows: $\mathrm{Cd}<\mathrm{Pb}<\mathrm{Ni}<\mathrm{Cr}<\mathrm{Zn}<\mathrm{Cu}<\mathrm{Mn}<\mathrm{As}<\mathrm{Fe}$.

Schoeller diagram depicting logarithmic data plots for the major ions investigated is shown in Figure 2. The peaks and troughs of the individual water samples indicate their dominant and less dominant ions respectively. A look at Figure 2 shows that $\mathrm{Mg}^{2+}$ is the most dominant ion followed by $\mathrm{Cl}^{-}$and $\mathrm{Ca}^{2+}$ in that order whereas $\mathrm{NO}_{3}{ }^{-}$and $\mathrm{PO}_{4}{ }^{3-}$ are the least dominant ions.

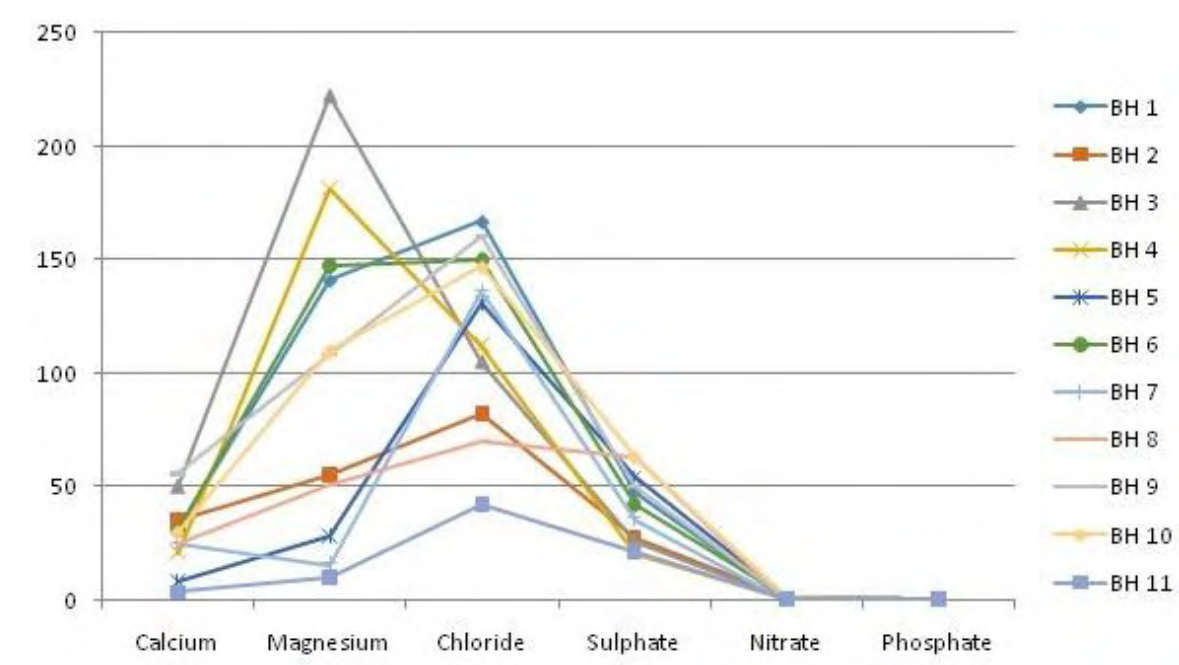

Figure 2. Schoeller Diagram of the major ionic species investigated.

A Pearson correlation analysis of the data generated (Table 5) was done using Microsoft EXCEL 2007 Statistical Tool Package. Positive correlations existed between the following parameters: $\mathrm{Temp}^{-\mathrm{NO}_{3}}$ (0.183), Temp-Mg (0.504), Temp-Ca (0.398), Temp-TH (0.517), Temp-DO (0.224), pH-DO (0.6), pH-TDS (0.906), pH-EC (0.788), pH-TA (0.625),

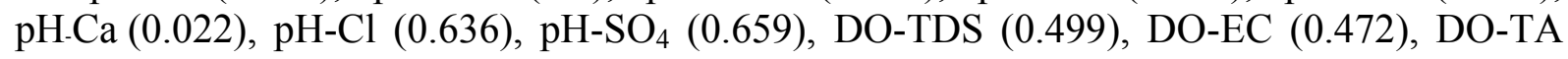
(0.601), DO-TH (0.484), DO-Ca (0.511), DO-Mg (0.442), DO-Cl (0.679), DO-SO 4 (0.55), $\mathrm{DO}_{-\mathrm{NO}_{3}}(0.147)$, TDS-EC (0.81), TDS-TA (0.584), TDS-Cl (0.528), $\mathrm{TDS}_{-\mathrm{SO}_{4}}(0.583)$, EC-TA (0.791), EC-Cl (0.599), ${\mathrm{EC}-\mathrm{SO}_{4}}^{(0.494),}$ TA-TH $(0.26), \quad \mathrm{TA}-\mathrm{Ca} \quad(0.207)$, TA-Mg (0.25), TA.Cl (0.61), TA-SO 4 (0.343), TH-Ca (0.7), TH-Mg (0.988), TH-Cl (0.405), $\mathrm{TH}_{-} \mathrm{NO}_{3}$ (0.474), Ca-Mg (0.581), Ca-Cl (0.429), Ca- $\mathrm{SO}_{4}$ (0.086), Ca- $\mathrm{NO}_{3}$ (0.086), $\mathrm{Mg}-\mathrm{Cl}(0.367), \mathrm{Mg}-\mathrm{NO}_{3}(0.524), \mathrm{Cl}-\mathrm{SO}_{4}(0.433), \mathrm{Cl}-\mathrm{NO}_{3}(0.158), \mathrm{SO}_{4}-\mathrm{PO}_{4}(0.223), \mathrm{NO}_{3}-\mathrm{PO}_{4}$ (0.141). This trend suggests that the pairs of concerned ions originated from common natural and anthropogenic sources. 
Table 4. Descriptive statistics of analyzed groundwater samples compared with NIS, ISI and WHO Standards for Drinking Water Quality.

\begin{tabular}{|c|c|c|c|c|c|c|c|}
\hline Parameter & Minimum & Maximum & Mean & $\begin{array}{l}\text { Standard } \\
\text { deviation }\end{array}$ & NIS: 2007 & $\begin{array}{c}\text { BIS: } \\
105001991\end{array}$ & WHO:2011 \\
\hline Temp. $\left({ }^{\circ} \mathrm{C}\right)$ & 27 & 29 & 28.45 & 0.820 & Ambient & - & - \\
\hline $\mathrm{pH}$ & 7.8 & 8.8 & 8.34 & 0.291 & $6.5-8.5$ & $6.5-8.5$ & $6.5-8.5$ \\
\hline $\mathrm{DO}(\mathrm{mg} / \mathrm{L})$ & 4 & 9.5 & 8.75 & 1.617 & - & - & 4 \\
\hline TDS (mg/L) & 10 & 786 & 336.91 & 235.183 & 500 & 500 & 1200 \\
\hline $\mathrm{EC}(\mu \mathrm{S} / \mathrm{cm})$ & 10 & 1754 & 718.45 & 500.448 & 1000 & - & 1250 \\
\hline $\mathrm{TA}(\mathrm{mg} / \mathrm{L})$ & 15 & 640 & 314.18 & 164.670 & - & 200 & - \\
\hline $\mathrm{TH}(\mathrm{mg} / \mathrm{L})$ & 14 & 271 & 125.64 & 80.557 & 150 & 300 & - \\
\hline $\mathrm{Ca}^{2+}(\mathrm{mg} / \mathrm{L})$ & 3.5 & 56 & 28.68 & 15.496 & - & 75 & 75 \\
\hline $\mathrm{Mg}^{2+}(\mathrm{mg} / \mathrm{L})$ & 10 & 222 & 97.18 & 70.836 & 0.2 & 30 & 50 \\
\hline $\mathrm{Cl}^{-}(\mathrm{mg} / \mathrm{L})$ & 42 & 167 & 118.36 & 40.128 & 250 & 250 & 250 \\
\hline $\mathrm{SO}_{4}{ }^{2-}(\mathrm{mg} / \mathrm{L})$ & 21 & 63 & 41.00 & 15.856 & 100 & 150 & 500 \\
\hline $\mathrm{NO}_{3}^{-}(\mathrm{mg} / \mathrm{L})$ & 0.47 & 0.76 & 0.66 & 0.104 & 50 & 45 & 50 \\
\hline $\mathrm{PO}_{4}{ }^{3-}(\mathrm{mg} / \mathrm{L})$ & 0.10 & 0.24 & 0.18 & 0.054 & - & - & 10 \\
\hline As (mg/L) & 0.35 & 1.20 & 0.67 & 0.283 & 0.01 & 0.05 & - \\
\hline $\mathrm{Cu}(\mathrm{mg} / \mathrm{L})$ & 0.016 & 0.158 & 0.056 & 0.0497 & 1 & 0.05 & - \\
\hline $\mathrm{Ni}(\mathrm{mg} / \mathrm{L})$ & 0.008 & 0.020 & 0.012 & 0.00335 & 0.02 & - & - \\
\hline $\mathrm{Fe}(\mathrm{mg} / \mathrm{L})$ & 0.362 & 2.890 & 0.792 & 0.803 & 0.3 & 0.3 & 0.3 \\
\hline $\mathrm{Pb}(\mathrm{mg} / \mathrm{L})$ & 0.00 & 0.058 & 0.008 & 0.0189 & 0.01 & 0.05 & - \\
\hline $\mathrm{Zn}(\mathrm{mg} / \mathrm{L})$ & 0.003 & 0.256 & 0.0445 & 0.0862 & 3 & 5 & - \\
\hline $\mathrm{Cr}^{6+}(\mathrm{mg} / \mathrm{L})$ & 0.000 & 0.088 & 0.0196 & 0.0313 & 0.05 & 0.05 & - \\
\hline $\mathrm{Cd}(\mathrm{mg} / \mathrm{L})$ & 0.000 & 0.002 & 0.000182 & 0.000603 & 0.003 & 0.01 & - \\
\hline $\mathrm{Mn}(\mathrm{mg} / \mathrm{L})$ & 0.000 & 0.330 & 0.129 & 0.115 & 0.200 & 0.1 & 0.1 \\
\hline
\end{tabular}

Note: DO-dissolved oxygen, TDS-total dissolved solids, EC-electrical conductivity, TA-total alkalinity, TH-total hardness as $\mathrm{CaCO}_{3}$.

On the other hand, negative correlations existed among the following pairs of parameters: Temp-pH (-0.537), Temp-TDS (-0.462), Temp-EC (-0.197), Temp-TA (-0.043), Temp-Cl (-0.088), Temp-SO 4 (-0.208), Temp- $\mathrm{PO}_{4}$ (-0.299), $\mathrm{pH}-\mathrm{TH}(-0.179), \mathrm{pH}-\mathrm{Mg}(-0.21)$,

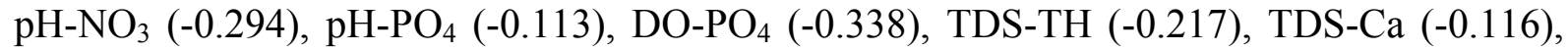
TDS-Mg (-0.225), TDS-NO 3 (-0.452), TDS-PO 4 (-0.221), EC-TH (-0.079), EC-Ca (-0.041), EC-Mg (-0.084), EC-NO (-0.393), EC-PO $_{4}(-0.424)$, TA-NO ${ }_{3}(-0.23), \mathrm{TA}_{3} \mathrm{PO}_{4}(-0.71)$, TH.SO 4 (-0.14), TH.PO 4 (-0.41), Ca-PO (-0.14), Mg-SO $(-0.09), \mathrm{SO}_{4}-\mathrm{NO}_{3}(-0.3)$. This trend infers that the pairs of concerned ions involved came from different natural and anthropogenic sources.

One-way analysis of variance (ANOVA) tests were conducted to determine whether significant differences existed between the physico-chemical parameters, cations/anions and trace metals data generated during this study. In the case of physico-chemical parameters, the ANOVA test at $\mathrm{P}=0.05$ shows that $\mathrm{F}=1.004<\mathrm{Fcrit}=1.977$, implying that no significant differences exist between the physico-chemical parameters in the groundwater samples, irrespective of the sampling location. Similarly, subjecting the data generated from determination of major cations and anions in the groundwater samples to the ANOVA test at 
$\mathrm{P}=0.05$ shows that $\mathrm{F}=0.547<\mathrm{F}$ crit $=2.008$, implying that no significant differences exist in the results, irrespective of sampling location. Further, with regard to trace metals, subjecting the data generated to the ANOVA test at $\mathrm{P}=0.05$ shows that $\mathrm{F}=0.772<$ Fcrit $=1.940$, suggesting that no significant differences exist in the results, regardless of borehole location.

Table 5. Pearson correlation coefficient matrix of measured parameters in groundwater samples.

\begin{tabular}{|c|c|c|c|c|c|c|c|c|c|c|c|c|c|}
\hline & Temp & pH & DO & TDS & EC & TA & TH & $\mathrm{Ca}^{2+}$ & $\mathbf{M g}^{2+}$ & $\mathrm{Cl}^{-}$ & $\mathrm{SO}_{4}{ }^{2-}$ & $\mathrm{NO}_{3}^{-}$ & $\mathrm{PO}_{4}{ }^{3-}$ \\
\hline Temp & 1 & & & & & & & & & & & & \\
\hline $\mathrm{pH}$ & -0.537 & 1 & & & & & & & & & & & \\
\hline DO & 0.224 & 0.600 & 1 & & & & & & & & & & \\
\hline TDS & -0.462 & 0.906 & 0.4991 & 1 & & & & & & & & & \\
\hline EC & -0.197 & 0.788 & 0.4724 & 0.810 & 1 & & & & & & & & \\
\hline TA & -0.043 & 0.625 & 0.6007 & 0.584 & 0.791 & 1 & & & & & & & \\
\hline $\mathrm{TH}$ & 0.517 & -0.179 & 0.4843 & -0.217 & -0.079 & 0.260 & 1 & & & & & & \\
\hline $\mathrm{Ca}^{2+}$ & 0.398 & 0.022 & 0.5105 & -0.116 & -0.041 & 0.207 & 0.700 & 1 & & & & & \\
\hline $\mathrm{Mg}^{2+}$ & 0.504 & -0.21 & 0.4419 & -0.225 & -0.084 & 0.250 & 0.988 & 0.581 & 1 & & & & \\
\hline $\mathrm{Cl}^{-}$ & -0.088 & 0.636 & 0.6789 & 0.528 & 0.599 & 0.610 & 0.405 & 0.429 & 0.367 & 1 & & & \\
\hline $\mathrm{SO}_{4}^{2-}$ & -0.208 & 0.659 & 0.5500 & 0.583 & 0.494 & 0.343 & -0.140 & 0.086 & -0.180 & 0.433 & 1 & & \\
\hline $\mathrm{NO}_{3}^{-}$ & 0.183 & -0.294 & 0.1469 & -0.452 & -0.393 & -0.230 & 0.474 & 0.086 & 0.524 & 0.158 & -0.300 & 1 & \\
\hline $\mathrm{PO}_{4}^{3-}$ & -0.299 & -0.113 & -0.338 & -0.221 & -0.424 & -0.710 & -0.410 & -0.140 & -0.430 & -0.090 & 0.223 & 0.1412 & 1 \\
\hline
\end{tabular}

\section{CONCLUSION}

Hydrochemical analyses revealed that the groundwater within Ngbo area of Ebonyi State and its environs is slightly alkaline, but mostly hard in nature and highly mineralized. All of the parameters investigated in this study fall within national (NIS, BIS) and international (WHO) standards for potable water except for total alkalinity, $\mathrm{Mg}, \mathrm{As}, \mathrm{Cu}$ and Fe levels in the samples. Magnesium and iron values were exceptionally high in some of the groundwater samples studied and a possible treatment procedure involves the periodic exposure of the water samples to air in order to allow the concerned ions to precipitate. Precipitates formed may then be filtered out to obtain potable water. Further, the slightly high average electrical conductivity $(718.45 \mu \mathrm{S} / \mathrm{cm})$ of the water samples implies that some of them are saline rather than fresh in nature. This implies that all the groundwater samples are suitable for irrigation purposes, though not all of them are suitable as a source of drinking water. Also, slightly high total dissolved solids and chloride concentrations imply possible encroachment of saltwater into the groundwater. To mitigate this challenge, regular flushing of boreholes is recommended in order to aid in the removal of mineralized deposits accumulated in the groundwater over time. In conclusion, there is a need for regular hydrogeochemical studies in the study area in order to detect any future deterioration of groundwater quality.

\section{REFERENCES}

ABAM, T. K. S. Regional hydrological research perspective in the Niger Delta. Hydrological Sciences Journal, v. 46, n. 1, 2001. http://dx.doi.org/10.1080/02626660109492797 
ACHI, C. Hydrocarbon exploitation, Environmental degradation and Poverty: The Niger Delta Experience. In: ENVIRONMENTAL POLLUTION CONFERENCE, 2003, Dublin. Proceedings... Dublin: [s.n.], 2003. p. 78-94.

AKUJIEZE, C. N.; COKER, S. J. L.; OTEZE, G. E. Groundwater in Nigeria-A millennium experience: distribution, practice, problems and solutions. Hydrogeology Journal, v. 11, n. 2, p. 259-274, 2003. http://dx.doi.org/10.1007/s10040-002-0227-3

AMADI, A. N.; OLASEHINDE, P. I.; YISA, J.; OKOSUN, E. A.; NWANKWOALA, H. O.; ALKALI, Y. B. Geostatistical assessment of groundwater quality of coastal aquifers of Eastern Niger Delta. Geosciences, v. 2, n. 3, p. 51-59, 2012. http://dx.doi.org/10.5923/j.geo.20120203.03

AMERICAN PUBLIC HEALTH ASSOCIATION - APHA; AMERICAN WATER WORKS ASSOCIATION - AWWA; WATER POLLUTION CONTROL FEDERATION WPCF. Standard methods for the examination of water and waste water. $22^{\text {th }}$ ed. Washington DC, 2012.

AMERICAN SOCIETY TESTING MATERIALS - ASTM Annual Book of ASTM Standards. 2012. Vol. 11.01. Available in: http://www.astm.org. Access in: Oct. 2014.

BOYLE, D. R. Application of groundwater geochemistry in mineral exploration. In: BERRY, M. J. (Ed.). Proceedings of Current Activities Forum. Dartmouth: Geological Survey of Canada, 1988.

FREEZE, R. A.; CHERRY, J. A. Groundwater. Englewood Cliffs: Prentice-Hall, 1979.

GILLY, G.; CORRAO, G.; FAVILLI, S. Concentrations of nitrates in drinking water and incidence of gastric carcinomas: First descriptive study of the Piemonate Region, Italy. Science of the Total Environment, v. 34, p. 35-37, 1984.

HANIDU, J. A. National growth, water demand and supply strategies in Nigeria in the 1990s. Water Resource Journal of Nigerian Association of Hydrogeologists, V. 2, n. 1, p. $1-6,1990$.

HEM, J. D. Study and interpretation of the chemical characteristics of natural water. Water Supply Paper, n. 2254, 1985. (Technical Paper n. 2254)

INDIA. Public Health Engineering Department. Bureau of Indian Standard. Specification for Drinking Water (BIS: 105001991). West Bengal, 2006.

LANGENEGGAR, O. Groundwater quality in rural areas of Western Africa. UNDPProject INT/81/026. Abdijan: World Bank; Regional Water and Sanitation Group, 1990. p.10

NATIONAL POPULATION COMMISSION - NPC. National Population and Housing Census. [S.I.], 2006.

STANDARDS ORGANIZATION OF NIGERIA - SON. Nigerian Standard for Drinking Water Quality: Nigerian Industrial Standard (NIS: 554). [S.1.], 2007. p. 30.

NWANKWOALA, H. O.; OKEKE E. V.; OKEREKE S. C. Groundwater quality in parts of Port-Harcourt, Nigeria: an overview, trends and concerns. International Journal of Biotechnology and Allied Sciences, v. 2, n. 3, p. 282-289, 2007. 
NWANKWOALA, H. O; UDOM, G. J. Investigation of hydrogeochemical characteristics of groundwater in Port- Harcourt City, Nigeria: implication for use and vulnerability. Journal of Applied Science and Environmental Management, v. 15, n. 3, p. 479488, 2011.

OBATOYINBO, O.; OYEDOTUN, T. D. T. Microbial/bacteriological analysis of water resources in Ikare and Arigidi Akoko of Ondo state, Nigeria. In: EIT INTERNATIONAL CONFERENCE ON WATER RESOURCES ENGINEERING, 18 19 August, Cha-Am. Proceedings... [S.1.: s.n.], 2011. Vol. 1. p. 279-284.

OKAGBUE, C. O. Hydrology and chemical characteristics of surface and groundwater resources of the Okigwe area and environs, Imo State, Nigeria. In: OFOEGBU, C. O. (Ed.). Groundwater and mineral resources of Nigeria. Braunschweig: Vieweg and Sohn, 1988. p. 3-16.

OKIONGBO, K. S.; DOUGLAS, R. Hydrogeochemical analysis and evaluation of groundwater quality in Yenagoa City and Environs, Southern Nigeria. Ife Journal of Science, v. 15, n. 2, p. 209-222, 2013.

OlATUNJI, A. S.; ABIMBOlA, A. F.; OlORUNTOLA, M. O.; ODEWADE, A. A. Hydrogeochemical evaluation of groundwater resources in shallow coastal aquifers around Ikorodu area, Southwestern Nigeria. Water Resources, v. 16, p. 65-71, 2005.

OMAKA O. N. Atmospheric and Metallic Pollutants and their impacts on the Environment: Case Study of Abakaliki Metropolis. Journal of Applied and Natural Sciences (JANS), v. 4, n. 1, p. 24-29, 2010.

OYEDOTUN, T. D. T.; OBATOYINBO, O. Hydrogeochemical evaluation of groundwater quality in Akoko North West Local Government Area of Ondo State, Nigeria. Revista Ambiente \& Água, Taubaté, v. 7, n. 1, p. 67-80, 2012. http://dx.doi.org/10.4136/ambiagua. 851

RAGUNATH, H. M. Groundwater. New Delhi: Wiley Eastern, 1987. p. 563.

RAMANATHAN, A. L. Water quality: guidelines and assessment of risk. New Delhi: School of Environmental Sciences, 2004. p. 110-167.

RIJSWIJK, K. Small community water supplies. The Hague: IRC, 1981 (Technical paper 18).

SALEH, A.; AL-RUWATH, F.; SHEHATA, M. Hydrogeochemical processes operating within the main aquifers of Kuwait. Journal of Arid Environment, v. 42, p. 195-209, 1999. http://dx.doi.org/10.1006/jare.1999.0511

TALABI A. O.; TIJANI, M. N. Assessment of groundwater quality in parts of the basement complex terrain of Southwestern Nigeria. GQ10: Groundwater quality management in a rapidly changing world. In: INTERNATIONAL GROUNDWATER QUALITY CONFERENCE, 7., 13-18 June 2010, Zurich. Proceedings...Zurich: IAHS, 2011. p. 503-506. (IAHS Publication, 342).

TIJANI, M. N. Hydrogeochemical assessment of groundwater in Moro are, Kwara State, Nigeria. Environmental Geology, v. 24, n. 3, p. 194-202, 1994. http://dx.doi.org/10.1007/BF00766889

TINDALL, J. A.; PETRUSAK, R. L.; MCMOHAN, P. B. Nitrate transport and transportation process in unsaturated porous media. Journal of Hydrology, v. 169, p. 51-94, 1995. 
TWORT, A. C.; RATNAYAKA, D. D.; BRADT M. J. Water Supply. $5^{\text {th }}$ ed. London: Arnold and IWA Publishing, 2000.

WORLD HEALTH ORGANIZATION - WHO Guidelines for drinking water quality criteria. $4^{\text {th }}$ ed. Geneva, 2011. p. 307-441. 


Ambiente \& Água - An Interdisciplinary Journal of Applied Science
ISSN 1980-993X - doi:10.4136/1980-993X
www.ambi-agua.net
E-mail: ambi-agua@agro.unitau.br

\title{
Induction of cytotoxic and genotoxic effects of Guandu River waters in the Allium cepa system
}

\author{
doi: 10.4136/ambi-agua.1487
}

Received: 15 Aug. 2014; Accepted: 23 Oct. 2014

\author{
Jennifer Vieira Gomes; Jéssica Tamara dos Santos Teixeira; \\ Viviane Moreira de Lima*; Helcio Resende Borba \\ Universidade Federal Rural do Rio de Janeiro, Seropédica, RJ, Brasil \\ Instituto de Biologia, Departamento de Biologia Animal \\ *Corresponding author: e-mail: vilima@ hotmail.com, \\ vieira.jb@hotmail.com, jteixeira2911@gmail.com, borba@ufrrj.br
}

\begin{abstract}
The Guandu River is the main source of water supply for the metropolitan region of Rio de Janeiro and has been facing serious environmental problems due to increasing population and industrial pollution, as well as the presence of polluted tributaries. This study analyzed the cytotoxic and genotoxic potential of the Guandu River's waters, through the use of the Allium cepa test system. Collection points were chosen at the greatest confluences of pollutant sources. The sampling included two different seasons: the rainy season (January and February) and the dry season (June and July). The analyses of 5000 cells per treatment showed that all the points studied had some degree of cytotoxicity and/or genotoxicity. Two sampling locations, which receive major influxes from the polluted waters of the Poços/Queimados and Cabuçu/Ipiranga Rivers, stood out for the strong presence of micronuclei, sticky chromosomes, mitotic spindle abnormalities, necrotic cells and nucleolar changes compared to the negative control. At least two locations also found changes in the mitotic index. The existence of variations in the number of cytotoxic and genotoxic changes between periods of rain and drought indicates that the cytotoxic and genotoxic potential of the water pollutants varies according to time, depending on the discharges of the tributary rivers and the increase of contaminated effluents. The results highlight the importance of bio-monitoring to assist managers in the control of effluent discharge.
\end{abstract}

Keywords: Allium cepa, cytotoxicity, genotoxicity.

\section{Indução de efeito citotóxico e genotóxico das águas do Rio Guandu em sistema Allium cepa}

\section{RESUMO}

O Rio Guandu é a principal fonte de água para abastecimento da região metropolitana do Rio de Janeiro e enfrenta sérios problemas ambientais devido à crescente poluição tanto de origem populacional quanto industrial acentuada pela presença de afluentes com águas poluídas. Este trabalho teve por objetivo analisar o potencial citotóxico e genotóxico das águas do Rio Guandu, utilizando para isto o sistema teste de Allium cepa. Foram escolhidos pontos de coleta nos quais ocorrem as maiores confluências de fontes poluidoras. As coletas 
foram realizadas em duas épocas distintas: na estação chuvosa (Janeiro e Fevereiro) e na estação de estiagem (Junho e Julho). As análises realizadas em 5000 células por tratamento demonstraram que todos os pontos estudados apresentaram algum nível de citotoxicidade e ou genotoxicidade. Dois locais de coleta, os quais recebem maiores influências das águas poluídas dos rios Poços/Queimados e Cabuçu/Ipiranga, se destacaram quanto à presença acentuada de micronúcleos, aderência cromossômica, anormalidades no fuso mitótico, células em necrose e alterações nucleolares em relação ao controle negativo. Foram encontradas também alterações no índice mitótico em pelo menos dois locais. A existência de variações no número de alterações citotóxicas e genotóxicas entre os períodos chuvosos e de seca indicam que o potencial citotóxico e ou genotóxico dos poluentes na água variam em função do tempo, dependendo das descargas vindas dos afluentes e do aumento na emissão de efluentes contaminados. Estes resultados ressaltam a importância de estudos de biomonitoramento que auxiliem as ações de manejo visando o controle do lançamento de efluentes.

Palavras-chave: Allium cepa, citotoxicidade, genotoxicidade.

\section{INTRODUCTION}

The Guandu River is the main source of water supply for the Rio de Janeiro metropolitan region. The Guandu River Basin, located west of the Guanabara Bay, covers an area of $3.600 \mathrm{~km}^{2}$, including 15 municipalities, and is the only water source for subsistence and for the development of the metropolitan region of the state (Bruno, 2012). Guandu River is $48 \mathrm{~km}$ long, and formed by the joining of the Santana and Ribeirão das Lages Rivers; it discharges into Sepetiba Bay. Much of its water comes from the Rio Paraiba do Sul, through a transposition that occurs in the municipality of Barra do Pirai (Salamene, 2007).

Despite its importance, the Guandu River faces serious environmental problems due to unplanned urban development in the municipalities of the Guandu Basin, the release of domestic and industrial effluents, illegal solid waste disposal and illegal sand extraction. This pollution is further enhanced by the joining of Guandu River with the Poços/Queimados and the Cabuçu/Ipiranga Rivers, the waters of which are very polluted. This joining occurs in the Guandu Lagoon, which is formed by CEDAE dams, and which is connected to the Guandu River and has low rate of water renewal due to its configuration (Serber, 2005; Salamene, 2007). In addition, the Paraíba do Sul River, the main water contributor to the Guandu River, is polluted with domestic and industrial untreated effluents as well as agricultural waste (Oliveira et al., 2011). All of these factors affect the quality of the water that is treated at the Guandu Water Treatment Station (WTS). There are also several quality of life issues. For example, industrial, agricultural and urban residues contain organic and inorganic compounds that often are not degraded during the water treatment process, and some of these are genotoxic (Nielsen and Rank, 1994; Oliveira et al., 2011). Also, the increasing reduction of water quality may in the near future render it very difficult to treat (Serber, 2005). The Allium cepa test system is used as a bioindicator in studies evaluating environmental genotoxic potential (Barbério et al., 2009; Leme and Marin-Morales, 2009) and was validated by the International Programme of Chemical Safety (IPCS, WHO) and the United Nations Environment Programme (UNEP) as an effective test for analysis and in situ monitoring of the cytotoxicity and genotoxicity of environmental substances (Bagatini et al., 2007), as indicated by several authors (Nielsen and Rank, 1994; Grover and Kaur, 1999; Leme and Marin-Morales, 2008; Hoshina and Marin-Morales, 2009; Barbério et al., 2009; Barbosa et al., 2010; Radic et al., 2010; Oliveira et al., 2011).

This study analyzed the water of Guandu River at specific locations during the dry and rainy seasons in order to evaluate cytotoxic and genotoxic effects. 


\section{MATERIAL AND METHODS}

\subsection{Collection sites}

Samples were collected at three locations prior to capitation by the Guandu Water Treatment Station (WTS) and where the Guandu River receives significant influxes of water from polluted tributaries as follows: Local A - the Ipiranga River at the point where it joins the Guandu Lagoon ( $22^{\circ} 48^{\prime} 10.2^{\prime}$ S, $43^{\circ} 37^{\prime} 24.0^{\prime}$ ' O); Local B - the Guandu Lagoon after the joining of the Poços and Ipiranga Rivers (22 $\left.48^{\prime} 20.9^{\prime \prime} \mathrm{S}, 43^{\circ} 37^{\prime} 38.8^{\prime \prime} \mathrm{O}\right)$ and Local $\mathrm{C}$ - the Ribeirão das Lages River following the influx of the Paraíba do Sul River

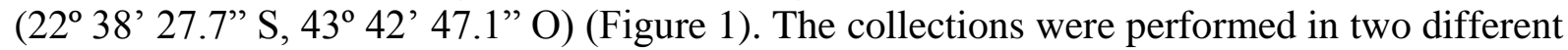
seasons: dry season (June and July 2012) and the rainy season (January and February 2013).
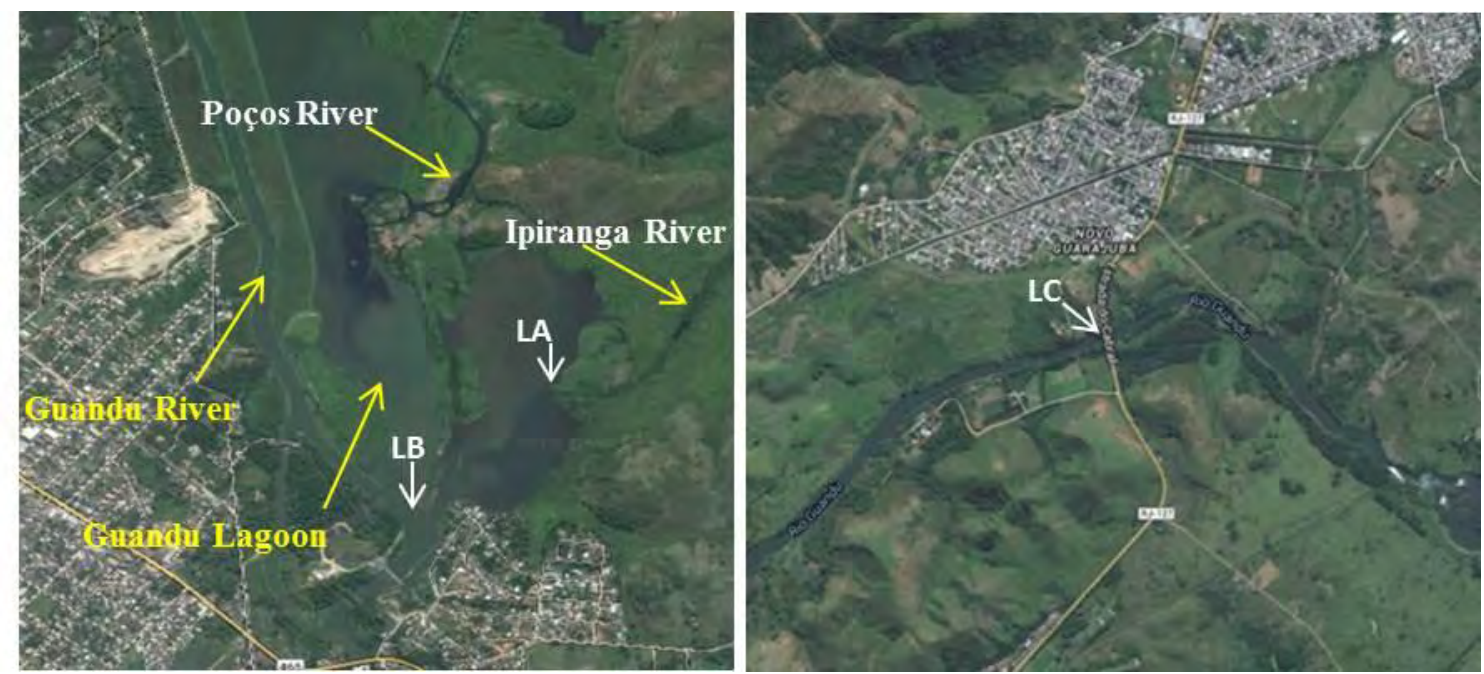

Figure 1. Collection sites - Local A (LA) - the Ipiranga River at the point that it joins the Guandu Lagoon; Local B (LB) - the Guandu Lagoon after the influx of the Poços and Ipiranga Rivers; Local C (LC) - the Ribeirão das Lages River after the influx of the Paraíba do Sul River.

\subsection{Sample collection}

Samples from each site were collected with the aid of a $10 \mathrm{~L}$ bucket and transferred to polyethylene flasks ( $1 \mathrm{~L})$ that were properly identified and kept in coolers with ice for transport to the laboratory, where they were kept under refrigeration $\left(4^{\circ} \mathrm{C}\right)$ for further genotoxicity and cytotoxicity analysis.

\subsection{Cytotoxicity and genotoxicity tests}

Commercially acquired organic cultured onion bulbs approximately $2.0 \mathrm{~cm}$ in diameter were used to test performance. A stylet was used to remove external catafilo from each bulb without damaging the root buds. Five onion bulbs were used for water samples from each locale, as well as positive and negative controls. Initially, the bulbs were placed into a container with distilled water for 48 hours, with daily water exchanges. The bulbs were then transferred to tubes containing water samples collected at the locales previously mentioned, where they remained for 48 hours. The bulbs used as negative controls were kept in distilled water and the positive ones were placed in an ethylmethane sulfonate (EMS, $25 \mathrm{mM}$ ) solution. The tests and the control bulbs were incubated at $25^{\circ} \mathrm{C}$ with daily water exchanges. For each treatment, 2 and $2.5 \mathrm{~cm}$ roots apical meristems were removed from the bulbs after 48 hours of exposure to the solution and were processed according Iganci et al. (2006) for slide preparation with some modifications. Meristems were fixed on ethanol: glacial acetic acid solution in a 3:1 ratio (V/V) and stored at $4^{\circ} \mathrm{C}$ until slide preparation. Five meristems 
were used to prepare five slides for each bulb. For slide performance, meristems were twice washed in distilled water for $5 \mathrm{~min}$, hydrolyzed with $5 \mathrm{~N} \mathrm{HCl}$ for $30 \mathrm{~min}$, then again twice washed in distilled water for $5 \mathrm{~min}$ and stained with $2 \%$ acetic orcein. Meristems regions were fragmented with a scalpel blade and the coverslip was placed and pressed over the material. Slides were observed on a 100X optical microscope. The presence of cellular and chromosomal abnormalities was analyzed at 1000 cells/bulb for a total of 5000 cell treatment.

\subsection{Analysis of the potential for the induction of cell death}

Onion roots submitted to various treatments, as described above, were analyzed for the ability to induce cell death using propidium iodide and the 33342 Hoescht staining technique (Locke et al., 1990). Longitudinal cuts were made in the roots' meristematic regions. A drop of Hoescht $\left(10 \mu \mathrm{g} \mathrm{mL}^{-1}\right)$ dye was added to each slide for the staining technique. After $10 \mathrm{~min}$, the material was transferred to another slide and submitted to a drop of propidium iodide $\left(30 \mu \mathrm{g} \mathrm{mL} \mathrm{m}^{-1}\right)$. Both incubations were protected from light. As a positive control, roots submitted to fixing (ethanol: glacial acetic acid on 3:1 ratio) were used, since fixing promotes cell death. Subsequently, the material was analyzed, evaluated and photographed by a fluorescence microscope (Zeiss Axid Lab A1) under $365 \mathrm{~nm}$ and $400 \mathrm{~nm}$ excitation filters. According to Locke et al. (1990) 33342 Hoescht stains intact membrane cells while propidium iodide stains only the compromised ones. The nuclei with fluorescent blue cells were considered alive and intact, while those with fluorescent red nuclei were considered not intact, in a process of cell death.

\subsection{Statistical design}

Statistical analyses of data obtained through the Allium cepa test for the assessment of cytotoxicity and genotoxicity of the water samples were evaluated by the $\chi^{2}$ test, using the Yates correction, with Bioestat 5.0 software.

\section{RESULTS AND DISCUSSION}

The principal use of the Guandu River waters is for the intake of drinking water for treatment. Water treated at the WTS (Water Treatment Station) has served $80 \%$ of the metropolitan region of Rio de Janeiro's population. However, for this treatment be effective, it is necessary that the so-called "raw water" does not reach certain levels of pollution (Oliveira et al., 2011).

\subsection{Cytotoxicity and genotoxicity}

Analysis showed the presence of micronuclei, chromosomal fragment loss, chromosomal bridges, sticky chromosomes and abnormalities in mitotic spindle, including in this group cells with c-metaphase, polyploide metaphase, irregular metaphase, multipolar anaphase, delayed anaphase, vagrant chromosome of the chromatinic group and binucleated cells. Necrotic cells and nucleolar changes, with the presence of large nucleoli cells, were also observed, this being one of the representative changes from the positive control (Figure 2).

During the dry season (June and July), at Locale B at the Guandu Lagoon, the mixture formed after the confluence of the Poços/Queimados and Ipiranga Rivers presented a higher number of necrotic and micronucleated cells in relation to the negative control (Table 1), which is indicative of the presence of pollutants with cytotoxic and genotoxic potential. Micronuclei are formed in dividing cells presenting acentric chromosome fragments and/or whole chromosomes that disrupted the ability to bind to mitotic spindle, indicating the presence of substances with clastogenic and aneugenic effects (Fenech, 2000). The locale where the Ipiranga River joins with the Guandu Lagoon (Locale A) presented a significant number of cells with nucleolar changes when compared to the negative control (Table 1). 

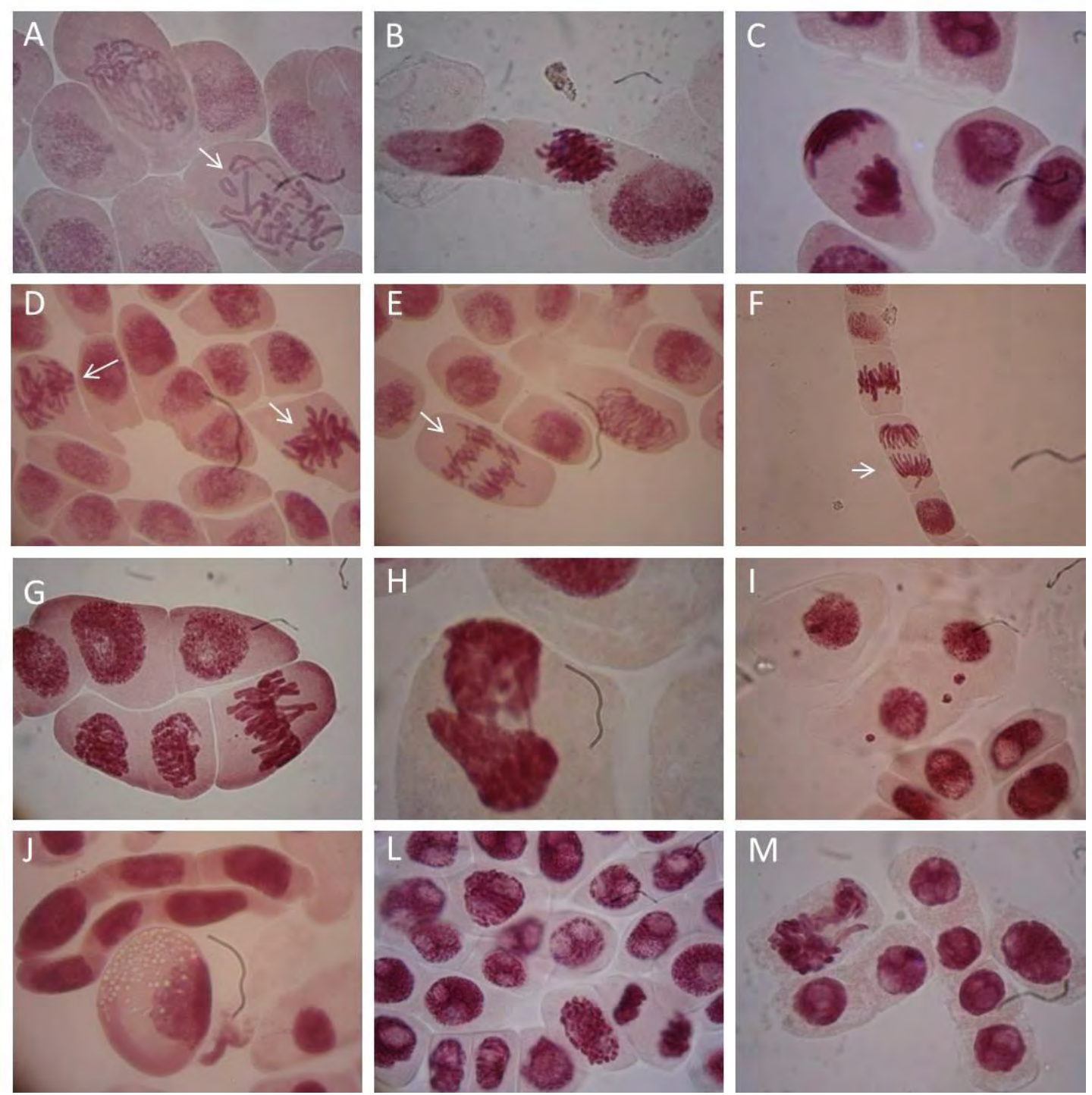

Figure 2. Allium cepa meristematic cells exposed to ethyl methane sulfonate (EMS) (M) and water samples collected at different sites (A-L); (A) c-metaphases (arrow); (B-C) sticky chromosomes; (D-E) spindle abnormalities (arrow); (F) vagrant chromosome (arrow); (G) irregular metaphase; (H) chromosomal bridge; (I) micronucleated cells; (J) necrotic cells; (L-M) nucleolar changes.

Nucleolar changes are characteristic in cells treated with the positive control EMS employed in the present survey. EMS is a highly efficient mutagenic agent directly acting on the DNA molecule through its alkylating activity. These sort of changes are the most common criteria used as an indication of malignancy, differentiating normal cells from the malignant ones with the malignant cells nuclei presenting a variable nucleoli number of different sizes and shapes (Oshima and Forones, 2001; Filippin et al., 2006; Montanaro et al., 2008). Locale C, the Ribeirão das Lages River following the influx of the Paraíba do Sul River showed no significant changes (Table 1).

During the rainy season (January and February), the sample that stood out with significant changes was Locale A, which showed a higher number of cells with chromosomal abnormalities in the mitotic spindle and sticky chromosomes compared to the negative control (Table 2). Sticky chromosomes are characterized by chromatic clustering involving few chromosomes or the whole genome, possibly resulting in chromatin degeneration and cell death. Thus, detection of such abnormalities has indicated the presence of toxic substances at 
this site. Locale $\mathrm{C}$ presented a greater number of nucleolar changes (Table 2). In this season, water samples collected at Locale B showed no significant changes when compared to the negative control (Table 2). This result was probably influenced by a high rainfall level during the collection time, since Locale B (the Guandu Lagoon) is formed by the confluence of the Guandu River with the Poços/Queimados and Ipiranga Rivers and thus, depending on the collection site, a greater influence of the Guandu River's waters might be possible, where pollution indicators are lower than those of the Guandu Lagoon's tributaries (Coelho et al., 2012).

Table 1. Cells changes and mitotic index in A. cepa roots submitted to different treatments during the dry season.

\begin{tabular}{llccccc}
\hline Treatment & Concentration & LN & LNB & NEC & MC & ID (\%) \\
\hline Negative control & Distilled water & $163^{(\mathrm{b})}$ & $1^{(\mathrm{b})}$ & 13 & 7 & 6.7 \\
EMS & $25 \mathrm{mM}$ & $1224^{(\mathrm{a})}$ & $169^{(\mathrm{a})}$ & $58^{(\mathrm{a})}$ & 7 & 4.5 \\
Local A & & $\mathbf{2 7 2}^{(\mathrm{a})}$ & 2 & 19 & 8 & 7.7 \\
Local B & & 183 & $\mathbf{9}^{(\mathrm{a})}$ & $\mathbf{3 0}^{(\mathrm{a})}$ & $\mathbf{2 0}^{\text {(b)(a) }}$ & 5.9 \\
Local C & & $95^{(\mathrm{a})(\mathrm{b})}$ & $0^{(\mathrm{b})}$ & 12 & 2 & $\mathbf{1 0 . 4}$ \\
\hline
\end{tabular}

Note: ${ }^{(a)}$ Significant difference compared to negative control $(\mathrm{P}<0,05)$ according to the $\chi^{2}$ test;

(b) Significant difference compared to positive control $(\mathrm{P}<0,05)$ according to the $\chi^{2}$ test; $\mathbf{L N}$ - one or more large nucleoli; LNB - one or more large nucleoli and nuclear bud; NEC - necrotic cells; MC - micronucleated cells; ID - mitotic index. 5000 cells for each treatment were analyzed.

Mitotic index analysis of the onions' meristematic cells submitted to different water samples in the dry and rainy seasons was also employed for cytotoxicity evaluation. In both seasons, Locale $\mathrm{C}$ presented a significantly greater mitotic index than that observed in the negative control (Table 1 and Table 2). However, Locale A presented a significantly greater mitotic index than that observed in the negative control, in the rainy season only (Table 2). No significative changes were reported in regard to the mitotic index for Locale B.

Table 2. Cells changes and mitotic index in A. cepa roots submitted to diferent treatments during the rainy season.

\begin{tabular}{llllllc}
\hline Treatment & Concentration & LN & LNB & SA & SC & ID (\%) \\
\hline Negative control & Distilled water & $229^{(\text {b) }}$ & $4^{(\mathrm{b})}$ & 8 & 2 & 2.3 \\
EMS & $25 \mathrm{mM}$ & $572^{(\mathrm{a})}$ & $27^{(\mathrm{a})}$ & 13 & 8 & 3.4 \\
Local A & & 198 & 13 & $\mathbf{2 9}^{(\text {a) }}$ & $\mathbf{1 3}^{(\mathrm{a})}$ & $\mathbf{4 . 3}$ \\
Local B & & 183 & 3 & 11 & 6 & 2.8 \\
Local C & & $\mathbf{3 0 0}^{(\mathrm{a})}$ & 9 & 14 & 8 & $\mathbf{5 . 5}$ \\
\hline
\end{tabular}

Note: ${ }^{\text {(a) }}$ Significant difference compared to negative control $(\mathrm{P}<0,05)$ according to $\chi^{2}$ test; (b) Significant difference compared to positive control $(\mathrm{P}<0,05)$ according to $\chi^{2}$ test; $\mathbf{L} \mathbf{N}$ - one or more large nucleoli; LNB - one or more large nucleoli and nuclear bud; SA - spindle abnormalities; SC - sticky chromosomes; ID - mitotic index. 5000 cells for each treatment were analyzed.

The increase or decrease of the mitotic index might be a relevant indicator for monitoring pollution levels of contaminated environments, especially those contaminated by potentially toxic and cytotoxic compounds (Fernandes et al., 2007; Caritá and Marin-Morales, 2008). 
Greater mitotic indices than those reported in the negative control are from the induction of increased cell division, which characterizes cell damage, producing uncontrolled proliferation and eventually causing further tumor development. Smaller mitotic indices than those in the negative control might indicate that the growth and development of organisms is being affected by components in the samples (Hoshina, 2002; Caritá and Marin-Morales, 2008).

Despite the alterations induced by the water samples from Locale B during the dry season, it no changes were observed in the mitotic index. This is because the cytotoxic agents are able to act on the cell in a broad manner, acting on its compartments, the organization of its components and can thus cause morphological, biochemical and physiological changes that can affect the cell cycle or induce death cell (Kaioumova et al., 2001; Costa and Huck, 2006; Capobiango et al., 2009). These changes may also lead indirectly to changes in the genetic material; however, a cytotoxic effect is not necessarily associated with a genotoxic effect (Oliveira et al., 2011), which also explains the fact that no genotoxic changes were detected in the meristematic cells treated with the water sample from the Locale $\mathrm{C}$ during the dry season, although there was a change in the mitotic index.

\subsection{Induction test for cell death}

Considering that water collected from Locale B during the dry period presented a significant number of necrotic cells when compared to the negative control (Table 1), the presence of components which induce cell death was confirmed through the 3342 Hoescht and propidium iodide dyes. Both dyes presented a specific staining, clearly observed by the colors red (for dead cells) and green/blue (for live cells) (Collins and Donoghue, 1999). In this analysis, Locale B presented more extensive cell death when compared to the negative control (Figure 3).
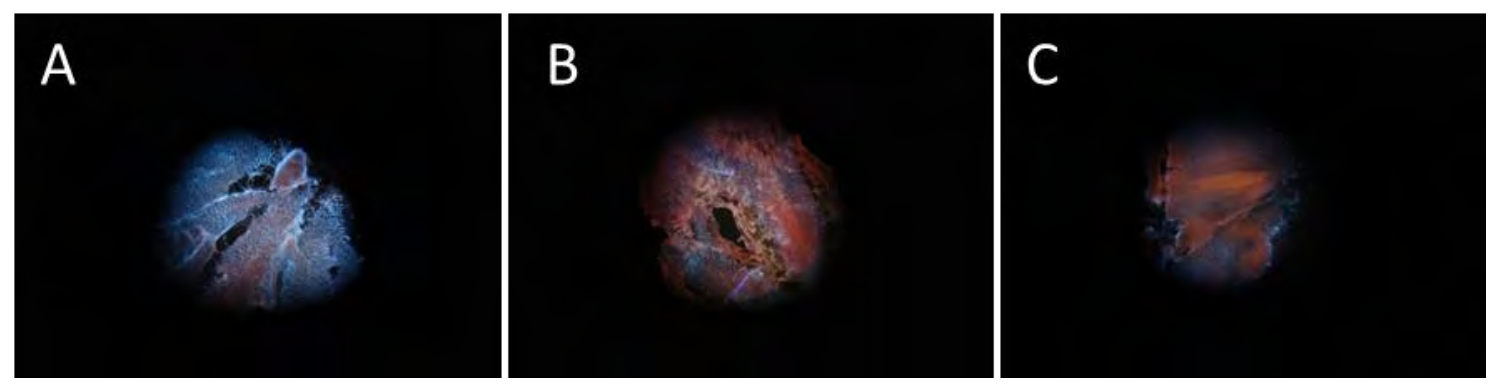

Figure 3. Allium cepa meristematic cells submitted to different treatments: (A) negative control; (B) fixed cells (positive control); (C) cells exposed to water collected in drought period from Locale B.

Necrosis is not determined by factor intrinsic to cells, but by environmental disturbances, indicating toxic chemicals or toxins promoting cells lysis (Hoshina and Marin-Morales, 2009).

Variations in the number of cytotoxic and genotoxic changes between both seasons, such as those observed on this survey, have also been described by other authors regarding river water from other Brazilian locations (Caritá and Marin-Morales, 2008; Oliveira et al., 2011; Monteiro et al., 2014). Cytotoxic and genotoxic water pollutant potentials have ranged in accordance with time, depending on the discharges of the tributary rivers, as well as increases in the emissions of contaminated effluents. Moreover, high rainfall in summer might promote significant increases in organic matter and toxic compounds discharged into rivers, causing increased cytoxicity and genotoxicity during this season (Monteiro et al., 2014).

The results obtained in this study reflect the level of pollution and indicate the presence of pollutants with cytotoxic and genotoxic effects in the waters of the analyzed locales. 
The Poços, Queimados and Ipiranga Rivers have water of rather dubious quality. The Queimados River receives, untreated, the entire sewage from the urban area of Queimados city, in addition to the effluents generated by industrial activities, and flows into the Poços River; while the Ipiranga River receives a significant portion of effluents from the urban areas of Nova Iguaçu and Queimados (Serber, 2005). These tributaries, which flow into the Guandu Lagoon, present poor quality water, with values outside the limits allowed in monitored parameters, including: dissolved oxygen, total phosphorus, nitrate, ammonia nitrogen and biochemical oxygen demand (Weinberg, 2013; INEA, 2014)

Locale C (the Ribeirão das Lajes River following the influx of the Paraiba do Sul River) showed fewer changes compared to locales A and B, which conforms with the report of Coelho et al. (2012), which noted that the values of the indicators of pollution are much higher in the tributaries of the Guandu Lagoon than in the Guandu River upstream of the main dam CEDAE. At this locale, the Guandu River receives a large volume of water from the Paraíba do Sul River, which at this point has already been polluted by domestic and industrial untreated effluents and waste from agricultural sources (Oliveira et al., 2011). However, although this pollution is present, as also indicated by the observed changes in the mitotic index, it may be mitigated to some extent due to the water's transit time sedimentation occurring in the reservoirs of Light in Barra do Piraí, where the confluence occurs.

\section{CONCLUSIONS}

This survey detected the existence of potential cytotoxic and genotoxic in the waters of the analyzed locales. The observed cytotoxic and genotoxic changes reflect the state of contamination of the tributaries and water contributors of the Guandu River, and emphasize the importance of bio-monitoring studies to aid managers in controlling the release of domestic and industrial effluents. These components exhibit potent cytotoxic and/or genotoxic properties, some of which cannot be degraded during the treatment process, and may thus render even treated water unsafe for human use or consumption.

\section{ACKNOWLEDGMENTS}

Committee of the Guandu Basin and Paraíba do Sul River Basin Agency (AGEVAP) for financial support.

\section{REFERENCES}

BAGATINI, M. D.; DA SILVA, A. C. F.; TEDESCO, S. B. Uso do sistema teste de Allium cepa como bioindicador de genotoxicidade de infusões de plantas medicinais. Revista Brasileira de Farmacognosia, Curitiba, v. 17, n. 3, p. 444-447, 2007. http://dx.doi.org/10.1590/S0102-695X2007000300019

BARBÉRIO, A.; BARROS, L.; VOLTOLINI, J. C.; MELLO, M. L. S. Evaluation of the cytotoxic and genotoxic potential of water from the River Paraíba do Sul, in Brazil, with the Allium cepa L. test. Brazilian Journal of Biology, São Carlos, v. 69, n. 3, p. 837842, 2009. http://dx.doi.org/10.1590/S1519-69842009000400010

BARBOSA, J. S.; CABRAL, T. M.; FERREIRA, D. N.; AGNEZ-LIMA, L. F.; DE MEDEIROS, S. R. Genotoxicity assessment in aquatic environment impacted by the presence of heavy metals. Ecotoxicology Environmental Safety, New York, v. 73, p. 320-325, 2010. http://dx.doi.org/10.1016/j.ecoenv.2009.10.008 
BRUNO, A. A. G. Água, fonte de vida. In: COMITÊ GANDU. Bacia hidrográfica dos rios Guandu, da Guarda e Guandu-Mirim: experiências para a gestão dos recursos hídricos. Rio de Janeiro: INEA, 2012. p. 11-15.

CAPOBIANGO, R. A.; VESTENA, S.; BITTENCOURT, A. H. C. Alelopatia de Joanesia princeps Vell. e Casearia sylvestris Sw. Sob espécies cultivadas. Revista Brasileira de Farmacognosia, Curitiba, v. 19, n. 4, p. 924-930, 2009. http://dx.doi.org/10.1590/S0102-695X2009000600023

CARITÁ, R.; MARIN-MORALES, M. A. Induction of chromosome aberrations in the Allium cepa test system caused by the exposure of seeds to industrial effluents contamined with azo dyes. Chemosphere, New York, v. 72, p. 722-725, 2008.

http://dx.doi.org/10.1016/j.chemosphere.2008.03.056

COELHO, F. M.; DE AZEVEDO, J. P. S.; JÚNIOR, I. V. Análise multicritério de propostas para a melhoria da qualidade da água captada para abastecimento da Região Metropolitana oeste do Rio de Janeiro. In: COMITÊ GUANDU. Bacia hidrográfica dos rios Guandu, da Guarda e Guandu-Mirim: experiências para a gestão dos recursos hídricos. Rio de Janeiro: INEA, 2012. p. 61-77.

COLLINS, A. M.; DONOGHUE, A.M. Viability assessment of honey bee Apis mellifera, sperm using dual fluorescent staining. Theriogenology, New York, v. 51, n. 8, p. 15131523, 1999. http://dx.doi.org/10.1016/S0093-691X(99)00094-1

COSTA, C. A. S.; HUCK, C. E. Efeitos citotóxicos e biocompatibilidade de agentes clareadores usados na odontologia. Uma revisão de literatura. Revista Odontológica do Brasil Central, Goiânia, v. 15, n. 39, p. 3-14, 2006.

FENECH, M. The in vitro micronucleus technique. Mutation Research, Amsterdam, v. 455, p. 81-95, 2000. http://dx.doi.org/10.1016/S0027-5107(00)00065-8

FERNANDES, T. C. C.; MAZZEO, D. E. C.; MARIN-MORALES, M. A. Mechanism of micronuclei formation in polyploidizated cells of Allium cepa exposed to trifluralin herbicide. Pesticide Biochemistry and Physiology, Massachusetts, v. 88, p. 252-259, 2007. http://dx.doi.org/10.1016/j.pestbp.2006.12.003

FILIPPIN, C.; CHISTOFOLETTI, L. D.; RIBEIRO, M. C. M.; VITURI, C. I. Determinação do número de regiões organizadoras de nucléolo (AgNOR) em lesões do epitélio cervical uterino. Revista Brasileira de Análises Clínicas, Rio de Janeiro, v. 38, n. 3, p. 133-139, 2006.

GROVER, I. S.; KAUR, S. Genotoxicity of wastewater samples from sewage and industrial effluent detected by the Allium root anaphase aberration and micronucleus assays. Mutation Research, Amsterdam, v. 426, p. 183-188, 1999. http://dx.doi.org/10.1016/S0027-5107(99)00065-2

HOSHINA, M. M. Avaliação da possível contaminação das águas do Ribeirão Claro, município de Rio Claro, pertencente à Bacia do Rio Corumbataí, por meio de testes de mutagenicidade em Allium cepa. 2002. 52 f. Monografia (Bacharel e Licenciatura em Ciências Biológicas) - Instituto de Biociências, Universidade Estadual Paulista, Rio Claro, SP. 
HOSHINA, M. M.; MARIN-MORALES, M. A. Micronucleus and chromosome aberrations induced in onion (Allium cepa) by a petroleum refinery effluent and by river water that receives this effluent. Ecotoxicology Environmental Safety, New York, v. 72, p. 20902095, 2009. http://dx.doi.org/10.1016/j.ecoenv.2009.07.002

IGANCI, J. R. V.; BOBROWSKI, V. L.; HEIDEN, G.; STEIN, V. C.; ROCHA, B. H. G. Efeito do extrato aquoso de diferentes espécies de boldo sobre a germinação e índice mitótico de Allium cepa L. Arquivos do Instituto Biológico, São Paulo, vol. 73, n.1, p. 79-82, 2006.

INSTITUTO ESTADUAL DO AMBIENTE (Rio de Janeiro). Boletim de qualidade das águas da região hidrográfica II - Guandu, 2014. Disponível em: http://www.inea.rj.gov.br/cs/groups/public/documents/document/zwff/mdi3/ edisp/inea _027650.pdf. Acesso em: 22 set. 2014.

KAIOUMOVA, D.; SÜSAL, C.; OPELZ, G. Induction of apoptosis in Human lymphocytes by the herbicide 2,4-dichlorophenoxyacetic acid. Human Immunology, California, v. 62, p. 64-74, 2001. http://dx.doi.org/10.1016/S0198-8859(00)00229-9

LEME, D. M.; MARIN-MORALES, M. A. Chromosome aberration and micronucleus frequencies in Allium cepa cells exposed to petroleum polluted water-a case study. Mutation Research, Amsterdam, v. 650, p. 80-86, 2008.

http://dx.doi.org/10.1016/j.mrgentox.2007.10.006

LEME, D. M.; MARIN-MORALES, M. A. Allium cepa test in environmental monitoring: a review on its application. Mutation Research, Amsterdam, v. 682, n. 1, p. 71-81, 2009. 10.1016/j.mrrev.2009.06.002

LOCKE, S. J.; PENG, Y. S.; CROOS, N .L. A supravital staining technique for honeybee spermatozoa. Physiological-Entomology, v. 15, n. 2, p. 187-192, 1990.

http://dx.doi.org/10.1111/j.1365-3032.1990.tb00506.x

MONTANARO, L.; TRERÉ, D.; DERENZINI, M. Nucleolus, ribosomes, and câncer. The Americam Journal of Patology, New York, v. 173, n. 2, p. 301-310, 2008. http://dx.doi.org/10.2353/ajpath.2008.070752

MONTEIRO, R. T. R.; SILVA, G. H., MESSIAS, T. G.; QUEIROZ, S. C. N.; ASSALIN, M. R.; CASSOLI, D. R. et al. Chemical and ecotoxicological assessments of water samples before and after being processed by a water treatment plant. Revista Ambiente \& Água - An interdisciplinary Journal of Applied Science, Taubaté, v. 9, n.1, p. 6-18, 2014. http://dx.doi.org/10.4136/ambi-agua.1292

NIELSEN, M. H.; RANK, J. Screening of toxicity and genotoxicity in waste water by the use of the Allium test. Hereditas, Lund, v. 121, n. 3, p. 249-254, 1994.

http://dx.doi.org/10.1111/j.1601-5223.1994.00249.x

OLIVEIRA, L. M.; VOLTOLINI, J. C.; BARBÉRIO, A. Potencial mutagênico dos poluentes da água do rio Paraíba do Sul em Tremembé, SP, Brasil, utilizando o teste Allium cepa. Revista Ambiente \& Água - An Interdisciplinary Journal of Applied Science, Taubaté, v. 6, n. 1, p. 90-103, 2011. http://dx.doi.org/10.4136/ambi-agua.176

OSHIMA, C. T. F.; FORONES, N. M. AgNOR em câncer gástrico. Arquivos de Gastroenterologia, São Paulo, v. 38, n. 2, p. 89-93, 2001. http://dx.doi.org/10.1590/S0004-28032001000200003 
RADIC, S.; STIPANICEV, D.; VUJCIC, V.; RAJCIC, M. M.; SIRAC, S.; PEVALEKKOZLINA, B. The evaluation of surface and wastewater genotoxicity using the Allium cepa test. Science of Total Environment, New York, v. 408, p. 1228-1233, 2010. http://dx.doi.org/10.1016/j.scitotenv.2009.11.055

SALAMENE, S. Estratificação e caracterização ambiental da área de preservação permanente do Rio Guandu, Rj. 2007. 84f. Dissertação (Mestrado em Ciências Ambientais e Florestais) - Universidade Federal Rural do Rio de Janeiro, Rio de Janeiro, 2007.

SERBER, J. B. Diagnóstico ambiental das atividades do pólo industrial de queimados como subsídio ao termo de ajustamento de conduta na gestão sustentável da bacia hidrográfica do Rio Guandu, RJ. 2005. 111f. Dissertação (Mestrado em Engenharia Ambiental) - Universidade do Estado do Rio de Janeiro, Rio de Janeiro, 2005.

WEINBERG, A. Uso de índices de qualidade de água para a caracterização da bacia hidrográfica do Rio Guandu. 2013. 166f. Projeto de Graduação (Curso de Engenharia Ambiental) - Escola Politécnica da Universidade Federal do Rio de Janeiro, Rio de Janeiro, 2013. 


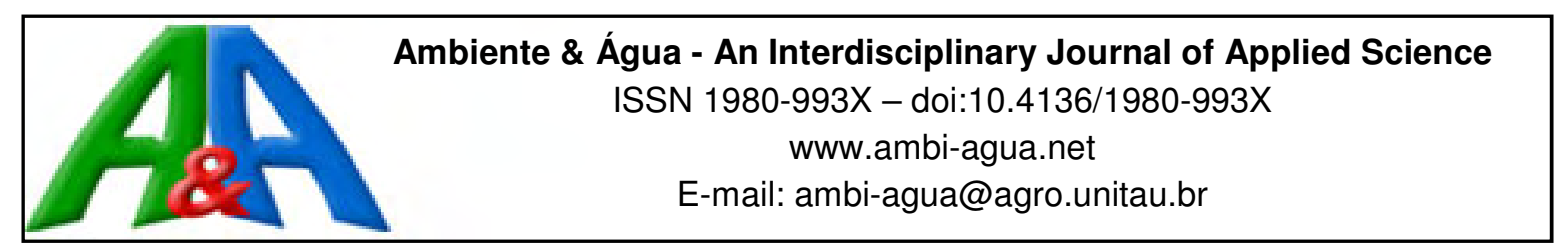

\title{
Remoción de plomo en soluciones acuosas empleando nanoaluminofosfatos amorfos
}

\author{
doi: 10.4136/ambi-agua.1437
}

Received: 24 Jun. 2014; Accepted: 25 Sep. 2014

\author{
Wendy Rondón $^{1}$; Ángela B. Sifontes ${ }^{1 *}$; David Freire ${ }^{1}$; Manuelita Quintal ${ }^{1}$; \\ Edgar Cañizales ${ }^{2}$; Franklin J. Méndez ${ }^{1}$; \\ Ligia Llovera $^{1}$; Yraida Diaz ${ }^{1}$; Joaquin L. Brito ${ }^{1}$ \\ ${ }^{1}$ Instituto Venezolano de Investigaciones Científicas (IVIC), Caracas, Venezuela \\ Centro de Química \\ ${ }^{2}$ Instituto de Tecnología Venezolana para el Petróleo (Intevep), Caracas, Venezuela \\ Petróleos de Venezuela, S.A. (PDVSA) \\ "Autor correspondiente: e-mail: asifonte @ivic.gob.ve, \\ wrondon@ivic.gob.ve,dfreire6@gmail.com, manolis45@yahoo.com, \\ canizalesej@pdvsa.com,fmendez@ivic.gob.ve, 1llovera@ivic.gob.ve, \\ ygdiaz@ivic.gob.ve, joabrito@ivic.gob.ve
}

\section{RESUMEN}

Se emplearon nanopartículas de aluminofosfatos amorfos entre 23 a $30 \mathrm{~nm}$ de diámetro para la adsorción de iones plomo $\left(\mathrm{Pb}^{2+}\right)$ en soluciones acuosas. Los materiales fueron sintetizados mediante biosíntesis utilizando como plantillas biológicas las células de levadura Saccharomyces cerevisiae. Los estudios de adsorción se llevaron a cabo empleando el método batch, utilizando $100 \mathrm{mg}$ del sólido nanométrico y $200 \mu \mathrm{g} \mathrm{\textrm {L } ^ { - 1 }}$ de iones plomo. La determinación cuantitativa de $\mathrm{Pb}^{+2}$ fue realizada mediante espectrometría de absorción atómica con atomización electrotérmica (EAA-HG). Los valores obtenidos para el porcentaje de remoción (\% EF), capacidad de adsorción $\mathrm{q}_{\mathrm{e}}\left(\mu \mathrm{g} \mathrm{g}^{-1}\right)$ y coeficiente de remoción $\mathrm{K}_{\mathrm{d}}\left(\mathrm{mL} \mathrm{g}^{-1}\right)$ fueron de 40; 90 y 800 en promedio, respectivamente. Los resultados obtenidos demostraron las potencialidades que presentan estos materiales para la adsorción de iones $\mathrm{Pb}^{2+}$, siendo excelentes candidatos para la remediación de aguas contaminadas.

Palabras clave: adsorción, biosíntesis, nanopartículas.

\section{Remoção de chumbo em soluções aquosas usando nanoaluminofosfatos amorfos}

\section{RESUMO}

Empregaram-se nanopartículas de aluminofosfatos amorfos, com diâmetro de 23 a $30 \mathrm{~nm}$, para adsorção de íons chumbo $\left(\mathrm{Pb}^{2+}\right)$ em soluções aquosas. Os materiais foram preparados por meio de biossíntese usando como modelos biológicos Saccharomyces cerevisiae. Os estudos de adsorção foram feitos empregando-se o método batch, usando 100 mg do sólido nanométrico e $200 \mu \mathrm{g} \mathrm{L}^{-1}$ de íons chumbo. A determinação quantitativa de $\mathrm{Pb}^{2+}$ foi realizada por meio de espectrometria de absorção atômica com atomização eletrotérmica (EAA-HG). Os valores obtidos para a percentagem de remoção (\% EF), 
capacidade de adsorção $\mathrm{q}_{\mathrm{e}}\left(\mu \mathrm{g} \mathrm{g}^{-1}\right)$ e grau de remoção $\mathrm{K}_{\mathrm{d}}\left(\mathrm{mL} \mathrm{g}^{-1}\right)$ foram de 40; 90 e 800 em média, respectivamente. Os resultados obtidos demonstraram as potencialidades destes materiais para a adsorção de íons $\mathrm{Pb}^{2+}$ e para a remediação de águas poluídas.

Palavras-chave: adsorção, biossíntese, nanopartículas.

\title{
Removal of lead in aqueous solutions using amorphous nanoaluminophosphates
}

\begin{abstract}
Amorphous aluminophosphates nanoparticles with diameter sizes from 23 to $30 \mathrm{~nm}$ were used for the adsorption of lead ions $\left(\mathrm{Pb}^{2+}\right)$ in aqueous solutions. The materials were biosynthesized using Saccharomyces cerevisiae as a biological template. Adsorption studies were conducted using the batch method, employing $100 \mathrm{mg}$ of the solid and $200 \mu \mathrm{g} \mathrm{L}^{-1}$ of lead ions. The quantitative determination of $\mathrm{Pb}^{2+}$ was carried out by electrothermal atomic absorption spectrometry (ETAAS). The percentage of removal (EF), adsorption capacity $\mathrm{q}_{\mathrm{e}}\left(\mu \mathrm{g} \mathrm{g}^{-1}\right)$ and removal coefficient $\mathrm{Kd}\left(\mathrm{mL} \mathrm{g}^{-1}\right)$ averaged 40, 90 and 800 , respectively. The results showed the potential of these materials for the adsorption of $\mathrm{Pb}^{2+}$ ions, and that these materials are good candidates for use in the remediation of contaminated water.
\end{abstract}

Keywords: adsorption, biosynthesis, nanoparticles.

\section{INTRODUCCIÓN}

En la actualidad, la contaminación por metales pesados en aguas subterráneas y superficiales, generada principalmente por actividades antropogénicas, representa una materia importante en el campo de la salud pública y el ambiente. Estas sustancias pueden ser causantes de graves daños en los seres vivos y son capaces de permanecer por largo tiempo en el entorno, circulando o acumulándose en sedimentos o incorporándose a la cadena alimenticia (Gupta et al., 1997; Sousa et al., 2010). La remoción de estos contaminantes por medio de procesos como el intercambio iónico y la adsorción, son considerados hoy en día como una alternativa eficiente. Sin embargo, en estos procesos se emplean materiales cuyo uso puede resultar prohibitivo debido a los altos costos asociados a su adquisición, implementación y operación (Gupta et al., 1997; Sousa et al., 2010). En este sentido, son muchas las investigaciones que se han desarrollado hacia la búsqueda de adsorbentes "no convencionales", de bajo costo y ambientalmente amigables (Gupta et al., 1997; Sousa et al., 2010). Por otra parte, en el área de purificación de aguas, la nanotecnología y la química verde están explorando nuevos campos asociados a la extracción eficiente de contaminantes y gérmenes. Las nanopartículas, nanomembranas y otros materiales nanoestructurados, están siendo utilizados para la detección y remoción de sustancias químicas y biológicas, incluyendo metales pesados (ejemplos: cadmio, cobre, plomo mercurio, níquel y cinc) (Tiwari et al., 2008). Básicamente cuatro tipos de materiales a escala nanométrica están siendo evaluados como materiales funcionales para aplicaciones en la purificación de aguas: nanopartículas que contienen metales, nanopartículas carbonáceas, zeolitas y dendrímeros (Tiwari et al., 2008). A este respecto, debido a su alta área superficial (relación superficie/volumen), los materiales a escala nanométrica exhiben mejores resultados en comparación con los adsorbentes convencionales, ofreciendo hoy en día nuevas perspectivas para su aplicación en los procesos de purificación de aguas (Gupta et al., 2011; Jia et al., 2009; Tiwari et al., 2008). En este particular, recientemente ha sido investigada la remoción de metales pesados en aguas empleando nanopartículas de aluminofosfatos mesoporosos 
amorfos. Estos materiales han demostrado excelente capacidad de adsorción para cationes de $\mathrm{Hg}, \mathrm{Cd}$, As y Fe (Das et al., 2012). No obstante, no han sido realizados estudios sobre la adsorción de iones plomo.

Los efectos tóxicos del plomo son bien conocidos y la máxima concentración aceptada, para el agua de consumo humano, está fijada en todos los países del mundo a niveles muy bajos $\left(\leq 50 \mu \mathrm{g} \mathrm{L}^{-1}\right)$ (Tiwari et al., 2008). Entre otras consideraciones, la contaminación con este elemento puede ocurrir cuando el agua fluye a través de tuberías que contienen plomo en su composición (Sublet et al., 2003). Es por ello, que la presente contribución exhibe un estudio preliminar a fin de explorar las propiedades que presentan los nanoaluminofosfatos amorfos en la remoción de iones plomo en medio acuoso. El adsorbente utilizado fue preparado mediante biosíntesis, empleando levadura, Saccharomyces cerevisiae, como plantilla biológica para la formación de la estructura inorgánica (Sifontes et al., 2013). Es importante destacar que esta nueva ruta de síntesis emplea materiales que presentan alta biodegrabilidad, escasa toxicidad y bajo costo (Sifontes et al., 2013).

\section{MATERIALES Y MÉTODOS}

Los nanoaluminofosfatos empleados en este estudio se sintetizaron mediante un procedimiento publicado en la literatura por Sifontes et al. (2013). El tamaño de partícula fue estimado por microscopía electrónica de transmisión, determinándose un rango entre 23 y $30 \mathrm{~nm}$ (Sifontes et al., 2013). El material biológico utilizado correspondió a células de levadura de tipo comercial. Todos los reactivos empleados fueron de grado analítico. Los sólidos preparados se caracterizaron utilizando un difractómetro de rayos-X (DRX) Siemens modelo D-5000, empleando radiación $\mathrm{CuK} \alpha$ en el rango $2 \theta$ entre $5^{\circ}$ y $70^{\circ}$ a fin de examinar su posible estructura cristalina. El área superficial se determinó por adsorción de nitrógeno a $-196{ }^{\circ} \mathrm{C}$, en un equipo Micromeritics ASAP 2010, empleando el método de BET. Las observaciones mediante microscopía electrónica de barrido (MEB-EDX) fueron realizadas en un instrumento HITACHI S-2400. Los espectros de infrarrojo con Transformada de Fourier (FTIR) se obtuvieron empleando un equipo Perkin Elmer Spectrom RX1. Los estudios por RMN de estado sólido (RMN-MAS de ${ }^{27} \mathrm{Al} \mathrm{y}{ }^{31} \mathrm{P}$ ) se realizaron en un espectrómetro Bruker Avance $300 \mathrm{MHz}$. Para la síntesis, procedimientos analíticos y los estudios de adsorción se empleó agua ultrapura $(18 \mathrm{M} \Omega \cdot \mathrm{cm})$ obtenida con un equipo desionizador marca Millipore modelo Milli-Q (Millipore Corporation). Los patrones acuosos necesarios para la cuantificación fueron preparados por dilución de las soluciones metálicas estándar de 1000 $\mathrm{mgL}^{-1}$ (Merck). Un equipo Varian Techtron modelo AA-875 de doble haz equipado con un horno de grafito GTA-95 (EAA-HG) fue requerido para la determinación de plomo. Las mediciones de $\mathrm{Al}$ y $\mathrm{P}$ se realizaron con un espectrómetro de emisión atómica con plasma inductivamente acoplado (ICP-OES), OPTIMA 3000 (Perkin Elmer).

\subsection{Estudio de adsorción}

Se estudió el efecto de captación de plomo en medio acuoso empleando los nanomateriales sintetizados previamente. Las medidas de adsorción se realizaron mediante la técnica de muestras por lotes (batch) a temperatura ambiente $\left(20 \pm 1^{\circ} \mathrm{C}\right)$. La cantidad de metal removido por gramo de adsorbente (capacidad de adsorción $\mathrm{q}_{\mathrm{e}}\left(\mu \mathrm{g} \mathrm{g}^{-1}\right)$ ) se evaluó mediante el estudio de tres muestras representativas de los materiales sintetizados (Singh et al., 2011). A tal fin, $100 \mathrm{mg}$ de cada muestra adsorbente fueron añadidos a $100 \mathrm{~mL}$ de una solución de 200 $\mu \mathrm{g} \mathrm{L}^{-1}$ de $\mathrm{Pb}^{2+}$ preparada a partir de una solución patrón de $1000 \mu \mathrm{g} \mathrm{L}^{-1}$ de $\mathrm{Pb}^{2+}$. La mezcla fue colocada en un agitador de tipo vortex $(1500 \mathrm{rpm})$ por periodos de 5 y $24 \mathrm{~h}$ para determinar el efecto del tiempo de contacto con el material adsorbente. Posteriormente cada solución fue centrifugada durante $1 \mathrm{~min}$ y el sobrenadante obtenido fue filtrado y 
diluido 5 veces. Se consideró como blanco (muestra control), una muestra del material sintetizado sin levadura, la cual fue sometida al mismo tratamiento de adsorción que los nanomateriales en estudio. La determinación de plomo en la solución final se realizó empleando EAA-HG. Se emplearon soluciones estándares para calibrar el instrumento. El valor de $\mathrm{q}_{\mathrm{e}}\left(\mu \mathrm{g} \mathrm{g}^{-1}\right)$ se determinó según la Ecuación 1 (Singh et al., 2011; Sprynskyy et al., 2006).

$$
q_{e}=\frac{\left(C_{i}-C_{f}\right) \times V_{s o l}}{m_{a d s}}
$$

donde:

$\mathrm{C}_{\mathrm{i}} \mathrm{y}_{\mathrm{f}}$ son las concentraciones inicial y final en equilibrio, de los iones metálicos en la solución en $\mu \mathrm{g} \mathrm{L}^{-1}$,

$\mathrm{V}_{\text {sol }}$ es el volumen de la solución en litros y

$\mathrm{m}_{\mathrm{ads}}$ es la masa del adsorbente en gramos.

Adicionalmente, también se calculó la constante de adsortividad o coeficiente de distribución $\mathrm{K}_{\mathrm{d}}\left(\mathrm{mL} \mathrm{g}^{-1}\right)$ relacionada con la selectividad y afinidad de la adsorción y el porcentaje de eficiencia o remoción \% EF, empleando las Ecuaciones 2 y 3, para todos los nanomateriales sintetizados con levadura y para el blanco (muestra control) (Singh et al., 2011; Sprynskyy et al., 2006).

$$
\begin{aligned}
& K_{d}=\frac{q_{e}}{C_{f}} \\
& \% E F=\frac{K_{d} \times m_{a d s}}{\left(K_{d} \times m_{a d s}+V_{s o l}\right)} \times 100
\end{aligned}
$$

\section{RESULTADOS Y DISCUSIÓN}

\subsection{Caracterización del material adsorbente}

Se realizó un estudio por DRX para verificar la presencia o ausencia de fases cristalinas en los sólidos sintetizados. La Figura 1 presenta los difractogramas para el blanco y la muestra preparada mediante biosíntesis. En ellos puede observarse una banda ancha en el rango $2 \theta$ de $15-40^{\circ}$, la cual es atribuida a la naturaleza amorfa de los materiales sintetizados (Liu et al., 2006a; Sifontes et al., 2013).

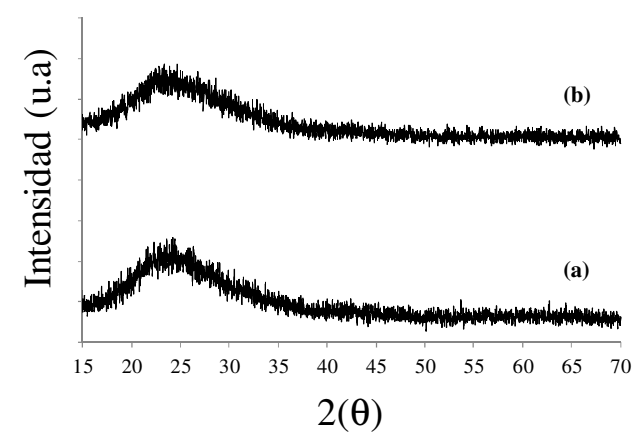

Figura 1. DRX de los aluminofosfatos sintetizados $\sin$ levadura (a) con levadura (b). 
En la Figura 2 es mostrado el espectro de ${ }^{27}$ Al-RMN-MAS correspondiente a los aluminofosfatos sintetizados con y sin la presencia de levadura. En la Tabla 1 se recopilan los valores de los desplazamientos químicos correspondientes.

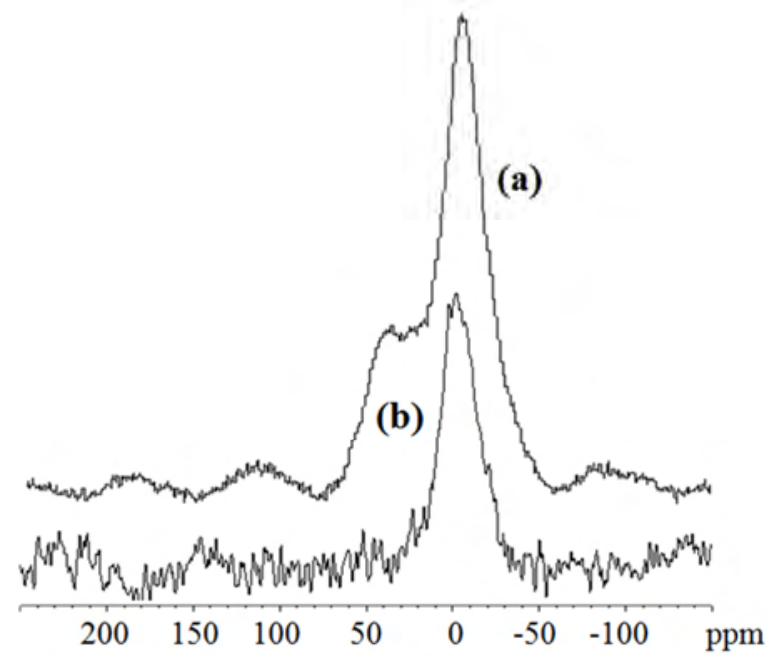

Figura 2. Espectro de ${ }^{27} \mathrm{Al}-\mathrm{RMN}-\mathrm{MAS}$ de los aluminofosfatos sintetizados sin levadura (a), con levadura (b).

La inexistencia de $\mathrm{Al}^{3+}$ en coordinación tetraédrica pareciera sugerir ausencia de material de tipo aluminofosfato, ya que como se indicó antes, la señal correspondiente a $\mathrm{Al}^{3+}$ tetraédrico es asignada a grupos $\left[\mathrm{Al}(\mathrm{PO})_{4}\right]$. Sin embargo, la existencia de cationes de aluminio en una coordinación diferente de cuatro ya ha sido postulada para los sistemas AlPO amorfos (Marquez et al., 1997). En este particular varios investigadores proponen que estos materiales amorfos están constituidos por una fase rica en alúmina, con disminución del contenido de fósforo $(\mathrm{Al} / \mathrm{P}>1)$ y que estos sistemas $\mathrm{AlPO}_{4}-\mathrm{Al}_{2} \mathrm{O}_{3}$ son construidos a partir de unidades de tetraedros $\left[\mathrm{PO}_{4}\right]$ y poliedros de $\left[\mathrm{AlO}_{4}\right]$, $\left[\mathrm{AlO}_{5}\right]$ y $\left[\mathrm{AlO}_{6}\right]$ (Liu et al., 2006a; Marquez et al., 1997).

Tabla 1. Resumen de los desplazamientos químicos obtenidos mediante los análisis de ${ }^{27} \mathrm{Al}-\mathrm{RMN}-\mathrm{MAS}$ y ${ }^{31} \mathrm{P}-\mathrm{RMN}-\mathrm{MAS}$ para los AlPOs sintetizados.

\begin{tabular}{lcc}
\hline \multirow{2}{*}{ Muestra } & \multicolumn{2}{c}{$\begin{array}{c}\text { Desplazamiento químico } \\
(\mathbf{p p m})\end{array}$} \\
\cline { 2 - 3 } & ${ }^{27} \mathbf{A l}$ & ${ }^{31} \mathbf{P}$ \\
\hline \multirow{3}{*}{ AlPO Control } & 38,67 & 29,94 \\
& & $-10,45$ \\
& & $-47,05$ \\
AlPO sintetizado & $-5,52$ & 28,04 \\
con levadura & 2,30 & $-16,16$ \\
& $-2,30$ & $-56,56$ \\
\hline
\end{tabular}

Esto pudiera dar soporte a los resultados obtenidos mediante ${ }^{27} \mathrm{Al}-\mathrm{RMN}-\mathrm{MAS}$. En este sentido, adicionalmente fue efectuada la determinación de la relación de Al/P mediante los estudios realizados por ICP-OES. El valor promedio obtenido fue $\mathrm{Al} / \mathrm{P}=1,6$, lo cual demuestra, en concordancia con lo anteriormente descrito, un enriquecimiento de aluminio 
para los AlPO obtenidos mediante biosíntesis. Por otra parte, se ha encontrado que las especies de fosfatos asociadas con átomos de aluminio generalmente presentan desplazamientos químicos ${ }^{31} \mathrm{P}$ en el rango de -7 a $-30 \mathrm{ppm}$, dependiendo del grado de cristalinidad, contenido de agua y grado de condensación (Duffy e VanLoon, 1995; Liu et al., 2006b; Marquez et al., 1997). Las señales ${ }^{31} \mathrm{P}$ con desplazamiento químico a campo bajo, de 0 a -13 ppm, pueden ser asignadas a las especies de P con muy bajo grado de condensación (Figura 3).

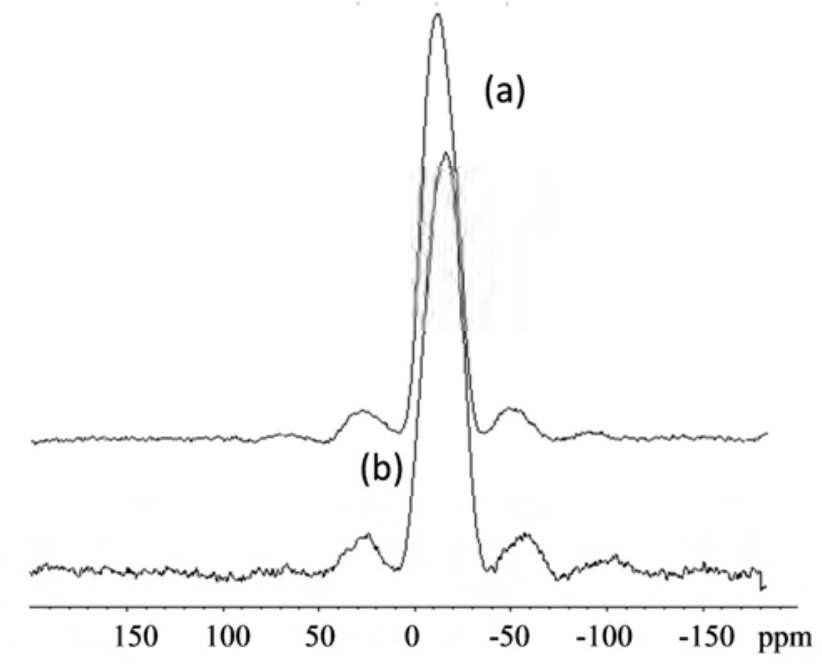

Figura 3. Espectro de ${ }^{31} \mathrm{P}-\mathrm{RMN}-\mathrm{MAS}$ de los aluminofosfatos sintetizados sin levadura (a), con levadura (b).

En los espectros de ${ }^{31}$ P-RMN-MAS mostrados en la Figura 3, se asignaron los desplazamientos químicos correspondientes al sólido sintetizado y la muestra control. Las señales identificadas se atribuyen a la presencia de especies de $\mathrm{P}$ en coordinación tetraédrica con enlaces $\mathrm{O}-\mathrm{Al}, \mathrm{P}(\mathrm{OAl})_{4}$. La señal de resonancia relativamente ancha podría implicar la presencia de pequeñas cantidades de especies de $\mathrm{P}$ parcialmente coordinadas con grupos $\mathrm{OH}$ o $\mathrm{H}_{2} \mathrm{O}$ como por ejemplo $\mathrm{P}(\mathrm{OAl})_{\mathrm{x}}(\mathrm{OH})_{4-\mathrm{x}}$ (Duffy e VanLoon, 1995; Liu et al., 2006b; Marquez et al., 1997). Así mismo, en la Tabla 1 son mostrados los valores de los desplazamientos químicos obtenidos del espectro de ${ }^{31} \mathrm{P}-\mathrm{RMN}-\mathrm{MAS}$, tanto para la muestra control como para la preparada con levaduras. En la Figura 4 se muestra el espectro FTIR correspondiente a los sólidos sintetizados con y sin levadura. Mediante este estudio se pudo confirmar la presencia de los grupos funcionales característicos de los aluminofosfatos, entre los cuales se pudieron determinar las señales más importantes. En las regiones alrededor de $3600 \mathrm{y} \mathrm{de} 1600 \mathrm{~cm}^{-1}$ se encuentran unas bandas atribuidas al estiramiento y flexión del enlace O-H de la molécula de agua (Kannan et al., 2013; Liu et al., 2006a; 2006b). Entre 1422-959 $\mathrm{cm}^{-1}$ fue observada una banda de fuerte intensidad, la cual se corresponde con las vibraciones de tensión P-O de los grupos $\mathrm{PO}_{4}{ }^{3-}$ (Kannan et al., 2013; Liu, et al., 2006a; 2006b). Del mismo modo, fueron asignadas las bandas ubicadas en la región de $712-469 \mathrm{~cm}^{-1}$ a las vibraciones simétricas de tensión P-O-Al. Una señal de moderada intensidad a $560 \mathrm{~cm}^{-1}$ fue atribuida a las vibraciones de flexión O-P-O de los grupos $\left(\mathrm{PO}_{4}\right)^{3-}$ (Kannan et al., 2013; Liu, et al., 2006a; 2006b). La ausencia de las bandas en la región $2000-2950 \mathrm{~cm}^{-1}$ para las dos muestras sintetizadas, las cuales se atribuyen a las vibraciones simétricas y asimétricas de los grupos $\mathrm{C}-\mathrm{H}$, indican la eliminación completa de la plantilla biológica (levadura).

En las Figuras 5 y 6 se presentan las micrografías (MEB) de las células de levadura antes y después del tratamiento de síntesis. Adicionalmente, es presentado un análisis por EDX el cual permite evidenciar claramente la naturaleza de los aluminofostatos sintetizados. 


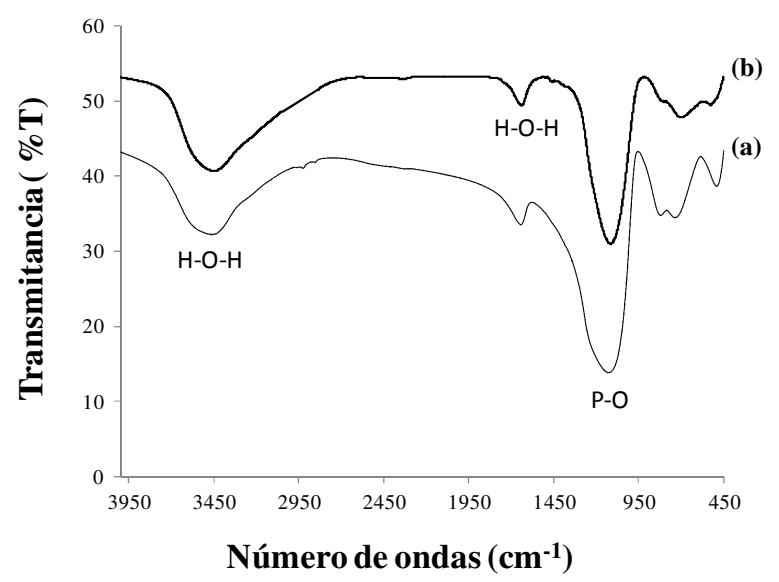

Figura 4. Espectro IR de los aluminofosfatos sintetizados sin levadura (a) y con levadura (b).

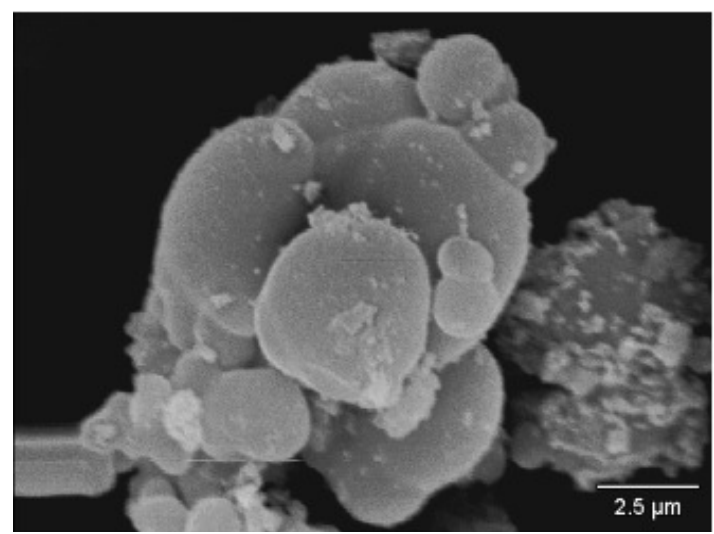

Figura 5. Imagen SEM de células de levadura de Saccharomyces cerevisiae.
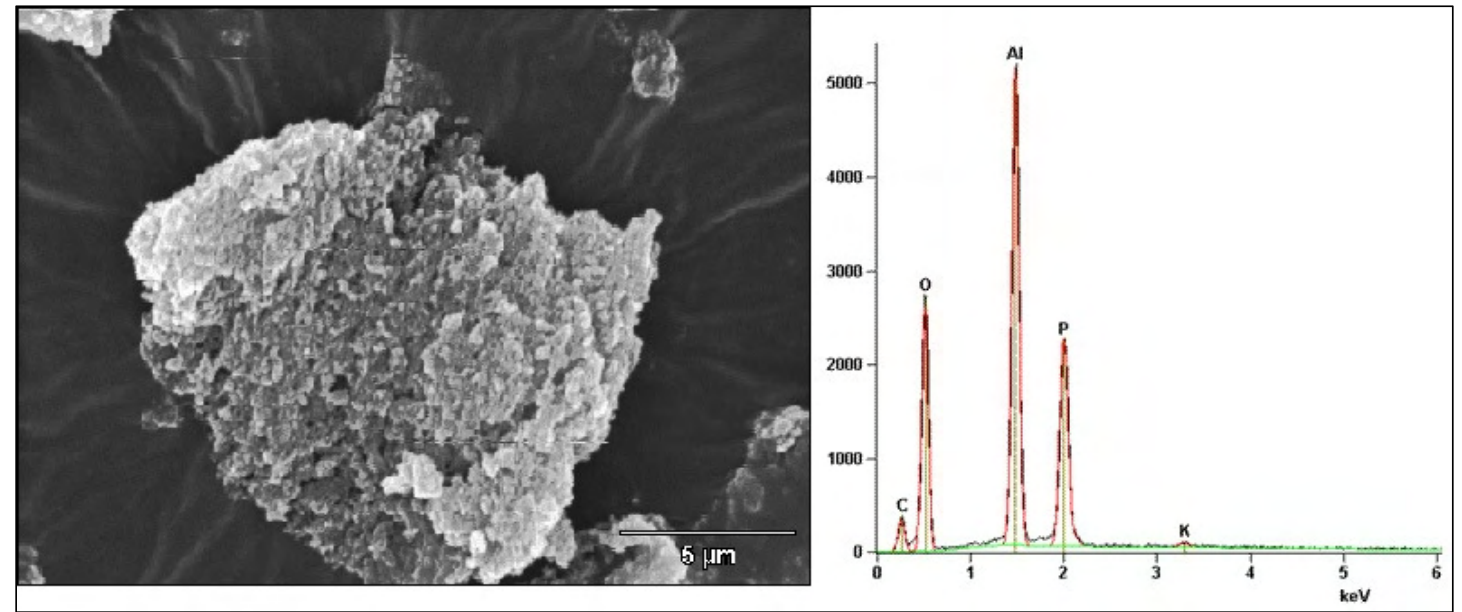

Figura 6. Panel izquierdo: Imagen SEM de las células de levadura Saccharomyces cerevisiae después del tratamiento de síntesis. Panel derecho: Espectro EDX de las células de levadura después del tratamiento de síntesis.

La Figura 7 muestra las isotermas resultantes del estudio por adsorción física de $\mathrm{N}_{2}$ para la muestra sintetizada con los microorganismos y la muestra control, calcinada a $650{ }^{\circ} \mathrm{C}$. Las isotermas observadas presentan un comportamiento tipo IV (Barrett et al., 1951) con una abertura de histéresis desplazada a altas presiones. Esto puede ser atribuido a la presencia de una mesoporosidad extra-estructural generada por los espacios ubicados entre los agregados 
de nanopartículas. En cuanto al volumen total de poros $\left(\mathrm{V}_{\mathrm{t}}\right)$, las muestras exhibieron valores que indican porosidad media con diámetros de poro promedios entre 10 y $20 \mathrm{~nm}$. En la Tabla 2 se muestran los valores de área superficial, diámetro y volumen de poro para los sólidos sintetizados, apreciándose las diferencias entre el material sintetizado y la muestra control.

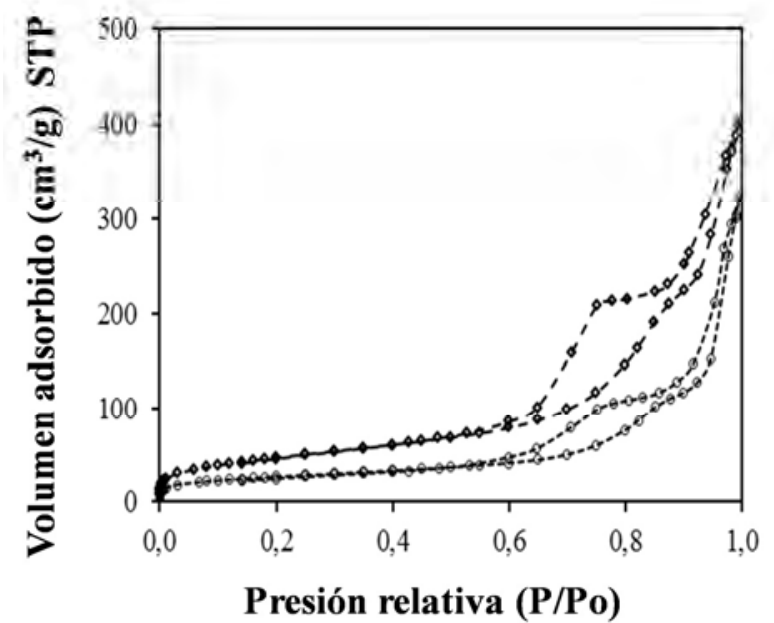

Figura 7. Isotermas de adsorción física de $\mathrm{N}_{2}$ para la muestra Control -o- y la sintetizada con los microorganismos $-\diamond-$.

Tabla 2. Área superficial y tamaños de los poros de las muestras.

\begin{tabular}{lccccc}
\hline Muestra & $\mathbf{S}_{\text {BET }}\left(\mathrm{m}^{2} \mathrm{~g}^{-1}\right)$ & $\mathbf{S}_{\text {micro }}\left(\mathrm{m}^{2} \mathrm{~g}^{-1}\right)$ & $\mathbf{S}_{\text {meso }}\left(\mathrm{m}^{2} \mathrm{~g}^{-1}\right)$ & $\mathbf{V}_{\mathbf{t}}\left(\mathrm{cm}^{3} \mathrm{~g}^{-1}\right)$ & $\mathbf{D p}(\mathrm{nm})$ \\
\hline Control & 93 & 13 & 80 & 0,5 & 17 \\
M & 164 & 0 & 164 & 0,6 & 13 \\
\hline
\end{tabular}

Nota: $\mathrm{S}$ : área superficial; $\mathrm{V}_{\mathrm{t}}$ : volumen total de poros; Dp: diámetro de poros, $\mathrm{M}$ : promedio de las muestras M1 y M2.

La porosidad observada en las muestras tratadas con las células de levadura es atribuida a la acción de las biomoléculas presentes en sus membranas celulares, las cuales pueden actuar diferentemente favoreciendo la fijación de precursores y actuando como centros de nucleación (Merroun et al., 2005). Estas contienen grupos funcionales, tales como grupos amino y carboxilato (entre otros), capaces de interaccionar con los precursores metálicos introducidos en el lecho de síntesis (Sifontes et al., 2013). La superficie de las células de levadura adquiere una carga eléctrica, debido a la ionización de estos grupos químicos superficiales lo cual le permitirá interactuar con los iones o moléculas presentes en el medio exterior (Sifontes et al., 2013; Pompe et al., 2013).

\subsection{Estudios de adsorción}

Debido a que recientemente ha sido investigada con éxito la remoción de metales pesados en aguas, empleando nanopartículas de aluminofosfatos mesoporosos amorfos (Gupta et al., 1997; Sousa et al., 2010) y dadas las ventajosas características estructurales y texturales observadas en los materiales sintetizados empleando levadura como plantilla biológica, fue considerado de particular interés, realizar algunos estudios sobre la adsorción de iones metálicos en medio acuoso. Esto permitiría evaluar las potencialidades de los nanoaluminofosfatos amorfos preparados mediante biosíntesis como material adsorbente. Para ello, se realizó la determinación de plomo por EAA-HG, en solución acuosa (Tabla 3) y se evaluaron los parámetros de adsorción, \% EF, qe y $\mathrm{K}_{\mathrm{d}}$ para ambos materiales (Tabla 4). Para 
verificar la dispersión experimental, se emplearon dos muestras separadas de material sintetizado por biosíntesis, M1 y M2 (réplicas).

Tabla 3. Determinación de la adsorción de iones de plomo en $100 \mathrm{mg}$ de aluminofosfatos.

\begin{tabular}{lcc}
\hline Adsorbentes & $\begin{array}{c}\text { Horas de } \\
\text { contacto }\end{array}$ & $\begin{array}{c}\text { Concentración } \mathbf{P b}^{\mathbf{2 +}} \\
\left(\boldsymbol{\mu g} \mathbf{~ L}^{-1}\right)^{\mathbf{*}}\end{array}$ \\
\hline M1 & 5 & 117,84 \\
M2 & 5 & 104,77 \\
Control & 5 & 140,56 \\
M1 & 24 & 54,21 \\
M2 & 24 & 68,41 \\
Control & 24 & 109,88 \\
\hline
\end{tabular}

Nota: M1 y M2: muestras sintetizadas con levadura, réplicas. Control: muestra sintetizada sin levadura. La concentración inicial de $\mathrm{Pb}^{2+}$ fue de $200 \mu \mathrm{g} \mathrm{L}{ }^{-1}$ en todos los experimentos.

"Calculado utilizando la curva de calibración lineal con ecuación de regresión lineal $\mathrm{Abs}=0,0040 \mathrm{C}+0,0120$ y $\mathrm{R}^{2}=0,9989$ obtenida con el EAA-HG.

Los resultados obtenidos mediante la determinación de plomo en solución acuosa permitieron estimar el porcentaje de eficiencia o remoción de los materiales adsorbentes. El sólido preparado mediante biosíntesis presentó una mayor eficiencia de remoción que la muestra control, preparada sin levadura. Los valores oscilaron entre 40-48 \% para 5 horas y de $65-73 \%$ para $24 \mathrm{~h}$ de exposición. Los valores obtenidos empleando la muestra control fueron de 30 y $45 \%$ para 5 y 24 h de exposición, respectivamente. Esto puede ser atribuido a la mayor área superficial que presentan los materiales preparados con levadura (ver Tabla 2). Se calculó el valor promedio de los parámetros de adsorción experimentales $\mathrm{q}_{\mathrm{e}} \mathrm{y} \mathrm{K}_{\mathrm{d}}$ obtenidos para las muestras de los materiales preparados mediante biosíntesis. Los resultados mostrados son los siguientes: $\mathrm{q}_{\mathrm{e}}(88,69 \pm 9,24) \mu \mathrm{g} \mathrm{g}^{-1}$ y $\mathrm{K}_{\mathrm{d}}(803,00 \pm 149,62) \mathrm{mL} \mathrm{g}^{-1}$ correspondientes para 5 horas de contacto y de $\mathrm{q}_{\mathrm{e}}(138,69 \pm 10,05) \mu \mathrm{g} \mathrm{g}^{-1}$ y $\mathrm{K}_{\mathrm{d}}(2306,66 \pm 541,74) \mathrm{mL} \mathrm{g}^{-1}$ para 24 horas de contacto. En la Tabla 5, se comparan estos resultados con los obtenidos por otros autores. Si bien es cierto que los materiales sintetizados no alcanzan una eficiencia del $90 \%$ para la remoción de $\mathrm{Pb}^{+2}$, ofrecen sin embargo, una alternativa interesante la cual es económica, viable y menos contaminante que las vías de síntesis convencionales. Los estudios realizados sugieren que las aplicaciones de estos materiales pudieran ser evaluadas también para otros iones metálicos como cobalto, zinc y cadmio.

Tabla 4. Parámetros de adsorción estimados para 100 mg de aluminofosfatos.

\begin{tabular}{lcccc}
\hline Adsorbentes & $\begin{array}{c}\text { Horas de } \\
\text { contacto }\end{array}$ & $\mathbf{q}_{\mathbf{e}}\left(\boldsymbol{\mu} \mathbf{g ~ g}^{-\mathbf{1}}\right)$ & $\mathbf{K}_{\mathbf{d}}\left(\mathbf{m L ~ \mathbf { g } ^ { - \mathbf { 1 } } )}\right.$ & $\boldsymbol{\%} \mathbf{E F}$ \\
\hline M1 & 5 & 82,16 & 697,20 & 41,08 \\
M2 & 5 & 95,23 & 908,80 & 47,61 \\
Control & 5 & 59,43 & 422,80 & 29,72 \\
M1 & 24 & 145,80 & 2689,73 & 72,90 \\
M2 & 24 & 131,59 & 1923,59 & 65,80 \\
Control & 24 & 90,12 & 820,01 & 45,06 \\
\hline
\end{tabular}

Nota: M1 y M2: muestras sintetizadas con levadura, réplicas. Control: muestra sintetizada sin levadura. 
Tabla 5. Comparación de diferentes materiales adsorbentes empleados en la remoción de iones plomo.

\begin{tabular}{lccc}
\hline \multicolumn{1}{c}{ Material } & Dosis $(\mathbf{m g})$ & $\begin{array}{c}\text { Tiempo de } \\
\text { Contacto }(\mathbf{h})\end{array}$ & $\mathbf{q}\left(\mathbf{m g ~ g}^{-\mathbf{1}}\right)$ \\
\hline Hongos Mucor rouxii & 50 & 7 & 35,69 \\
Fosfato natural & 100 & 3 & 115,34 \\
Fosfato activado & 100 & 3 & 155,04 \\
Magnetita/Hidroxiapatita & 50 & 24 & 598,80 \\
AlPO Control & 100 & 5 & 95,23 \\
AlPO sintetizado con levadura & 100 & 5 & 59,43 \\
\hline
\end{tabular}

\section{CONCLUSIONES}

Mediante el procedimiento de síntesis utilizado, se generó un material nanométrico de elevada porosidad (nanoaluminofosfatos amorfos), característica obtenida como producto de las interacciones entre la pared celular y el precursor inorgánico. Se realizaron los estudios de adsorción de iones plomo en soluciones acuosas obteniendo eficiencias entre 40 y $70 \%$, capacidad de adsorción entre 50 y $145 \mu \mathrm{g} \mathrm{g}^{-1}$ y una constante de distribución en el orden de los 400 a $2600 \mathrm{~mL} \mathrm{~g}^{-1}$. Estos resultados evidencian que los sólidos preparados mediante biosíntesis pueden ser empleados para la remoción de trazas de iones plomo y podrían ser aplicados como materiales adsorbentes de otros iones metálicos en soluciones acuosas.

\section{AGRADECIMIENTOS}

Los autores agradecen al Instituto Venezolano de Investigaciones Científicas (IVIC) por el apoyo a través de los proyecto No 1077 y No 1112 y al Laboratorio de Análisis Químico Estructural de Materiales de la Universidad de Los Andes (LAQUEM-ULA).

\section{REFERENCIAS}

BARRETT, E. P.; JOYNER, L. G.; HALENDA, P. P. The determination of pore volume and area distributions in porous substances. I. Computations from Nitrogen Isotherms. Journal of the American Chemical Society, v. 73, n. 1, p. 373-380, 1951. http://dx.doi.org/10.1021/ja01145a126

DAS, S. K.; BHUNIA, M. K.; BHAUMIK, A. Solvothermal synthesis of mesoporous aluminophosphate for polluted water remediation. Microporous and Mesoporous Materials, v. 155, p. 258-264, 2012.

http://dx.doi.org/10.1016/j.micromeso.2012.01.034

DUFFY, S. J.; VANLOON, G. W. Investigations of aluminum hydroxyphosphates and activated sludge by27Al and31P MAS NMR. Canadian Journal of Chemistry, v. 73, n. 10, p. 1645-1659, 1995. http://dx.doi.org/10.1139/v95-204

GUPTA, V. K.; AGARWAL, S.; SALEH, T. A. Synthesis and characterization of aluminacoated carbon nanotubes and their application for lead removal. Journal of hazardous materials, v. 185, n. 1, p. 17-23, 2011. http://dx.doi.org/10.1016/j.jhazmat.2010.08.053 
GUPTA, V. K.; SRIVASTAVA, S. K.; MOHAN, D.; SHARMA, S. Design parameters for fixed bed reactors of activated carbon developed from fertilizer waste for the removal of some heavy metal ions. Waste Management, v. 17, n. 8, p. 517-522, 1997. http://dx.doi.org/10.1016/s0956-053x(97)10062-9

JIA, K.; PAN, B.; LV, L.; ZHANG, Q.; WANG, X.; PAN, B.; ZHANG, W. Impregnating titanium phosphate nanoparticles onto a porous cation exchanger for enhanced lead removal from waters. Journal of Colloid and Interface Science, v. 331, n. 2, p. 453457, 2009. http://dx.doi.org/10.1016/j.jcis.2008.11.068

KANNAN, C.; MUTHURAJA, K.; DEVI, M. R. Hazardous dyes removal from aqueous solution over mesoporous aluminophosphate with textural porosity by adsorption. Journal of hazardous materials, v. 244-245, p. 10-20, 2013.

http://dx.doi.org/10.1016/j.jhazmat.2012.11.016

LIU, G.; JIA, M.; ZHOU, Z.; WANG, L.; ZHANG, W.; JIANG, D. Synthesis and pore formation study of amorphous mesoporous aluminophosphates in the presence of citric acid. Journal of Colloid and Interface Science, v. 302, n. 1, p. 278-286, $2006 \mathrm{a}$. http://dx.doi.org/10.1016/j.jcis.2006.06.026

LIU, G.; WANG, Z.; JIA, M.; ZOU, X.; ZHU, X.; ZHANG, W. et al. Thermally stable amorphous mesoporous aluminophosphates with controllable P/Al ratio: synthesis, characterization, and catalytic performance for selective O-methylation of catechol. The Journal of Physical Chemistry B, v. 110, n. 34, p. 16953-16960, 2006 b. http://dx.doi.org/10.1021/jp062824u

MARQUEZ, A. M.; OVIEDO, J.; SANZ, J. F.; BENITEZ, J. J.; ODRIOZOLA, J. A. Geometric and electronic structure of amorphous aluminophosphates. Ab initio and experimental studies. The Journal of Physical Chemistry B, v. 101, n. 46, p. 95109516, 1997. http://dx.doi.org/10.1021/Jp972147r

MERROUN, M. L.; RAFF, J.; ROSSBERG, A.; HENNIG, C.; REICH, T.; SELENSKAPOBELL, S. Complexation of Uranium by Cells and S-Layer Sheets of Bacillus sphaericus JG-A12. Applied and Environmental Microbiology, v. 71, p. 5532, 2005. http://dx.doi.org/10.1128/AEM.71.9.5532-5543.2005.

POMPE, W.; RÖDEL, G.; WEISS H-J.; MERTIG, M. Bio-nanomaterials, designing materials inspired by nature. [S.1.]: Wiley-VCH Verlag GmbH \& Co.KGaA, 2013.

SIFONTES, Á. B., GONZÁlEZ, G., TOVAR, L. M., MÉNDEZ, F. J., GOMES, M. E., CAÑIZALES, E. et al. Biosynthesis of amorphous mesoporous aluminophosphates using yeast cells as templates. Materials Research Bulletin, v. 48, n. 2, p. 730-738, 2013. http://dx.doi.org/10.1016/j.materresbull.2012.11.016

SINGH, B. K.; BHADAURIA, J.; TOMAR, R.; TOMAR, B. S. Effect of humic acid on sorption of trace metal ions by sodium aluminosilicate. Desalination, v. 268, n. 1-3, p. 189-194, 2011. http://dx.doi.org/10.1016/j.desal.2010.10.022

SOUSA, F. W.; OLIVEIRA, A. G.; RIBEIRO, J. P.; ROSA, M. F.; KEUKELEIRE, D.; NASCIMENTO, R. F. Green coconut shells applied as adsorbent for removal of toxic metal ions using fixed-bed column technology. Journal of Environmental Management, v. 91, n. 8, p. 1634-1640, 2010. http://dx.doi.org/10.1016/j.jenvman.2010.02.011 
SPRYNSKYY, M.; BUSZEWSKI, B.; TERZYK, A. P.; NAMIESNIK, J. Study of the selection mechanism of heavy metal $\left(\mathrm{Pb}^{2+}, \mathrm{Cu}^{2+}, \mathrm{Ni}^{2+}\right.$, and $\left.\mathrm{Cd}^{2+}\right)$ adsorption on clinoptilolite. Journal of Colloid and Interface Science, v. 304, n. 1, p. 21-28, 2006. http://dx.doi.org/10.1016/j.jcis.2006.07.068

SUBLET, R.; SIMONNOT, M. O.; BOIREAU, A.; SARDIN, M. Selection of an adsorbent for lead removal from drinking water by a point-of-use treatment device. Water Research, v. 37, n. 20, p. 4904-4912, 2003. http://dx.doi.org/10.1016/j.watres.2003.08.010

TIWARI, D. K.; BEHARI, J.; SEN, P. Application of nanoparticles in waste water treatment. World Applied Sciences Journal, v. 3, n. 3, p. 417-433, 2008. 


Ambiente \& Água - An Interdisciplinary Journal of Applied Science
ISSN 1980-993X - doi:10.4136/1980-993X
www.ambi-agua.net
E-mail: ambi-agua@agro.unitau.br

\section{Construção e emprego de diagramas de coagulação como ferramenta para o monitoramento contínuo da floculação em águas de abastecimento}

doi: 10.4136/ambi-agua.1239

Received: 06 Nov 2013; Accepted: 21 Oct. 2014

Daniel Bartiko*; Marcelo De Julio

Instituto Tecnológico de Aeronáutica (ITA), São José dos Campos, SP, Brasil
*Autor correspondente: e-mail: danielbartiko@hotmail.com,
dejulio@ita.br

\section{RESUMO}

Neste trabalho foram construídos diagramas de coagulação a partir de ensaios em bancada com auxílio de um equipamento jartest para a avaliação da turbidez remanescente de uma água de estudo preparada com a adição de suspensão de caulinita (até atingir turbidez de $25 \pm 2$ uT) submetida à coagulação, floculação e sedimentação. Foi utilizado sulfato de alumínio isento de ferro como coagulante, sendo variada sua dosagem $\left(5 \mathrm{mg} \mathrm{L}^{-1}\right.$ a $\left.100 \mathrm{mg} \mathrm{L}^{-1}\right)$ e o pH de coagulação (3 a 9). Foram estudadas três velocidades de sedimentação (3,0; 1,5 e $\left.0,5 \mathrm{~cm} \mathrm{~min}^{-1}\right)$. Com o auxílio dos diagramas de coagulação, observou-se resultados satisfatórios para a remoção de turbidez com valores de pH de coagulação entre 6,5 e 7,5 e dosagem de coagulante a partir de $15 \mathrm{mg} \mathrm{L}^{-1}$; dosagens elevadas de coagulante não levaram, necessariamente, aos menores valores de turbidez remanescente. A eficiência de remoção da turbidez foi maior para velocidades de sedimentação mais baixas, chegando a valores menores que 1 uT na menor velocidade de sedimentação estudada. A construção de diagramas de coagulação com base em ensaios em equipamento jartest demonstrou ser uma ferramenta eficaz para a otimização das etapas de coagulação, floculação e sedimentação do tratamento da água, possibilitando produzir flocos com elevado potencial para terem suas características analisadas por meio de técnicas de monitoramento contínuo da floculação.

Palavras-chave: estudos de tratabilidade, jar test, sedimentação, sulfato de alumínio, tratamento de água.

\section{Construction and use of coagulation diagrams as a tool for continuous monitoring of flocculation in water supply}

\section{ABSTRACT}

In this work coagulation diagrams were constructed from bench tests with the aid of jartest equipment in order to evaluate the remaining turbidity of water prepared by adding kaolin suspension (until reaching a turbidity of $25 \pm 2 \mathrm{uT}$ ). The water was then subjected to coagulation, flocculation and sedimentation. Iron-free aluminum sulfate was used as a coagulant in dosages varying from $5 \mathrm{mg} \mathrm{L}^{-1}$ to $100 \mathrm{mg} \mathrm{L}^{-1}$ and coagulation $\mathrm{pH}$ varying from 3 to 9 . Three different rates of sedimentation velocities were investigated $(3.0 ; 1,5$; and 
$0.5 \mathrm{~cm} \mathrm{~min}^{-1}$ ). Based upon the coagulation diagrams, satisfactory results for turbidity removal were observed with coagulation $\mathrm{pH}$ values between 6.5 and 7.5 and coagulant dosages greater than $15 \mathrm{mg} \mathrm{L}^{-1}$; high dosages of coagulant did not necessarily lead to lower remaining turbidity values. Turbidity removal efficiency was higher in lower rates of sedimentation velocities, reaching values less than $1 \mathrm{uT}$ at the lowest sedimentation rate studied. The construction of coagulation diagrams using jartest equipment proved to be an effective tool for the optimization of the coagulation phases, flocculation and sedimentation in water treatment, producing flocs whose characteristics were more easily analyzed using techniques of continuous monitoring flocculation.

Keywords: aluminum sulfate, jar test, treatability studies, sedimentation, water treatment.

\section{INTRODUÇÃO}

Estações de Tratamento de Água (ETA's) que empregam o tratamento em ciclo completo (que usa os processos e operações de coagulação, floculação, decantação e filtração para a clarificação da água, seguida da desinfecção, fluoração e estabilização final) estão presentes em $63,3 \%$ dos municípios brasileiros. Este índice sobe para 80,7\% naqueles com população superior a 100 mil habitantes, segundo a última Pesquisa Nacional de Saneamento Básico (IBGE, 2008). Logo, considerando que grande parte da população brasileira reside em capitais, regiões metropolitanas e cidades de maior porte, é de se esperar que o índice da população abastecida por água tratada em ETA's de ciclo completo seja elevado.

Estes dados mostram a importância do controle e otimização dos processos e operações de coagulação, floculação, decantação e filtração nas ETA's para a produção de água com qualidade adequada ao consumo e atividades humanas.

A coagulação consiste na desestabilização de partículas em suspensão presentes na água (que possuem sua superfície carregada com cargas elétricas negativas), pela adição, na maioria dos casos, de sais de ferro ou alumínio, os quais em contato com a água formam espécies hidrolisadas que, por sua vez, adsorvem nas partículas coloidais presentes na água e as desestabilizam (Kim et al., 2001). Di Bernardo e Dantas (2005) citam que a etapa de coagulação depende basicamente das características da água como $\mathrm{pH}$, alcalinidade, cor verdadeira, turbidez, temperatura, mobilidade eletroforética, força iônica, concentração de sólidos totais dissolvidos, tamanho e distribuição de tamanhos das partículas em estado coloidal e em suspensão, entre outros.

Segundo Di Bernardo e Dantas (2005) o processo de coagulação pode ser considerado como a atuação individual ou conjunta de quatro mecanismos diferentes: compressão da dupla camada elétrica; adsorção e neutralização de cargas; varredura (recomendada para ETA's de ciclo completo) e adsorção e formação de pontes. No mecanismo de varredura, maiores dosagens de coagulante são adicionadas à água em relação ao mecanismo de adsorção e neutralização de cargas, o que leva à formação de precipitados do tipo $\mathrm{Al}(\mathrm{OH})_{3}$ ou $\mathrm{Fe}(\mathrm{OH})_{3}$. Segundo Pavanelli (2001) o mecanismo de varredura vem sendo bastante empregado em estações de tratamento em ciclo completo devido aos flocos formados serem maiores do que aqueles formados pelo mecanismo de adsorção e neutralização de cargas; consequentemente suas velocidades de sedimentação são maiores.

A floculação, por sua vez, ocorre em tanques denominados floculadores, onde as partículas previamente desestabilizadas (na etapa de coagulação) recebem agitação lenta controlada por um tempo relativamente longo para que se aumente a probabilidade de ocorrência dos choques entre as mesmas. O desempenho da etapa da floculação depende diretamente da eficiência do processo de coagulação acrescida de alguns parâmetros inerentes a esta fase: gradiente de velocidade médio e tempo de floculação e o tipo e geometria do equipamento utilizado. A fenomenologia da floculação baseia-se em dois mecanismos 
complementares: adesão e transporte. O primeiro está relacionado às cargas superficiais das partículas, que devem ser alteradas durante a coagulação para que os choques sejam efetivos. $\mathrm{O}$ segundo mecanismo refere-se à agitação introduzida ao meio e pode ser divido em outros três: movimento Browniano (floculação pericinética), movimento do fluído (floculação ortocinética) e sedimentação diferencial (Santos et al., 2004).

Uma maneira simples de se obter os parâmetros para projeto e operação de ETA's que empregam as etapas de coagulação, floculação, sedimentação e filtração são ensaios de tratabilidade da água em equipamento jar test. A otimização das etapas descritas anteriormente, com auxílio de ensaios em equipamento jartest, além de resultar em maior eficiência global da ETA, pode levar a minimização da dosagem de coagulante aplicada à água bruta, fato que, segundo Nam et al. (2013) é valioso, pois resíduos químicos de coagulantes a base de alumínio podem ter efeitos maléficos sobre a saúde humana, principalmente ao sistema nervoso, como o surgimento da Doença de Alzheimer. Os mesmos autores ainda citam que o jartest é comumente usado na determinação da dosagem de coagulante que deve ser aplicado à água bruta por meio de ensaios laboratoriais.

As informações obtidas através dos ensaios em jar test podem ser dispostas em diagramas de coagulação (Kim et al., 2001; Lin et al., 2008; Bongiovani et al., 2010; Selhorst Filho et al., 2011; Ferrari et al., 2012), no qual os valores da dosagem de coagulante, pH de coagulação e turbidez remanescente podem ser facilmente visualizados graficamente e então identificados o par de valores "dosagem de coagulante x pH de coagulação" que conduziram aos melhores resultados na remoção da turbidez ou outro parâmetro de interesse da massa líquida. Amirtharajah e Mills (1982) foram os primeiros a propor o uso de diagramas de coagulação na avaliação da eficiência de remoção de turbidez em amostras de água submetidas à coagulação, floculação e decantação.

O objetivo deste trabalho foi construir diagramas de coagulação para avaliar a turbidez remanescente de uma água de estudo preparada a partir de uma suspensão de caulinita, variando-se a dosagem do coagulante e o pH de coagulação para três diferentes velocidades de sedimentação. Os dados encontrados neste trabalho serviram de base para ensaios em bancada com o auxílio de um equipamento de monitoramento contínuo da floculação (EMCF), desenvolvido recentemente por pesquisadores vinculados ao Departamento de Recursos Hídricos e Saneamento Ambiental do ITA.

Basicamente, estudos com o EMCF são realizados de forma acoplada a ensaios em jar test modificado (Yu et al., 2009; Yukselen e Gregory, 2004); no EMCF é possível avaliar o crescimento dos flocos formados na etapa de floculação do tratamento de águas de abastecimento, sua resistência ao cisalhamento e a capacidade do floco cisalhado (quebrado) de retornar ao seu tamanho original. Estas informações podem resultar na otimização da dosagem de coagulante, bem como o tempo e gradiente de floculação médio, parâmetros fundamentais para o bom desempenho das ETA's que empregam o tratamento em ciclo completo na potabilização de águas distribuídas à população. O princípio de funcionamento do EMCF pode ser detalhadamente consultado no trabalho de Gregory (1984).

\section{MATERIAL E MÉTODOS}

\subsection{Suspensão de caulinita}

A metodologia adotada para a preparação da suspensão-mãe de caulinta foi semelhante à adotada por Yukselen e Gregory (2004). Duzentos gramas de caulinita (Synth ${ }^{\circledR}$ ) foram adicionadas em $500 \mathrm{~mL}$ de água ultrapura com condutividade de $1,5 \mu \mathrm{S} \mathrm{cm}^{-1}$ (obtida através de um equipamento Gehaka Master System pelo sistema Millipore) e, então, a solução resultante foi agitada por $10 \mathrm{~min}$ a $1200 \mathrm{~s}^{-1} \mathrm{com}$ o auxílio de um equipamento jar test. Para obter-se a dispersão total da solução foi necessário elevar o $\mathrm{pH}$ da suspensão ao valor 7,5; o 
qual foi alcançado pela adição de $5 \mathrm{~mL}$ de $\mathrm{NaOH} 0,1 \mathrm{~N}$. Após agitar a solução por 10 min a $1200 \mathrm{~s}^{-1}$, a suspensão de caulinita foi diluída em $1 \mathrm{~L}$ de água ultrapura, homogeneizada e transferida a um cilindro graduado no qual permaneceu em repouso durante $12 \mathrm{~h} 00$.

Com o auxílio de uma bomba de baixa vazão e um tubo plástico de pequeno diâmetro acondicionado dentro do cilindro, com uma de suas extremidades no fundo do mesmo, foi descartado parte do volume da solução contida no recipiente até se obter $800 \mathrm{~mL}$ do sobrenadante; este foi encaminhado para análise de concentração de sólidos totais. A concentração de sólidos totais foi determinada gravimetricamente e encontrou-se o valor de $132 \mathrm{~g} \mathrm{~L}^{-1}$, muito próximo do valor $135 \mathrm{~g} \mathrm{~L}^{-1}$, reportado na literatura (Yukselen e Gregory, 2002 ; 2004) para o mesmo procedimento. Logo depois, a amostra foi diluída em água ultrapura até fornecer uma concentração de sólidos totais de $50 \mathrm{~g} \mathrm{~L}^{-1}$, determinada gravimetricamente e então armazenada em recipientes de plástico sobre refrigeração até seu posterior uso.

\subsection{Coagulante}

Foi utilizado o mesmo coagulante aplicado na ETA da cidade de São José dos Campos, operada pela Companhia de Saneamento Básico do Estado de São Paulo (SABESP), sulfato de alumínio isento de ferro $-\mathrm{Al}_{2}\left(\mathrm{SO}_{4}\right)^{3} \mathrm{x} 14,3 \mathrm{H}_{2} \mathrm{O}$; com teor de $\mathrm{Al}_{2} \mathrm{O}_{3}$ de $8,35 \%$. Como se trata do tratamento de água em escala de bancada, o coagulante foi diluído, sendo preparada uma solução dosando-se $4 \mathrm{~g}$ do produto comercial em $1 \mathrm{~L}$ de água ultrapura.

\subsection{Caracterização da água do poço}

Água de um poço artesiano localizado nas dependências do Departamento de Ciência e Tecnologia Aeroespacial (DCTA) em São José dos Campos/SP foi usada para diluir a suspensão mãe de caulinita, sendo que o $\mathrm{pH}$ foi quantificado com o auxílio de um pHmetro QX 1500 Plus Qualxtron. Para a determinação do $\mathrm{pH}$ as amostras foram agitadas com o auxílio de um agitador magnético FISATOM 752.

A turbidez e a condutividade da água do poço foram quantificadas com o auxílio de um turbidímero de bancada HACH 2100Q e um Condutívimetro Marconi, respectivamente. Cor aparente e cor verdadeira foram avaliadas com o emprego de um Espectrofotômetro UV-VIS HACH DR 6000. Já a dureza e alcalinidade total foram determinadas por titulometria, metodologia descrita no Standard Methods For the Examination of Water and Wastewater (APHA et al., 2012). Todas as demais análises de turbidez e pH realizadas neste trabalho também seguiram a metodologia descrita nesta subseção.

\subsection{Procedimento de ensaio}

Para os ensaios em equipamento jar test (Nova Ética, modelo LDB especial), a suspensão mãe de caulinita foi diluída diariamente em água proveniente do poço artesiano até obter valor de turbidez de $25 \pm 2 \mathrm{uT}$, configurando a água de estudo. Devido a pequenas mudanças nas características da água, como também na sensibilidade do turbidímetro usado na quantificação da turbidez, avaliada em cada batelada de ensaios, admitiu-se trabalhar com o valor de turbidez entre 23 e $27 \mathrm{uT}$.

Usando o procedimento tradicional de ensaio em equipamento jar test, os jarros foram preenchidos com $2 \mathrm{~L}$ da água de estudo preparada a partir da suspensão-mãe de caulinita ( $25 \pm 2$ uT) e então variou-se o par de valores "dosagem de coagulante x pH de coagulação", sendo que o objetivo foi variar o valor do $\mathrm{pH}$ de coagulação de 3 até 9 (valores próximos). Já a dosagem do coagulante foi variada de $5 \mathrm{mg} \mathrm{L}^{-1}$ a $100 \mathrm{mg} \mathrm{L}^{-1}$.

Foram empregados os seguintes parâmetros de mistura rápida e floculação: gradiente de velocidade médio de mistura rápida $\left(G_{m r}\right)$ de $1000 \mathrm{~s}^{-1}$ e tempo de mistura rápida $\left(\mathrm{T}_{\mathrm{mr}}\right)$ de $10 \mathrm{~s}$; gradiente de velocidade médio de floculação $\left(G_{f l}\right)$ de $20 \mathrm{~s}^{-1}$ e tempo de floculação $\left(\mathrm{T}_{\mathrm{fl}}\right)$ de 20 min. Após isso, a água floculada foi mantida em repouso para que os flocos 
sedimentassem, sendo que foram empregadas velocidades de sedimentação de 3,$0 ; 1,5$ e $0,5 \mathrm{~cm} \mathrm{~min}^{-1}$, após os quais foram coletadas amostras dos sobrenadantes para as medidas de turbidez e coraparente. A metodologia para a execução dos ensaios em jar test é descrita em Di Bernardo et al. (2011).

Para se variar o $\mathrm{pH}$ foi empregado agente alcalinizante (hidróxido de sódio $0,1 \mathrm{~N}$ ) ou acidificante (ácido clorídrico $0,1 \mathrm{~N}$ ). Depois de dosado o alcalinizante ou acidificante à solução, foi aguardado aproximadamente $30 \mathrm{~s}$ para a estabilização do $\mathrm{pH}$. A temperatura da água de estudo foi ajustada a $20 \pm 1^{\circ} \mathrm{C}$.

Para as diferentes velocidades de sedimentação foi avaliada a turbidez e cor aparente remanescentes da amostra e, de posse destes dados, foram construídos os diagramas de coagulação. As curvas foram construídas como conjuntos, onde as linhas delimitam a região dos pares de dosagem de coagulante $\mathrm{x}$ pH de coagulação que possuem turbidez menor ou igual a indicada pela curva, conforme metodologia adotada no trabalho de De Julio et al. (2009). Também foram construídos diagramas de coagulação para a cor aparente, no entanto, os dados não foram apresentados neste trabalho para não torná-lo muito extenso.

\subsection{Monitoramento contínuo da floculação}

Com o par de valores "dosagem de coagulante x pH de coagulação" definidos anteriormente, foram realizados ensaios de monitoramento contínuo da floculação com o auxílio do EMCF, nesta etapa foi adotado gradiente de velocidade e tempos de quebra de 913 $\mathrm{s}^{-1}$ e 5, 60 e $300 \mathrm{~s}$, respectivamente, gradiente de velocidade de mistura rápida e de floculação de 1000 e $25 \mathrm{~s}^{-1}$, respectivamente. O procedimento de ensaio, resumidamente, consistiu em coagular a água nas condições operacionais definidas anteriormente $(6 \mathrm{mg} / \mathrm{L}$ de hidróxido de sódio como agente alcalinizante e $30 \mathrm{mg} / \mathrm{L}$ de sulfato de alumínio como coagulante $1,32 \mathrm{mg} / \mathrm{L} \mathrm{de} \mathrm{Al}^{3+}$ ), efetuou-se a mistura rápida por $10 \mathrm{~s}$ e se iniciou a etapa da floculação (gradiente de velocidade de floculação de $25 \mathrm{~s}^{-1}$ ). Durante a etapa de mistura rápida, houve a coleta de amostra da água para leitura do pH de coagulação.

Passados 25 minutos de floculação, o valor do gradiente de velocidade foi automaticamente aumentado para $913 \mathrm{~s}^{-1}$ durante os tempos de 5, 60 ou $300 \mathrm{~s}$ a fim de verificar a quebra dos flocos e sua potencialidade de recrescimento para cada situação. Após o tempo de quebra correspondente a cada ensaio, novamente, o gradiente de velocidade foi reduzido a $25 \mathrm{~s}^{-1}$ durante 25 minutos a fim de permitir a refloculação. Passado esta etapa, mais uma vez, o gradiente de velocidade foi aumentado para $913 \mathrm{~s}^{-1}$, promovendo desta forma, o segundo evento de quebra dos flocos por tempo previamente determinado e igual ao da quebra anterior, seguida pela refloculação.

\section{RESULTADOS E DISCUSSÃO}

\subsection{Caracterização da água do poço}

Na Tabela 1 são apresentados os valores obtidos na caracterização da água do poço artesiano do DCTA utilizada na diluição da suspensão-mãe de caulinita. Como a coleta da água do poço para a realização dos ensaios foi feita diariamente, são apresentados os valores médios e seus respectivos desvio padrão, valores máximo e mínimo obtidos para os parâmetros medidos, aqueles que apresentam apenas um valor não sofreram mudanças ao longo das coletas.

Pode-se verificar na Tabela 1 o caráter levemente ácido da água do poço utilizada para a preparação da água de estudo a partir da suspensão-mãe da caulinita. Como era esperado, os valores de turbidez, cor aparente e cor verdadeira foram baixos devido à origem da água. Não houve variação expressiva na maioria dos parâmetros analisados, com exceção de cor 
aparente e verdadeira, porém sem mudança da magnitude dos valores; já a alcalinidade e dureza total não sofreram variações ao longo das coletas, apresentando baixos valores.

Tabela 1. Caracterização da água do poço artesiano utilizada nos ensaios.

\begin{tabular}{lccc}
\hline \multicolumn{1}{c}{ Parâmetro } & Valor médio & Desvio padrão & Min. - Máx. \\
\hline $\mathrm{pH}$ & 6,33 & $\pm 0,04$ & $6,30-6,41$ \\
Condutividade $\left(\mu \mathrm{S} \mathrm{cm}^{-1}\right)$ & 35,77 & $\pm 0,59$ & $35,05-36,63$ \\
Turbidez $(\mathrm{uT})$ & 1,13 & $\pm 0,09$ & $1,01-1,27$ \\
Cor aparente $(\mathrm{uH})$ & 8,25 & $\pm 3,3$ & $3-12$ \\
Cor verdadeira $(\mathrm{uH})$ & 3,75 & $\pm 2,4$ & $1-7$ \\
Alcalinidade total $\left(\mathrm{mgCaCO}_{3} \mathrm{~L}^{-1}\right)$ & 12,8 & - & - \\
Dureza total $\left(\mathrm{mgCaCO}_{3} \mathrm{~L}^{-1}\right)$ & 3,5 & - & - \\
\hline
\end{tabular}

\subsection{Diagramas de coagulaçãa}

Nas Figuras 1, 2 e 3 são apresentados os diagramas de coagulação construídos para a avaliação da remoção de turbidez da água de estudo em três diferentes velocidades de sedimentação, sendo elas 3,$0 ; 1,5$ e $0,5 \mathrm{~cm} \mathrm{~min}^{-1}$, (taxas de aplicação superficial de 43,2;21,6 e $7,2 \mathrm{~m}^{3} \mathrm{~m}^{-2} \mathrm{dia}^{-1}$ ), respectivamente.

$\mathrm{O}$ valor médio e respectivo desvio padrão da turbidez da água de estudo utilizada nos ensaios foi de $25,5 \pm 1,1 \mathrm{uT}$, quantificada em cada batelada de ensaios.

Analisando as Figuras 1, 2 e 3 observa-se, conforme esperado, que houve melhoria significativa no valor da turbidez remanescente ao passo em que a velocidade de sedimentação diminuiu. No entanto, na prática, velocidades de sedimentação muito baixas resultam em decantadores com dimensões elevadas devido à necessidade de maior tempo de detenção hidráulica da água a ser decantada, o que pode tornar o projeto de uma ETA inviável economicamente.

Para o sulfato de alumínio líquido isento de ferro foram obtidos resultados satisfatórios nos valores de turbidez remanescenteda água decantada, em alguns casos, abaixo de $1 \mathrm{uT}$, sendo que inúmeros valores apresentaram turbidez remanescente menor que $3 \mathrm{uT}$, valor máximo recomendado para água decantada visando aumentar a duração das carreiras de filtração (Ferrari et al., 2012). Pode-se observar também que alguns valores de turbidez remanescente $(0,71$ e $0,8 \mathrm{uT})$ obtidos para a água decantada chegaram a valores próximos do exigido pelo Padrão de Potabilidade Brasileiro (Portaria MS N 2914/2011) para a água após passar por polimento final em filtros rápidos de areia utilizados nas ETA's (Brasil, 2011).

Os melhores resultados encontraram-se na faixa de $\mathrm{pH}$ de coagulação entre 6,5 e 7,5 com

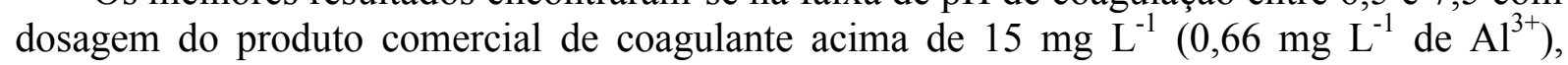
caracterizando o mecanismo de coagulação da varredura. Embora bons resultados tenham sido obtidos também para elevadas dosagens de coagulante, esta não é a alternativa mais viável para a rotina operacional de uma ETA devido aos custos com o coagulante e agente alcalinizante requerido no tratamento, além da maior geração de lodo a ser tratado e disposto. Exemplificando, para a velocidade de sedimentação de $1,5 \mathrm{~cm} \mathrm{~min}^{-1}$ foram obtidos valores de turbidez remanescente de $1,57 \mathrm{uT}$ com a adição de $30 \mathrm{mg} \mathrm{L}^{-1}$ de coagulante e de $5 \mathrm{mg} \mathrm{L}^{-1}$ de $\mathrm{NaOH}$. Enquanto que a turbidez remanescente com valor pouco menor, 1,41 uT, foi obtida com a adição de $50 \mathrm{mg} \mathrm{L}^{-1}$ de coagulante e $20 \mathrm{mg} \mathrm{L}^{-1}$ do agente alcalinizante. Neste caso não justifica usar uma dosagem $2 / 3$ maior de coagulante e quatro vezes maior de agente alcalinizante para obter-se resultados ligeiramente melhores para a turbidez remanescente.

Para a maior dosagem de coagulante, $100 \mathrm{mg} \mathrm{L}^{-1}$, foi necessário $28 \mathrm{mg} \mathrm{L}^{-1} \mathrm{de} \mathrm{NaOH}$ para chegar-se ao $\mathrm{pH}$ que levou aos melhores resultados (turbidez remanescente de 7,61; 2,44 e $1,57 \mathrm{uT}$ para as velocidades de 3,$0 ; 1,5$ e $0,5 \mathrm{~cm} \mathrm{mim}^{-1}$, respectivamente). Pode ser constatado 
neste caso, que, apesar de serem obtidos bons resultados de remoção de turbidez, os diagramas de coagulação demonstram eficiência inferior aos exemplos citados anteriormente, onde se obteve valores de turbidez mais satisfatórios com menores dosagens de coagulante e de agente alcalinizante. Logo, elevadas dosagens de coagulante não se traduzem, necessariamente, em melhores resultados. Esta mesma conclusão foi obtida por Pavanelli (2001), que em seu trabalho avaliou a eficiência de remoção de turbidez e cor aparente de uma água que apresentava estes parâmetros com valor elevado, com o uso de sulfato de alumínio, cloreto férrico, hidroxicloreto de alumínio e sulfato férrico.

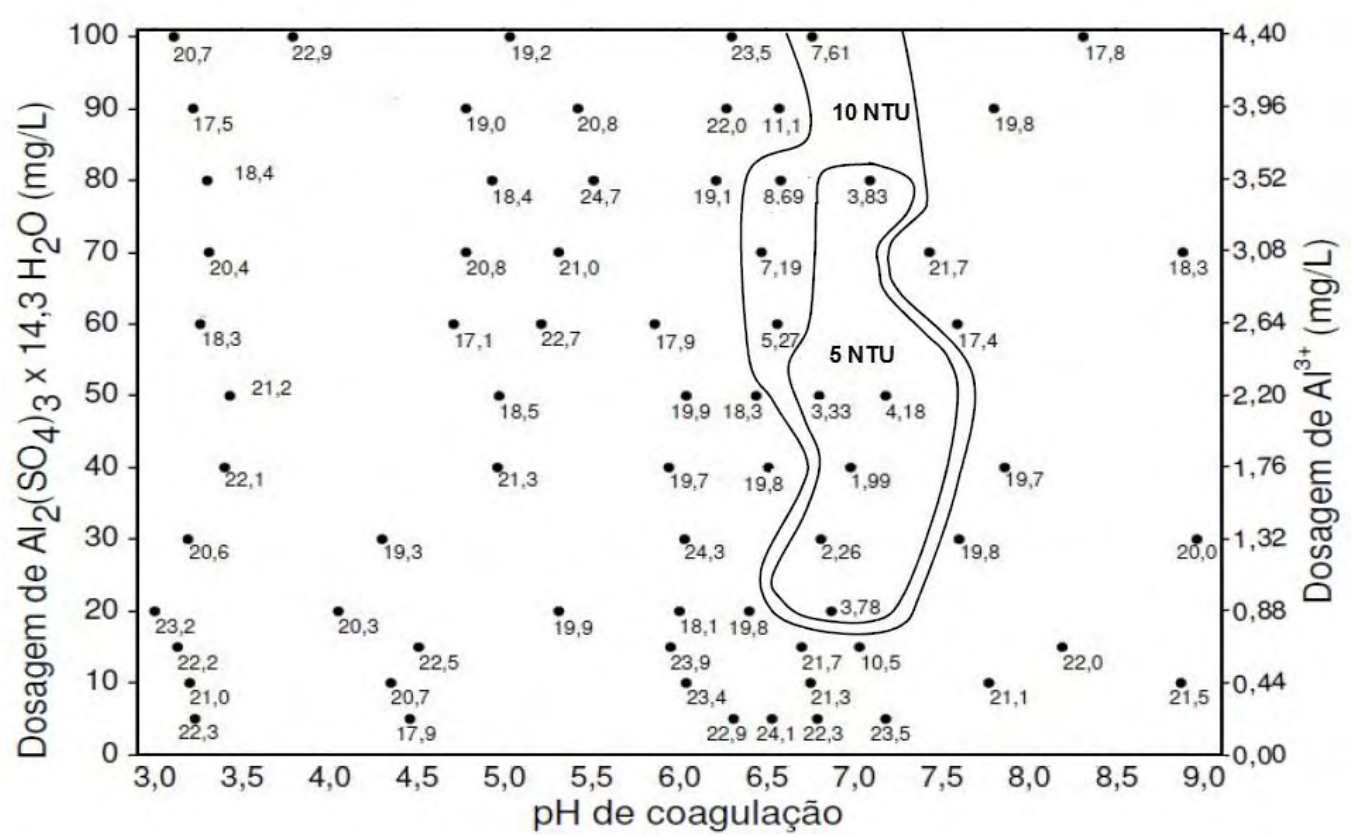

Figura 1. Diagrama de coagulação contendo as curvas de mesma turbidez remanescente (uT) em função da dosagem de coagulante $\mathrm{x} \mathrm{pH}$ de coagulação para a água decantada com velocidade de $3,0 \mathrm{~cm} \mathrm{~min}^{-1}$.

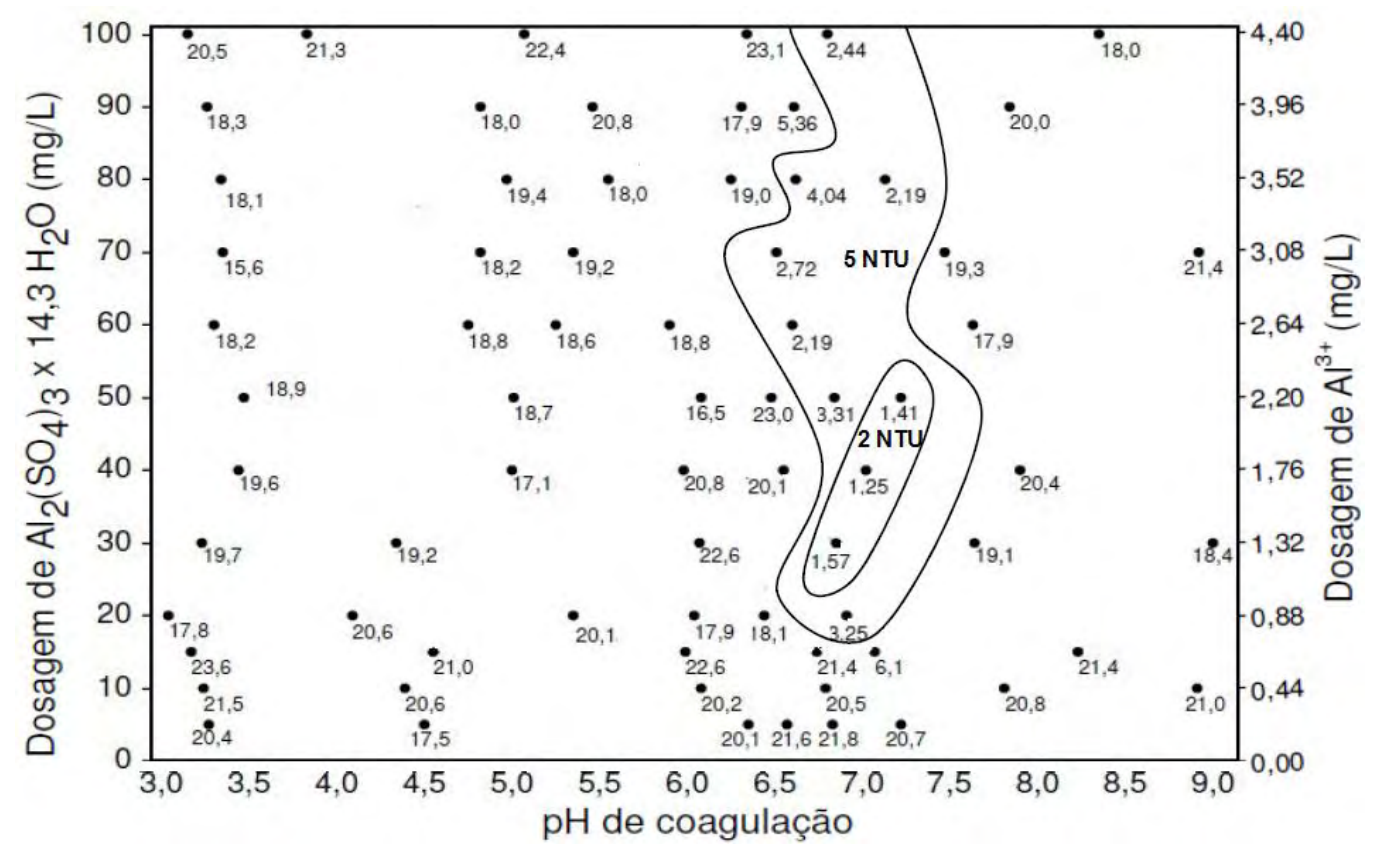

Figura 2. Diagrama de coagulação contendo as curvas de mesma turbidez remanescente (uT) em função da dosagem de coagulante $\mathrm{x} \mathrm{pH}$ de coagulação para a água decantada com velocidade de $1,5 \mathrm{~cm} \mathrm{~min}^{-1}$. 


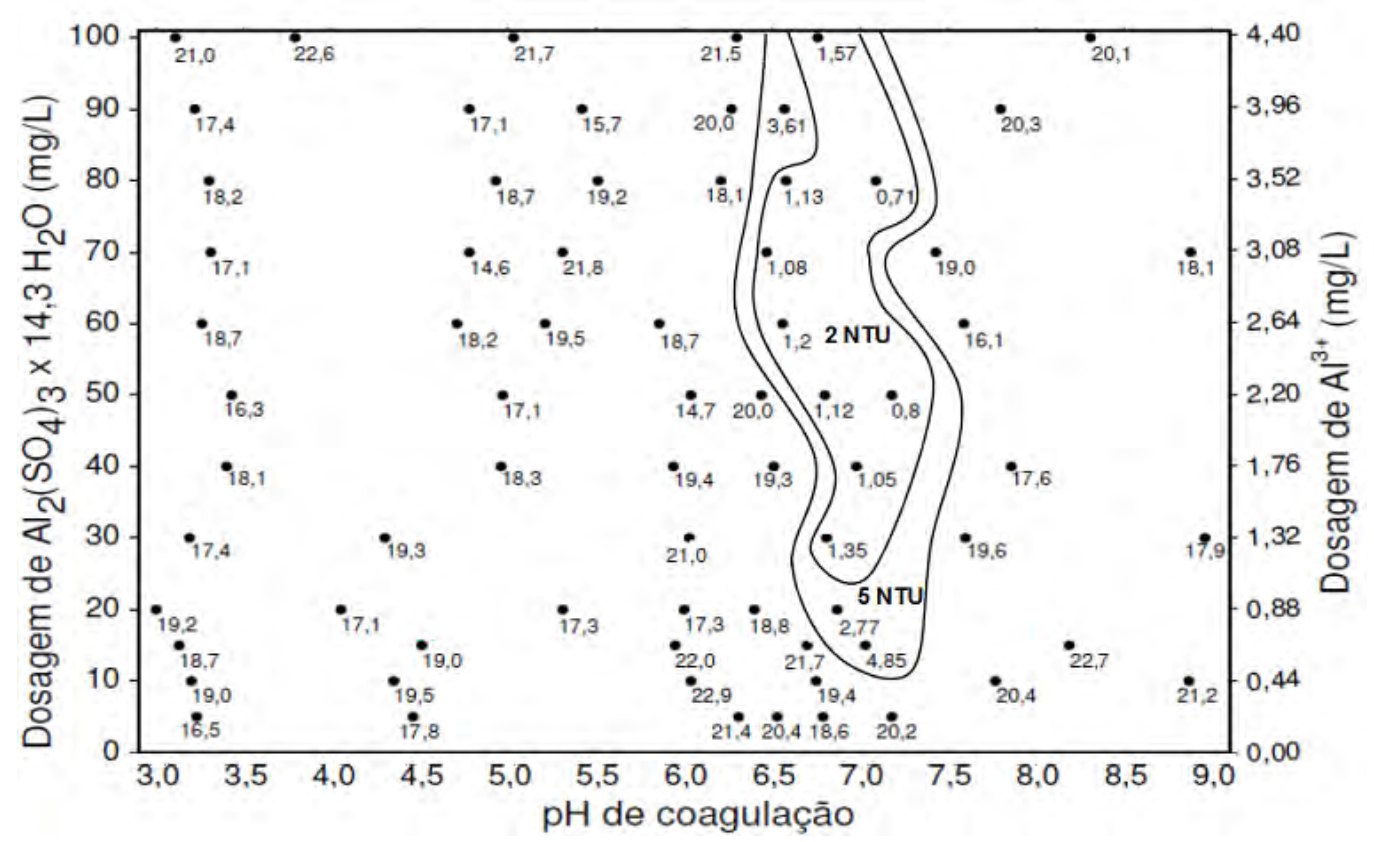

Figura 3. Diagrama de coagulação contendo as curvas de mesma turbidez remanescente (uT) em função da dosagem de coagulante $\mathrm{x}$ pH de coagulação para a água decantada com velocidade de $0,5 \mathrm{~cm} \mathrm{~min}^{-1}$.

Para os estudos posteriores, no EMCF, objeto de prosseguimento desta pesquisa, foi avaliado o crescimento dos flocos formados na etapa de floculação, sua resistência ao cisalhamento e a capacidade do floco cisalhado (quebrado) de retornar ao seu tamanho original (reversibilidade) para o par de valores "dosagem de coagulante x pH de coagulação" que apresentou valores de turbidez remanescente de 2,26; 1,57 e 1,35 uT para as velocidades de sedimentação de 3,$0 ; 1,5$ e $0,5 \mathrm{~cm} \mathrm{~min}^{-1}$, respectivamente.

Chegou-se aos resultados mencionados acima para turbidez remanescente por meio da aplicação de $30 \mathrm{mg} \mathrm{L}^{-1}$ de sulfato de alumínio líquido isento de ferro $\left(1,32 \mathrm{mg} \mathrm{L}^{-1}\right.$ de $\left.\mathrm{Al}^{3+}\right)$ e pH de coagulação igual a 6,81, alcançado através da adição de $6 \mathrm{mg} \mathrm{L}^{-1}$ de $\mathrm{NaOH}$ à água de estudo. Esta configuração permitiu a obtenção de turbidez remanescente menor que 3 uT nas três velocidades de sedimentação estudadas, além da adição de dosagens de coagulante e agente alcalinizante à água de estudo relativamente baixas quando comparadas aos demais dados apresentados nas Figuras 1, 2 e 3.

\subsection{Ensaios de monitoramento contínuo da floculação}

$\mathrm{Na}$ Figura 4, está representada a variação do Índice de Floculação (IF) em função do tempo (min) durante as etapas de floculação e refloculação, destaque para os valores de IF quando aumentado o gradiente de velocidade para $913 \mathrm{~s}^{-1}$ durante os tempos de 5,60 e $300 \mathrm{~s}$ (gradiente de velocidade e tempos de quebra). Resumidamente, o valor de IF presente na Figura 4, dá uma indicação do tamanho das partículas/flocos no decorrer dos ensaios, sendo que quanto maior o tamanho dos flocos presentes em determinada suspensão, maior é o valor de IF correspondente (Gregory e Chung, 1995).

Fica evidente ao analisar a Figura 4, que os flocos formados a partir da adição de sulfato de alumínio como agente coagulante e submetidos a um gradiente de velocidade de quebra de $913 \mathrm{~s}^{-1}$, durante três tempos distintos, não apresentaram capacidade de voltar ao tamanho observado antes da quebra, mesmo que dada a condição favorável de tempo para tal fato ocorrer (tempo de refloculação de $25 \mathrm{~min}$ ). Este fato já foi descrito na literatura por Yukselen e Gregory (2002; 2004), que também obtiveram estes resultados por meio de ensaios de monitoramento contínuo da floculação. 


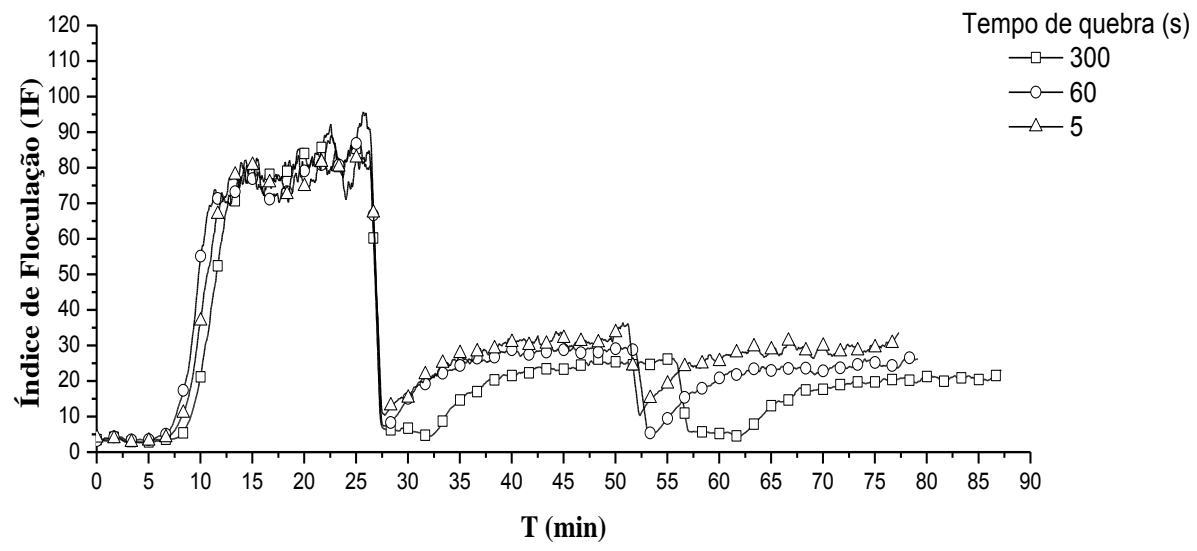

Figura 4. Variação do valor do Índice de Floculação (IF) em função do tempo (min) para o gradiente de velocidade de quebra de $913 \mathrm{~s}^{-1}$ e tempos de quebra de 5, 60 e 300 s. Dosagem de coagulante e alcalinizante aplicada: $30 \mathrm{mg} \mathrm{L}^{-1}$ e $6 \mathrm{mg} \mathrm{L}^{-1}$, respectivamente. Gradiente de velocidade de floculação: $25 \mathrm{~s}^{-1}$. Gradiente de velocidade e tempo de mistura rápida: $1000 \mathrm{~s}^{-1}$ e $10 \mathrm{~s}$, respectivamente.

\section{CONCLUSÕES}

Os diagramas obtidos e apresentados neste trabalho demonstraram valores satisfatórios para turbidez remanescente de uma água de estudo preparada a partir de uma suspensão-mãe de caulinita submetida à coagulação, floculação e sedimentação em escala laboratorial com auxílio de um equipamento jar test. Estes dados indicam que ocorreu formação de flocos com tamanho suficiente para serem removidos com eficiência pelo mecanismo de sedimentação.

$\mathrm{O}$ crescimento dos flocos obtidos neste estudo tem potencialidade para ser avaliado através de técnica de monitoramento contínuo da floculação, com o auxílio da qual pode alcançar-se flocos com tamanhos otimizados e que são removidos da massa líquida com velocidades de sedimentação ajustadas às condições da água floculada resultando, na prática, em decantadores de menor área e maior eficiência.

Os resultados obtidos demonstram que a coagulação da água de estudo ocorreu pelo mecanismo de varredura, com resultados satisfatórios a partir da dosagem de $15 \mathrm{mg} \mathrm{L}^{-1}$ de coagulante e $\mathrm{pH}$ na faixa de 6,5 a 7,5.

Este trabalho foi de fundamental importância para a avaliação do comportamento da água de estudo em relação à coagulação e à formação dos flocos, bem como a capacidade dos mesmos sedimentar-se. Desta forma, houve informações suficientes para dar prosseguimento aos ensaios de monitoramento contínuo da floculação para as melhores configurações obtidas e descritas neste trabalho, ensaios em que ficou claro que o EMCF foi capaz de fornecer informações contundentes sobre o crescimento, quebra e recrescimento dos flocos, formados a partir de água de estudo com turbidez de $25 \mathrm{uT}$. Ficou claro, nos ensaios de monitoramento contínuo da floculação, que os flocos formados durante a etapa de floculação do tratamento de águas de abastecimento, uma vez quebrados, possuem capacidade limitada de alcançar tamanho semelhante ao original, comprometendo significativamente a remoção deles durante a etapa seguinte, decantação.

\section{AGRADECIMENTOS}

Os autores agradecem à Fundação de Amparo à Pesquisa do Estado de São Paulo (FAPESP), financiadora deste projeto de pesquisa, por meio do edital FAPESP/SABESP (Processo 10/50694-0), e da bolsa de mestrado (Processo 11/22685-0). Também agradecem à 
Companhia de Saneamento Básico do Estado de São Paulo (SABESP), empresa parceira e financiadora deste projeto.

\section{REFERÊNCIAS}

AMERICAN PUBLIC HEALTH ASSOCIATION; AMERICAN WATER WORKS ASSOCIATION; WATER POLLUTION CONTROL FEDERATION. Standard methods for the examination of water and wastewater. 21. ed. Washington, 2005.

AMIRTHARAJH, A.; MILLS, K. M. Rapid mix design for mechanisms of alum coagulation. Journal of the American Water Works Association, v. 74. n. 4, p. 210-216, 1982. http://www.jstor.org/stable/41271001

BONGIOVANI, M. C.; KONRADT-MORAES, L. C.; BERGAMASCO, R.; LOURENÇO, B. S. S.; TAVARES, C. R. G. Os benefícios da utilização de coagulantes naturais para a obtenção de água potável. Acta Scientiarum Technology, v. 32, n. 2, p. $167-170$, 2010. http://dx.doi.org/10.4025/actascitechnol.v32i2.8238

BRASIL. Ministério da Saúde. Portaria No 2914/2011. Dispõe sobre os procedimentos de controle e de vigilância da qualidade da água para consumo humano e seu padrão de potabilidade. Diário Oficial [da] União, 12 dez. 2011.

DE JULIO, M.; FIOVARANTE. D. A.; SELHORST FILHO, O.; De JULIO, T. S.; OROSKI, F. I. Avaliação da Remoção de Cianobactérias e Saxitoxinas da água bruta afluente a ETA Pitangui de Ponta Grossa/PR, utilizando os diagramas de coagulação para o cloreto férrico e o reagente de Fenton. Holos Environment, v. 9, n. 2, p. $254-273$, 2009.

DI BERNARDO, L.; DANTAS, A. D. B. Métodos e técnicas de tratamento de água. 2. Ed. São Carlos: RiMA, 2005. 1565p.

DI BERNARDO, L.; DI BERNARDO, A.; VOLTAN, P. L. Tratabilidade de água e dos resíduos gerados em estações de tratamento de água. [S.1.]: LdiBe, 2011. 454p.

FERRARI, T. N.; DE JULIO, M.; DE JULIO, T. S.; SOUSA JÚNIOR, W. C. Estudos de Tratabilidade das Águas do Rio Paraíba do Sul que Abastecem o Município de São José dos Campos/SP. Revista AIDIS de Ingeniería y Ciencias Ambientales, v. 5, n. 2, p. $45-58,2012$.

GREGORY, J. Turbidity fluctuations in flowing suspensions. Journal of Colloid and Interface Science, v. 105 , p. 357-371, 1984. http://dx.doi.org/10.1016/00219797(85)90309-1

GREGORY, J.; CHUNG, H. Continuous monitoring of floc properties in stirred suspensions. Journal of Water Supply: Research and Technology, v. 44, n. 3, p. 125-131, 1995.

INSTITUTO BRASILEIRO DE PESQUISA E ESTATÍSTICA - IBGE. Pesquisa Nacional de saneamento básico 2008. Rio de Janeiro, 2010. 219 p. Disponível em: $<$ http://www.ibge.gov.br/home/estatistica/

populacao/condicaodevida/pnsb2008/PNSB_2008.pdf> Acesso em: 14 set. 2012.

KIM, S.; MOON, B.; LEE, H. Effects of $\mathrm{pH}$ and dosage on pollutant removal and floc structure during coagulation. Michochemical Journal, v. 68, p. 197 - 203, 2001. http://dx.doi.org/10.1016/S0026-265X(00)00146-6 
LIN, J.; HUANG, C.; PAN, J. R.; WANG, D. Effect of Al(III) speciation on coagulation of highly turbid water. Chemosphere, v. 72, p. $189-196,2008$. http://dx.doi.org/10.1016/j.chemosphere.2008.01.062

NAM, S.; JO, B.; KIM, M.; KIM, W.; ZOH, K. Streaming current titration for coagulation of high turbidity water. Colloids and surfaces A: Phisicochemical and Engineering Aspects, v. 419, p. 133 - 139, 2013. http://dx.doi.org/10.1016/j.colsurfa.2012.11.051

PAVANELLI, G. Eficiência de diferentes tipos de coagulantes na coagulação, floculação e sedimentação de água com cor ou turbidez elevada. 2001. 216f. Dissertação (Mestrado) - Escola de Engenharia de São Carlos, Universidade de São Paulo, São Carlos, 2001.

SANTOS, H. R.; PRADO, G. S.do; VIDAL, C. M. de S.; MORUZZI, R. B.; CAMPOS, J. R. Aplicabilidade das técnicas de determinação de tamanho de partículas em sistemas de tratamento de água e esgoto sanitário. Revista Engenharia Sanitária e Ambiental, v. 9, n. 4, p. $291-300,2004$.

SELHORST FILHO, O.; WIECHETECK, G. K.; ANDRADE FILHO, A. G.; DÖLL, M. M. R. Otimização de parâmetros de classificação de água do sistema de tratamento de água de Ponta Grossa (PR). Revista de Engenharia e Tecnologia, v. 3, n. 2, p. 35-46, 2011.

YU, W.; GUIBAI, L.; XU, Y.; YANG, X. Breakage and re-growth of flocs formed by alum and PACl. Powder Technology, n. 189, p. 439-443, 2009.

http://dx.doi.org/10.1016/j.powtec.2008.07.008

YUKSELEN, M. A.; GREGORY, J. Breakage and re-formation of alum flocs. Environmental Engineering Science, v. 19, n. 4, p. 229 - 236, 2002. http://dx.doi.org/10.1089/109287502760271544

YUKSELEN, M. A.; GREGORY, J. The reversibility of floc breakage. International $\begin{array}{lllllll}\text { Journal of Mineral Processing, v. 73, p. 251-259, } 2004 . & \text {. }\end{array}$ http://dx.doi.org/10.1016/S0301-7516(03)00077-2 


Ambiente \& Água - An Interdisciplinary Journal of Applied Science
ISSN 1980-993X - doi:10.4136/1980-993X
www.ambi-agua.net
E-mail: ambi-agua@agro.unitau.br

\title{
Análise de tendência em séries históricas de vazão e precipitação: uso de teste estatístico não paramétrico
}

\author{
doi: 10.4136/ambi-agua.1427
}

Received: 16 Jun. 2014; Accepted: 07 Nov. 2014

\author{
Eduardo Morgan Uliana ${ }^{1 *}$; Demetrius David da Silva ${ }^{2}$; Estevão Morgan Uliana ${ }^{3}$; \\ Beatriz Silveira Rodrigues ${ }^{2}$; Lucas de Paula Corrêdo \\ ${ }^{1}$ Universidade Federal de Mato Grosso (UFMT), Sinop, MT, Brasil \\ ${ }^{2}$ Universidade Federal de Viçosa (UFV), Viçosa, MG, Brasil \\ ${ }^{3}$ Instituto Federal do Espírito Santo (IFES), Colatina, ES, Brasil \\ *Autor correspondente: e-mail: morganuliana@gmail.com, \\ demetrius@ufv.br, estevaomorgan@hotmail.com, \\ biasrodrigues23@hotmail.com, lucascorredo@gmail.com
}

\section{RESUMO}

A detecção de tendência em séries de vazão e precipitação apresenta expressiva importância para a gestão de recursos hídricos e ela pode ser feita por meio de testes estatísticos não paramétricos. $\mathrm{O}$ objetivo do trabalho foi avaliar a significância das tendências em série temporais de vazões e precipitações mensal e anual, por meio do teste de Pettitt. Foram utilizadas séries históricas de precipitações e vazões correspondentes ao período de 1939 a 2005, registradas nas estações 02041003 e 57450000, pertencentes à rede hidrometeorológica da Agência Nacional de Águas (ANA), localizadas no município de Alegre-ES. Foi possível identificar, com o teste de Pettitt, mudança de tendência na vazão média mensal do mês de outubro a partir do ano 1963. A vazão média deste mês aumentou $34,2 \%$ a partir do ano 1963. Já a série de precipitações apresentou tendência positiva nos meses de agosto e setembro a partir dos anos 1967 e 1964, respectivamente. O aumento da vazão média do mês de outubro pode ser consequência do aumento da precipitação nos meses de agosto e setembro, havendo a necessidade de estudos adicionais que levem em consideração a influência de fatores climáticos e antrópicos neste componente do ciclo hidrológico. Após a análise dos resultados obtidos concluiu-se que o teste de Pettit apresentou desempenho satisfatório para a detecção de tendências nas séries históricas de vazões e de precipitações.

Palavras-chave: chuva, estacionariedade, série temporal, teste de Pettitt, vazão média.

\section{Trend analysis of an historical series of flow and precipitation using a non-parametric statistical test}

\section{ABSTRACT}

Detecting trends in flow and precipitation series has great importance for the management of water resources and can be accomplished using statistic non-parametric tests. This work evaluated the significance of trends in flow and temporal series of precipitation as well as monthly and annual precipitation trends using Pettitt's test. The study used historical 
series of flow and precipitation corresponding to the period of 1939 to 2005, recorded at stations 02041003 and 57450000 of the hydro-meteorological network of Water National Agency (ANA), located in the municipality of Alegre-ES. Using the Pettitt test, it was possible to identify a change in the trend of October average flow since the year 1963. The average for this month increased $34.2 \%$ since 1963 . However, the rainfall series had a positive trend in August and September since the years 1967 and 1964, respectively. The increase in the October average flow may be a consequence of an increase in rainfalls in August and September; this requires further studies which may consider the influence of climate and anthropic factors in this hydrologic cycle component. Based on the results of the study, it was concluded that the Pettitt test performed satisfactorily in detecting trends in the historic series of flow and precipitation.

Keywords: average flow, Pettitt test, rain, stationarity, time series.

\section{INTRODUÇÃO}

A disponibilidade de recursos hídricos é uma questão de importância global e seu conhecimento é fundamental para o desenvolvimento da sociedade. Dentre as variáveis para avaliar a disponibilidade hídrica destaca-se a vazão de cursos d'água, a qual, nas últimas décadas tem sido influenciada expressivamente por mudanças no clima e também no uso e ocupação do solo em bacias hidrográficas. Atividades antrópicas intensivas como, por exemplo, irrigação e construção de barragens tem resultado em grandes impactos sobre a vazão, principalmente em sua magnitude (Wei et al., 2013).

$\mathrm{O}$ interesse em estudos de tendência dos componentes do ciclo hidrológico tem aumentado devido às controvérsias sobre as mudanças climáticas. De acordo com Joseph et al. (2013), a identificação de tendências sazonais de precipitação e vazão contribui para a compreensão da variabilidade climática global e é essencial para o desenvolvimento de modelos hidrológicos, previsão hidrológica e gestão dos recursos hídricos.

Hamed (2008), ao analisar a vazão anual de 35 rios em todo o mundo, verificou tendência positiva em 11 e negativa em seis considerando o nível de $5 \%$ de significância. $O$ mesmo autor, ao analisar a tendência das vazões de 12 rios dos Estados Unidos, verificou redução de vazões em um rio e aumento em três rios.

Além de verificar se a não estacionariedade de uma série hidrológica está relacionada com variações do clima ou alterações antrópicas, a realização da análise de tendência é importante na maioria das aplicações de hidrologia estatística, visto que as séries hidrológicas devem ter como pré-requisito os atributos de estacionariedade e homogeneidade (Naghettini e Pinto, 2007).

A identificação de tendência em séries históricas pode ser feita por meio de análise estatística, sendo o teste de Pettitt (Pettitt, 1979) um dos mais utilizados. Este teste não paramétrico permite confirmar a estacionariedade da série histórica, ou seja, excetuando as flutuações aleatórias, as observações são invariantes com relação à cronologia de suas ocorrências.

Com isso, o objetivo do trabalho foi analisar a tendência de séries histórica de vazão e precipitação por meio de teste estatístico não paramétrico, de forma que as informações obtidas possam ser incorporadas em estudos relacionados com a modelagem hidrológica e gestão de recursos hídricos.

\section{MATERIAL E MÉTODOS}

Foram utilizadas séries históricas de vazões e precipitações correspondentes ao período de 1939 a 2005, registradas nas estações 57450000 e 02041003, pertencente à rede 
hidrometeorológica da Agência Nacional de Águas (ANA), ambas localizadas nas

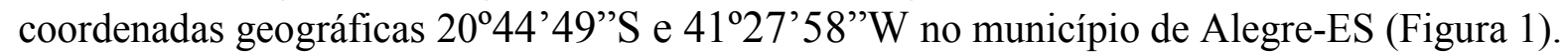

A bacia hidrográfica possui área e perímetro iguais a $2.218,0 \mathrm{~km}^{2}$ e $407,0 \mathrm{~km}$, respectivamente.

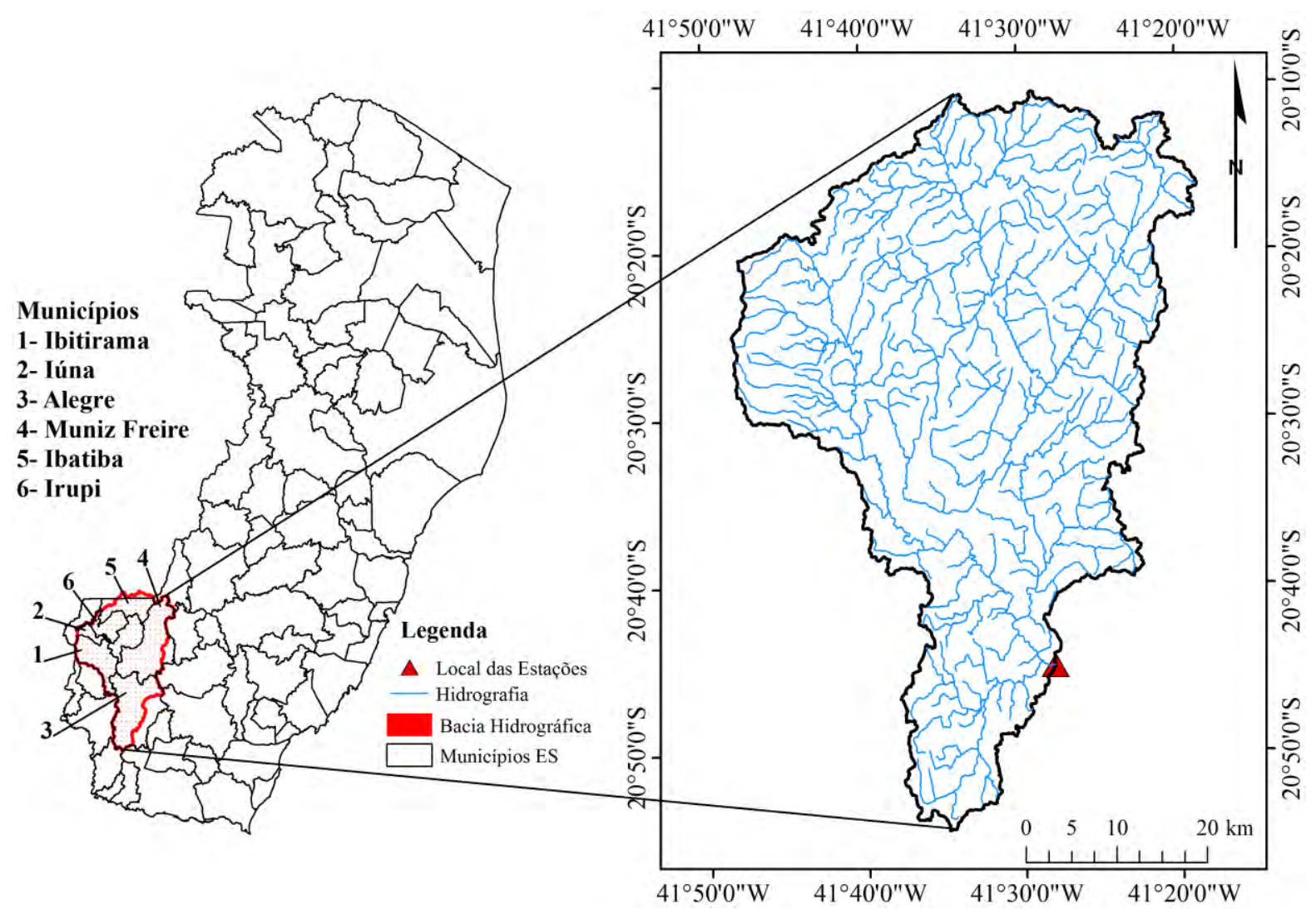

Figura 1. Localização da bacia hidrográfica e da estação fluviométrica utilizada no estudo.

Para identificação de tendência nas séries históricas de vazão média e precipitação mensal e anual foi utilizado o teste de Pettitt (Pettitt, 1979; Liu et al., 2013) ao nível de 5\% de significância. Este é um teste não paramétrico que utiliza uma versão da estatística de Mann-Whitney $U_{t, N}$, a qual verifica se duas amostras $x_{1}, \ldots, x_{t}$ e $x_{t+1}, \ldots, x_{N}$, são da mesma população ou não. A estatística de teste $U_{t, N}$ foi calculada com as Equações 1 e 2 .

$$
\mathrm{U}_{\mathrm{t}, \mathrm{N}}=\mathrm{U}_{\mathrm{t}-1, \mathrm{~N}}+\sum_{\mathrm{j}=1}^{\mathrm{N}} \operatorname{sgn}\left(\mathrm{x}_{\mathrm{t}}-\mathrm{x}_{\mathrm{j}}\right) \quad \text { para } \mathrm{t}=2,3, \ldots, \mathrm{N}
$$

em que:

$$
\operatorname{sgn}=(x)\left\{\begin{array}{c}
1 \text { se } x>0 \\
0 \text { se } x=0 \\
-1 \text { se } x<0
\end{array}\right\}
$$

A estatística de teste conta o número de vezes que o valor da primeira amostra excede o valor da segunda amostra. A hipótese nula do teste de Pettitt admite ausência de um ponto de mudança (change point) na série. A estatística $\mathrm{k}(\mathrm{t})$ representa $\mathrm{o}$ ponto de mudança t significativo no qual o valor de $\left|\mathrm{U}_{\mathrm{t}, \mathrm{N}}\right|$ é máximo e está associado a um nível de significância (P) calculado pelas Equações 3 e 4. 


$$
\begin{aligned}
& \mathrm{k}_{(\mathrm{t})}=\operatorname{máx}_{1 \leq \mathrm{t} \leq \mathrm{N}}\left|\mathrm{U}_{\mathrm{t}, \mathrm{N}}\right| \\
& \mathrm{P}=2 \mathrm{e}^{\left\{\frac{-6\left(\mathrm{~K}_{\mathrm{N}}\right)^{2}}{\left(\mathrm{~N}^{3}+\mathrm{N}^{2}\right)}\right\}}
\end{aligned}
$$

em que:

P é o nível de significância,

$\mathrm{K}_{\mathrm{N}}$ é o valor crítico e

$\mathrm{N}$ é o número de anos da série histórica.

\section{RESULTADOS E DISCUSSÃO}

Para vazões médias mensais $\left(\mathrm{m}^{3} \mathrm{~s}^{-1}\right)$, verifica-se na Figura 2 que apenas no mês de outubro foi detectada mudança de tendência na série. Neste mês, o ponto de mudança (change point) ocorreu no ano de 1963. A vazão média anual $\left(\mathrm{m}^{3} \mathrm{dia}^{-1}\right)$ apresentou estacionariedade.
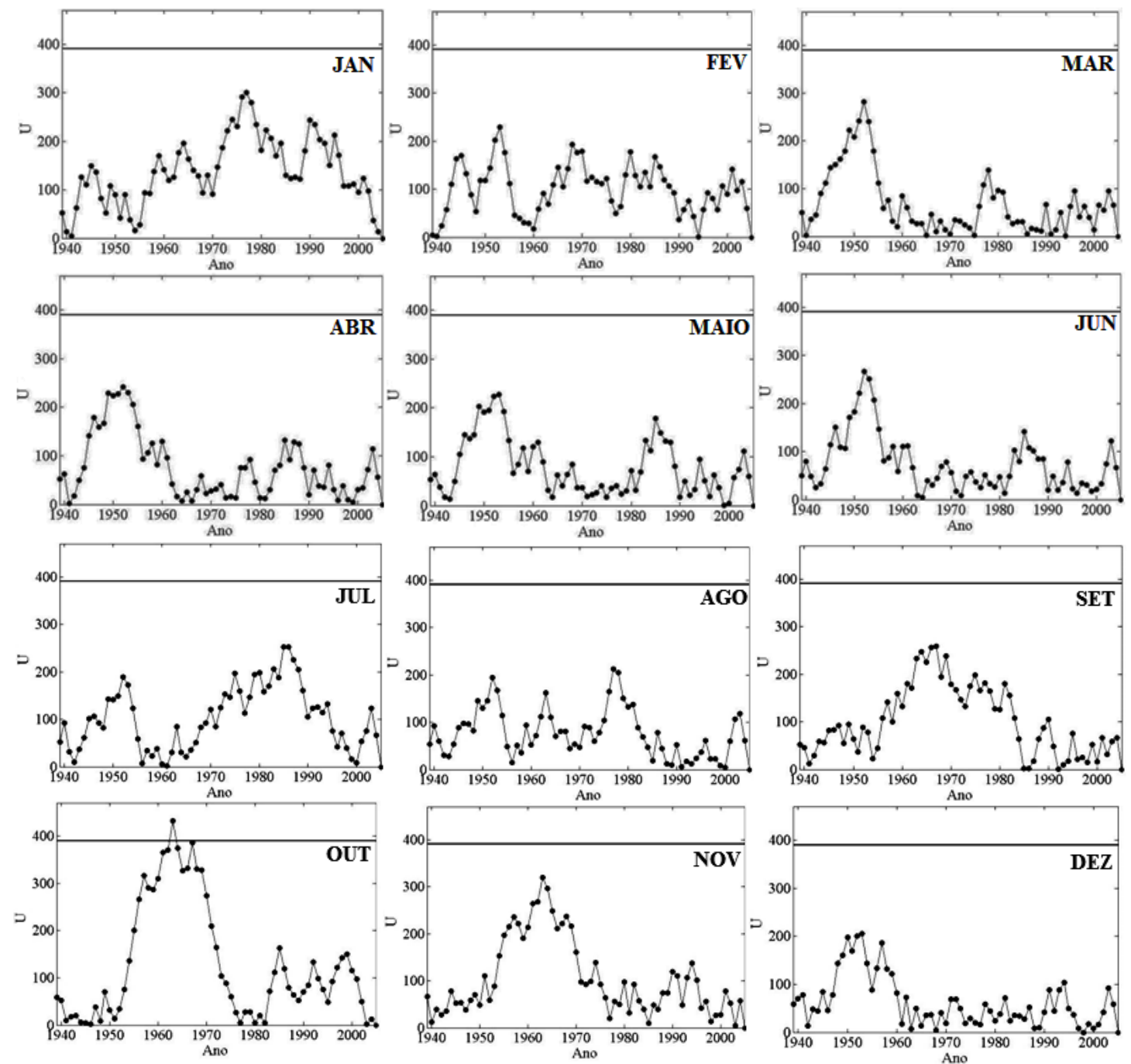

Figura 2. Resultado do teste de Pettitt para os dados de vazão média mensal $\left(\mathrm{m}^{3} \mathrm{~s}^{-1}\right)$, para o posto 57450000 . 
Na Figura 3 apresenta-se a divisão da série de vazões no ano de 1963, onde se verifica que a vazão média do mês de outubro no período de 1963 a 2005 aumentou 34,2 \% em relação ao período de 1939 a 1962.

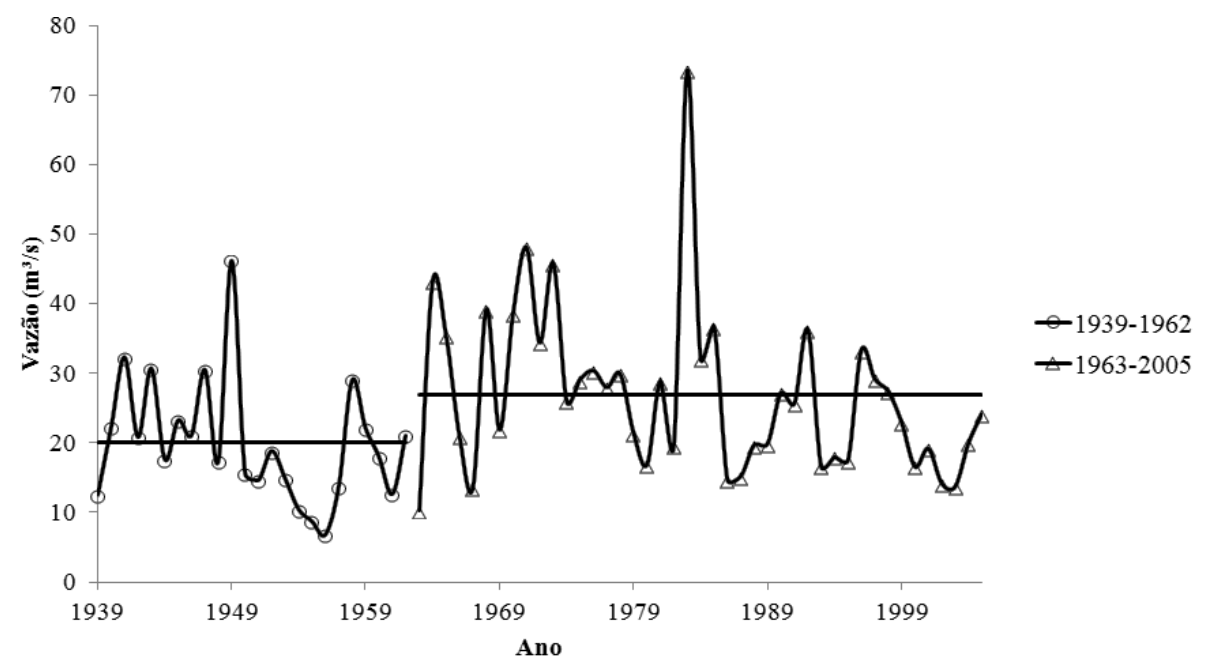

Figura 3. Série histórica da vazão média do mês de outubro, para o posto 57450000 .

Quanto as prováveis precipitações mensal e anual no estado do Espírito Santo, foram identificados por Uliana et al. (2013) dois períodos distintos: o primeiro entre outubro e abril que concentra grande parte da precipitação e o segundo entre maio e setembro com decréscimo acentuado da pluviosidade. Por meio da análise da distribuição espacial da precipitação obtida pelos autores, verifica-se que o final do período seco e o início do período chuvoso ocorrem nos meses de setembro e outubro respectivamente. $\mathrm{O}$ solo neste período encontra-se com baixa umidade e com máxima capacidade de infiltração e retenção de água.

$\mathrm{Na}$ Figura 4 são apresentados os resultados para a precipitação mensal (mm mês ${ }^{-1}$ ) no estado do Espírito Santo. Verifica-se na Figura 4 que nos meses de agosto e setembro foram detectadas mudanças de tendência na série, cujos pontos de mudança foram os anos de 1967 e 1964, respectivamente. Já a precipitação anual apresentou estacionariedade.

O aumento da vazão média do mês de outubro pode estar associado também a mudanças no uso e ocupação do solo que tenham reduzido a capacidade de infiltração da água no solo, causando o consequente aumento do escoamento superficial. Tucci e Clarke (1997) realizaram revisão sobre os impactos da cobertura vegetal no escoamento e verificaram, em grande parte dos estudos, que a vazão média aumenta com a redução da cobertura de florestas na bacia hidrográfica.

Com o teste de Pettitt é possível afirmar apenas que a vazão ou precipitação associada a determinado período apresenta tendência significativa, sendo necessário realizar estudos adicionais para identificação das causas deste comportamento. Estes estudos são complexos, visto ser grande o número de combinações entre os fatores envolvidos.

Outro ponto importante a ser considerado é a influência dos moduladores climáticos na precipitação e nas vazões dos cursos d'água. Wei et al. (2013) analisou o impacto da monção de verão do leste asiático (EASM) e do El Niño - Oscilação Sul (ENOS) na mudança das vazões do rio Chang Jiang e concluiu que: eventos relativamente fracos de EASM e de La Niña podem induzir aumento na vazão; e eventos relativamente fortes de EASM e El Niño podem causar redução da vazão. Com isso, o estudo de frequência destes fatores climáticos torna-se também item relevante para justificativa da mudança de tendência nas séries históricas de vazão. 

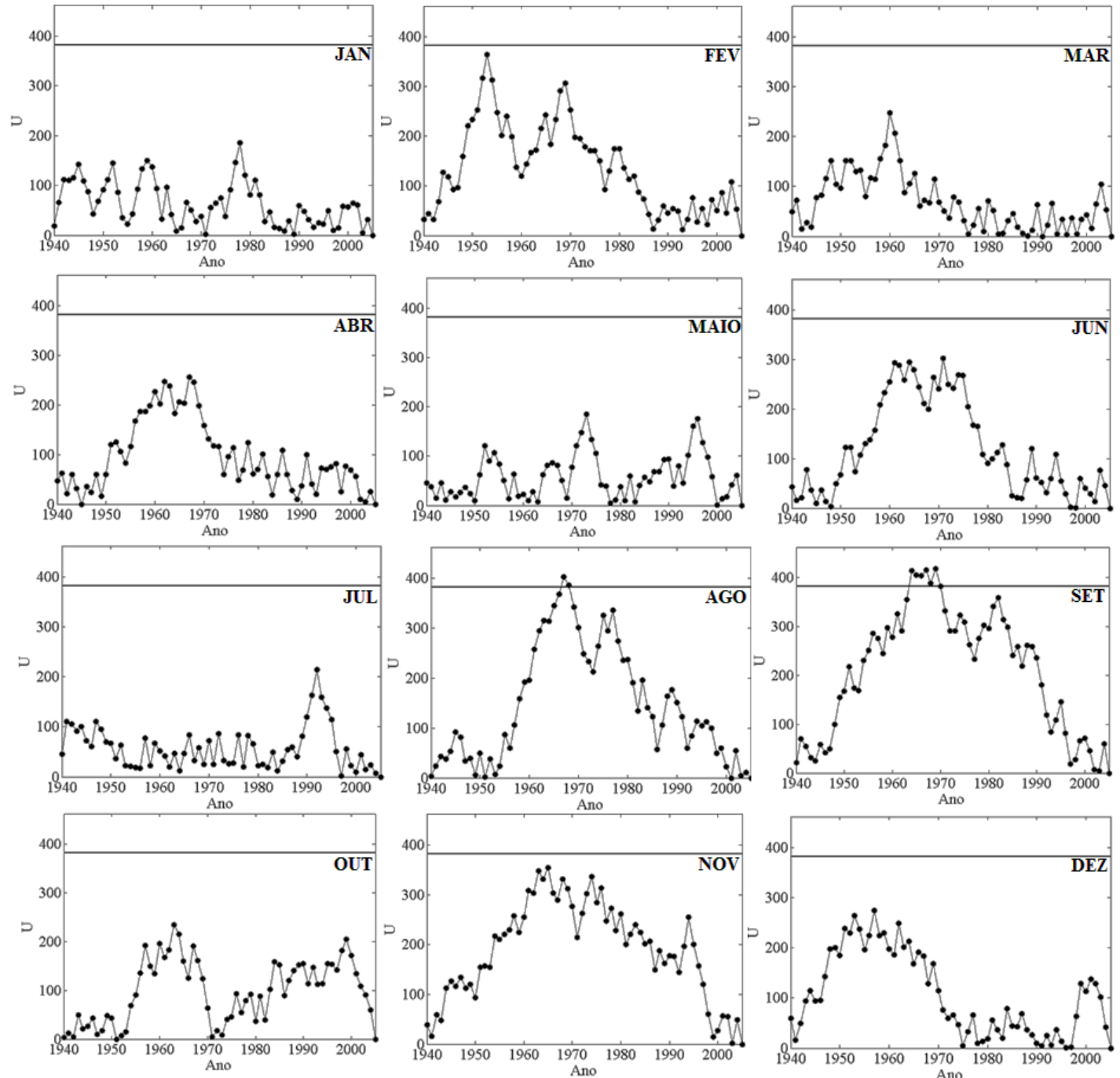

Figura 4. Resultado do teste de Pettitt para os dados de precipitação mensal (mm mês ${ }^{-1}$ ), para a estação 02041003 .

Por meio da divisão da série de precipitação no ano de 1967 para o mês de agosto e no ano de 1964 para o mês de setembro foi possível verificar que a precipitação média aumentou $50,8 \%$ e $61,2 \%$ respectivamente (Figura 5). O aumento da vazão média do mês de outubro pode ser consequência do aumento da precipitação nestes dois meses antecedentes.
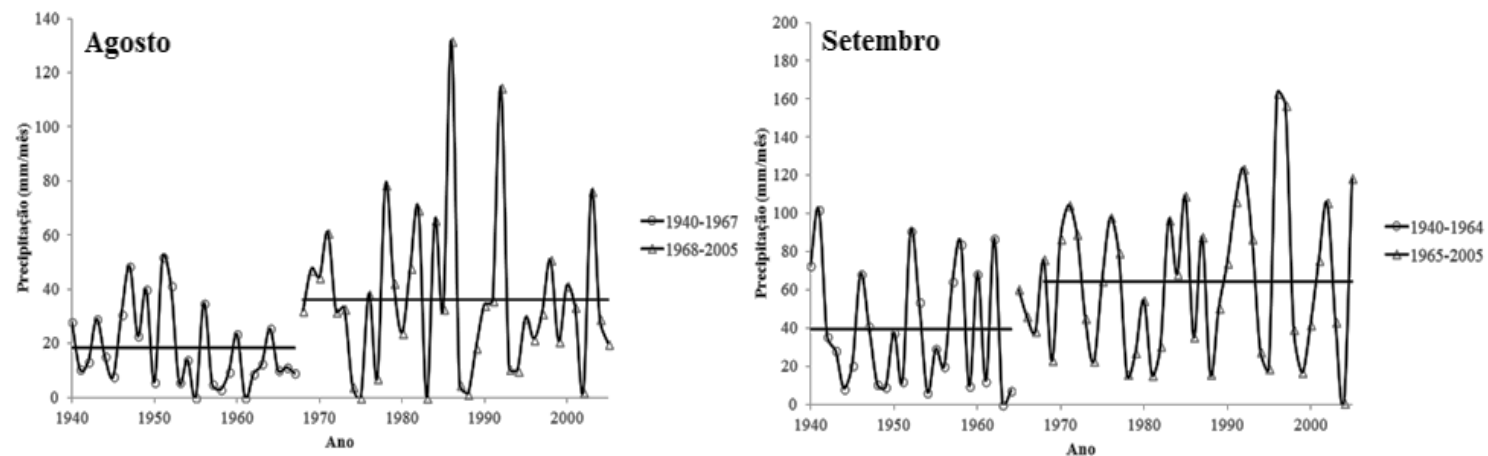

Figura 5. Séries históricas de precipitação dos meses de agosto e setembro, para a estação 02041003. 


\section{CONCLUSÕES}

O teste de Pettitt possibilitou a identificação de tendência significativa nas séries históricas de vazões e precipitações mensais, bem como do ponto de mudança. Na série de vazões médias foi identificada tendência positiva no mês de outubro com ponto de mudança no ano de 1963. Nas séries de precipitação ocorreu tendência positiva nos meses de agosto e setembro e os pontos de mudança foram os anos de 1967 e 1964, respectivamente.

$\mathrm{O}$ aumento da precipitação nos meses de agosto e setembro, dentre outros possíveis fatores, pode ter causado influência no incremento da vazão média do mês de outubro.

\section{AGRADECIMENTOS}

Os autores agradecem a Fundação de Amparo à Pesquisa do Estado de Minas Gerais (FAPEMIG) e ao Conselho Nacional de Desenvolvimento Científico e Tecnológico (CNPq) pela concessão das bolsas de doutorado e iniciação científica, bem como a Agência Nacional de Águas (ANA) pela disponibilidade dos dados hidrológicos.

\section{REFERÊNCIAS}

HAMED, K. H. Trend detection in hydrologic data: The Mann-Kendall trend test under the scaling hypothesis. Journal of Hydrology, v. 349, n. 3-4, p. 350-363, 2008. http://dx.doi.org/10.1016/j.jhydrol.2007.11.009

JOSEPH, J. F.; FALCON, H. E.; SHARIF, H. O. Hydrologic Trends and Correlations in South Texas River Basins: 1950-2009. Journal of Hydrologic Engineering, v. 18, n. 2, p. 1653-1662, 2013. http://dx.doi.org/10.1061/(ASCE)HE.1943-5584.0000709

LIU, X.; DAI, X.; ZHONG, Y.; LI, J.; WANG, P. Analysis of changes in the relationship between precipitation and streamflow in the Yiluo River, China. Theoretical and Applied Climatology, Vienna, v. 114, n. 1-2, p. 183-191, 2013. http://dx.doi.org/10.1007/s00704-013-0833-0

NAGUETTINI, M.; PINTO, E. J. A. Hidrologia estatística. Belo Horizonte: CPRM, 2007. $552 \mathrm{p}$.

PETTITT, A. N. A Non-Parametric Approach to the Change-Point Problem. Applied Statistics, v. 28, n. 2, p. 126-135, 1979. http://www.jstor.org/stable/2346729

TUCCI, E. M.; CLARKE, R. T. Impactos das mudanças da cobertura vegetal no escoamento: revisão. Revista Brasileira de Recursos Hídricos, v. 2, n. 1, p. 135-152, 1997.

ULIANA, E. M.; REIS, E. F.; SILVA, J. G. F.; XAVIER, A. C. Precipitação Mensal e Anual Provável para o Estado do Espírito Santo. Irriga, Botucatu, v. 18, n. 1, p. 139-147, 2013. http://irriga.fca.unesp.br/index.php/irriga/article/view/493/344

WEI, W.; CHANG, Y.; DAI, Z. Streamflow changes of the Changjiang (Yangtze) River in the recent 60 years: Impacts of the East Asian summer monsoon, ENSO, and human activities. Quaternary International, p. 1-10, 2013.

http://dx.doi.org/10.1016/j.quaint.2013.10.064 


Ambiente \& Água - An Interdisciplinary Journal of Applied Science
ISSN 1980-993X - doi:10.4136/1980-993X
www.ambi-agua.net
E-mail: ambi-agua@agro.unitau.br

\title{
Modelagem neural e análise estatística do processo de degradação de chorume por foto-Fenton solar
}

\author{
doi: 10.4136/ambi-agua.1341
}

Received: 13 Mar. 2014; Accepted: 19 Nov. 2014

\author{
Alessandro Sampaio Cavalcanti ${ }^{1 *}$; Hélcio José Izário Filho; \\ Oswaldo Luiz Cobra Guimarães ${ }^{2}$; Adriano Francisco Siqueira ${ }^{2}$ \\ Universidade de São Paulo (EEL-USP), Lorena, SP, Brasil \\ ${ }^{1}$ Departamento de Química \\ ${ }^{2}$ Departamento de Ciências Básicas e Ambientais \\ *Autor correspondente: e-mail: alessandro@dequi.eel.usp.br, \\ oswaldocobra@debas.eel.usp.br, helcio@dequi.eel.usp.br, \\ adriano@debas.eel.usp.br
}

\section{RESUMO}

O chorume em estudo é oriundo da cidade de Cachoeira Paulista-SP, apresentando baixa biodegradabilidade, devendo ser pré-tratado por um processo oxidativo. O objetivo desse trabalho foi obter um modelo neural em conjunto com análises estatísticas que representassem a degradação do chorume in natura pelo processo foto-Fenton solar, em função da redução da demanda química de oxigênio (DQO). Redes neurais apresentam-se como uma alternativa para a modelagem de processos não-lineares como os processos oxidativos avançados, que envolvem um grande número de variáveis de controle e complexas reações envolvidas. O processo fotocatalítico foi otimizado por um planejamento fatorial fracionado $\left(2^{4-1}\right)$ em duplicata e triplicata no ponto central, sendo as variáveis de entrada em três níveis: $\mathrm{pH}$, radiação solar e concentrações de $\mathrm{H}_{2} \mathrm{O}_{2}$ e $\mathrm{Fe}^{2+}$. $\mathrm{O}$ sistema de tratamento em escala de bancada utilizou um reator batelada, com volume constante $(3 \mathrm{~L})$ de chorume, vazão $13 \mathrm{~L} \mathrm{~min}^{-1}$ e 2-h de reação. A otimização do processo mostrou que o efeito individual de cada variável de entrada deve operar em seu maior nível, sendo que a variável $\mathrm{Fe}^{2+}$ teve significância na forma combinada. A redução percentual de DQO do melhor experimento foi de $88,7 \%$, sendo esta avaliada em um custo de $\mathrm{R} \$ 126,67 \mathrm{~m}^{-3}$ de chorume. O processo de degradação foi modelado via redes neurais feedforward backpropagation, com coeficientes de correlação linear para os conjuntos de treinamento, validação e teste acima de 0,9 , indicando alta predição e generalização do modelo neural proposto. A rede neural proposta mapeou um espaço multidimensional da forma $\triangle D Q O=f\left(t, p H,\left[\mathrm{H}_{2} \mathrm{O}_{2}\right], P_{U V},\left[\mathrm{Fe}^{2+}\right]\right)$.

Palavras-chave: aterro sanitário, análise econômica, processo oxidativo avançado.

\section{Neural modeling and statistical analysis of the degradation process of leachate by the solar photo-Fenton process}

\section{ABSTRACT}

This study sought to obtain a neural model and statistical analyzes that represented the degradation of leachate in natura by the solar photo-Fenton process, according to the 
reduction of chemical oxygen demand (COD). The study used leachate from the town of Cachoeira Paulista-SP, which had low biodegradability and required pre-treatment by an oxidative process. Neural networks are presented as an alternative for the modeling of nonlinear processes such as advanced oxidation processes, which involve a large number of control variables and complex reactions. The photo-catalytic process was optimized by a fractional $\left(2^{4-1}\right)$ factorial design in duplicate and triplicate, with the center point being the input variables at three levels: $\mathrm{pH}$, solar radiation and concentrations of $\mathrm{H}_{2} \mathrm{O}_{2}$ and $\mathrm{Fe}^{2+}$. The treatment system used an open reactor in bench scale with a constant volume $(3 \mathrm{~L})$ of leachate flow of $13 \mathrm{~L} \mathrm{~min}^{-1}$ and a 2 -h reaction. The optimization process showed that the individual effect of each input variable should operate at its highest level, and that the variable $\mathrm{Fe}^{2+}$ was significant for the combination. The percentage reduction of COD of the best experiment was $88.7 \%$, which is valued at a cost of $\mathrm{R} \$ 126.67 \mathrm{~m}^{-3}$. The degradation process was modeled via feedforward backpropagation neural networks with linear correlation coefficients for the training sets, validation and test above 0.9 , indicating high prediction and generalization of the proposed neural model. The proposed neural network mapped a multidimensional space of the form $\triangle D Q O=f\left(t, p H,\left[\mathrm{H}_{2} \mathrm{O}_{2}\right], P_{U V},\left[\mathrm{Fe}^{2+}\right]\right)$.

Keywords: advanced oxidation, economic analysis, landfill.

\section{INTRODUÇÃO}

\subsection{Processo foto-Fenton}

Um dos grandes problemas encontrados pela sociedade moderna é resolver a questão do lixo urbano e seu consequente depósito no ambiente. O destino impróprio dos resíduos sólidos, em áreas que não possuem uma infraestrutura adequada para o controle dos poluentes, poderá causar uma degradação ambiental (poluição do solo, do ar, das águas superficiais e subterrâneas, proliferação de vetores, contaminação da biota e desequilíbrio ecológico), acarretando riscos à saúde humana. E esse problema vem sendo discutido nos mais variados recursos midiáticos, pois são sentidas diretamente na qualidade do ar, das águas superficiais e subterrâneas e dos solos (Vilela-Ribeiro et al., 2009).

A fração líquida, conhecida como chorume ou lixiviado, é originada pela umidade natural presente nos resíduos, pelo processo de decomposição da matéria orgânica e pelas águas das chuvas percoladas sobre a massa de resíduo, onde várias substâncias orgânicas e inorgânicas são arrastadas e farão parte da composição do chorume, que é um líquido escuro e apresenta um sério problema ambiental, devido ao seu alto potencial de contaminação. Os resíduos sólidos dispostos nos lixões ou nos aterros sofrem os processos de decomposição física, química e biológica, dando origem a efluentes líquidos e gasosos (Kjeldsen et al., 2002).

A complexidade da composição do lixiviado gera grandes dúvidas na técnica de seu tratamento; no caso dos lixiviados novos ( $<2$ anos), onde predomina alta biodegradabilidade, ou seja alta razão de Demanda Química de Oxigênio em relação a Demanda Bioquímica de Oxigênio $\left(\mathrm{DBO}_{5} / \mathrm{DQO}>0,6\right)$, o processo biológico tem sido recomendado. Entretanto, para os lixiviados maduros ( $>10$ anos), onde predominam a baixa biodegradabilidade $\left(\mathrm{DBO}_{5} / \mathrm{DQO}<0,2\right)$, é constituída de substâncias refratárias e com alto peso molecular (húmicas e fúlvicas), que dificultam o processo biológico (Zhang et al., 2005).

A característica físico-química do lixiviado é influenciada por alguns fatores, como: variações climáticas, técnica de aterramento e método de compactação, os quais influenciam na quantidade e na qualidade do lixiviado produzido no aterro. $\mathrm{O}$ seu envelhecimento é caracterizado pela presença de substâncias recalcitrantes (Telles, 2010).

Justifica-se o estudo de diferentes processos oxidativos avançados no tratamento do lixiviado por se tratar de uma mistura complexa, sendo muitas vezes, necessário o emprego de 
duas ou mais tecnologias de tratamento para se obter um lixiviado dentro dos padrões para descarte (Telles, 2010).

A oxidação de compostos orgânicos sob irradiação UV na presença de íon de ferro, em meio ácido, foi verificada nas décadas anteriores, quando foi postulado que a transferência eletrônica iniciada pela irradiação resultava na geração de $\bullet \mathrm{OH}$, responsável pelas reações de oxidação. Com o aumento do $\mathrm{pH}$, ocorre hidrólise formando espécies hidroxiladas, como mostra a Equação 1.

$$
\mathrm{Fe}^{3+}+\mathrm{H}_{2} \mathrm{O} \rightarrow \mathrm{Fe}(\mathrm{OH})^{2+}+\mathrm{H}^{+}
$$

Quando complexos de Fe (III) são irradiados, ocorre a promoção de um elétron de um orbital centrado no ligante para um orbital centrado no metal, chamada de transferência de carga ligante-metal ("ligand to metal charge transfer", LMCT), que implica na redução de $\mathrm{Fe}(\mathrm{III})$ a $\mathrm{Fe}(\mathrm{II})$ e oxidação do ligante (Equação 2), formando o radical hidroxila (Nogueira et al., 2007).

$$
\mathrm{Fe}(\mathrm{OH})^{2+}+h v \rightarrow \mathrm{Fe}^{2+}+\cdot \mathrm{OH}
$$

$\mathrm{O} \mathrm{Fe}^{2+}$ gerado durante a irradiação da luz solar, quando na presença de peróxido de hidrogênio $\left(\mathrm{H}_{2} \mathrm{O}_{2}\right)$, reage com este dando seqüência à reação de Fenton (Equação 3). Assim, a reação é catalítica e é estabelecido um ciclo em que $\mathrm{Fe}^{2+}$ (íon ferroso) é regenerado. Otimizado o custo de reagente e aliado à irradiação solar, abundante e intensa na região Sudeste, onde se desenvolveu esse projeto, o processo oxidativo pode ser viabilizado economicamente (Vilar et al., 2011).

$$
\mathrm{Fe}^{2+}+\mathrm{H}_{2} \mathrm{O}_{2} \rightarrow \mathrm{Fe}^{3+}+\mathrm{HO}^{-}+\cdot \mathrm{OH}
$$

Neste contexto, este trabalho tem como objetivo obter um modelo neural que represente a degradação do chorume in natura em função da redução de DQO, utilizando-se o processo foto-Fenton solar, além de analisar estatisticamente os resultados obtidos do processo de degradação.

\subsection{Redes Neurais}

As Redes neurais artificiais (RNA) podem ser definidas como sendo sistemas paralelos distribuídos, compostos por unidades de processamento simples que computam determinadas funções matemáticas (normalmente não-lineares). Tais unidades são dispostas em uma ou mais camadas e interligadas por um grande número de conexões, geralmente unidirecionais (Haykin, 1999).

Cerqueira et al. (2001) definem redes neurais "como um conjunto de métodos matemáticos e algoritmos computacionais, especialmente projetados para simular o processamento de informações e aquisição de conhecimento do cérebro humano". Os autores observam que se pode considerar uma rede neural como uma "caixa de processamento", que pode ser treinada para que, a partir de um conjunto de entradas (input), o mesmo forneça um conjunto de saída (output).

Uma rede neuronal é um processador distribuído massivamente em paralelo, que tem a propensão natural para armazenar conhecimentos experimentais e torná-los disponíveis para uso. Em uma rede neural conhecimento é adquirido pela rede através do processo de aprendizagem, e os pesos das conexões entre neurônios, conhecido como sinapses, são usados para armazenar o conhecimento (Haykin, 1999). 
As RNA possuem a característica de serem consideradas como aproximadores universais de funções, podendo realizar em mapeamento de um espaço vetorial em outro espaço vetorial (Svozil et al., 1997).

De forma geral, uma rede neural artificial pode ser vista como uma estrutura interconectada formada por uma camada de neurônios de entrada, um número de camadas ocultas e uma camada de saída. Fundamentalmente, redes neurais são aproximadores universais de funções com poder de classificação, associação e predições.

A função de transferência é necessária para a transformação da soma dos pesos ponderados dos sinais de entrada nos neurônios, de forma a determinar o valor ou intensidade do sinal de saída. As funções de transferência, também chamadas funções de ativação, limitam a amplitude do intervalo do sinal de saída do neurônio para algum valor finito, geralmente num intervalo normalizado.

Elmolla et al. (2010) realçam a dificuldade de modelamento matemático devido à complexidade química dos processos oxidativos avançados e, desta forma, redes neurais podem ser utilizadas na modelagem matemática do processo de degradação de efluentes, em função de sua simplicidade de simulação, predição e modelagem. As vantagens da modelagem neural envolvem o fato de que a descrição do fenômeno de degradação não é necessária e tempo menor é requerido para o desenvolvimento do modelo, comparados aos modelos matemáticos tradicionais. Neste sentido, Guimarães et al. (2008) utilizaram uma rede neural feedforward backpropagation para predição do ponto crítico de adição de peróxido de hidrogênio, no processo de descoloração de azo corantes através da utilização de $\mathrm{UV} / \mathrm{H}_{2} \mathrm{O}_{2}$.

$\mathrm{Na}$ maioria dos modelos, estas conexões estão associadas a pesos que armazenam o conhecimento representado no modelo, e servem para ponderar a entrada recebida por cada neurônio da rede. A solução de problemas com RNA passa inicialmente por uma fase de aprendizagem, onde um conjunto de exemplos é apresentado para a rede, que extrai automaticamente as características necessárias para representar a informação fornecida. Essas características são utilizadas posteriormente para gerar respostas para o problema.

Kato et al. (2001) aplicaram a técnica de redes neurais com algoritmo de correção de pesos do tipo backpropagation, na predição de DQO de efluentes sintéticos contendo fenol, ácido salicílico, anilina e lignina. Estes efluentes continham substâncias que poderiam influir no processo de determinação de DQO. Espécies inorgânicas como halogênios e sulfato ferroso possuem caráter redutor para o dicromato de potássio usado na determinação da DQO, interferindo no resultado de análise. Na etapa de validação, os autores alcançaram um erro médio de 12,67 \% para amostras contendo interferentes inorgânicos. A presença destes interferentes reveste o processo de medição de DQO de não linearidades.

Os modelos do tipo entrada-saída são úteis na representação matemática de processos complexos, para os quais o desenvolvimento de modelos fenomenológicos seria uma tarefa complexa e potencialmente revestida de consumo de tempo e investimento financeiro apreciável.

Diversos estudos indicam a potencialidade de aplicação de redes neurais, independente de sua arquitetura (como por exemplo redes feedfoward ou Mapas de Kohonen), em processos de tratamento de efluentes, sendo indiferente ao método de mineralização utilizado, sejam eles processos oxidativos ou biológicos. Çinar (2005) utilizou uma rede do tipo Mapas de Auto-Organização de Kohonen, para classificar dados de operação de tratamento de plantas de Pelham e determinar as razões para as altas concentrações de demanda bioquímica de oxigênio (DBO), sólidos suspensos totais (SST) e coliformes fecais neste estudo. A rede de Kohonen é uma rede neural não supervisionada que pode ser utilizada para reduzir a quantidade de dados por clusterização e projetar a não-linearidade dos dados em um espaço dimensional de menor ordem. No trabalho, dados operacionais de 400 dias foram utilizados para alimentar a rede e clusterizar o conjunto amostral, indicando altos valores de parâmetros 
de demanda bioquímica de oxigênio (DBO), TSS e coliformes fecais. Os autores consideram que o uso da rede de Kohonen à avaliação de plantas de tratamento de efluentes é uma maneira eficiente de determinar as complexas dependências das variáveis de processo e, desta forma, resolver problemas operacionais em plantas de tratamento de efluentes.

Em relação à aplicação de redes neurais na modelagem de processos oxidativos avançados, também pode ser citado o trabalho de Pareek et al. (2002), no qual ocorreu a modelagem da fotodegradação do denominado "Bayer liquor", pela catálise do dióxido de titânio. Neste trabalho, os autores modelaram a razão de reação com coeficientes de correlação acima de 0.99, e estudaram a influência dos parâmetros de entrada pela utilização da partição dos pesos de conexão de Garson. A rede neural proposta pelos autores seguiu uma arquitetura feedforward, com uma camada oculta.

Algumas características do processo foto-Fenton indicam as vantagens da utilização de redes neurais em seu processo de modelagem. Os radicais hidroxila formados reagem com espécies orgânicas, e o mecanismo que envolve o processo foto-Fenton é formado por inúmeras reações elementares com diversos radicais e formação de complexos de ferro com água, além de espécies intermediárias e diversos interferentes. Realça-se a complexidade das matrizes químicas de percolados de aterro sanitário. Desta forma, mecanismo de degradação de efluentes orgânicos não obedecem a uma ordem de reação definida (Nadtochenco e Kiwi, 1998; Wang e Lemley, 2001; Giroto, 2002).

Giroto (2002) relata que a complexidade do mecanismo fotoquímico induz a grandes dificuldades na determinação de modelos cinéticos. O radical hidroxila é não seletivo, dificultando a descrição por modelos cinéticos de forma simples.

Giordani (2003) salienta a capacidade de trabalho das redes neurais com fenômenos não lineares, e que esta capacidade é uma poderosa ferramenta para a indústria de processos químicos, onde a não-linearidade é quase constante, sendo que na rede neural as não-linearidades são introduzidas pelas funções de transferência.

No presente trabalho, a rede neural utilizada foi uma rede do tipo feedforward, contendo três camadas (entrada, oculta e de saída).

$\mathrm{O}$ critério de ajuste da rede neural feedforward usando o algoritmo bach-propagation foi a minimização do erro quadrático médio para os conjuntos de treinamento, validação e teste.

O comportamento funcional da rede feedforward (Hamed et al., 2004) pode ser definido conforme Equação 4.

$$
\bar{Y}_{i}=f_{o}\left[\sum_{j=1}^{h_{n}} W_{o_{j}} * f_{h}\left(\sum_{i=1}^{m} W H_{i j} \cdot X_{i}+b_{j}\right)+b_{o}\right]
$$

$\mathrm{Na}$ Equação 4, $\mathrm{WH}_{\mathrm{ij}}$ representa os pesos entre a j-ésima entrada e a j-ésimo neurônio oculto, m é o número de neurônios de entrada, Woj representa os pesos entre o j-ésimo neurônio oculto e o neurônio de saída, $\mathrm{f}_{\mathrm{h}}$ representa a função de ativação do neurônio oculto e $f_{o}$ a função de ativação do neurônio de saída, $b_{j}$ representa a bias do j-ésimo neurônio oculto, $b_{o}$ a bias do neurônio de saída e $h_{n}$ é o número de neurônios ocultos. Nesta notação, fica explícito o caráter funcional da modelagem neural. Desta forma, $y_{i}$ pode representar um valor de predição, no sentido funcional ou de classificação.

O objetivo básico da modelagem neural é a minimização de uma função erro. Neste desenvolvimento, a função Erro Quadrático Médio é representada pela (Equação 5), em que N representa o número de amostras do experimento.

$$
M S E=\sum_{i=1}^{N}\left(Y_{i}-\bar{Y}_{i}\right)^{2} / N
$$




\section{MATERIAL E MÉTODOS}

A descrição de Materiais e Métodos foi subdivida em 4 subseções de forma a um melhor entendimento dos processos de caracterização analítica, experimental e modelagem neural.

\subsection{Amostragem e preservação}

O chorume utilizado nos experimentos foi proveniente do aterro sanitário da cidade de Cachoeira Paulista localizado na Estrada Municipal de Fiuta, km 4 - Bairro do Jardim no interior de São Paulo, região do vale do Paraíba, Brasil. O aterro sanitário está em operação desde o ano de 2006 e recebe um volume médio de lixo de 100 ton $\mathrm{dia}^{-1}$.

A coleta do chorume foi realizada na caixa de mistura (tipo tanque equalizador), antes deste entrar na lagoa de estabilização. Foram coletados $80 \mathrm{~L}$ de chorume em um dia ensolarado e seco numa temperatura de $23{ }^{\circ} \mathrm{C}$, em agosto de 2011 . O mesmo foi homogeneizado e acondicionado em uma câmara fria a $4{ }^{\circ} \mathrm{C}$ do LOT (Departamento de Biotecnologia) da EEL-USP, para minimizar possíveis alterações das propriedades físicoquímicas (APHA et al., 1999).

\subsection{Metodologias analíticas}

As metodologias utilizadas para as caracterizações analíticas do chorume in natura e após tratamentos oxidativos foram adaptadas segundo Standard Methods (APHA et al., 1999). As determinações de DQO foram realizadas com digestão em tubo fechado, seguida de determinação espectrofotométrica (espectrofotômetro SP 1105) em $620 \mathrm{~nm}$. As determinações de $\mathrm{DBO}_{5}$ (Demanda Bioquímica de Oxigênio 5 dias) foram incubadas (SP 500) por um período de 5 dias com inoculação de microorganismos tipo Seed, a $20 \pm 3{ }^{\circ} \mathrm{C}$, onde a medida de oxigênio, antes e após a incubação, foi feita pelo método titrimétrico de Winkler modificado pela azida sódica (Lima et al., 2006). As análises de sólidos seguiram procedimento gravimétrico clássico, em cápsulas de porcelana com massas previamente estabilizadas. $\mathrm{O}$ processo de secagem, a $105^{\circ} \mathrm{C}$, foi realizado em estufa (SP LABOR) e o processo de calcinação, a $650{ }^{\circ} \mathrm{C}$, foi realizado em um forno tipo mufla (SP LABOR). As pesagens foram feitas em balança analítica marca SHIMADZU, modelo AY 220. As análises de Nitrogênio Amoniacal e Orgânico utilizaram a prévia destilação para sua posterior determinação espectrofotométrica. A concentração de óleo e graxa foi determinada pelo método de extração por solvente (hexano) em um sistema de Soxhlet, sendo previamente adsorvido em diatomácea, em meio ácido clorídrico. Fosfato, após digestão ácida para a forma solúvel, e cloreto foram determinadas por métodos espectrofotométricos. As determinações de carbono orgânico foram realizadas em um analisador de carbono orgânico total da Shimadzu, modelo TOC-VCPH, fundamentado na oxidação catalítica a elevadas temperaturas e determinação de $\mathrm{CO}_{2}$ por espectroscopia no infravermelho. Para determinação do carbono orgânico, a curva de calibração foi preparada a partir de um padrão de biftalato de potássio, na faixa linear de $0-1000 \mathrm{mg} \mathrm{L}^{-1}$. As determinações analíticas dos elementos metálicos nas amostras, previamente digeridas em meio ácido e em sistema fechado, foram realizadas em um espectrômetro de absorção atômica, marca PerkinElmer, modelo Aanalyst 800. Para a determinação do peróxido de hidrogênio residual foi utilizado o método espectrofotométrico, com o reagente complexante metavanadato de amônio. A concentração de peróxido de hidrogênio foi monitorada pela formação de um complexo com absorvidade em $450 \mathrm{~nm}$, pela reação de $\mathrm{H}_{2} \mathrm{O}_{2}$ com metavanadato de amônio $\left(\mathrm{NH}_{4} \mathrm{VO}_{3}\right)$ (Guimarães et al., 2007).

\subsection{Procedimento experimental}

Os experimentos foram realizados em um reator semi-batelada aberto para a absorção das radiações UV solar, conforme mostra a Figura 1, constituído basicamente de uma bomba 
centrífuga (BOMAX, Modelo NH-30PX-T e vazão de $13 \mathrm{~L} \mathrm{~min}^{-1}$ ), um reservatório cilíndrico $(19,9 \mathrm{~cm}$ x 23,9 cm) e uma placa metálica $(25 \mathrm{~cm}$ de largura e $75 \mathrm{~cm}$ de comprimento, com uma área útil de $1875 \mathrm{~cm}^{2}$ ), sem pigmentação denominada branco. $\mathrm{O}$ controle e ajuste do $\mathrm{pH}$ durante os experimentos foram realizados no reservatório, com um pHmetro (PH21 pH/mV HANNA) e a intensidade da radiação solar foi medida através de um radiômetro portátil (IL 1430 UV Health Hazard - Actinic). No sistema fotocatalítico, o chorume era bombeado até a parte superior da placa, na qual era distribuído por toda a placa, através de um tubo de PVC perfurado, que por gravidade percolava por toda a extensão da placa, sob uma fina e uniforme camada, enquanto recebia a radiação solar. Esta foi colocada sobre um suporte de madeira em sentido ao equador, com um ângulo de inclinação de $23^{\circ}$ (aproveitamento melhor a incidência da radiação na fotocatálise). Concomitantemente, o volume do reagente de $\mathrm{FeSO}_{4} \cdot 7 \mathrm{H}_{2} \mathrm{O}\left(0,82 \mathrm{~mol} \mathrm{~L}^{-1}\right)$ foi adicionado totalmente no início da reação e $\mathrm{o}_{2} \mathrm{O}_{2}\left(30 \% \mathrm{~m} \mathrm{~m}^{-1}\right)$ durante $60 \mathrm{~min}$ do tempo total de 2-h. Durante a reação fotocatalítica utilizou-se $\mathrm{NaOH}$ e $\mathrm{H}_{2} \mathrm{SO}_{4}$ (ambos a $5 \mathrm{eq} \mathrm{L}^{-1}$ ) para ajustar e controlar o $\mathrm{pH}$ do processo.

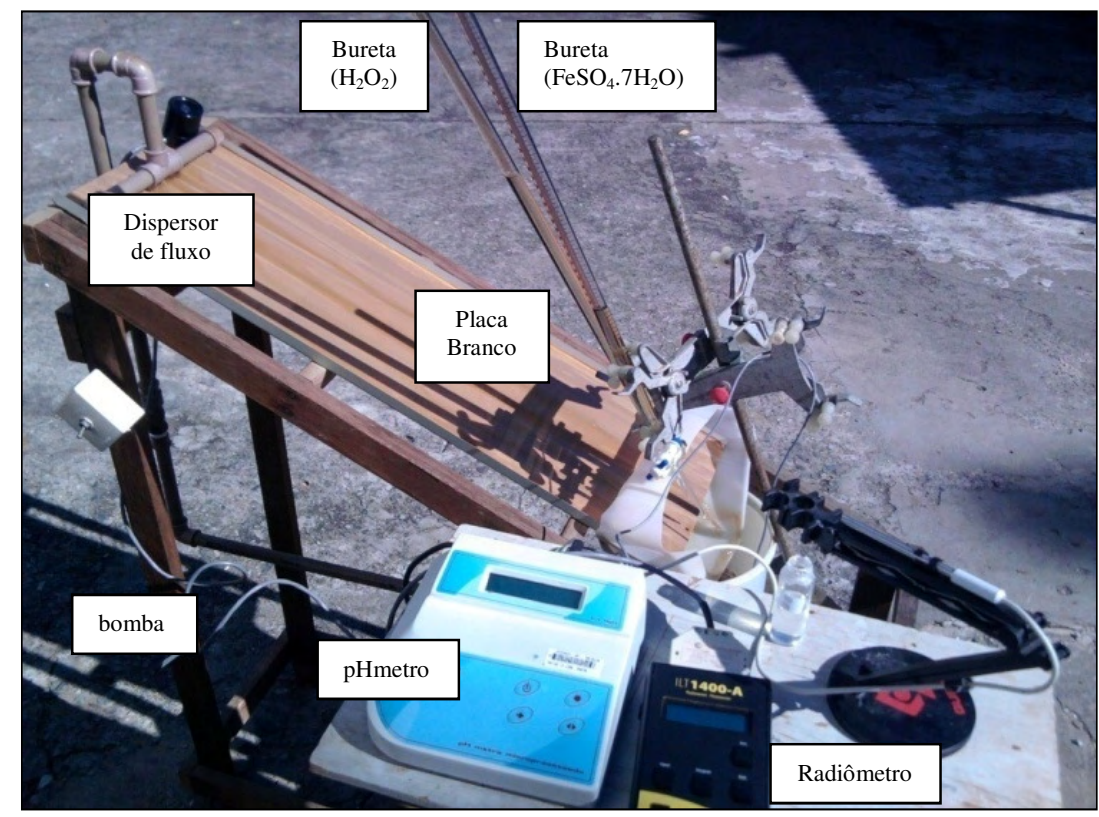

Figura 1. Layout do Reator Solar.

O processo fotocatalítico foi otimizado por um planejamento fatorial fracionado $\left(2^{4-1}\right)$, com duplicata e triplicata no ponto central (19 experimentos), sendo as variáveis de entrada: $\mathrm{pH}$, intensidade média de radiação solar em três períodos do dia (manhã das 8:00 às 10:00 h; almoço das 12:00 às 14:00; tarde das 15:00 às 17:00 h), quantidades de $\mathrm{H}_{2} \mathrm{O}_{2}$ e Fe ${ }^{2+}$, como mostrado na Tabela 1. Para a variável resposta foi utilizada a redução percentual da carga orgânica em função de DQO. O modelo neural foi implementado utilizando o software MatLab R2010 e a análise estatística o Minitab.

Tabela 1. Fatores e níveis para o planejamento de experimento foto-Fenton de $3 \mathrm{~L}$ do chorume.

\begin{tabular}{lcccc}
\hline Variáveis de Entrada & Notação & Nível 1 & Nível 2 & Nível 3 \\
\hline $\mathbf{p H}$ & $\mathrm{A}$ & 2 & 3 & 4 \\
{$\left[\mathbf{H}_{\mathbf{2}} \mathbf{O}_{2}\right](\mathbf{g})$} & $\mathrm{B}$ & 48,5 & 60,6 & 72,8 \\
${\mathbf{R a d i a c ̧ a ̃ o ~ S o l a r ~}(\mathbf{U V})\left(\boldsymbol{\mu W} \mathbf{~ c m}^{-2}\right)}_{\left[\mathbf{F e}^{\mathbf{2}}\right](\mathbf{g})}^{\mathrm{C}}$ & $\mathrm{C}$ & 719 & 1012 & 860 \\
\hline
\end{tabular}

Por ser um sistema semi-batelada aberto, a taxa de evaporação de 216,2 $\mathrm{mL} \mathrm{h}^{-1}$ (média) foi minimizada com adição de água (COT $<1 \mathrm{mg} \mathrm{L}^{-1}$ ), evitando-se uma pré-concentração do 
chorume durante o tratamento oxidativo. Para a medição e maximização da radiação solar, o radiômetro era posicionado na mesma inclinação do reator, onde o suporte de madeira era rotacionado constantemente para a incidência da máxima radiação do sol.

\subsection{Procedimento relacionado ao modelo neural}

A rede feedforward com algoritmo de aprendizagem backpropagation mapeou um espaço multi-dimensional da forma representada pela (Equação 6).

$$
\triangle D Q O=f\left(t, p H,\left[\mathrm{H}_{2} \mathrm{O}_{2}\right], P_{U V},\left[\mathrm{Fe}^{2+}\right]\right)
$$

A variável de saída $\triangle_{D_{\ell}}$ representa o decréscimo da DQO calculada pela (Equação 7), em que $\mathrm{DQO}_{\mathrm{o}}$ representa Demanda Química de Oxigênio do chorume in natura, e $\mathrm{DQO}_{\mathrm{t}}$ o teor da DQO após t minutos de tratamento. No modelo neural proposto, os neurônios da camada de entrada representaram as variáveis independentes ou variáveis de entrada e o neurônio da camada de saída representou a variável dependente $\Delta_{\mathrm{D} Q \mathrm{O}}$.

$$
\Delta D Q O=\frac{D Q O_{o}-D Q O_{t}}{D Q O_{o}}
$$

Durante o processo de treinamento da rede foram efetuadas várias configurações quanto ao número de neurônios existentes na camada oculta. Dentre os resultados foi escolhida a configuração que atuou com nove (9) neurônios na camada oculta, pois foi a que apresentou o melhor coeficiente de correlação para os conjuntos de treinamento, validação e teste. $\mathrm{O}$ ambiente computacional utilizado foi a toolbox NNtool do software MatLab.

\section{RESULTADOS E DISCUSSÃO}

\subsection{Caracterização do chorume in natura e tratado}

A caracterização do chorume foi realizada segundo aos parâmetros físico-químicos mais relevantes, como pH, DQO, $\mathrm{DBO}_{5}$, Nitrogênio amoniacal e orgânico, Carbono Orgânico Total (COT), Sólidos Totais, fixos e dissolvidos, óleos e graxas, razão de biodegradabilidade $\left(\mathrm{DBO}_{5} / \mathrm{DQO}\right)$, alguns íons específicos e metais sendo mostrados na Tabela 2. Ressalta-se que para a caracterização do chorume tratado foi utilizado o que apresentou o maior percentual de redução de DQO na reação foto-Fenton solar, após o ajuste do $\mathrm{pH}(9,0)$ para a floculação e precipitação.

As concentrações dos parâmetros encontrados no chorume quando comparados àquelas permitidas pela legislação, indicou que há a necessidade de um tratamento antes do descarte deste lixiviado ao rio, destacando os seguintes parâmetros: concentrações de $\mathrm{DQO}, \mathrm{DBO}_{5}$, biodegradabilidade, óleos e graxas, nitrogênio amoniacal e do metal Prata (Ag).

A alta concentração de Prata (Ag) apresentada na caracterização do chorume, ocorreu possivelmente devido ao descarte das chapas de raio-x no lixo urbano, que contém esse metal em sua composição, e que não foram reciclados em consultórios médicos e hospitais, apenas separados dos lixos hospitalares classificados como perigosos (classe 1).

Em relação aos parâmetros sem limitação de concentração máxima, destaca-se a diminuição significativa dos sólidos (fixos e voláteis) e a alteração acentuada nas características visuais (cor e turbidez).

O resíduo gerado após a precipitação do íon ferroso/férrico em meio alcalino, após o processo de oxidação, pode ser reaproveitado como reagente no processo novamente. Logo, otimizar o custo do processo. A segunda alternativa é reutilizar esse lodo formado para indústria de materiais cerâmicos, como: telhas e tijolos, para suprir a demanda da indústria 
civil. Sabendo que um resíduo formado deixa de ser um passivo ambiental quando transformado em subproduto no processo (CETESB, 1993).

De acordo com a lei Federal e Estadual (governo de São Paulo) é recomendado o mínimo na redução dos valores de $\mathrm{DBO}_{5}\left(<60 \mathrm{mg} \mathrm{O}_{2} \mathrm{~L}^{-1}\right)$ ou $80 \%$ na sua remoção.

Tabela 2. Caracterização Química do Chorume in natura e pré-tratado do aterro sanitário.

\begin{tabular}{|c|c|c|c|}
\hline Parâmetros & in natura & Pré-tratado & Legislação $^{(*)}$ \\
\hline $\mathrm{COT}\left(\mathrm{mg} \mathrm{C} \mathrm{L}^{-1}\right)$ & 368,6 & 65,9 & - \\
\hline $\mathrm{DQO}\left(\mathrm{mg} \mathrm{O}_{2} \mathrm{~L}^{-1}\right)$ & 3552,2 & 402,8 & - \\
\hline $\mathrm{DBO}\left(\mathrm{mg} \mathrm{O}_{2} \mathrm{~L}^{-1}\right)$ & 397,4 & 166,4 & 60 \\
\hline DBO/DQO & 0,11 & 0,41 & - \\
\hline $\mathrm{pH}$ & 8,23 & 9,34 & 5,0 a 9,0 \\
\hline Óleos e Graxas (mg L $\left.{ }^{-1}\right)$ & 165,1 & 12,0 & 100 \\
\hline Sólidos Totais $\left(\mathrm{mg} \mathrm{L}^{-1)}\right.$ & 6035 & 1134 & - \\
\hline Sólidos Fixos $\left(\mathrm{mg} \mathrm{L}^{-1}\right)$ & 1032 & 139 & - \\
\hline Sólidos Voláteis ( $\mathrm{mg} \mathrm{L}^{-1}$ ) & 5003 & 995 & - \\
\hline Turbidez (NTU) & 630,0 & 3,95 & 40 \\
\hline Cor (mg Pt-Co L $\left.{ }^{-1}\right)$ & 1763,2 & 13,7 & 100 \\
\hline Cloreto $\left(\mathrm{mg} \mathrm{Cl}^{-} \mathrm{L}^{-1}\right)$ & 246,1 & 114,2 & - \\
\hline 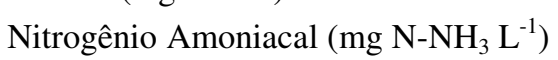 & 1582,3 & 425,3 & 20 \\
\hline Nitrogênio Orgânico (mg Norg $\left.\mathrm{L}^{-1}\right)$ & 860,0 & 652,1 & - \\
\hline Nitrito $\left(\mathrm{mg} \mathrm{NO}_{2}{ }^{-} \mathrm{L}^{-1}\right)$ & 0,30 & 0,22 & - \\
\hline Nitrato $\left(\mathrm{mg} \mathrm{NO}_{3}^{-} \mathrm{L}^{-1}\right)$ & 2,10 & 1,45 & - \\
\hline Nitrogênio Total $\left(\mathrm{mg} \mathrm{N}_{\mathrm{T}} \mathrm{L}^{-1}\right)$ & 2444,7 & 1079,1 & - \\
\hline Fósforo Total (mg P L $\left.{ }^{-1}\right)$ & 2,10 & 0,90 & - \\
\hline Arsênio (mg As L ${ }^{-1}$ ) & 0,016 & 0,009 & 0,2 \\
\hline Cádmio (mg Cd L $\left.{ }^{-1}\right)$ & 0,091 & 0,083 & 0,2 \\
\hline Cromo Total (mg Cr L $\left.{ }^{-1}\right)$ & 0,256 & 0,016 & 5,0 \\
\hline Chumbo (mg Pb L ${ }^{-1}$ ) & 0,209 & 0,062 & 0,5 \\
\hline Ferro total $\left(\mathrm{mg} \mathrm{Fe} \mathrm{L}^{-1}\right)$ & 4,300 & 0,391 & 15,0 \\
\hline Manganês (mg Mn L ${ }^{-1}$ ) & 0,037 & 0,022 & 1,0 \\
\hline Níquel (mg Ni L ${ }^{-1}$ ) & 0,073 & 0,042 & 2,0 \\
\hline Prata $\left(\mathrm{mg} \mathrm{Ag} \mathrm{L}^{-1}\right)$ & 0,72 & 0,63 & 0,02 \\
\hline Zinco $\left(\mathrm{mg} \mathrm{Zn} \mathrm{L}^{-1}\right)$ & 0,089 & 0,049 & 5,0 \\
\hline
\end{tabular}

*Valores permitidos, quando apresentados, referem-se aos limites permitidos pela resolução do CONAMA 430/2011 (Brasil, 2011).

Os resultados mostraram a eficiência de $58,2 \% \mathrm{DBO}_{5}$ e $88,7 \%$ DQO no processo foto-Fenton solar, onde ainda não atendem a legislação. No entanto, o processo mostrou viabilidade técnica, onde utilizando a luz solar concomitante ao reagente de Fenton, conseguiu obter um aumento na biodegradabilidade, elevando a razão $\mathrm{DBO}_{5} / \mathrm{DQO}$ de 0,11 a 0,41 (com um incremento de $273 \%$ ). Após o tratamento foto-oxidativo, este resultado indica que o chorume aumentou significativamente a sua biodegradabilidade (Malato et al., 2002), mostrando, assim, condições físico-químicas favoráveis para ser tratado biologicamente, com melhoras nas etapas operacionais como a diminuição do tempo de retenção hidráulico e da formação de lodo no tratamento por lodo ativado. 


\subsection{Custo do processo}

A avaliação econômica (consumo energético e reagentes) no tratamento do chorume, de acordo com o planejamento fracionado $\left(2^{4-1}\right)$, considerou apenas o processo foto-Fenton Solar. A energia consumida pelos equipamentos no processo foto-Fenton com duração de 2 horas foram: pHmetro (PH21 pH/mV HANNA) e a bomba centrífuga (BOMAX, Modelo NH-30PX-T).

Essa estimativa do consumo energético dos equipamentos do processo foto-Fenton solar foi realizada através da medida direta da respectiva energia consumida, pelo equipamento ICEL ME-2500 (220V e $60 \mathrm{~Hz})$, certificado de conformidade com a numeração (201111011512) e seu custo calculado através da cotação da potência nacional (KWh) (Portal Business Brasil, 2012). E a estimativa de consumo de reagentes, de acordo com os níveis da planilha (Tabela 1), foi calculado considerando-se os seguintes reagentes e concentrações: $\mathrm{H}_{2} \mathrm{SO}_{4}\left(98 \% \mathrm{~m} \mathrm{~m}^{-1}\right), \mathrm{NaOH}\left(98 \% \mathrm{~m} \mathrm{~m}^{-1}\right), \mathrm{H}_{2} \mathrm{O}_{2}\left(30 \% \mathrm{~m} \mathrm{~m}^{-1}\right)$ e $\mathrm{FeSO}_{4} \cdot 7 \mathrm{H}_{2} \mathrm{O}\left(98 \% \mathrm{~m} \mathrm{~m}^{-1}\right)$, com base de cotação no Guia do Químico (2012).

O pHmetro consumiu $0,00166 \mathrm{KWh}$ para o controle operacional e a bomba centrífuga $0,0276 \mathrm{KWh}$ para o fluxo do efluente sobre o reator, totalizando $0,02926 \mathrm{KWh}$, considerandose o tempo total de reação para todos os experimentos realizados.

A otimização do processo foto-Fenton, em função da redução de DQO, foi avaliada através do cálculo final do consumo total (energia + reagente) à relação custo/benefício (menor é melhor), realizado em cada experimento, conforme mostrado na Tabela 3.

Os resultados obtidos da Tabela 3 foram realizados em duplicatas e apresentaram valores de redução de DQO com baixo desvio padrão $(\mathrm{d}<0,05)$, obtendo, assim, uma maior confiabilidade nos resultados. A redução percentual de DQO do experimento 13 (o melhor com redução da concentração de DQO de 88,7 \%) foi avaliada através do cálculo final do consumo total (energia + reagente), onde o custo de energia ( $\mathrm{R} \$ 0,0095)$ e o consumo de reagente $(\mathrm{R} \$ 0,3705)$ totalizaram $\mathrm{R} \$ 0,38$ para os $3 \mathrm{~L}$ do reator, durante as 2 horas de reação fotocatalítica. Também, os resultados da Tabela 3 mostram que o melhor resultado custo/benefício da planilha não está relacionado com o melhor rendimento de degradação (redução da DQO). O melhor desempenho na degradação fotocatalítica e o seu respectivo resultado do custo/benefício não é o melhor da planilha, devido ao seu alto consumo de reagentes, principalmente $\mathrm{o}_{2} \mathrm{O}_{2}\left(30 \% \mathrm{~m} \mathrm{~m}^{-1}\right)$, obtendo a $4^{\mathrm{a}}$ menor relação custo/benefício $(4,28)$ entre todos os experimentos.

Em relação ao melhor custo/benefício, o melhor experimento foi o 1, cujo valor foi de 3,26 (sendo a sua duplicata com 3,34, experimento 11), com uma redução na DQO de 84,4 \%. $\mathrm{Na}$ avaliação geral, buscando-se uma viabilidade econômica no processo foto-Fenton, é melhor utilizar as variáveis utilizadas nos experimentos 1 e 11 , mesmo com uma menor redução da DQO (diferença de 4,3 \%), mas com uma economia de $\mathrm{R} \$ 35,67 \mathrm{~m}^{-3}$ de chorume tratado (cerca de $28,1 \%$ ). Os níveis das variáveis para esse experimento com melhor custo/benefício, considerando-se $3 \mathrm{~L}$ de chorume, são: $\mathrm{pH}$ em 4, quantidades dos reagentes $\mathrm{H}_{2} \mathrm{O}_{2}$ de 48,5 g e Fe ${ }^{2+}$ de $6,1 \mathrm{~g}$ e UV no período da tarde.

\subsection{Modelo neural proposto}

Numa fase anterior à implementação do modelo de redes neurais e sua otimização, os dados foram transformados de modo que as variáveis dependentes e independentes exibissem características de distribuições particulares (Olden e Jackson, 2002). No presente trabalho, os dados foram normalizados de forma a possuírem média zero e desvio padrão igual a 1.

Os resultados e os níveis dos fatores de entrada do planejamento fatorial fracionado $2^{4-1}$ são apresentados na Tabela 4. Realça-se que os 247 pontos experimentais componentes do modelo neural foram obtidos através da planilha experimental, com coleta de pontos em 
tempos de 10 minutos durante cada experimento, tendo como base a matriz experimental da Tabela 4.

Tabela 3. Custo ( $\mathrm{R} \$$ ) dos reagentes e energia do processo foto-Fenton solar para o tratamento de $3 \mathrm{~L}$ de chorume em 2-h de reação, segundo planejamento de experimentos.

\begin{tabular}{|c|c|c|c|c|c|c|c|c|}
\hline \multirow{3}{*}{ Exp. } & \multicolumn{4}{|c|}{ Reagentes } & \multirow{3}{*}{ Energia } & \multirow{3}{*}{$\begin{array}{c}\text { Custo } \\
\text { Total } \\
\text { (CT) } \mathbf{R} \$\end{array}$} & \multirow{3}{*}{$\begin{array}{c}\text { Redução } \\
\text { DQO } \\
\left(\%_{\text {red }}\right)\end{array}$} & \multirow{3}{*}{ 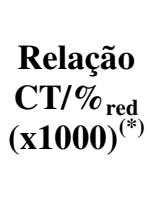 } \\
\hline & \multicolumn{2}{|c|}{ Ajuste de pH } & \multicolumn{2}{|c|}{ Reagente de Fenton } & & & & \\
\hline & $\mathrm{NaOH}$ & $\mathrm{H}_{2} \mathrm{SO}_{4}$ & $\mathbf{H}_{2} \mathbf{O}_{2}$ & $\mathrm{FeSO}_{4} \cdot 7 \mathrm{H}_{2} \mathrm{O}$ & & & & \\
\hline 1 & 0,0036 & 0,0649 & 0,147 & 0,050 & 0,0095 & 0,275 & 84,4 & 3,26 \\
\hline 2 & 0,0074 & 0,0629 & 0,147 & 0,050 & 0,0095 & 0,277 & 54,6 & 5,07 \\
\hline 3 & 0,0074 & 0,0629 & 0,147 & 0,079 & 0,0095 & 0,306 & 67,9 & 4,50 \\
\hline 4 & 0,0054 & 0,0619 & 0,185 & 0,065 & 0,0095 & 0,327 & 75,3 & 4,34 \\
\hline 5 & 0,0036 & 0,0649 & 0,147 & 0,079 & 0,0095 & 0,304 & 47,0 & 6,47 \\
\hline 6 & 0,0074 & 0,0629 & 0,147 & 0,050 & 0,0095 & 0,277 & 52,3 & 5,29 \\
\hline 7 & 0,0074 & 0,0629 & 0,223 & 0,079 & 0,0095 & 0,382 & 72,3 & 5,28 \\
\hline 8 & 0,0074 & 0,0629 & 0,223 & 0,050 & 0,0095 & 0,353 & 70,1 & 5,03 \\
\hline 9 & 0,0054 & 0,0619 & 0,185 & 0,065 & 0,0095 & 0,327 & 74,3 & 4,40 \\
\hline 10 & 0,0074 & 0,0629 & 0,223 & 0,079 & 0,0095 & 0,382 & 71,1 & 5,37 \\
\hline 11 & 0,0036 & 0,0649 & 0,147 & 0,050 & 0,0095 & 0,275 & 82,2 & 3,34 \\
\hline 12 & 0,0036 & 0,0649 & 0,223 & 0,079 & 0,0095 & 0,380 & 87,6 & 4,33 \\
\hline 13 & 0,0036 & 0,0649 & 0,223 & 0,079 & 0,0095 & 0,380 & 88,7 & 4,28 \\
\hline 14 & 0,0036 & 0,0649 & 0,223 & 0,050 & 0,0095 & 0,351 & 69,0 & 5,08 \\
\hline 15 & 0,0036 & 0,0649 & 0,147 & 0,079 & 0,0095 & 0,304 & 49,0 & 6,21 \\
\hline 16 & 0,0074 & 0,0629 & 0,223 & 0,050 & 0,0095 & 0,353 & 66,3 & 5,32 \\
\hline 17 & 0,0074 & 0,0629 & 0,147 & 0,079 & 0,0095 & 0,306 & 68,5 & 4,46 \\
\hline 18 & 0,0054 & 0,0619 & 0,185 & 0,065 & 0,0095 & 0,327 & 78,4 & 4,17 \\
\hline 19 & 0,0036 & 0,0649 & 0,223 & 0,050 & 0,0095 & 0,351 & 66,1 & 5,31 \\
\hline
\end{tabular}

(*) Fator para avaliar a relação custo-benefício.

O número ótimo de neurônios da camada oculta foi determinado baseado no valor mínimo do MSE (Mean Square Error) dos conjuntos de treinamento, validação e teste, variando-se o número de neurônios de 1 a 25 . A Figura 2 apresenta as relações entre MSE e o número de épocas necessários para se obter o valor mínimo de MSE.

As Figuras 3A, 3B, 3C e 3D apresentam os ajustes obtidos respectivamente para os conjuntos de treinamento, teste, validação e totalidade dos pontos experimentais.

A matriz de entrada foi composta por 247 amostras e este conjunto foi dividido em subconjuntos de treinamento $(50 \%)$, de validação $(25 \%)$ e de teste $(25 \%)$, com o objetivo de verificação do poder de generalização da rede neural obtida.

Observa-se resultados de coeficientes de correlação acima de 0,94 para os conjuntos de treinamento, validação e teste, confirmando o poder de generalização do modelo neural obtido. 
Tabela 4. Variação percentual de DQO para cada experimento foto-Fenton solar.

\begin{tabular}{|c|c|c|c|c|c|}
\hline \multicolumn{6}{|c|}{ Fatorial Fracionado $2^{4-1}$} \\
\hline \multirow{2}{*}{$\operatorname{Exp}$} & $\mathbf{p H}$ & $\mathbf{H}_{2} \mathbf{O}_{2}{ }^{(*)}$ & $\mathbf{U V}$ & $\mathbf{F e}^{2+(*)}$ & DQO \\
\hline & (A) & (B) & (C) & (D) & $(\%)$ \\
\hline 1 & 4 & 48,5 & Tarde & 6,1 & 84,4 \\
\hline 2 & 2 & 48,5 & Manhã & 6,1 & 54,6 \\
\hline 3 & 2 & 48,5 & Tarde & 9,7 & 67,9 \\
\hline 4 & 3 & 60,6 & Almoço & 7,9 & 75,3 \\
\hline 5 & 4 & 48,5 & Manhã & 9,7 & 47,0 \\
\hline 6 & 2 & 48,5 & Manhã & 6,1 & 52,3 \\
\hline 7 & 2 & 72,8 & Manhã & 9,7 & 72,3 \\
\hline 8 & 2 & 72,8 & Tarde & 6,1 & 70,1 \\
\hline 9 & 3 & 60,6 & Almoço & 7,9 & 74,3 \\
\hline 10 & 2 & 72,8 & Manhã & 9,7 & 71,1 \\
\hline 11 & 4 & 48,5 & Tarde & 6,1 & 82,2 \\
\hline 12 & 4 & 72,8 & Tarde & 9,7 & 87,6 \\
\hline 13 & 4 & 72,8 & Tarde & 9,7 & 88,7 \\
\hline 14 & 4 & 72,8 & Manhã & 6,1 & 69,0 \\
\hline 15 & 4 & 48,5 & Manhã & 9,7 & 49,0 \\
\hline 16 & 2 & 72,8 & Tarde & 6,1 & 66,3 \\
\hline 17 & 2 & 48,5 & Tarde & 9,7 & 68,5 \\
\hline 18 & 3 & 60,6 & Almoço & 7,9 & 78,4 \\
\hline 19 & 4 & 72,8 & Manhã & 6,1 & 66,1 \\
\hline
\end{tabular}

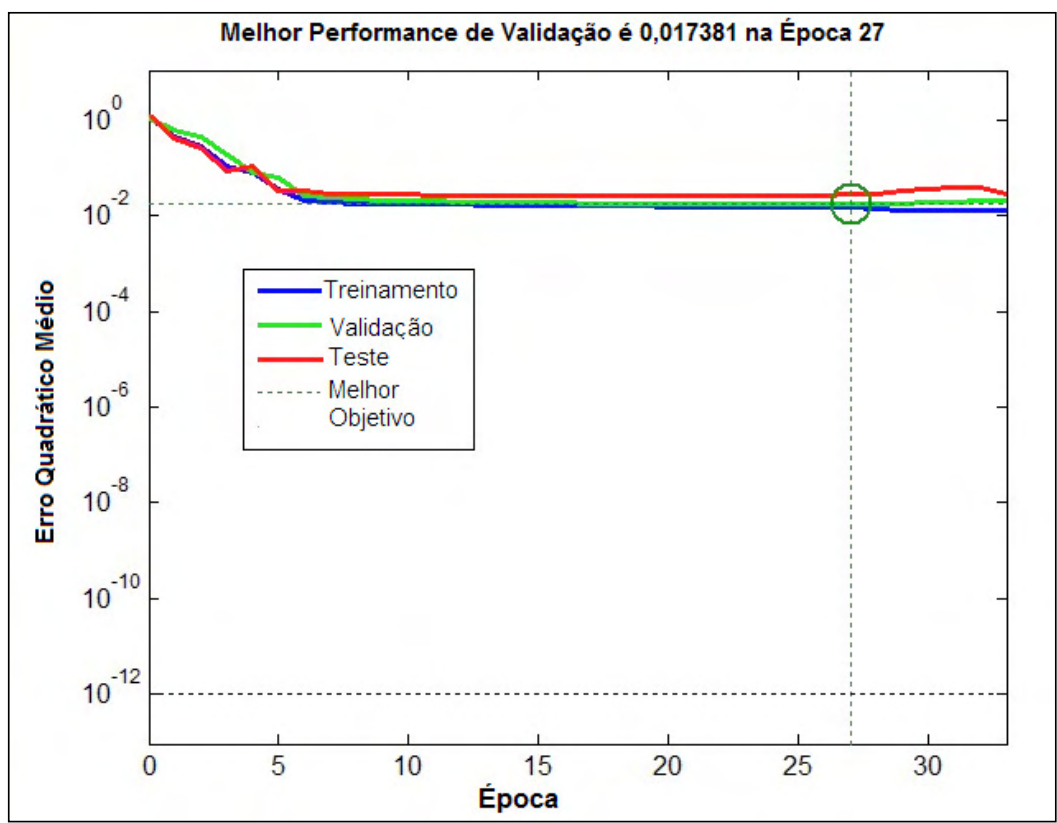

Figura 2. Erro Quadrático Médio. 

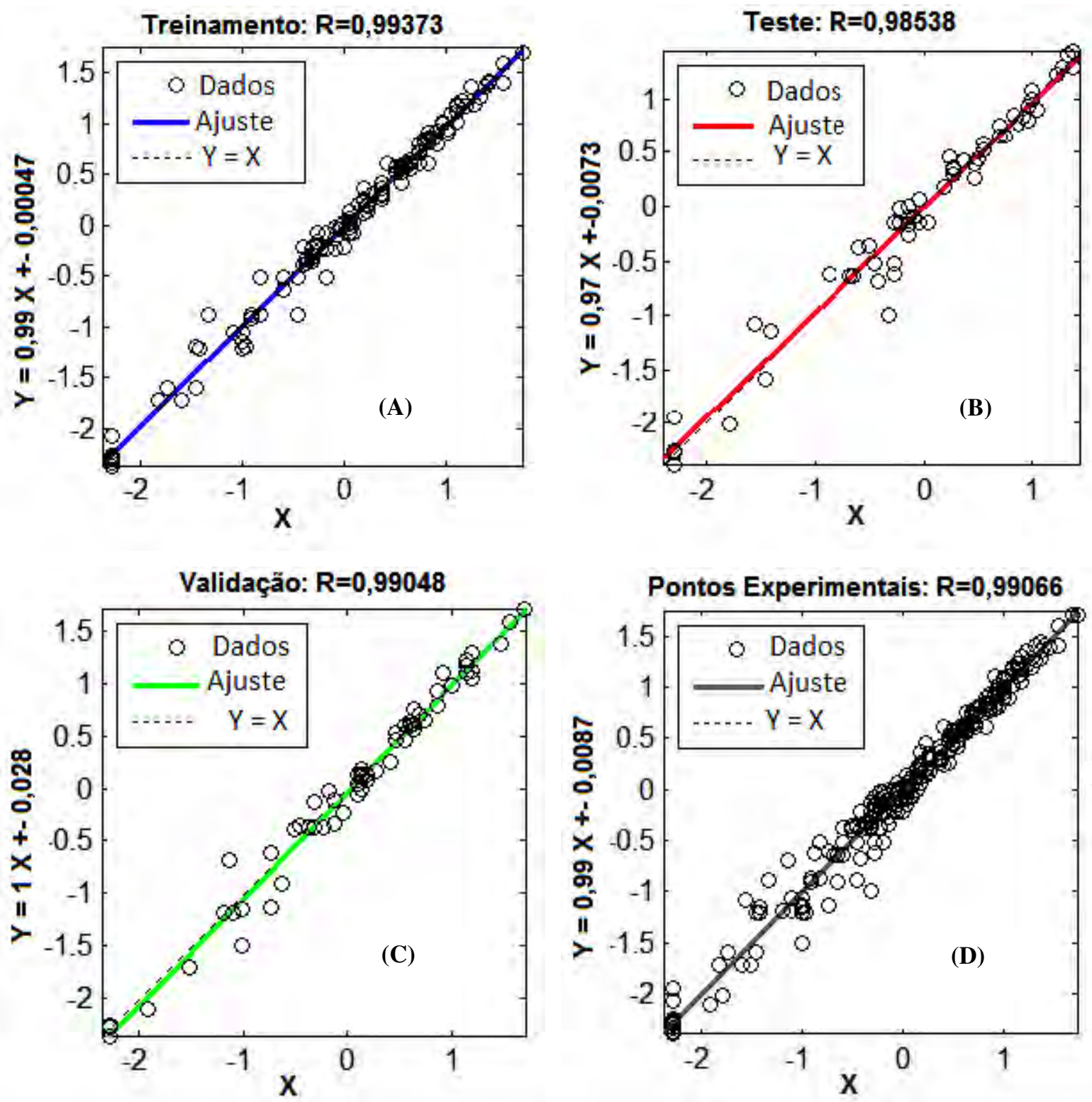

Figura 3. Ajuste dos conjuntos em função de: (A) Treinamento; (B) Teste; (C) Validação; (D) Pontos Experimentais, onde Y são os valores preditos pelo modelo neural e $\mathrm{X}$ os valores reais dos experimentos.

\subsection{Análise dos efeitos}

A Tabela 5 apresenta a análise de variância dos fatores envolvidos no tratamento do chorume com o processo foto-Fenton solar em função da \% redução de DQO.

Pela análise de variância, verificou-se que os fatores 1, 2 e 3 apresentaram efeito significativo $(\mathrm{P}<0,05)$ na redução percentual DQO. $\mathrm{O}$ fator 4 não apresentou significância no processo, dentro do intervalo de confiança de $95 \%$.

A Figura 4 proporciona uma noção qualitativa da importância dos efeitos dos fatores principais. $\mathrm{O}$ gráfico dos efeitos principais mostra que deve existir curvatura para a variação de DQO, visto que a média dos pontos centrais não está posicionada próxima da reta dos valores médios de variação da DQO. 
Tabela 5. Análise de variância (ANOVA) obtida a partir dos valores médios de redução percentual de DQO.

\begin{tabular}{lccccc}
\hline \multicolumn{1}{c}{ Termo } & Efeito & Coeficiente & $\begin{array}{c}\text { Erro } \\
\text { Quadrático }\end{array}$ & $\mathbf{T}$ & $\mathbf{P}$ \\
\hline Constante & 68,601 & 0,4312 & 159,09 & 0,000 & \\
$\mathbf{p H}$ & 6,416 & 3,208 & 0,4312 & 7,44 & 0,000 \\
$\mathbf{H}_{\mathbf{2}} \mathbf{O}_{2}$ & 10,719 & 5,359 & 0,4312 & 12,43 & 0,000 \\
$\mathbf{U V}$ & 16,844 & 8,422 & 0,4312 & 19,53 & 0,000 \\
$\mathbf{F e}^{2+}$ & 0,926 & 0,463 & 0,4312 & 1,07 & 0,308 \\
$\mathbf{p H} \mathbf{H}_{2} \mathbf{O}_{2}$ & 1,564 & 0,782 & 0,4312 & 1,81 & 0,100 \\
$\mathbf{p H} \mathbf{U V}^{2}$ & 11,214 & 5,607 & 0,4312 & 13,00 & 0,000 \\
$\mathbf{p H} \mathbf{F e}^{2+}$ & $-8,204$ & $-4,102$ & 0,4312 & $-9,51$ & 0,000 \\
$\mathbf{P o n t o}$ Central & & 7,393 & 1,0852 & 6,81 & 0,000 \\
\hline
\end{tabular}

Nota: Coeficiente de Determinação = 98,90\%; Coeficiente de Determinação Ajustado = 98,03\%.

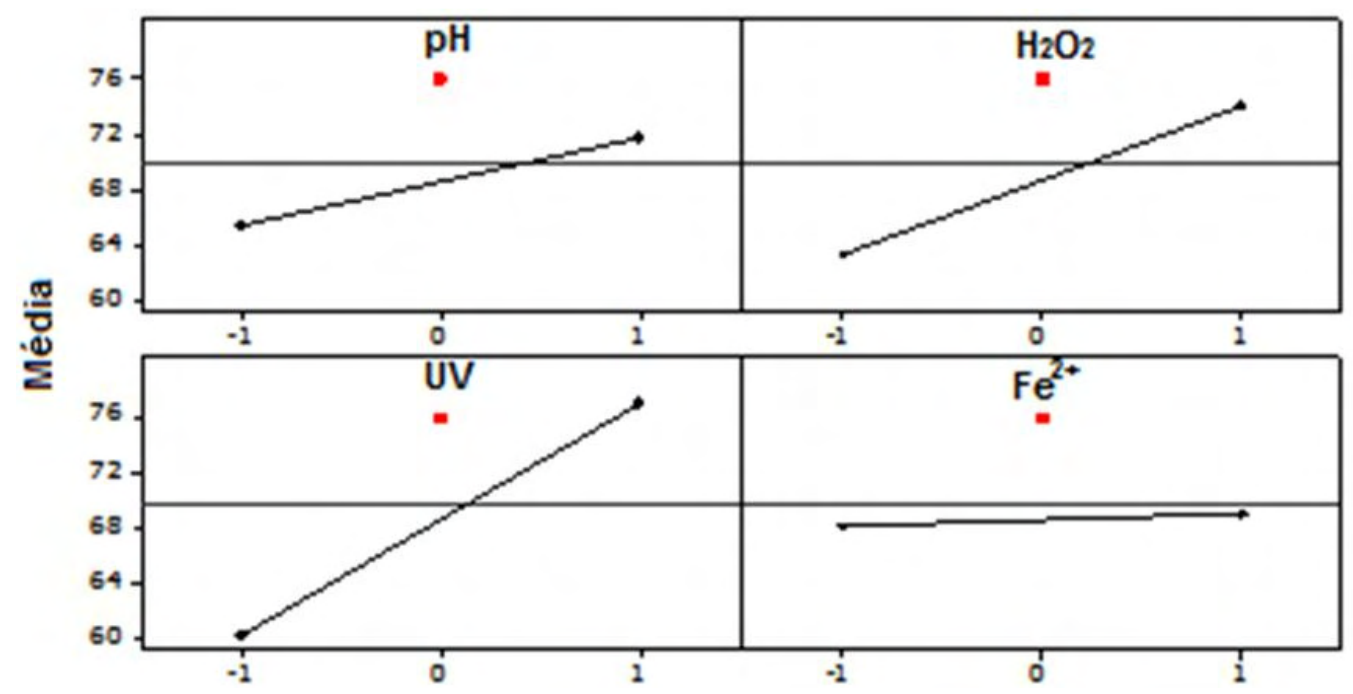

Figura 4. Gráfico dos efeitos em relação a \% redução de DQO.

$\mathrm{O}$ fator "D" referente à quantidade $\mathrm{Fe}^{2+}$ obteve uma baixa significância, possivelmente devido à inerente turvação verificada após a sua adição durante o processo de oxidação, em todos os níveis, dificultando a ação da incidência da luz solar. Mesmo assim, este termo não deve ser retirado do modelo, visto que existe uma significância deste fator interagindo com o fator $\mathrm{pH}$.

No rendimento do processo foto-Fenton solar foi relacionado com a quantidade ótima de oxidante $\left(\mathrm{H}_{2} \mathrm{O}_{2}\right)$, utilizado no processo oxidativo, bem como o período de reação. Por esse foco podemos notar que os experimentos realizados nos período da tarde e almoço, apresentaram valores de porcentagem de redução de DQO na faixa de $80 \%$, mostrando que a presença do UV potencializa a reação de formação do radical hidroxila (Loures, 2011).

A Figura 5 mostra o gráfico de contorno da variável resposta DQO em função das concentrações de $\mathrm{Fe}^{2+}$ e do $\mathrm{H}_{2} \mathrm{O}_{2}$ no tratamento foto-Fenton solar, onde pode ser observado que no nível mais alto de suas quantidades obtêm-se reduções acima de $80 \%$. 


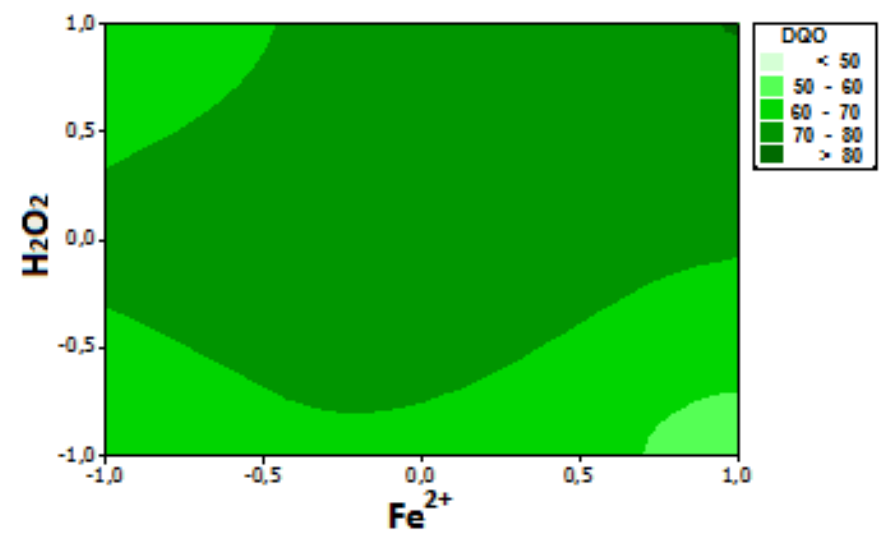

Figura 5. Variação da DQO em função do $\mathrm{Fe}^{2+} \mathrm{e}$ $\mathrm{H}_{2} \mathrm{O}_{2}$ do processo foto-Fenton solar no tratamento de chorume.

\section{CONCLUSÃO}

Uma rede neural feedforward backpropagation com 3 camadas foi otimizada para predizer o grau de degradação de chorume in natura utilizando processo foto-fenton solar. A configuração do modelo neural que proporcionou o menor valor de MSE utilizou uma função de transferência logaritmo sigmoidal (logsig) na camada oculta com 9 neurônios e função de transferência linear (purelin) na camada de saída. O algoritmo de treinamento utilizado foi Levenberg-Marquardt backpropagation.

Além disso, a proposta de um tratamento ou pré-tratamento de chorume pelo processo foto-Fenton Solar teve como sua maior eficiência em $88 \%$ na redução de DQO. Segundo legislação ambiental, o parâmetro de controle para descarte em água superficial (DBO) não apresentou a concentração estabelecida após a oxidação do chorume, o que mostra que o chorume deve passar por um outro tipo de tratamento. Como a relação DBO/DQO teve um incremento de 0,3 unidades, mostra uma viabilidade para o tratamento biológico por lodo ativado.

Dentro do delineamento do planejamento de experimentos avaliado para a degradação de chorume in natura pelo processo foto-Fenton, o melhor resultado em função da redução de DQO obtido foi de 88,7 \% . Mas, a viabilidade econômica e operacional do processo fotooxidativo tendeu a uma eficiência de $84,4 \%$ na redução de DQO, chegando-se num valor de $\mathrm{R} \$ 91,67 \mathrm{~m}^{-3}$, com uma economia de $\mathrm{R} \$ 35,67$ para cada $\mathrm{m}^{3}$ de chorume tratado. Em relação ao custo de cada parâmetro avaliado, como em todo o processo Fenton, a variável peróxido de hidrogênio foi a mandatória. Com esse tipo de tratamento realizado, segundo os dados do planejamento de experimentos propostos, não foi possível otimizar a relação entre $\mathrm{H}_{2} \mathrm{O}_{2} \mathrm{e}$ $\mathrm{Fe}^{2+}$, que deve ser criteriosamente avaliado em função dos seus elevados valores comerciais.

Os resultados preditos pela rede neural aproximaram-se dos resultados experimentais com coeficientes de correlação acima de 0,94 para os conjuntos de treinamento, validação e teste, evidenciando o poder de generalização do modelo proposto.

A utilização de redes neurais no processo de degradação de efluentes por processos foto oxidativos pode ser acoplada à análise estatística, fornecendo uma forma de modelagem matemática entre representatividade do modelo e tempo computacional. Realça-se, desta forma, a aplicabilidade de técnicas de redes neurais e análises estatísticas no equacionamento dos problemas da área ambiental, em particular na predição e análises de processos de tratamento de efluentes.

Algumas características dos modelos neurais apresentam-se como vantagens na análise de processos de degradação de efluentes, sendo destacadas a capacidade de modelar processos 
não-lineares e complexos, como o processos foto-Fenton, revestidos de não-linearidades com a ação de interferentes na análise de parâmetros ambientais como a DQO. A influência aleatória da radiação ultravioleta em função de condições ambientais apresenta-se como forte fator não linear em dados empíricos, sendo parâmetros incertos que variam com o tempo. A utilização de modelos que envolvem processos de radiação de caráter determinístico reveste de complexidade matemática a modelagem de processos foto-fenton, pois envolvem a solução de equações de energia, balanços de massa e de energia térmica. Neste sentido, a modelagem neural pode ser aplicada facilmente em ambientes computacionais como o software Matlab. A rede neural proposta mapeou um espaço multidimensional da forma $\triangle D Q O=f\left(t, p H,\left[\mathrm{H}_{2} \mathrm{O}_{2}\right], P_{U V},\left[\mathrm{Fe}^{2+}\right]\right)$. Entretanto, realça-se que modelos neurais são baseados em dados históricos do processo em estudo e que esta base de conhecimento não é desperdiçada podendo ser inserida em novos processos de modelagem do efluente objeto de estudo deste trabalho. Em particular, poderíamos inserir no modelo proposto dados de um processo de degradação que envolvesse uma nova variável de entrada, ou seja, uma camada de entrada com um número de neurônios diferentes da base de dados inicial.

\section{AGRADECIMENTOS}

Agradecimentos a CAPES (Coordenação de Aperfeiçoamento de Pessoal de Nível Superior) pelo apoio financeiro. E aos autores do projeto de pesquisa de tratamento de chorume financiado pela FAPESP (Fundação de Amparo à Pesquisa do Estado de São Paulo) 2009/17650-2 que forneceram o apoio técnico ao trabalho.

\section{REFERÊNCIAS}

AMERICAN PUBLIC HEALTH ASSOCIATION - APHA; AMERICAN WATER WORKS ASSOCIATION - AWWA; WATER ENVIRONMENTAL FEDERATION - WEF. Standard methods for the examination of water and wastewater. 22. ed. Washington, DC., 2009.

BRASIL. Conselho Nacional do Meio Ambiente. Resolução no 430 de 13 de maio de 2011. Dispõe sobre as condições e padrão de lançamento de efluentes complementa e altera a Resolução no 357, de 17 de março de 2005. Brasília: MMA, 2011. 9p.

CERQUEIRA, E. O.; ANDRADE, J. C.; POPPI, R. J. Redes neurais e suas aplicações em calibração multivariada. Química Nova, v. 24, n. 6, 864-873, 2001. http://dx.doi.org/10.1590/S0100-40422001000600025

ÇINAR, O. New tool for evaluation of performance of wastewater treatment plant: artificial neural network. Process Biochemistry, v. 40, n. 3, p 2980-2984, 2005. http://dx.doi.org/10.1016/j.procbio.2005.01.012

COMPANHIA DE TECNOLOGIA DE SANEAMENTO AMBIENTAL - CETESB. Resíduos sólidos industriais. 1993. Disponível em: <http://www.cetesb.sp.gov.br>. Acesso em: 12 dez. 2011.

ELMOLLA, E. S.; CHAUDHURI, M.; ELTOUKHY, M. M. The use of artificial neural network (ANN) for modeling of COD renoval from antibiotic aqueous solution by the Fenton process. Journal of Hazardous Material, v. 179, n.1, p 127-134, 2010. http://dx.doi.org/10.1016/j.jhazmat.2010.02.068 
GIORDANI, D. S Estudo comparativo entre observador de estado não-linear de alto ganho e calorimetria associada a redes neurais artificiais para previsão de composição de copolímeros produzidos em emulsão com altos teores sólido. 2003. 90f. Tese (Doutorado) - Faculdade de Engenharia Química, Universidade Estadual de Campinas, Campinas, 2003.

GIROTO, J. A. Estudo do processo de degradação fotoquímica de soluções aquosas de álcool polivinílico. 2002. 93f. Dissertação (Mestrado) - Escola Politécnica de São Paulo, Universidade de São Paulo, São Paulo, 2002.

GUIA DO QUÍMICO. Cotação de reagentes Químicos. 2012. Disponível em: <http://www.guiaquimico.com.br>. Acesso em: 29 fev. 2012.

GUIMARÃES, O. L. C.; AQUINO, H. O. Q; OLIVEIRA, I. S.; SILVA, M. B.; IZÁRIO FILHO, H. J. et al. Prediction via Neural Networks of the Residual Hydrogen Peroxide used in Photo-Fenton Processes for Effluent Treatment. Chemical Engineering Technology, v. 30, n. 8, p. 1134-1139, 2007. http://dx.doi.org/10.1002/ceat.200700113

GUIMARÃES, O. L. C.; VILLELA FILHO, D. N.; SIQUEIRA, A. F.; IZÁRIO FILHO, H. J.; SILVA, M. B. Optimization of the AZO dyes decoloration process through neural networks: determination of the $\mathrm{H}_{2} \mathrm{O}_{2}$ addition critical point. Chemistry Engineering Journal, v. 141, n. 1/3, p. 35-41, 2008. http://dx.doi.org/10.1016/j.cej.2007.10.017

HAMED, M. M.; KHALAFALLAH, M. G.; HASSANIEN, E. A. Prediction of wastewater treatment plant performance using artificial neural networks. Environmental Modelling \& Software, v. 19, n. 4, p. 919-928, 2004. http://dx.doi.org/10.1016/j.envsoft.2003.10.005

HAYKIN, S. S. Redes neurais: princípios e prática. 2. ed. São Paulo: Artmed, 1999.

KATO, Y.; KUMAGAI, T.; NISHIOKA, H.; SUGIE, Y. The prediction of Chemical Oxygen Demand (COD) in waste water by UV-visible absorption spectrum - neural network. Oriental Journal of Chemistry, v. 17, n. 1, p. 1-8, 2001.

KJELDSEN P. I.; BARLAZ, M. A.; ROOKER, A. P.; BAUN, A.; LEDIN, A.; CHRISTENSEN, T. H. Present and long-term composition of MSW landfill leachate: a review. Critical Reviews in Environmental Science and Technology, v. 32, n. 5, p. 297-336, 2002. http://dx.doi.org/10.1080/10643380290813462

LIMA, L. S.; IZÁRIO FILHO, H. J.; CHAVES, F. J. M. Determinação da demanda bioquímica de oxigênio para teores $\leq 5 \mathrm{mg} \mathrm{L}^{-1} \mathrm{O}_{2}$. Revista Analítica, v. 25, n. 2, p. 52 57, 2006.

LOURES, C. C. A. Estudo da Aplicação de UV/FENTON $\left(\mathrm{Fe}^{2+}+\mathrm{H}_{2} \mathrm{O}_{2}\right)$ no Tratamento de Efluentes de Laticínio. 2011. 209 f. Dissertação (Mestrado em ciências) - Escola de Engenharia de Lorena, Universidade de São Paulo, Lorena, 2011.

MALATO, S.; BLANCO, J.; FERNANDEZ-ALBA, A. R.; AGUERA, A. Solar photocatalytic mineralization of commercial pesticides: acrinathrin. Chemosphere, v. 40, n. 8, p. 403-409, 2002. http://dx.doi.org/10.1016/S0045-6535(99)00267-2

NADTOCHENCO, V.; KIWI, J. Photoinduced Mineralization of Xylidine by the Fenton Reagent 2. Implications of precursors formed in the dark. Environmental Science \& Technology, v. 32, n. 21, p. 3282-3285, 1998. http://dx.doi.org/10.1021/es9709637 
NOGUEIRA, R. F. P.; TROVÓ, A. G.; SILVA, M. R. A.; VILLA, R. D. Fundamentos e Aplicações dos Processos Fenton e Foto-Fenton. Química Nova, v. 30, n. 2, p. 400-408, 2007. http://dx.doi.org/10.1590/S0100-40422007000200030

OLDEN, J. D.; JACKSON, D. A. Illuminating the "black box": a randomization approach for understanding variable contributions in artificial neural networks. Ecological Modelling, v. 154, n. 1, p. 135-150, 2002. http://dx.doi.org/10.1016/S03043800(02)00064-9

PAREEK, V. K.; BRUNGS, M. P.; ADESINA, A. A.; SHARAMA, R. Artificial neural network modeling of a multiphase photodegradation system. Journal of Photochemistry and Photobiology A: Chemistry, v. 149, n. 3, p. 139-146, 2002. http://dx.doi.org/10.1016/S1010-6030(01)00640-2

PORTAL BUSINESS BRASIL. Energia elétrica a terceira mais cara do país. 2012. Disponível em: http://sites.google.com/site/portalbusinessbrasil/home/energiabrasileira . Acesso em: 29/07/2012.

SVOZIL, D.; KVASNICKA. A; POSPÍCHAl, J. Introduction to multi-layer feed-forward neural networks. Chemometrics and Intelligent Systems, v. 39, n. 1, p. 43-62, 1997. http://dx.doi.org/10.1016/S0169-7439(97)00061-0

TELLES, C. A. S. Processos combinados para o tratamento de lixiviado de aterro sanitário. 2010. Dissertação (Mestrado em Engenharia Química) - Universidade do Rio de Janeiro, Universidade Federal do Rio de Janeiro, Rio de Janeiro, 2010.

VILAR, J. P. V; MOREIRA, J. M. S; BOAVENTURA, R. A. R. Solar photo-Fenton as a preoxidation step for biological treatment of landfill leachate in a plot with CPCs.

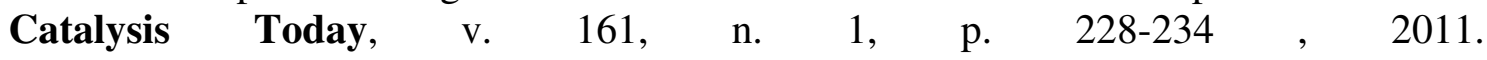
http://dx.doi.org/10.1016/j.cattod.2010.08.025

VILELA-RIBEIRO, E. B; COSTA L. S. O; LIMA-RIBEIRO M. S; SOUSA M. H. Uma abordagem normativa dos resíduos sólidos de saúde e a questão ambiental. REMEA Revista Eletrônica do Mestrado em Educação Ambiental, v. 22, n. 1, p. 168-176, 2009.

WANG, Q.; LEMLEY, A. T. Kinetic model and optimization of 2,4-D degradation by anodic Fenton treatment. Environmental Science \& Technology, v. 35, n. 15, p. 4509-4514, 2001. http://dx.doi.org/10.1021/es0109693

ZHANG, H.; CHOI, H. J.; HUANG, C. Optimization of Fenton process for the treatment fo landfill leachate. Journal of Hazardous Materials, v. 125, n. 1, p. 166-174, 2005. http://dx.doi.org/10.1016/j.jhazmat.2005.05.025 


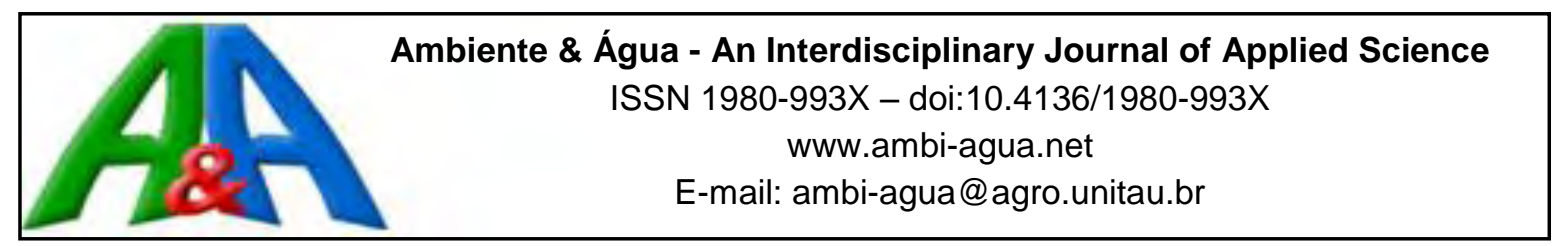

\title{
Tratamento de lixiviados de aterros de resíduos sólidos utilizando Processos Fenton e Foto-Fenton Solar
}

\author{
doi: 10.4136/ambi-agua.1483 \\ Received: 12 Aug. 2014; Accepted: 15 Sep. 2014 \\ Fábio Moraes da Costa ${ }^{1}$; Juacyara Carbonelli Campos ${ }^{2 *}$; \\ Fabiana Valéria da Fonseca ${ }^{2}$; Daniele Maia Bila ${ }^{3}$ \\ ${ }^{1}$ Universidade Castelo Branco (UCB), Rio de Janeiro, RJ, Brasil \\ Programa de Pós-Graduação em Tecnologia de Processos Químicos e Bioquímicos da Escola de Química - UFRJ \\ ${ }^{2}$ Universidade Federal do Rio de Janeiro (UFRJ), Rio de Janeiro, RJ, Brasil \\ Departamento de Processos Inorgânicos \\ ${ }^{3}$ Universidade do Estado do Rio de Janeiro (UERJ), Rio de Janeiro, RJ, Brasil \\ Departamento de Engenharia Sanitária e do Meio Ambiente \\ *Autor correspondente: e-mail: juacyara@eq.ufrj.br, \\ fmcchemist@gmail.com, fabiana@eq.ufrj.br, danielebila@yahoo.com.br
}

\section{RESUMO}

O lixiviado de aterros de resíduos sólidos urbanos é uma complexa mistura de substâncias orgânicas e inorgânicas, que em sua composição causam danos ao meio ambiente, devido à elevada concentração de matéria orgânica recalcitrante e à toxicidade presente. A proposta deste trabalho envolve a aplicação dos processos oxidativos avançados (POA), como tratamento de lixiviado oriundos de dois aterros sanitários, Gericinó (Rio de Janeiro/RJ) e Gramacho (Duque de Caxias/RJ), dois aterros com mais de 30 anos de operação, sendo que o último encontra-se encerrado há 2 anos. O objetivo da utilização dos POA é melhorar a qualidade do lixiviado para um tratamento biológico subsequente, para isso foi utilizado o ensaio com a bactéria Alivibrio fischeri para avaliação da toxicidade. Para o Processo Fenton-escuro, realizado em escala de bancada, nos experimentos realizados a $\mathrm{pH}=3,0$ e razão $\mathrm{Fe}^{+2}: \mathrm{H}_{2} \mathrm{O}_{2}$ igual a 1:5 foram obtidas remoções de $56 \%$ e $19 \%$ de DQO para os lixiviados oriundos de Gericinó e Gramacho, respectivamente. Para o Processo Foto-Fenton Solar, realizado em uma planta piloto, nos testes a $\mathrm{pH}=3,0$ e razão $\mathrm{Fe}^{+2}: \mathrm{H}_{2} \mathrm{O}_{2}$ igual a $1: 5$, foram alcançadas $88 \%$ e $78 \%$ de remoção de DQO. Essas condições permitiram uma diminuição da toxicidade dos lixiviados para valores de coeficiente de efeito igual a 50\% (CE 50\%) para Aliivibrio fischeri maior que 50\%, indicando um lixiviado mais adequado para tratamento biológico posterior.

Palavras-chave: Aliivibrio fischeri, aterro sanitário, processos oxidativos avançados, toxicidade.

\section{Treatment of landfill leachate using Fenton Processes and Photo.Fenton Solar}

\section{ABSTRACT}

The leachate from municipal solid waste landfills is a complex mixture of organic and inorganic substances, which can damage the environment due to toxicity 
and the high concentration of recalcitrant organic matter. This work applied advanced oxidation processes (AOP) to the treatment of leachate from the Gericinó (Rio de Janeiro / RJ) and Gramacho (Duque de Caxias / RJ) landfills. These landfills were both in operation for more than 30 years; the latter closed two years ago. The purpose of using AOP is to improve the leachate quality prior to a subsequent biological treatment. The bacteria Aliivibrio fischeri was used as a biological process for evaluation of toxicity. The Dark-Fenton Process employed in bench scale experiments with a $\mathrm{pH}$ of 3.0 and $\mathrm{Fe}^{+2}: \mathrm{H}_{2} \mathrm{O}_{2}$ ratio $=1: 5$ resulted in removals of $56 \%$ and $19 \%$ for COD leachate of the Gericinó and Gramacho leachates, respectively. The Solar Photo-Fenton Process conducted in pilot plant tests at $\mathrm{pH}=3.0$ and $\mathrm{Fe}^{+2}: \mathrm{H}_{2} \mathrm{O}_{2}$ ratio $=1: 5$ generated COD removal $88 \%$ and $78 \%$, respectively. These conditions led to a decrease in the toxicity of leachates' coefficient values to a degree equal to $50 \%$ - for Alivibrio fischeri greater than $50 \%$ - indicating a more suitable leachate for further biological treatment.

Keywords: Aliivibrio fischeri, advanced oxidation processes, landfill, toxicity.

\section{INTRODUÇÃO}

O tratamento para os lixiviados de aterros tem recebido significante atenção nos últimos anos, em especial as áreas municipais. Conforme Campos et al. (2013), os lixiviados apresentam elevadas concentrações de matéria orgânica recalcitrante (acima de $2000 \mathrm{mg} \mathrm{L}^{-1}$ de DQO e relação DBO/DQO menores que 0,05) e nitrogênio amoniacal (acima de $1000 \mathrm{mg} \mathrm{L}^{-1}$ ) e são tratados com a combinação de técnicas físico-químicos (coagulação/floculação) e biológicos, que segundo alguns autores (Campos et al., 2013; Gotvajn et al., 2009) os resultados apresentados não têm sido satisfatórios. Uma forma de tratamento que tem sido avaliada é o uso de Processos Oxidativos Avançados (POA) para pré-tratamento de lixiviado, para degradar quimicamente a matéria orgânica recalcitrante e aumentar a biodegradabilidade do lixiviado.

Os POA constituem um grupo de técnicas que envolvem a geração de espécies altamente oxidantes, de radicais hidroxila (Araujo et al., 2011). A aplicação dos POA geralmente visa à transformação oxidativa dos componentes, em substâncias biologicamente degradáveis, ou em substâncias mais facilmente elimináveis por processos físico-químicos convencionais, como as moléculas de corantes em efluentes têxteis (Kunz et al., 2002).

$O$ radical hidroxila é um agente com elevado potencial de oxidação $\left(E^{o}=2,8 \mathrm{~V}\right)$, extremamente reativo e de pouca seletividade, que o torna capaz de atacar uma diversidade de moléculas orgânicas (Tell et al., 2001). A grande vantagem dos Processos Oxidativos Avançados reside no fato dela ser um tipo de tratamento destrutivo, ou seja, o contaminante não é simplesmente transferido de fase, mas sim degradado através de uma seqüência de reações químicas (Hassemer, 2006).

Entre os Processos Oxidativos Avançados, grande destaque tem sido dado ao uso do peróxido de hidrogênio em presença de sais de ferro, conhecido como o Processo Fenton, devido principalmente ao seu baixo custo e alta eficiência na degradação dos compostos recalcitrantes. O Processo Fenton consiste em um conjunto de reações cíclicas, que utilizam íons $\mathrm{Fe}^{+2}$ e $\mathrm{Fe}^{+3}$ como catalisadores, para decompor o peróxido de hidrogênio e produzir radicais hidroxila. $\mathrm{O}$ íon ferroso $\left(\mathrm{Fe}^{2+}\right)$ inicia e catalisa a decomposição do $\mathrm{H}_{2} \mathrm{O}_{2}$, resultando na geração de radicais hidroxila (Araujo et al., 2011), (Reação 1). A reação de íons férricos com peróxido de hidrogênio, conhecida como tipo-Fenton (Reação 2) permite a regeneração dos íons 
ferrosos, porém sua cinética é mais lenta. De acordo com Sun et al. (2007), as constantes de reação são $k_{1}=76,5 \mathrm{~L} \mathrm{~mol}^{-1} \mathrm{~s}^{-1}$ e $\mathrm{k}_{2}=0,01-0,02 \mathrm{~L} \mathrm{~mol}^{-1} \mathrm{~s}^{-1}$ para reações Fenton e Tipo-Fenton, respectivamente.

$$
\begin{aligned}
& \mathrm{Fe}^{+2}+\mathrm{H}_{2} \mathrm{O}_{2} \rightarrow \mathrm{Fe}^{+3}+\mathrm{H}^{-}+{ }^{\circ} \mathrm{OH}(\text { em meio ácido) } \\
& \mathrm{Fe}^{+3}+\mathrm{H}_{2} \mathrm{O}_{2} \rightarrow \mathrm{HO}_{2} \bullet+\mathrm{Fe}^{+2}+\mathrm{H}^{+}
\end{aligned}
$$

Além do Processo Fenton tradicional, que daqui por diante será denominado "Fenton-escuro", existe processos que utilizam a radiação ultravioleta, como os processos Foto-Fenton e Foto-Fenton solar (este último daqui por diante denominado "Fenton-solar"). No Fenton-solar, a luz solar atua como fonte de radiação que atua aumentando a regeneração do $\mathrm{Fe}^{+2}$ e a produção adicional de radicais hidroxilas (Reação 3). A utilização da radiação solar tem como principal objetivo a redução dos custos e aplicações em escala industrial. A maior eficiência deste POA em relação ao Fenton-escuro ocorre devido à regeneração do íon ferroso (Reação 4), dos quelatos orgânicos intermediários de $\mathrm{Fe}^{+3}$ e da fotólise direta do peróxido de hidrogênio, Reação (5) (Pignatello, 1992).

$$
\begin{aligned}
& \mathrm{Fe}^{+3}+\mathrm{H}_{2} \mathrm{O}+h v \longrightarrow \mathrm{Fe}^{+2}+\mathrm{H}^{+}+{ }^{\circ} \mathrm{OH} \\
& \mathrm{Fe}^{+3}\left(\mathrm{RCO}_{2}\right)^{+3}+h v \longrightarrow \mathrm{Fe}^{+2}+\mathrm{CO}_{2}+\mathrm{R}^{\cdot} \\
& \mathrm{H}_{2} \mathrm{O}_{2}+h v \longrightarrow 2{ }^{\circ} \mathrm{OH}
\end{aligned}
$$

A avaliação da ação dos POA no aumento da biodegradabilidade e na formação de intermediários tóxicos em efluentes líquidos pode ser realizada pela avaliação de testes de toxicidade utilizando bactérias, tais como a Aliivibrio fischeri. O princípio dos ensaios de toxicidade consiste em expor organismos-teste representativos no ambiente a várias concentrações de uma ou mais substâncias, durante determinado período de tempo, para avaliação de seu potencial tóxico, sendo os efeitos detectados através de respostas nos organismos (CETESB, 1990).

O presente estudo tem como objetivo avaliar o desempenho da aplicação dos Processos Fenton-escuro e Fenton-solar em lixiviados oriundos de dois aterros sanitários do Estado do Rio de Janeiro (Aterro Metropolitano de Gramacho e Aterro de Gericinó).

\section{MATERIAL E MÉTODOS}

\subsection{Breve descrição dos aterros geradores de lixiviados do presente estudo}

O Aterro Metropolitano de Gramacho situa-se em Duque de Caxias, município do Estado do Rio de Janeiro e ocupa uma área de $1,3 \mathrm{~km}^{2}$, margeando a Baía da Guanabara. Segundo Campos et al. (2013), o aterro operou de 1978 a 2012 e recebeu uma média de 9.500 ton dia $^{-1}$ de resíduos urbanos da Região Metropolitana do Rio de Janeiro. Ainda segundo os autores, no início da sua operação, a área era um lixão e recebia resíduos industriais; a partir de 1995, a área foi recuperada e desde então foi operado como aterro sanitário, recebendo apenas resíduos urbanos.

O Aterro Sanitário de Gericinó, no bairro de Bangu, RJ, foi constituído para as atividades de disposição de resíduos sólidos de origem residencial, comercial e resíduo de varrição pública e recebe em torno de 2.000 ton de resíduos diariamente e opera desde 1985. 
Ambos aterros são considerados antigos, pois possuem mais de 25 de operação, entretanto, o Aterro de Gramacho encontra-se desativado e o de Gericinó, ainda em operação.

As amostras de ambos lixiviados foram trazidas ao laboratório e mantidas sob refrigeração até o momento do processamento e análise.

\subsection{Processo Fenton-escuro}

Os experimentos do processo Fenton-escuro foram realizados em escala de bancada. Foram tratados $500 \mathrm{~mL}$ de amostra de lixiviado. A reação se iniciou com a adição de sulfato ferroso e $\mathrm{H}_{2} \mathrm{O}_{2}$. A concentração de peróxido de hidrogênio foi estabelecida com base na DQO inicial das amostras ( 1 de $\mathrm{H}_{2} \mathrm{O}_{2}$ para 1 de DQO) e a reação de decomposição do peróxido, considerando o oxigênio produzido equivale à demanda de oxigênio da amostra. A concentração de sulfato ferroso foi determinada de acordo com a dosagem de peróxido de hidrogênio, de forma a atender às razões mássicas $\mathrm{Fe}^{+2}: \mathrm{H}_{2} \mathrm{O}_{2}$ de 1:2, 1:5 e 1:10. Após adição de peróxido e sulfato ferroso $\mathrm{o}$ $\mathrm{pH}$ foi ajustado com adição de $\mathrm{H}_{2} \mathrm{SO}_{4}\left(1 \mathrm{~mol} \mathrm{~L}^{-1}\right)$ para os valores de 3 e 5 . Após 60 minutos, a reação foi interrompida com o ajuste de $\mathrm{pH}$ para 7,5 com adição de $\mathrm{NaOH}$ (50\%), ocorrendo assim a precipitação dos íons férricos. Os ensaios foram realizados com solução aquosa de sulfato ferroso heptahidratado P.A (Merck) e peróxido de hidrogênio 50\% v/v (Peróxidos do Brasil).

\subsection{Processo Foto-Fenton solar}

Os experimentos do processo Foto-Fenton solar foram realizados em uma unidade piloto acoplados a um reator solar de filme descendente, instalada na Universidade Federal do Rio de Janeiro (Figuras 1a e b). A composição da unidade piloto consiste em uma placa de polietileno com $1,37 \mathrm{~m}$ de comprimento por $0,82 \mathrm{~m}$ de largura e espessura de $0,01 \mathrm{~m}$. As tubulações são de meia polegada com 52 pontos de distribuição, calha receptora, bomba centrífuga modelo SHP - 35,05 V (0,37 kW), três tanques de tratamento: TQI (tanque alimentação, $20 \mathrm{~L}$ de capacidade), TQII (tanque de mistura com agitação mecânica, 20L), TQIII (tanque de armazenamento do efluente tratado, 50 litros de capacidade). A amostra de lixiviado foi colocada no TQ1 e com o auxílio de uma bomba centrifuga direcionada ao TQII, onde eram adicionados os reagentes. Após a passagem pela placa de polietileno (Figura 1b) e contato com o sol, a amostra era recirculada para o TQII. Ao final do tempo pré-estabelecido, coletava-se o lixiviado tratado no tanque TQIII.

A solução de $\mathrm{H}_{2} \mathrm{O}_{2}$ foi adicionada no TQII utilizando uma bomba peristáltica com vazão pré-determinada de forma a atender as concentrações desejadas. Após 60

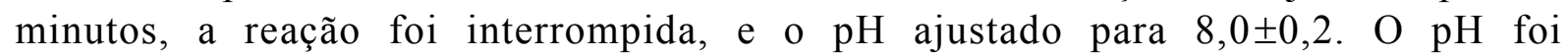
monitorado a cada minuto por medidor portátil Tecnopon, Modelo MPA 210P. A irradiação solar foi medida por um radiômetro KIMO, modelo SL100, durante toda a reação. As alíquotas do efluente tratado foram coletadas em 20, 40 e 60 minutos durante a reação.

Tal como no processo Fenton-escuro, a concentração de peróxido de hidrogênio foi estabelecida com base na DQO inicial das amostras, atendendo uma relação 1:1, da mesma maneira anterior. A concentração de sulfato ferroso foi determinada de acordo com a dosagem de peróxido de hidrogênio, de forma a atender as razões estudadas.

Todos os ensaios foram realizados em dias de sol e céu claro, e no horário de maior incidência de radiação, entre as $12 \mathrm{~h} 00$ e $14 \mathrm{~h} 00$, no período de agosto a outubro de 2013. A energia média acumulada foi medida em todos os experimentos de acordo com método baseado em Malato et al. (2002). 

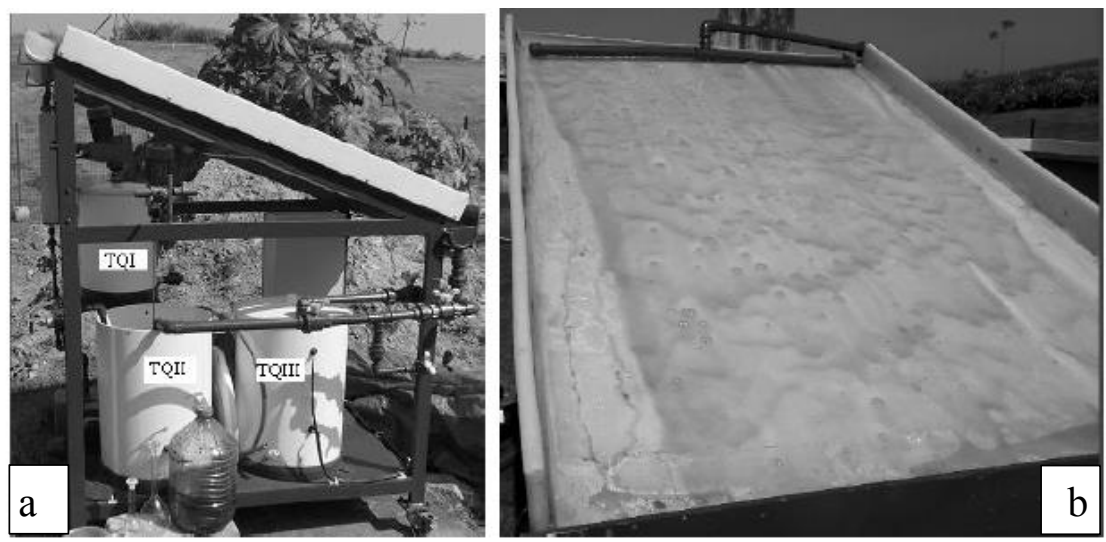

Figura 1. Unidade piloto solar. (a) detalhamento dos tanques (b) detalhamento do reator, tipo filme descendente.

Fonte: Freire (2012).

\subsection{Metodologia analítica}

\subsubsection{Análises físico-químicas}

A DQO foi determinada pelo método colorimétrico, descrito pela AWWA (APHA et al., 2005), refluxo fechado. Foi utilizado para a digestão das amostras o reator digital de DQO marca Hach, modelo DRB20025, e um espectrofotômetro marca Hach, modelo DR-2800 para a leitura da absorvância, após a etapa da digestão.

A concentração residual de peróxido de hidrogênio interfere na determinação da DQO, assim, é necessário quantificar o peróxido de hidrogênio residual e subtrair a contribuição de DQO relativa ao peróxido ao valor final de DQO. O peróxido de hidrogênio residual foi determinado de acordo com o procedimento baseado na reação entre o peróxido de hidrogênio e o íon metavanadato $\left(\mathrm{VO}^{-3}\right)$ (Oliveira et al., 2001).

\subsubsection{Toxicidade com Allivibrio fischeri}

O procedimento para a realização do ensaio foi realizado através Norma Técnica NBR 15411-3 (ABNT, 2012). Os resultados foram expressos em coeficiente de efeito a 50\% (CE 50\%). Os valores de CE50\% acima de 50\% foram considerados com baixa toxicidade para esse trabalho, seguindo recomendação de procedimento de TRIBEL (2002).

\section{RESULTADOS E DISCUSSÃO}

\subsection{Processo Fenton-Escuro}

$\mathrm{Na}$ Figura 2 são apresentados os resultados de DQO dos lixiviados dos aterros de Gericinó e Gramacho, respectivamente, após o tratamento com Processo Fenton-escuro. Para ambos os lixiviados, o $\mathrm{pH}$ igual a 3 produziu melhores resultados em 60 minutos de reação: as amostras do lixiviado de Gericinó tratadas por processo Fenton apresentaram eficiência de remoção de 49 a $56 \%$ enquanto para o lixiviado de Gramacho, foram obtidas remoções de 16 a $19 \%$ de DQO. A razão $\mathrm{Fe}^{+2}: \mathrm{H}_{2} \mathrm{O}_{2}$ igual a 1:5 foi a condição que gerou melhores resultados nestes experimentos para ambos os lixiviados

Sendo assim, em ambos lixiviados, o Fenton-escuro obteve melhor desempenho em pH igual 3 e razão $\mathrm{Fe}^{+2}: \mathrm{H}_{2} \mathrm{O}_{2}$ igual a $1: 5$, gerando resultados de eficiências de remoção de $56 \%$ e $19 \%$, respectivamente para os lixiviados de Gericinó e Gramacho. 
Campos et al. (2013) relataram que o lixiviado de Gramacho é de difícil tratabilidade devido à deposição de resíduos industriais no início de sua operação. Esse fato explica porque o Processo Fenton não foi tão efetivo para a sua redução de DQO, quanto foi para o outro lixiviado. Além disso, os mesmos autores destacam que o lixiviado de Gramacho apresenta elevadas concentrações de alcalinidade e cloreto, que podem agir interferindo na reação de Fenton, pois agem como "sequestrantes" de radicais hidroxila (Lin et al., 1999).

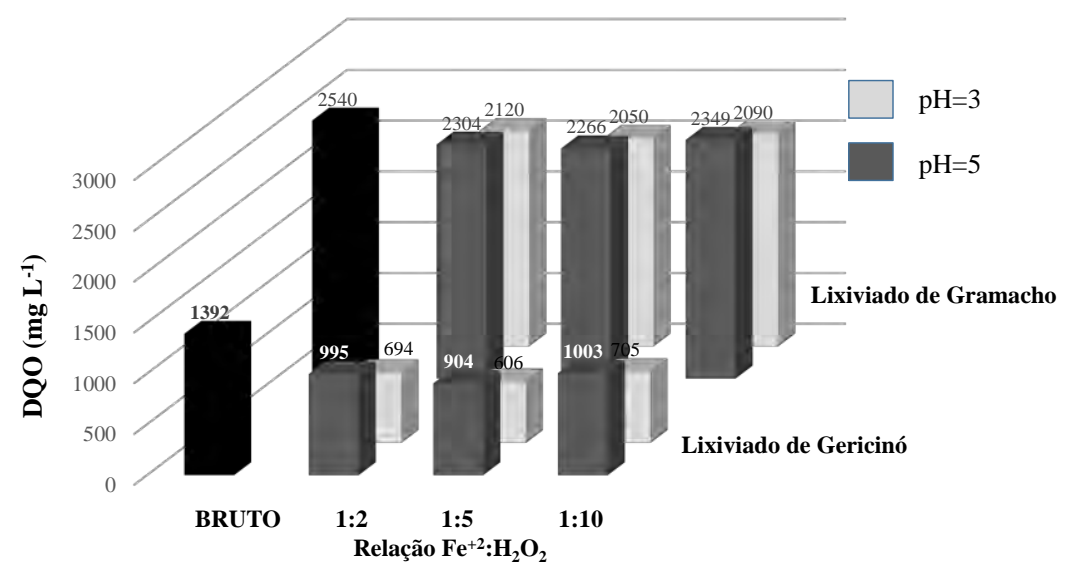

Figura 2. Resultados de DQO dos lixiviados de Gericinó e Gramacho bruto e tratados por processo Fenton -Escuro em diferentes razões $\mathrm{Fe}^{+2}: \mathrm{H}_{2} \mathrm{O}_{2}$. Condições: $\mathrm{H}_{2} \mathrm{O}_{2 \text { GERICINÓ }}=2958$ $\mathrm{mg} \mathrm{L}^{-1} ; \mathrm{H}_{2} \mathrm{O}_{2 \mathrm{GRAMACHO}}=5398 \mathrm{mg} \mathrm{L}^{-1}$ e tempo de reação= $=60 \mathrm{~min}$.

O tempo de reação foi avaliado nessa condição que obteve os melhores resultados, como ilustrado na Figura 3.

Em até 20 minutos, a reação se realizou, não observando remoções adicionais significativas nos tempos posteriores. No processo Fenton, a queda inicial de DQO é mais rápida, em que tem-se uma maior disponibilidade dos íons $\mathrm{Fe}^{2+}$ para catalisar a decomposição do $\mathrm{H}_{2} \mathrm{O}_{2}$ em radicais hidroxilas (reação 1). Após um determinado período de reação, os íons ferrosos $\left(\mathrm{Fe}^{+2}\right)$ são oxidados a íons férricos $\left(\mathrm{Fe}^{+3}\right)$, formando complexos que podem levar a interrupção da reação de Fenton.

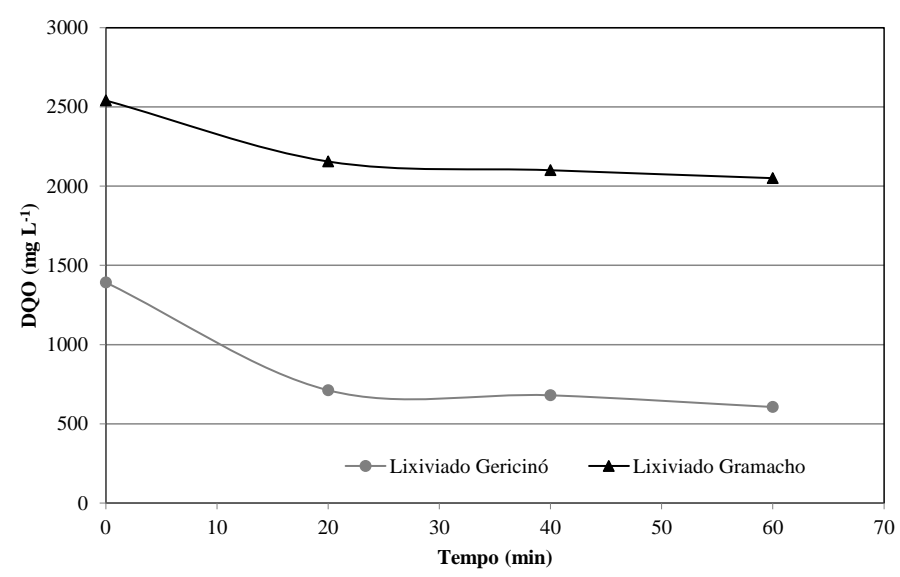

Figura 3. Resultados de DQO com o tempo de reação dos lixiviados de Gericinó e Gramacho tratados por processo Fenton-Escuro. Condições: $\mathrm{pH}$ 3,0, razão mássica $\mathrm{Fe}^{+2}: \mathrm{H}_{2} \mathrm{O}_{2}=1: 5 \mathrm{e} \mathrm{H}_{2} \mathrm{O}_{2 \text { GERICINÓ }}=2958 \mathrm{mg} \mathrm{L}^{-1} \mathrm{e}$ $\mathrm{H}_{2} \mathrm{O}_{2 \mathrm{GRAMACHO}}=5398 \mathrm{mg} \mathrm{L}^{-1}$. 


\subsection{Processo Fenton-Solar}

$\mathrm{Na}$ Figura 4 são apresentados os resultados de DQO dos lixiviados dos aterros de Gericinó e Gramacho, respectivamente, após o tratamento com o Processo Fenton-solar.

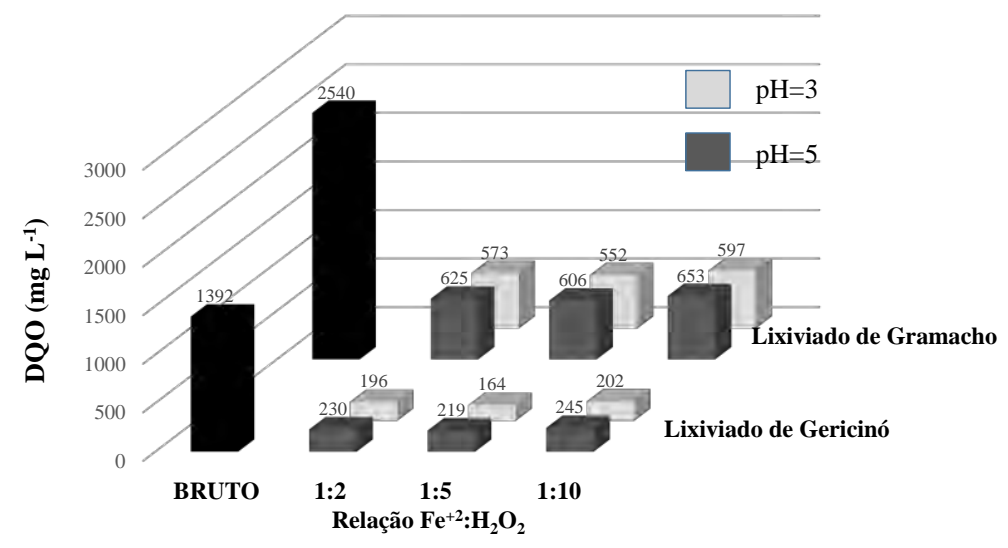

Figura 4. Resultados de DQO dos lixiviados de Gericinó e Gramachado bruto e tratados por processo Fenton-Solar em diferentes razões $\mathrm{Fe}^{+2}: \mathrm{H}_{2} \mathrm{O}_{2}$. Condições: $\quad \mathrm{H}_{2} \mathrm{O}_{2 \text { GERICINÓ }}=2958 \mathrm{mg} \mathrm{L}^{-1} \mathrm{e}$ $\mathrm{H}_{2} \mathrm{O}_{2 \text { GRAMACHO }}=5398 \mathrm{mg} \mathrm{L}^{-1}$ e tempo de reação $=60 \mathrm{~min}$.

Como pode ser verificado, o processo Fenton-solar mostrou-se bastante efetivo na redução na DQO dos lixiviados estudados em diferentes condições experimentais. As mesmas condições que geraram os melhores desempenhos para o Fenton-escuro também foram as melhores para o Fenton-solar $\left(\mathrm{Fe}^{+2}: \mathrm{H}_{2} \mathrm{O}_{2}=1: 5 \mathrm{e} \mathrm{pH}=3\right)$. Nestas condições, os resultados de eficiência de remoção para a DQO foram de $88,2 \%$ e $78,2 \%$, respectivamente, para os lixiviados de Gericinó e Gramacho. Sendo assim, foi avaliado o tempo de reação nestas condições, conforme ilustrado na Figura 5.

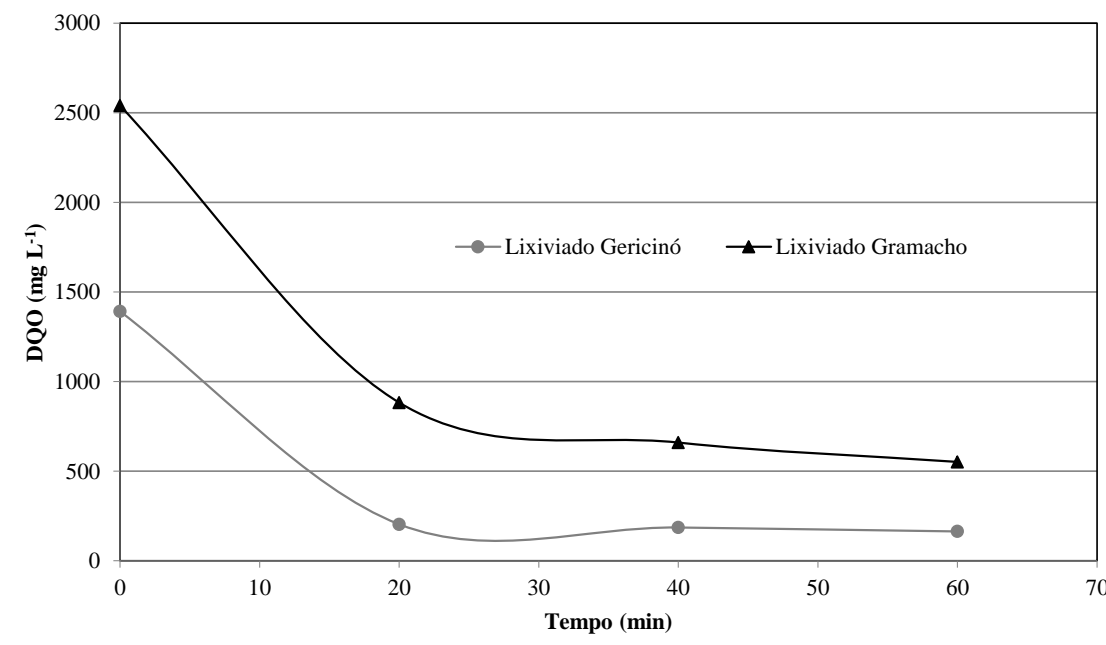

Figura 5. Resultados de DQO com o tempo de reação dos lixiviados de Gericinó e Gramacho tratados por processo Fenton-solar. Condições: $\mathrm{pH} 3,0$, razão mássica $\mathrm{Fe}^{+2}: \mathrm{H}_{2} \mathrm{O}_{2}=1: 5$ e $\mathrm{H}_{2} \mathrm{O}_{2 \text { GERICINÓ }}=2958 \mathrm{mg} \mathrm{L}^{-1}$ e $\mathrm{H}_{2} \mathrm{O}_{2 \text { GRAMACHO }}=5398 \mathrm{mg} \mathrm{L}^{-1}$. 
Da mesma maneira que o Fenton-escuro, houve a maior remoção de DQO nos primeiros 20 minutos de reação. A incidência da radiação UV sobre os complexos férricos favorece a regeneração de novos íons ferrosos, que continuam com o processo de decomposição do $\mathrm{H}_{2} \mathrm{O}_{2}$ (Araujo et al., 2011; Primo et al., 2008). A geração de mais radicais hidroxilas que oxidam a matéria orgânica, reação (3), favorecem a reação, enquanto que no processo Fenton-escuro ocorre a formação de substâncias estáveis complexadas pelos íons férricos, interrompendo rapidamente a reação. O que provavelmente levou a melhores resultados para a remoção da matéria orgânica nos dois lixiviados tratados no presente estudo pelo processo Fenton-solar. A energia média acumulada medida em todos os experimentos foi $643 \mathrm{~kJ} \mathrm{~L}^{-1}$.

\subsection{Avaliação de toxicidade com bactéria Allivibrio fischeri}

A Tabela 1 apresenta os resultados de toxicidade aguda com Allivibrio fischeri para as amostras brutas e tratadas por processo Fenton. Os valores indicam o percentual de cepas bioluminescentes remanescentes após contato com a amostra.

Verifica-se que o processo Fenton foi efetivo para diminuir a toxicidade do lixiviado às cepas bioluminescentes, o que pode indicar que um processo biológico de tratamento posterior seja possível. Comparando os dois lixiviados, o valor de toxicidade é maior para o lixiviado de Gramacho, tanto bruto quanto tratado por POA. Esses resultados são corroborados por Campos et al. (2013), que relataram a presença de DQO mais recalcitrante no lixiviado de Gramacho, o que dificulta a ação oxidativa do processo Fenton.

Tabela 1. Toxicidade CE $50 \%$ para as amostras do lixiviado de Gericinó e Gramacho. Condições: pH 3,0, razão mássica $\mathrm{Fe}^{+2}: \mathrm{H}_{2} \mathrm{O}_{2}=1: 5$ e $\mathrm{H}_{2} \mathrm{O}_{2 \text { GERICINó }}=2958 \mathrm{mg} \mathrm{L}^{-1} \mathrm{e}$ $\mathrm{H}_{2} \mathrm{O}_{2 \text { GRAMACHO }}=5398 \mathrm{mg} \mathrm{L}^{-1}$.

\begin{tabular}{lccc}
\hline & $\begin{array}{c}\text { Toxicidade CE 50\% } \\
\text { Amostra bruta (\%) }\end{array}$ & $\begin{array}{c}\text { Toxicidade CE 50\% } \\
\text { Fenton escuro (\%) }\end{array}$ & $\begin{array}{c}\text { Toxicidade CE 50\% } \\
\text { Fenton solar (\%) }\end{array}$ \\
\hline Lixiviado de Gericinó & 27 & 76 & 79 \\
Lixiviado de Gramacho & 13 & 54 & 59 \\
\hline
\end{tabular}

Ao contrário da DQO, não houve diferença de eficiência para os processos Fenton escuro e solar, para ambos lixiviados. No entanto, ambos processos só mostraram efeito de remoção de toxicidade no tempo de 60 minutos para os dois lixiviados. As alíquotas das amostras que foram retiradas em períodos intermediários, em 20 e 40 minutos, não demostraram eficiência no tratamento, mantendo a toxicidade dos compostos presentes nas amostras (resultados não apresentados).

\section{CONCLUSÕES}

Os resultados obtidos neste estudo revelaram que os processos Fenton e Foto Fenton solar são promissores no tratamento dos lixiviados dos aterros em estudo. Foi possível observar a redução da toxicidade com Allivibrio fischeri, o que indica que um tratamento biológico posterior seja aplicável e da DQO nas amostras de lixiviados tratadas dos aterros de Gericinó e Gramacho nas condições de pH igual a 3,0 , razão mássica $\mathrm{Fe}^{+2}: \mathrm{H}_{2} \mathrm{O}_{2}=1: 5$ e 60 min de reação.

Embora os processos avaliados tenham apresentado boas eficiências de tratamento, comparativamente o processo Foto-Fenton se mostrou mais eficiente na remoção de DQO e toxicidade, indicando a importância da radiação UV solar na 
degradação da matéria orgânica recalcitrante e tóxica presente nos lixiviados estudados.

\section{AGRADECIMENTOS}

Apoio financeiro da FAPERJ (Fundação Carlos Chagas Filho de Amparo à Pesquisa do Estado do Rio de Janeiro) e CNPq (Conselho Nacional de Desenvolvimento Científico e Tecnológico). À Haztec Ambiental, unidade Belford Roxo, RJ, pela realização dos ensaios de toxicidade e à COMLURB (Companhia de Limpeza Urbana do Município do Rio de Janeiro) pelo envio de amostras de lixiviados.

\section{REFERÊNCIAS}

ASSOCIAÇÃO BRASILEIRA DE NORMAS TÉCNICAS - ABNT. NBR 15411-3. Determinação do efeito inibitório de amostras de água sobre a emissão de luz de Aliivibrio fischeri: método com utilização de bactérias liofilizadas. Rio de Janeiro, 2012.

AMERICAN PUBLIC HEALTH ASSOCIATION - APHA; AMERICAN WATER WORKS ASSOCIATION - AWWA; WATER ENVIRONMENT FEDERATION - WEF. Standard methods for the examination of water and wastewater. $21^{\text {th }}$ ed. Washington, DC, 2005.

ARAUJO, F. V. F.; YOKOYAMA, L.; TEIXEIRA, L. A. C.; CAMPOS, J. C. Heterogeneous fenton process using the mineral hematite for the discolouration of a reactive dye solution. Brazilian Journal of Chemical Engineering, v. 28, p. 605-616, 2011. http://dx.doi.org/10.1590/S0104-66322011000400006

CAMPOS, J. C.; MOURA, D.; COSTA, A. P.; YOKOYAMA, L.; ARAUJO, F. V. F.; CAMMAROTA, M. C. et al. Evaluation of $\mathrm{pH}$, alkalinity and temperature during air stripping process for ammonia removal from landfill leachate. Journal of Environmental Science and Health, Part A: Toxic/Hazardous Substances and Environmental Engineering, v. 48, n. 9, p. 1105-1113, 2013. http://dx.doi.org/10.1080/10934529.2013.774658

COMPANHIA DE TECNOLOGIA DE SANEAMENTO AMBIENTAL - CETESB. Procedimentos para utilização de testes de toxicidade no controle de efluentes líquidos. São Paulo, 1990. 17p.

FREIRE, L.F.A. Estudo do sistema Fenton-Solar aplicado a remoção de fenol, 2012. 94f. Dissertação (Mestrado em Tecnologia de Processos Químicos e Bioquímicos) - Escola de Química - UFRJ, Rio de Janeiro, 2012.

GOTVAJN, A. Z.; TISLER, T.; KONCAN, J. Z. Comparison of different treatment strategies for industrial landfill leachate. Journal of Hazardous Materials, v. 162, p. 1446 - 1456, 2009. http://dx.doi.org/10.1016/j.jhazmat.2008.06.037

HASSEMER, M. E. N. Oxidação fotoquímica - UV/ $\mathbf{H}_{2} \mathbf{O}_{2}$ - para degradação de poluentes em efluentes da indústria têxtil. 2006. 175f. Tese (Doutorado em Engenharia Ambiental) - UFSC, Florianópolis, 2006. 
KUNZ, A.; PERALTA-ZAMORA, P.; MORAES, S. G.; DURÁN, N. Novas tendências no tratamento de efluentes têxteis. Química Nova, v. 25, n. 1, p. 78 82, 2002. http://dx.doi.org/10.1590/S0100-40422002000100014

LIN, SHENG H.; LIN, C. M.; LEU, H. G. Operating characteristics and kinetics studies of surfactant wastewater treatment by Fenton oxidation. Water Research, v. 33, n. 7, p. 1735-1741, 1999. http://dx.doi.org/10.1016/S00431354(98)00403-5

MALATO, S.; BLANCO, J.; VIDAL, A.; RICHTER, C. Photocatalysis with solar energy at a pilot- plant scale: An overview. Applied Catalysis B: Environmental, v. 37, n. 1, p. 1-15, 2002. http://dx.doi.org/10.1016/S09263373(01)00315-0

OLIVEIRA, M. C.; NOGUEIRA, R. F. P.; GOMES NETO, J. A.; JARDIM, W. F.; ROHWEDDER, J. J. R. Sistema de injeção em fluxo espectrofotométrico para monitorar peróxido de hidrogênio residual em processo de fotodegradação por reação foto-Fenton. Química Nova, v. 24, n. 2, p. 188 - 190, 2001. http://dx.doi.org/10.1590/S0100-40422001000200007

PIGNATELlO, J. J. Dark and photoassisted $\mathrm{Fe}^{3+}$ - catalysed degradation of chlorophenoxy herbicides by hydrogen peroxide. Environmental Science \& Technology, v. 26, n. 5, p. 944-951, 1992. http://dx.doi.org/10.1021/es00029a012

PRIMO, O.; RIVERO, M. J.; ORTIZ, I. Photo-Fenton process as an efficient alternative to the treatment of landfill leachates. Journal of Hazardous Materials, v. 153, n. 1-2, p. 834-842, 2008. http://dx.doi.org/10.1016/j.jhazmat.2007.09.053

SUN, J. H.; SUN, S. P.; WANG, G. L.; QIAO, L-P. Degradation of azo dye amido black $10 \mathrm{~B}$ in aqueous solution by Fenton oxidation process. Dyes and Pigments, v.74, 647-652, 2007. http://dx.doi.org/10.1016/j.dyepig.2006.04.006

TELL, A. L.; WARBERG, C. R.; ATKINSON, D. A.; WATTS, R. J. Comparison of mineral and soluble iron Fenton catalysts for the treatment of trichloroetilene. Water Research, v. 35, n. 4, p. 977-984, 2001.

http://dx.doi.org/10.1016/S0043-1354(00)00332-8

TRATAMENTO DE RESÍDUOS INDUSTRIAIS DE BELFORD ROXO - TRIBEL. Procedimento TRLAB-MA-02. Determinação da toxicidade aguda de substâncias químicas e efluentes para bactérias marinhas Vibrio fischeri. Belford Roxo, 2002. 


Ambiente \& Água - An Interdisciplinary Journal of Applied Science
ISSN 1980-993X - doi:10.4136/1980-993X
www.ambi-agua.net
E-mail: ambi-agua@agro.unitau.br

\title{
Uso de biomarcadores para monitoramento das águas do Córrego Arara no município de Rio Brilhante, MS, Brasil
}

\author{
doi: 10.4136/ambi-agua.1500
}

Received: 12 Sep. 2014; Accepted: 27 Nov. 2014

\author{
Elisangela Bortoluci Maceda ${ }^{1 *}$; Alexéia Barufatti Grisolia ${ }^{1}$; \\ Jussara Oliveira Vaini ${ }^{2}$; Liliam Silvia Candido ${ }^{1}$ \\ Universidade Federal da Grande Dourados (UFGD), Dourados, MS, Brasil \\ ${ }^{\mathbf{1}}$ Faculdade de Ciências Biológicas e Ambientais \\ ${ }^{2}$ Faculdade de Ciências Exatas e Tecnológicas \\ *Autor correspondente: e-mail: elisangelabmac@gmail.com, \\ alexeiagrisolia@ufgd.edu.br,jussaravaini@hotmail.com, \\ liliamcandido@ufgd.edu.br
}

\section{RESUMO}

O objetivo deste trabalho foi avaliar a mutagenicidade, genotoxicidade e citotoxicidade de amostras de água provenientes do Córrego Arara no município de Rio Brilhante, MS, Brasil, por meio de testes toxicológicos utilizando biomarcadores. Para avaliar a mutagenicidade utilizou-se o teste de micronúcleo, para a genotoxicidade o ensaio cometa, ambos com Astyanax altiparanae, e para analisar a citotoxicidade utilizou-se o teste de Allium cepa. As coletas foram realizadas nos meses de abril e junho de 2013, em três pontos distintos (pontos 1, 2 e 3) do Córrego Arara e no Tanque de Irrigação da UFGD (ponto 4). Os resultados indicaram aumento significativo na frequência de micronúcleos pisceos na coleta do mês de junho quando comparada com a realizada em abril. No teste de Allium cepa, o efeito citotóxico apresentou-se significativamente maior na coleta de abril, porém a genotoxicidade das águas não apresentou diferença estatística entre os meses de coleta. $\mathrm{O}$ maior número de danos no ensaio cometa ocorreu no ponto 4 , em ambos períodos de coleta. A análise química da água indicou níveis de cádmio, cromo, cobre, chumbo e níquel, com valores superiores ao permitido pela legislação vigente. A presença de metais acima dos níveis permitidos nas amostras de água analisadas do Córrego Arara indica possíveis potenciais mutagênicos, genotóxicos e citotóxicos aos organismos devido a esses poluentes derivados ou não de ações antrópicas. Assim sendo, este trabalho pode contribuir como ferramenta adicional ao controle da qualidade da água do Córrego Arara, considerando sua utilização e importância para a região.

Palavras-chave: ambiente aquático, anormalidade cromossômica, contaminação.

\section{Use of biomarkers for monitoring of waters from the Arara Stream in the city of Rio Brilhante, MS, Brazil}

\section{ABSTRACT}

This study evaluated the mutagenicity, genotoxicity and cytotoxicity of water samples from the Arara Stream in the city of Rio Brilhante, MS, Brazil, by means of toxicological 
tests using biomarkers. The micronuclei test was used to evaluate mutagenicity, and the comet assay was used to evaluate genotoxicity; both tests used Astyanax altiparanae. The Allium cepa test was used to analyze cytotoxicity. The collections were performed in April and June, 2013, at three different points (points 1,2 and 3) of the Arara Stream and at the Irrigation Tank of UFGD (point 4). The results indicated greater frequency of piscine micronucleus in the June collection than in that of April. In the Allium cepa test, the cytotoxic effect was significantly greater in the collection of April, but the genotoxicity of water showed no statistical difference between the months of collection. The greatest amount of damage in the comet assay occurred at point 4 , in both collection periods. The chemical analyses revealed that the levels of cadmium, chromium, copper and nickel were higher than the values allowed by legislation. The presence of pollutants above the permitted level in the analyzed samples of the Arara Stream shows possible potential mutagenic, genotoxic and cytotoxic effects to the water bodies due to these pollutants which may or may not be caused by human action. This work may thus contribute an additional tool to the quality control of water of the Arara Stream, considering its use and importance to the city.

Keywords: aquatic environment, chromosomal abnormality, contamination.

\section{INTRODUÇÃO}

A contaminação dos ecossistemas aquáticos é problema crescente proveniente de ações antrópicas, em consequência de atividades inadequadas por liberação de efluentes domésticos, industriais ou agrícolas sem tratamento adequado, uso indiscriminado de substâncias químicas, lixo urbano e descarte indevido de materiais e resíduos (Gupta et al., 2014).

Algumas das substâncias químicas liberadas no ambiente aquático, como compostos orgânicos e metais pesados, são capazes de inibir a atividade celular ou alterar o material genético podendo comprometer a fisiologia e sobrevivência de organismos aquáticos, transmitindo esta contaminação aos níveis tróficos superiores, incluindo o ser humano (Bianchi et al., 2011).

Desta forma, o uso de peixes, como bioindicadores de contaminação aquática, é explicado pela resposta destes aos tóxicos de maneira similar aos grandes vertebrados (Scalon et al., 2010). Os peixes são capazes de sofrer bioacumulação, assim como os mamíferos, respondendo a agentes mutagênicos mesmo em baixas concentrações (Mansouri et al., 2012).

Segundo Sánchez-Galán et al. (1998), uma espécie de peixe considerada ideal, para estudos de genotoxicidade e mutagenicidade, necessita ser: distribuída em vários ecossistemas; sensível para detectar poluentes em baixas concentrações; e de fácil manuseio no laboratório. Assim sendo, o Astyanax sp (lambari) tem sido o biomarcador amplamente utilizado para estes testes, pois é comum, pequeno, detritívoro, possui considerável importância econômica e sensível para detectar poluentes em baixas concentrações. Este pode ser utilizado para identificar a ação de agentes genotóxicos por meio de danos cromossômicos, como os micronúcleos (Ribeiro et al., 2014).

O teste de micronúcleo em peixe desenvolvido inicialmente por Schmid (1975) é amplamente utilizado, pois é uma técnica vantajosa, simples, sensível e de baixo custo para detecção de danos mutagênicos causados em organismos aquáticos e diversos trabalhos avaliaram a frequência de anormalidades como parâmetro de genotoxicidade (Russo et al. 2004; Hoshina et al., 2008; Melo et al., 2014). A avaliação de danos no material genético de peixes pode ser avaliada também pelo ensaio cometa. Este ensaio apresenta diversas vantagens e características quando comparado a outros testes genotóxicos, pois exige menor número de células para a análise, é sensível à detecção de danos no material genético, mesmo em baixa quantidade, possui custo relativamente baixo, necessita de curto período de tempo para sua realização (Tice et al., 2000). 
O ensaio cometa pode ser utilizado para avaliar a potencialidade de água contaminada por agentes genotóxicos provenientes de efluentes industriais, domésticos e agrícolas de, induzir danos no DNA monitorar a qualidade da água (White e Rasmussen, 1998; Matsumoto et al., 2003), que podem resultar em mutações. Estes danos observados com o ensaio cometa, diferente das mutações, podem ser reparados (Gontijo e Tice, 2003).

Outro teste utilizado para avaliar a qualidade da água é o teste de Allium cepa, uma vez que ensaios utilizando modelos vegetais podem ser considerados mais simples quando comparado aos testes utilizando modelos animais (Radić et al., 2010). O teste de Allium cepa possui curta duração, baixo custo devido este organismo possuir cromossomos grandes e em pequena quantidade, deixando visível assim os danos cromossômicos e os distúrbios do ciclo mitótico (Rank e Nielsen, 1998; Alvim et al., 2011).

O município de Rio Brilhante, localizado no Estado do Mato Grosso do Sul, Brasil, caracteriza-se por representar região agropecuária, sucroalcooleira de grande importância no Estado. Está situado entre dois rios, Vacaria e Brilhante, pertencentes à Bacia Rio do Ivinhema, e possui diversos cursos d'água, sendo o seu principal, o Córrego Arara. As águas deste são utilizadas, principalmente, para irrigação de lavouras e dessedentação de animais. Também recebe efluentes da estação de tratamento de esgoto da cidade e apresenta degradação da sua mata ciliar original ao longo do percurso (IMAP/MS, 2006).

A avaliação da qualidade da água do Córrego Arara é necessária em decorrência da sua importância para o município, onde até o momento não há registros de pesquisas realizadas para este fim. Dessa forma, o objetivo deste trabalho foi avaliar a mutagenicidade, genotoxicidade e citotoxicidade dos elementos presentes na água em diferentes pontos do Córrego Arara no município de Rio Brilhante, MS, Brasil, por meio do teste de micronúcleo e ensaio cometa em Astyanax altiparanae, teste de Allium cepa e análise química da água. Assim sendo, espera-se que essa pesquisa ressalte a importância de adoção de planos de medidas de conservação dos mananciais de água que recebem efluentes decorrentes das diversas atividades humanas.

\section{MATERIAL E MÉTODOS}

\subsection{Local de coleta}

Amostras de água do Córrego Arara, situado na Bacia do Rio Paraná e pertencente ao município de Rio Brilhante, MS, Brasil, foram coletadas em meses de maior e menor regime de pluviosidade (abril e junho respectivamente) no ano de 2013 , em três pontos distintos (1,2 e 3), conforme ilustrado na Figura 1. O ponto 1 ( $\left.21^{\circ} 47^{\prime} 40.60^{\prime \prime S} 54^{\circ} 31^{\prime} 58.37^{\prime \prime O}\right)$ caracteriza-se por possuir resquícios de poluição decorrente da ação humana, como resíduos sólidos e compostos orgânicos, o ponto 2 (21 $\left.{ }^{\circ} 48^{\prime} 20.77^{\prime \prime S} 54^{\circ} 31^{\prime} 38.12^{\prime \prime O}\right)$ localiza-se 100 metros após o efluente da unidade de Estação de Tratamento de Esgoto (ETE) do município, estando próximo a propriedades agrícolas, e o ponto $3\left(21^{\circ} 48^{\prime} 45.32^{\prime \prime S} 54^{\circ} 31^{\prime} 44.60^{\prime \prime O}\right)$ caracteriza-se pela ausência de mata ciliar ao redor de suas margens e áreas de lavouras em suas proximidades. Também foi realizada a coleta de água subterrânea proveniente de poço artesiano no Tanque de Irrigação da Universidade Federal da Grande Dourados (UFGD), caracterizado como ponto 4, sendo utilizado para fins comparativos. Aproximadamente 20 litros de água foram coletados em cada ponto de coleta em profundidade de aproximadamente $10 \mathrm{~cm}$ da superfície do espelho d'água. As amostras coletadas foram armazenadas em galões de polietileno e encaminhadas para o Laboratório de Mutagênese da Faculdade de Ciências Biológicas e Ambientais (FCBA) da UFGD. 


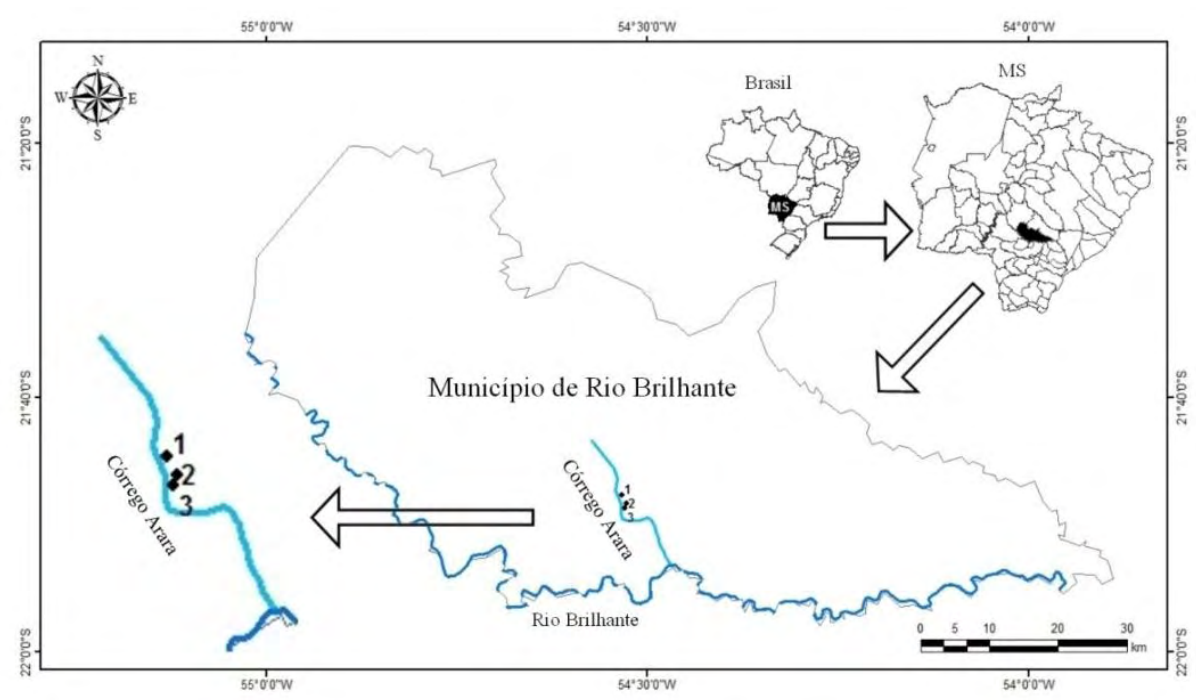

Figura 1. Mapa dos locais de coleta no Córrego Arara, município de Rio Brilhante, MS, Brasil. 1 (Ponto 1), 2 (Ponto 2), 3 (Ponto 3).

\subsection{Testes de mutagenicidade e genotoxicidade em animal}

As amostras de água coletadas foram acondicionadas em quatro aquários, sendo três deles para as amostras de águas superficiais provenientes do Córrego Arara (pontos 1, 2 e 3) e outro para a amostra de água subterrânea proveniente de poço artesiano no Tanque de irrigação da UFGD (ponto 4). O preparo consistiu em colocar a água coletada em aquários de vidro (40 × 30 × $20 \mathrm{~mm})$, devidamente aerados, no Laboratório de Mutagênese da FCBA.

Após este período, dez espécimes de Astyanax altiparanae, fornecidos por uma piscicultura do município de Dourados, MS, foram adicionados em cada aquário, onde ficaram expostos pelo período de 72-h, com aeração e em temperatura ambiente. Dentre esses, 5 espécimes foram utilizados para o ensaio do comenta e os outros 5 para o teste do micronúcleo.

\subsubsection{Teste de micronúcleo utilizando Astyanax altiparanae}

O teste do Micronúcleo Písceo foi realizado para verificar a frequência de micronúcleos empregando-se a técnica descrita por Schmid (1975), com modificações. Após 72-h cinco espécimes de exposição à agua do córrego nos aq uários Astyanax altiparanae foram retiradas de cada aquário e utilizados para o teste. Para isso, eles foram anestesiados em benzocaína $1 \%$, e em seguida colheu-se o sangue periférico retirado da veia caudal e procedeu-se com a extensão sanguínea em lâminas de microscopia. De cada peixe montou-se uma lâmina, que após secas foram fixadas em etanol $96 \%$ durante 30 minutos e coradas com Panótico Rápido. Para análise de presença ou ausência de micronúcleos foram observadas 2000 células de cada peixe, no aumento total de 400X em microscópio óptico (Bioval).

\subsubsection{Ensaio cometa utilizando Astyanax altiparanae}

Para o ensaio do cometa foram utilizados $6 \mathrm{~mL}$ de sangue, coletados por punção branquial e diluídos em $2000 \mu \mathrm{L}$ de solução salina (PBS). Duas lâminas de cada peixe foram confeccionadas com $20 \mu \mathrm{L}$ de suspensão celular e $120 \mu \mathrm{L}$ de agarose de baixo ponto de fusão $0,5 \%(\mathrm{v} / \mathrm{v})$ a $37^{\circ} \mathrm{C}$. As lâminas permaneceram em solução de lise, à $4{ }^{\circ} \mathrm{C}$, por 1 hora. Após a lise, as lâminas foram armazenadas em tampão $\mathrm{NaOH} 0,3 \mathrm{~mol} \mathrm{~L}^{-1}$ e EDTA $0,001 \mathrm{~mol} \mathrm{~L}^{-1}$ $(\mathrm{pH}>13)$ por 20 minutos e submetidas à eletroforese a $25 \mathrm{~V}, 300 \mathrm{~mA}$, por 20 minutos. Em seguida, foram neutralizadas com Tris $0,4 \mathrm{~mol} \mathrm{~L}^{-1}$ por 15 minutos, fixadas em etanol por 10 minutos e coradas com brometo de etídeo $\left(0,02 \mathrm{~mol} \mathrm{~L}^{-1}\right)$. Um total de 100 nucleóides de cada 
lâmina foi observado em microscópio de fluorescência (Labomed - T121100) na objetiva de 400x.

Para avaliação da genotoxicidade realizou o ensaio cometa adaptado da metodologia de Ventura et al. (2008), onde foram utilizados os cinco espécimes expostos por $72 \mathrm{~h}$ à agua do Córrego Arara de Astyanax altiparanae provenientes de cada aquário contendo as amostras dos quatro pontos analisados. Foram confeccionadas duas lâminas por peixe. Os procedimentos anestésicos foram similares aos anteriormente citados. Foram colhidos $6,0 \mu \mathrm{L}$ de sangue da região brânquial de cada animal e eluídos em 2,0 mL de PBS 1x. Dessa suspensão celular, 20,0 $\mu \mathrm{L}$ foram adicionados em 120,0 $\mu \mathrm{L}$ de agarose Low Melting de baixo ponto de fusão $(0,5 \%)$, a $37^{\circ} \mathrm{C}$. A suspensão foi aplicada sobre a lâmina de microscopia, previamente gelatinizada com agarose comum e identificada, coberta com lamínula e acondicionada sob-refrigeração durante 15 minutos. Em seguida, as lâminas foram transferidas para solução de lise final e mantidas por 1 hora na geladeira. Após a lise, as lâminas foram armazenadas em tampão $\mathrm{NaOH} 0,3 \mathrm{~mol} \mathrm{~L}^{-1}$ e EDTA $0,001 \mathrm{~mol} \mathrm{~L}^{-1}(\mathrm{pH}>13)$ por 20 minutos e submetidas à eletroforese a $25 \mathrm{~V}, 300 \mathrm{~mA}$, por 20 minutos. para desnaturação do DNA. As lâminas foram neutralizadas com Tris $0,4 \mathrm{M}$ por 15 minutos, fixadas em etanol $100 \%$ por 10 minutos e coradas com brometo de etídeo $\left(0,02 \mathrm{mg} \mathrm{mL}^{-1}\right)$ e em seguida observadas os nucleóides em microscópio de fluorescência Nikon, em aumento total de 200X. Para cada lâmina foram contados 100 nucleóides, e classificados de acordo com o seu grau de dano.

\subsection{Teste de citotoxicidade e genotoxicidade em vegetais}

\subsubsection{Teste de Allium cepa}

A citotoxicidade da água foi avaliada por meio do índice mitótico e a genotoxicidade através do índice de anormalidades cromossômicas, em radículas de Allium cepa, variedade Baia Periforme. As análises citogenéticas foram conduzidas de acordo com o procedimento descrito por Guerra e Souza (2002). Aproximadamente 15 sementes foram submetidas à germinação em placas de Petri com papel-filtro, contendo as amostras de água de cada ponto $(1,2,3$ e 4), sendo uma placa para cada ponto de estudo. Após $72 \mathrm{~h}$ de germinação, as sementes com as radículas foram fixadas em Carnoy 3:1 (etanol/ácido acético glacial) durante 6-h a temperatura ambiente e em seguida, transferidas para uma nova solução de Carnoy 3:1, mantendo-se as mesmas na geladeira até sua utilização. As radículas foram submetidas à hidrólise com ácido clorídrico $(\mathrm{HCl}) 1 \mathrm{~N}$ a $60{ }^{\circ} \mathrm{C}$ durante 10 minutos, lavadas com água destilada e coradas em Reativo de Schiff durante um período de 2-h, no escuro. Confeccionou-se 5 lâminas por ponto de cada coleta realizada, e a montagem das mesmas foi realizada por meio de esmagamento do meristema radicular. Um total de 1000 células por lâmina foram contadas e analisadas em microscópio óptico (Nikon Eclipse E200), no aumento total de $400 \mathrm{X}$.

\subsection{Análise química da água}

A análise química das amostras de água foi realizada no Laboratório de Espectrometria e Cromatografia Aplicada (LECA) da Faculdade de Ciências Exatas e Tecnologia (FACET) da UFGD. Para isso, coletou-se $1000 \mathrm{~mL}$ de água de cada ponto de coleta em frasco âmbar, e aproximadamente $0,5 \mathrm{~mL}$ de ácido nítrico foram acrescentadas (para deixar o $\mathrm{pH}$ da água inferior à 2,0). Posteriormente, as amostras de água foram filtradas e armazenadas sobre refrigeração. As análises foram realizadas por meio do método de Espectrometria de Absorção Atômica com Chama (FAAS - "Flame Atomic Absorption Spectrometry"). Os elementos químicos analisados foram: cádmio, cálcio, chumbo, cobalto, cobre, cromo, ferro, magnésio, manganês, níquel e zinco. 


\subsection{Análise estatística}

O número de micronúcleos $(\mathrm{MCN})$, proveniente do teste de micronúcleo e os índices mitóticos (IM) e índices de anormalidades cromossômicas (IAC) provenientes do teste de Allium cepa, foram submetidos à análise de variância (ANOVA) e posteriormente, as médias foram comparadas por meio do Teste Tukey $(p<0,05)$, sendo realizadas com auxílio do pacote computacional SAS ${ }^{\circledR}$ (SAS 9.1, SAS Institute, Cary, NC, USA). Os dados do ensaio cometa foram avaliados pelo teste qui-quadrado $\left(\chi^{2}\right)$, por meio do Programa BioEstat 4.0 (Ayres et al., 2005), a fim de se comparar o total de núcleos alterados de peixes expostos aos pontos de coleta.

\section{RESULTADOS E DISCUSSÃO}

Os resultados do teste de micronúcleo (MCN) em Astyanax altiparanae estão apresentados na Tabela 1. Na coleta realizada no mês de abril, não foi verificada diferença estatística na frequência de micronúcleos entre os pontos de coleta. Entretanto, na coleta realizada em junho, o ponto 2 apresentou maior número de micronúcleos, que os demais pontos, apesar da média não ter sido estatisticamente diferente dos pontos 1 e 3 . O ponto 4 foi o que apresentou menor média de micronúcleos.

Tabela 1. Médias \pm desvio padrão do teste de micronúcleo em Astyanax altiparanae e do teste de Allium cepa, observados nos dois períodos de coleta.

\begin{tabular}{|c|c|c|c|c|c|c|}
\hline & & & \multicolumn{4}{|c|}{ Allium cepa } \\
\hline & \multicolumn{2}{|c|}{ MCN } & \multicolumn{2}{|c|}{ IM } & \multicolumn{2}{|c|}{ IAC } \\
\hline & Abril & Junho & Abril & Junho & Abril & Junho \\
\hline Ponto 1 & $3,10 \pm 0,65^{a}$ & $3,70 \pm 1,03^{\mathrm{ab}}$ & $4,68 \pm 1,81^{\mathrm{b}}$ & $6,70 \pm 1,73^{a}$ & $0,20 \pm 0,24^{b}$ & $0,30 \pm 0,10^{\mathrm{a}}$ \\
\hline Ponto 2 & $2,70 \pm 0,57^{\mathrm{a}}$ & $4,50 \pm 1,17^{\mathrm{a}}$ & $8,74 \pm 3,00^{a b}$ & $4,90 \pm 0,97^{\mathrm{a}}$ & $1,86 \pm 0,69^{a}$ & $0,72 \pm 0,38^{\mathbf{a}}$ \\
\hline Ponto 3 & $1,50 \pm 1,06^{\mathrm{a}}$ & $3,00 \pm 0,93^{\mathrm{ab}}$ & $11,64 \pm 2,52^{a}$ & $4,28 \pm 0,68^{a}$ & $0,68 \pm 0,17^{b}$ & $0,88 \pm 0,38^{\mathbf{a}}$ \\
\hline Ponto 4 & $2,30 \pm 0,90^{\mathrm{a}}$ & $2,30 \pm 0,67^{b}$ & $8,94 \pm 4,58^{a b}$ & $6,20 \pm 1,08^{a}$ & $0,64 \pm 0,55^{b}$ & $0,60 \pm 0,48^{a}$ \\
\hline
\end{tabular}

Nota: MCN: Micronúcleo; IM: Índice Mitótico; IAC: Índice de Anormalidades Cromossômicas. MCN, IM e IAC seguida pela mesma letra minúscula em determinada coluna não difere estatisticamente pelo teste Tukey $(\mathrm{p}<0,05)$.

Provavelmente, o número de micronúcleos dos pontos 1 e 3 foram estatisticamente iguais ao ponto 2, devido a elevada pluviosidade $(263,2 \mathrm{~mm})$ do mês de junho no município de Rio Brilhante, MS (Embrapa, 2013), visto que tanto o ponto 1 quanto o ponto 3 apresentam resquícios de poluição decorrente da ação humana, seja por meio de resíduos (orgânicos ou inorgânicos) ou uso indiscriminado de defensivos agrícolas.

Em relação à citotoxicidade avaliada por meio do teste de Allium cepa, no mês abril foi verificado que o ponto 3 apresentou maior índice mitótico (IM), embora a média não tenha sido estatisticamente diferente das obtidas nos pontos 2 e 4 . O ponto 1 apresentou menor frequência de IM. Já para a coleta realizada em junho não foram verificadas diferenças significativas entre os pontos de coleta (Tabela 1).

Costa et al. (2008) apontam que não é possível identificar e determinar a toxicidade de determinadas substâncias presentes no meio, uma vez que seus efeitos podem ser influenciados por outras substâncias ou componentes, como propriedades químicas e físicas do ambiente, pluviosidade, temperatura, entre outros. Segundo Bollmann e Marques (2006), os danos celulares ocasionados podem ainda estar relacionados a outros fatores como, por exemplo, a contaminação do meio por agrotóxicos, poluentes atmosféricos, e materiais orgânicos e inorgânicos, provenientes basicamente do escoamento das águas da chuva. 
A avaliação do IAC no mês de abril revelou que o ponto 2 foi o que apresentou maiores danos cromossômicos, possivelmente devido à presença do efluente da Estação de Tratamento de Esgoto e seus componentes, o que resultou em maior taxa de danos. Esse resultado complementado pela análise química, sugere que os micronúcleos em Astyanax altiparanae podem ter sido induzidos pelas elevadas concentrações de cádmio e níquel $\left(0,087 \mathrm{mg} \mathrm{L}^{-1} \mathrm{e}\right.$ $0,600 \mathrm{mg} \mathrm{L}^{-1}$ respectivamente), que segundo Dias et al. (2003), estes metais presentes em efluentes domésticos em elevadas concentrações estão associados a danos em plantas e animais.

Para a genotoxicidade, não foi verificado diferença estatística para o índice de anormalidades cromossômicas (IAC) entre os pontos de coleta, no mês junho de 2013 (Tabela1).

Segundo Silva (2002), testes toxicológicos utilizando diferentes organismos vivos, como o teste de Allium cepa e teste de micronúcleo, podem apresentar respostas diferentes a um mesmo composto químico ou mistura, podendo este ser um fator para a divergência obtida entre os meses de coleta.

Na Tabela 2 estão apresentados os tipos de anormalidades cromossômicas encontradas nas células meristemáticas das raízes de Allium cepa. Foram observadas anáfases com pontes cromossômicas e com quebras cromossômicas, c-metáfases, intérfases com micronúcleos e telófases com pontes cromossômicas. Para Grant (1982) as anormalidades das células de Allium cepa são maneiras eficientes de investigar possíveis efeitos citotóxicos e genotóxicos causados por substâncias químicas em ambientes aquáticos contaminados, seja por efluentes industriais ou domésticos.

Tabela 2. Análise de anormalidades cromossômicas do teste de Allium cepa nos dois períodos de coleta (média \pm desvio padrão).

\begin{tabular}{|c|c|c|c|c|c|c|c|c|c|c|}
\hline & & & \multicolumn{4}{|c|}{ Anormalidades Cromossômicas } & & & & \\
\hline & \multicolumn{2}{|c|}{$\begin{array}{l}\text { Anáfase com ponte } \\
\text { cromossômica }\end{array}$} & \multicolumn{2}{|c|}{$\begin{array}{l}\text { Anáfase com quebra } \\
\text { cromossômica }\end{array}$} & \multicolumn{2}{|c|}{ C-metáfase } & \multicolumn{2}{|c|}{$\begin{array}{l}\text { Intérfase com } \\
\text { micronúcleo }\end{array}$} & \multicolumn{2}{|c|}{$\begin{array}{l}\text { Telófase com ponte } \\
\text { cromossômica }\end{array}$} \\
\hline & Abril & Junho & Abril & Junho & Abril & Junho & Abril & Junho & Abril & Junho \\
\hline Ponto 1 & $0,2 \pm 0,44$ & $2,8 \pm 1,09$ & $0,2 \pm 0,44$ & - & $0,2 \pm 0,44$ & - & - & - & - & $0,2 \pm 0,44$ \\
\hline Ponto 2 & $2,0 \pm 2,34$ & $4,0 \pm 2,34$ & $1,2 \pm 1,64$ & $0,2 \pm 0,44$ & $3,2 \pm 1,64$ & $1,8 \pm 1,64$ & $5,8 \pm 4,43$ & - & $2,0 \pm 1,58$ & $1,2 \pm 1,30$ \\
\hline Ponto 3 & $3,6 \pm 1,94$ & $1,0 \pm 1,00$ & $0,4 \pm 0,89$ & - & $1,8 \pm 1,09$ & $2,4 \pm 0,89$ & - & $3,4 \pm 2,07$ & $0,2 \pm 0,44$ & $2,0 \pm 0,70$ \\
\hline Ponto 4 & $0,4 \pm 0,89$ & $2,6 \pm 2,07$ & - & - & $0,8 \pm 0,83$ & $0,8 \pm 1,09$ & $1,4 \pm 1,67$ & $2,2 \pm 4,91$ & $1,6 \pm 1,34$ & $0,4 \pm 0,89$ \\
\hline
\end{tabular}

Na coleta do mês de abril, o maior número de anormalidades encontradas situam-se no ponto 2 (Tabela 2), com exceção das células que apresentaram anáfase com ponte cromossômica, sendo maior em junho. A quantidade de cromo observada no mês de abril, encontra-se superior no ponto 2, quando comparada aos demais pontos, explicando assim, a presença de células com c-metáfase. Matsumoto (2006) obteve resultado semelhante a esse trabalho, onde relatou a ação do cromo sobre o fuso mitótico, o principal causador de anormalidades cromossômicas do tipo c-metáfase. Segundo Sahi et al. (1998) o cromo tem potencial genotóxico, podendo induzir quebras e perdas cromossômicas, e resultar em anormalidades nas células dos organismos teste.

Os resultados do ensaio cometa estão apresentados na Tabela 3. Considerando o total de células analisadas com dano, foram constatadas diferenças estatisticamente significativas do ponto 1 em relação ao ponto 4 (tanque de irrigação da UFGD), em ambos os períodos de coleta (Tabela 3). Diferentemente dos demais testes, no ensaio cometa a maior frequência de danos no mês de abril ocorreu no ponto 1, enquanto no mês de junho ocorreu nos pontos 2 e 4. Isso possivelmente pode estar relacionado à natureza dos testes utilizados. Segundo 
Hartmann et al. (2001), o ensaio cometa apresenta-se mais sensível, quando comparado ao teste de micronúcleo, por detectar danos reversíveis no DNA.

Tabela 3. Médias das classes de dano obtidas através do ensaio cometa em Astyanax altiparanae em dois períodos de coleta.

\begin{tabular}{lllccccc}
\hline & \multicolumn{7}{c}{ Classes de Dano } \\
\cline { 3 - 8 } & & TCA & Classe 1 & Classe 2 & Classe 3 & Classe 4 & CS \\
\hline Abril & Ponto 1 & $8,8^{*}$ & 4,2 & 0,4 & 2,4 & 1,8 & 19,4 \\
& Ponto 2 & 17,2 & 8,0 & 6,4 & 2,0 & 0,8 & 30,0 \\
& Ponto 3 & $9,8^{*}$ & 6,0 & 1,6 & 1,2 & 1,0 & 16,8 \\
& Ponto 4 & 22,6 & 17,2 & 4,0 & 0,0 & 1,4 & 30,8 \\
\multirow{6}{*}{ Junho } & Ponto 1 & $17,4^{*}$ & 11,2 & 2,4 & 0,8 & 3,0 & 30,4 \\
& Ponto 2 & 23,6 & 4,0 & 5,2 & 8,2 & 6,2 & 63,8 \\
& Ponto 3 & $14,6^{*}$ & 7,0 & 5,4 & 0,8 & 1,4 & 25,8 \\
& Ponto 4 & 33,0 & 5,2 & 5,4 & 10,0 & 12,4 & 95,6 \\
\hline
\end{tabular}

Nota: TCA: Total de células analisadas com dano; CS: Pontuação do cometa. Classe 1= danos mínimos; Classe 2= danos médios; Classe 3= danos intensos; Classe $4=$ danos máximos. "Significativamente diferente em relação ao ponto 4 , por meio do teste $\chi^{2} \operatorname{com} p<0,05$.

Os resultados do ensaio do cometa estão apresentados na Tabela 3. Considerando o total de células analisadas com dano, foram constatadas diferenças estatisticamente significativas dos pontos 1 e 3 em relação ao ponto 4 (tanque de irrigação da UFGD), em ambos os períodos de coleta (Tabela 3). A maior frequência de danos, nos dois períodos de coleta foi verificada no ponto 4 .

Deve-se considerar ainda que a água proveniente do tanque de irrigação da UFGD possui origem subterrânea e sua classificação é diferenciada dos demais pontos. A elevada quantidade de elementos químicos acima do permitido pode explicar o alto número de danos neste ponto, apresentados pelo ensaio cometa. Segundo Salvadori et al. (2003), os danos avaliados por este teste aparecem antes que no teste de micronúcleo, pois é possível visualizar o micronúcleo somente após um ou mais ciclos de divisão celular.

Os pontos de coleta 1 e 3 apresentaram menor quantidade de células danificadas, para ambos os períodos de coleta (Tabela 3). De acordo com Belpaeme et al. (1998), os danos apresentados no ensaio cometa dependem não somente da amostra a ser analisada, mas também dos agentes genotóxicos presentes na mesma, do período de exposição, da espécie de peixe a ser utilizada e das condições experimentais.

As diferenças apresentadas entre os pontos e meses de coleta podem estar relacionadas a diversos fatores aos quais as águas estão expostas. Segundo Handy et al. (2003), as diferentes respostas apresentadas no biomarcador podem ser influenciadas por fatores abióticos como temperatura, $\mathrm{pH}$ e oxigênio, e fatores bióticos como microrganismos, animais e vegetais presentes naquele ambiente.

Os resultados positivos apresentados em ambos períodos de coleta sugerem que a provável presença de agentes genotóxicos nas amostras analisadas podem ter influenciado na boa qualidade da água. Porém, estes agentes não necessariamente são representados por compostos químicos. Segundo Rojas et al. (1999), os danos analisados através do ensaio cometa podem ser formados por diversos mecanismos, entre eles Mitchelmore e Chipman (1998) citam além dos compostos químicos, a digestão do DNA durante o processo de apoptose.

Entre os elementos químicos analisados, as concentrações de cádmio, cromo, cobre, chumbo e níquel, apresentaram-se acima da concentração máxima $\left(0,01 \mathrm{mg} \mathrm{L}^{-1}, 0,05 \mathrm{mg} \mathrm{L}^{-1}\right.$, $0,013 \mathrm{mg} \mathrm{L}^{-1}, 0,033 \mathrm{mg} \mathrm{L}^{-1}$ e $0,025 \mathrm{mg} \mathrm{L}^{-1}$, respectivamente) permitida para classe 3 de água 
superficial, que são destinadas para abastecimento para consumo humano, após tratamento convencional ou avançado; à irrigação de culturas arbóreas, cerealíferas e forrageiras; à pesca amadora; à recreação de contato secundário; e à dessedentação de animais, de acordo com a Resolução $\mathrm{n}^{\circ}$ 357, de 17 de março de 2005, do CONAMA (Brasil, 2005), em todas as amostras analisadas do Córrego Arara, das coletas realizadas em abril e junho.

Os resultados obtidos no presente trabalho concordam com os dados obtidos por Cavas et al. (2005), em que observaram a formação de micronúcleos em peixes quando expostos a cádmio e cobre, indicando sua ação genotóxica para estes organismos. Tagliari et al. (2006) verificaram um aumento de danos no material genético em bioindicadores expostos a elevadas concentrações de cromo, como demonstrado em estudo através do teste de Allium cepa.

A maior concentração de chumbo foi verificada nos ponto 4 , para ambos períodos de coleta. Duffus (2002) propôs a capacidade deste metal se acumular no interior das células e consequentemente apresentar toxicidade às mesmas. Porém, sua toxicidade pode ser alterada em função do $\mathrm{pH}$ e dos teores de carbono presente no meio, formando complexos ou sendo adsorvidos (Baird e Cann, 2011).

Em relação ao níquel, Marques e Chierice (1993) demonstram que seu potencial genotóxico no ambiente aquático depende de fatores como $\mathrm{pH}$ e oxigênio dissolvido. Sua elevada concentração é ocasionada principalmente por efluentes domésticos, assim como pela erosão do solo e de rochas (Sawasdee e Köhler, 2009), concordando com os resultados apresentados pelo teste de micronúcleo.

Entre as amostras analisadas do ponto 4, nos meses de abril e junho, todos os elementos químicos analisados, com exceção do cálcio, magnésio e manganês, apresentaram-se acima da concentração máxima permitida pela Resolução $\mathrm{n}^{\mathbf{0}}$ 396, de 03 de abril de 2008, do CONAMA, para as águas subterrâneas utilizadas para irrigação (Brasil, 2008).

$\mathrm{O}$ armazenamento dessa água a céu aberto antes da sua distribuição às lavouras submete a mesma à exposição aos fatores externos. Chaim (1995) apresenta a deriva dos agrotóxicos, durante sua aplicação, uma das formas de contaminação e diminuição da qualidade das águas, considerando-se a presença de pequenas plantações em suas proximidades. Walls et al. (1996) demonstraram que a presença de resíduos de agrotóxicos nas águas podem gerar um elevado grau de toxicidade aos organismos expostos.

Os valores máximos permitidos de cálcio e magnésio não se encontram nos padrões de qualidade de água superficial e subterrânea, estabelecidos pelo CONAMA através da Resolução 357/2005 (Brasil, 2005) e Resolução 396/2008 (Brasil, 2008), respectivamente. Sendo assim, não foi possível compará-los aos demais elementos químicos analisados.

De acordo com os resultados obtidos neste trabalho, pôde-se perceber a importância da utilização de múltiplos biomarcadores para avaliação a toxicidade em ambientes aquáticos. Pesquisas realizadas com estes organismos indicadores da qualidade da água podem servir de parâmetros aos demais estudos relacionados à preservação e conservação do ambiente.

Pesquisas futuras que possibilitem verificar a possível origem desses poluentes seriam de grande interesse para melhor explicar os resultados de alterações genotóxicas e mutagênicas observadas no presente estudo.

\section{CONCLUSÃO}

O presente estudo demonstrou que todas as amostras de água analisadas do Córrego Arara apresentaram possível potencial mutagênico, genotóxico e citotóxico à animais e vegetais, que estão em contato com a mesma, devido à presença de substâncias e materiais poluentes derivados ou não de ações antrópicas.

Considerando-se a utilização e importância das águas deste córrego para o município de Rio Brilhante, MS, esse trabalho pode contribuir como ferramenta de suporte ao controle da 
qualidade da água a fim de auxiliar ações e medidas para recuperação do Córrego Arara (Rio Brilhante, MS).

\section{AGRADECIMENTOS}

As autoras agradecem à Universidade Federal da Grande Dourados pelo apoio financeiro e logístico, à Douradense Piscicultura pelo fornecimento dos peixes, a todos os alunos do Laboratório de Biotecnologia Aplicada a Produção Animal pelo auxílio na realização dos testes, ao professor Dr. Joelson Gonçalves Pereira e sua aluna Chun Pun Hung por terem ajudado na elaboração do mapa e ao professor Dr. Jorge Luiz Raposo Júnior por ter realizado as análises químicas das amostras de água.

\section{REFERÊNCIAS}

ALVIM, L. B.; KUMMROW, F.; BEIJO, L. A.; LIMA, C. A. A.; BARBOSA, S. Evaluation of the cytogenotoxicity of textile effluents using Allium cepa L. Revista Ambient. Água, v. 6, n. 2, p. 255-265, 2011. http://dx.doi.org/10.4136/ambi-agua.198

AYRES, M.; AYRES JR, M.; AYRES, D. L.; SANTOS, A. A. S. BioEstat 4.0 aplicações estatísticas nas áreas das ciências biomédicas. Belém: ONG Mamirauá, 2005.

BAIRD, C.; CANN, M. Química ambiental. 4. ed. Porto Alegre: Bookman, 2011. p. 844.

BELPAEME, K.; COOREMAN, K.; ZHU, L.; KIRSCH-VOLDERS, M. Development and validation of the in vivo alkaline Comet assay for detecting genomic damage in marine flat fish. Mutation Research, v. 415, p. 167-184, 1998.

http://dx.doi.org/10.1016/S1383-5718(98)00062-X

BIANCHI, J.; ESPINDOLA, E. L. G.; MARIN-MORALES, M. A. Genotoxicity and mutagenicity of water samples from the Monjolinho River (Brazil) after receiving untreated effluents. Ecotoxicology \& Environmental Safety, v. 74, n. 4, p. 826-833, 2011. http://dx.doi.org/10.1016/j.ecoenv.2010.11.006

BOLLMANN, H. A.; MARQUES, D. M. L. M. Influência da densidade populacional nas relações entre matéria orgânica carbonácea, nitrogênio e fósforo em rios urbanos situados em áreas com baixa cobertura sanitária. Revista Engenharia Sanitária e Ambiental, v. 11, p. 343-352, 2006.

BRASIL. Ministério do Desenvolvimento Urbano e Meio Ambiente. Conselho Nacional do Meio Ambiente - CONAMA. Resolução no 357, de 17 de março de 2005. Dispõe sobre a classificação dos corpos de água e diretrizes ambientais para o seu enquadramento, bem como estabelece as condições e padrões de lançamento de efluentes, e dá outras providências. Diário Oficial [da] República Federativa do Brasil, Brasília, DF, 2005.

BRASIL. Ministério do Desenvolvimento Urbano e Meio Ambiente. Conselho Nacional do Meio Ambiente - CONAMA. Resolução no 396, de 03 de abril de 2008. Dispõe sobre a classificação e diretrizes ambientais para o enquadramento das águas subterrâneas e dá outras providências. Diário Oficial [da] República Federativa do Brasil, Brasília, DF, 2008.

CAVAS, T.; GARANKO N. N.; ARKHIPCHUK, V. V. Induction of micronuclei in blood, gill and liver cells of fishes subchronically exposed to cadmium chloride and copper sulphate. Food and Chemical Toxicology, v. 43, p. 569-574, 2005. http://dx.doi.org/10.1016/j.fct.2004.12.014 
CHAIM, A. Impacto ambiental de agrotóxicos e biopesticidas. Revista Brasileira de Toxicologia, v. 8, n. 1, p. 9-10, 1995.

COSTA, C. R.; OlIVI, P.; BOtTA, C. M. R.; ESPINDOLA, E. I. G. A toxicidade em ambientes aquáticos: discussão e métodos de avaliação. Química Nova, v. 31, n. 7, p. 1820-1830, 2008.

DIAS, N. M. P.; ALlEONI, L. R. F.; CASAGRANDE, J. C.; CAMARGO, O. A. Energia livre da reação de adsorção de cádmio em latossolos ácricos. Revista Ciência Rural, v. 33, n. 5, p. 829-834, 2003.

UFFUS, J. H. "Heavy metals" - A meaningless term? Pure and Applied Chemistry, v. 74, n. 5, p. 793-807, 2002.

EMPRESA BRASILEIRA DE PESQUISA AGROPECUÁRIA - EMBRAPA. Guia clima: Estação da Embrapa Agropecuária Oeste. 2013. Disponível em: < http://www.cpao.embrapa.br/clima/?lc=site/estatisticas/estatisticas $>$. Acesso em: $11 \mathrm{de}$ fev de 2014.

GONTIJO, A. M. M. C.; TICE, R. Teste do cometa para a detecção de dano no DNA e reparo em células individualizadas. In: RIBEIRO, L. R.; SALVADORI, D. M. F.; MARQUES, E. K. (Org.). Mutagênese ambiental. Canoas: Ulbra, 2003. p. 173-200.

GRANT, W. F. Chromosome Aberration Assays in Allium. Mutation Research, Orlando, v. 99, n.3, p. $273-29,1982$. http://dx.doi.org/10.1016/0165-1110(82)90046-X

GUERRA, M.; SOUZA, M. J. Como observar cromossomos: um guia de técnica em citogenética vegetal, animal e humana. São Paulo: FUNPEC, p. 131, 2002.

GUPTA, A. K.; AHMAD, I.; AHMAD, M. Genotoxicity of refinery waste assessed by some DNA damage tests. Ecotoxicology \& Environmental Safety, in press, 2014. http://dx.doi.org/10.1016/j.ecoenv.2014.03.032

HANDY, R. D.; GALlOWAY, T. S.; DEPLEDGE, M. H. A proposal for the use f biomarkers for the assessment of chronic pollution and in regulatory toxicology. Ecotoxicology, v. 12, p. 331-343, 2003.

HARTMANN, A.; PLAPPERT, U.; RADDATZ, K.; GRÜNERT-FUCHS, M.; SPEIT, G. Does physical activity induced DNA damage? Mutagenesis, v. 9, p. 269-272, 2001.

HOSHINA, M. M.; DE ANGELIS, D. DE F.; MARIN-MORALES, M. A. Induction of micronucleus and nuclear alterations in fish (Oreochromis niloticus) by a petroleum refinery effluent. Mutation Research/Genetic Toxicology and Environmental Mutagenesis, v. 656, n. 1-2, p. 44-48, 2008.

http://dx.doi.org/10.1016/j.mrgentox.2008.07.004

INSTITUTO DO MEIO AMBIENTE DO PANTANAL DE MATO GROSSO DO SUL IMAP MS. Bacia do Rio Ivinhema: diagnóstico hidroambiental e socioeconômico. Campo Grande: Secretaria de Estado de Meio Ambiente e Recursos Hídricos/Instituto de Meio Ambiente Pantanal. Gerência de Recursos Hídricos, 2006.

MANSOURI, B.; EBRAHIMPOUR, M.; BABAEI, H. Bioaccumulation and elimination of nickel in the organs of black fish (Capoeta fusca). Toxicology and Industrial Health, v. 28 , n. 4 , p. 361-368, 2012. http://dx.doi.org/10.1177/0748233711412425 
MARQUES, A. L. B.; CHIERICE, G. O. Trace nickel determination with phenyl dithiocarbamate in sea water, by adsorptive stripping voltammetry. Journal of the Brazilian Chemical Society, v. 4, n.1, p.17-19, 1993.

MATSUmOTO, S. T.; MANTOVANI, M. S.; MALAGUTTI, M. I. A.; DiAS, A. L.; FONSECA, I. C.; MARIN-MORALES, M. A. Genotoxicity and mutagenicity of water contaminated with tannery effluents, as evaluated by the micronucleus test and comet assay using the fish Oreochromis niloticus and chromosome aberrations in onion roottips. Genetics and Molecular Biology, v. 29, p. 148-158, 2006.

http://dx.doi.org/10.1590/S1415-47572006000100028

MATSUMOTO, S. T.; MANTOVANI, M. S.; MALLAGUTI, M. I.; MARIN-MORALES, M. A. Investigation of the genotoxic potential of the waters of a river receiving tannery effluents by means of the in vitro comet assay. Cytologia, v. 68, p. 395-401, 2003. http://dx.doi.org/10.1590/S1415-47572006000100028

MELO, K. M.; GRISOLIA, C. K.; PIECZARKA, J. C.; SOUZA, L. R.; FILHO, J. S.; NAGAMACHI, C. Y. FISH in micronucleus test demonstrates aneugenic action of rotenone in a common freshwater fish species, Nile tilapia (Oreochromis niloticus). Mutagenesis, v. 29, n. 3, p. 215-219, 2014.

http://dx.doi.org/dx.doi.org/10.1093/mutage/geu005

MITCHELMORE, C. L.; CHIPMAN, J. K. DNA strand breakage in aquatic organisms and the potencial value of the comet assay in environmental monitoring. Mutation Research, Amsterdam, v. 399, p. 135-147, 1998. http://dx.doi.org/10.1016/S00275107(97)00252-2

RADIĆ, S.; STIPANICER, D.; VUJCIĆ, V.; RAJCIĆ, M. M.; SIRAC, S.; PEVALEKKOZLINA, B. The evaluation of surface and wastewater genotoxicity using the Allium cepa test. Science of the Total Environment, v. 408, p. 1228-1233, 2010. http://dx.doi.org/10.1016/j.scitotenv.2009.11.055

RANK, J.; NIELSEN, M. H. Genotoxicity testing of wastewater sludge using the Allium cepa anaphase-telophase chromosome aberration assay. Mutation Research, v. 418, n. 2-3, p. 113-119, 1998. http://dx.doi.org/10.1016/S1383-5718(98)00118-1

RIBEIRO, D. L.; BARCELOS, G. R. M.; D’ARCE, L. P. G. Genotoxic Effects of Water from São Francisco River, Brazil, in Astyanax paranae. Bulletin of Environmental

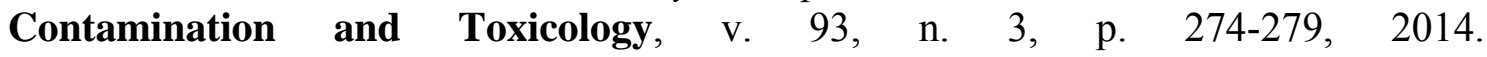
http://dx.doi.org/10.1007/s00128-014-1291-9

ROJAS, E.; LOPEZ, M. C.; VALVERDE, M. Single cell gel electrophoresis assay: methodology and applications. Journal of Chromatography B, v. 722, p. 25-254, 1999. http://dx.doi.org/10.1016/S0378-4347(98)00313-2

RUSSO, C.; ROCCO, L.; MORESCALCHI, M. A.; STINGO, V. Assessment of environmental stress by the micronucleus test and the Comet assay on the genome of teleost populations from two natural environments. Ecotoxicology and Environmental Safety, v. 57, n. 2, p. 168-174, 2004. http://dx.doi.org/10.1016/S0147-6513(03)00027-7

SAHI, A. N.; SINGH, S. K.; SEM, P. K.; SINGH, R. N. Cytogenetic response of hexavalent chromium-induced somatic cell abnormalities in Allium cepa. Cytobios, v. 96, p. 71-79, 1998. 
SALVADORI, D. M. F.; RIBEIRO, L. R.; FENECH, M. Teste de micronúcleo em células humanas in vitro. In: RIBEIRO, L. R.; SALVADORI, D. M. F.; MARQUES, E. K. (Org.). Mutagênese Ambiental. Canoas: Ulbra, p. 201-220, 2003.

SÁNCHEZ-GALÁN, S.; LINDE, A. R.; IZQUIERDO, J. I.;GARCÍA-VÁSQUEZ, E. Micronuclei and fluctuating asymmetry in brown trout (Salmo trutta): complementary methods to biomonitor freshwater ecosystems. Mutation Research, v. 412, p. 219-225, 1998. http://dx.doi.org/10.1016/S1383-5718(97)00186-1

SAWASDEE B.; KÖHLER, H. R. Embryo toxicity of pesticides and heavy metals to the ramshorn snail, Marisa cornuarietis (Prosobranchia). Chemosphere, v. 75, n. 11, p. 1539-1547, 2009. http://dx.doi.org/10.1016/j.chemosphere.2009.01.08

SCALON, M. C. S.; RECHENMACHER, C.; SIEBEL, A. M.; KAYSER, M. L.; RODRIGUES, M. T.; MALUF, S. W.; RODRIGUES, M. A. S.; SILVA, L. B. Evaluation of Sinos River water genotoxicity using the comet assay in fish. Brazilian Journal of Biology, v.70, n.4, p.1217-1222, 2010. http://dx.doi.org/10.1590/S151969842010000600011

SCHMID, W. The micronucleus test. Mutation Research, v. 31, p. 9-15, 1975. http://dx.doi.org/10.1016/0165-1161(75)90058-8

SILVA, A. C. Tratamento do percolado de aterro sanitário e avaliação da toxicidade do efluente bruto e tratado. 2002. Dissertação (Mestrado em Engenharia Civil) - Rio de Janeiro: Universidade Federal do Rio de Janeiro, 2002.

TAGLIARI, K. C.; CECCHINI, R.; VAZ ROCHA, J. A.; VARGAS, V. M. F. Mutagenicidade do Sedimento e Estresse Oxidativo Hepático em Peixes sob a Influência de Curtumes. Journal of the Brazilian Society of Ecotoxicology, v.1, p. 57 61, 2006.

TICE, R. R.; AGURELL, E.; ANDERSON, D.; BURLINSON, B.; HARTMANN, A.; KOBAYASHI, H. et al. Single cell gel/Comet Assay: guidelines for in vitro and in vivo genetic toxicology testing. Environmental and Molecular Mutagenesis, v. 35, n. 3, p. 206-221, 2000.

VENTURA, B. C.; ANGELIS, D. F.; MARIN-MORALES, M. A. Mutagenic and genotoxic effects of the Atrazine herbicide in Oreochromis niloticus (Perciformes, Cichlidae) detected by the micronuclei test and the comet assay. Pesticide Biochemistry and Physiology, v. 90, p. 42-51, 2008. http://dx.doi.org/10.1016/j.pestbp.2007.07.009

WALLS, D.; SMITH, P. G.; MANSELL, M. G. Pesticides in groundwater in Britain. International Journal of Environmental Health Research, v. 6, p. 55-62, 1996.

WHITE, P. A.; RASMUSSEN, J. B. The genotoxic hazards of domestic wastes in surface waters. Mutation Research, v. 410, p. 223-236, 1998. http://dx.doi.org/10.1016/S13835742(98)00002-7 


Ambiente \& Água - An Interdisciplinary Journal of Applied Science
ISSN 1980-993X - doi:10.4136/1980-993X
www.ambi-agua.net
E-mail: ambi-agua@agro.unitau.br

\title{
Citotoxicidade e genotoxicidade da água do rio Subaé (Humildes, Bahia, Brasil) usando Allium cepa $\mathrm{L}$. como bioindicador
}

\author{
doi: 10.4136/ambi-agua.1459
}

Received: 09 Jul. 2014; Accepted: 06 Nov. 2014

\author{
Jacqueline Ramos Machado Braga*; Diêgo Menezes Lopes \\ Universidade Federal do Recôncavo da Bahia (UFRB), Cruz das Almas, Bahia, Brasil \\ Centro de Ciências Agrárias, Ambientais e Biológicas \\ Setor de Biologia, Laboratório IMUNOBIO \\ *Autor correspondente: e-mail: jacquebraga@globo.com, \\ dmlopes-gen@hotmail.com
}

\section{RESUMO}

Ao longo dos anos, o rio Subaé na Bahia tem sido impactado por atividade industrial pelo lançamento de efluentes tóxicos em suas águas. O presente estudo analisou os efeitos citotóxicos e genotóxicos provocados pela água do trecho deste rio que banha o município de Humildes-BA, utilizando o teste do Allium cepa. Amostras de água foram obtidas em três pontos de coleta (PI, PII e PIII) para as análises de parâmetros fisico-químicos. Bulbos de $A$. cepa foram colocados por $72 \mathrm{~h}$ para germinação nas amostras de água em triplicata. As radículas foram submetidas por 12 -h a $4{ }^{\circ} \mathrm{C}$, fixadas em Carnoy por 12 -h e coradas com técnica de Feulgen. Foram analisadas 6.500 células/tratamento. A citotoxicidade foi avaliada pelo índice mitótico (IM) e a genotoxicidade, por anormalidades cromossômicas (AC). Os testes fisico-químicos revelaram que PI e PIII apresentavam valores de $\mathrm{OD}, \mathrm{DBO}_{5}$ e DQO alterados. O teste de toxicidade indicou que houve inibição do crescimento das raízes nos pontos PI (10,3\%) e PII (10,6 \%), quando comparados ao controle negativo (11,9\%). No PI, foram encontrados $6,2 \%$ de ACs, $6 \%$ em PII e 5,4\% em PIII, valores acima do controle positivo (3\%), evidenciando o efeito genotóxico nos pontos estudados. A partir dos resultados, pode-se inferir que as águas do Rio Subaé podem conter substâncias que provavelmente estão afetando o ciclo celular do $A$. cepa, sendo importante que outras análises sejam realizadas em diferentes estações do ano, para melhor monitoramento das condições ambientais locais.

Palavras-chave: anormalidades cromossômicas, ecotoxicologia, índice mitótico, monitoramento ambiental.

\section{Cytotoxicity and genotoxicity in water of the Subaé River (Humildes, Bahia, Brazil) using Allium cepa $\mathbf{L}$. as a bioindicator}

\section{ABSTRACT}

Over the years, the Subae River in Bahia has been polluted by the discharge of toxic industrial waste into its waters. This study used the Allium cepa test to investigate the cytotoxic and genotoxic effects caused by the river water that that runs through the city of Humildes-BA. Water samples were collected in three points (PI, PII and PIII) for physical and 
chemical analysis. Onions bulbs were immersed in triplicate water samples for $72 \mathrm{~h}$ to induce germination. The root tips were kept at $4{ }^{\circ} \mathrm{C}$ for $12-\mathrm{h}$, then fixed in Carnoy for 12-h and stained using the Feulgen technique. We analyzed 6500 cells of each treatment. Cytotoxicity was assessed by the mitotic index (MI) and genotoxicity by chromosomal abnormalities (CA). The tests revealed that PI and PIII had DO values, $\mathrm{BOD}_{5}$ and COD changed. The toxicity test indicated that root growth was inhibited by the water drawn from PI $(10.3 \%)$ and PII $(10.6 \%)$ compared to the negative control (11.9\%). CAs were found in $6.2 \%$ of the cells relating to PI, $6.0 \%$ relating to PII, and $5.4 \%$ relating to PIII, respectively. These values were higher than those found in the positive control (3\%), indicating a genotoxic effect at the points studied. From the results, it can be inferred that the waters of the Rio Subaé may contain substances that are probably affecting the cell cycle of $A$. cepa. It is important that other analyses be conducted in different seasons, in order to better monitor local environmental conditions.

Keywords: chromosomal abnormalities, ecotoxicology, environmental monitoring, mitotic index.

\section{INTRODUÇÃO}

A água é um elemento de extrema importância para a dinâmica de sobrevivência do planeta. No entanto, diversos setores da indústria lançam nos rios resíduos oriundos de suas atividades que são considerados tóxicos por afetarem o equilíbrio do meio ambiente, prejudicando não somente a saúde humana, mas também de animais e plantas (Ferreira et al., 2012).

Em 1960, a Companhia Brasileira de Chumbo (COBRAC/Plumbum) estabeleceu-se na periferia da cidade de Santo Amaro da Purificação, região do Recôncavo da Bahia. A Usina Plumbum localiza-se a 300 metros da margem do rio Subaé, principal rio da bacia hidrográfica do Subaé (Carvalho et al., 1984). Segundo ensaios realizados conforme a NBR 10.004 (ABNT, 2004), da Associação Brasileira de Normas Técnicas - ABNT, na escória deixada pela fundição, desativada desde 1993, predominam os metais traço chumbo $(\mathrm{Pb})$ e cádmio $(\mathrm{Cd})$. Os dados ambientais existentes sobre a área onde se localizam as instalações da usina, desativada no ano de 1993, indicam que as águas superficiais também estão contaminadas por estes metais traço na área denominada "zona alagadiça" (Anjos, 2003), os solos apresentam elevadas concentrações destes metais nos locais onde ocorre a deposição da escória, e as águas subterrâneas apresentam concentrações de $\mathrm{Pb}$ e $\mathrm{Cd}$ acima dos limites determinados pela legislação vigente (Adorno et al., 2013).

Moulis e Thevénod (2010) sugerem que a bioacumulação de metais traço em organismos vivos é um dos efeitos mais sérios de contaminação ambiental porque tanto animais quanto plantas podem concentrar estes elementos em níveis muito superiores aos encontrados no meio ambiente, promovendo o transporte nos vários níveis da cadeia alimentar.

O Rio Subaé é vizinho do Centro Industrial do Subaé, implantado em 1970. Atualmente as residências avançaram sobre a área industrial, aumentando a poluição e prejudicando a vazão do rio. O problema da contaminação do rio Subaé não se restringe apenas à degradação da fauna e flora local ou à contaminação por metais traço. A saúde humana também é afetada pela carga de efluentes líquidos, lançados por fábricas de beneficiamento de frutas, e hidrocarbonetos de petróleo (Andrade e Moraes, 2013). Um exemplo da extensão da contaminação do Subaé é o município de Humildes, distrito de Feira de Santana-BA, onde em junho de 2010 surgiram milhares de peixes mortos. Entre as possíveis causas da mortandade dos peixes, levantadas pela Secretaria do Meio Ambiente (IMA), estão a contaminação por produtos químicos, a diminuição do oxigênio pela redução do fluxo de água por falta de chuva ou o acúmulo de matéria orgânica (Feira de Santana, 2011).

Ainda segundo o SEMMAM (Feira de Santana, 2011), o prejuízo para os produtores é grande, visto que muitos utilizam as águas do rio para irrigar plantações de hortaliças. 
Conforme relatos, a água já vinha escurecendo há algum tempo, o odor é muito forte e os dados coletados no local indicam que a contaminação pode advir do lançamento de efluentes líquidos de uma fábrica de polpas de frutas. Como o rio Subaé sofreu severas interferências em decorrência da influência antrópica, é de suma importância a avaliação do comprometimento ambiental gerado.

Inúmeros testes toxicológicos são utilizados para avaliar as concentrações e o tempo de exposição necessário para que os agentes tóxicos possam produzir efeitos adversos sobre os organismos. Carneiro Jr. (2010) sugere que dentre estes testes, o cultivo direto de bulbos, em soluções preparadas com químicos diversos ou em amostras de águas naturais contaminadas, caracteriza-se como um interessante teste de toxicidade, pois permite avaliar os efeitos de compostos químicos, além de monitorar ambientes com suspeita de contaminação.

Plantas superiores são reconhecidas como excelentes modelos para detectar mutágenos ambientais, sendo por isso frequentemente utilizadas em estudos de monitoramento. Dentre as espécies de plantas, a cebola (Allium cepa) tem sido usada para avaliar danos ao DNA, como anormalidades cromossômicas e distúrbios no ciclo mitótico. O teste genotóxico que utiliza o A. cepa é validado pelo Programa Internacional de Segurança Química e pelo Programa Ambiental das Nações Unidas (Grippa et al., 2010). O teste do A. cepa é caracterizado como padrão ouro para avaliação de anormalidades cromossômicas, índice mitótico, formação de micronúcleos e anormalidades nucleares na detecção de contaminantes, além de permitir a avaliação da ação de um agente e seus efeitos clastogênicos ou aneugênicos sobre o material genético (Auib e Felzenswalb, 2011; Feretti et al., 2007).

A partir de ensaios de biomonitoramento e genotoxicidade será possível avaliar as consequências do impacto gerado no trecho do rio Subaé no município de Humildes-BA, promovendo um delineamento mais especifico das ações que futuramente poderão ser realizadas no sentido de minimizar os efeitos da poluição para a população local.

\section{METODOLOGIA}

As amostras de água foram coletadas ao longo do rio Subaé, no trecho que passa pelo município de Humildes, distrito de Feira de Santana, Bahia, Brasil (Figura 1). Foram estabelecidos três pontos de coleta, com três repetições cada, realizadas entre os meses de junho a agosto de 2013. Todas as amostras tiveram dados fisico-químicos avaliados in loco através de sonda multiparâmetros. As amostras foram armazenadas em garrafas estéreis e mantidas a $4{ }^{\circ} \mathrm{C}$ até o momento de realização dos testes.

Com relação às características dos pontos de coleta, verificou-se que o ponto PI, localizado nas proximidades de uma fábrica de polpas de frutas, apresentava uma grande quantidade de taboás, plantas indicadoras de poluição, o que dificultava a visualização do leito do rio. Os pontos PII e PIII estavam cada um, distantes cerca de $6 \mathrm{~km}$ do PI e também apresentavam ao longo das margens lixo e indícios de influência antrópica.

A avaliação da toxicidade foi realizada seguindo os procedimentos descritos por Fiskesjö (1985) e de acordo com protocolo de Barbério et al. (2011), com adaptações. Para cada ponto de coleta, foram selecionados três bulbos de tamanho semelhante, ausência de traumas, deformidades naturais ou contaminação por fungos. O anel radicular foi lavado em água corrente e água destilada antes do início do teste. Os bulbos foram transferidos para recipientes com $200 \mathrm{~mL}$ de cada amostra de água coletada e mantidos à temperatura ambiente para germinação. A água era trocada após 24-h, 48-h e 72-h. Os controles foram realizados em água destilada (controle negativo) e $25 \mathrm{mg} \mathrm{L}^{-1}$ de solução de sulfato de cobre (controle positivo). As radículas foram medidas $(\mathrm{cm})$ após $72-\mathrm{h}$ de germinação. O sulfato de cobre, usado como controle positivo, além de atuar no ciclo redox, produzindo espécies reativas de oxigênio (EROs) que induzem a peroxidação de lipídios, e alterações no sistema de reparo do 
DNA, pode ser tóxico para plantas e animais, sendo comprovadamente genotóxico para a amostra testada (Prá et al., 2006).

As radículas retiradas foram submetidas ao "choque frio" em banho de gelo por 12-h sob refrigeração. Para a fixação foi utilizado o fixador Carnoy à temperatura ambiente (12-h), sendo trocado o fixador após 6-h. Após isso, o material foi estocado em etanol $(70 \%)$ sob refrigeração.

As radículas foram retiradas do fixador, enxutas e submetidas à hidrólise ácida em $\mathrm{HCl}$ $\left(1 \mathrm{~N}\right.$ a $\left.60{ }^{\circ} \mathrm{C}\right)$ de 10 a 12 -min em banho-maria, sendo depois lavadas em água destilada. Foi realizada a reação de Feulgen e coloração com o Reativo de Schiff. O meristema e a região F1 foram cortados e colocados numa lâmina limpa onde foi seccionada apenas a região do meristema apical. O fragmento foi recoberto com uma gota de carmim acético (2\%), esmagado com lamínula e submetido à microscopia de luz.
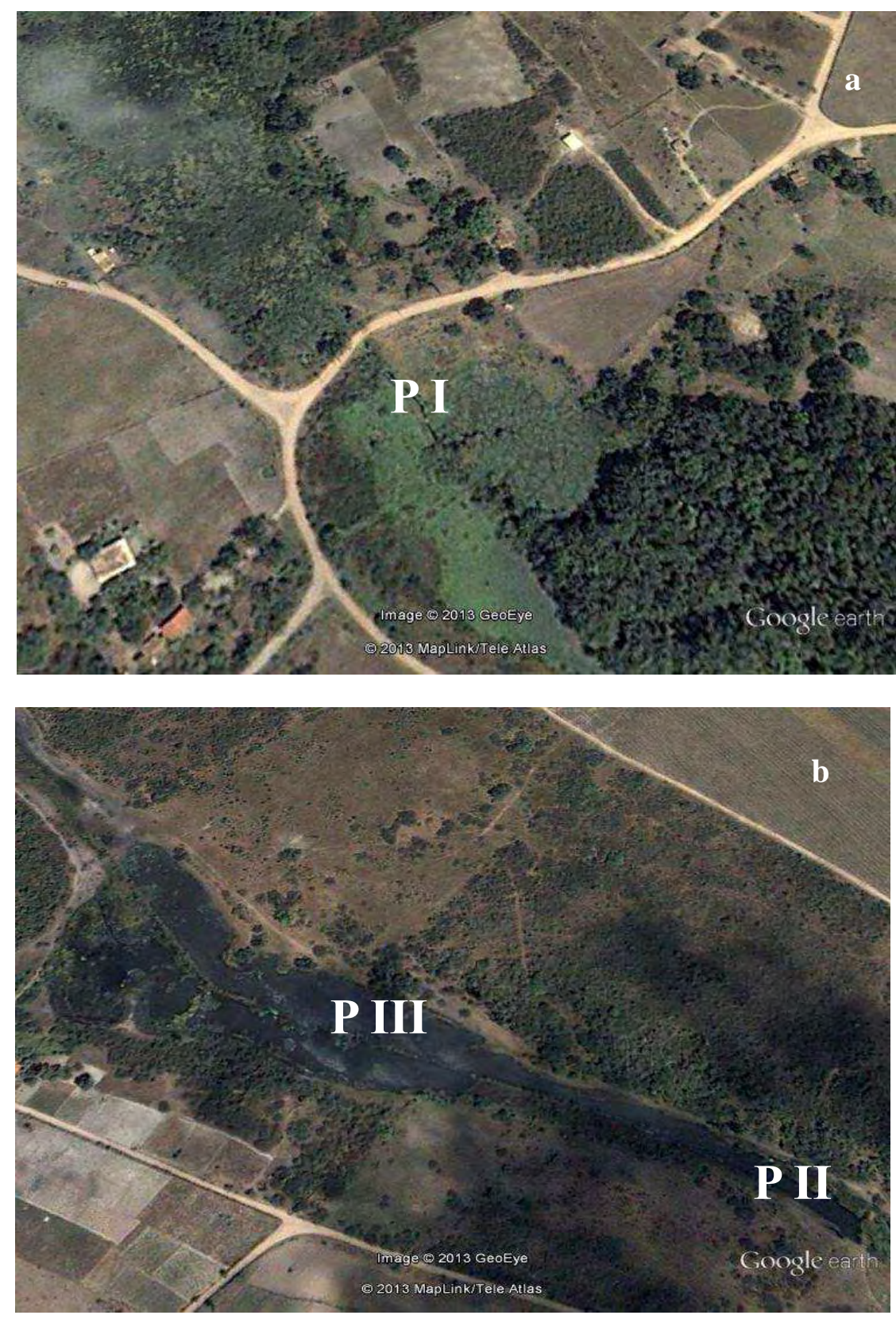

Figura 1. Pontos de coleta de coleta de água I (a), II e III (b) no Rio Subaé, Município de Humildes, Bahia, Brasil.

Fonte: Google Earth. 
Foram confeccionadas três lâminas/bulbo/amostra de água. De cada lâmina, foram analisadas em "teste cego" 500 células, perfazendo um total de 1500 células/bulbo/amostra. Para a avaliação dos efeitos citotóxicos, foram verificadas as alterações no Índice Mitótico (IM), calculado pelo número de células em divisão dividido pela soma do número das células em interfase e das células em divisão. Os efeitos genotóxicos foram analisados pelas anormalidades cromossômicas (AC) encontradas, considerando a porcentagem de irregularidades em metáfases, anáfases e presença de células com fragmentos cromossômicos e binucleações. As AC foram então fotodocumentadas e o tratamento estatístico foi realizado por pelo Teste do Qui-Quadrado $(\alpha=0,05)$.

\section{RESULTADOS E DISCUSSÃO}

\subsection{Análise fisico-química}

Estudos de monitoramento da qualidade da água requerem dados preliminares sobre condições da água a ser estudada. Temperatura, $\mathrm{pH}$, salinidade, Oxigênio Dissolvido (OD), Demanda Química de Oxigênio (DQO), Demanda Bioquímica de Oxigênio (DBO) dentre outros, são rotineiramente utilizados para o monitoramento da qualidade em amostras de águas, fornecendo dados importantes sobre o objeto de estudo (Oliveira et al., 2012). A DQO é um parâmetro que estima o potencial poluidor (consumidor de oxigênio) dos efluentes domésticos e industriais, assim como o impacto dos mesmos sobre os ecossistemas aquáticos (Zuccari et al., 2005).

Segundo a Resolução 357/2005 (Brasil, 2005), que classifica as águas de acordo com os valores de salinidade, as amostras dos pontos I e II (Tabela 1) são classificadas como sendo água salobra (acima de $0.5 \%$ ). Entretanto, o ponto III foi classificado com água doce (inferior a $0.5 \%$ ).

Os dados fisico-químicos da Tabela 1, revelaram ainda alterações em todos os pontos coletados, considerando os valores de referência estabelecidos para OD $\left(\mathrm{mg} \mathrm{L}^{-1}\right)$. O índice de OD é um dos parâmetros mais importantes para avaliar a capacidade de um corpo hídrico em suportar a atividade biológica de organismos aquáticos. Índices de 5 a $6 \mathrm{mg} \mathrm{L}^{-1}$ já suportam uma variada população de peixes. De acordo com o CONAMA (Resolução 357/2005) a quantidade ideal de OD $\left(\mathrm{mg} \mathrm{L}^{-1}\right)$ em águas de rio salobra de classe 2 não pode ser inferior a $4 \mathrm{mg} \mathrm{L}^{-1}$. No presente estudo, os valores de OD registrados nos pontos coletados não se enquadram de acordo com a resolução do órgão regulador.

Tabela 1. Parâmetros físico-químicos em média \pm desvio padrão das amostras de água coletadas nos pontos I, II e III do Rio Subaé, Município de Humildes - BA, Brasil, 2013.

\begin{tabular}{lcccc}
\hline \multicolumn{1}{c}{ Parâmetros } & Ponto I & Ponto II & Ponto III & Referência* $^{*}$ \\
\hline Salinidade $(\%)$ & $0,75 \pm 0,15$ & $0,74 \pm 0,11$ & $0,11 \pm 0,07$ & 0,5 \\
Temperatura $\left({ }^{\circ} \mathrm{C}\right)$ & $25,0 \pm 1,02$ & $26,3 \pm 1,15$ & $26,1 \pm 1,11$ & - \\
$\mathrm{pH}$ & $6,6 \pm 0,2$ & $7,3 \pm 0,4$ & $7,3 \pm 0,3$ & $6,0-9,0$ \\
$\mathrm{OD}\left(\mathrm{mgL}^{-1}\right)$ & $1,4 \pm 0,3$ & $3,45 \pm 0,86$ & $1,8 \pm 0,44$ & Não $<5$ \\
$\mathrm{DBO}_{5}\left(\mathrm{mgL}^{-1}\right)$ & $4,35 \pm 0,4$ & $3,2 \pm 0,7$ & $4,0 \pm 0,9$ & Não $>5$ \\
$\mathrm{DQO}\left(\mathrm{mgL}^{-1}\right)$ & $15,4 \pm 2,3$ & $10,3 \pm 1,2$ & $13,2 \pm 1,4$ & $3,0-5,0$ \\
\hline
\end{tabular}

* Segundo Resolução 357/2005 do CONAMA.

A $\mathrm{DBO}_{5}$ correlaciona-se à quantificação indireta de matéria orgânica presente no corpo hídrico, associada à carga poluidora orgânica antrópica, enquanto que a DQO seria a 
quantidade de $\mathrm{O}_{2}$ necessária para a oxidação da matéria orgânica através de um agente químico (Naime e Nascimento, 2009). No presente estudo, apesar dos dados de OD não estarem dentro dos limites estabelecidos pelo CONAMA, os valores referentes à $\mathrm{DBO}_{5}$, encontram-se dentro do limite de referência. Segundo Ferreira et al. (2012), a $\mathrm{DBO}_{5}$ revela a quantidade de oxigênio para que os microorganismos biodegradem a matéria orgânica, o que resulta no decréscimo da concentração de OD no meio hídrico, como confirmam nossos achados.

Os dados do presente estudo revelam ainda que, considerando a relação $\mathrm{DQO} / \mathrm{DBO}_{5}$, verificou-se que em PI, esta relação foi de 3,54, seguida de 3,21 (PII) e 3,3 (PIII). Nesta relação $\mathrm{DQO} / \mathrm{DBO}_{5}$, Ferreira et al. (2012) sugerem que valores entre 1,7 e 2,4 indicam poluição por esgoto doméstico, enquanto que dejetos industriais mostram variação mais ampla. Se for considerado ainda que o aumento de $\mathrm{DBO}_{5}$ está relacionado a despejos de origem orgânica, e DQO a despejos de origem industrial, nossos achados indicam que a água do Rio Subaé, no trecho que passa pelo município de Humildes-BA está sofrendo a influência de efluentes de origem industrial.

\subsection{Teste de citotoxicidade}

Os resultados da Tabela 2 demonstram que no ponto PI não houve crescimento de raízes dentro das 72-h. A germinação desta amostra só iniciou uma semana após a montagem do experimento. De acordo com Leme e Marin-Morales (2008) e Souza et al. (2014), em estudo realizado com células meristemáticas de $A$. сеpa, expostas às amostras de água e sedimento do Rio Corumbataí - SP, os contaminantes presentes em algumas amostras podem ter uma ação persistente, enquanto que em outras, a depender do tipo de contaminante ou da quantidade presente na amostra, as células meristemáticas podem ter capacidade de recuperação dos efeitos dos agentes mutagênicos. Nos pontos PII e PIII, as raízes cresceram dentro das $72 \mathrm{~h}$ previstas, entretanto todas as amostras mostraram inibição em seu crescimento e consequente redução do IM.

Tabela 2. Resultados dos valores médios de Índice Mitótico (IM), crescimento e percentual de inibição de crescimento das raízes de A. cepa submetidas aos diferentes tratamentos com a água do Rio Subaé.

\begin{tabular}{cccc}
\hline Tratamentos & IM (\%) & Crescimento $(\mathbf{c m})$ & \% Inibição \\
\hline Ponto I & - & 0,0 & 100 \\
Ponto II & 10,6 & $2,06 \pm 0,6$ & 22,6 \\
Ponto III & 9,8 & $1,63 \pm 0,2$ & 38,7 \\
Controle & 11,9 & $2,66 \pm 0,5$ & 0,0 \\
\hline
\end{tabular}

Nota: IM (\%) de 6.500 células por ponto.

O Índice Mitótico (IM) é um dado expresso em porcentagem que é utilizado para avaliar a taxa de divisão celular. Ele tem se mostrado um parâmetro importante para se avaliar os efeitos que agentes químicos causam no ciclo celular (Smaka-Kincl et al., 1996; Sobral et al., 2013). Alvin et al. (2011) relatam que qualquer mudança nas condições ambientais, principalmente por causa da poluição, pode ser observada nas plantas pela redução do crescimento radicular. Isto levanta a suspeita que algum ou vários poluentes possam estar presentes na área de estudo. 
Os valores obtidos no teste de toxicidade nos três pontos, por meio da visualização das fases da divisão celular, indicaram que houve inibição do crescimento das raízes e consequente redução do IM. Segundo Circunvis et al. (2012), a análise do IM constitui uma ferramenta confiável para estudos de monitoramento de águas superficiais, pois o nível de citotoxicidade de águas poluídas pode ser determinado pela taxa de redução deste índice. No presente estudo, a análise dos índices de divisão das células de $A$. cepa mostrou que os resultados obtidos mostraram relevância estatística entre os grupos tratados com amostras de água do Rio Subaé $(\mathrm{p}<0,05)$, ou seja, a água apresentou ação citotóxica.

\subsection{Teste de genotoxicidade}

Estudo anterior de genotoxicidade, realizado na região, revelou elevada frequência de anormalidades cromossômicas em sangue periférico de bovinos expostos ao $\mathrm{Pb}$ e $\mathrm{Cd} \mathrm{da}$ escória da fábrica Plumbum (Braga, 2013). No presente estudo, anormalidades cromossômicas foram verificadas em todas as amostras $(\mathrm{p}<0,05)$, indicando a presença de substãncias clastogênicas (indutoras de quebras) sobre o DNA. A Tabela 3 revela o resultado do teste de genotoxicidade, onde foram encontradas anormalidades cromossômicas dos tipos binucleação, stickness (cromossomos pegajosos), pontes anafásicas e metáfases com cromossomos atrasados, indicando também alterações em nível de fuso mitótico (Figuras 2 e 3).

Tabela 3. Número total de Anormalidades Cromossômicas, e frequência de cada tipo de alteração, nas raízes de $A$. cepa submetidas aos diferentes tratamentos com a água do Rio Subaé.

\begin{tabular}{lccccc}
\hline Tratamentos & Total de AC & BN $(\%)$ & Sn $(\%)$ & PA $(\%)$ & Ca $(\%)$ \\
\hline Ponto I & 62 & 16,1 & 12,9 & 25,8 & 45,2 \\
Ponto II & 60 & - & 10,0 & 35,0 & 55,0 \\
Ponto III & 54 & 11,1 & 16,7 & 46,3 & 25,9 \\
Controle (+) & 30 & 16,7 & 16,7 & 33,3 & 33,3 \\
Controle (-) & 0 & - & - & - & - \\
\hline
\end{tabular}

Nota: AC (Anormalidades Cromossômicas); BN (Binucleação); $\mathbf{S n}$ (Stickness); PA (Ponte Anafásica); Ca (Cromossomo atrasado).

De acordo com Galindo et al. (2012), substâncias contaminantes tóxicas liberadas na água podem não mostrar efeitos agudos em organismos expostos, porém podem causar redução de sobrevivência, danos teciduais, danos genéticos de células somáticas e germinativas, acumulação de contaminantes persistentes e neoplasias. $\mathrm{O}$ estudo de Peron et al. (2009) indica que em geral, alterações no índice de divisão celular e do material genético podem ser deletérias para o organismo e indutoras de consequências severas e irreversíveis à saúde humana ou animal.

Os resultados sugerem que no trecho do Rio Subaé que banha o município de Humildes-Ba, existem substâncias indutoras de efeito citotóxico e genotóxico que promoveram alterações no organismo-teste. Entretanto, fazem-se necessários estudos na região em outras épocas do ano, principalmente no verão, haja vista que em estações mais secas existe uma maior potencialização da ação tóxica de poluentes nos corpos hídricos. Estudos anteriores revelaram que as diferenças na genotoxicidade entre os pontos de coleta em um rio podem ter relação com o volume de água, devido à influência das diferenças 
sazonais de precipitação pluviométrica, da concentração das substâncias tóxicas, do local e da época das amostragens (Scalon, 2009; Oliveira et al., 2012).

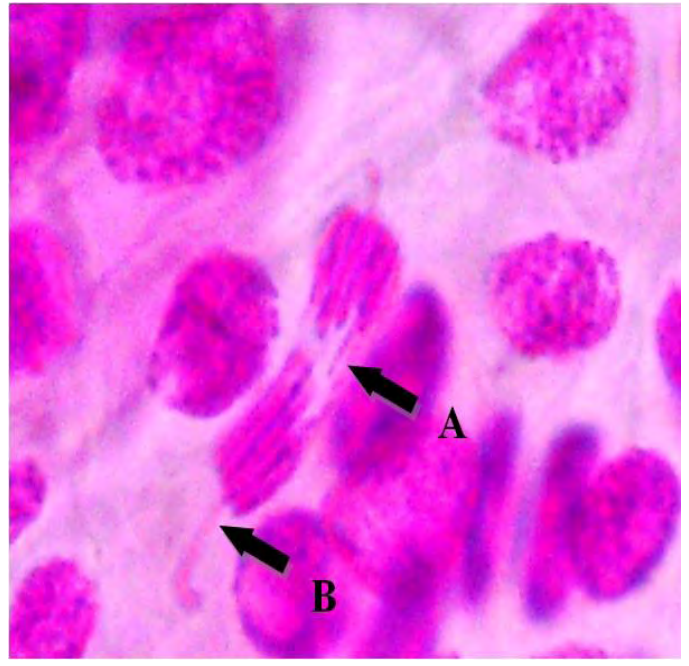

Figura 2. Ponte anafásica (A) e Stickness (B). Aumento 1000X.

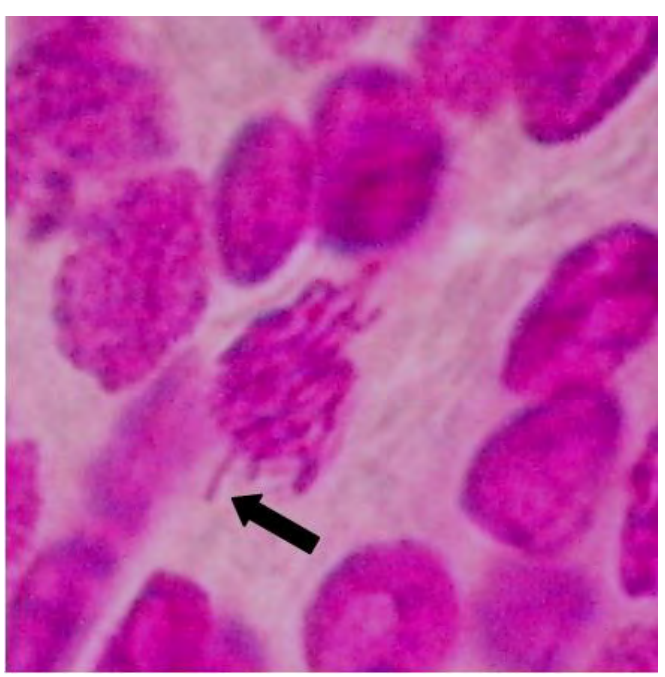

Figura 3. Metáfase com cromossomo atrasado em A. cepa. Aumento 1000X.

\section{CONSIDERAÇÕES FINAIS}

A partir dos dados observados neste estudo, pode-se inferir que a avaliação dos parâmetros físicoquímicos e citogenotóxicos da água do Rio Subaé, indicam que o este rio pode conter possíveis contaminantes de origem industrial, que podem estar afetando o ciclo celular, a morfologia cromossômica e o crescimento das raízes de $A$. cepa. Nesse sentido, é relevante que estudos de sazonalidade adicionais sejam realizados, para um monitoramento periódico das condições ambientais do local, no intuito de garantir água de qualidade à população ribeirinha, do município de Humildes, e monitorar o equilíbrio ambiental do Rio Subaé.

\section{REFERÊNCIAS}

ADORNO, E. V.; SANTOS, E. S. dos; JESUS, T. B. SIG e agressão linear para avaliação ambiental das nascentes do rio Subaé em Feira de Santana-BA. Boletim Goiano de Geografia, Goiânia, v. 33, n. 2, p. 63-80, 2013.

AIUB, C. A. F.; FELZENSWALB, I. O uso de Allium cepa como modelo experimental para investigar genotoxicidade de substâncias usadas em conservantes alimentares. Revista Genética na Escola, v. 6, n. 1, p. 12-15, 2011.

ALVIN, L. B.; KUMMROW, F.; BEIJO, L. A.; LIMA, C. A. de A.; BARBOSA, S. Avaliação da citogenotoxicidade de efluentes têxteis utilizando Allium cepa L. Rev. Ambient. Água, v. 6, n.2, p. 255-265, 2011. http://dx.doi.org/10.41368/ambi-agua.198

ANDRADE, M. F.; MORAES, L. R. S. Contaminação por chumbo em santo amaro desafia décadas de pesquisas e a morosidade do poder público. Ambiente \& Sociedade, v. 16, n. 2, p. 63-80, 2013. http://dx.doi.org/10.1590/S1414-753X2013000200005 
ANJOS, J. Â. S. A. Avaliação da eficiência de uma zona alagadiça (wetland) no controle da poluição por metais pesados: o caso da Plumbum em Santo Amaro da Purificação/BA. 2003. 130f. Tese (Doutorado em Engenharia Mineral) - Universidade de São Paulo, São Paulo, 2003. Disponível em: <http://www.teses.usp.br/teses /disponiveis/3/3134/tde-28042004-094552/ >. Acesso em: 8 dez. 2013.

ASSOCIAÇÃO BRASILEIRA DE NORMAS TÉCNICAS - ABNT. Nbr 10.004: resíduos sólidos - Classificação. Rio de Janeiro, 2004. 71p.

BARBÉRIO, A.; VOLTOLINI, J. C.; MELLO, M. L. S. Standardization of bulb and root sample sizes for the Allium cepa test. Ecotoxicology, v. 20, n. 4, p. 927-935, 2011. http://dx.doi.org/10.1007/s10646-011-0602-8

BRAGA, J. R. M. Efeito genotóxico de matais-traço em área contaminada na Bahia. In: CONGRESSO NACIONAL DE MEIO AMBIENTE, 10, Poços de Caldas-MG. Anais... 2013. 1 CD-ROM.

BRASIL. Conselho Nacional Do Meio Ambiente - CONAMA. Resolução nr. 357, de 17 de março de 2005. Disponível em:

$<$ http://www.mma.gov.br/port/conama/res/res05/res35705.pdf $>$. Acesso em: $10 \mathrm{dez}$. 2013 .

CARVAlHO, F.; TAVARES, T. M.; SOUZA, S. P.; LINHARES, P. Lead and cadmium concentrations in the hair of fisherman from the Subaé River basin, Brazil. Environmental Research, v. 33, n. 2, p. 300-306, 1984. http://dx.doi.org/10.1016/0013-9351(84)90027-6

CARNEIRO JR., I. N. Relatório do Grupo de Trabalho / Justiça Ambiental. GT Justiça Ambiental - Frente Parlamentar Ambientalista. Santo Amaro da Purificação: AVICCA, 2010. 74p.

CIRCUNVIS, B. C.; HECK, M. C.; VICENTINI, V. E. P. Investigação do potencial citotóxico das águas superficiais do Ribeirão Atlântico (Madaguaçu-PR) em Allium cepa L. Revista Saúde e Biologia, v. 7, n. 3, p. 7-14, 2012.

FEIRA DE SANTANA. Secretaria Municipal De Meio Ambiente. A Secretaria de Municipal de Meio Ambiente está investigando poluição de um riacho no distrito de Humildes. 2011. Disponível em:

$<$ http://semmamfeiradesantana.blogspot.com.br/2011/12/secretaria-de-municipal-demeio.html> Acesso em: 04 jul. 2014.

FERETTI, D.; ZERBINI, I.; ZANI, C.; CERETTI, E.; MORETTI, M.; MONARCA, S. Allium cepa chromosome abberation and micronucleus tests applied to study genotoxicity of extracts from pesticide-treated vegetables and grapes. Food Additives \& Contaminants, v. 24, n. 6, p. 561-572, 2007.

http://dx.doi.org/10.1080/02652030601113602

FERREIRA, C. F.; FRUEH, A. B.; DÜSMAN, E.; HECK, M. C.; VICENTINI, V. E. P. Avaliação da citoxicidade das águas dos ribeirões Varginha (Califórnia - PR) e Tabatinga (Mandaguari - PR) em Alluim cepa L. Revista Saúde e Biologia, v. 7, n. 2, p. 46-54, 2012.

FISKESJÖ, G. The Allium test as a standard in environmental monitoring. Hereditas, v. 102, n. 1, p. 99-112, 1985. http://dx.doi.org/10.1111/j.1601-5223.1985.tb00471.x 
GALINDO, T.; SILVA, E.; ROSÁRIO, I. Indução de micronúcleos e toxicidade por efluente doméstico em duas populações de Bathygobius soporator (Valenciennes, 1837) (Teleostei, Gobbidae) no Litoral de Salvador (BA), Brasil. Brazilian Journal of Aquatic Science and Technology, v. 15, n. 1, p. 1-7, 2012.

http://dx.doi.org/10.14210/bjast.v16n1.p1-7

GRIPPA, G. A.; MOROZESK, M.; NATI, N.; MATSUMOTO, S. T. Estudo genotóxico do surfactante Tween 80 em Allium cepa. Revista Brasileira de Toxicologia, v. 23, n. 1-2, p. 11-16, 2010.

LEME, D. M.; MARIN-MORALES, M. A. Chromosome aberration and micronucleus frequencies in Allium cepa cells exposed to petroleum polluted water - a case study. Mutation Research, v. 650, n. 1, p. 80-86, 2008.

http://dx.doi.org/10.1016/j.mrgentox.2007.10.006

MOULIS, J. M.; THEVÉNOD, F. New perspectives in cadmium toxicity: an introduction. Biometals, v. 23, n. 5, p. 763-768, 2010. http://dx.doi.org/10.1007/s10534-010-9365-6

NAIME, R.; NASCIMENTO, C. A. Monitoramento de $\mathrm{pH}$, temperatura, OD, DBO e condições microbiológicas das águas do arroio Pampa em Novo Hamburgo (RS). Estudos Tecnológicos, v. 5, p. 228-246, 2009.

OLIVEIRA, J. P. W.; DOS SANTOS, R. N.; PIBERNAT, C. C.; BOEIRA, J. M. Genotoxicidade e análises físico-químicas das águas do rio dos Sinos (RS) usando Allium cepa e Eichhornia crassipes como bioindicadores. Biochemistry and Biotechnology Reports, v. 1, n. 1, p. 15-22, 2012. http://dx.doi.org/10.5433/2316$5200.2012 \mathrm{v} 1 \mathrm{n} 1 \mathrm{p} 15$

PERON, A. P.; CANESIN, E. A.; CARDOSO, C. M. V. Potencial mutagênico das águas do Rio Pirapó (Apucarana, Paraná, Brasil) em células meristemáticas de raiz de Allium cepa L. Revista Brasileira de Biociências, v. 7, n. 2, p. 155-159, 2009.

PRÁ, D.; GUECHEVA, T.; FRANKE， I. R.; KNAKIEVICZ，T.; ERDTMANN, B.; HENRIQUES, J. A. P. Toxicidade e genotoxicidade do sulfato de cobre em planárias de água doce e camundongos. Journal of the Brazilian Society Ecotoxicology, v. 1, n. 2, p. 171-175, 2006.

SCALON, M. C. S. Avaliação dos efeitos genotóxicos da água do rio dos Sinos sobre peixes e vegetais. 2009. Dissertação (Mestrado em Qualidade Ambiental) - Centro Universitário Feevale, Novo Hamburgo, 2009.

SMAKA-KINCL, V.; STEGNARB, P.; LOVKAC, M.; TOMAND, M. J. The evaluation of waste, surface and ground water quality using the Allium test procedure. Mutation Research/Genetic Toxicology, v. 368, n. 3-4, p. 171-179, 1996. http://dx.doi.org/10.1016/S0165-1218(96)90059-2

SOBRAL, O.; MARIN-MORALES, M. A.; RIBEIRO, R. Could contaminat induced mutations lead to a genetic diversity overestimulation? Ecotoxicology, v. 22, p. 838846, 2013.

SOUZA, L. L.; ROCHA, P. S.; MARIN-MORALES, M. A. Avaliação in vivo e in vitro do potencial citotóxico, genotóxico e mutagênico da água e sedimento do rio Corumbataí (São Paulo - Brasil). 2014. Dissertação (Mestrado em Ciências Biológicas) - Instituto de Biociências, UNESP, Rio Claro, 2014. 
ZUCCARI, M. L.; GRANER, C. A. F.; LEOPOLDO, P. F. Determinação da demanda química de oxigênio (DQO) em águas e efluentes por método colorimétrico alternativo. Engenharia Agrícola, v. 20, n. 4, p. 69-82, 2005. 


Ambiente \& Água - An Interdisciplinary Journal of Applied Science
ISSN 1980-993X - doi:10.4136/1980-993X
www.ambi-agua.net
E-mail: ambi-agua@agro.unitau.br

\title{
Vulnerabilidade à contaminação das águas subterrâneas do município de Nova Palma, Rio Grande do Sul, Brasil
}

\author{
doi: 10.4136/ambi-agua.1390
}

Received: 05 May 2014; Accepted: 02 Oct. 2014

\author{
Carlos Alberto Löbler*; José Luiz Silvério da Silva \\ Universidade Federal de Santa Maria (UFSM), Santa Maria, RS, Brasil \\ *Autor correspondente: e-mail: carloslobler@gmail.com, \\ silverioufsm@gmail.com
}

\section{RESUMO}

A água subterrânea é um componente muito importante do ciclo hidrológico e exerce papel relevante, muitas vezes imperceptível aos olhos humanos. Esta pesquisa apresenta um estudo de caso da aplicação do sistema GOD na avaliação da vulnerabilidade do aquífero à contaminação. Foram pesquisados 36 poços tubulares de captação de água subterrânea do município de Nova Palma, cadastrados e situados na borda da Bacia Sedimentar do Paraná na região central do estado do Rio Grande do Sul, Brasil. Ocorrem dois sistemas de aquíferos na área em estudo: um constituído pelos derrames vulcânicos de rochas básicas e ácidas formadores do Sistema Aquífero Serra Geral (SASG) e outro, constituído de formações sedimentares arenosas, denominado de Sistema Aquífero Guarani (SAG), tanto em sua zona de afloramentos quanto na de confinamento. A água subterrânea no município é utilizada no abastecimento humano e outros usos. A aplicação do Sistema GOD indicou 8\% das captações em área da classe de vulnerabilidade insignificante; $15 \%$ baixa; $31 \%$ média; $31 \%$ alta e ainda, $15 \%$ da classe extrema. Os pontos potenciais de contaminação das águas subterrâneas identificados foram sete cemitérios e dois postos de combustíveis, os quais foram espacializados em cartogramas. Estes resultados poderão ser aplicados no planejamento do uso e ocupação dos solos do município, além de contribuir para a preservação dos recursos hídricos subterrâneos.

Palavras-chave: Geotecnologias, Sistema Aquífero Guarani, Sistema GOD.

\section{Contamination vulnerability of groundwater in the municipality of Nova Palma, Rio Grande do Sul, Brazil}

\begin{abstract}
While often imperceptible to the human eye, groundwater has a relevant role as a very important component of the hydrological cycle. This research presents a case study of the GOD system assessing the vulnerability of the aquifer to contamination. The study surveyed thirty-six groundwater extraction wells in the municipality of Palma Nova, registered and located on the edge of the Paraná Sedimentary Basin in the central region of Rio Grande do Sul, Brazil. Two aquifer systems occur in this area in both outcropping and confined zones; one is composed of volcanic flows known as the Serra Geral Aquifer System (SGAS) and the other of sedimentary sandstone rocks, known as the Guarani Aquifer System (SAG). The
\end{abstract}


GOD method indicates that $8 \%$ of the wells are in the insignificant class of vulnerability, $15 \%$ in the low class, $31 \%$ in the moderate class, $31 \%$ in the high class and $15 \%$ in the extreme vulnerability class. Seven cemeteries and two gas stations were identified as pollution source points. These results could be used for land use planning by the municipality's administration as well as for the preservation of groundwater quality.

Keywords: GIS technology, GOD method, Guarani aquifer.

\section{INTRODUÇÃO}

A interação homem natureza ocasiona modificações no meio. O homem detentor de técnicas, que se aprimoraram com o passar dos anos, busca alternativas para planejar o melhor uso e ocupação do espaço. Atividades como a indústria e a agricultura podem, no entanto, ocasionar danos ao meio ambiente e aos recursos hídricos, tanto superficiais como subterrâneos.

$\mathrm{Na}$ agricultura os diversos tipos de cultivos utilizam águas subterrâneas para irrigação e ainda podem gerar elevadas concentrações de alguns elementos químicos, pelo uso de fertilizantes e defensivos agrícolas.

O declínio da qualidade da água tornou-se uma questão global, relacionado ao crescimento da população e, especialmente, à expansão das atividades industriais e agrícolas. As mudanças climáticas também causando importantes alterações no ciclo hidrológico (UM-Water, 2011). A Comunidade Europeia estabeleceu normas importantes, como a Diretiva Quadro da Água (DQA) e a Diretiva das Águas Subterrâneas (DAS) que reconhecem as águas subterrâneas como um recurso natural valioso que, enquanto tal, deverá ser protegido da deterioração e da poluição química. Tal proteção é particularmente importante aos ecossistemas dependentes das águas subterrâneas e à utilização destas águas para o abastecimento destinado ao consumo humano. Salienta-se o intensivo uso da água subterrânea na agricultura no continente Europeu.

A valorização das águas subterrâneas ocorre em nível global, já que são essenciais para a manutenção da vida na Terra. As águas subterrâneas são reservas importantes por estarem disponíveis a qualquer momento, além de apresentarem baixo custo de armazenamento, constituindo-se em alternativa às águas superficiais, sujeitas a alterações mais frequentes na sua qualidade natural. As águas subterrâneas também são fonte de abastecimento em períodos de estiagens para rios e outros mananciais superficiais como nascentes, lagos e banhados. A água subterrânea é uma parte essencial do ciclo hidrológico, que tem importante papel na manutenção da biodiversidade (Custodio e Silva Junior, 2008).

Segundo Eckhardt et al. (2009), as águas subterrâneas vêm assumindo uma importância cada vez mais relevante como fonte de abastecimento, devido a uma série de fatores que restringem a utilização das águas superficiais. Muitos municípios brasileiros possuem seu abastecimento unicamente por meio de águas subterrâneas, pela facilidade de acesso ou mesmo por sua melhor qualidade física e química.

As águas subterrâneas são, contudo, também susceptíveis à contaminação. Segundo Foster et al. (2006), no mundo inteiro, os aquíferos estão sob perigo cada vez maior de contaminação em decorrência da urbanização, do desenvolvimento industrial, das atividades agrícolas e de mineração. Os custos para realizar a descontaminação de aquíferos são elevados tornando o processo inviável, assim é muito mais sensato preservar este recurso natural e renovável.

Dentre as fontes potenciais de poluição de aquíferos estão, também, os cemitérios, os quais liberam um liquido escuro de composição química potencialmente contaminante denominado necrochorume. Esse pode migrar no subsolo em diferentes escalas e com distintas composições físico-químicas e bacteriológicas. Também, os postos de combustíveis, 
que no país operam com óleo Diesel, gasolina e álcool, podendo liberar os BTEX (Benzeno, Tolueno, Etileno e Xileno), além dos hidrocarbonetos totais do petróleo. As lavagens e os lava-a-jato de veículos automotores são potencialmente contaminantes do meio ambiente subterrâneo, necessitando de monitoramento constante em poços construídos para esse fim, buscando identificar e evitar vazamentos de distintos produtos químicos como os resíduos de óleos e graxas (Kemerich et al., 2011).

O município de Nova Palma conta com uma população de 6.342 habitantes, com ocupação média de 20,23 hab. / $\mathrm{km}^{2}$ na área total do Município (IBGE, 2010). De acordo com OEA e GEF (2009), a distribuição da população na área de ocorrência do SAG não é homogênea, variando de 2,5 hab. $/ \mathrm{km}^{2}$ no estado do Mato Grosso a 118,6 hab. $/ \mathrm{km}^{2}$ no estado de São Paulo.

O meio urbano e rural do Município de Nova Palma é abastecido por águas subterrâneas, sendo que a captação, o tratamento com cloro e flúor, a distribuição e a administração na área urbana do município são de responsabilidade da Companhia Rio-Grandense de Saneamento (CORSAN). Uma vez que toda a população urbana do município depende desta fonte subterrânea para as suas múltiplas atividades diárias, esse recurso merece atenção especial e deve ser levado em conta no gerenciamento e gestão, no que diz respeito ao planejamento de uso e ocupação do solo, justificando-se assim a realização deste trabalho.

Ribeiro (2005) discorre sobre índices de vulnerabilidade: "A grande maioria dos métodos de avaliação da vulnerabilidade possuem uma natureza empírica, o que é veículo para o surgimento de um vasto leque de diferentes interpretações por diferentes técnicos. Essa subjetividade vai repercutir forçosamente na atribuição de valores numéricos a entidades de natureza descritiva". Na opinião desse autor torna-se necessário distinguirem-se dois tipos de vulnerabilidade: a intrínseca e a específica. A intrínseca considera unicamente as características geológicas, hidrológicas e hidrogeológicas do meio, sendo por isso independente do tipo de poluente gerado pelas atividades antropogênicas.

Já a vulnerabilidade específica deve ser utilizada sempre que se consideram também as propriedades de um poluente específico e as suas relações com os componentes de vulnerabilidade intrínseca. Neste trabalho, foi considerada a vulnerabilidade como sendo intrínseca.

Foster et al. (2013) consideram que a vulnerabilidade do aquífero se destina a representar a variação do nível de proteção natural ou a capacidade de atenuação do contaminante na zona de solo insaturado ou zona de semi-confinamento da água, localizadas acima do aquífero. Essa contaminação advém de processos físicos e químicos (filtração, biodegradação, hidrólise, adsorção, neutralização, e volatilização dispersão)

O Sistema GOD já foi utilizado na avaliação da vulnerabilidade de aquíferos à contaminação, em rochas sedimentares arenosas formadoras de aquíferos porosos intergranulares, na zona de afloramento (ZA), do Sistema Aquífero Guarani (SAG): OEA e GEF (2009); Silvério da Silva et al. (2007) em Santana do Livramento/Rivera; Martinez et al. (2008) no Município de Santa Cruz do Sul; Marion et al. (2010) no Campus da UFSM; Silvério da Silva e Descovi Filho (2010) na bacia do rio Santa Maria; Kemerich et al. (2011) no Município de Santa Maria; Ertel et al. (2012) no Município de Rosário do Sul; Terra et al. (2013) no Município de Santiago - todos no Estado do Rio Grande do Sul, Brasil.

Adotando a escala de município Löbler et al. (2014) realizaram um estudo de caso no Município de Nova Palma comparando duas bases de dados: uma do Sistema de Informações de Águas Subterrâneas/SIAGAS/CPRM e Imagens STRM (Shuttle Radar Topography Mission) considerando que as duas bases de dados podem ser utilizadas com eficácia no mapeamento de águas subterrâneas em escala local.

De acordo com OEA e GEF (2009), o SAG é a unidade hidroestratigráfica mais importante da porção meridional do continente sul-americano e está associado ao conjunto de 
rochas formadas por sedimentos originados da acumulação mecânica de partículas detríticas (partículas produzidas pela decomposição de rochas preexistentes e denominadas de "siliciclastos" - cascalho, areia, silte e argila) da Bacia do Paraná (Brasil e Paraguai), Bacia Chacoparanaense (Argentina) e Bacia Norte (Uruguai). Ele ocorre em parte de quatro países: na Argentina $\left(228.225 \mathrm{~km}^{2}\right)$, no Brasil $\left(735.918 \mathrm{~km}^{2}\right)$, no Paraguai $\left(87.536 \mathrm{~km}^{2}\right)$ e no Uruguai $\left(36.170 \mathrm{~km}^{2}\right)$.

Já as rochas vulcânicas que cobrem mais da metade do Estado do Rio Grande do Sul constituem aquíferos cristalinos fraturados, pertencentes ao Sistema Aquífero Serra Geral (SASG II) (Machado e Freitas, 2005; Scheibe e Hirata, 2008). Nesse sistema aquífero há aplicações do Sistema GOD no município de Erexim Silvério da Silva et al. (2013); Terra et al. (2013) em Santiago; Löbler et al. (2013), no município de Restinga Seca; e Silvério da Silva et al. (2014), no Município de Boa Vista do Cadeado - todos, também, no estado do Rio Grande do Sul, Brasil.

De acordo com Machado e Freitas (2005), o SASG II ocupa a parte oeste do estado do Rio Grande do Sul, e ocorre na Argentina (Curuzú Cuatiá) e no Uruguai com a denominação de Formação Arapey (OEA e GEF, 2009). Suas litologias são predominantemente constituídas por riólitos, riodacitos e em menor proporção basaltos fraturados. A capacidade específica é inferior a $0,5 \mathrm{~m}^{3} / \mathrm{h} / \mathrm{m}$, entretanto, excepcionalmente em áreas mais fraturadas ou com arenitos na base do sistema, podem ser encontrados valores superiores a $2 \mathrm{~m} / \mathrm{h} / \mathrm{m}$.

O presente trabalho teve como objetivo elaborar um estudo sobre a vulnerabilidade à contaminação das águas subterrâneas do Município de Nova Palma, com uso do sistema GOD de Foster et al. (2006), aliado ao uso de geotecnologias. Também se realizou o mapeamento dos pontos contaminantes (cemitérios e postos de combustíveis) dentro do município.

O sistema GOD tem fácil aplicabilidade, por ter plena sintonia com os dados disponíveis e ainda, em uma revisão da literatura, se constatou ser um método de bons e confiáveis resultados. Segundo Foster et al. (2006), esse método é adaptado às condições brasileiras e caribenhas, sendo necessário no mínimo o conhecimento de três parâmetros do aquífero. $\mathrm{Na}$ literatura existem vários outros métodos de avaliação da vulnerabilidade de aquíferos à contaminação, como o DRASTIC de Aller et al. (1987), que utiliza sete parâmetros, e o Índice de Susceptibilidade (IS) de Ribeiro (2005), que inclui o uso do solo.

\section{MATERIAIS E MÉTODOS}

\subsection{Coleta de dados}

Para a elaboração do presente trabalho utilizaram-se dados secundários, construindo-se um banco de dados referente às captações por poços no município de Nova Palma. Foram reunidos dados através de pesquisas no sítio da Companhia de Pesquisas de Recursos Minerais/CPRM e no Sistema de Informações de Águas Subterrâneas/SIAGAS. Inicialmente se identificou o número de cadastro do poço (ID de 10 dígitos, com o número do poço), as coordenadas no sistema Universal Transversa de Mercator (UTM), a cota altimétrica do terreno $(\mathrm{m})$, a(s) formação(ões) geológica(s) penetrada(s) na perfuração do poço, a condição do meio aquífero (se é livre, confinado ou semi-confinado), o nível estático da água (m) (condição sem bombeamento, ou se é surgente) e os usos da água subterrânea.

Estão cadastrados 36 poços para o município de Nova Palma na base de dados CPRM/SIAGAS, sendo que desses apenas 13 possuíam todos os parâmetros catalogados necessários para aplicar-se o sistema GOD (Foster et al., 2006). Esses parâmetros são: $\mathbf{G}=$ Grau de confinamento do poço, $\mathbf{O}=$ ocorrência de estratos de cobertura litológica penetrados (ex. rochas vulcânicas e sedimentares do Sistema Aquífero Serra Geral/SASG e Sistema Aquífero Guarani/SAG) e $\mathbf{D}=$ nível estático ou nível da água, conforme é apresentado na Figura 1. 
Também foram coletados dados em campo com visitas aos proprietários dos poços, os quais foram sistematizados, e então usados nas avaliações espaciais através de geotecnologias. Estes dados são referentes ao nível estático, para atualizar e mesmo completar as informações disponíveis. Também foram registradas imagens das condições atuais das captações, dos pontos potenciais de contaminação e dos principais usos da água.

Os pontos potencialmente contaminantes foram localizados no território do município, com uso do Global Position System/GPS Garmim Etrex manual. Adotou-se o sistema de coordenadas UTM, com o fuso 22, o Datum horizontal SAD69, e o Datum vertical Porto de Imbituba/Santa Catarina. Esse sistema também é usado pelo SIAGAS da CPRM. No presente estudo foram mapeados dois postos de combustíveis e sete cemitérios no meio urbano e rural do município.

\subsection{Descrição do método}

Foster et al. (2006) caracterizam a vulnerabilidade intrínseca (natural) do aquífero à contaminação, adotando os seguintes parâmetros: (G) Tipo de ocorrência da água subterrânea; (O) Classificação dos estratos acima da zona saturada do aquífero, em termos do grau de consolidação e caráter litológico; (D) Determinação da profundidade do nível freático. A figura 1 descreve passo a passo os procedimentos utilizados, explanando as etapas que compõem o sistema GOD.

Após a pontuação de cada etapa é feito o produto dos três valores, obtendo-se a(s) classe(s) de vulnerabilidade à(s) qual/quais pertence(m) o aquífero. Esta vulnerabilidade pode ser classificada como: desprezível $(0$ a 0,1$)$, baixa $(0,1$ a 0,3$)$, média $(0,3$ a 0,5$)$, alta $(0,5$ a $0,7)$ e extrema $(0,7$ a 1,0$)$.

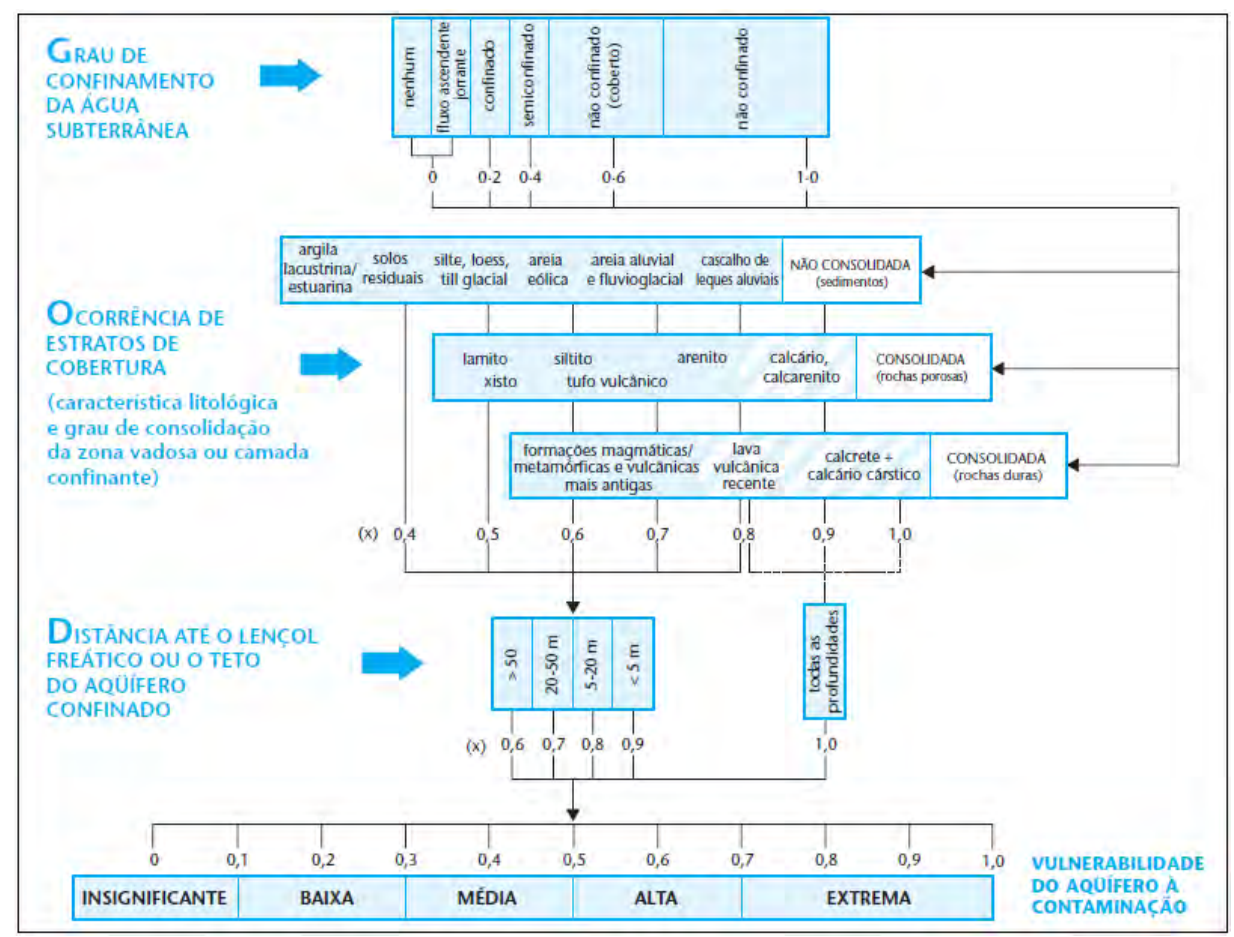

Figura 1. Sistema $G O D$ de avaliação da vulnerabilidade do aquífero à contaminação.

Fonte: Foster et al. (2006).

\subsection{Espacialização dos dados}

A aplicação de geotecnologias permite a espacialização dos pontos de captação de água e ainda a localização do ponto contaminante no mapa das classes de vulnerabilidade à 
contaminação intrínseca, tornando-se possível assim sugerir áreas mais adequadas para instalação de empreendimentos potencialmente poluidores do meio subterrâneo. Esses estudos devem ser acompanhados do uso dos Sistemas de Informações Geográficas/SIGs, que segundo Muradas et al. (2010) permitem a manipulação e atualização dos dados de forma dinâmica e constante.

Para a delimitação da área do estudo, optou-se pelos limites políticos do município de Nova Palma, disponíveis na malha digital do IBGE (2005), com limites municipais e urbanos, e plotados com uso do Programa ArcGIS 10. Após a obtenção dos dados no sistema SIAGAS/CPRM (CPRM, 2013) e em pesquisa de campo, foi realizado o seu tratamento em ambiente SIG.

Para a interpolação dos dados foi usado o programa Surfer 8 e o método de krigagem. Esse foi o que melhor representou a realidade espacial dos pontos distribuídos em vários locais da área avaliada. O Surfer 8 é uma importante ferramenta que utiliza algoritmos matemáticos para gerar suas curvas, otimizando o trabalho do usuário e permitindo sobrepor mapas com diferentes planos de informações. Após isso, foi feito o Overlay (ferramenta desse programa que permite cruzar mapas dentro de uma mesma projeção cartográfica).

Para a construção do mapa de tendência de direção de fluxo das águas subterrâneas, foram usadas as cotas altimétricas extraídas da base de dados CPRM/SIAGAS. Utilizou-se o programa Surfer8 espacializando-se a Superfície Potenciométrica (avaliada pela diferença entre a cota da "boca" do poço e o nível da água em cada poço tubular), indicando a tendência de fluxo das águas subterrâneas. A tendência de direção de fluxo subterrâneo é fundamental para entendermos a dinâmica subterrânea e avaliar possíveis empreendimentos poluidores.

\section{RESULTADOS E DISCUSSÃO}

No município de Nova Palma os principais usos da água subterrânea são para o abastecimento humano (doméstico, urbano e em comunidades rurais) e usos na indústria (múltiplos). Já as águas superficiais possuem uso também na irrigação, principalmente na produção de arroz, e na produção de energia elétrica, com destaque para a Usina Hidroelétrica de Dona Francisca, e ainda para o lazer (recreação, pesca e esportes náuticos).

Conforme se observa na Figura 2, os poços da CORSAN seguem padrões de construção e proteção da ABNT/NBR 12.244/2006 (ABNT, 2006). Estes poços também se encontram de acordo com as normas da legislação estadual que trata da outorga, contidas no Decreto Estadual No 42.047/2002 (Rio Grande do Sul, 2002), e segundo as quais, o poço deve dispor de tubo lateral (tubo guia) para medições de nível da água, hidrômetro, selo sanitário em concreto com no mínimo $1 \mathrm{~m}^{2}$ de área e uma área cercada com cerca de $10 \mathrm{~m}$, que visa impedir o acesso de pessoas não autorizadas e de animais.
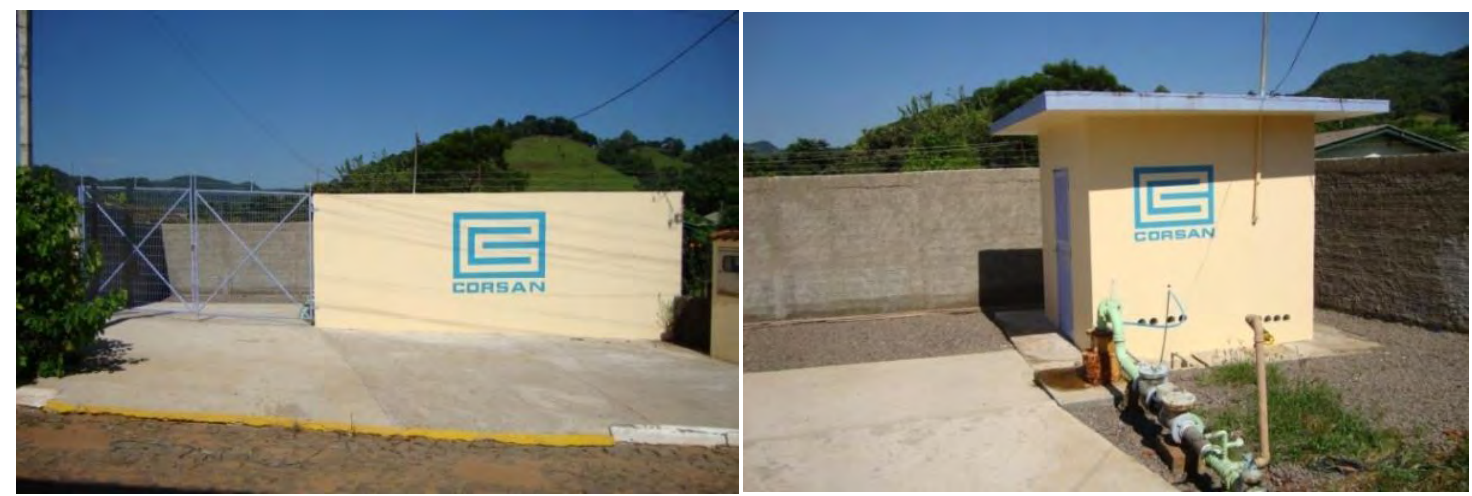

Figura 2. Imagens do poço 4300007677/SIAGAS/CPRM, localizado na área urbana do município de Nova Palma, RS. 
Após serem elaborados os parâmetros e intervalos de valores no sistema $G O D$ para as captações de água subterrânea, conforme apresentado na Figura 1 e Tabela 1, constatou-se que o município de Nova Palma apresenta em seu território cinco níveis de vulnerabilidade: $7,69 \%$ dos poços estão em área da classe de vulnerabilidade insignificante e de $15,38 \%$ na de vulnerabilidade baixa. Já 30,76\% estão em área de vulnerabilidade média, 30,76\% em área de vulnerabilidade alta. E finalizando, na área da classe de vulnerabilidade extrema situam-se 15 , $38 \%$ das captações do município.

$\mathrm{Na}$ Tabela 1 são apresentados alguns aspectos relativos às captações, selecionados para aplicação do sistema GOD. Observa-se que o nível estático dos poços varia entre um mínimo de $0,90 \mathrm{~m}$, quase surgente (aflorante), até cerca de 80,00 m. Também é apresentado o principal uso da água, que ocorre na categoria de abastecimento público (doméstico e/ou urbano) coincidindo com outras áreas do SAG no Brasil, de acordo com OEA e GEF (2009).

Tabela 1. Captações em 13 poços tubulares selecionados para aplicação do Sistema GOD.

\begin{tabular}{cclccc}
\hline $\begin{array}{c}\mathbf{N}^{\circ} \text { Cadastro } \\
\text { CPRM }\end{array}$ & $\begin{array}{c}\text { Nível Estático } \\
\text { (metros) }\end{array}$ & $\begin{array}{c}\text { Uso Água } \\
\text { (Abastecimento) }\end{array}$ & $\begin{array}{c}\text { Nota } \\
\text { GOD }\end{array}$ & $\begin{array}{c}\text { Classe de } \\
\text { Vulnerabilidade }\end{array}$ & $(\%)$ \\
\hline 4300007696 & 80,00 & Doméstico & 0,07 & Insignificante & 7,69 \\
4300002707 & 5,50 & Urbano & 0,29 & Baixa & - \\
4300007703 & 20,00 & Doméstico & 0,29 & Baixa & 15,38 \\
4300002705 & 2,29 & Urbano & 0,32 & Média & - \\
4300007678 & 1,39 & Múltiplo & 0,38 & Média & - \\
4300007715 & 20,00 & Doméstico & 0,34 & Média & 30,76 \\
4300002706 & 1,39 & Urbano & 0,38 & Média & - \\
4300002703 & 1,33 & Urbano & 0,54 & Alta & - \\
4300007675 & 1,33 & Múltiplo & 0,63 & Alta & - \\
4300007679 & 5,50 & Urbano & 0,64 & Alta & 30,76 \\
4300007677 & 1,89 & Urbano & 0,63 & Alta & - \\
4300007676 & 0,90 & Múltiplo & 0,81 & Extrema & - \\
4300002704 & 0,90 & Urbano & 0,72 & Extrema & 15,38 \\
\hline
\end{tabular}

Fonte: Organizado a partir da CPRM (2013).

As principais fontes pontuais potencialmente poluidoras encontradas no município foram: sete cemitérios de diferentes dimensões e dois postos de combustíveis, como se pode observar no mapa temático da Figura 3.

Com base nos dados coletados foi possível elaborar um mapa de pontos potenciais de contaminação localizados dentro do Município de Nova Palma. Na Figura 3 observa-se que a maioria dos poços está localizada em áreas de alta e média vulnerabilidade. Levando-se em conta que a maioria das fontes potenciais de poluição, tais como postos de combustíveis, cemitérios, despejos de esgotos estão concentradas na área urbana, deve-se manter um acompanhamento e monitoramento frequente para proteger as captações de águas subterrâneas, uma vez que essas são muito importantes no abastecimento doméstico e urbano do Município de Nova Palma, representando mais de 76\% dos usos (Tabela 1).

Observa-se ainda no mapa da Figura 3 que existem duas áreas de alta vulnerabilidade a oeste e a leste, com uma faixa extensa que vai de sul a norte apresentando uma vulnerabilidade baixa e insignificante. As áreas de maior vulnerabilidade geralmente se encontram próximas aos maiores rios que drenam a Bacia Hidrográfica onde se insere o município (Bacia G50/Alto Jacuí, classificação da Secretaria Estadual de Meio 
Ambiente/SEMA - RS), o rio Soturno e o rio Jacuí (figuras 3 e 4). Geralmente as planícies aluviais destes cursos de água apresentam nível freático próximo da superfície do terreno, caracterizando-as como mais vulneráveis à contaminação difusa. Vale ressaltar que essas áreas ribeirinhas são usadas para o cultivo do arroz irrigado, onde se utilizam fertilizantes a base de Nitrogênio, Fósforo e Potássio (NPK), além de agrotóxicos.
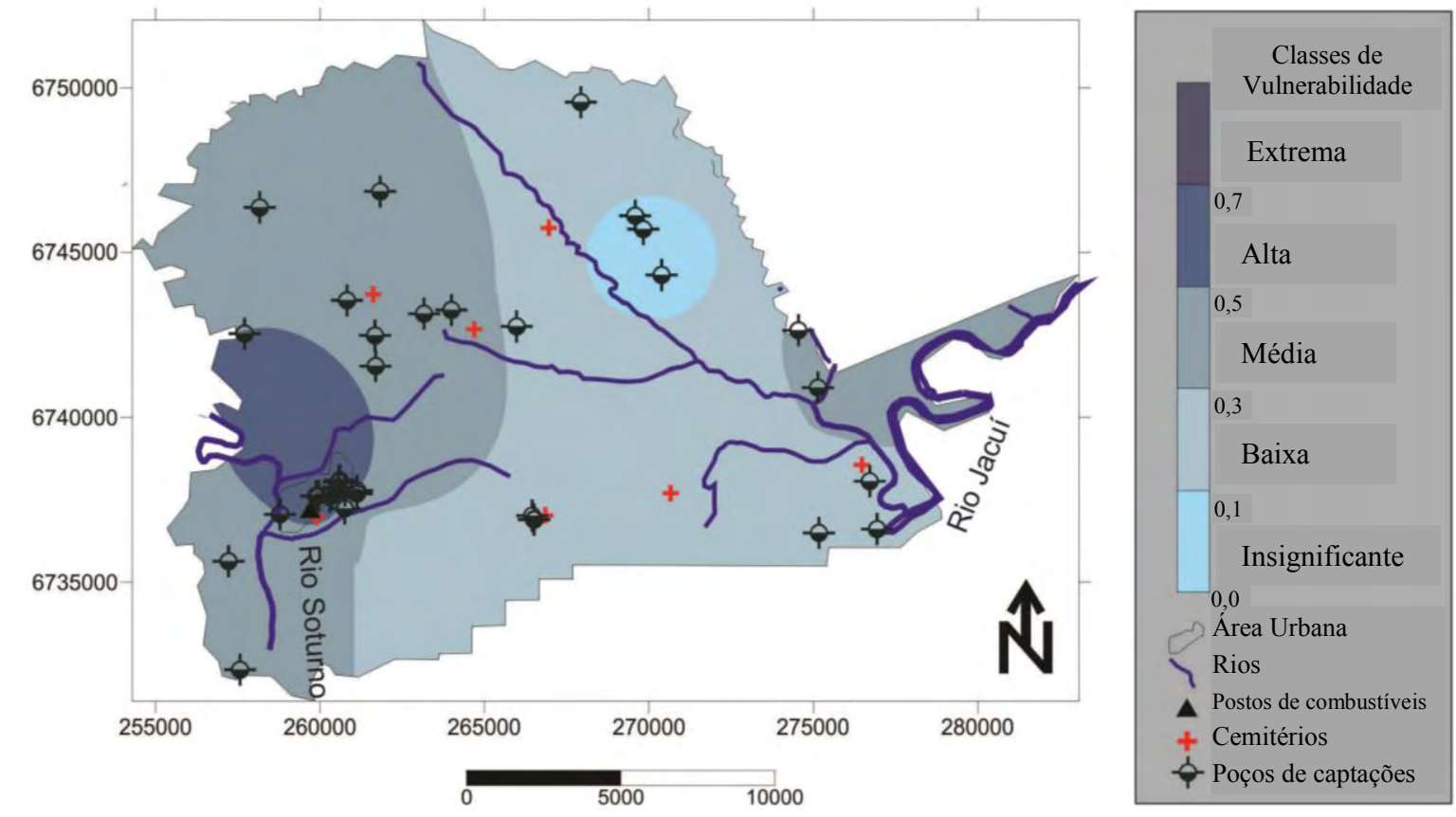

Figura 3.Mapa dos pontos potenciais de contaminação, poços tubulares de abastecimento, drenagem e vulnerabilidade GOD para o município de Nova Palma, RS.

Fonte: Malha digital de limite municipal e urbana do IBGE, dados CPRM/SIAGAS (sistema de coordenadas UTM, fuso 22) e trabalhos de campo.

Analisando-se o mapa de tendência de direção de fluxo subterrâneo (com base na superfície potenciométrica) apresentado na Figura 4, observa-se que os vetores indicam o fluxo da água subterrânea em direção aos principais cursos de água (Rio Jacuí e Rio Soturno), evidenciando uma contribuição do aquífero na perenização do fluxo de base dos rios, mesmo em períodos de estiagem. Esse comportamento está descrito em OEA e GEF (2009). Segundo Feitosa e Manoel Filho (2008), "em zonas de fluxo local de águas subterrâneas, parte da recarga alimenta o fluxo de base da rede de drenagem existente. Este tipo de contribuição denomina-se curso efluente, onde o aquífero cede água para o rio".

Como a água subterrânea assume principalmente dois sentidos, um sul e sudeste em direção à calha do Rio Jacuí e outro, oeste, em direção à calha do Rio Soturno, estima-se que as captações de água subterrânea localizadas próximas às calhas desses rios apresentem menor probabilidade de ficar sem água em períodos menos chuvosos (estiagens), devido à resposta aquífera ser suficiente para sua manutenção.

Também se deve levar em conta que há tendência de a direção de fluxo ser indicativa do movimento subterrâneo de água de um ponto com extrema ou alta vulnerabilidade para outros locais, podendo aumentar os riscos de contaminação, pois também se deve considerar o movimento lateral, que também é fonte de recarga de aquíferos (Custódio e Silva Jr., 2008). 


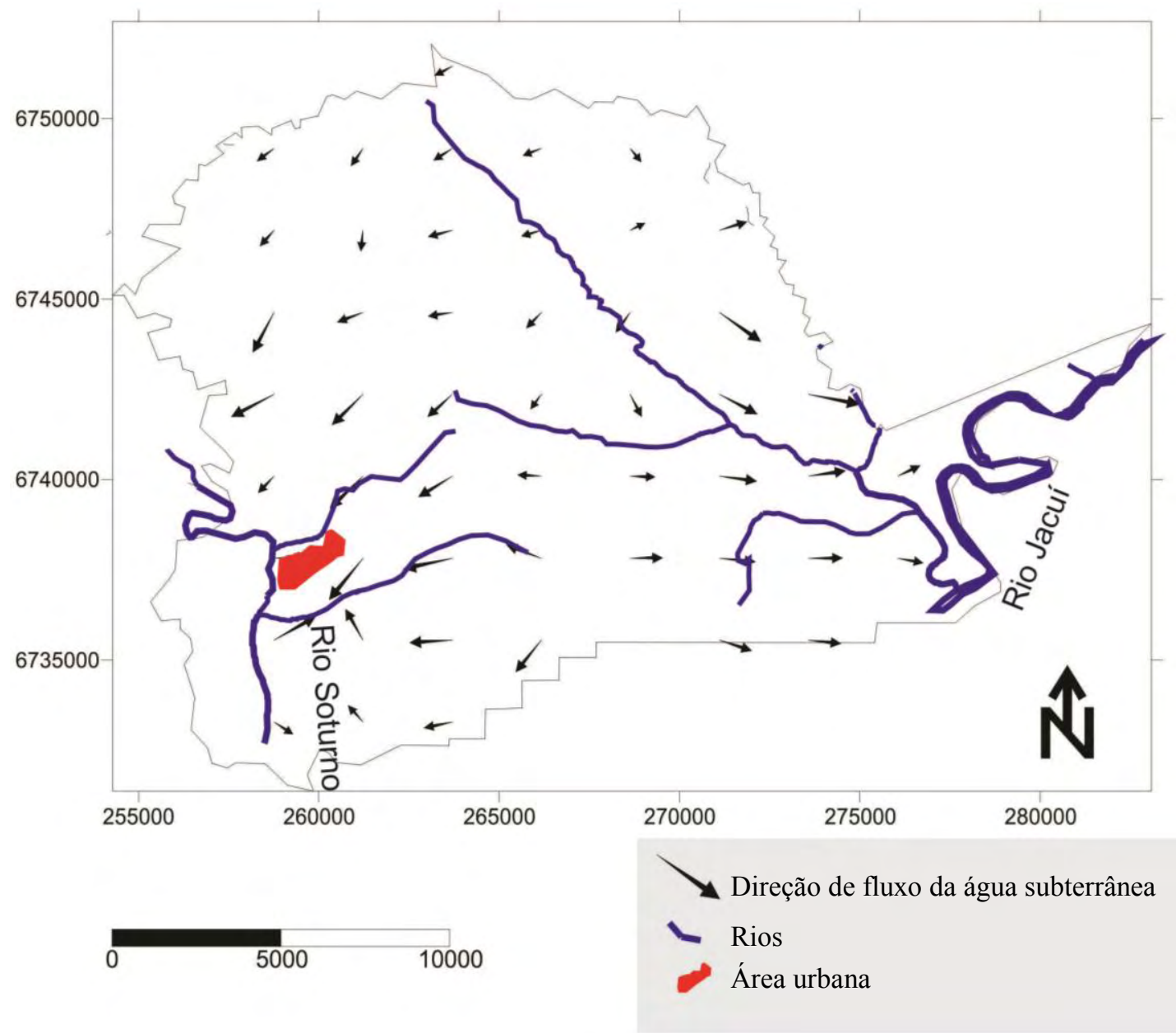

Figura 4. Mapa das tendências de direção dos fluxos das águas subterrâneas no Município de Nova Palma. Inclui seus principais rios, da Bacia Hidrográfica do Alto Jacuí (G50).

Fonte: Malha digital de limite municipal e urbana do IBGE e dados CPRM/SIAGAS (sistema de coordenadas UTM, fuso 22).

\section{CONCLUSÕES}

Os derrames de rochas vulcânicas exercem papel importantíssimo para o SAG na região: servem como proteção natural dos arenitos que estão confinados e que produzem água subterrânea com altas vazões.

As classes de vulnerabilidade predominantes foram a média e a alta, sugerindo-se atenção especial dos órgãos gestores em relação ao uso e à ocupação dos solos, na implantação de empreendimentos que possam causar algum tipo de contaminante no meio subterrâneo, e monitoramento dos já existentes. Portanto, sugere-se precaução no licenciamento ambiental do município.

A pesquisa contribuiu para a ampliação dos conhecimentos relativos às captações de água subterrânea no Município de Nova Palma, subsidiando assim a sua preservação ambiental e seu gerenciamento tanto na Zona de Afloramentos do Sistema Aquífero Guarani quanto na sua Zona de Confinamento, pelo Sistema Aquífero Serra Geral (OEA e GEF, 2009). Portanto, o sistema GOD apresentou resultados aproveitáveis para a área. 


\section{AGRADECIMENTOS}

Ao CNPq pela bolsa de iniciação cientifica no período da graduação e a FAPERGS/CAPES pela bolsa de mestrado conforme Edital 14/2012 concedidas ao autor principal. À CAPES pela bolsa de estágio sênior, Processo BEX 3954/13-7 concedida ao co-autor.

\section{REFERÊNCIAS}

ALLER, L. et al. DRASTIC: a standardized system for evaluating groundwater pollution potential using hydrogeologic setting. Washington: USEPA, 1987. $58 \mathrm{p}$.

ASSOCIAÇÃO BRASILEIRA DE NORMAS TÉCNICAS - ABNT. NBR 12244:2006. Poço tubular - construção de poço tubular para captação de água subterrânea. Rio de Janeiro, 2006.

COMPANHIA DE PESQUISA EM RECURSOS MINERAIS. Sistema de Informações de Águas Subterrâneas. Cadastro de poços tubulares do município de Nova Palma. Disponível em: <http://siagasweb.cprm.gov.br/layout/>. Acesso em: 20 jun. 2013.

CUSTODIO, E.; SILVA JUNIOR, G. C. da. Conceptos básicos sobre o papel ambiental das águas subterrâneas e os efeitos da sua explotação. Boletín Geológico y Minero, v. 119, p. 93-106, 2008.

ECKHARDT, R. R.; DIEDRICH, V. L.; FERREIRA, E. R.; STROHSCHOEN, E.; DEMAMAN, L. C. Mapeamento e avaliação da potabilidade da água subterrânea do Município de Lajeado, RS, Brasil. Revista Ambiente \& Água, Taubaté, v. 4, n. 1, p. 58-80, 2009. http://dx.doi.org/10.4136/ambi.agua.74

ERTEL, T.; LÖBLER, C. A.; SILVÉRIO DA SILVA, J. L. Índice de Vulnerabilidade das Águas Subterrâneas no Município de Rosário do Sul, Rio Grande do Sul. Revista Eletrônica em Gestão, Educação e Tecnologia Ambiental, Santa Maria, v. 7, p. 1400 -1408, 2012. http://dx.doi.org/10.5902/223611705962

FEITOSA, F. A. C.; MANOEL FILHO, J. Hidrogeologia: conceitos e aplicações. 3. ed., rev. e ampl. Rio de Janeiro: CPRM; LABHIH, 2008. 812 p.

FOSTER, S.; HIRATA, R.; GOMES, D.; D’ELIA, M.; PARIS, M. Proteção da qualidade da água subterrânea: um guia para empresas de abastecimento de água, órgãos municipais e agências ambientais. São Paulo: Servemar, 2006.

FOSTER, S.; HIRATA, R.; ANDREO, B. The aquifer pollution vulnerability concept: aid or impediment in promoting groundwater protection? Hydrogeology Journal, p. 737-750, 2013. http://dx.doi.org/10.1007/s10040-013-1019-7

INSTITUTO BRASILEIRO DE GEOGRAFIA E ESTATÍSTICA - IBGE. Censo demográfico 2010. Disponível em: http://www.censo2010.ibge.gov.br>. Acesso em: 21 jul. 2013.

INSTITUTO BRASILEIRO DE GEOGRAFIA E ESTATÍSTICA - IBGE. Malha municipal digital do Brasil: situação em 2005. Rio de Janeiro, 2007. 1 CD-ROM 
KEMERICH, P. D. C.; SILVÉRIO DA SILVA, J. L.; DESCOVI FILHO, L. L. V.; VOLPATTO, F.; SAUCEDO, E. M. Determinação da vulnerabilidade natural à contaminação da água subterrânea no Bairro Nossa Senhora do Perpétuo Socorro em Santa Maria-RS. Engenharia Ambiental, v. 1, p. 85-98, 2011.

LÖBlER, C. A.; SILVÉRIO DA SILVA, J. L.; MARTELli, G. V.; ERTEL, T. Pontos Potenciais de Contaminação e Vulnerabilidade Natural das Águas Subterrâneas do Município de Restinga Seca - RS. Revista Brasileira de Geografia Física, v. 6, n. 3, p. 500-509, 2013.

LÖBLER, C. A.; TERRA, L. G.; SILVÉRIO DA SILVA, J. L. Dados da CPRM/SIAGAS e Imagens STRM como Base Cartográfica na Elaboração de Mapeamento em Recursos Hídricos Subterrâneos em Escala Municipal: o Caso de Nova Palma, RS. Revista Brasileira de Geografia Física, v. 7, n. 3, p. 513-523, 2014.

MACHADO, J. L. F.; FREITAS, M. A. Projeto mapa hidrogeológico do estado do Rio Grande do Sul: escala 1:750.000: relatório final. Porto Alegre: CPRM, 2005.

MARION, F. A.; MELlO FILHO, J. A. S.; SILVÉRIO DA SILVA, J. L. Análise da vulnerabilidade natural das águas subterrâneas por geoprocessamento no Campus da UFSM/RS. Terr@ Plural, v. 4, p. 65-76, 2010.

http://dx.doi.org/10.5212/TerraPlural.v.4i1.065076

MARTINEZ, M. M.; SILVÉRIO DA SILVA, J. L.; LOPES, G. N. Avaliação da vulnerabilidade das águas subterrâneas no município de Santa Cruz do Sul, RS/Brasil. Agro@mbiente On-line, v. 2, p. 1-8, 2008.

MURADÁS, K.; COELHO, O. G. W.; WOJAHN, D. Levantamento de dados geomorfológicos e hidrogeológicos para mapeamento de vulnerabilidade de contaminação do Aquífero Guarani nos municípios de Portão e Estância Velha/RS utilizando o método DRASTIC. Revista Ambiente \& Água, Taubaté, v. 5, n. 3, p. $172-$ 194, 2010. http://dx.doi.org/10.4136/ambi-agua.161

ORGANIZAÇÃO DOS ESTADOS AMERICANOS - OEA; GLOBAL ENVIRONMENT FACILITY - GEF. Projeto de proteção ambiental e desenvolvimento sustentável do Sistema Aquífero Guarani. Programa Estratégico de Ação - PEA. [S.1.], 2009. 102 p. (Manuais e Documentos Técnicos, 5)

RIBEIRO, L. F. T. Um novo índice vulnerabilidade específico de aquíferos: formulação e aplicações. In: SIMPÓSIO DE HIDRÁULICA E RECURSOS HÍDRICOS DOS PAÍSES DE LÍNGUA OFICIAL PORTUGUESA (SILUSBA), 7., 2005, Évora. Anais... Évora: [s.n.], 2005. p. 16.

RIO GRANDE DO SUL. Secretaria do Meio Ambiente. Decreto n. 42.047, de 26 de dezembro de 2002. Disponível em: http:/www.legislacao.sefaz.rs.gov.br/Site/ Document.aspx?inpKey=106552\&inpCodDispositive $=$ \&inpDsKeywords $=10350$. Acesso em: out. 2014.

SCHEIBE, L. F.; HIRATA, R. O contexto tectônico dos sistemas aquíferos Guarani e Serra Geral em Santa Catarina: uma revisão. In: CONGRESSO BRASILEIRO DE ÁGUAS SUBTERRÂNEAS, 15., 2008, Natal. Anais... Natal: ABAS, 2008. 1 CD-ROM. 
SILVÉRIO DA SILVA, J. L.; BESSOUAT, C.; CAMPONOGARA, I.; FRANTZ, L. C.; GUIMARAENS, M.; FLORES, E. M. et al. Caracterização de áreas de recarga e descarga do SAG em Santana-Rivera e Quarai-Artigas. Estudo da vulnerabilidade na área de influência de Quarai-Artigas. In: ROBERTO MONTES, R. Aqüífero Guarani: avanços no conhecimento para sua gestão sustentável. Montevideu: Secretaria General del Proyecto Aquifero Guarani/OEA, 2007. v. 1, p. 1-20.

SILVÉRIO DA SILVA, J. L.; DESCOVI FILHO, L. L. V. Vulnerabilidade das águas subterrâneas na bacia hidrográfica do rio Santa Maria-RS. Revista Águas Subterrâneas, v. 24, p. 1-17, 2010.

SILVÉRIO DA SILVA, J. L.; DESCOVI FILHO, L. L. V.; LORENSI, R. P.; CRUZ, J. C.; ELTZ, F. L. Vulnerabilidade do aquífero serra geral à contaminação no município de Erechim - Rio Grande do Sul - Brasil. Ciência e Natura, v. 35, p. 10-23, 2013. http://dx.doi.org/10.5902/2179-460X0746

SILVÉRIO DA SILVA, J. L.; NASCIMENTO, L. M.; LÖBLER, C. A. Mapeamento das águas subterrâneas do município de Boa Vista do Cadeado/RS. Revista Monografias Ambientais, v. 14, p. 3061-3074, 2014. http://dx.doi.org/10.5902/223613085688

TERRA, L. G.; LÖBLER, C. A.; SILVÉRIO DA SILVA, J. L. Estimativa da vulnerabilidade à contaminação dos recursos hídricos subterrâneos do município de Santiago-RS. Revista Eletrônica em Gestão, Educação e Tecnologia Ambiental, v. 10, p. 22082218, 2013. http://dx.doi.org/10.5902/223611707887

UN-WATER. Policy brief: water quality. 2011. 22 p. Disponível em: $<$ http://www.unwater.org/> Acesso em: 20 dez. 2013. 


Ambiente \& Água - An Interdisciplinary Journal of Applied Science
ISSN 1980-993X - doi:10.4136/1980-993X
www.ambi-agua.net
E-mail: ambi-agua@agro.unitau.br

\title{
Manganês, zinco, cádmio, chumbo, mercúrio e crômio no chorume de aterro sanitário em Dourados, MS, Brasil
}

\author{
doi: 10.4136/ambi-agua. 1538
}

Received: 10 Oct. 2014; Accepted: 21 Nov. 2014

\author{
Priscilla Fracalossi Riguetti ${ }^{1}$; Cláudia Andréa Lima Cardoso ${ }^{2}$; \\ Alberto Adriano Cavalheiro'; Ervim Lenzi ${ }^{3}$; Antonio Rogério Fiorucci ${ }^{2}$; \\ Margarete Soares da Silva ${ }^{2^{*}}$ \\ ${ }^{1}$ Centro Universitário da Grande Dourados (UNIGRAN), Dourados, MS, Brasil \\ ${ }^{2}$ Universidade Estadual de Mato Grosso do Sul (UEMS), Dourados, MS, Brasil \\ Programa de Pós Graduação em Recursos Naturais (PGRN) \\ ${ }^{3}$ Universidade Estadual de Maringá (UEM), Maringá, PR, Brasil \\ Departamento de Química (DQI) \\ *Autor correspondente: e-mail: margaret@uems.br, \\ priscillariguetti@hotmail.com, claudia@uems.br, albecava@uems.br, \\ ervimlenzi@gmail.com, arfiorucci@yahoo.com.br
}

\section{RESUMO}

Neste estudo analisaram-se as concentrações de manganês, zinco, cádmio, chumbo, mercúrio e crômio, em amostras de chorume coletado no aterro sanitário de Dourados/MS. Foram retiradas amostras de dois reservatórios, um com tratamento aeróbio e outro com tratamento anaeróbio. Foram realizadas quatro coletas de 2012 a 2013, compreendendo períodos de seca e de chuvas. As concentrações dos metais foram determinadas por espectrometria de absorção atômica, modalidade chama. A comparação de resultados entre os dois reservatórios e entre as coletas foi realizada empregando-se análise de variância com nível de confiança de 95\%. A análise dos resultados mostrou diferenças, quanto à concentração dos metais, somente entre as coletas. Os elementos manganês, zinco e chumbo apresentaram maiores concentrações nos períodos chuvosos e os demais elementos nos períodos de seca. De forma geral, apenas o índice pluviométrico interferiu nas concentrações dos metais estudados, também não se observaram variações de $\mathrm{pH}$ e condutividade elétrica ao longo do experimento.

Palavras-chave: condutividade, índice pluviométrico, metais pesados.

\section{Manganese, zinc, cadmium, lead, mercury and chromium in leachate from a landfill in Dourados, MS, Brazil}

\begin{abstract}
This study analyzed concentrations of manganese, zinc, cadmium, lead, mercury and chrome in leachate samples collected in the landfill of Dourados, Mato Grosso do Sul, Brazil. Samples from two ponds were taken, one undergoing aerobic treatment and the other undergoing anaerobic treatment. Four collections were made between 2012 and 2013, during dry seasons and rainy seasons. Metal concentrations were determined by atomic absorption
\end{abstract}


spectrometry, flame mode. Comparison between the results for both ponds and between the sample collections was done using analysis of variance with a 95\% accuracy level. Analysis results revealed differences in metal concentration within the samples, with the elements manganese, zinc and lead displaying higher concentrations during rainy periods and the other elements during droughts periods. Only the pluviometric index interfered in the studied metal concentrations and there were no variations in $\mathrm{pH}$ and electrical conductivity noticed throughout the experiment.

Keywords: conductivity, heavy metals, pluviometric index.

\section{INTRODUÇÃO}

Atualmente, a quantidade de resíduos sólidos urbanos (RSU) gerados pela sociedade é motivo de preocupação social, ambiental e de saúde pública. Esses resíduos são formados, basicamente, por matéria orgânica, papéis, vidros, plásticos e metais, sendo que muitos desses materiais são ricos em substâncias nocivas ao meio ambiente e à vida (Celere et al., 2007).

A quantidade de RSU gerados no Brasil, em 2013, foi de 76 milhões de toneladas. Desse montante, apenas $58,3 \%$ foi depositado em aterros sanitários, o restante foi depositado em aterros controlados e lixões. Isso ocorreu em todos os Estados, sendo que nas regiões Norte, Nordeste e Centro Oeste estão $74 \%$ dos municípios que ainda utilizaram lixões e aterros controlados (ABRELPE, 2014). Considerando o Estado de Mato Grosso do Sul, apenas 27\% dos RSU foram destinados a aterros sanitários, $49 \%$ e $24 \%$, respectivamente, foram enviados a aterros controlados e lixões.

O aterro sanitário é mais adequado porque nele o solo é impermeabilizado, há sistemas de drenagem para líquidos e gases produzidos, o lixo é coberto com terra e compactado, entre outras medidas (Moreira et al., 2011). Nos aterros controlados e nos lixões não há impermeabilização e as substâncias provenientes da decomposição dos resíduos infiltram no solo atingindo águas subterrâneas e são carreadas pelas chuvas contaminando o meio ambiente. Outro agravante é que esses contaminantes irão persistir no ambiente até décadas após o fechamento do aterro (Cavallet et al., 2013; Santana et al., 2007).

No aterro sanitário a maior dificuldade é quanto ao destino do chorume coletado, pois é um efluente altamente contaminado e de difícil tratamento. Normalmente, o chorume é recirculado ou enviado para estações de tratamento de esgoto onde é submetido à degradação microbiológica e descartado, com o esgoto, em águas superficiais. Esse tratamento não é eficiente para chorume maduro, que apresenta nitrogênio amoniacal e substâncias húmicas, que são de difícil degradação e vários tipos de metais pesados (Morais et al., 2006).

A recirculação reduz a quantidade de compostos orgânicos e inorgânicos devido aos processos de adsorção, precipitação e complexação que ocorrem quando o chorume entra em contato com os resíduos aterrados e com o solo de cobertura, mas também não é uma solução permanente (Hipólito e Ezaki, 2006).

A grande dificuldade no tratamento do chorume deve-se ao fato dele ter composição química muito complexa. Ela pode variar de acordo com a natureza, forma de disposição e manejo dos resíduos, com a idade do aterro, com fatores climáticos, entre outros. De forma geral, pode-se dividir sua composição em quatro frações: matéria orgânica dissolvida, compostos orgânicos xenobióticos, macrocompostos inorgânicos como $\mathrm{Ca}, \mathrm{Mg}, \mathrm{Na}, \mathrm{K}, \mathrm{NH}^{4+}$, $\mathrm{Fe}, \mathrm{Cl}, \mathrm{SO}_{4}{ }^{2-}, \mathrm{HCO}_{3}{ }^{-}$, metais pesados e outros elementos potencialmente tóxicos como $\mathrm{Cd}, \mathrm{Cr}, \mathrm{Cu}, \mathrm{Pb}, \mathrm{Mn}, \mathrm{Ni}, \mathrm{Zn}, \mathrm{Hg}, \mathrm{As}, \mathrm{Ti}$, Se e Te (Morais et al., 2006; Moreira et al., 2011).

Os metais pesados reagem com ligantes difusores e com macromoléculas, o que lhes confere propriedades de bioacumulação, de biomagnificação na cadeia alimentar e persistência no ambiente, causando distúrbios nos processos metabólicos e danos aos sistemas 
biológicos de todos os seres vivos. Todos os metais bivalentes reagem facilmente com as proteínas através dos grupos tiois e amino, competindo com nutrientes essenciais.

O chumbo é absorvido pela proteína ligante do cálcio depositando-se nos ossos. Ele afeta todos os sistemas e órgãos do corpo humano, inclusive o sistema nervoso central e periférico (Moreira e Moreira, 2004).

O cádmio entra nas células sanguíneas, acumula-se nos rins, no fígado e nos músculos, podendo provocar lesão renal, irritação grave no estômago, enfraquecimento dos ossos e deformidades no esqueleto (Tavares e Carvalho, 1992).

O cromo, o manganês e zinco são elementos essenciais, mas, quando em excesso, são perigosos à saúde e ao equilíbrio ambiental. $\mathrm{O}$ cromo, no estado de oxidação hexavalente $\left(\mathrm{Cr}^{6+}\right)$ não tem função metabólica e é extremamente tóxico. Excesso de manganês pode causar distúrbios da fala e de memória e doenças degenerativas e excesso de zinco leva à interação competitiva com outros minerais essenciais à saúde (Cruz e Soares, 2011; Gomes et al., 2005; Sisinno, 2003).

Sendo assim, é necessário monitorar a concentração de metais pesados, como alerta ao descarte do chorume no ambiente. Na literatura encontraram-se estudos sobre metais pesados em chorume, mas não se encontrou monitoramento de forma sazonal. Assim, este estudo teve como objetivo monitorar as concentrações dos íons metálicos: cádmio $\left(\mathrm{Cd}^{2+}\right)$, chumbo $\left(\mathrm{Pb}^{2+}\right)$, cromo (Cr total), manganês $\left(\mathrm{Mn}^{2+}\right)$, mercúrio $\left(\mathrm{Hg}^{2+}\right)$ e zinco $\left(\mathrm{Zn}^{2+}\right)$, em amostras de chorume coletadas no aterro sanitário de Dourados, em épocas com diferentes índices pluviométricos.

O município de Dourados tem população estimada em 207.498 habitantes, com mais de 181.000 residindo em área urbana. O aterro sanitário foi construído em 2004, até então os RSU eram depositados num lixão. Em 2011 ele recebeu 64.422 toneladas de RSU, coletados de forma indiferenciada e produziu aproximadamente $12.480 \mathrm{~m}^{3}$ de chorume.

\section{MATERIAL E MÉTODOS}

No aterro, os resíduos dos serviços da saúde são aterrados em vala séptica e os demais são aterrados em trincheiras impermeabilizadas e com ductos para escoamento do chorume (Figura 1). O chorume bruto é conduzido até um tanque de homogeneização, na sequência ele passa por duas lagoas anaeróbias, para tratamento biológico e depois escoa para a lagoa facultativa, onde o tratamento é semiaeróbio, passando então para a lagoa 4, de maturação, onde o tratamento é aeróbio. Sempre que o volume da lagoa 4 atinge o limite máximo, o chorume é retirado e despejado sobre o aterro, passando pelo processo de recirculação. $\mathrm{O}$ escoamento entre as lagoas é permanente e a vazão varia de acordo com o índice pluviométrico.

A amostragem foi feita na lagoa anaeróbial e na lagoa 4. Em cada coleta foram preenchidos três frascos de $2000 \mathrm{~mL}$ para cada lagoa (triplicatas). O chorume depositado em cada frasco foi coletado de forma representativa: em diversos pontos da superfície da lagoa. As coletas ocorreram em outubro/2012 ( $1^{\mathrm{a}}$ coleta), fevereiro/2013 ( $2^{\mathrm{a}}$ coleta), agosto/2013 ( $3^{\text {a }}$ coleta) e novembro/2013 ( $4^{\mathrm{a}}$ coleta) (Instituto Adolfo Lutz, 2008).

Imediatamente após a coleta, mediu-se a temperatura, a condutividade elétrica e o pH das amostras, em cada frasco. As medidas de temperatura e condutividade foram realizadas num condutivímetro Digimed DM31, com compensação automática de temperatura com referência de $25,0^{\circ} \mathrm{C}$. As medidas de $\mathrm{pH}$ foram feitas potenciometricamente em um pHmetro Micronal B474, com eletrodo de vidro combinado.

Do volume coletado foram retirados $1000 \mathrm{~mL}$ de cada frasco, para digestão. $\mathrm{O}$ procedimento de ataque químico foi: para cada $1000 \mathrm{~mL}$ de amostra foi acrescentado $5 \mathrm{~mL}$ de $\mathrm{HNO}_{3} 65 \%$, PA, Vetec. As soluções foram aquecidas em chapa elétrica, à temperatura de 
$80^{\circ} \mathrm{C}$, até volume de $40 \mathrm{~mL}$, sendo transferido, quantitativamente, para um balão volumétrico de $50 \mathrm{~mL}$ e o volume aferido com água deionizada (Instituto Adolfo Lutz, 2008).

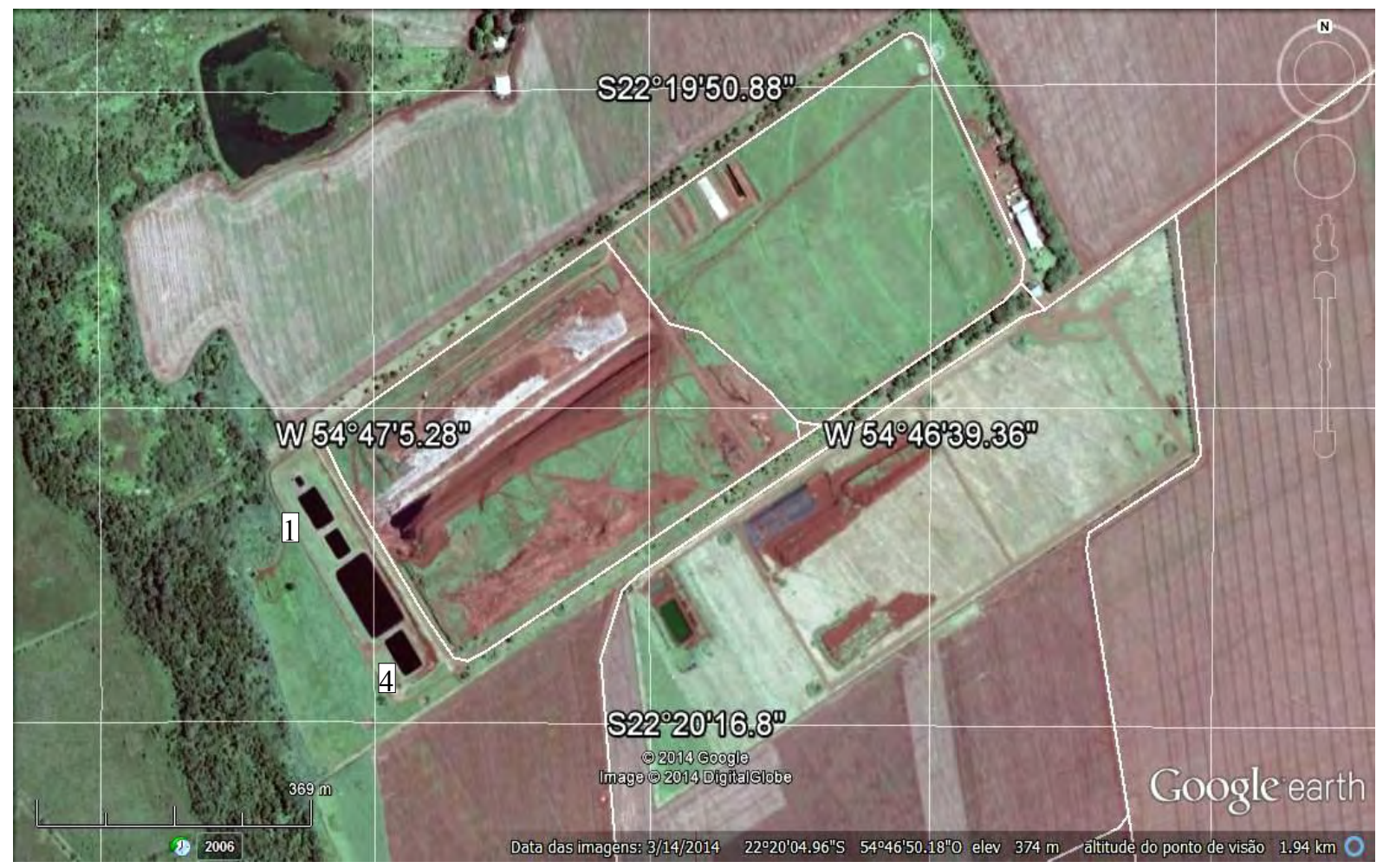

Figura 1. Vista aérea do aterro sanitário de Dourados, MS, Brasil. O reservatório menor é o tanque de homogeneização, na sequência têm-se as quatro lagoas de tratamento.

Fonte: Google Earth (2014).

A determinação da concentração dos metais foi feita por Espectrometria de Absorção Atômica, num espectrômetro Perkin Elmer AAnalyst 200, chama ar/acetileno, com lâmpadas de catodo oco, nos comprimentos de onda (nm): $\mathrm{Mn}=279,5 ; \mathrm{Zn}=213,9 ; \mathrm{Cd}=228,8 ; \mathrm{Pb}=$ 244,$8 ; \mathrm{Hg}=253,7$ e $\mathrm{Cr}=357,9$. Cada metal foi determinado por curva de calibração com os seguintes limites de detecção $\left(\mathrm{mg} \mathrm{L}^{-1}\right): \mathrm{Mn}=0,04 ; \mathrm{Zn}=0,020 ; \mathrm{Cd}=0,009 ; \mathrm{Pb}=0,025 ; \mathrm{Hg}=$ 0,016 e $\mathrm{Cr}=0,05$ (Keith, 1991).

O tratamento estatístico dos resultados foi realizado por análise de variância (ANOVA). Para comparação de resultados foi aplicado o teste de Tukey com nível de confiança de 95\%. Os cálculos foram realizados pelo Programa Estatístico Assistat 7.6 beta 2014.

\section{RESULTADOS E DISCUSSÃO}

Nas tabelas de resultados, a comparação estatística está indicada por vogais. Vogais iguais indicam que não houve diferença significativa entre os valores comparados. Há também os valores máximos permitidos para concentração de metais em efluentes líquidos, segundo Resolução $n^{0}$. 430/2011 do Conselho Nacional do Meio Ambiente - CONAMA, que dispõe sobre as condições e padrões de lançamento de efluentes (Brasil, 2011).

A concentração de metais, o $\mathrm{pH}$ e a condutividade elétrica do chorume podem variar em função da quantidade de água de chuvas que percola o aterro e do nível de decomposição da matéria orgânica presente no lixo. A degradação química e microbiológica dos resíduos acontece por etapas, que duram cerca de 15 anos (Cort et al., 2008).

$\mathrm{O}$ chorume novo (até 5 anos) é levemente ácido, tem $\mathrm{pH}$ menor que 7,0, pois predominam os ácidos voláteis e produtos nitrogenados sendo altamente biodegradável. Em 
aterros na fase metanogênica (acima de 5 anos) o pH é alcalino, superior a 8,0. Nesta fase, a decomposição da matéria orgânica é intensa com formação de gás carbônico $\left(\mathrm{CO}_{2}\right)$ e sais, principalmente bicarbonatos e sulfetos. Quanto maior for a quantidade de Sólidos Totais Dissolvidos (STD), maior será a condutividade do chorume (Athayde Júnior et al., 2009).

Todas as amostras apresentaram valores elevados de condutividade elétrica (Tabela 1), acima de $500 \mathrm{mS} \mathrm{m}^{-1}$. Isso indica que esse chorume é altamente poluidor podendo, ao longo do tempo, provocar a contaminação e a salinização de um provável ambiente receptor.

Tabela 1. Valores médios de temperatura, condutividade elétrica e $\mathrm{pH}$ das amostras de chorume coletadas no aterro sanitário de Dourados, MS, Brasil.

\begin{tabular}{|c|c|c|c|c|}
\hline Coletas & Lagoas & $\begin{array}{c}\text { Temperatura } \\
\left({ }^{\circ} \mathrm{C}\right)\end{array}$ & $\begin{array}{c}\text { Condutividade } \\
\text { Elétrica }\left(\mathrm{mS} \mathrm{m}^{-1}\right)\end{array}$ & $\mathbf{p H}$ \\
\hline \multirow[t]{2}{*}{$1^{\mathrm{a}}$ coleta } & Lagoa 1 & $29,7^{\circ} \pm 0,1$ & $838 \pm 1$ & $8,16 \pm 0,01$ \\
\hline & Lagoa 4 & $29,2^{\circ} \pm 0,1$ & $585 \pm 4$ & $8,27 \pm 0,01$ \\
\hline \multirow[t]{2}{*}{$2^{\mathrm{a}}$ coleta } & Lagoa 1 & $28,4 \pm 0,0$ & $603 \pm 14$ & $8,07 \pm 0,00$ \\
\hline & Lagoa 4 & $28,4 \pm 0,0$ & $592 \pm 10$ & $8,47 \pm 0,00$ \\
\hline \multirow[t]{2}{*}{$3^{\mathrm{a}}$ coleta } & Lagoa 1 & $25,8 \pm 0,4$ & $719 \pm 12$ & $8,15 \pm 0,01$ \\
\hline & Lagoa 4 & $25,7 \pm 0,3$ & $510 \pm 11$ & $8,20 \pm 0,01$ \\
\hline \multirow[t]{2}{*}{$4^{\mathrm{a}}$ coleta } & Lagoa 1 & $29,3 \pm 0,1$ & $651 \pm 7$ & $8,13 \pm 0,07$ \\
\hline & Lagoa 4 & $28,8 \pm 0,1$ & $541 \pm 1$ & $8,19 \pm 0,02$ \\
\hline CONAMA & & 40 & --- & 5 a 9 \\
\hline
\end{tabular}

Nota: --- Valor não referenciado na Resolução nº. 430/2011 (Brasil, 2011).

Vários autores avaliaram áreas contaminadas por lixões, por aterros e ambientes que receberam despejo de chorume. Todos detectaram condutividade elevada (Moreira et al., 2011; Sisinno e Moreira, 1996).

Os valores de $\mathrm{pH}$ entre 8,0 e 8,5 indicam a predominância de chorume proveniente de resíduos em estado avançado de decomposição. Como o aterro ainda está em operação, o chorume provém de resíduos em diferentes estágios de decomposição, mas a recirculação favorece o envelhecimento do mesmo.

Para se verificar a influência do índice pluviométrico (Figura 2) na concentração dos metais, no $\mathrm{pH}$ e na condutividade elétrica do chorume, as coletas foram realizadas em épocas com diferentes índices: outubro/2012 (139,6mm), fevereiro/2013 (223,5 mm), agosto/2013 $(4,2 \mathrm{~mm})$ e novembro/2013 $(74,8 \mathrm{~mm})$.

Os resultados não apontaram diferenças significativas de $\mathrm{pH}$ nem de condutividade elétrica, somente de concentração.

Conforme Hipólito e Ezaki (2006), o conteúdo total de metais num aterro corresponde ao somatório dos íons presentes no lixo, no chorume e no solo de cobertura. Eles estão, em grande parte, nos resíduos de origem e dependendo do tipo de resíduo, a lixiviação é muito lenta. No chorume bruto os íons ficam associados às partículas coloidais (orgânicas e inorgânicas) e na forma de complexos, o pH alcalino torna o meio redutor fazendo com que os íons metálicos sejam precipitados na forma de sulfetos, sulfatos, carbonatos e oxi-hidróxidos. Quando há recirculação também pode ocorrer retenção de metais no solo de cobertura por processos de adsorção e precipitação resultante do contato chorume/solo/lixo. 


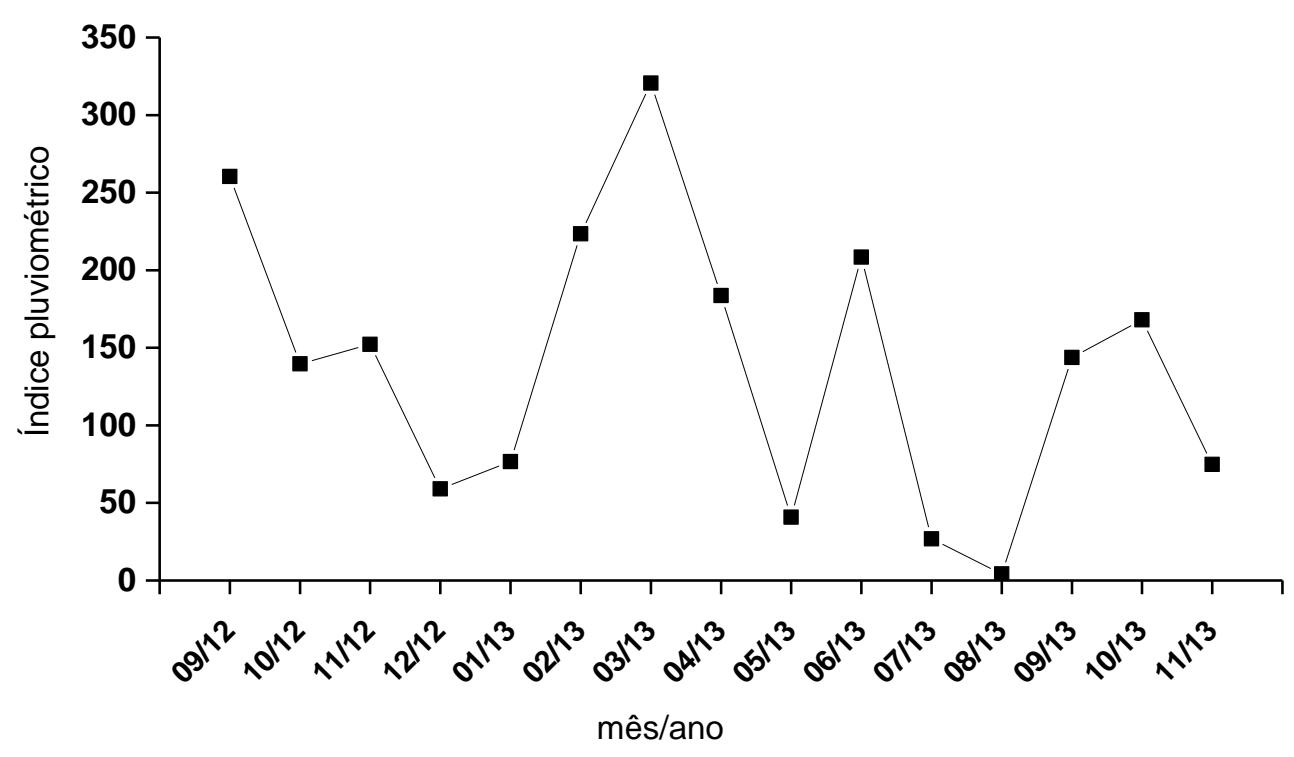

Figura 2. Variação do índice pluviométrico no município de Dourados, no período compreendido entre as coletas de chorume no aterro sanitário (EMBRAPA, 2014).

A comparação de resultados de concentração, entre as coletas (Tabela 2), foi feita com o intuito de se analisar a influência do índice pluviométrico sobre as mesmas. Nas coletas realizadas em outubro de 2012 e fevereiro de 2013, meses com maior precipitação, foram determinadas concentrações acima do limite de detecção, somente para manganês, zinco, e chumbo e nas coletas realizadas em agosto e novembro de 2013, meses com menor precipitação foram determinados também traços de cádmio, mercúrio e crômio.

Tabela 2. Comparação, entre as coletas, dos valores médios determinados para os metais, nas amostras de chorume coletadas no aterro sanitário de Dourados, MS, Brasil.

\begin{tabular}{|c|c|c|c|c|c|c|}
\hline \multirow[t]{2}{*}{ Amostras } & \multicolumn{6}{|c|}{ Concentrações $\left(\mathrm{mg} \mathrm{L}^{-1}\right)$} \\
\hline & Mn & $\mathbf{Z n}$ & $\mathbf{P b}$ & $\mathbf{C d}$ & Hg & Cr (total) \\
\hline \multicolumn{7}{|l|}{ Lagoa 1} \\
\hline $1^{\mathrm{a}}$ coleta & $1,97 \mathrm{a}$ & $0,270 \mathrm{a}$ & $0,335 \mathrm{~b}$ & nd & nd & nd \\
\hline $2^{\mathrm{a}}$ coleta & $1,34 \mathrm{~b}$ & $0,127 \mathrm{~b}$ & $0,587 \mathrm{a}$ & nd & nd & nd \\
\hline $3^{a}$ coleta & $0,83 \mathrm{c}$ & $0,099 \mathrm{~b}$ & $0,066 \mathrm{c}$ & 0,010 & $0,018 \mathrm{~b}$ & 0,02 \\
\hline $4^{\mathrm{a}}$ coleta & $0,06 \mathrm{~d}$ & $0,002 \mathrm{c}$ & $0,006 \mathrm{~d}$ & nd & $0,165 \mathrm{a}$ & 0,01 \\
\hline $\mathrm{CV} \%$ & 1 & 11 & 3 & --- & 3 & -- \\
\hline \multicolumn{7}{|l|}{ Lagoa 4} \\
\hline $1^{\mathrm{a}}$ coleta & $2,59 \mathrm{a}$ & $0,075 \mathrm{a}$ & $0,078 \mathrm{~b}$ & nd & nd & nd \\
\hline $2^{\mathrm{a}}$ coleta & $0,77 \mathrm{c}$ & $0,053 \mathrm{~b}$ & $1,280 \mathrm{a}$ & nd & nd & nd \\
\hline $3^{\mathrm{a}}$ coleta & $0,81 \mathrm{~b}$ & $0,044 \mathrm{~b}$ & $0,021 \mathrm{c}$ & 0,005 & 0,008 & 0,02 \\
\hline $4^{\mathrm{a}}$ coleta & $0,06 \mathrm{~d}$ & $0,001 \mathrm{c}$ & $0,004 \mathrm{c}$ & nd & 0,092 & 0,01 \\
\hline $\mathrm{CV} \%$ & 1 & 9 & 5 & --- & --- & --- \\
\hline CONAMA* & 1,0 & 5,0 & 0,5 & 0,2 & 0,01 & 0,05 \\
\hline
\end{tabular}

Nota: nd: elemento não detectado. CV\%: coeficiente de variação. --- Valores não calculados devido a várias amostras apresentarem concentração abaixo do limite de detecção do método de análise.

*Valores máximos permitidos pela Resolução nº. 430/2011 (Brasil, 2011). 
Na maioria das análises, as concentrações de manganês, zinco e chumbo foram maiores na $1^{\mathrm{a}}$ e na $2^{\mathrm{a}}$ coleta, para as duas lagoas, indicando que o maior volume de água no aterro influenciou na disponibilidade e mobilidade desses elementos.

Verifica-se que o manganês apresentou diferença de concentração entre todas as coletas, em ambas as lagoas. Os maiores valores foram determinados na $1^{\text {a }}$ coleta: $1,97 \mathrm{mg} \mathrm{L}^{-1}$ na lagoa 1 e $2,59 \mathrm{mg} \mathrm{L}^{-1}$ na lagoa 4 . A $1^{\text {a }}$ coleta ocorreu no mês com $2^{\circ}$ maior índice pluviométrico, indicando que o aumento da concentração foi proporcional ao volume de água no aterro até certo limite pois, diminuiu na $2^{\circ}$ coleta, que foi um mês com volume de chuva muito superior ao da $1^{\text {a }}$ coleta. $\mathrm{O}$ volume de água que percolou o aterro aumentou a disponibilidade e mobilidade desse metal, mas verifica-se também a influência da diluição do chorume, que foi muito maior na $2^{\circ}$ coleta.

Para o zinco, observou-se diferença de concentrarão entre as $1^{\mathrm{a}}, 2^{\mathrm{a}}$ e $4^{\mathrm{a}}$ coletas, não havendo diferença entre a $2^{\mathrm{a}}$ e $3^{\mathrm{a}}$ coletas, tanto na lagoa 1 quanto na lagoa 4 . Os maiores valores foram determinados na lagoa 1 sendo, $0,270 \mathrm{mg} \mathrm{L}^{-1}$ na $1^{\mathrm{a}}$ coleta e $0,127 \mathrm{mg} \mathrm{L}^{-1}$ na $2^{\mathrm{a}}$ coleta. Para a lagoa 4, o maior valor foi $0,075 \mathrm{mg} \mathrm{L}^{-1}$ na $1^{\text {a }}$ coleta. A influência do volume de chuvas sobre a concentração do zinco foi idêntica à do manganês.

Para o chumbo foi observado diferença de concentração entre todas as coletas realizadas na lagoa 1 e entre a $1^{a}, 2^{a}$ e $3^{a}$ coleta da lagoa 4 , não havendo diferença entre a $3^{\mathrm{a}}$ e $4^{\mathrm{a}}$ coleta. Diferentemente, os maiores valores foram determinados na $2^{\mathrm{a}}$ coleta: $0,587 \mathrm{mg} \mathrm{L}^{-1}$ na lagoa 1 e $1,280 \mathrm{mg} \mathrm{L}^{-1}$ na lagoa 4 . Neste caso, a diluição do chorume não reduziu a concentração indicando que a disponibilidade e mobilidade do chumbo foi maior que a do manganês e do zinco. Oliveira e Santana (2010) observaram aumento da concentração de chumbo em águas contaminadas por aterro, em períodos de chuva, também mostrando maior mobilidade desse elemento nessa época.

Verifica-se também que os menores valores determinados para o manganês, o zinco e o chumbo foram os da $4^{\text {a }}$ coleta, que ocorreu após o período de seca. Ocorreu que, no final do período de chuvas (após a $2^{\mathrm{a}}$ coleta) houve retirada de chorume da lagoa 4 então, o aumento do índice pluviométrico nos dois meses que antecederam a $4^{\mathrm{a}}$ coleta provocou diluição do chorume das lagoas, mas não foi suficiente para carreá-los do aterro, diminuindo a concentração desses metais no chorume. Além desse fator, o $\mathrm{pH}$ alcalino, continuamente, favorece a precipitação dos metais para o fundo das lagoas e a imobilização deles no lodo já que o mesmo é rico em matéria orgânica persistente à degradação microbiológica (Morais et al., 2006).

Os metais que, em algumas coletas, apresentaram concentração acima do estabelecido pela legislação foram: manganês, chumbo e mercúrio. $O$ manganês excedeu na $1^{\mathrm{a}}$ e na $2^{\mathrm{a}}$ coleta, na lagoa 1 e o chumbo excedeu na $2^{\mathrm{a}}$ coleta, nas duas lagoas, ambos em período de maior precipitação. O mercúrio apresentou concentrações acima da legislação na $3^{\mathrm{a}}$ e na $4^{\mathrm{a}}$ coleta, sendo o que mais excedeu o limite, com concentração 16,5 vezes superior a do limite estabelecido, na $4^{\mathrm{a}}$ coleta da lagoa 1 . Oliveira e Santana (2010) também verificaram excesso de chumbo e manganês, inclusive em períodos mais chuvosos, mas não de mercúrio.

A principal fonte de mercúrio, de chumbo e de zinco é a matéria orgânica resultante da decomposição do lixo. Os metais ferrosos e galvanizados, os plásticos e os papéis também são fontes de chumbo, de zinco e cádmio. O manganês é utilizado em baterias, porcelanas, etc. Os metais estão presentes também em lâmpadas, pilhas, restos de produtos químicos, eletrônicos e vários outros resíduos que vão para o aterro por coleta indiferenciada (Celere et al., 2007; Cort et al., 2008).

Quanto à comparação entre as lagoas 1 e 4 (Tabela 3), não foi possível fazer o cálculo estatístico para todas as coletas, devido às concentrações estarem muito próximo ou abaixo do limite de detecção. Considerando as coletas individualmente, observa-se que houve diferença entre as lagoas, para todos os metais determinados, exceto para o manganês na $3^{\text {a }}$ coleta. 
Tabela 3. Comparação, entre lagoas, dos valores médios determinados, para os metais, em cada coleta, nas amostras de chorume coletadas no aterro sanitário de Dourados, MS, Brasil.

\begin{tabular}{|c|c|c|c|c|c|c|c|}
\hline \multirow[t]{2}{*}{ Coletas } & \multirow[t]{2}{*}{ Lagoas } & \multicolumn{6}{|c|}{ Concentrações (mg L $\left.{ }^{-1}\right)$} \\
\hline & & Mn & $\mathbf{Z n}$ & $\mathbf{P b}$ & $\mathbf{C d}$ & $\mathrm{Hg}$ & Cr (total) \\
\hline \multirow[t]{3}{*}{$1^{\mathrm{a}}$ coleta } & Lagoa 1 & $1,97 \mathrm{~b}$ & $0,270 \mathrm{a}$ & $0,335 \mathrm{a}$ & nd & nd & nd \\
\hline & Lagoa 4 & $2,59 \mathrm{a}$ & $0,075 \mathrm{~b}$ & $0,078 \mathrm{~b}$ & nd & nd & nd \\
\hline & $\mathrm{CV} \%$ & 0,8 & 9 & 3 & --- & --- & --- \\
\hline \multirow[t]{3}{*}{$2^{a}$ coleta } & Lagoa 1 & $1,34 \mathrm{a}$ & $0,127 \mathrm{a}$ & $0,586 \mathrm{~b}$ & nd & nd & nd \\
\hline & Lagoa 4 & $0,77 \mathrm{~b}$ & $0,053 \mathrm{~b}$ & $1,280 \mathrm{a}$ & nd & nd & nd \\
\hline & $\mathrm{CV} \%$ & 1 & 12 & 2 & --- & --- & --- \\
\hline \multirow[t]{3}{*}{$3^{a}$ coleta } & Lagoa 1 & $0,83 \mathrm{a}$ & --- & $0,066 \mathrm{a}$ & --- & --- & --- \\
\hline & Lagoa 4 & $0,81 \mathrm{a}$ & --- & $0,021 b$ & --- & --- & --- \\
\hline & $\mathrm{CV} \%$ & 2 & --- & 5 & --- & --- & --- \\
\hline \multirow[t]{3}{*}{$4^{\mathrm{a}}$ coleta } & Lagoa 1 & --- & --- & --- & --- & $0,165 \mathrm{a}$ & --- \\
\hline & Lagoa 4 & --- & --- & --- & --- & $0,092 \mathrm{~b}$ & --- \\
\hline & $\mathrm{CV} \%$ & --- & --- & --- & --- & 2 & --- \\
\hline CONAMA* & & 1,0 & 5,0 & 0,5 & 0,2 & 0,01 & 0,05 \\
\hline
\end{tabular}

Nota: nd: elemento não detectado. CV\%: coeficiente de variação. --- Valores não calculados pelo programa devido às amostras apresentarem valores de concentração muito próximos ou abaixo do limite de detecção. *Valores máximos permitidos pela Resolução n ${ }^{\circ}$. 430/2011 (Brasil, 2011).

Para a maioria das coletas, a concentração dos metais determinados na lagoa 1, que contem o chorume em sua primeira fase de tratamento, foram superiores às concentrações determinadas na lagoa 4, exceto para o manganês, nas $1^{\mathrm{a}}$ e $3^{\mathrm{a}}$ coletas e para o chumbo na $2^{\mathrm{a}}$ coleta. Cort et al. (2008) também verificaram concentrações maiores de zinco, de manganês, de cádmio, de chumbo e de crômio no chorume bruto em relação ao chorume tratado.

Apesar de, na análise individual das coletas, se observarem diferenças entre as lagoas, quando se considera a média de todas as coletas (Tabela 4), verifica-se pouca influência do tratamento ao qual a lagoa é submetida, na concentração dos metais. Nesta comparação, somente o zinco apresentou maior concentração na lagoa 1.

Tabela 4. Comparação entre as lagoas, considerando o valor médio de todas as coletas, para cada metal determinado nas amostras de chorume do aterro sanitário de Dourados, MS, Brasil.

\begin{tabular}{lcccccc}
\hline \multirow{2}{*}{ Lagoas } & $\mathbf{7}$ Concentrações $\left(\mathbf{m g ~ L}^{-\mathbf{1}}\right)$ \\
\cline { 2 - 7 } & $\mathbf{M n}$ & $\mathbf{Z n}$ & $\mathbf{P b}$ & $\mathbf{C d}$ & $\mathbf{H g}$ & $\mathbf{C r}$ (total) \\
\hline Lagoa 1 & $1,05 \mathrm{a}$ & $0,124 \mathrm{a}$ & $0.248 \mathrm{a}$ & $0.002 \mathrm{a}$ & $0,045 \mathrm{a}$ & $0,007 \mathrm{a}$ \\
Lagoa 4 & $1,06 \mathrm{a}$ & $0,043 \mathrm{~b}$ & $0.345 \mathrm{a}$ & $0.001 \mathrm{a}$ & $0,025 \mathrm{a}$ & $0,007 \mathrm{a}$ \\
CV (\%) & 81 & 95 & 146 & 190 & 166 & 115 \\
CONAMA* & 1,0 & 5,0 & 0,5 & 0,2 & 0,01 & 0,05 \\
\hline
\end{tabular}

Nota: CV\%: coeficiente de variação. *Valores máximos permitidos pela Resolução ${ }^{\circ}$. 430/2011 (Brasil, 2011).

Considerando a média de todas as coletas, somente o mercúrio apresentou concentração muito superior ao limite estabelecido pela legislação. 


\section{CONCLUSÃO}

Devido ao tempo de funcionamento do aterro, agravado pelo processo de recirculação, o chorume apresentou $\mathrm{pH}$ básico, o que favorece a precipitação e complexação de metais. Esse fato influencia na concentração dos metais no chorume e pode torná-la não representativa do montante que realmente há no aterro.

O índice pluviométrico interfere na concentração dos metais no chorume, devido as suas diferenças de mobilidade e disponibilidade no material aterrado.

Considerando as coletas individualmente, os metais chumbo, manganês e mercúrio excederam os limites estabelecidos pela legislação, o que mais excedeu foi o mercúrio, atingindo concentração 16,5 vezes superior a do limite estabelecido. Isso mostra a relevância de uma análise prévia do chorume e tratamento do mesmo, antes de qualquer destino que possa impactar o meio, seja como fertilizante, despejo em ambientes aquáticos ou estações de tratamento de esgoto, entre outros.

Quanto ao tratamento anaeróbio-aeróbio, de forma geral, não houve diferenciação entre as lagoas, exceto para o zinco, que apresentou concentração maior na lagoa 1, que tem tratamento anaeróbio. Conclui-se que os processos de degradação biológica da matéria orgânica não interferiram, de maneira relevante, na disponibilização dos metais determinados.

Apesar da concentração da maioria dos elementos estarem abaixo do estabelecido, o descarte do chorume no ambiente, sem tratamento, é preocupante, levando-se em consideração o efeito cumulativo e tóxico de todos os metais determinados.

Todas as amostras analisadas apresentaram condutividade elevada, indicando a presença de grande quantidade de sólidos totais dissolvidos no chorume.

Para que o efluente analisado neste estudo seja descartado no ambiente é necessário fazer correções de $\mathrm{pH}$, de condutividade elétrica, controlar as quantidades de STD e de metais pesados, dentre outras substâncias nocivas à saúde e ao meio ambiente.

\section{AGRADECIMENTOS}

À administração do Aterro Sanitário de Dourados que permitiu acesso ao local e material de estudo, ao Laboratório de Agroquímica e Meio Ambiente da Universidade Estadual de Maringá-PR, que realizou as análises por espectrometria de absorção atômica, ao Programa de Pós Graduação em Recursos Naturais - PGRN/UEMS onde a pesquisa foi desenvolvida e à CAPES pelo suporte financeiro PROAP/CAPES n802116/2014.

\section{REFERÊNCIAS}

ASSOCIAÇÃO BRASILEIRA DE EMPRESAS DE LIMPEZA PÚBLICA E RESÍDUOS ESPECIAIS - ABRELPE. Panorama de resíduos sólidos no Brasil 2013. São Paulo: Grapa, 2014. 114p.

ATHAYDE JÚNIOR, G. B.; NÓBREGA, C. C.; GADELHA, C. L. M.; SOUZA, I. M. F.; FAGUNDES, G. S. Efeito do antigo Lixão do Roger, João Pessoa, Brasil, na qualidade de água subterrânea local. Revista Ambient. Água, Taubaté, v. 4, n. 1, p. 142-155, 2009. http://dx.doi.org/10.4136/ambi-agua.79

BRASIL. Resolução CONAMA n 430, de 13 de maio de 2011. Dispõe sobre condições e padrão de lançamento de efluentes, complementa e altera a Resolução ${ }^{\circ} 357$, de 17 de março de 2005, do Conselho Nacional do Meio Ambiente - CONAMA. Diário Oficial [da] União. Brasília, 16 mai. 2011. 
CAVAlLET, L. E.; CARVALHO, S. G. de; FORTES NETO, P. Metais pesados no rejeito e na água em área de descarte de resíduos sólidos urbanos. Revista Ambient. Água, Taubaté, v. 8 n. 3, p 229-238, 2013. http://dx.doi.org/10.4136/ambi-agua.1155

CELERE, M. S.; OLIVEIRA, A. S.; TREVIlATO, T. M. B.; SEGURA-MUÑOZ, S. I. Metais pesados presentes no chorume coletado no aterro sanitário de Ribeirão Preto, São Paulo, Brasil e sua relevância para saúde pública. Cadernos de Saúde Pública, Rio de Janeiro, v. 23, n. 4, p. 939-947, 2007. http://dx.doi.org/10.1590/S0102$311 \times 2007000400021$

CORT, E. P. D.; ALBERTI, V.; ROTTA, M.; BECEGATO, V.; MACHADO, W. C. P.; ONOFRE, S. B. Níveis de metais pesados presentes no chorume produzido em aterros sanitários da região sudoeste do Paraná. Geoambiente, Jataí, n. 11, p. 103-116, 2008.

CRUZ, J. B. F.; SOARES, H. F. Uma revisão sobre o zinco. Ensaios e Ciência: Ciências Biológicas, Agrárias e da Saúde, Valinhos, v. 15, n. 1, p. 207-222, 2011.

EMPRESA BRASILEIRA DE PESQUISA AGROPECUÁRIA - EMBRAPA. Guia clima. Estação da Embrapa Agropecuária Oeste - Dourados/MS. Disponível em: $<$ http://www.cpao.embrapa.br/clima/?lc=site/banco-dados/base_dados $>$. Acesso em: 10 nov. 2014.

GOOGLE EARTH. Vista aérea do aterro sanitário de Dourados, MS, Brasil. Imagem de 14/03/2014. Coordenadas: 22²0’04,96”S E 5446’50,18”O. Elevação 375 M.

GOMES, M. R.; ROGERO M. M.; TIRAPEGUI, J. Considerações sobre cromo, insulina e exercício físico. Revista Brasileira de Medicina do Esporte, São Paulo, v. 11, n. 5, p. 262-266, 2005. http://dx.doi.org/10.1590/S1517-86922005000500003

HYPÓLITO, R.; EZAKI, S. Íons de metais pesados em sistema solo-lixo-chorume-água de aterros sanitários da região metropolitana de São Paulo. Águas Subterrâneas, São Paulo, v. 20, n. 1, p. 99-114, 2006.

INSTITUTO ADOLFO LUTZ. Métodos físico-químicos para análise de alimentos. 4. ed. São Paulo: Instituto Adolfo Lutz, 2008. 1020 p.

KEITH, L. H. Compilation of EPA's Sampling and analysis methods. 2. ed. New York: Lewis Publishers, 1991. $803 \mathrm{p}$.

MORAIS, J. L.; SIRTORI, C.; PERALTA-ZAMORA, G. Tratamento de chorume de aterro sanitário por fotocatálise heterogênea integrada a processo biológico convencional. Química Nova, São Paulo, v. 29, n. 1, p. 20-23, 2006. http://dx.doi.org/10.1590/S010040422006000100005

MOREIRA, C. A.; OIVEIRA, M. T. de; SILVA, A. C. da. Estudo geofísico em aterro controlado disposto sobre rochas fraturadas. Revista Ambient. Água, Taubaté, v. 6, n. 2, p. 180-190, 2011. http://dx.doi.org/10.4136/ambi-agua.193

MOREIRA, F. R.; MOREIRA J. C. Os efeitos do chumbo sobre o organismo humano e seu significado para a saúde. Revista Panamericana de Salud Pública, Washington, v. 15, n. 2, p. 119-29, 2004.

OLIVEIRA, D. L.; SANTANA, G. P. Influência do aterro municipal de Manaus sobre as águas superficiais da circunvizinhança: um enfoque ao estudo de metais pesados. Caminhos de Geografia, Uberlândia, v. 11, n. 34, p. 75-83, 2010. 
SANTANA, G. P.; BARRONCAS, P. S. R. Estudo de metais pesados (Co, $\mathrm{Cu}, \mathrm{Fe}, \mathrm{Cr}, \mathrm{Ni}$, $\mathrm{Mn}, \mathrm{Pb}$ e $\mathrm{Zn}$ ) na Bacia do Tarumã-Açu Manaus-AM. Acta Amazônica, Manaus, v. 37, n. 1, p. 111-118, 2007. http://dx.doi.org/10.1590/S0044-59672007000100013

SISINNO, C. L. S. Disposição em aterros controlados de resíduos sólidos industriais nãoinertes: avaliação dos componentes tóxicos e implicações para o ambiente e para a saúde humana. Cadernos de Saúde Pública, Rio de Janeiro, v. 19, n. 2, p. 369-374, 2003. http://dx.doi.org/10.1590/S0102-311X2003000200003

SISINNO, C. L. S.; MOREIRA, J. C. Avaliação da contaminação e poluição ambiental na área de influência do aterro controlado do Morro do Céu, Niterói, Brasil. Cadernos de Saúde Pública, Rio de Janeiro, v. 12, n. 4, p. 515-523, 1996. http://dx.doi.org/10.1590/S0102-311X1996000400010

TAVARES, T. M.; CARVALHO, F. M. Avaliação de exposição de populações humanas a metais pesados no ambiente: exemplos do Recôncavo Baiano. Química Nova, São Paulo/SP, v. 15, n. 2, p. 147-154, 1992. 


Ambiente \& Água - An Interdisciplinary Journal of Applied Science
ISSN 1980-993X - doi:10.4136/1980-993X
www.ambi-agua.net
E-mail: ambi-agua@agro.unitau.br

\title{
Avaliação de sistemas de gestão ambiental em granjas de suínos
}

\author{
doi: 10.4136/ambi-agua.1504
}

Received: 14 Sep. 2014; Accepted: 22 Oct. 2014

\author{
Bruno Vieira de Carvalho; Angelita Pereira Melo e Sousa; \\ Francisco Rafael Martins Soto ${ }^{*}$ \\ Instituto Federal de Educação, Ciência e Tecnologia de São Paulo (IFSP-SRQ), São Roque, SP, Brasil \\ *Autor correspondente: e-mail: chicosoto34@gmail.com, \\ brunov.carvalho94@gmail.com, angelmelo83@gmail.com
}

\section{RESUMO}

Considerando os impactos ambientais causados pela criação intensiva de suínos, a importância econômica e crescimento desta atividade, este estudo teve como objetivo avaliar o uso de boas práticas de gestão ambiental em granjas localizadas no Estado de São Paulo/Brasil. Para isto, entrevistas exploratórias foram realizadas com gerentes ou proprietários de 37 granjas escolhidas aleatoriamente. A partir dos dados obtidos, foi possível elaborar um questionário com oito perguntas e criar um banco de dados eletrônico contendo perguntas e respostas. Para cada processo realizado nas granjas, foram atribuídos pesos de 1 a 10 de acordo com sua importância em prevenir ou atenuar a degradação ambiental. As granjas foram, então, classificadas como satisfatórias, satisfatórias com restrições e insatisfatórias se suas pontuações fossem maiores ou iguais a 70 pontos, entre a 51 e 69 pontos ou inferiores a 51 pontos, respectivamente. Os resultados mostraram que aproximadamente $62 \%$ das granjas investigadas foram consideradas insatisfatórias uma vez que tiveram pontuação média de 43,61 pontos. Aproximadamente $11 \%$ e $27 \%$ foram satisfatórias com restrições e satisfatórias, respectivamente, à medida que tiveram pontuações de 59,50 e 84,40 pontos. Para os itens considerados importantes, como o uso de biodigestores e práticas de compostagem, 51,35\% não possuíam biodigestores e $67,55 \%$ e $62,15 \%$, respectivamente, não utilizavam práticas de compostagem ou o faziam de forma insatisfatória. Ficou evidente então a necessidade de criação de programas de incentivo para melhoria no manejo dos resíduos de suínos em granjas do estado de São Paulo.

Palavras-chave: biodigestor, compostagem, dejeto suíno.

\section{Evaluation of environmental management systems on swine farms}

\begin{abstract}
Due to the environmental impacts caused by intensive swine farming and the economic importance and growth of this activity, this study evaluated environmental management practices on swine farms located in the state of São Paulo/Brazil. In order to accomplish this, exploratory interviews were conducted with the managers or owners of 37 randomly selected farms. The resulting data was then used to create a questionnaire that contained eight questions, and to also create an electronic database containing questions and answers. Weights, varying from 1 to 10 based upon their importance in preventing or mitigating
\end{abstract}


environmental degradation, were assigned for each process carried out on the farm. The farms were then classified as satisfactory, satisfactory with restrictions and unsatisfactory, if their scores were greater than or equal to 70 points, between 51 and 69 points or lower than 51 points, respectively. The results showed that approximately $62 \%$ of the swine farms investigated were considered unsatisfactory, since their average score was 43.61. Approximately $11 \%$ and $27 \%$ were deemed satisfactory with restrictions and satisfactory, respectively, since their scores were 59.50 and 84.40 . For items considered important, such as the use of biodigesters and compost, $51.35 \%$ used no biodigesters and $67.55 \%$ and $62.15 \%$, respectively, used no compost or their practices were unsatisfactory. The need for incentive programs to improve swine waste management on farms in São Paulo State was therefore made evident.

Keywords: biodigester, composting, swine waste.

\section{INTRODUÇÃO}

O Brasil é o quinto maior consumidor de carne suína do mundo e o quarto maior exportador deste produto (ABIPECS, 2014). A expectativa do mercado suinícola brasileiro para os anos vindouros é de crescimento, principalmente da exportação (ABCS, 2014).

A produção intensiva da suinocultura e a constante busca pelo incremento de produtividade contribuem de forma decisiva no aumento da poluição por dejetos, o que têm causado desequilíbrios ecológicos em diversos municípios brasileiros.

Segundo a ISO 14.001, que é uma norma internacionalmente aceita, Sistema de Gestão Ambiental é uma estrutura desenvolvida para que uma organização possa consistentemente controlar seus impactos significativos sobre o meio ambiente e melhorar continuamente as operações e negócios. Particularmente, Sistema de Gestão Ambiental em Suinocultura (SGAS) pode ser definido como um conjunto de atividades econômicas e sociais que utilizam os recursos naturais de forma racional com o uso de tecnologias que proporcionem a produção de suínos de forma sustentável com a minimização dos impactos ambientais gerados principalmente pela elevada produção de efluentes e de gases do efeito estufa (Honeyman, 1996). Neste contexto, o crescimento da suinocultura demandará maior investimento, principalmente, em SGAS, requisito fundamental para a conquista de mercados internacionais.

Um suíno produz em média 2,35 kg de efluente sólido por dia, e quando se considera o dejeto sólido associado à urina, esse total se eleva para $5,80 \mathrm{~kg}$, fazendo de sistemas intensivos de produção com alta densidade de animais por metro quadrado, uma atividade altamente poluidora (Beli et al., 2010).

A maior parte do impacto ambiental gerado pela suinocultura provém da falta do manejo adequado dos resíduos sólidos (Williams, 2008) e líquidos gerados pela atividade, como a produção de efluentes com alta carga orgânica que é lançada em corpos d'água e que podem chegar aos lençóis freáticos, o que causa a poluição dos recursos hídricos da região em torno da granja; a poluição do ar e a destruição da camada de ozônio majoritariamente ocasionada pela emissão de gás metano e óxido nitroso (Barbosa e Langer, 2011).

Apesar disso, há medidas que podem ser tomadas em um SGAS a fim de mitigar os impactos gerados pela atividade, como a implantação de biodigestores (Vanotti et al., 2007), compostagem e politica de recursos hídricos com economia de água de consumo e consequente redução de produção de efluentes (Berto e Miranda, 2007). Entretanto, o conceito de SGAS ainda é novo na produção de suínos, e os componentes que o constituem são poucos aplicados na suinocultura tecnificada de forma satisfatória, o que faz com que a crescente exigência do consumidor interno como externo sobre a qualidade de produção da carne suína brasileira, uma mudança de paradigma por parte dos produtores. 
O Estado de São Paulo é o $7^{\circ}$ maior produtor de carne suína do país, com 85.406 matrizes alojadas (APCS, 2014). Tais números, juntamente com o histórico nacional da falta de cuidado com os impactos ambientais gerados pela atividade suinícola acabam por justificar a necessidade do desenvolvimento de um trabalho que apresente um diagnóstico do que vem sendo feito pelos produtores em relação ao SGAS em granjas do Estado de São Paulo.

Tendo em vista o potencial poluidor da atividade suinícola, o seu crescimento, a sua importância econômica e poucos estudos de diagnóstico de SGAS, este trabalho teve por objetivo avaliar o SGAS em granjas de suínos do Estado de São Paulo.

\section{MATERIAL E MÉTODOS}

O trabalho foi efetuado em 37 granjas de suínos tecnificadas de ciclo completo do Estado de São Paulo escolhidas de forma aleatória (Figura 1). A pesquisa foi realizada durante os meses de outubro de 2013 a maio de 2014, com 51.030 matrizes investigadas, que representou $60 \%$ do total de matrizes alojadas no Estado de São Paulo (APCS, 2014).

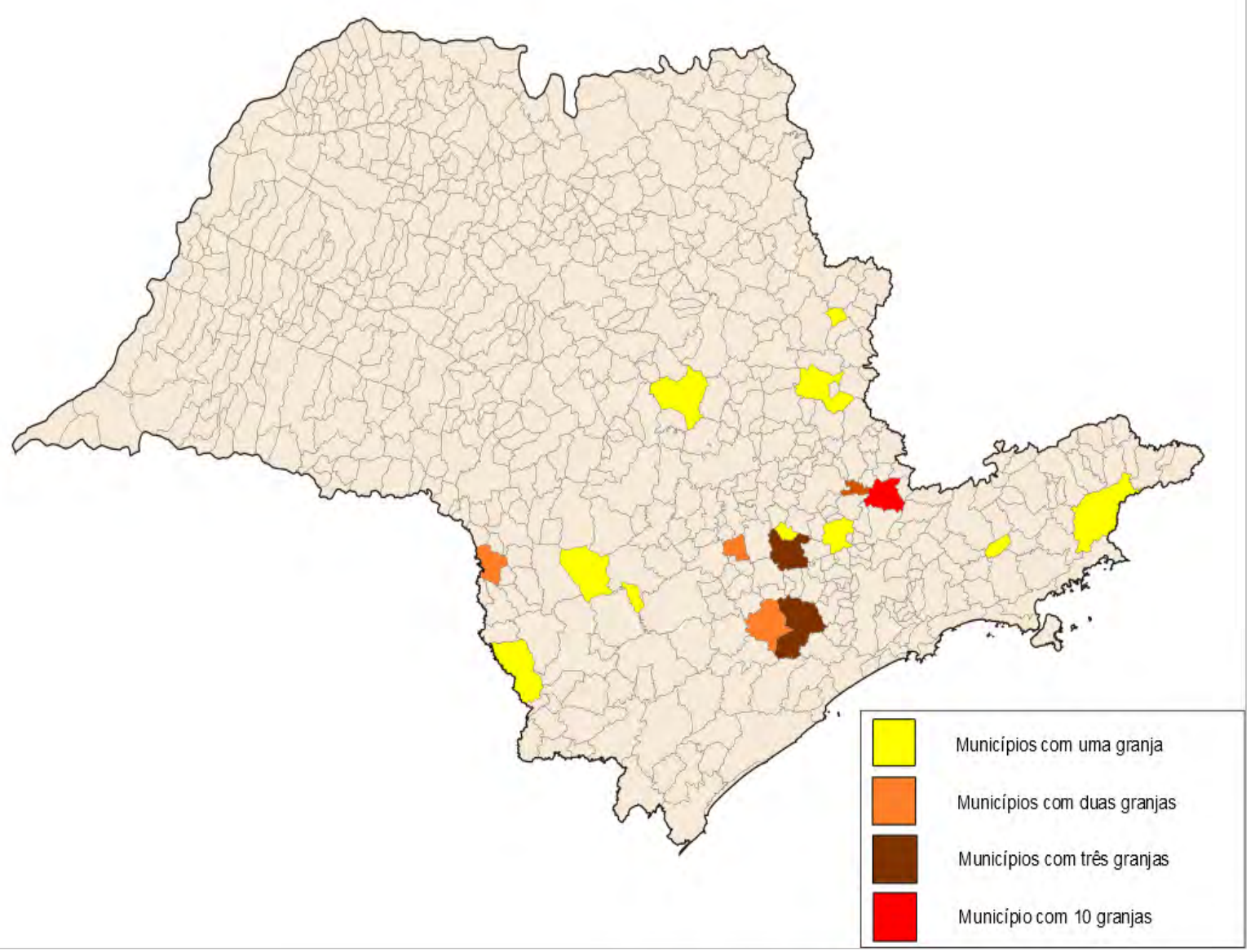

Figura 1. Mapa geopolítico do Estado de São Paulo e os respectivos municípios onde estavam localizadas as granjas pesquisadas.

Para a coleta de informações a respeito do SGAS nas granjas avaliadas e seu armazenamento foi elaborado um banco de dados eletrônico com a utilização do programa Google drive ${ }^{\circledR}$. Neste banco de dados foi inserido um questionário desenvolvido conforme metodologia proposta por Soto et al. (2006) e adaptada para esta investigação a fim de obter informações a respeito do SGAS nas respectivas granjas. 
Foram elaboradas oito questões abertas e fechadas para que as mesmas pudessem abranger todos os itens possíveis de estarem presentes em um SGAS e assim classificar os respectivos quesitos. As perguntas versavam sobre a existência ou não de tratamento de dejetos sólidos e carcaças por sistema de compostagem, uso de tratamento do efluente por sistema de lagoa anaeróbia integrada com a utilização da tecnologia de biodigestor, gestão de recursos hídricos com economia de água, planejamento de compras ecoeficientes (produtos produzidos com menor consumo de recursos naturais, espaço físico e menor geração de poluentes), gestão de resíduos sólidos de serviços de saúde animal, licenciamento e certificação ambiental, outorga de uso e lançamento de efluentes e geração ou não de produtos de valor agregado a partir dos diferentes sistemas de tratamento de dejetos sólidos e efluentes nas granjas investigadas.

Para cada questão foram atribuídos pesos distintos de um a 10 (Soto et al., 2006), de acordo com a importância e o impacto ambiental de cada item realizado ou não pela granja, e variando de dois (para a presença de produto com valor agregado resultante de algum processo) a 10 (atribuído aos itens considerados fundamentais para um eficiente SGAS). Neste trabalho as granjas foram classificadas em três categorias, tanto na somatória de todos os itens avaliados, como também de forma individual de cada quesito presente ou não no SGAS em: insatisfatórias com pontuação abaixo de 50 pontos, satisfatórias com restrição, entre 51 e 70 pontos e satisfatórias com pontuação acima ou igual a 70 pontos (Soto et al., 2006).

O questionário foi aplicado por meio de entrevistas diretamente com o gerente e ou proprietário das granjas investigadas e os resultados analisados com a utilização do software BrOffice Calc.

\section{RESULTADOS E DISCUSSÃO}

Na Tabela 1, estão apresentados os resultados totais obtidos em relação à pontuação nos SGAS das 37 granjas investigadas.

Tabela 1. Classificação das granjas em relação ao sistema de gestão ambiental empregado e as respectivas porcentagens, pontuações totais e médias obtidas.

\begin{tabular}{lccccc}
\hline Classificação & $\mathbf{N}^{\circ}$ de granjas & $\mathbf{\%}$ & MEP & MAP & PM \\
\hline Insatisfatórias & 23 & 62,16 & 23 & 49 & 43,61 \\
$\begin{array}{l}\text { Satisfatórias com } \\
\text { restrição }\end{array}$ & 04 & 10,81 & 56 & 62 & 59,50 \\
Satisfatórias & 10 & 27,03 & 73 & 97 & 84,40 \\
\hline Total & $\mathbf{3 7}$ & $\mathbf{1 0 0 , 0 0}$ & $\mathbf{5 0 , 6 6}$ & $\mathbf{6 9 , 3 3}$ & $\mathbf{6 2 , 5 0}$ \\
\hline
\end{tabular}

Nota: $\mathrm{N}^{\circ}$ - número; \% - porcentagem; MEP- menor pontuação; MAP- maior pontuação, PM- pontuação média.

Foram classificadas como insatisfatórias $23(62,16 \%)$ granjas do total de 37 avaliadas, com valor médio de 43,61 pontos, e satisfatórias com restrição, quatro $(10,81 \%)$, com valor médio de 59,50 pontos (Tabela1). Estes dois itens classificatórios juntos representaram $72,97 \%$ de granjas potencialmente geradoras de riscos ambientais, relacionados principalmente a disposição e destino inadequado de efluentes e resíduos sólidos no meio ambiente. Considerando as menores (23) e maiores (49) pontuações nas granjas classificadas como insatisfatórias, foram encontrados valores médios de 43,61 pontos, aquém do mínimo necessário para um SGAS que ofereça minimização de riscos ao meio ambiente. A maior 
pontuação média, obtida nas três classificações, apresentou valor de 69,33 pontos, considerado satisfatório com restrição (Tabela 1).

Das granjas consideradas como satisfatórias, obteve-se o resultado de $27,03 \%$, valor considerado baixo, quando se considera que nas 37 unidades investigadas o nível de tecnologia de produção zootécnica de suínos foi alto. Observa-se com estes resultados, que ainda persiste na maioria das unidades de produção de suínos do Estado de São Paulo avaliadas, uma maior preocupação com resultados zootécnicos, relacionados principalmente a maximização do desempenho produtivo e reprodutivo dos planteis, e a gestão ambiental ficou em segundo plano na lista de prioridades da administração do empreendimento.

Em relação à licença e certificação ambiental, somente $10(27,03 \%)$ granjas classificadas como satisfatórias eram licenciadas e certificadas (Tabelas 1 e 2) e geravam no SGAS pelo menos um produto de valor agregado como: biofertilizante, energia elétrica e ou térmica e créditos de carbono. Para a outorga de uso e captação de água e lançamento de efluentes, $11(29,72 \%)$ granjas possuíam o documento (Tabela 2). Estes resultados reforçam a necessidade por parte dos órgãos governamentais do Estado de São Paulo, o desenvolvimento de legislação mais rigorosa e especifica para a atividade suinícola. Ela deve prever a obrigatoriedade de SGAS que sejam capazes de sequestrar a produção de gás metano (Secco et al., 2014) e tratar os resíduos sólidos de modo que os tornem produtos de baixo impacto sanitário e ambiental (Daga et al., 2008) e que gerem valor agregado, como por exemplo, a tecnologia de biodigestor associada a um motogerador de energia elétrica (Ferrarez et al., 2010) e de compostagem (Bernal et al., 2009). Aliado a esta obrigatoriedade, é necessário que seja construída uma estrutura que ofereça maior poder de fiscalização e abrangência, com punição eficiente para as granjas infratoras.

Na Tabela 2 estão apresentados os resultados obtidos em relação ás 37 granjas investigadas e a respectiva classificação por questão relacionada ao SGAS.

Tabela 2. Resultados obtidos em relação às 37 granjas investigadas e a respectiva classificação por questão relacionada ao sistema de gestão ambiental em suinocultura. Valores expressos numericamente e em porcentagem $(\%)$.

\begin{tabular}{|c|c|c|c|c|}
\hline Questão & AS & IN & SR & SA \\
\hline Compostagem de carcaças e placentas & $\begin{array}{l}18 \\
(48,64 \%)\end{array}$ & $\begin{array}{l}05 \\
(13,51 \%)\end{array}$ & $\begin{array}{l}04 \\
(10,81 \%)\end{array}$ & $\begin{array}{l}10 \\
(27,03 \%)\end{array}$ \\
\hline Compostagem de dejetos sólidos & $\begin{array}{l}11 \\
(29,72 \%)\end{array}$ & $\begin{array}{l}14 \\
(37,83 \%)\end{array}$ & $\begin{array}{l}02 \\
(5,40 \%)\end{array}$ & $\begin{array}{l}10 \\
(27,03 \%)\end{array}$ \\
\hline Biodigestor & $\begin{array}{l}19 \\
(51,35 \%)\end{array}$ & $\begin{array}{l}07 \\
(18,91 \%)\end{array}$ & $\begin{array}{l}01 \\
(2,70 \%)\end{array}$ & $\begin{array}{l}10 \\
(27,03 \%)\end{array}$ \\
\hline $\begin{array}{l}\text { Gestão de resíduos sólidos de serviços de } \\
\text { saúde animal }\end{array}$ & $\begin{array}{l}13 \\
(35,13 \%)\end{array}$ & $\begin{array}{l}13 \\
(35,13 \%)\end{array}$ & $\begin{array}{l}03 \\
(8,10 \%)\end{array}$ & $\begin{array}{l}08 \\
(21,62 \%)\end{array}$ \\
\hline Planejamento de compras ecoeficientes & $\begin{array}{l}25 \\
(67,56 \%)\end{array}$ & $\begin{array}{l}01 \\
(2,70 \%)\end{array}$ & $\begin{array}{l}03 \\
(8,10 \%)\end{array}$ & $\begin{array}{l}08 \\
(21,62 \%)\end{array}$ \\
\hline $\begin{array}{l}\text { Gestão de recursos hídricos como economia } \\
\text { de água de consumo }\end{array}$ & $\begin{array}{l}18 \\
(48,64 \%)\end{array}$ & $\begin{array}{l}11 \\
(29,72 \%)\end{array}$ & $\begin{array}{l}01 \\
(2,70 \%)\end{array}$ & $\begin{array}{l}07 \\
(18,91 \%)\end{array}$ \\
\hline Licença e certificação ambiental & $\begin{array}{l}27 \\
(72,98 \%)\end{array}$ & $\begin{array}{l}00 \\
\text { (zero) }\end{array}$ & $\begin{array}{l}00 \\
\text { (zero) }\end{array}$ & $\begin{array}{l}10 \\
(27,03 \%)\end{array}$ \\
\hline $\begin{array}{l}\text { Outorga de uso e captação de água e } \\
\text { lançamento de efluentes }\end{array}$ & $\begin{array}{l}26 \\
(70,27 \%)\end{array}$ & $\begin{array}{l}00 \\
\text { (zero) }\end{array}$ & $\begin{array}{l}00 \\
\text { (zero) }\end{array}$ & $\begin{array}{l}11 \\
(29,72 \%)\end{array}$ \\
\hline
\end{tabular}

Nota: AS-Ausência, IN- Insatisfatória, SR- Satisfatória com restrição, SA- Satisfatória . 
Para o item compostagem de carcaças e placenta, considerando a soma das granjas que não praticavam esta tecnologia $(48,64 \%)$ ou a faziam de forma insatisfatória $(13,51 \%)$ (Tabela 2), obteve-se o resultado de $62,15 \%$. Evidenciou-se neste quesito, que apesar de poder ser facilmente cumprido de forma satisfatória pelo seu baixo custo de implantação e simplicidade operacional com a geração de resultados positivos tanto no aspecto ambiental como sanitário (Soto et al., 2010), ainda não é uma prática realizada pela maioria das granjas.

Em relação à compostagem de dejetos sólidos, $29,72 \%$ das granjas avaliadas apresentaram ausência do sistema e 37,83\%, foram classificadas como insatisfatórias, somando 67,55\%. Estes resultados são preocupantes, devido ao fato do alto risco de contaminação ambiental que a ausência desta pratica ou a sua utilização de forma insatisfatória pode representar para o meio ambiente (Kunz et al., 2008). Ademais, a produção de compostagem a partir de resíduos sólidos podem gerar outros lucros como a produção de biofertilizante, um produto de valor agregado para a suinocultura (Sardá et al., 2010).

Para o quesito biodigestor, $51,35 \%$ das granjas avaliadas ainda não dispunham desta tecnologia para o SGAS, considerada como protagonista para o tratamento de efluentes, sequestro de gás metano (Vanotti et al., 2008) e produção de biocombustível para a geração de produtos de valor agregado como energia elétrica e térmica (Cervi et al., 2010).

Em relação à gestão de resíduos sólidos de serviços de saúde animal, considerando a soma das granjas que não praticavam este tipo de atividade, ou faziam de forma insatisfatória, obteve-se o valor de $70,26 \%$, o que demonstrou que a prática de gerenciamento deste tipo de resíduo ainda é incipiente nos sistemas intensivos de produção animal (Reis et al., 2013).

No item planejamento de compras ecoeficientes, considerando também a soma das granjas que não praticavam este tipo de atividade, ou faziam de forma insatisfatória observou-se que $70,26 \%$ das granjas ainda não realizavam esta prática ou a faziam de forma insatisfatória, o que reforça a necessidade de todos os setores envolvidos em gestão ambiental em suinocultura implantarem esta filosofia de trabalho. Observou-se também o pouco conhecimento por parte dos suinocultores desta prática, ainda distante do seu cotidiano na atividade suinícola, o que mostra a necessidade de processos de capacitação de gestão ambiental, em todos os seus componentes.

Para o quesito gestão de recursos hídricos como economia de água de consumo, quase metade das granjas avaliadas $(48,64 \%)$ não tinham esta ação contemplada no SGAS e $29,72 \%$ realizavam de forma insatisfatória. Este fato gera preocupação ambiental pelo fato da água ser um recurso natural escasso (Mckeon, 2008) e quanto maior o seu consumo no sistema produtivo de suínos, maior será o volume de efluentes produzido diariamente, com aumento de custos operacionais para o seu tratamento adequado (Palhares e Calijuri, 2007).

\section{CONCLUSÕES}

Das granjas de suínos avaliadas em relação ao SGAS, $62,16 \%$ foram consideradas insatisfatórias e classificadas como potencialmente geradoras de riscos ambientais. Este resultado reforça a necessidade do real cumprimento da legislação, com maior controle por parte das autoridades a fim de evitar a deterioração ambiental no entorno da atividade suinícola.

Dos itens considerados importantes em um SGAS, 51,35\% e 67,55\% respectivamente, das granjas investigadas, não dispunham da tecnologia de biodigestor e não praticavam a compostagem de resíduos sólidos e de carcaças ou as faziam de forma insatisfatória.

A preocupação com a gestão do uso eficiente da água de consumo na atividade suinícola paulista, ainda é incipiente. Há a necessidade de um processo de capacitação e conscientização continuado aos proprietários e funcionários que trabalham com suinocultura, em virtude do potencial dano que esta gestão inadequada pode gerar ao meio ambiente. 


\section{REFERÊNCIAS}

ASSOCIAÇÃO BRASILEIRA DE CRIADORES DE SUÍNOS - ABCS. Estatísticas 2014.

Disponível em: <http://www.abcs.org.br/>. Acesso em: 12 jun. 2014.

ASSOCIAÇÃO BRASILEIRA DA INDÚSTRIA PRODUTORA E EXPORTADORA DE CARNE SUÍNA - ABIPECS. Estatísticas 2014. Disponível em:

http://www.abipecs.com.br/. Acesso em: 12 jun. 2014.

ASSOCIAÇÃO PAULISTA DE CRIADORES DE SUÍNOS - APCS. Estatísticas, 2014. Disponível em: <http://www.apcs.com.br/portal/>. Acesso em: 12 jun. 2014.

BARBOSA, G.; LANGER, M. Uso de biodigestores em propriedades rurais: uma alternativa à sustentabilidade ambiental. Unoesc \& Ciência ACSA, v. 2, n. 1, p. 87-96, 2011.

BELI, E.; HUSSAR, G. J.; HUSSAR, D. H. Redução de DQO e turbidez de efluente de uma unidade suinícola empregando Reator Anaeróbio Compartimentado (RAC) seguido de filtro biológico e filtro de areia. Engenharia Ambiental, v. 7, n. 1, p. 5-19, 2010.

BERNAL, M. P.; ALBURQUERQUE, J. A.; MORAL, R. Composting of animal manures and chemical criteria for compost maturity assessment. A review. Bioresource Technology, v. 10, n. 22, p. 5444-5453, 2009.

http://dx.doi.org/10.1016/j.biortech.2008.11.027

BERTO, J. L.; MIRANDA, C. R. Sustentabilidade ambiental das propriedades suinícolas da microrregião do Meio Oeste Catarinense: uma avaliação com base no balanço de nutrientes. Revista Brasileira de Agroecologia, v. 2, n. 1, p. 1589-1592, 2007.

CERVI, R. G.; ESPERANCINI, M. S. T.; BUENO, O. C. Viabilidade econômica da utilização do biogás produzido em granja suinícola para geração de energia elétrica. Engenharia Agrícola, v. 30, n. 5, p. 831-844, 2010.

DAGA, J.; CAMPOS, A. T.; FEIDEN, A.; KLOSOWSKI, E. S.; CÂMARA, R. J. Análise da adequação ambiental e manejo dos dejetos de instalações para suinocultura em propriedades na região oeste do Paraná. Engenharia Agrícola, v. 27, n. 3, p.1-11, 2008. http://dx.doi.org/10.1590/S0100-69162007000400001

FERRAREZ, A. H.; FILHO, D. O.; TEIXEIRA, C. A. Independência energética de granja suinícola a partir do uso de biogás. Engenharia na Agricultura, v. 18, n. 3, p. 248-257, 2010 .

HONEYMAN, M. S. Sustainability issues of U. S. swine production. Journal of Animal Science, v. 74, p. 1410-1417, 1996.

KUNZ, A.; BORTOLI, M.; HIGARASHI, M. M. Avaliação do manejo de diferentes substratos para compostagem de dejetos líquidos de suínos. Acta Ambiental Catarinense, v. 5. n. 1/2, p. 8-19, 2008.

MCKEON, M. Cut your slurry costs: new calculations show the difference in menure handling water economies reduce the volume for disposal. Pig International, v. 5, p. 22-24, 2008.

PALHARES, J. C. P.; CALIJURI, M. C. Caracterização dos afluentes e efluentes suinícolas em sistemas de crescimento/terminação e qualificação do seu impacto ambiental. Ciência Rural, v. 37, n. 2, p. 502-509, 2007. http://dx.doi.org/10.1590/S010384782007000200032 
REIS, M. A.; RANGEL, M. L.; MATTOS, C. M.; FRANKE, C. R. Conhecimento, prática e percepção sobre o gerenciamento de resíduos de serviços de saúde em estabelecimentos médicos veterinários de Salvador, Bahia. Revista Brasileira de Saúde e Produção Animal, v. 14, n.2 p. 287-298, 2013.

SARDÁ, L. G.; HIGARASHI, M. M.; MUller, S.; OliVEIRA, P. A.; COMIN, J. J. Redução de emissão de $\mathrm{CO}_{2}, \mathrm{CH}_{4}$ e $\mathrm{H}_{2} \mathrm{~S}$ através da compostagem de dejetos de suínos. Revista Brasileira de Engenharia Agrícola e Ambiental, v. 9, p. 1008-1013, 2010.

SECCO, M. A. G.; KUNZ, A.; EDWIGES, T.; JUNIOR, A. M.; FRIGO, E. P.; ALVES, E. J. Performance of up-flow anaerobic digester in solids removal and biogas production. Journal of Food, Agriculture \& Environment, v. 12, n. 2, p. 1135-1139, 2014.

SOTO, F. R. M.; RISSETO, M. R.; CAZZOLA, C. P. B. Proposta e análise crítica de um protocolo de inspeção e de condições sanitárias em supermercados do município de Ibiúna- SP. Revista Brasileira de Epidemiologia, v. 9, n. 2, p. 235-241, 2006.

SOTO, F. R. M.; COELHO, C. P.; BENITES, N. R.; BONAMIN, L.V.; MELVILlE, P. A. Elimination of $E$. coli from piglet carcases using an on-farm composting system. Veterinary Records, v. 166, p. 564-565, 2010.

VAnOTTI, M. B.; SzOGI, A. A.; HUNT, P. G.; Millner, P. D.; HUMENIK, F. J. Development of environmentally superior treatment system to replace anaerobic swine lagoons in the USA. Bioresource Technology, v. 98, n. 17, p. 3184-3194, 2007. http://dx.doi.org/10.1016/j.biortech.2006.07.009

VANOTTI, M. B.; SZOGI, A. A.; VIVES, C. A. Greenhouse gas emission reduction and environmental quality improvement from implementation of aerobic waste treatment systems in swine farms. Waste Management, v. 28, n. 4, p. 759-766, 2008. http://dx.doi.org/10.1016/j.wasman.2007.09.034

WILLIAMS, C. M. M. Technologies to mitigate environmental impact of swine production. Revista Brasileira de Zootecnia, v. 37, p. 253-259, 2008. http://dx.doi.org/10.1590/S1516-35982008001300029 


Ambiente \& Água - An Interdisciplinary Journal of Applied Science
ISSN 1980-993X - doi:10.4136/1980-993X
www.ambi-agua.net
E-mail: ambi-agua@agro.unitau.br

\title{
Análise probabilística da distribuição de dados diários de chuva no estado do Paraná
}

\author{
doi: 10.4136/ambi-agua.1489
}

Received: 17 Aug. 2014; Accepted: 12 Oct. 2014

Airton Kist; Jorim Sousa das Virgens Filho*

Universidade Estadual de Ponta Grossa (UEPG), Ponta Grossa, PR, Brasil

Departamento de Matemática e Estatística

*Autor correspondente: e-mail: jvirgens@uepg.br, kist@uepg.br

\section{RESUMO}

O regime de chuvas no Brasil tem uma distribuição diferente dos países do hemisfério norte onde em pesquisas de natureza hidrológica, utilizam-se os simuladores de dados climáticos desenvolvidos e calibrados para a Europa e /ou EUA. Assim, ao serem aplicados os dados de estações meteorológicas brasileiras podem produzir resultados não muito satisfatórios. Com o intuito de introduzir a distribuição de probabilidade Exponencial Mista, como alternativa para modelar dados de chuva no Brasil, este trabalho objetivou analisar probabilisticamente a distribuição de dados diários de chuva no estado do Paraná, determinando, dentre várias funções densidade de probabilidade, a que melhor se ajusta mensalmente às séries históricas. Para isso foram usadas séries históricas de trinta anos (1980-2009) de 29 localidades, nas quais foram avaliadas as aderências das distribuições de probabilidade Exponencial, Gama, Weibull, Log-Normal, Pareto Generalizada e Exponencial Mista com base nos testes não-paramétricos de Anderson-Darling e Qui-Quadrado. Na análise sem a distribuição Exponencial Mista a distribuição Gama foi a que teve o maior número de vezes o maior valor-p nos dois testes, seguida pela Weibull. Quando a Exponencial Mista foi incluída na análise, ela obteve o maior número de vezes o maior valor-p nos testes de aderência, chegando a 73,85\% das vezes no teste de Anderson-Darling e 71,84\% das vezes no teste Qui-Quadrado.

Palavras-chave: dados de chuva, distribuição de probabilidade, distribuição exponencial mista.

\section{Probabilistic analysis of the distribution of daily rainfall in the State of Paraná}

\begin{abstract}
Rainfall in Brazil has a different distribution compared with northern hemisphere countries where hydrological research employs climatic data simulators developed and calibrated for Europe and/or the USA. Thus, these simulators do not produce very satisfactory results when applied to data of Brazilian weather stations. With the aim of introducing the Mixed Exponential probability distribution as an alternative to model rainfall data in Brazil, this work probabilistically analyzed the distribution of daily rainfall data in the State of Paraná, by determining which probability density functions best fit the historical monthly
\end{abstract}


series. The historical series of thirty years (1980-2009) of 29 locations were used, in order to evaluate the fit of the Exponential, Gamma, Weibull, Log-Normal, Mixed Exponential and Generalized Pareto probability distributions, based on the non-parametric Anderson-Darling and Chi-Square tests. In the analyses without the Mixed Exponential distribution, the largest p-value in the two tests occurred most frequently in the Gamma distribution, followed by the Weibull distribution. When the Mixed Exponential was included in the analysis, the largest p-value occurred most frequently in the tests of adhesion, reaching $73.85 \%$ of the time in the Anderson-Darling test and $71.84 \%$ of the time in the Chi-Square test.

Keywords: mixed exponential distribution, probability distribution, rainfall data.

\section{INTRODUÇÃO}

A chuva (precipitação pluvial) é um dos fatores climáticos que mais favorece alterações nas condições ambientais de um agroecossistema, pois é grande a influência da sua ocorrência na caracterização do microclima local. As peculiaridades do regime de chuva proporcionam as variações observadas na temperatura do ar, umidade do ar, nebulosidade e na quantidade de radiação solar incidente na superfície, que afetam diretamente o crescimento e desenvolvimento das plantas. Do ponto de vista socioeconômico, o excesso ou escassez da chuva pode interferir profundamente em atividades econômicas e na vida do cidadão comum. Casos como o racionamento de energia em 2001 no Brasil e as frequentes enchentes em zonas urbanas mostram a importância da pluviosidade e de seu monitoramento mais sistemático e detalhado (Salgado et al., 2007).

Uma das preocupações quanto à chuva é a intensidade e a frequência de sua ocorrência, pelos seus efeitos potencialmente danosos, quando em excesso ou por escassez. Assim, o conhecimento das probabilidades de ocorrência de chuva torna-se de suma importância no planejamento de atividades relacionadas à agricultura ou no monitoramento de processos hidrológicos concernentes às bacias hidrográficas. Neste contexto, a chuva de um determinado local pode ser estimada, dentre outras formas, em termos probabilísticos, mediante modelos teóricos de distribuição ajustados a uma série histórica.

Existem várias pesquisas realizadas sobre a aderência de funções densidade de probabilidade aos dados de chuva de regiões ou cidades do Brasil. Algumas dessas pesquisas se referem à avaliação da chuva total mensal, outras por decêndios e/ou diário mensal. Catalunha et al. (2002), por exemplo, testaram a aderência de cinco funções densidade de probabilidade (Exponencial, Gama, Log-Normal, Normal e Weibull) às séries de chuva para 982 estações meteorológicas do estado de Minas Gerais nas escalas diária do decêndio, diária mensal, total do decêndio e total mensal. Silva et al. (2007) também avaliaram o comportamento destas cinco distribuições para a chuva mensal para Santa Maria, RS.

Com intuito semelhante no estado do Paraná, Sampaio et al. (2007) avaliaram a distribuição de chuva decendial para o estado do Paraná testando as distribuições Gama e Log-Normal em 22 postos de medição com um mínimo de 12 anos de observação. Pedron e Klosowski (2008) efetuaram o ajuste da distribuição Exponencial para descrever a distribuição de frequências de totais diários de chuva de 18 estações meteorológicas paranaenses. Outras pesquisas também têm sido conduzidas com o objetivo de avaliar os totais mensais de chuva. Souza et al. (2010) avaliaram o ajuste de seis distribuições de probabilidade a dados de 28 municípios de Pernambuco. Lyra et al. (2006) efetuaram também o ajuste de seis distribuições de probabilidade aos dados de chuva total mensal de 25 estações climatológicas do estado de Táchira, Venezuela.

A análise, entendimento, e simulação de dados climáticos são de grande importância nas avaliações envolvendo tomadas de decisões em atividades humanas estratégicas como a agricultura e o monitoramento ambiental. Diante disso, vários pesquisadores têm dedicado 
esforços nessa área e com isso têm sido propostos vários modelos computacionais de simulação de séries sintéticas diárias de dados de chuva, temperatura do ar, umidade relativa do ar, radiação solar, vento e outras variáveis climáticas. Na literatura são descritos vários geradores de dados climáticos diários, nos quais são gerados dados de chuva com as mais variadas distribuições de probabilidade. Na simulação dos dados de chuva pelo LARS-WG (Semenov e Barrow, 1997) é utilizada uma distribuição semi-empírica ajustada a partir dos dados observados. O ClimGen (Stockle et al., 1999) simula a chuva através da distribuição Weibull. O WeaGETS (Chen et al., 2012) gera dados de chuva a partir da distribuição Exponencial ou Gama. No Brasil, o PGECLIMA_R (Virgens Filho et al., 2013) utiliza a distribuição Gama na simulação de séries diárias de chuva.

Ao longo do tempo, comparações vêm sugerindo que uma mistura de duas distribuições de probabilidade exponenciais, ajusta melhor as distribuições observadas de chuva diária do que as alternativas mais comumente usadas, tais como a Exponencial Simples e a Gama (Richardson, 1981; Woolhiser e Roldan, 1982; Foufoula-Georgiou e Lettenmaier, 1987; Wilks, 1999). Além disso, a variabilidade interanual da chuva mensal é fortemente influenciada por eventos extremos raros. Por representar melhor esses eventos extremos, a distribuição Exponencial Mista de três parâmetros, também conhecida como Hiper-Exponencial (Feldmann e Whitt, 1998), melhora a variabilidade interanual da chuva mensal total em relação à distribuição Gama de dois parâmetros que é mais frequentemente utilizada (Wilks, 1999).

Sendo assim, a partir destas considerações acerca dos resultados encontrados nestes trabalhos, justifica-se testar a distribuição Exponencial Mista para dados de chuva diária em localidades brasileiras em detrimento das distribuições usualmente testadas como a Exponencial, Gama, Weibull, Log-Normal e Pareto Generalizada, visto que a avaliação desta distribuição de probabilidade para dados de chuva no Brasil ainda é incipiente. É de se destacar que o regime de chuvas no Brasil tem um comportamento diferente dos países do hemisfério norte. Por isso, os simuladores de variáveis climáticas desenvolvidos e calibrados para a Europa e/ou EUA ao serem aplicados a dados de estações brasileiras podem produzir resultados não muito satisfatórios.

Com o intuito de introduzir a distribuição Exponencial Mista, como alternativa para modelar dados de chuva no Brasil, este trabalho objetiva analisar probabilisticamente a distribuição de dados diários de chuva no estado do Paraná, determinando dentre os vários modelos probabilísticos usuais, o que melhor se ajusta mensalmente às séries históricas.

\section{MATERIAIS E MÉTODOS}

Esta pesquisa foi conduzida no Laboratório de Estatística Computacional e AplicadaLECA da Universidade Estadual de Ponta Grossa. Foram utilizados dados climatológicos de 29 estações meteorológicas espalhadas pelo estado do Paraná, pertencentes ao Instituto Agronômico do Paraná-IAPAR. Considerou-se, para fins de análise, a chuva diária mensal de janeiro a dezembro de um período de trinta anos (1980 a 2009) para todas as localidades. Informações básicas, incluindo o total médio de chuva anual, coordenadas geográficas para estas estações são apresentadas na Tabela 1.

É importante destacar que o total médio de chuva anual nestas estações variou de 1411 mm em Cambará a 2619 mm em Antonina, que representa de forma sucinta a distribuição e a variabilidade natural da pluviometria no Paraná. O tamanho da série histórica, integridade, consistência dos dados, cobertura espacial (Figura 1) e diversidade climática foram fatores relevantes na escolha dos locais. 
Tabela 1. Identificador da localidade (ID), coordenadas geográficas e totais médios de chuva anual das estações analisadas.

\begin{tabular}{|c|c|c|c|c|c|}
\hline ID & Nome da Estação & Latitude & Longitude & $\begin{array}{l}\text { Altitude } \\
\quad(\mathbf{m})\end{array}$ & $\begin{array}{c}\text { Chuva } \\
\text { (mm) }\end{array}$ \\
\hline 29 & Antonina & $-25^{\circ} 13^{\prime}$ & $-48^{\circ} 48^{\prime}$ & 60 & 2619 \\
\hline 16 & Apucarana & $-23^{\circ} 30^{\prime}$ & $-51^{\circ} 32^{\prime}$ & 746 & 1680 \\
\hline 18 & Bandeirantes & $-23^{\circ} 6^{\prime}$ & $-50^{\circ} 21^{\prime}$ & 440 & 1446 \\
\hline 20 & Bela Vista do Paraíso & $-22^{\circ} 57^{\prime}$ & $-51^{\circ} 12^{\prime}$ & 600 & 1526 \\
\hline 17 & Cambará & $-23^{\circ} 0^{\prime}$ & $-50^{\circ} 2^{\prime}$ & 450 & 1411 \\
\hline 6 & Cascavel & $-24^{\circ} 56^{\prime}$ & $-53^{\circ} 26^{\prime}$ & 760 & 2033 \\
\hline 25 & Cerro Azul & $-24^{\circ} 49^{\prime}$ & $-49^{\circ} 15^{\prime}$ & 360 & 1426 \\
\hline 2 & Cianorte & $-23^{\circ} 40^{\prime}$ & $-52^{\circ} 35^{\prime}$ & 530 & 1669 \\
\hline 12 & Clevelândia & $-26^{\circ} 25^{\prime}$ & $-52^{\circ} 21^{\prime}$ & 930 & 2053 \\
\hline 23 & Fernandes Pinheiro & $-25^{\circ} 27^{\prime}$ & $-50^{\circ} 35^{\prime}$ & 893 & 1624 \\
\hline 10 & Francisco Beltrão & $-26^{\circ} 5^{\prime}$ & $-53^{\circ} 4^{\prime}$ & 650 & 2073 \\
\hline 22 & Guarapuava & $-25^{\circ} 21^{\prime}$ & $-51^{\circ} 30^{\prime}$ & 1058 & 1956 \\
\hline 28 & Guaraquecaba & $-25^{\circ} 16^{\prime}$ & $-48^{\circ} 32^{\prime}$ & 40 & 2423 \\
\hline 14 & Ibiporã & $-23^{\circ} 16^{\prime}$ & $-51^{\circ} 1^{\prime}$ & 484 & 1503 \\
\hline 19 & Joaquim Távora & $-23^{\circ} 30^{\prime}$ & $-49^{\circ} 57^{\prime}$ & 512 & 1441 \\
\hline 8 & Laranjeiras Do Sul & $-25^{\circ} 25^{\prime}$ & $-52^{\circ} 25^{\prime}$ & 880 & 2056 \\
\hline 15 & Londrina & $-23^{\circ} 22^{\prime}$ & $-51^{\circ} 10^{\prime}$ & 585 & 1616 \\
\hline 27 & Morretes & $-25^{\circ} 30^{\prime}$ & $-48^{\circ} 49^{\prime}$ & 59 & 1997 \\
\hline 5 & Nova Cantu & $-24^{\circ} 40^{\prime}$ & $-52^{\circ} 34^{\prime}$ & 540 & 1994 \\
\hline 13 & Palmas & $-26^{\circ} 29^{\prime}$ & $-51^{\circ} 59^{\prime}$ & 1100 & 2099 \\
\hline 4 & Palotina & $-24^{\circ} 18^{\prime}$ & $-53^{\circ} 55^{\prime}$ & 310 & 1667 \\
\hline 1 & Paranavaí & $-23^{\circ} 5^{\prime}$ & $-52^{\circ} 26^{\prime}$ & 480 & 1477 \\
\hline 11 & Pato Branco & $-26^{\circ} 7^{\prime}$ & $-52^{\circ} 41^{\prime}$ & 700 & 2080 \\
\hline 26 & Pinhais & $-25^{\circ} 25^{\prime}$ & $-49^{\circ} 8^{\prime}$ & 930 & 1469 \\
\hline 9 & Planalto & $-25^{\circ} 42^{\prime}$ & $-53^{\circ} 47^{\prime}$ & 400 & 1961 \\
\hline 24 & Ponta Grossa & $-25^{\circ} 13^{\prime}$ & $-50^{\circ} 1^{\prime}$ & 880 & 1636 \\
\hline 7 & Quedas do Iguaçu & $-25^{\circ} 31^{\prime}$ & $-53^{\circ} 1^{\prime}$ & 513 & 2086 \\
\hline 21 & Telêmaco Borba & $-24^{\circ} 20^{\prime}$ & $-50^{\circ} 37^{\prime}$ & 768 & 1619 \\
\hline 3 & Umuarama & $-23^{\circ} 44^{\prime}$ & $-53^{\circ} 17^{\prime}$ & 480 & 1637 \\
\hline
\end{tabular}

Foram considerados apenas os dias com alguma quantidade de chuva, sendo que o dia a mais dos anos bissextos foi desconsiderado. Os dados faltantes nas séries históricas foram preenchidos com o auxilio do software PGECLIMA_R (Virgens Filho et al., 2013).

Para testar a hipótese de distribuições semelhantes, ou seja, verificar o nível de aderência entre a distribuição empírica dos dados e distribuição teórica (parâmetros estimados a partir dos dados observados) usou-se os testes não-paramétricos Anderson-Darling (A-D) e Qui-Quadrado (Q-Q) ao nível de significância de 5\%. As distribuições de probabilidade avaliadas (resumidas na Tabela 2) nesta pesquisa foram a Exponencial, Gama, Weibull, Log-Normal, Pareto Generalizada e Exponencial Mista. 


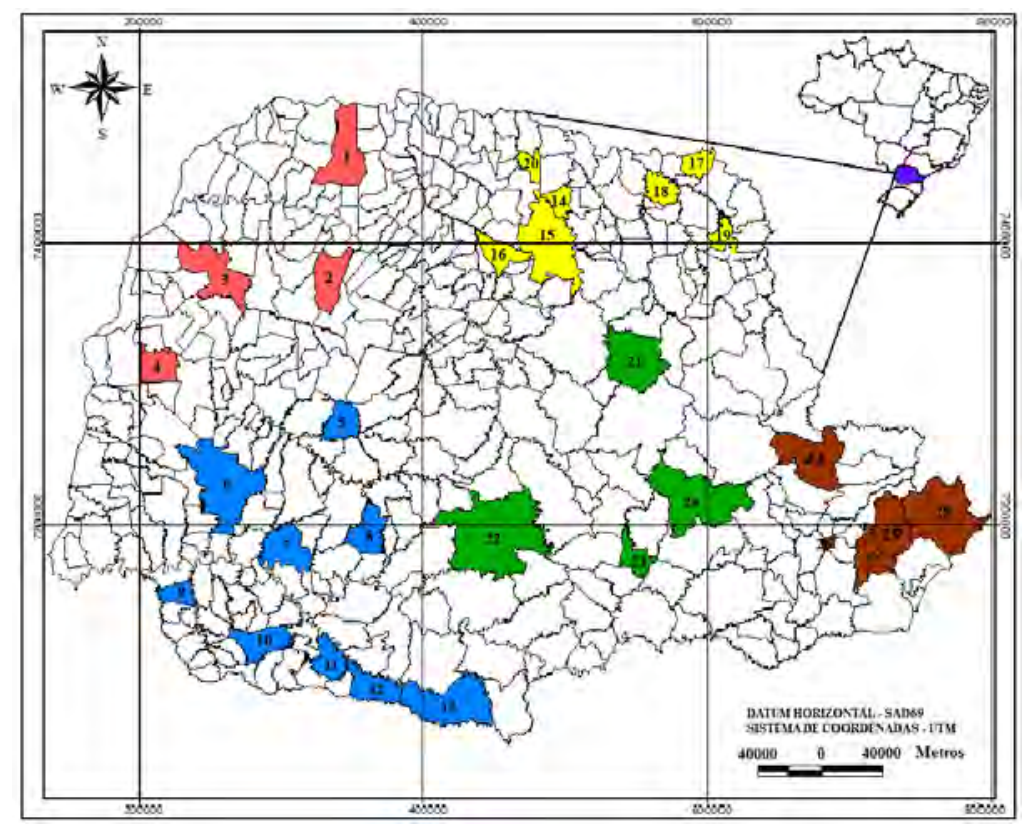

Figura 1. Cobertura espacial das estações meteorológicas, pertencentes ao IAPAR.

Tabela 2. Distribuições de probabilidade e suas respectivas funções densidade de probabilidade.

\begin{tabular}{ll}
\hline $\begin{array}{c}\text { Distribuição de } \\
\text { Probabilidade }\end{array}$ & Função densidade de probabilidade \\
\hline Exponencial $(\lambda)$ & $\lambda e^{-\lambda x}, x \geq 0 ; \lambda>0$, \\
Gama $(\alpha, \beta)$ & $\frac{1}{\beta^{\alpha} \Gamma(\alpha)} x^{\alpha-1} e^{-x / \beta}, \quad x>0 ; \alpha>0, \beta>0$ \\
Weibull $(\alpha, \beta)$ & $\frac{\alpha}{\beta^{\alpha}} x^{\alpha-1} e^{-(x / \beta)^{\alpha}}, \quad x>0 ; \alpha>0, \beta>0$ \\
Log-Normal $(\mu, \sigma)$ & $\frac{1}{x \sigma \sqrt{2 \pi}} e^{-\frac{(\ln (x)-\mu)^{2}}{2 \sigma^{2}}}, \quad x>0 ;-\infty<\mu<\infty, \sigma>0$ \\
$\begin{array}{l}\text { Pareto Generalizada } \\
(\xi, \mu, \sigma)\end{array}$ & $\frac{1}{\sigma}\left(1+\frac{\xi(x-\mu)}{\sigma}\right)^{-1-\frac{1}{\xi}}, x \geq \mu$ quando $\xi \geq 0 \mathrm{e}$ \\
$\begin{array}{l}\text { Exponencial Mista } \\
\left(\alpha, \lambda_{1}, \lambda_{2}\right)\end{array}$ & $\alpha \leq x \leq \mu-\frac{\sigma}{\xi}$ quando $\xi<0 ;-\infty<\mu, \xi<\infty, \sigma>0$ \\
\hline
\end{tabular}

Para análise dos resultados foram elaborados gráficos e tabelas, apresentando mensalmente as aderências das distribuições de probabilidade avaliadas nos testes estatísticos realizados nas séries históricas.

\section{RESULTADOS E DISCUSSÃO}

Nas Tabelas 3 e 4 são apresentados o número de aderências de cada distribuição, para cada mês com base nas 29 estações analisadas segundo os testes A-D e Q-Q, respectivamente. 
As distribuições Gama, Weibull e Exponencial Mista são as distribuições que tiveram o maior número de aderências nos dois testes aplicados. $\mathrm{O}$ teste $\mathrm{A}-\mathrm{D}$ foi o teste que apresentou $\mathrm{o}$ maior número de aderências.

Tabela 3. Número de aderências de cada distribuição por mês no teste A-D.

\begin{tabular}{lcccccccccccc}
\hline DistribuiçãolMês & Jan & Fev & Mar & Abr & Mai & Jun & Jul & Ago & Set & Out & Nov & Dez \\
\hline Exponencial & 21 & 19 & 13 & 10 & 8 & 11 & 12 & 18 & 27 & 26 & 22 & 20 \\
Gama & 29 & 29 & 29 & 29 & 29 & 29 & 28 & 29 & 29 & 29 & 29 & 29 \\
Weibull & 29 & 29 & 29 & 29 & 28 & 29 & 29 & 29 & 29 & 29 & 29 & 29 \\
Log-Normal & 16 & 19 & 26 & 26 & 25 & 22 & 27 & 28 & 25 & 12 & 19 & 21 \\
Pareto Generalizada & 29 & 29 & 29 & 28 & 27 & 26 & 26 & 27 & 29 & 29 & 29 & 29 \\
Exponencial Mista & 29 & 29 & 29 & 29 & 29 & 29 & 29 & 29 & 29 & 29 & 29 & 29 \\
\hline
\end{tabular}

Tabela 4. Número de aderências de cada distribuição por mês no teste Q-Q.

\begin{tabular}{lcccccccccccc}
\hline DistribuiçãolMês & Jan & Fev & Mar & Abr & Mai & Jun & Jul & Ago & Set & Out & Nov & Dez \\
\hline Exponencial & 2 & 4 & 1 & 0 & 0 & 3 & 1 & 2 & 5 & 3 & 3 & 4 \\
Gama & 29 & 29 & 29 & 28 & 28 & 26 & 26 & 27 & 29 & 29 & 29 & 29 \\
Weibull & 29 & 29 & 28 & 28 & 27 & 27 & 27 & 28 & 29 & 27 & 29 & 29 \\
Log-Normal & 15 & 18 & 23 & 20 & 20 & 13 & 22 & 28 & 22 & 16 & 18 & 17 \\
Pareto Generalizada & 20 & 20 & 20 & 12 & 9 & 11 & 18 & 18 & 19 & 12 & 12 & 19 \\
Exponencial Mista & 29 & 29 & 29 & 29 & 29 & 28 & 28 & 29 & 29 & 29 & 29 & 29 \\
\hline
\end{tabular}

O resultado do teste A-D, apresentado na Tabela 3, mostrou muito bom desempenho para as distribuições Gama, Weibull, Pareto Generalizada e Exponencial Mista, enquanto que as distribuições Exponenciais e Log-Normal tiveram redução no número de aderências, 59,48\% (207 de 348 testes) e 76,44\%, respectivamente. As distribuições testadas mantiveram a mesma tendência no teste Q-Q (Tabela 4), em relação ao teste A-D. Pelo teste Q-Q a distribuição Exponencial Mista teve 99,43\% de aderências e as distribuições Gama e Weibull tiveram $97,13 \%$ e $96,84 \%$ de aderências, respectivamente. As distribuições Log-Normal e Pareto Generalizada tiveram $66,67 \%$ e $54,60 \%$ de aderências, respectivamente, e a distribuição Exponencial teve apenas $8,05 \%$ de aderências. No teste Q-Q, não houve aderência de nenhuma distribuição testada nos meses de abril e maio para as localidades avaliadas.

Considerando os dois testes, a distribuição que teve o melhor desempenho foi a Exponencial Mista. No teste A-D houve aderência de 100\% e em apenas dois meses (junho e julho) não houve aderência de $100 \%$ pelo teste Q-Q. Em termos de número de aderências as distribuições Gama e Weibull tiveram desempenhos próximos. Assim, considerando a quantidade de aderências das distribuições testadas percebe-se que a distribuição Exponencial Mista é a que mais aderiu, seguida pela Gama e Weibull. A distribuição Exponencial foi a que 
teve pior desempenho nos dois testes. As distribuições Log-Normal e Pareto Generalizada tiveram desempenho melhor que a Exponencial, porém inferior a Exponencial Mista, Gama e Weibull.

A aderência de uma distribuição aos dados não significa necessariamente que o ajuste é bom. Apenas não teve evidência suficiente na série para essa rejeição. Como foram testadas seis distribuições diferentes, e algumas tiveram alto índice de aderência, fez-se uma análise da qualidade desses ajustes através da probabilidade de significância ou valor-p dos testes.

Dado que existem vários geradores de dados climáticos calibrados para gerar dados de chuva a partir da distribuição Gama, Weibull, Exponencial, por exemplo, e nenhum gera a partir da Exponencial Mista, fez-se inicialmente um estudo do desempenho das distribuições nos testes de aderência com base no maior valor-p. Assim também pode-se comparar este trabalho com o de Catalunha et al. (2002) que estudou a distribuição da chuva para o estado e Minas Gerais.

Os resultados da análise do valor-p, sem considerar a distribuição Exponencial Mista, estão na Figura 2, onde estão representadas a proporção de vezes que cada distribuição teve o maior valor-p em cada um dos testes de aderência (Figura 2(A) e 2(B)). Em todos os meses, nos dois testes, apenas as distribuições Gama e Weibull tiveram proporcionalmente o maior valor-p nas aderências dentre as cinco distribuições analisadas, sendo a Gama a distribuição com o maior número de meses o maior valor-p nos dois testes. A Gama teve em oito meses o maior valor-p nos dois testes e a Weibull nos demais meses.

Nos meses onde a Weibull teve o maior número de aderências a diferença em relação a Gama é em geral menor do que nos meses onde a Gama teve mais aderências. Em sete meses (maio, junho, agosto, setembro, outubro, novembro e dezembro) a Gama teve a maior proporção de vezes o maior valor-p nos dois testes enquanto a Weibull em três meses (janeiro, março e julho). A Gama teve em vários meses mais de $60 \%$ das vezes o maior valor-p nos dois testes, chegando a 90\% em outubro, a Weibull teve o melhor desempenho em março chegando a $76 \%$, ambos no teste A-D.

Em uma análise geral, a distribuição Exponencial não teve o maior valor-p em nenhum mês, nas localidades analisadas. A distribuição Pareto Generalizada teve menos de $2 \%$ de vezes o maior valor-p nos dois testes. A Log-Normal teve o maior valor-p apenas no teste Q-Q (Figura 2(B)), 6,03\% das vezes. Dentre as cinco distribuições aqui consideradas, nos testes A.D e Q-Q, a distribuição Gama teve $57,76 \%$ e $52,87 \%$ das vezes o maior valor-p, respectivamente e a Weibull teve $41,09 \%$ e $39,65 \%$ das vezes o maior valor-p, respectivamente.

Quando se inclui na análise a distribuição Exponencial Mista (Figura 3) os resultados são bem diferentes. Nos testes A-D (Figura 3(A)) e Q-Q (Figura 3(B)) a Exponencial Mista teve em todos os doze meses a maior proporção de vezes o maior valor-p, tendo em nove meses (fevereiro, abril, junho, julho, agosto, setembro, outubro, novembro, dezembro), no teste A-D, mais de $70 \%$ das vezes o maior valor-p e em oito meses (abril, maio, julho, agosto, setembro, outubro, novembro, dezembro) no teste Q-Q. Isso evidencia que a Exponencial Mista tem boa aderência em todos os meses sendo, portanto, a distribuição que melhor descreve a distribuição da chuva do período de 1980 a 2009 para as 29 localidades do Paraná.

Em termos gerais, a distribuição Exponencial Mista teve nos doze meses o maior valor-p nos dois testes de aderência, enquanto que a distribuição Exponencial também em nenhum dos testes teve o maior valor-p e as distribuições Log-Normal e Pareto Generalizada tiveram menos de $1 \%$ das vezes o maior valor-p. As distribuições Gama e Weibull, por sua vez, tiveram $9,48 \%$ e $16,67 \%$ no teste A-D e $10,34 \%$ e $16,09 \%$ das vezes no teste Q-Q o maior valor-p, respectivamente. A distribuição Exponencial Mista teve a maior proporção de vezes o maior valor-p nos dois testes: 73,85\% no teste A-D e 71,84\% no teste Q-Q. 
( A )

(B)
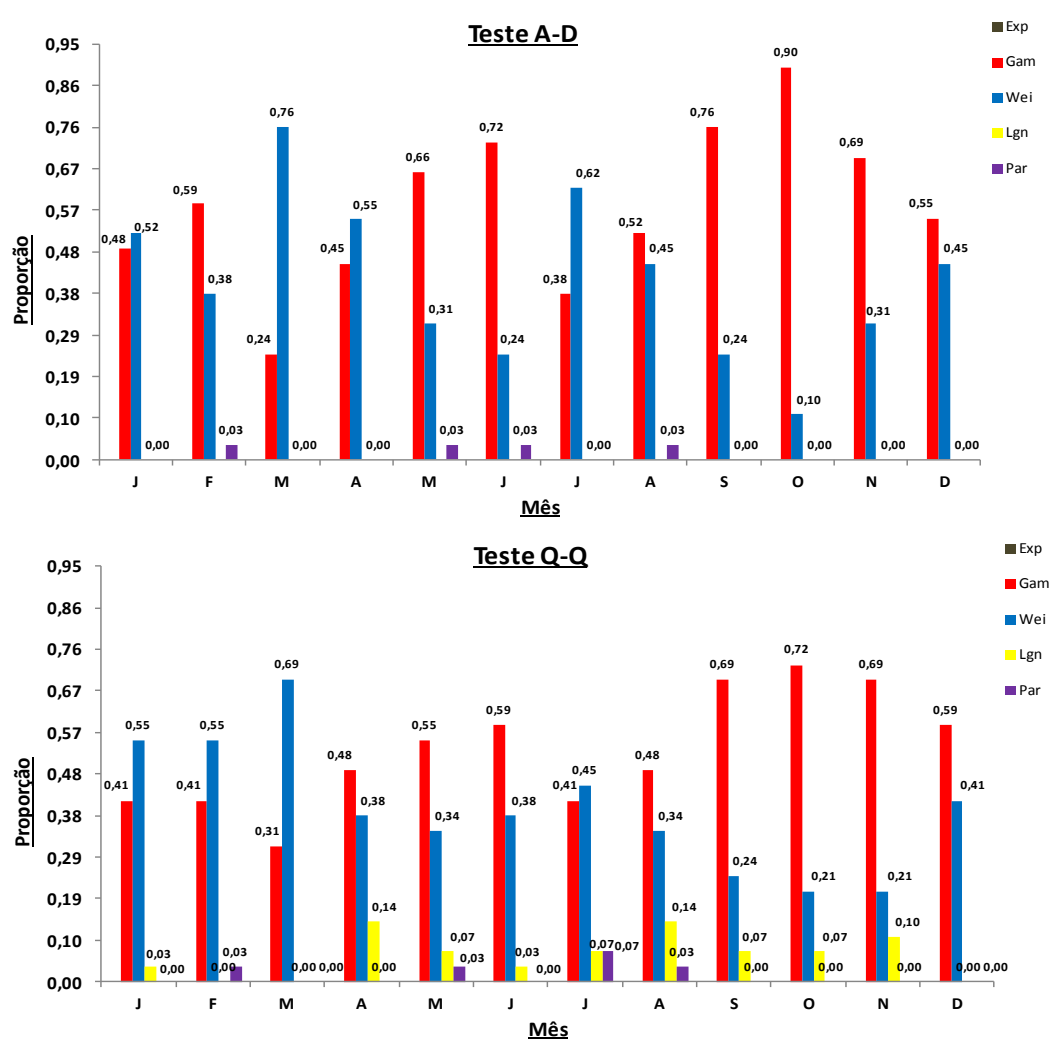

Figura 2. Proporção de aderências com maior valor-p de cada distribuição por mês no teste A-D (A) e teste Q-Q (B), sem se considerar a distribuição Exponencial Mista.

(A)

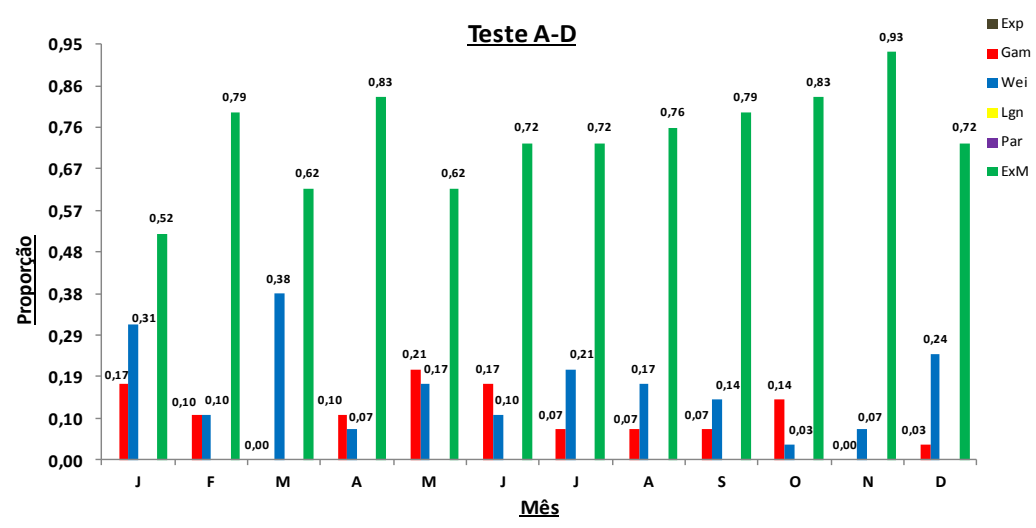

( B )

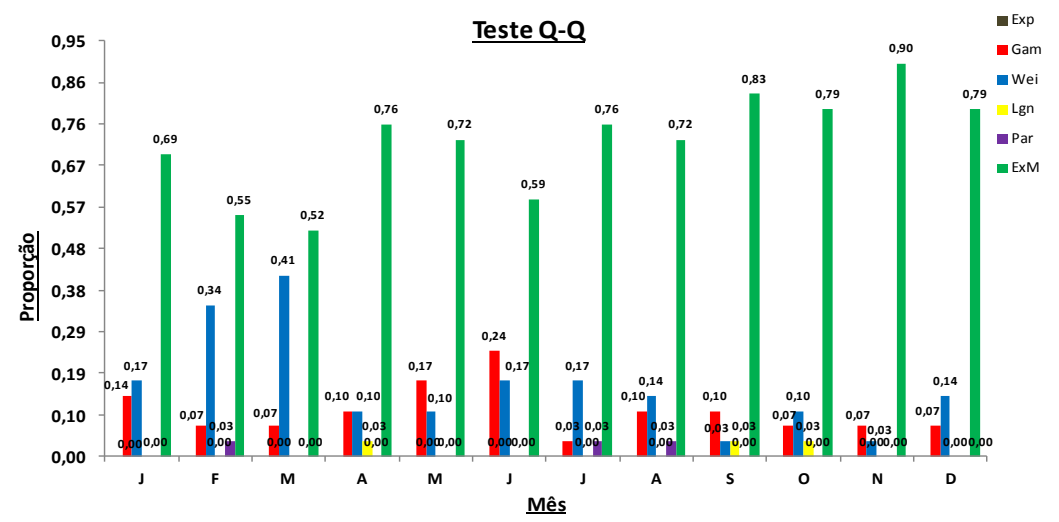

Figura 3. Proporção de aderências com maior valor-p de cada distribuição por mês no teste A-D (A) e teste Q-Q (B) considerando-se na análise a distribuição Exponencial Mista. 
Catalunha et al. (2002) analisaram 982 estações meteorológicas do estado de Minas Gerais e concluíram que a distribuição Weibull foi a que melhor ajustou-se aos dados de chuva diária mensal com base no teste Q-Q. Porém, utilizando o teste Kolmogorov-Smirnov (K-S) a distribuição Gama teve o melhor ajuste em sete meses e a Weibull nos outros cinco meses. Dessa forma, concluíram que a distribuição Weibull foi a que melhor aderiu aos dados de chuva diária mensal pelo teste Q-Q e a Gama no teste K-S. Os autores não explicam qual o critério usado para a escolha de uma ou outra, se foi pelo maior valor-p ou o maior número de aderências. Comparando os resultados encontrados para Minas Gerais com os resultados para o estado do Paraná, nota-se que no Paraná tanto pelo teste A-D como pelo teste Q-Q, quando desconsiderado a distribuição Exponencial Mista, a distribuição Gama apresenta melhor aderência, enquanto que para Minas Gerais, segundo Catalunha et al. (2002), a Weibull é melhor no teste K-S e a Gama é melhor no teste Q-Q, fazendo com que este resultado condicione o uso da distribuição levando-se em consideração o poder do teste de aderência.

\section{CONCLUSÃO}

Dentre as distribuições de probabilidade comumente utilizadas para ajuste de dados diários de chuva, a distribuição Exponencial Mista foi a que melhor se ajustou aos dados no estado do Paraná independente do teste de aderência usado. $\mathrm{Na}$ análise realizada sem a distribuição Exponencial Mista a distribuição Gama teve o melhor desempenho seguido pela distribuição Weibull. Dessa forma, sugere-se que na simulação de dados de chuva para localidades do Paraná, sejam utilizados preferencialmente geradores de dados climáticos que estejam calibrados para a distribuição Exponencial Mista. Porém, caso os gerador não disponibilizem esta distribuição, os dados podem ser melhor simulados a partir da distribuição Gama.

\section{REFERÊNCIAS}

CATAlUNHA, M. J.; SEDIYAMA, G. C.; LEAL, B. G.; SOARES, C. P. B.; RIBEIRO, A. Avaliação de cinco funções densidade de probabilidade a séries de precipitação pluvial no Estado de Minas Gerais. Revista Brasileira de Agrometeorologia, v. 10, n. 1, p. 153-162, 2002.

CHEN, J.; BRISSETTE, F. P.; LECONTE, R. WeaGETS-a Matlab-based daily scale weather generator for generating precipitation and temperature. Procedia Environmental Sciences, v. 13, p. 2222-2235, 2012. http://dx.doi.org/10.1016/j.proenv.2012.01.211

FELDMANN, A.; WHITT, W. Fitting mixtures of exponentials to long-tail distributions to analyze network performance models. Performance Evaluation, v. 31, p. 245279, 1998. http://dx.doi.org/10.1109/INFCOM.1997.631130

FOUFOULA-GEORGIOU, E.; LETTENMAIER, D. P. A Markov renewal model for rainfall occurrences. Water Resources Research, v. 23, n. 5, p. 875-884, 1987. http://dx.doi.org/10.1029/WR023i005p00875

LYRA, G. B.; GARCIA, B. I. L.; PIEDADE, S. D. S.; SEDIYAMA, G. C.; SENTELHAS, P. C. Regiões homogêneas e funções de distribuição de probabilidade da precipitação pluvial no Estado de Táchira, Venezuela. Pesquisa Agropecuária Brasileira, v. 41, n. 2, p. 205-215, 2006. http://dx.doi.org/10.1590/S0100-204X2006000200004

PEDRON, I. T.; KLOSOWSKI, E. S. Distribuição de frequência de chuvas diárias no Estado do Paraná. Scientia Agraria Paranaensis, v. 7, n. 1-2, p. 55-63, 2008. 
RICHARDSON, C. W. Stochastic simulation of daily precipitation, temperature, and solar radiation.Water Resources Research, v. 17, n. 1, p. 182-190, 1981. http://dx.doi.org/10.1029/WR017i001p00182

SAlGADO, C. M.; SILVA, T. C; SOUZA, G. C. A.; FREITAS, H. C. Caracterização temporal e espacial da precipitação no entorno do município de São Gonçalo (RJ) considerando a série histórica de 1968 a 2002. Sociedade \& Natureza, v. 19, n. 1, p. 19-31, jun. 2007.

SAMPAIO, S. C.; QUEIROZ, M. D.; FRIGO, E. P.; LONGO, A. J.; SUSZEK, M. Estimativa e distribuição de precipitações decendiais para o Estado do Paraná. Irriga, v. 12, n. 1, p. 38-53, 2007.

SILVA, J. C. da; HELDWEIN, A. B.; MARTINS, F. B.; TRENTIN, G.; GRIMM, E. L. Análise de distribuição de chuva para Santa Maria, RS. Revista Brasileira de Engenharia Agrícola e Ambiental, v. 11, n. 1, p. 67-72, 2007. http://dx.doi.org/10.1590/S1415-43662007000100009

SEMENOV, M. A.; BARROW, E. M. Use of a stochastic weather generator in the development of climate changes scenarios. Climatic Changes, v. 35, p. 397-414, 1997. http://dx.doi.org/10.1023/A:1005342632279

SOUZA, I. F.; LUNDGREN, W. J. C.; NETTO, A. O. A. Comparação entre distribuições de probabilidades da precipitação mensal no estado de Pernambuco. Scientia Plena, v. 6, n. 6,2010 .

STOCKLE, C. O.; CAMPBELL, G. S.; NELSON, R. ClimGen manual. Pullman: Biological Systems Engineering Department; Washington State University 1999.

VIRGENS FILHO, J. S.; OLIVEIRA, R. B.; LEITE, M. L.; TSUKAHARA, R. Y. Desempenho dos modelos CLIGEN, LARS-WG e PGECLIMA_R na simulação de séries diárias de temperatura máxima do ar para localidades do estado do Paraná. Engenharia Agrícola, v. 33, n. 3, p. 538-547, 2013. http://dx.doi.org/10.1590/S010069162013000300010

WILKS, D. S. Interannual variability and extreme-value characteristics of several stochastic daily precipitation models. Agricultural and Forest Meteorology, v. 93, p. 153-169, 1999. http://dx.doi.org/10.1016/S0168-1923(98)00125-7

WOOLHISER, D. A.; ROLDAN, J. Stochastic daily precipitation models: 2. A comparison of distribution of amounts. Water Resources Research, v. 18, n. 6, p. 1461-1468, 1982.

http://dx.doi.org/10.1029/WR018i005p01461 


Ambiente \& Água - An Interdisciplinary Journal of Applied Science
ISSN 1980-993X - doi:10.4136/1980-993X
www.ambi-agua.net
E-mail: ambi-agua@agro.unitau.br

\title{
Eventos extremos de precipitação em Belém-PA: uma revisão de notícias históricas de jornais
}

\author{
doi: 10.4136/ambi-agua.1433
}

Received: 19 Jun. 2014; Accepted: 03 Nov. 2014

\section{Thamiris Luisa de Oliveira Brandão Campos ${ }^{1}$; Maria Aurora Santos da Mota²; Sergio Rodrigo Quadros dos Santos ${ }^{1}$}

\author{
${ }^{1}$ Instituto Nacional de Pesquisas Espaciais (INPE), São José dos Campos, SP, Brasil \\ ${ }^{2}$ Universidade Federal do Pará (UFPA), Belém, PA, Brasil \\ *Autor correspondente: e-mail: brandao.thamiris@gmail.com, \\ aurora_ufpa@yahoo.com.br, sergiosanntos@yahoo.com.br
}

\section{RESUMO}

Os impactos socioeconômicos dos Eventos Extremos de Precipitação Diária em Belém-Pará foram estudados com base em histórias relatadas nas notícias dos jornais da cidade durante o período de 1987 a 2009. Estes eventos foram caracterizados como extremos por meio da Técnica do Decil. O objetivo foi identificar as consequências e os principais bairros afetados pelos eventos extremos de precipitação diária. Os anos que estiveram sob influência do fenômeno El Niño (La Niña) foram os que apresentaram menor (maior) frequência de Eventos Extremos de Precipitação e de noticias publicadas nos jornais da cidade. A variação mensal mostrou que os Eventos Extremos e as notícias dos jornais foram maiores no período "mais chuvoso" (verão/outono austral). As histórias relatadas nas notícias dos jornais da cidade indicaram que o alagamento das ruas é a principal consequência dos eventos extremos para a cidade de Belém-Pará e os bairros mais afetados são Cremação, Jurunas e Batista Campos. Esses bairros são vizinhos e tem como fator comum a presença da bacia hidrográfica da Travessa Quintino Bocaiúva, a qual apresenta baixa declividade e dificulta o escoamento da água, favorecendo a formação de pontos de alagamentos. Identificou-se que a falta de infraestrutura da cidade é o fator predominante quanto à consequência dos eventos extremos de precipitação diária.

Palavras-chave: cidade, eventos extremos, jornal, precipitação diária.

\section{Extreme precipitation events in Belém-Pará: a historical review of newspaper articles}

\begin{abstract}
This study examined the socioeconomic impacts of Daily Extreme Precipitation Events (hereafter "DEPEs") in Belem-Pará during the period 1987-2009. The DEPEs were characterized as "extreme" using the Decile technique. The objective of the study was to identify the consequences and the main neighborhoods affected by these events. The years influenced by occurrences of El Niño or La Niña had the lowest or the highest rates of DEPEs, respectively, and as well as the number of articles published in the city's newspapers. The monthly variation showed that the DEPEs and resulting newspaper articles were higher in
\end{abstract}


the "wettest" (summer / austral autumn) period. The newspaper articles indicated that street flooding is the main consequence of DEPEs in the city of Belém-Pará, and that the most affected districts are Cremação, Jurunas and Batista Campos. These neighborhoods are near to one another and are all located in the Travessa Quintino Bocaiúva watershed, which has a low slope that delays the flow of water, favoring flooding. The city's lack of infrastructure is the main reason for the severe consequences of DEPEs.

Keywords: city, daily precipitation, extreme events, newspapers.

\section{INTRODUÇÃO}

Valores discrepantes de um estado climático médio (doravante chamado de "eventos extremos") ocorrem em escalas temporais que variam de dias a milênios, embora os mais importantes para as atividades humanas sejam os eventos extremos de curta duração (no máximo alguns minutos ou poucas horas), os quais cobrem uma área horizontal de centenas a poucos metros. Incluem-se nessa escala as tempestades convectivas, rajadas de ventos, tornados, chuvas torrenciais, granizo e ocorrência de descargas atmosféricas (Malone, 1951; Marengo, 2009).

A intensidade dos eventos extremos de precipitação tem aumentado ao redor do mundo, preocupando tanto a comunidade científica quanto as autoridades, por causarem intensos danos à sociedade assim como ao ecossistema. Bryan et al. (2009) identificaram que as tendências demográficas deve ser uma questão a ser abordada nos debates políticos, o autor destaca que o rápido crescimento populacional agrava os efeitos das mudanças climáticas ou impede a capacidade adaptação. Pode ser notado que as consequências dos eventos extremos de precipitação têm chamado atenção, haja visto que em distintas regiões do planeta ocorrem prejuízos como danos à infraestrutura das cidades, agricultura, turismo, além de perdas de bens materiais e de vidas humanas.

Os Sistemas Meteorológicos e Mecanismos de Interações Oceano-Atmosfera influenciam nas ocorrências e intensificação dos eventos extremos, como por exemplo, os sistemas de meso (Linhas de Instabilidade) e de grande escala (Zona de Convergência Intertropical, Oscilação de Madden Julian, Padrão de Dipolo no Oceano Atlântico, El Niño-Oscilação Sul/ENOS), que de acordo com a atividade/fase agem ora acelerando ora enfraquecendo o desenvolvimento das nuvens e consequentemente a quantidade de chuva, ocasionando grandes inundações ou secas prolongadas em diferentes partes do planeta (Chu et al., 2009; Truong et al., 2009).

A cidade de Belém, capital do Estado do Pará, localizada na região Amazônica (Zona Equatorial) possui uma homogeneidade espacial e sazonal de temperatura, o que não acontece em relação à pluviosidade, pois sofre influência dos sistemas meteorológicos de meso e grande escalas mencionados anteriormente. Com precipitação média anual de 3000 $\mathrm{mm}$, precipitações diárias maiores que $25 \mathrm{~mm}$ são comuns na cidade e causam grandes prejuízos e transtornos para sociedade, como alagamentos e falta de energia elétrica. Neste contexto, o objetivo do presente estudo é identificar as consequências e os principais bairros afetados pelos eventos extremos de precipitação diária. Além de mostrar que veículos de comunicação, como os jornais, podem ser uma importante ferramenta para este tipo de análise histórica que identifica os desastres físicos, econômicos e sociais que a população enfrenta por conta das chuvas.

\section{MATERIAL E MÉTODOS}

A cidade de Belém-PA (Figura 1) está situada na Zona Equatorial ( $1^{\circ} 28^{\prime} \mathrm{S}$ e $\left.48^{\circ} 29^{\prime} \mathrm{W}\right)$. A baía do Marajó a banha ao norte, a leste é delimitada pelos municípios de Benevides e 
Ananindeua, o rio Guamá é o seu limite ao sul e as baías do Guajará e do Marajó limitam a cidade a oeste (Silva, 2008). O clima da cidade é tropical chuvoso de monção (segundo a classificação de Köppen (1900-1936)), com média anual da temperatura do ar de $26,0^{\circ} \mathrm{C}$, com máximas e mínimas variando de 31,5 a $22,0^{\circ} \mathrm{C}$ respectivamente (INMET, 1992) e o acumulado anual de precipitação varia de $2500 \mathrm{~mm}$ a $3900 \mathrm{~mm}$ (resultado será mostrado na seção 3.1).

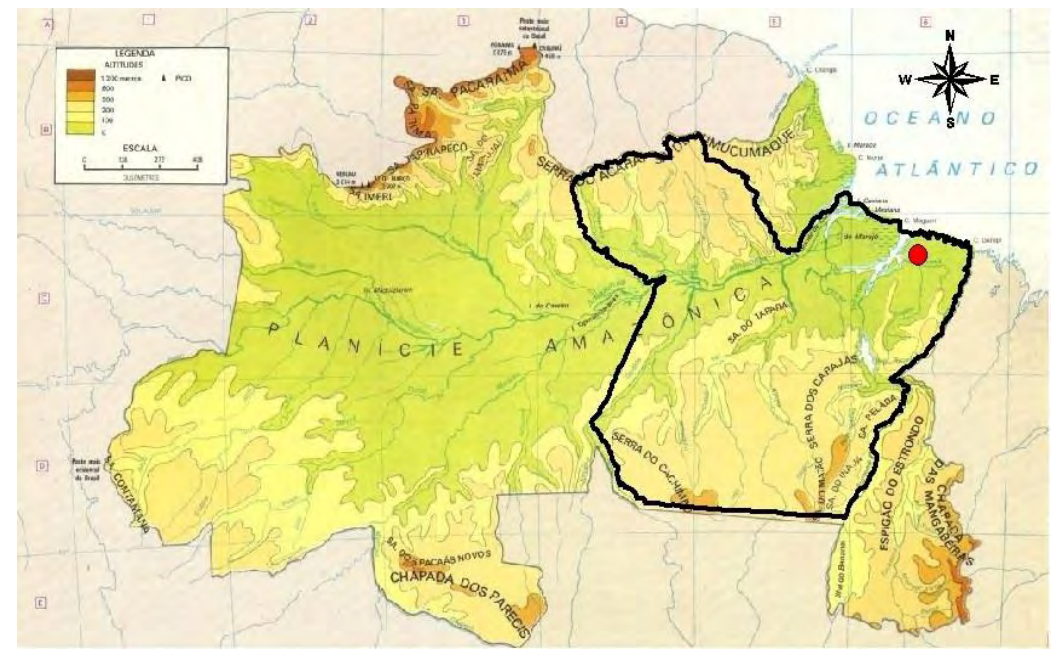

Figura 1. Região da Amazônia brasileira. O ponto em vermelho sinaliza a cidade de Belém - Pará. Figura adaptada de Simielli (1991).

Neste estudo foram utilizados dados pluviométricos, coletados nas estações climatológicas da Empresa Brasileira de Pesquisa Agropecuária (EMBRAPA- Amazônia Oriental), no período de 1987 a 2005, e do Instituto Nacional de Meteorologia (INMET) nos anos de 2006 a 2009. Estes dados são de precipitações acumuladas de 24 horas, observações feitas todo o dia às 1200 UTC (09h00 horas local). O índice do Niño Oceânico (INO) do "Climate Prediction Center" do "National Oceanic and Atmospheric Administrat (NOAA), disponível no site (http://www.cpc.ncep.noaa.gov/products/analysis_monitoring/ensostuff/ ensoyears.shtml), foi utilizado para comparação das fases do ENOS com a variabilidade interanual da precipitação e dos eventos extremos de precipitação diária (EEPD). Este índice define as fases com base na anomalia da temperatura da superfície do mar (TSM) em intensidade fraca (anomalia de TSM de $0,5^{\circ} \mathrm{C}$ a $0,9^{\circ} \mathrm{C}$ ), moderada $\left(1,0^{\circ} \mathrm{C}\right.$ a $1,4^{\circ} \mathrm{C}$ ) e forte $\left(\geq 1.5^{\circ} \mathrm{C}\right)$.

As notícias dos principais jornais sobre as chuvas que afetaram a cidade e/ou sociedade foram obtidas no Arquivo Público, Biblioteca Arthur Vianna, da Fundação Cultural do Pará Tancredo Neves (CENTUR) e nos jornais online. Para efeito da pesquisa nos jornais foram consideradas que todas as precipitações maiores que $20 \mathrm{~mm}$, no período de 1987 a 2009 , poderiam causar impactos a cidade de Belém-Pará. Este limiar foi usado antes da determinação estatística de eventos extremos de precipitação diária (EEPD) para colher o máximo das notícias, possíveis, nos jornais e assim avaliar o impacto das chuvas desde a intensidade moderada. Importante ressaltar que as 296 notícias que foram catalogadas neste período estão disponíveis fisicamente para pesquisa no prédio da Meteorologia na Universidade Federal do Pará.

A detecção dos efeitos negativos que os eventos extremos de precipitação de curto prazo podem gerar a uma cidade é bastante importante para a mitigação destes transtornos e as notícias de jornais tem se mostrado um método respeitável para análise, pois abordam e exibem as consequências que os eventos extremos de precipitação tenham acarretado a 
diversas cidades e/ou sociedades. Desta maneira, as notícias foram catalogadas a partir de caracterização dos bairros afetados e dos tipos de problemas causados à população devido à ocorrência das chuvas, tais como: alagamentos, congestionamento no trânsito, queda de árvores, destelhamentos de casas e interrupção de energia elétrica.

A técnica de Decil, conforme proposto por Xavier et al. (2007), foi aplicada a série de dados diários de chuva (1 de janeiro de 1987 a 31 de dezembro de 2009), para definir os eventos extremos de precipitação diária (EEPD). Este método consiste em dividir a série, organizada em ordem crescente, em dez partes iguais $(10 \%, 20 \%, \ldots, 90 \%)$, considerando o nono Decil como precipitação extrema, aqueles que, embora sejam em número muito reduzido, correspondem a $10 \%$ do total de chuva de toda a série. Os decis foram calculados da seguinte maneira (1):

$$
D_{k}=l_{i}+\frac{\left[\left(\frac{k \sum f_{i}}{10}\right)-f_{a n t}\right]}{f_{i}} \times h
$$

em que:

$\mathrm{Dk}=$ é o Decil que se pretende calcular,

li = é o limite inferior da classe,

$\mathrm{k}=$ é o número de ordem do Decil,

$\mathrm{fi}=$ é a frequência da classe da posição do Decil,

fant $=$ é a frequência da classe anterior do Decil e

$\mathrm{h}=$ é a amplitude da distribuição

Os eventos extremos de precipitação diária (EEPD) foram considerados precipitações maiores ou iguais a $24 \mathrm{~mm}$, valor correspondente ao nono Decil da série. Após a caracterização dos EEPD, calculou-se o coeficiente de determinação $\left(\mathrm{R}^{2}\right)$ entre a série de EEPD (frequência do acumulado mensal de EEPD, independente se houve notícia ou não) com as notícias dos jornais (considerando o limiar inicial de $20 \mathrm{~mm}$ ). Para verificar se a ocorrência de EEPD gera impacto a cidade de Belém, com publicação de notícias de chuva a partir de $20 \mathrm{~mm}$.

$\mathrm{O} \mathrm{R}^{2}$ é um indicador usado para medir a qualidade do ajustamento de uma linha de regressão, isto é, em que proporções a variável dependente (y) é explicada pela variável independente (x). Seu valor está no intervalo de 0 a 1 , se $\mathrm{R}^{2}$ for igual a 1 , isto significa que todos os pontos observados se situam "exatamente" sobre a reta de regressão. Tendo-se, neste caso, um ajuste perfeito. As variações da variável Y são $100 \%$ explicadas pelas variações da variável $\mathrm{X}$, não ocorrendo desvios em torno da função estimada. Por outro lado, se $\mathrm{R}^{2}=0$, isto quer dizer que as variações de $\mathrm{Y}$ são exclusivamente aleatórias e explicadas pelas variações de outros fatores que não X. Mais detalhes sobre o coeficiente de determinação podem ser obtidos em Wilks (2006).

\section{RESULTADOS E DISCUSSÃO}

\subsection{Análise interanual e mensal da precipitação e dos EEPD em Belém-Pará para os anos de 1987 a 2009.}

A Figura 2 apresenta a variabilidade interanual do acumulado da precipitação e da frequência dos EEPD durante os anos de 1987 a 2009. O acumulado anual de precipitação variou de $2500 \mathrm{~mm}$ a $3900 \mathrm{~mm}$ e a frequência dos EEPD foi de 22 a 54 eventos por ano. Os 
anos que apresentaram maiores acumulados de precipitações foram em 1989 com $3.890 \mathrm{~mm}$ de chuva (54 eventos de EEPD), 1988 com 3.697,7 mm (49 eventos de EEPD) e 2006 apresentou 3.663,8 $\mathrm{mm}$ (51 eventos de EEPD). Excetuando o ano de 2006 que foi um ano neutro, os anos de 1989 e 1988 estiveram sob influência do fenômeno La Niña que aumenta as chuvas na cidade de Belém. Os menores volumes de precipitações e de EEPD ocorreram nos anos de 1991 com 2513,3 mm (30 eventos de EEPD), 1997 com 2528,4 mm (35 EEPD) e 1990 com 2528,9 mm (22 eventos de EEPD). Excetuando o ano de 1990, que foi um ano neutro, os anos de 1991 e 1997 estiveram sob a influência do fenômeno El Niño que inibe as chuvas na cidade de Belém. Estes resultados corroboram com Marengo e Nobre (2000) que observaram o episódio de El Niño 1997/98 o mais intenso dos últimos 118 anos e as condições mais úmidas foram encontradas durante o episódio de La Niña 1988/89.

Embora o ENOS tenha um papel importante na interação entre a TSM e a precipitação da Amazônia, ele não é o único modulador da precipitação nesta região, por isso que nem todos os eventos de maiores/menores precipitações estão associados a este padrão. Os eventos ocorridos em 2006 e 1990 estiveram entre os três anos de maiores e menores volumes de precipitação anuais, porém o fenômeno ENOS estava em neutralidade, indicando que existiu influência de outro padrão para a variação acentuada da precipitação nestes anos. Souza e Nobre (1998) sugerem que a TSM do Oceano Atlântico interfere na ocorrência das chuvas na Amazônia, onde o gradiente térmico meridional e inter-hemisférico sobre o Atlântico Equatorial exerce influência no deslocamento norte-sul da Zona de Convergência Intertropical (ZCIT), que constitui o principal sistema meteorológico indutor de chuvas no nordeste da Amazônia. Grimm (2011) indica que o impacto do ENSO na chuva do nordeste da América do sul é mais forte se as anomalias de TSM tiverem o mesmo sinal no Pacifico Tropical leste e no Atlântico Tropical norte, devido ao efeito direto da relação ENSO via anomalias atmosféricas.

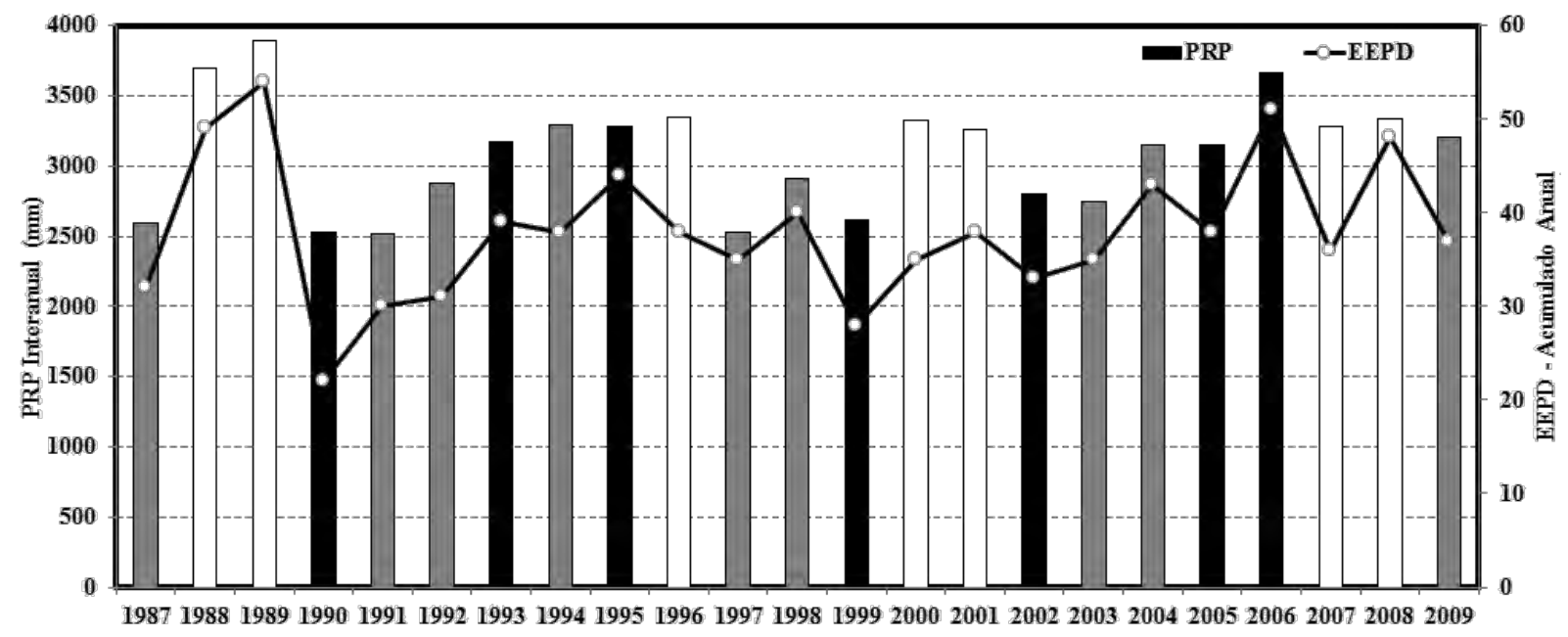

Figura 2. Variabilidade interanual da precipitação (barras) e da frequência dos EEPD (linha) em Belém para 1987 - 2009. As barras na cor cinza representam os anos que estiveram sob a influência do fenômeno El Niño, as barras em branco estiveram sob a influência do fenômeno La Niña e as pretas foram anos que não estiveram sob a influência do ENOS.

Analisando a precipitação média mensal em $\mathrm{mm} \mathrm{dia}^{-1}$ e número de casos mensais de EEPD no período de 1987-2009 (Figura 3) é possível observar que nos meses de dezembro a maio, período "mais chuvoso" da Região, ocorreu uma média mensal de $12,2 \mathrm{~mm} \mathrm{dia}{ }^{-1}$ de precipitação (71,9\% do total da média mensal) e 693 EEPD (79,3\% do total). Os meses com maior precipitação foram março e abril, a média mensal desses dois meses foi de 14,6 mm $\operatorname{dia}^{-1}$ de chuva (28,6\% do total da média mensal). Enquanto que no período "menos chuvoso" 
(junho a novembro) a média mensal foi de $4,8 \mathrm{~mm} \mathrm{dia}^{-1}$, o que representou $28,1 \%$ do total da média mensal, com 181 EEPD (20,7\% do total).

A variabilidade da precipitação entre os dois períodos (chuvoso e menos chuvoso) mostra a influência dos fenômenos de meso e grande escala no aumento e na redução de precipitação e consequentemente nos EEPD. O volume de precipitação e a quantidade elevada de EEPD, no período chuvoso, estão relacionados ao período de atuação da ZCIT e da MJO na região (Grimm, 2011; De Souza e Ambrizzi, 2006). Enquanto que no período menos chuvoso, as Linhas de Instabilidade são responsáveis por cerca de $45 \%$ da ocorrência das chuvas (Cohen et al., 1995).

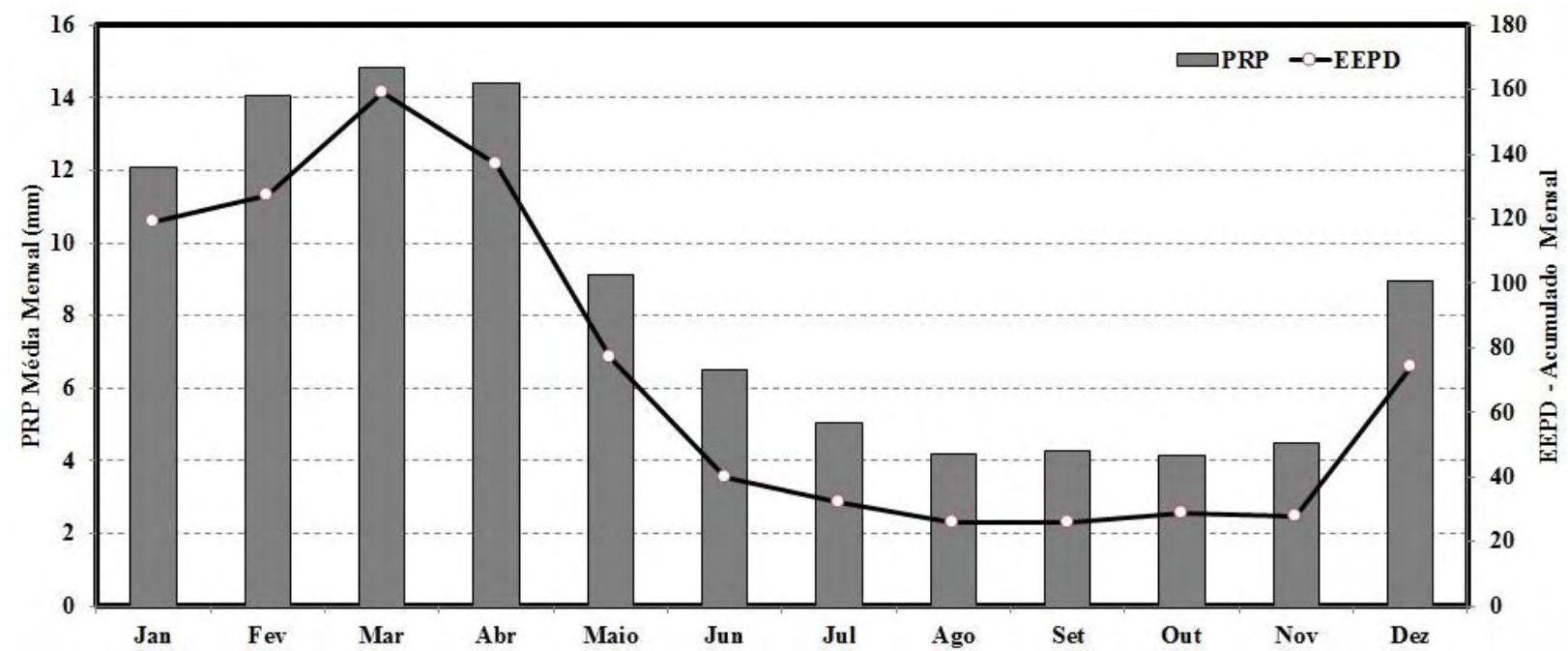

Figura 3. Variabilidade média mensal em $\mathrm{mm} \mathrm{dia}^{-1}$ (barra) e número de casos mensais de EEPD (linha) entre 1987 a 2009.

\subsection{Caracterização dos tipos de consequências e dos principais bairros afetados pelos EEPD na cidade de Belém-Pará}

A Figura 4 apresenta uma análise de regressão linear simples entre o total mensal de EEPD e o total mensal das notícias publicadas para os anos de 1987 a 2009. Esta regressão exibe uma alta correlação $\left(\mathrm{R}^{2}=0,99\right)$ entre o acumulado mensal de EEPD e de notícias publicadas, indicando que o limiar de $24 \mathrm{~mm}$ que classifica EEPD está associado a falta de infraestrutura da cidade em receber chuvas, haja visto que os impactos das chuvas a partir de $24 \mathrm{~mm}$ estão bem correlacionados com as notícias dos jornais. Os jornais evidenciam um 'acontecimento infeliz' (causal ou não) do qual resulta o dano, estrago, avaria, destruição, perdas humanas, chegando até o desastre (Monteiro, 1991). Ressalta-se que a variabilidade interanual e mensal da quantidade de notícias, que foram publicadas em consequência dos EEPD, estão diretamente associadas aos anos de maiores volumes pluviométricos (Figura 2 e 3 ).

As principais consequências que os EEPD geraram à cidade e/ou sociedade, publicadas nos jornais da cidade de Belém no período de 1987 a 2009, são apresentadas na Figura 5. Os alagamentos representam $65 \%$ das notícias que foram divulgadas na imprensa (Figura 5 - painel à direita), com os máximos nos anos 1988, 2008 e 2009 (Figura 5 - painel à esquerda). O congestionamento no trânsito representa $15 \%$ das notícias (Figura 5 - painel à direita), sendo considerado a segunda maior consequência que ocorre na cidade.

A cidade de Belém representa a experiência de maior adensamento urbano em toda a região amazônica. Comportam aproximadamente 3 milhões de habitantes, o que foi considerada uma das maiores taxa de ocupação humana na Amazônia (Castro, 2003). Logo, o quadro de ocupação urbana intensa e desordenada, junto com as condições de terrenos alagadiços da cidade, faz com que, em qualquer período, uma precipitação extrema provoque 
sérios problemas a população. Além disso, o processo de urbanização impermeabiliza o solo proporcionando o aumento dos problemas relacionados ao alagamento da cidade. Então, toda vez que acontece uma chuva de intensidade moderada a forte, grande parte da cidade fica alagada devido à falta de uma rede pública de esgoto sanitário adequada. O percentual de domicílios que utilizam rede de esgoto em Belém sofreu redução entre 2001 a 2006. Em 2001, $13 \%$ dos domicílios eram servidos pela rede de esgoto, enquanto em 2006 essa proporção caiu para apenas $9 \%$. A redução decorreu do aumento do número de domicílios e da desativação de redes antigas. Além disso, os municípios de Marituba, Benevides e Santa Bárbara não possuem rede pública de esgoto (Leão et al., 2008).

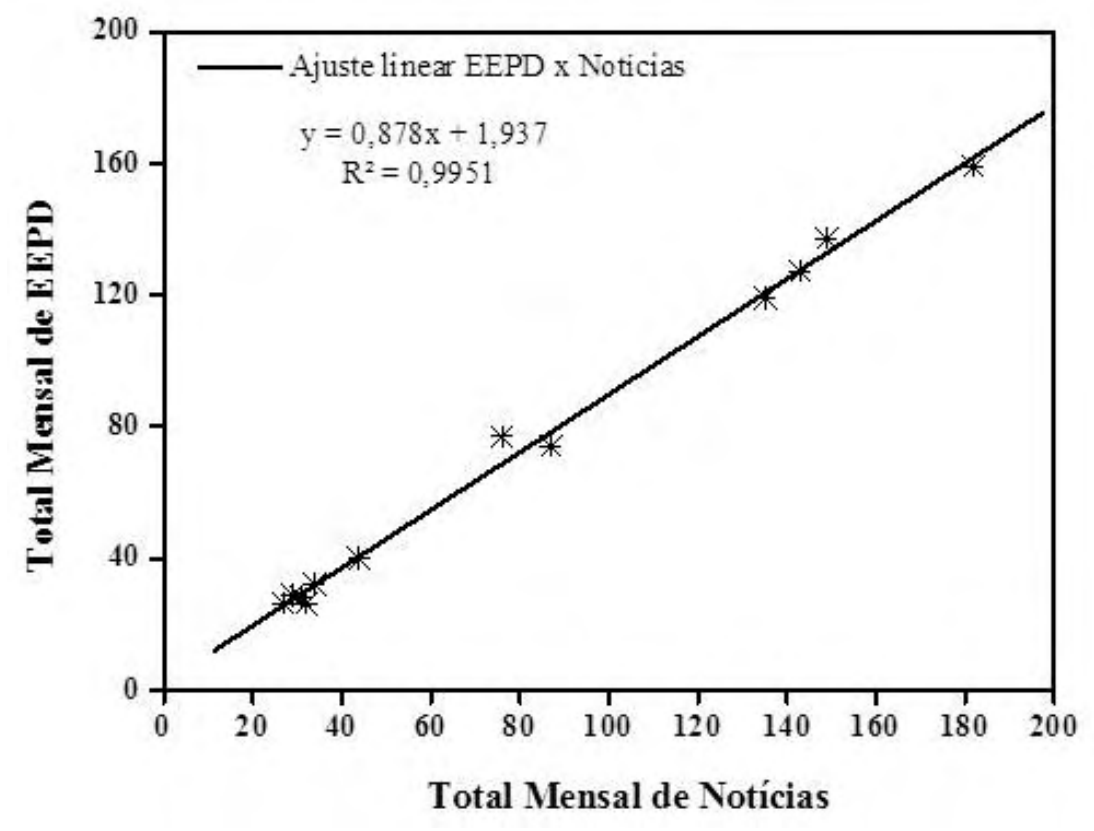

Figura 4. Regressão linear simples entre o acumulado mensal de EEPD e das notícias publicadas nos jornais durante os anos de 1987 a 2009.

Uma particularidade significativa da cidade é a sua arborização composta por diversos túneis de mangueiras seculares, que resistem ao passar do tempo e são elementos presentes nos espaços públicos. As quedas de árvores representam $12 \%$ das principais consequências (Figura 5 - painel à direita) e ocorrem devido os ventos de intensidade moderado/forte. A maioria das nuvens formadas em Belém é do tipo Cumuloninbus de grande desenvolvimento vertical, caracterizadas por gerar ventos fortes, chuvas duram até 2 horas. Precipitações desse tipo causam muitos transtornos, chegando em alguns casos a provocar interrupção de energia, devido ao desligamento de transmissão e queda de raios. Os ventos moderado-fortes também ocasionam destelhamentos e queda de placas; este tipo de consequência representa $5 \%$ das notícias (Figura 5 - painel à direita).

As descargas atmosféricas são intensas descargas elétricas que ocorrem na atmosfera em um curto intervalo de tempo, realizando um percurso de alguns quilômetros. Essas descargas elétricas podem ocorrer associadas às explosões nucleares, erupções vulcânicas e tornados. Contudo, geralmente, se originam em nuvens do tipo Cumulonimbus, ou seja, nuvens de tempestades (Uman, 1987). As descargas atmosféricas representam 3\% das notícias (Figura 5 - painel à direita). 


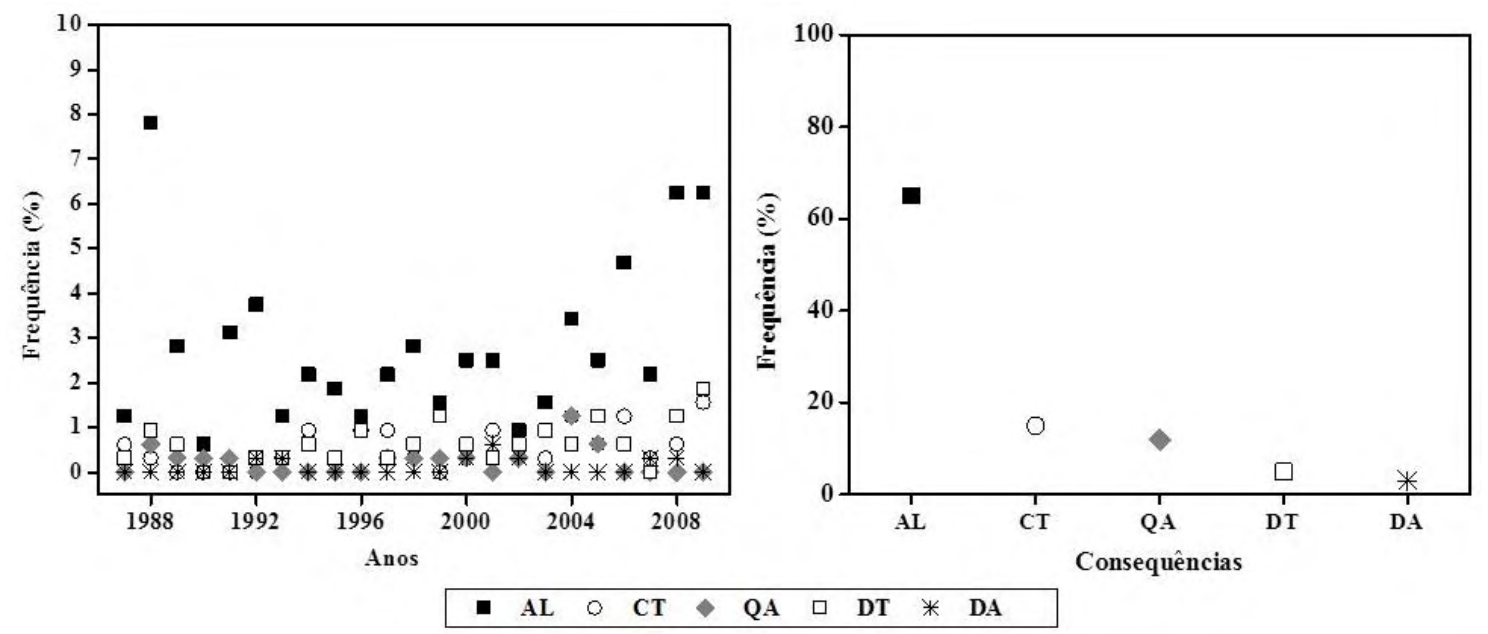

Figura 5. Principais consequências que os EEPD geraram à cidade e/ou à sociedade de Belém.Pará nos anos de 1987 a 2009. O painel à esquerda exibe a frequência interanual das consequência dos EEPD e o painel à direita apresenta a frequência do total de casos das consequência dos EEPD para o período em estudo. AL (alagamento); CT (congestionamento no trânsito); QA (queda de árvores); DT (destelhamentos); DA (descargas atmosféricas).

Os principais bairros afetados por alagamentos foram Cremação, Jurunas, Batista Campos, Pedreira, Guamá, Marco e Campina (Figura 6). Os três bairros mais afetados (Cremação, Jurunas, Batista Campos) estão localizados um ao lado do outro (Figura 7) e a bacia hidrográfica da Travessa Quintino Bocaiúva estende-se entre eles. Santos (2010) estudou esta bacia e constatou que o índice de drenagem é de aproximadamente $0,71 \mathrm{~km} / \mathrm{km}^{2}$, o qual é considerado muito baixo segundo a classificação de Sthraler (1957), tornando esta bacia muito suscetível a alagamentos e inundações. $\mathrm{O}$ autor ainda evidencia o fato de que a maior parte da topografia da bacia apresenta baixa declividade, onde $53,97 \%$ da área apresenta declividade abaixo de $0,5 \%$, dificultando o escoamento da água e favorecendo a formação de pontos de alagamentos.

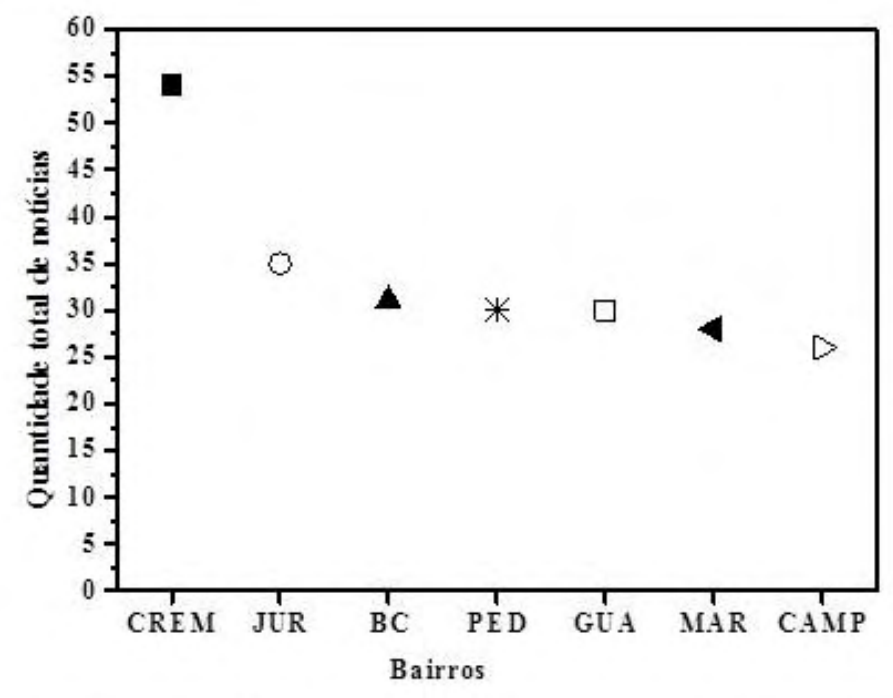

- CREM O JUR

Figura 6. Bairros que mais apresentaram notícias em consequência dos EEPD na cidade de Belém-Pará nos anos de 1987 a 2009. CREM (Cremação); JUR (Jurunas); BC (Batista Campos); PED (Pedreira); GUA (Guamá); MAR (Marco); CAMP (Campina). 


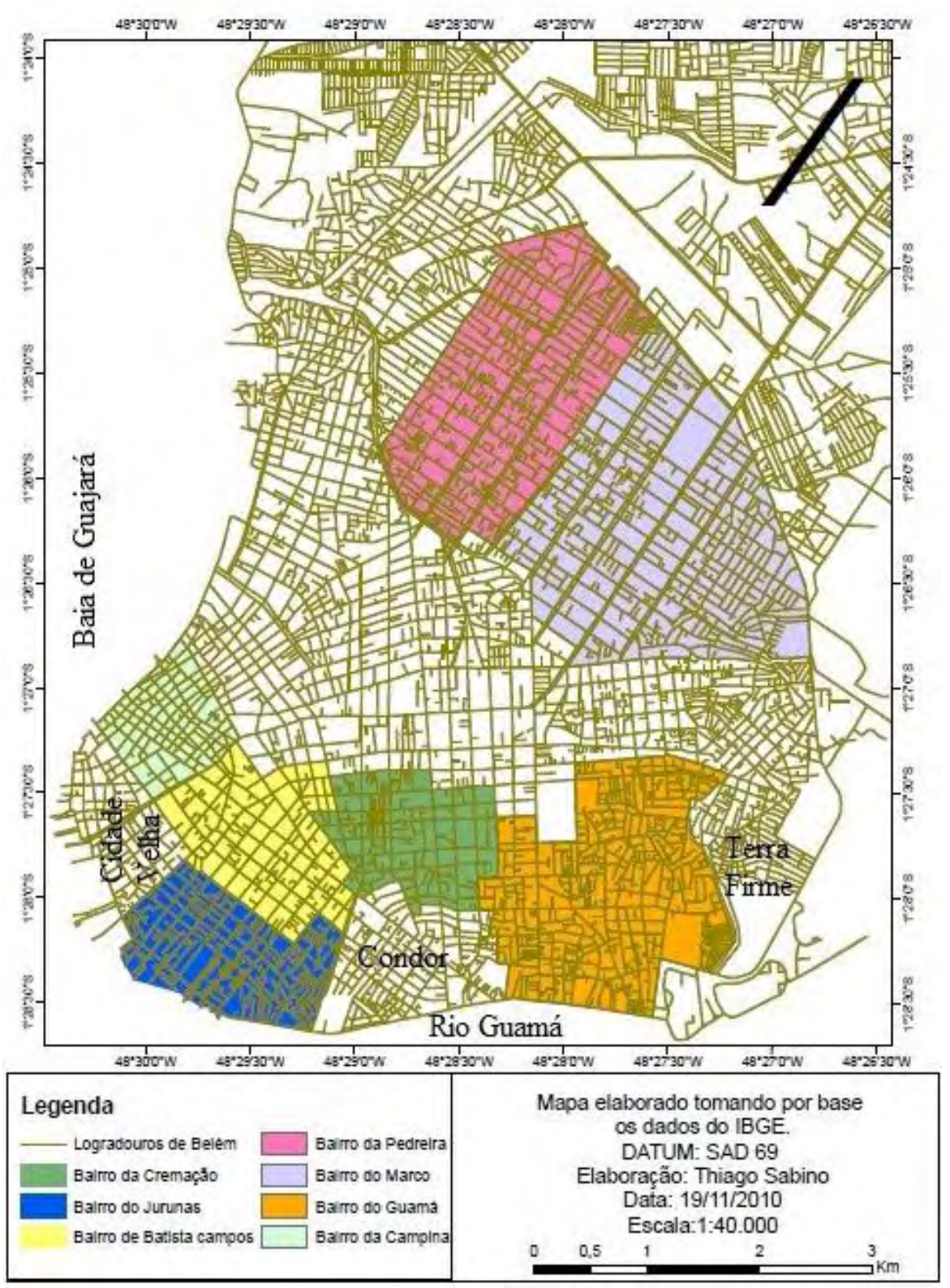

Figura 7. Localização dos principais bairros afetados por pontos alagamentos.

O bairro da Cremação foi o que mais sofreu com os eventos extremos de precipitação (54 notícias/Figura 6). Este bairro possui vários canais e mesmo que a chuva não seja muito forte, causa prejuízos à sociedade, como exemplifica as notícias dos dias 4 de Dezembro de 2009 e 1 de Abril de 2009 (ver com maiores detalhes na Tabela 1). O segundo bairro mais prejudicado foi o bairro do Jurunas (35 notícias/Figura 6), pois, além deste possuir canais, ele está localizado na Região costeira do rio Guamá e o transporte de umidade em direção ao bairro favorece ainda mais os eventos extremos (verificar notícia do dia 28 de Fevereiro de 1991 na Tabela 1). O bairro da Batista Campos, que faz fronteira com Jurunas e Cremação, é o terceiro com maior quantidade de notícias sobre o alagamento (31 notícias/Figura 6), apesar de ser um bairro nobre da cidade de Belém. Neste bairro ocorrem alagamentos nas ruas e casas próximas ao canal localizado na Rua Quintino Bocaiúva, como mostra a notícia do dia 28 de Abril de 1998 (Tabela 1).

Os bairros da Pedreira e do Guamá sofrem com alagamentos devido à falta de tratamento adequado do esgoto, neste bairros foram reportados 30 notícias cada (Figura 6), como 
mostram as reportagens dos dias 26 de Março de 1987 e 3 de Dezembro de 2007, vide na Tabela 1.

O bairro do Marco foi o quinto que mais sofreu com alagamentos (28 notícias/Figura 6), este fica vizinho ao bairro da Pedreira (semelhante aos três bairros, Cremação, Batista Campos e Jurunas, que também são vizinhos). Neste bairro há o canal Visconde de Inhaúma que ao transbordar prejudica os moradores, como mostra a notícia do dia 25 de Abril de 2005 (Tabela 1).

Por último, o bairro de Campinas foi o sexto bairro a sofrer com alagamentos (26 notícias/Figura 6). Este bairro se localiza na Região costeira da Baía do Guajará. SANTOS (2010) indicou que existe uma ação potencializada quando coincide ocorrência dos eventos pluviométricos intensos com a maré alta do Rio Guamá. Salienta-se ainda que neste bairro ocorreram graves alagamentos, porém não com tanta frequência, pois o problema maior se dá ao coincidir chuva forte com a maré alta do rio. Os alagamentos ocorrem principalmente no mercado do Ver-o-Peso, como mostra a notícia de 10 de Março de 2001 (Tabela 1).

Tabela 1. Principais publicações de notícias nos jornais da cidade de Belém durante os anos de 1987 a 2009.

\begin{tabular}{|c|c|c|}
\hline DIAS & PRP & NOTÍCIAS \\
\hline $26 / 03 / 1987$ & $71,8 \mathrm{~mm}$ & $\begin{array}{l}\text { "Na travessa Estrela, bairro da Pedreira, os moradores passaram a } \\
\text { madrugada ilhados em suas próprias casas." } \\
\text { Fonte: Chuva caiu sem... (1987). }\end{array}$ \\
\hline $28 / 02 / 1991$ & $48,2 \mathrm{~mm}$ & $\begin{array}{l}\text { "Os moradores da Dr. Moraes entraram em pânico com esgotos } \\
\text { entupidos devido as fortes chuva." } \\
\text { Fonte: Queixas (1991). }\end{array}$ \\
\hline $28 / 04 / 1998$ & $126,8 \mathrm{~mm}$ & $\begin{array}{l}\text { "A Rua Fernando Guilhon apresentou grande quantidade de lama } \\
\text { pela falta de drenagem dos canais." } \\
\text { Fonte: Periferia submerge com... (1998). }\end{array}$ \\
\hline $10 / 03 / 2001$ & $31,8 \mathrm{~mm}$ & $\begin{array}{l}\text { "Baía do Guajará transbordou e águas de } 3,5 \text { metros invadiram casas } \\
\text { comerciais nas avenidas Marechal Hermes, Castilho de França e } \\
\text { Ver-o-Peso." } \\
\text { Fonte: São as águas... (2001). }\end{array}$ \\
\hline $25 / 04 / 2005$ & $183,3 \mathrm{~mm}$ & $\begin{array}{l}\text { "Eletrodomésticos boiaram no bairro do Marco, os moradores da } \\
\text { Visconde de Inhaúma entre Mauriti e Barão do Triunfo fizeram } \\
\text { protesto, pois sete casas foram alagadas." } \\
\text { Fonte: Chuva alaga cidade... (2005). }\end{array}$ \\
\hline $03 / 12 / 2007$ & $73,7 \mathrm{~mm}$ & $\begin{array}{l}\text { "Na Avenida Conselheiro Furtado, bairro do Guamá, a água que } \\
\text { cobriu completamente a via, invadiu várias casas, deixando muitas } \\
\text { famílias ilhadas." } \\
\text { Fonte: Dias (2007). }\end{array}$ \\
\hline $01 / 04 / 2009$ & $53,6 \mathrm{~mm}$ & $\begin{array}{l}\text { "O canal transbordou, ocasionando alagamento acima do normal na } \\
\text { Rua dos Pariquis, o nível da água subiu tanto que chegou à altura do } \\
\text { pescoço de alguns moradores." } \\
\text { Fonte: Noguchi (2009). }\end{array}$ \\
\hline $04 / 12 / 2009$ & $22,6 \mathrm{~mm}$ & $\begin{array}{l}\text { "Chuva durou pouco tempo, mas foi o suficiente para alagar várias } \\
\text { ruas e casas. O bairro da Cremação foi um dos mais afetados." } \\
\text { Fonte: Noguchi (2009). }\end{array}$ \\
\hline
\end{tabular}




\section{CONCLUSÕES}

Os impactos dos eventos extremos de precipitação diária na cidade de Belém-Pará foram estudados com base em notícias de jornais no período de 1987 a 2009. Notou-se que a maioria dos EEPD geraram problemas para a cidade e viraram notícias nos jornais.

As notícias indicaram que os EEPD causaram desastres físicos, econômicos e sociais devido à falta de infraestrutura da cidade, ocupação urbana desordenada, impermeabilização do solo e falta de rede de esgoto adequada. Isto foi evidenciado, pois a principal consequência que os EEPD ocasionaram a cidade foi o alagamento. Os bairros localizados na bacia hidrográfica da Travessa Quintino Bocaiúva são os que mais ficam alagados, pois observa-se que o canal da Quintino Bocaiúva ainda sofre influência da maré alta do rio Guamá e quando chove, a vazão do escoamento superficial do canal é prejudicada.

As publicações dos jornais mostraram que os congestionamentos do trânsito, as quedas de árvores, os destelhamentos e as descargas atmosféricas também ocorreram na cidade, principalmente devido às nuvens de chuva que são formadas na cidade (cumulonimbus) com grande desenvolvimento vertical, altamente eletrificada, e associada a ventos fortes.

Portanto, a cidade necessita com urgência de um gerenciamento ambiental, principalmente devido ao crescimento exponencial da população e as necessidades de serviços urbanos. Pois apesar da precipitação anual na cidade ser em média $3000 \mathrm{~mm}$ com máximo de $3900 \mathrm{~mm}$, ela não possui rede de drenagem (esgoto) adequada o que provoca todo o problema de alagamento da cidade, agravada pela falta de educação ambiental da população.

Outro fato é a importância das notícias registradas nos veículos de comunicações. A partir das últimas décadas do século XX começou a haver um debate acerca do uso de jornais como fontes de pesquisa e no Brasil alguns trabalhos científicos foram elaborados com base no acervo dos impressos. Desta forma, as notícias dos jornais mostram sua importância dentro da sociedade, servindo, como nesta pesquisa, de fonte fundamental para um estudo mais detalhado sobre as consequências dos EEPD na cidade de Belém - Pará.

\section{AGRADECIMENTOS}

Os autores agradecem a Financiadora de Estudos e Projetos (FINEP) pelo apoio financeiro ao Projeto Rede de Monitoramento e Pesquisa de Fenômenos Meteorológicos Extremos da Amazônia - 1a e $2^{\mathrm{a}}$ fase. Thamiris L. de O. Brandão Campos agradece ao Conselho Nacional de Desenvolvimento Científico e Tecnológico (CNPq), a Universidade Federal do Pará pela Bolsa PIBIC concedida e ao Thiago Sabino pela elaboração do mapa utilizado.

\section{REFERÊNCIAS}

BRYANT, L.; CARVER, L.; BUTLER, C. D.; ANAGE, A. Climate change and family planning: least-developed countries define the agenda. Bulletin of the World Health Organization, v. $87, \quad$ p. $852-857, \quad 2009 . \quad$ http://dx.doi.org/10.1590/S004296862009001100014

CASTRO, E. Geopolítica da água e novos dilemas a propósito da Amazônia e seus recursos naturais. In: ARAGON L. E.; CLÜSENER-GODT, M. (Orgs.) Problemática do uso local e global da água da Amazônia. Belém: NAEA, 2003. p. 334.

CHU, P. S.; ZHAO, X.; RUAN, Y.; GRUBBS, B. Extreme rainfall events in the Hawaiian Islands. Journal of Applied Meteorology and Climatology, v. 48, p. 502-516, jul. 2009. http://dx.doi.org/10.1175/2008JAMC1829.1 
CHUVA alaga cidade e traz muitos problemas. O Liberal, Belém, 25 abr. 2005. Cidades, p. 4.

CHUVA caiu sem qualquer trégua. O Liberal, Belém, 27 mar. 1987. Cidades, p. 08.

COHEN, J.; SILVA DIAS, M. A. F.; NOBRE, C. A. Environmental conditions associated with Amazonian squall lines: A case study. Monthly Weather Review, v. 123, p. 31633174, 1995. http://dx.doi.org/10.1175/1520-0493(1995)123\%3C3163:ECAWAS\% 3E2.0.CO;2

DE SOUZA, E. B.; AMBRIZZI, T. Modulation of the intraseasonal rainfall over tropical Brazil by the Madden-Julian oscillation. International Journal of Climatology. São Paulo, v. 26, p. 1759-1776, 2006. http://dx.doi.org/10.1002/joc.1331

DIAS, D. Canais transbordam e trânsito fica lento. O Liberal, Belém, 3 dez. 2007. Cidades, p. A10.

GRIMM, A. M. Interannual climate variability in South America: impacts on seasonal precipitation, extreme events, and possible effects of climate change. Stochastic Environmental Research and Risk Assesment, v. 25, p. 537-554, 2011. http://dx.doi.org/10.1007/s00477-010-0420-1

INSTITUTO NACIONAL DE METEOROLOGIA - INMET. Normais climatológicas 1961 a 1990. Brasília, DF, 1992.

LEÃO, N.; ALENCAR, C.; VERÍSSIMO, A. Belém sustentável 2007. Belém: Instituto do Homem e Meio Ambiente da Amazônia, 2008. 140 p.

MALONE, T. F. Compendium of meteorology.Washington: American Meteorological Society, 1951.p. 1334.

MARENGO, J. A; NOBRE, C. A. The hydroclimatological frame work of Amazonia. In: RICHEY, J.; MAC CLAINE, M.; VICTORIA, R. (Orgs.). Biogeochemistry of Amazonia. New York: Cambridge University Press, 2000.

MARENGO, J. A. Impactos de extremos relacionados com o tempo e o clima - Impactos sociais e econômicos. Boletim do Grupo de Pesquisa em Mudanças Climáticas GPMC, Edição Especial, 2009.

MONTEIRO, C. A. de F. Clima e excepcionalismo: conjecturas sobre o desempenho da atmosfera como fenômeno geográfico Florianópolis: Ed. UFSC, 1991. 241 p.

NOGUCHI, M. Chuva de uma hora causa novos alagamentos. Diário do Pará, Belém, 04 dez. 2009. Belém, p. A6.

PERIFERIA submerge com a chuva. O Liberal, Belém, 28 abr. 1998. Atualidades.

QUEIXAS. O Liberal, Belém, 28 fev. 1991. Cidades, p. 08.

SANTOS, F. A. A. Alagamento e inundação urbana: modelo experimental de avaliação de risco. 2010. 165f. Dissertação (Mestrado em Ciências Ambientais)-Universidade Federal do Pará/Museu Paraense Emílio Goeldi/Embrapa, Belém, 2010.

SÃO as águas de março. O Liberal, Belém, 10 mar. 2001. Cidade, p. 10.

SILVA, K. Capital do Pará: Belém. Disponível em:

$<$ http://www.amazonia.com.br/turismo_novo/para/belem2.asp>. Acesso em: 27 fev. 2008. 
SIMIELLI, M. E. Geoatlas. São Paulo: Ática, 1991.

SOUZA, E. B.; NOBREGA, P. Uma revisão sobre o padrão de dipolo no Atlântico Tropical. Revista Brasileira de Meteorologia, v. 13, n. 3, p. 1-44, 1998

STHRALER, A. N. Quantitative analysis of watershed geomorphology. Transactions, American Geophysical Union, v. 38, p. 913-920, 1957.

TRUONG, N. M.; TIEN, T. T.; ROGER A. PIELKE SR.; CASTRO, C. L.; LEONCINI, G. A modified Kain-Fritsch scheme and its application for the simulation of an extreme precipitation event in Vietnam. Monthly Weather Review, v. 137, p. 766-789, 2009. http://dx.doi.org/10.1175/2008MWR2434.1

UMAN, M. A. The lightning discharge. Orlando: Academic Press, 1987. 370 p.

WILKS, D. S. Statistical methods in the atmospheric sciences. 2. ed. San Diego: Elsevier Science \& Technology Books, 2006.

XAVIER, T. M.; XAVIER, A. F. S.; ALVES, J. M. B. Quantis e eventos extremos aplicações em ciências da terra e ambientais. Fortaleza: RDS; Livro Técnico, 2007. $278 \mathrm{p}$. 


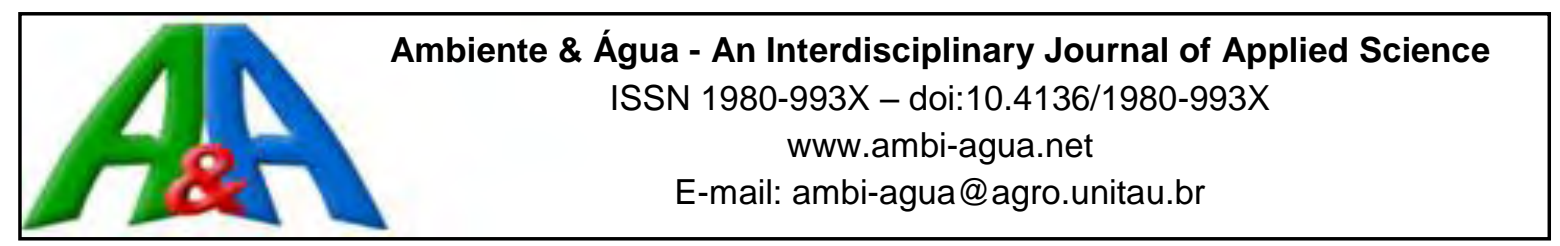

\title{
Influência das características morfométricas da bacia hidrográfica do rio Benevente nas enchentes no município de Alfredo Chaves-ES
}

\author{
doi: 10.4136/ambi-agua.1475 \\ Received: 01 Aug. 2014; Accepted: 07 Nov. 2014 \\ Alexandre Simões Lorenzon ${ }^{1 *}$; Micael de Souza Fraga ${ }^{2}$; \\ Amanda Rodrigues Moreira ${ }^{2}$; Eduardo Morgan Uliana ${ }^{2}$; \\ Demetrius David da Silva ${ }^{2}$; Carlos Antonio Alvares Soares Ribeiro ${ }^{1}$; \\ Alisson Carraro Borges ${ }^{2}$ \\ Universidade Federal de Viçosa (UFV), Viçosa, MG, Brasil \\ ${ }^{1}$ Departamento de Engenharia Florestal (DEF/UFV) \\ ${ }^{2}$ Departamento de Engenharia Agrícola (DEA/UFV) \\ *Autor correspondente: e-mail: alelorenzon@yahoo.com.br, \\ micaelfraga@yahoo.com.br, amanda.eaa2008@gmail.com, \\ morganuliana@gmail.com,demetrius@ufv.br, cribeiro@ufv.br, \\ borges@ufv.br
}

\section{RESUMO}

As enchentes afetam a vida de milhares de pessoas em todo o mundo e suas causas podem estar relacionadas tanto com fatores naturais como antrópicos. $\mathrm{O}$ objetivo do trabalho foi avaliar se a morfometria da bacia do rio Benevente tem relação com as enchentes ocorridas no município de Alfredo Chaves, ES. A base de dados consistiu de um modelo digital de elevação (ASTER), da rede hidrográfica vetorial do IBGE e de uma série histórica de 40 anos de precipitação diária. Os parâmetros morfométricos foram obtidos por meio de Sistemas de Informações Geográficas (SIG). A área de estudo possui uma forma alongada, relevo fortemente ondulado e declividade média da bacia de $42,75 \%$. A altitude variou de 480 a $1.591 \mathrm{~m}$. Novembro, dezembro e janeiro foram os meses de maior precipitação, com média mensal de 246,7, 261,5 e 204,2 mm, respectivamente. Conclui-se que a alta declividade da bacia e do rio principal, aliada à intensificação do uso e ocupação do solo, exerce expressiva influência sobre o escoamento superficial na bacia, aumentando as chances de ocorrência de picos de enchentes.

Palavras-chave: gestão de recursos hídricos, hidrologia, morfometria.

\section{Influence of morphometric characteristics of the Benevente River watershed in Alfredo Chaves Municipality - Espírito Santo State}

\begin{abstract}
Floods affect the lives of thousands of people around the world and their causes may be related to both natural and anthropogenic factors. The objective of this study was to evaluate the relationship between the Benevente River watershed morphometry and the occurrence of floods in the town of Alfredo Chaves, Espírito Santo State, Brazil. The database consisted of a digital elevation model (ASTER), a vector hydrographic network of IBGE and a 40-year
\end{abstract}


period series of daily precipitation. The morphometric parameters were obtained using Geographic Information Systems (GIS). The study area has an elongated shape, with a high drainage density, strongly undulate topography and an average slope of $42.75 \%$. The altitude ranged from 480 to $1591 \mathrm{~m}$. November, December and January were the wettest months, with a monthly average rainfall of 246.7, 261.5 and $204.2 \mathrm{~mm}$, respectively. It was concluded that high slopes of the basin and of the main river, coupled with increased use and occupation of the land, exerts significant influence on the runoff in the basin, increasing the chances of the occurrence of flood peaks.

Keywords: hydrology, morphometry, water resources management.

\section{INTRODUÇÃO}

Dentre os inúmeros fenômenos naturais, as enchentes afetam a vida de 102 milhões de pessoas a cada ano no mundo (UN, 2012). A maior parte da população atingida encontra-se em espaços geográficos vulneráveis, normalmente, próximo a cursos d'água. Suas causas, bem como as respostas e as ações para prevenção e mitigação, é tema de grande interesse público após inúmeros eventos de chuva resultar em perdas de âmbito econômico, ambiental e social (Freitas e Ximenes, 2012).

Segundo Tucci (2001), a ocorrência das enchentes pode estar relacionada tanto com fatores naturais como antrópicos. As condições naturais são aquelas cujas ocorrências são causadas pela bacia em seu estado natural, como: relevo, cobertura vegetal, capacidade de drenagem, forma da bacia e intensidade, duração e frequência das precipitações. As condições antrópicas são aquelas provocadas pela ação do homem, como: urbanização, obras hidráulicas, desmatamento, manejo inadequado do solo, entre outras.

O processo de ocupação e desenvolvimento da sociedade, seja na área urbana ou rural, tem gerado uma intensa degradação do ambiente (Carvalho et al., 2012), que ao longo do tempo tem contribuído, consideravelmente, para maior frequência e magnitude das enchentes (Righi e Robaina, 2010).

As bacias hidrográficas integram uma visão conjunta do comportamento das condições naturais, das atividades humanas e das mudanças nelas desenvolvidas (Guerra e Cunha, 2003). O conhecimento das características morfométricas de uma bacia hidrográfica é imprescindível para a conservação dos recursos hídricos, pois torna possível a compreensão do comportamento hidrológico e possibilita acompanhar as interferências nos processos do ciclo hidrológico e as respectivas respostas da natureza (Ferreira et al, 2012). Desta forma, o conhecimento das características morfométricas pode garantir maior eficiência das intervenções que venham a ser realizadas na bacia, facilitando o seu planejamento, de modo a minimizar impactos ambientais e desastres naturais (Carelli e Lopes, 2011).

O município de Alfredo Chaves é frequentemente atingido pelas enchentes do rio Benevente. No ano de 2012, o município sofreu a pior inundação de sua história, a chuva fez o rio Benevente transbordar e subir mais de 10 metros, alagando mais de $80 \%$ das ruas da cidade. Muitos moradores perderam casas, assim como destruição de lavouras, estradas e pontes (Huber, 2012; Scalzer, 2013).

Nesse contexto, o objetivo do trabalho foi realizar a caracterização morfométrica da bacia hidrográfica do rio Benevente, a montante da estação fluviométrica Matilde (código 57250000), com a finalidade de verificar a relação dos parâmetros físicos e climáticos da bacia com as enchentes ocorridas no município de Alfredo Chaves. 


\section{MATERIAL E MÉTODOS}

\section{1. Área de estudo}

A área de estudo compreende a bacia hidrográfica do rio Benevente (Figura 1), a montante da estação fluviométrica Matilde (código 57250000) pertencente à rede hidrometeorológica da Agência Nacional de Águas (ANA), localizada no município de Alfredo Chaves, a $88 \mathrm{~km}$ da capital do Estado do Espírito Santo.

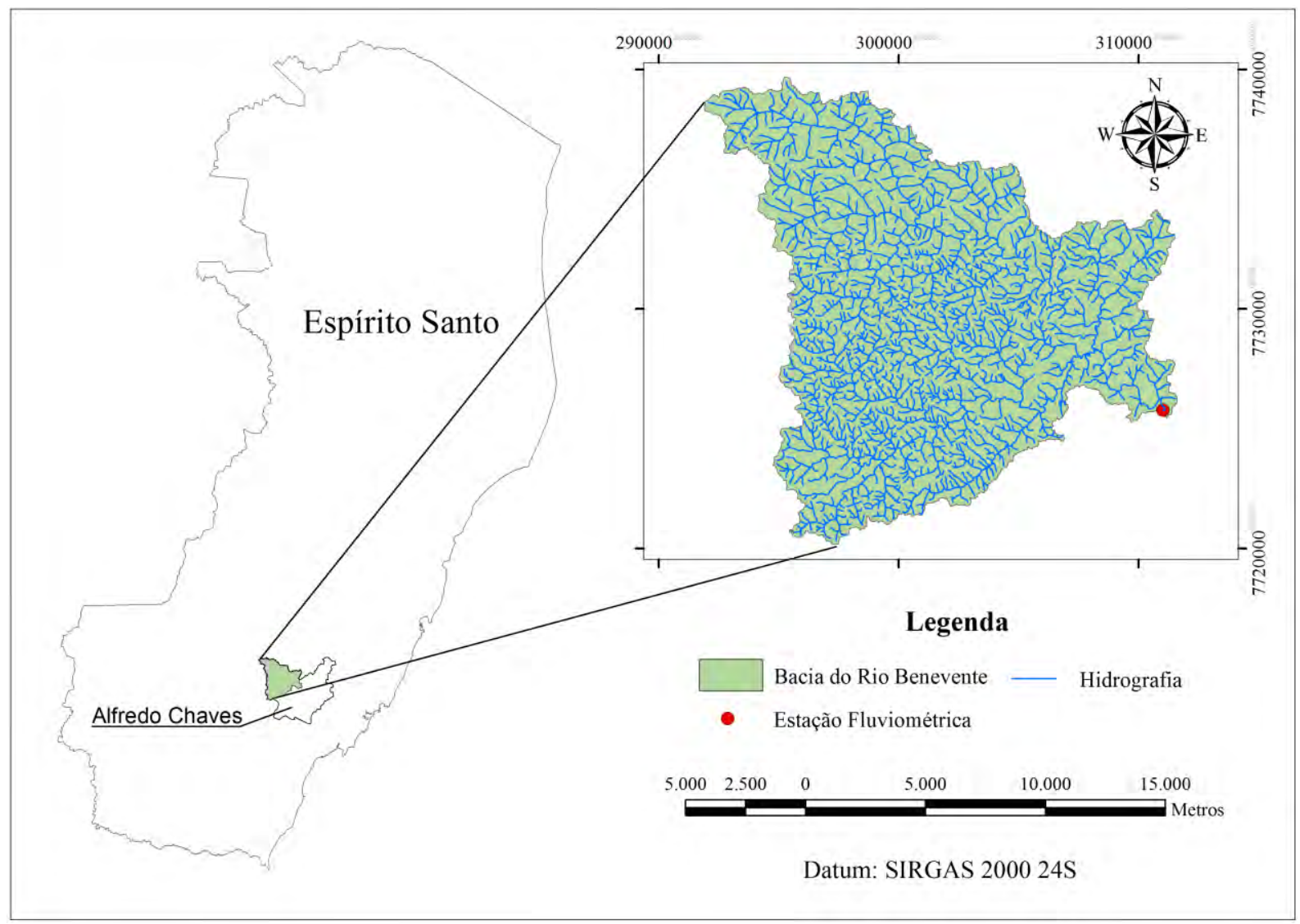

Figura 1. Localização da bacia hidrográfica do rio Benevente, Alfredo Chaves-ES.

A cabeceira da área de drenagem que forma a nascente do rio Benevente encontra-se na Serra do Tamanco, Distrito de São Bento de Urânia. O rio percorre cerca de $79 \mathrm{~km}$ até sua foz no município de Anchieta. Seus principais afluentes são os rios Batatal, Caco do Pote, Corindiba, Conceição, Crubixá, Grande, Iriritimirim, Joeba, Maravilha, Pongal, Salinas e São Joaquim (Espírito Santo, 2013).

Segundo a classificação climática de Köppen, o clima da região é classificado como tropical úmido com estação seca de inverno (Aw). A precipitação média anual e a umidade relativa são iguais a $1.566 \mathrm{~mm}$ e $83 \%$, respectivamente, sendo a temperatura média anual igual a $22{ }^{\circ} \mathrm{C}$.

O município de Alfredo Chaves possui um relevo montanhoso com picos e vales, tais como: Pico do Tamanco, Pico do Gururu, Salto D’água, Serra Pão Doce, Serra do Batatal, Serra Richmont e Serra Boa Vista. Os solos predominantes da região são os classificados como Latossolo Vermelho Amarelo Distrófico, com fertilidade variando de média a baixa e pH moderadamente ácido em torno de 5,0 (Alfredo Chaves, 2013).

As principais atividades econômicas desenvolvidas no município são a agricultura, com destaque para a cultura do café, banana e inhame, a pecuária e o turismo, em virtude do rico potencial de atrativos naturais (Alfredo Chaves, 2013). 


\subsection{Modelo Digital de Elevação (MDE)}

Utilizou-se o Modelo Digital de Elevação (MDE) Advanced Spaceborne Thermal Emission and Reflection Radiometer Global Digital Elevation Model (ASTERGDEM), com resolução espacial de $30 \mathrm{~m}$. Originalmente, esse modelo possui sistema de coordenadas geográficas e datum WGS84. Procedeu-se, então, à conversão do sistema de projeção para UTM e Datum SIRGAS 2000, fuso 24S. O software utilizado para manipulação dos dados foi o ArcGIS 10.1/ArcMap ${ }^{\circledR}$ do ESRI.

Segundo Ribeiro et al. (2002), a maioria dos modelos digitais de elevação possuem irregularidades que inviabilizam a correta delimitação da área de contribuição a montante do ponto de interesse, comprometendo seu uso para estudos hidrológicos. Assim, segundo metodologia descrita em Elesbon et al. (2011), foram realizadas operações de pós-processamento com a finalidade de eliminar essas imperfeições e gerar o Modelo Digital de Elevação Hidrograficamente Condicionado (MDEHC), objetivando garantir a convergência do escoamento superficial até a foz da bacia.

\subsection{Precipitação}

Foram utilizados dados de precipitações diárias da estação pluviométrica Matilde (código 02040011), pertencente à rede hidrometeorológica da Agência Nacional de Águas (ANA). As observações foram de uma série histórica de 40 anos (período-base de 1976 até 2013, exceto 1989 pela falta de informação). Para análise de consistência dos dados, utilizou-se o software Hidro 1.2.1 para gerar uma base de dados para o programa Hidro-Plu Beta 4.1.

\subsection{Características morfométricas}

A obtenção das características morfométricas também foi realizada em ambiente de SIG, utilizando o software ArcGIS 10.1/ArcMap ${ }^{\circledR}$ do ESRI.

\subsection{1. Área de drenagem e perímetro}

Utilizou-se o comando watershed da extensão Spatial Analyst, disponível na interface ArcToolbox, para delimitar a bacia de contribuição, considerando o MDEHC e um arquivo contendo a localização do exutório da mesma. Uma vez delimitada a bacia, a área de drenagem e o perímetro foram gerados automaticamente pelo software.

\subsubsection{Comprimento do rio}

$\mathrm{Na}$ determinação desse parâmetro utilizou-se a malha hidrográfica da bacia do rio Benevente obtida da base de dados digital do IBGE na escala 1:50.000. De posse da hidrografia, foi mensurado o comprimento total dos cursos d'água e o comprimento do rio principal. Este foi considerado como aquele que apresenta maior área de drenagem.

\subsubsection{Ordem dos cursos d'água}

Para a hierarquização dos cursos d'água, foi utilizado o método proposto por Strahler (1957), sendo esse o mais utilizado por possuir melhor compreensão (Oliveira e Borsato, 2011). Para classificar os cursos d'água por este método foi utilizado o comando Stream Order da extensão Spatial Analyst, disponível na interface ArcToolbox.

\subsubsection{Densidade de drenagem}

O sistema de drenagem é formado pelo rio principal e seus tributários ou afluentes e indica a maior ou menor ramificação da rede de drenagem na bacia hidrográfica, fornecendo uma indicação da eficiência da drenagem da bacia (Cardoso et al., 2006). A densidade de drenagem foi calculada de acordo com a Equação 1. 


$$
D d=\frac{L_{t}}{A}
$$

em que:

Dd $=$ densidade de drenagem $\left(\mathrm{km} \mathrm{km}^{-2}\right)$,

$\mathrm{Lt}=$ comprimento total dos cursos d'água $(\mathrm{km})$, e

$\mathrm{A}=$ área de drenagem $\left(\mathrm{km}^{2}\right)$.

\subsubsection{Declividade da bacia e altitude}

A partir do MDEHC, foi gerado um mapa de declividade utilizando-se o comando Slope disponível na extensão Spatial Analist da interface ArcToolbox. Em seguida, foi feita uma reclassificação das classes de valores de declividade em seis intervalos distintos, conforme proposto por Embrapa (1979). Para isso, utilizou-se o comando Reclassify da extensão Spatial Analyst. Uma vez gerado o mapa de declividade, o software automaticamente determina alguns parâmetros relativos ao mesmo, entre estes, a declividade média. Os valores de altitude foram obtidos diretamente do MDEHC.

\subsubsection{Fator de forma}

Obteve-se o fator de forma por meio da relação entre a área e o comprimento axial da bacia (Equação 2).

$$
K f=\frac{A}{L^{2}}
$$

em que:

$$
\begin{aligned}
& \mathrm{Kf}=\text { fator de forma (adimensional), } \\
& \mathrm{A}=\text { área de drenagem }\left(\mathrm{L}^{2}\right), \mathrm{e} \\
& \mathrm{L}=\text { comprimento axial da bacia }(\mathrm{L}) .
\end{aligned}
$$

\subsubsection{Coeficiente de compacidade}

O coeficiente de compacidade foi obtido por meio da relação entre o perímetro e a área da bacia (Carvalho et al., 2009), conforme a Equação 3.

$$
K c=0,28 \frac{P}{\sqrt{A}}
$$

em que:

$\mathrm{Kc}=$ coeficiente de compacidade,

$\mathrm{P}=$ perímetro (L), e

$\mathrm{A}=$ área de drenagem $\left(\mathrm{L}^{2}\right)$.

\subsection{8. Índice de circularidade}

Similarmente ao coeficiente de compacidade, o índice de circularidade tende para a unidade à medida que a bacia se aproxima da forma circular (Cardoso et al., 2006). Este índice é calculado de acordo coma Equação 4. 


$$
I C=\frac{12,57 A}{P^{2}}
$$

em que:

IC = índice de circularidade,

$\mathrm{A}=$ área de drenagem $\left(\mathrm{L}^{2}\right)$, e

$\mathrm{P}=$ perímetro (L).

\subsubsection{Tempo de concentração}

Para determinar o tempo de concentração foi utilizada equação de Giandotti (Pruski et al., 2004), conforme Equação 5.

$$
t c=\frac{4 \sqrt{A}+1,5 L}{0,8 \sqrt{H}}
$$

em que:

tc = tempo de concentração $(h)$,

$\mathrm{A}=$ área da bacia $\left(\mathrm{km}^{2}\right)$,

$\mathrm{L}=$ comprimento do talvegue $(\mathrm{km}) \mathrm{e}$

$\mathrm{H}=$ diferença de nível entre o ponto mais remoto da bacia e a seção de deságue (m).

\subsubsection{Declividade do rio principal}

Para o cálculo da declividade do rio principal foi levado em consideração o tempo de percurso da água ao longo da extensão do perfil longitudinal, obtendo-se a declividade equivalente constante (Villela e Mattos, 1975), como pode ser visto na Equação 6.

$$
S=\left(\frac{\sum L_{i}}{\sum\left(\frac{L_{i}}{\sqrt{D_{i}}}\right)}\right)^{2}
$$

em que:

$\mathrm{S}=$ declividade equivalente $\left(\mathrm{m} \mathrm{m}^{-1}\right)$,

$\mathrm{Li}=$ distância em cada trecho considerado $(\mathrm{m}) \mathrm{e}$

$\mathrm{Di}=$ declividade em cada trecho considerado $\left(\mathrm{m} \mathrm{m}^{-1}\right)$.

\section{RESULTADOS E DISCUSSÃO}

Na Tabela 1 são apresentados os parâmetros morfométricos obtidos pelo estudo. 
Tabela 1. Características morfométricas da bacia hidrográfica do rio Benevente, Alfredo Chaves-ES, Brasil.

\begin{tabular}{lc}
\hline Características morfométricas & Resultados \\
\hline Área de drenagem $\left(\mathrm{km}^{2}\right)$ & 210,49 \\
Perímetro $(\mathrm{km})$ & 121,70 \\
Comprimento do rio principal $(\mathrm{km})$ & 35,15 \\
Declividade do rio principal $(\%)$ & 1,69 \\
Comprimento total dos cursos d'água $(\mathrm{km})$ & 768,86 \\
Ordem da bacia & 6 \\
Densidade de drenagem (km km $\left.{ }^{-2}\right)$ & 3,65 \\
Declividade média (\%) & 42,75 \\
Altitude máxima (m) & 1591,00 \\
Altitude média (m) & 903,21 \\
Altitude mínima (m) & 480,00 \\
Fator de forma & 0,17 \\
Coeficiente de compacidade & 2,35 \\
Índice de circularidade & 0,18 \\
Tempo de concentração (h) & 4,68 \\
\hline
\end{tabular}

$\mathrm{O}$ fator de forma e o índice de circularidade apresentaram resultados muito baixos. Esses valores indicam que a bacia possui uma forma mais alongada. Ademais, o coeficiente de compacidade foi alto, sendo este um número adimensional que varia com a forma da bacia, independentemente do seu tamanho (Villela e Mattos, 1975). Quanto mais a forma da bacia se distancia do formato circular, maior deverá ser este índice. Analisando-se isoladamente esses resultados, pode-se inferir, pela forma da bacia, que a área de estudo é menos propensa à ocorrência de enchentes em condições normais de precipitação. Segundo Cardoso et al. (2006), as bacias com forma alongada possuem baixa probabilidade de chuvas intensas ocorrerem simultaneamente em toda a sua extensão. Além disso, quanto mais irregular for a forma da bacia maior será o coeficiente de compacidade e menor a tendência para enchentes em condições normais de precipitação. Destaca-se, entretanto, que não se pode avaliar a propensão às enchentes em uma bacia com base exclusivamente na sua forma.

A forma da bacia hidrográfica também influencia o tempo de concentração, ou seja, o tempo necessário para que toda a bacia contribua para a saída da água após uma precipitação (Tonello et al., 2006). Nas bacias alongadas, os afluentes atingem o curso d'água principal em vários pontos ao longo do mesmo, diferentemente das bacias circulares, na qual a concentração do deflúvio se dá em um só ponto diminuindo o tempo de concentração e aumentando as chances de ocorrência de enchentes (Villela e Mattos, 1975). Por isso, o tempo de concentração foi alto para a área de estudo, sendo este um reflexo da forma alongada da bacia.

Apesar da forma alongada, a bacia tem sofrido com constantes enchentes que atingem o município quase todos os anos. O estado do Espírito Santo possui dois períodos de precipitação bem distintos, um chuvoso, entre outubro e março (verão), e outro mais seco, com totais médios mensais inferiores a $30 \mathrm{~mm}$, entre abril e setembro (inverno) (De Mello 
et al., 2012). Os autores supracitados verificaram também que a região de Alfredo Chaves possui uma precipitação média mensal maior que as demais regiões do Estado.

Pela análise dos dados da estação pluviométrica utilizada no estudo verifica-se que o período de chuvas concentrou-se entre outubro e março no município de Alfredo Chaves. Os meses de novembro, dezembro e janeiro apresentaram um período contínuo de muita chuva, com média histórica que varia de 204,2 a 261,5 mm (Tabela 2).

Tabela 2. Precipitação média mensal dos seis meses mais chuvosos no município de Alfredo Chaves-ES, Brasil.

\begin{tabular}{lc}
\hline \multicolumn{1}{c}{ Mês } & Média $(\mathbf{m m})$ \\
\hline Outubro & 148,8 \\
Novembro & 246,7 \\
Dezembro & 261,5 \\
Janeiro & 204,2 \\
Fevereiro & 120,9 \\
Março & 221,6 \\
\hline
\end{tabular}

Segundo Reboita et al. (2010) e De Mello et al. (2012), a estação chuvosa do sudeste do Brasil é causada pela zona de convergência do atlântico sul, a qual é a principal causadora de precipitações que ocorrem especialmente entre novembro e janeiro, transportando calor e umidade do oceano atlântico para o interior do Brasil. Esses fatores climáticos, associados à ocorrência de serras e picos do município de Alfredo Chaves, com altitudes que chegam a $1.591 \mathrm{~m}$, estabelecem condições apropriadas para a formação de chuvas orográficas, as quais apresentam alto potencial para causar enchentes devido à sua curta duração e alta intensidade.

As chuvas orográficas são causadas pela ascensão do ar úmido e quente sobre regiões que apresentam elevada variação de altitude (Silva et al., 2012). Esse fenômeno atmosférico foi observado por Kruk et al. (2006), na Serra do Mar no Estado de São Paulo e por Silva et al. (2012), na Serra do Mar no Estado do Paraná. Esses autores destacam ainda a influência da zona de convergência do atlântico sul nas chuvas orográficas ocorridas nessas regiões.

A declividade da bacia e do rio principal são outros fatores com relevante influência nas enchentes que ocorrem no município de Alfredo Chaves. A área de estudo apresentou elevada variação de altitude, com valores entre 480 e $1.591 \mathrm{~m}$ (Tabela 1). Por isso, a declividade média foi alta e o relevo classificado como fortemente ondulado (EMBRAPA, 1979). As áreas planas ( 0 a $3 \%$ ) ocupam apenas $1,24 \%$ da área total da bacia, enquanto que as áreas com relevo mais acentuado ( $>45 \%)$ ocupam quase que a metade da bacia, $42,47 \%$. Observa-se, ainda, que $74,01 \%$ da área correspondem ao relevo fortemente ondulado e montanhoso (Tabela 3).

Tabela 3. Distribuição das classes de declividade da bacia hidrográfica do rio Benevente, Alfredo Chaves-ES, Brasil, segundo Embrapa (1979).

\begin{tabular}{clcc}
\hline Declividade (\%) & \multicolumn{1}{c}{ Relevo } & Área $\left.\mathbf{( k m}^{2}\right)$ & Área $(\%)$ \\
\hline $0-3$ & Plano & 2,61 & 1,24 \\
$3-8$ & Suavemente ondulado & 5,82 & 2,76 \\
$8-20$ & Ondulado & 26,94 & 12,80 \\
$20-45$ & Fortemente ondulado & 85,74 & 40,73 \\
$45-75$ & Montanhoso & 70,03 & 33,28 \\
$>75$ & Fortemente & 19,35 & 9,19 \\
\hline
\end{tabular}


Em áreas com declividade acentuada, a água da chuva concentra-se mais rapidamente nos cursos dos rios aumentando as chances de ocorrer picos de enchentes. A declividade afeta a velocidade do escoamento superficial, diminui a infiltração da água da chuva (Cardoso et al., 2006; Tonello et al., 2006) e potencializa o processo erosivo do solo (Pissarra et al., 2010; Santos et al., 2012). De acordo com Calil et al. (2012), em locais de chuvas intensas, onde a infiltração é dificultada por altas declividades, ocorre maior ramificação da rede hidrográfica, cuja consequência são bacias com densidade de drenagem mais alta e escoamento superficial mais eficiente.

Segundo Villela e Mattos (1975), a densidade de drenagem fornece a eficiência de drenagem da bacia. Para os autores supracitados esse índice pode variar de $0,5 \mathrm{~km} \mathrm{~km}^{-2} \mathrm{em}$ bacias com drenagem pobre, a $3,5 \mathrm{~km} \mathrm{~km}^{-2}$ ou mais, para bacias bem drenadas, considerando a escala de 1:50.000. Para a bacia em estudo, a densidade de drenagem e o comprimento total dos cursos d'água apresentaram valores bem elevados, caracterizando a bacia como bem drenada (Tabela 1). Confirmando esta tendência, a bacia apresentou grau de ramificação de sexta ordem pela hierarquização de Strahler (1957).

Outra característica morfométrica muito importante para fins de análise da propensão à ocorrência de enchentes é a declividade do rio principal, que no caso em questão foi de $1,69 \%$, valor extremamente elevado para canais naturais e, certamente, um dos principais responsáveis pelos picos de enchentes na bacia do rio Benevente.

A magnitude dos picos de enchentes pode ainda ser intensificada quando altas declividades estão relacionadas com a ausência de cobertura vegetal, tipo de solo e intensidade de precipitação, uma vez que esses fatores aumentam a rapidez com que ocorre o escoamento superficial no terreno (Villela e Mattos, 1975; Tonello et al., 2006).

Segundo Soprani e Reis (2007), dentre os principais problemas da região de Alfredo Chaves, destacam-se o acelerado processo de ocupação do solo, nascentes e cursos d'água desprovidos de mata ciliar, processos erosivos decorrentes do uso de encostas para plantio, lançamentos de efluentes e resíduos sólidos nos cursos d'água.

\section{CONCLUSÕES}

Conclui-se que, dentre as características morfométricas avaliadas, os elevados valores de declividade do rio principal e de declividade da bacia, decorrentes da grande variação de altitude na bacia do rio Benevente, assim como dos expressivos valores de densidade de drenagem, são os principais causadores das enchentes do município de Alfredo Chaves. Destaca-se, ainda, que na região ocorrem maiores índices pluviométricos do que em outras regiões do estado, decorrente da maior propensão à ocorrência de chuvas orográficas, potencializando ainda mais a ocorrência do escoamento superficial e, consequentemente, os picos de enchentes.

\section{REFERENCIAS}

ALFREDO CHAVES. Prefeitura. Disponível em: <www.alfredochaves.es.gov.br>. Acesso em: 25 set. 2013.

CALIL, P. M.; OliVEIRA, L. F. C. de; KLIEMANN, H. J.; OLIVEIRA, V. A. de. Caracterização geomorfométrica e do uso do solo da bacia hidrográfica do Alto Meia Ponte, Goiás. Revista Brasileira de Engenharia Agrícola e Ambiental, Campina Grande, v. 16, n. 4, p. 433-442, 2012. http://dx.doi.org/10.1590/S141543662012000400014 
CARDOSO, C. A.; DIAS, H. C. T; SOARES, C. P. B.; MARTINS, S. V. Caracterização morfométrica da bacia hidrográfica do Rio Debossan, Nova Friburgo, RJ. Revista Árvore, Viçosa, v. 30, n.2, p. 241-248, 2006. http://dx.doi.org/10.1590/S010067622006000200011

CARELLI, L.; LOPES, P. P. Caracterização fisiográfica da bacia Olhos D’água em Feira de Santana/BA: Geoprocessamento aplicado à análise ambiental. Boletim Goiano de Geografia, Goiânia, v. 31, n. 2, p. 43-54, 2011.

CARVALHO, A. P. V.; BRUMATTI, D. V.; DIAS, H. C. T. Importância do manejo da bacia hidrográfica e da determinação de processos hidrológicos. Revista Brasileira de Agropecuária Sustentável, Viçosa, v. 2, n. 2, p. 148-156, 2012.

CARVAlHO, W. M.; VIEIRA, E. O.; ROCHA, J. M. J.; PEREIRA, A. K. S.; CARMO, T. V. B. do. Caracterização fisiográfica da bacia hidrográfica do Córrego do Malheiro, no município de Sabará-MG. Irriga, Botucatu, v. 14, n. 3, p. 389-412, 2009.

DE MELLO, C. R.; VIOLA, M. R; CURI, N.; SILVA, A. M. da. Distribuição espacial da precipitação e da erosividade da chuva mensal e anual no estado do Espírito Santo. Revista Brasileira de Ciência do Solo, Viçosa, v. 36, p. 1878-1891, 2012. http://dx.doi.org/10.1590/S0100-06832012000600022

ELESBON, A. A. A.; GUEDES, H. A. S.; DA SILVA, D. D.; OLIVEIRA, I. C. Uso de dados SRTM e plataforma SIG na caracterização morfométrica da bacia hidrográfica do Braço Norte do Rio São Mateus - Brasil. Revista Escola de Minas, Ouro Preto, v. 64, n. 3, p. 281-288, 2011.

EMPRESA BRASILEIRA DE PESQUISA AGROPECUÁRIA - EMBRAPA. Serviço nacional de levantamento e conservação de solos. In: REUNIÃO TÉCNICA DE LEVANTAMENTO DE SOLOS, 10., 1979, Rio de Janeiro. Súmula... Rio de Janeiro: EMBRAPA; SNLCS, 1979. 83 p.

ESPÍRITO SANTO. Secretaria de Estado de Meio Ambiente e Recursos Hídricos - SEAMA. Website. Disponível em: <www.meioambiente.es.gov.br>. Acesso em: 30 jun. 2013.

FERREIRA, R. G.; MOURA, M. C. O.; CASTRO, F. S. Caracterização morfométrica da subbacia do Ribeirão Panquinhas, ES. Enciclopédia Biosfera, Goiânia, v. 8, n. 15, p. 2247 2256, 2012.

FREITAS, C. M; XIMENES, E. F. Enchentes e saúde pública - uma questão na literatura científica recente das causas, consequências e respostas para prevenção e mitigação.

Ciência \& Saúde Coletiva, Rio de Janeiro, v. 17, n. 6, p. 1601-1615, 2012. http://dx.doi.org/10.1590/S1413-81232012000600023

GUERRA, A. J. T.; CUNHA, S. B. Geomorfologia e meio ambiente. 4. ed. Rio de Janeiro: Bertrand Brasil, 2003. 394 p.

HUBER, J. Alfredo Chaves sofreu a pior enchente de sua história. Montanhas Capixabas, Alfredo Chaves, 2012. Disponível em: <www.montanhascapixabas.com.br/?x=materia \&codItem $=5503 \& \operatorname{cod}$ Area $=1>$. Acesso em: 30 jan. 2014.

KRUK, N. S.; HOFFMANN, P.; RAABE, A. Modeling of orographic precipitation events in South America to couple hydrological and atmospheric models. Part I: The simulation of rain with the Mesoscale Model GESIMA. Wissenschaftliche Mitteilungen Aus Dem Institut Für Meteorologie Der Universität Leipzig, v. 37, p. 137-147, 2006. 
OLIVEIRA, E. D.; BORSATO, V. A. Propriedades morfométricas da bacia hidrográfica do Córrego Marumbizinho, Jandaia do Sul/PR. Revista Geografar, Curitiba, v. 6, n. 1, p. 76-94, 2011.

PISSARRA, T. C. T.; RODRIGUES, F. M.; POLITANO, W.; GAlBiATTI, J. A. Morfometria de microbacias do Córrego Rico, afluente do Rio Mogi-Guaçu, Estado de São Paulo, Brasil. Revista Árvore, Viçosa, v. 34, n. 4, p. 669-676, 2010. http://dx.doi.org/10.1590/S0100-67622010000400011

PRUSKI, F. F.; BRANDÃO, V. D. S.; SILVA, D. D. D. Escoamento superficial. 2. ed. Viçosa: Editora UFV, 2004. 87 p.

REBOITA, M. S.; GAN, M. A.; ROCHA, R. P.; AMBRIZZI, T. Regimes de precipitação na América do Sul: uma revisão bibliográfica. Revista Brasileira de Meteorologia, São José dos Campos, v. 25, n. 2, p. 185-204, 2010.

RIBEIRO, C. A. A. S.; CHAVES, M. A.; SOARES, V. P.; EUCLYDES, H. P. Modelos digitais de elevação hidrologicamente consistentes para a Amazônia legal. In: SIMPÓSIO DE RECURSOS HÍDRICOS DO CENTRO-OESTE, 2., 2002, Campo Grande. Anais... Campo Grande: ABRH, 2002.

RIGHI, E.; ROBAINA, L. E. S. Enchentes do Rio Uruguai no Rio Grande do Sul entre 1980 e 2005: uma análise geográfica. Sociedade \& Natureza, Uberlândia, v. 22, n. 1, p. 35-54, 2010. http://dx.doi.org/10.1590/S1982-45132010000100004

SANTOS, D. B.; VIDOTTO, M. L.; BERTINATTO, R.; MARCON, G. R. S.; FRIGO, E. P. Caracterização morfométrica da bacia hidrográfica do Rio São José, Cascavel, PR. Revista Brasileira de Tecnologia Aplicada nas Ciências Agrárias, Guarapuava, v. 5, n. 2, p. 7-18, 2012.

SCALZER, P. Um ano depois, lembranças da grande enchente ainda atormentam moradores. CBN Vitória, Vitória, 2013. Disponível em: <http:/gazetaonline.globo.com/_conteudo/ 2013/10/cbn_vitoria/reportagens/1464907-um-ano-depois-lembrancas-da-grandeenchente-ainda-atormentam-moradores.html>. Acesso em: 30 jan. 2014.

SILVA, A. M. A.; OLIVEIRA, A. G.; LOEZER, T. L.; SOUZA, R. M.Avaliação do comportamento da precipitação entre o primeiro planalto paranaense e o litoral do Paraná no ano hidrológico 2010/2011. Revista Geonorte, Manaus, v. 2, n. 5, p. 967 974, 2012.

SOPRANI, M. A; REIS, J. A. T. Proposição de equações de intensidade-duração-frequência de precipitações para a bacia do Rio Benevente, ES. Revista Capixaba de Ciência e Tecnologia, Vitória, v. 2, n. 1, p. 18-25, 2007.

STRAHLER, A. N. Quantitative analysis of watershed Geomorphology.Transactions. American Geophysical Union, v. 38, n. 6, p. 913-920, 1957.

TONELlO, K. C.; DIAS, H. C. T.; SOUZA, A. L.; RIBEIRO, C. A. A. S.; LEITE, F. P. Morfometria da bacia hidrográfica da Cachoeira das Pombas, Guanhães - MG. Revista Árvore, Viçosa, v. 30, n. 5, p. 849-857, 2006. http://dx.doi.org/10.1590/S010067622006000500019

TUCCI, C. E. M. Hidrologia: ciência e aplicação. 2. ed. Porto Alegre: UFRGS; Edusp; ABRH, 2001. 943 p. 
VILLELA, S. M; MATTOS, A. Hidrologia aplicada. São Paulo: McGraw-Hill do Brasil, 1975. $245 \mathrm{p}$.

UNITED NATIONS-UN. Disaster-resilient Societies - Facts and figures. 2012. Disponível em: <www.un.org/en/sustainablefuture/disasters.shtml>. Acesso em: 22 jan. 2014. 


Ambiente \& Água - An Interdisciplinary Journal of Applied Science
ISSN 1980-993X - doi:10.4136/1980-993X
www.ambi-agua.net
E-mail: ambi-agua@agro.unitau.br

\title{
Caracterização de ambientes aquáticos no rio de Ondas, cerrado baiano
}

\author{
doi: 10.4136/ambi-agua.1535
}

Received: 20 Oct. 2014; Accepted: 21 Nov. 2014

\author{
Elis Regina Rodrigues de Souza Castro ${ }^{1 *}$; Michel Castro Moreira ${ }^{2}$; \\ Luís Gomes Carvalho ${ }^{2}$; Demetrius David da Silva ${ }^{3}$ \\ ${ }^{1}$ Universidade Federal do Oeste da Bahia (UFOB), Barreiras, BA, Brasil \\ Centro das Ciências Biológicas e da Saúde \\ ${ }^{2}$ Universidade Federal do Oeste da Bahia (UFOB), Barreiras, BA, Brasil \\ Centro das Ciências Exatas e das Tecnologias \\ ${ }^{3}$ Universidade Federal de Viçosa (UFV), Viçosa, MG, Brasil \\ Departamento de Engenharia Agrícola \\ *Autor correspondente: e-mail: errscbio@gmail.com, \\ michelcm@ufob.edu.br, carvalholg70@hotmail.com, \\ demetrius@ufv.br
}

\section{RESUMO}

O objetivo do presente trabalho foi realizar a caracterização da disponibilidade dos ambientes aquáticos presentes no rio de Ondas, visando obter informações sobre a complexidade estrutural e subsídiar políticas públicas de gestão dos recursos hídricos que permitam o uso compartilhado da água e a proteção da biodiversidade da bacia. O rio de Ondas drena área de $5.559 \mathrm{~km}^{2}$, localizada no oeste baiano, em uma região de cerrado. Para a caracterização, foram delimitados dois trechos: no alto e no baixo curso da bacia, onde foram realizados um levantamento topobatimétrico, caracterização do leito e hidráulica nos períodos seco e chuvoso. No levantamento topobatimétrico, verificou-se que no alto curso, as altitudes variaram de $699,82 \mathrm{~m}$ a $714,06 \mathrm{~m}$ e no baixo curso de 439,45 m a 446,91 m. Os perfis batimétricos permitiram a visualização da estrutura do leito e da distribuição dos ambientes no canal. $\mathrm{Na}$ análise granulométrica do alto curso predominou areia $(88,94 \%)$, enquanto no baixo curso matacão $(76,9 \%)$ e blocos $(23,1 \%)$. No alto curso, a vazão variou de $5,20 \mathrm{~m}^{3} \mathrm{~s}^{-1}$ (seco) a $9,07 \mathrm{~m}^{3} \mathrm{~s}^{-1}$ (chuvoso); e no baixo curso de $25,73 \mathrm{~m}^{3} \mathrm{~s}^{-1}$ (seco) a $66,44 \mathrm{~m}^{3} \mathrm{~s}^{-1}$ (chuvoso). A complexidade dos ambientes dos trechos favorece a diversidade biológica no rio de Ondas, resultando na disponibilidade de ambientes com diferentes características que beneficiam as aptidões das espécies aquáticas à estrutura do canal. $\mathrm{O}$ estudo das características físicas do rio de Ondas possibilitou um amplo conhecimento dos micro-ambientes nos trechos estudados, subsidiando estudos relativos à biota aquática e à gestão de recursos hídricos.

Palavras-chave: ADCP, biota aquática, granulometria, topobatimetria. 


\title{
Characterization of aquatic environments in the De Ondas River, Bahian Cerrado
}

\begin{abstract}
This work sought to characterize the types of environments present in the De Ondas River, with the aim of obtaining information on their structural complexity and supporting public policies for water resource management that will allow the shared water use and will protect the biodiversity of the river basin. The De Ondas River watershed is located in the Cerrado Region, in the western part of Bahia State, and covers an area of $5.559 \mathrm{~km}^{2}$. In order to perform the characterization, two stretches were demarcated, one in the upper part of the basin and another in the lower. Afterwards, a topobathymetric survey was conducted, and characterizations of the bottom and of hydraulic properties were done for both the rainy and dry seasons. The topobathymetric survey in the upper course showed altitudes ranging from $699.82 \mathrm{~m}$ to 714.06 , whereas in the lower course these varied from $439.45 \mathrm{~m}$ to $446.91 \mathrm{~m}$. Bathymetric profiles allowed the visualization of the structure and distribution of the bottom of the channel environments. The granulometric analysis of the higher part of the basin showed that sand was predominant $(88.94 \%)$, while in the lower part boulders $(76.9 \%)$ and cobble $(23.1 \%)$ predominated. In the higher part of the basin, the water flow varied from $5.20 \mathrm{~m}^{3} \mathrm{~s}^{-1}$ (dry period) to $9.07 \mathrm{~m}^{3} \mathrm{~s}^{-1}$ (rainy period); and in the lower part, from $25.73 \mathrm{~m}^{3} \mathrm{~s}^{-1}$ (dry period) to $66.44 \mathrm{~m}^{3} \mathrm{~s}^{-1}$ (rainy period). The complexity of the environments of these stretches favors biological diversity in the river, resulting in different environments that aid aquatic species in adapting to the channel structure. The study of the physical characteristics of the De Ondas River produced a great amount of information regarding the microenvironments of the stretches examined, and will support studies related to aquatic biota and water resource management.
\end{abstract}

Keywords: ADCP, aquatic biota, granulometry, topobathymetry.

\section{INTRODUÇÃO}

O estudo das características ecológicas de um rio se configura como a melhor maneira de compreender quais fatores interferem na biodiversidade aquática, possibilitando a compreensão da estrutura do ambiente aquático (Ferreira e Castro, 2005). O estudo da estrutura do ambiente aquático corresponde à análise das alterações geológicas, vegetacionais, climáticas e topográficas, as quais associadas e ao longo do tempo, contribuem para a evolução da paisagem de um rio (Beschta e Platts, 1986).

Os fatores abióticos agem sobre as espécies, servindo como um filtro que determina, a princípio, a abundância de indivíduos em um local com determinadas características. Fundamentando-se em tais aspectos, pode-se afirmar que uma maior complexidade física do ambiente promoverá um aumento da riqueza de espécies, devido à maior disponibilidade e combinações de ambientes correlacionados com as aptidões das espécies (Allan e Castillo, 2007).

Fundamentando-se nas relações entre a biota aquática e o ambiente físico do rio, Vannote et al. (1980) propuseram a Teoria do Rio Continuum. Esta teoria considera os rios como sendo sistemas que possuem gradientes contínuos de características físicas ao longo de seus cursos, que resultaram nas adaptações evolutivas das espécies aquáticas. Uma extensão desta teoria foi postulada por Ward e Stanford (1983), que previram a descontinuidade serial resultante da ação antrópica ao longo do gradiente longitudinal da bacia. Nesta descontinuidade, consideraram que as interferências ao longo do contínuo fluvial irão definir as modificações no gradiente das características físicas e da biota aquática do rio. 
A estrutura do ambiente aquático está associada aos componentes do canal, como o material presente no leito (Boëchat et al., 2013; Hawkins et al., 1993); sua morfologia, representada pelas características do leito, como a profundidade, a largura e a forma do canal; e o estudo de parâmetros hidráulicos, que possuem grande importância na descrição de ambientes (Jowett, 1993).

Dentre os diversos parâmetros utilizados na caracterização da estrutura do ambiente, destaca-se o estudo do material presente no leito do rio. Realizada através da análise granulométrica, a caracterização do material do leito constitui em importante indicador da capacidade de transporte dos canais, dos ambientes deposicionais e, principalmente, das mudanças ocorridas na dinâmica e morfologia fluvial (Leandro e Souza, 2012). Além disso, a caracterização do leito fornece informações sobre as interações entre os parâmetros limnológicos e bióticos nos ecossistemas aquáticos, correspondendo a um dos fatores responsáveis pela distribuição das comunidades aquáticas (Queiroz e Rocha, 2010; Rodrigues et al., 2010).

Melhor entendimento da configuração dos ambientes aquáticos é dado pelo estudo da morfologia do canal, feito através do levantamento da topografia do leito (Jowett, 1993), que possibilita o conhecimento de suas características morfométricas por meio da representação do leito por perfis batimétricos, os quais são gerados por georreferenciamento da superfície d'água e pelas medições das profundidades do canal (Krueger et al., 2003; Resck et al., 2007). Os levantamentos topobatimétricos, subsidiam estudos relacionados com a ecologia dos organismos aquático, tornando-se essencial no estabelecimento de planos de gestão sustentável dos ecossistemas aquáticos (Bezerra-Neto e Pinto-Coelho, 2008).

As características hidráulicas descrevem também muitos dos parâmetros relacionados à estrutura do ambiente, pois permitem estimar a dimensão da bacia, os tipos de escoamentos, além de subsidiar a criação de modelos que as representem. Modificações nessas características interferem decisivamente no escoamento, na qualidade da água e, consequentemente, na biota aquática. Dessa forma, o estudo das condições hidráulicas dos rios é essencial para a verificação das suas condições ambientais (Martins e Honda, 2004).

Rodrigues et al. (2010) destacam a importância dos estudos da estrutura dos ambientes para a conservação e o gerenciamento dos recursos hídricos, os quais fornecem dados que possibilitam avaliar os níveis de impactos antrópicos nas bacias hidrográficas. Além disso, os rios são ecossistemas abertos, com elevada conectividade longitudinal, lateral e vertical (Allan e Castillo, 2007), que estão sempre recebendo pressões externas, comprometedoras da sua qualidade (Barbour et al., 1999).

$\mathrm{Na}$ bacia do rio de Ondas, localizada no oeste do Estado da Bahia e caracterizada pelo bioma cerrado, verifica-se acelerado crescimento econômico advindo da agricultura irrigada. Devido ao intenso uso da água, podem ter ocorrido perdas ambientais relacionadas à disponibilidade hídrica e ao ecossistema aquático, tendo em vista a demanda dos recursos hídricos sem um gerenciamento integrado que vise à conservação da integridade das espécies aquáticas.

Além da constatação do intenso uso da água, na região é evidenciada grande carência de trabalhos que contemplem suas características ambientais. A falta dessas informações indica a necessidade de estudos que subsidiem políticas que contemplem as particularidades locais. Deste modo, o objetivo do presente trabalho foi realizar a caracterização da disponibilidade dos ambientes aquáticos presentes no rio de Ondas, visando obter informações sobre a sua complexidade estrutural e subsidiar políticas públicas de gestão dos recursos hídricos que permitam o uso compartilhado da água e a proteção da biodiversidade aquática da bacia. 


\section{MATERIAL E MÉTODOS}

\section{1. Área de estudo}

A bacia hidrográfica do rio de Ondas, localizada nos municípios de Barreiras e Luís Eduardo Magalhães, região oeste do Estado da Bahia, possui área de aproximadamente $5.559 \mathrm{~km}^{2}$ (Figura 1). O rio de Ondas apresenta comprimento de $144 \mathrm{~km}$, altitude variando de $900 \mathrm{~m}$ a $400 \mathrm{~m}$, e possui como principais tributários os rios das Pedras, Borá e riacho Cabeceira das Lajes (Damasceno, 2011; Soares Neto e Souza, 2007).

Visando realizar a caracterização da disponibilidade dos ambientes aquáticos no rio de Ondas, foram selecionados dois trechos de $300 \mathrm{~m}$ de extensão, distantes entre si em $100 \mathrm{~km}$, com condições ambientais distintas, sendo um localizado no alto curso e outro no baixo curso da bacia (Figura 1).
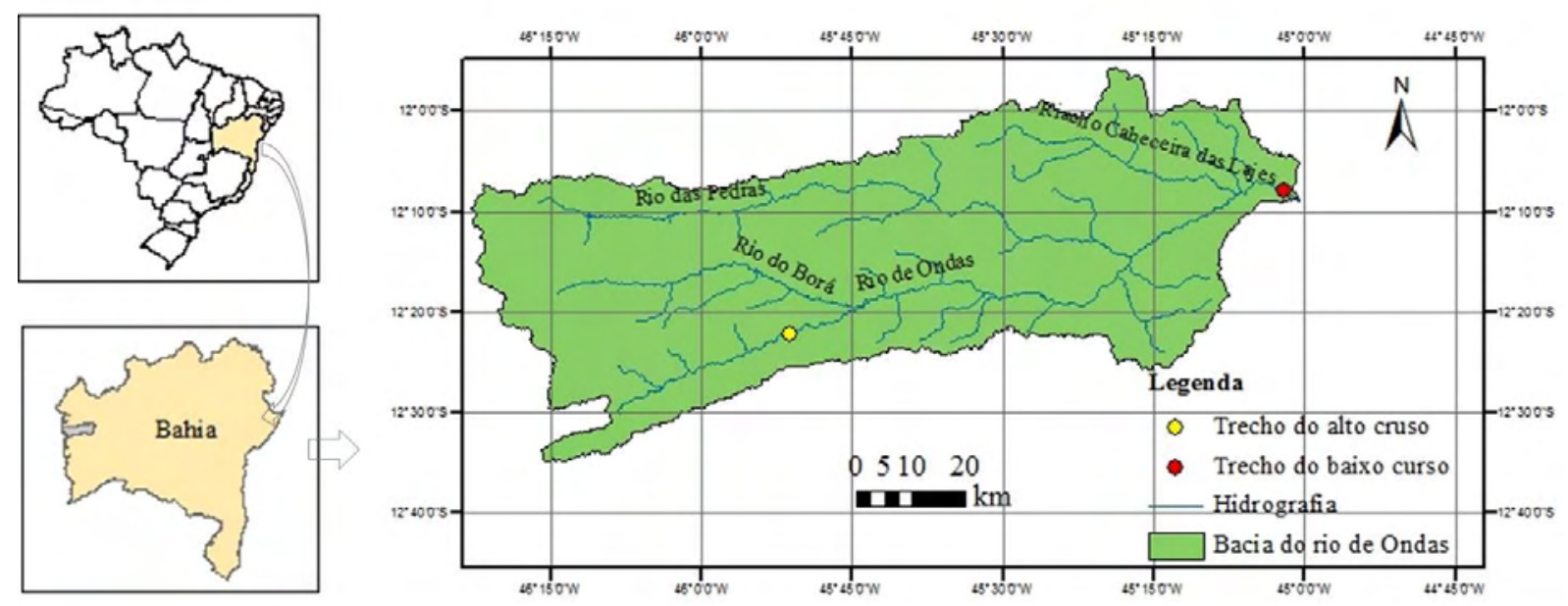

Figura 1. Localização da bacia do rio de Ondas e dos trechos de estudo.

\subsection{Coleta de dados}

Para caracterização dos ambientes aquáticos dos trechos do rio de Ondas, foram realizadas as seguintes atividades: levantamento topobatimétrico; caracterização do material do leito; e caracterização hidráulica.

\subsubsection{Levantamento topobatimétrico}

O levantamento topográfico das margens dos trechos foi realizado utilizando-se GPS Geodésico (Leica, modelo ATX900), contendo dois receptores geodésicos de dupla frequência L1/L2, conforme metodologia proposta pelo IBGE (2009); e uma estação total (Nikon, modelo DT332) com dois prismas.

Os dados obtidos pelo GPS Geodésico foram processados no programa computacional Leica GeoOffice, enquanto os dados levantados pela estação total foram processados no programa computacional GeoOffice Topográfico Profissional.

A batimetria no trecho do alto curso do rio de Ondas foi realizada com a utilização do Acoustic Doppler Current Profiler (ADCP) (Sontek, modelo M9) composto de dois conjuntos de transdutores para medições de velocidade, um feixe acústico vertical de 0,50 MHz para medição da profundidade e um sistema de GPS e RTK acoplado para determinação do posicionamento dos equipamentos (Sontek, 2009).

No levantamento com o ADCP, as linhas batimétricas foram definidas em zigue-zague por toda a área do trecho (Bezerra-Neto e Pinto-Coelho, 2008), sendo um condutor colocado em cada margem para guiar o deslocamento do equipamento. 
Os dados coletados foram processados utilizando-se o programa computacional SonTek RiverSurveyor Live, sendo posteriormente exportados e editados na planilha no Microsoft Excel para pós-processamento no programa computacional ESRI ArcGis $10^{\circledR}$.

No trecho do baixo curso do rio de Ondas, devido à elevada quantidade de material grosseiro, o levantamento batimétrico foi realizado com a utilização de estação total e bastão de altura fixa de $2 \mathrm{~m}$ com prisma. Os pontos de apoio e de distribuição das linhas batimétricas foram materializados através dos marcos de concreto instalados nas seções transversais. Realizou-se o levantamento concentrando a coleta nas áreas onde ocorreram mudanças na topografia do leito do canal.

\subsubsection{Caracterização hidráulica}

Para a medição dos parâmetros hidráulicos dos trechos foi utilizado o ADCP. As medições foram realizadas durante os períodos seco e chuvoso, característicos da região, em três seções transversais em cada trecho delimitadas para este procedimento. Em cada seção transversal foram realizadas oito medições dos parâmetros, correspondendo à profundidade $(\mathrm{m})$, à largura $(\mathrm{m})$, à velocidade $\left(\mathrm{m} \mathrm{s}^{-1}\right)$ e à vazão $\left(\mathrm{m}^{3} \mathrm{~s}^{-1}\right)$.

A análise do desvio padrão e do coeficiente de variação dos parâmetros obtidos pelo ADCP foi realizada no programa SonTek RiverSurveyor, sendo aceitas as leituras que apresentassem um coeficiente de variação de até 5\% (Sontek, 2009). Os valores dos parâmetros foram obtidos a partir das médias referentes às oito medições.

\subsubsection{Caracterização do leito}

Devido à heterogeneidade das partículas distribuídas nos trechos de estudo, a caracterização do leito do canal foi realizada empregando-se diferentes técnicas. Em locais que apresentavam partículas de menor diâmetro, como areia e seixo, foram coletadas 18 amostras ao longo de cada trecho do material de fundo com amostrador de arrasto. As amostras foram armazenadas em sacos plásticos identificados por trecho e ponto de coleta e transportadas para o Laboratório de Engenharia Civil da Universidade Federal do Oeste da Bahia.

A preparação das amostras baseou-se nas metodologias propostas pela NBR 6457/86 (ABNT, 1986) e NBR 7181/84 (ABNT, 1984). Para separação das frações de argila, silte e areia, foi utilizado o método de pipetagem (EMBRAPA, 1997), e para separação das frações grosseiras foi utilizado o método do peneiramento (ABNT, 1986). Além disso, para a determinação da umidade e do fator de correção das amostras, foram utilizados os procedimentos propostos pela EMBRAPA (1997).

No trecho do baixo curso, onde há predominância de partículas grosseiras, estas foram coletadas para realização de medições dos seus eixos, visando à obtenção do diâmetro médio, conforme a técnica de medição e cálculo do diâmetro médio das partículas (Cunha e Guerra, 2011). Nesta análise foi utilizada a classificação granulométrica proposta por Wentworth (1922).

\subsection{Análise da disponibilidade de ambientes para os organismos aquáticos}

A análise da disponibilidade de ambientes aquáticos foi realizada a partir da correlação da topobatimetria com os parâmetros hidrodinâmicos (vazão, profundidade e velocidade do escoamento), da granulometria do material do leito, e a vegetação superficial e aquática encontrados nos trechos do rio de Ondas.

A caracterização dos ambientes deu-se a partir da análise das diferentes características ambientais de cada trecho e de suas alterações ao longo dos períodos sazonais. Observaram-se, também, a estruturação, a distribuição e a abundância da condição do ambiente imposta através da associação dos parâmetros, que correspondem a uma fonte potencial de limitação para a biota aquática (Barbour et al., 1999). 


\section{RESULTADOS E DISCUSSÃO}

\subsection{Levantamento topobatimétrico}

Na Figura 2 apresenta-se a distribuição dos pontos levantados, bem como a superfície batimétrica de montante a jusante vista em 3D do trecho do alto curso. Neste trecho foram levantados 1.550 pontos, com altitudes variando entre $699,82 \mathrm{~m}$ e 714,06 m. Na Figura 2 verifica-se menor concentração de pontos coletados na margem esquerda, fato associado à extensa mata de galeria inundável, que dificultou o acesso e a realização de um estudo mais detalhado dessa margem.

Pela análise da Figura 2, verifica-se na superfície batimétrica em 3D que o canal deste trecho possui uma distribuição descontínua das profundidades, entre locais rasos e profundos, sendo que os pontos mais profundos do trecho localizam-se próximos à região mais a jusante do canal.

Pode-se verificar também a presença de ambientes de erosão e sedimentação localizados principalmente na margem direita do canal, na qual ocorrem áreas com topografia irregular ao longo do trecho, como mostrado na superfície 3D (Figura 2). Na margem esquerda, de forma contrária, observa-se que o canal é mais profundo em relação à margem direita.
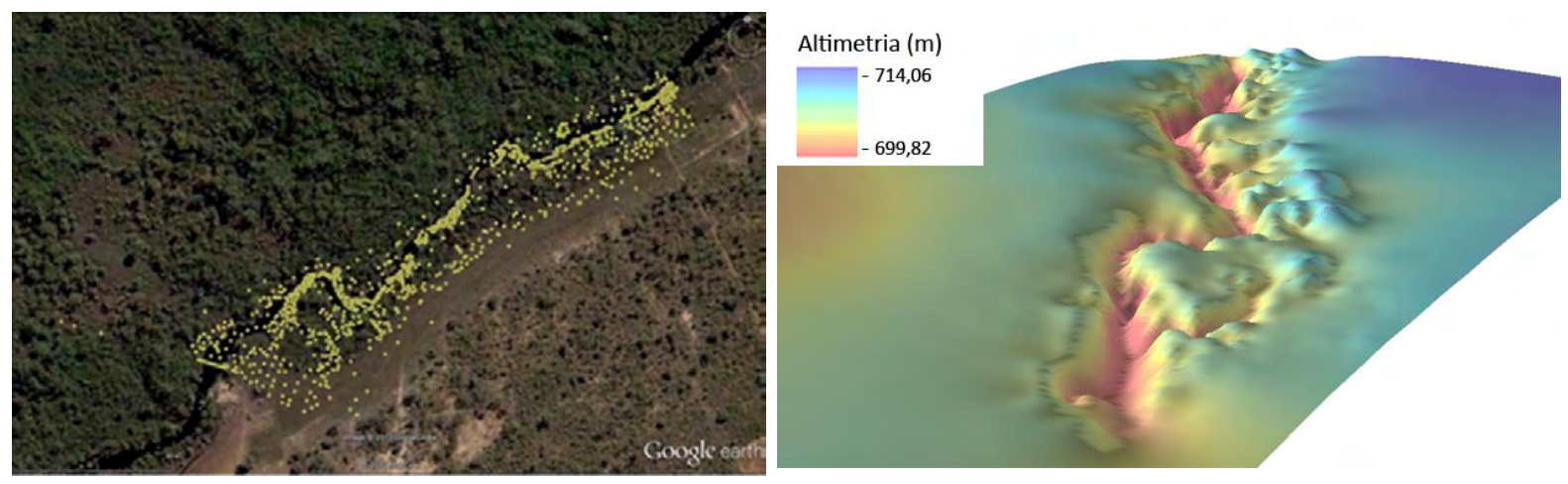

Figura 2. Distribuição dos pontos do levantamento batimétrico no trecho do alto curso do rio de Ondas e vista em 3D da superfície batimétrica de montante para jusante.

Fonte: Adaptado de Google Earth (2013).

Na Figura 3 apresenta-se a distribuição dos pontos levantados no trecho do baixo curso, bem como sua superfície batimétrica de montante a jusante em 3D. Neste trecho foram levantados 1.326 pontos, com altitude variando de $439,45 \mathrm{~m}$ a $446,91 \mathrm{~m}$. Pela análise da Figura 3, percebe-se que os pontos foram levantados de forma distribuída em todo o trecho. Em alguns locais, notadamente os mais profundos e turbulentos, devido à dificuldade de se fixar o bastão para a leitura da altitude, há menor concentração de pontos em relação às demais áreas.

Os resultados do levantamento topobatimétrico do baixo rio de Ondas demostraram o detalhamento da superfície batimétrica, advinda principalmente da extensa malha amostral. $\mathrm{Na}$ representação da superfície do canal, percebe-se que as maiores profundidades ocorrem na região à jusante do trecho, em local que se verifica um estreitamento do canal. Além disso, pode-se constatar que o leito é mais regular no início do trecho, sendo que para jusante aumentam-se as irregularidades no leito, ocorrendo zonas descontínuas com altas variações na profundidade.

Essas variações nos perfis mostram a diversidade na disponibilidade de ambiente nesse trecho, com depressões próximas às margens, formando remansos, e corredeiras nos locais mais profundos no centro do canal, podendo contribuir também para a diversidade dos organismos aquáticos, como explicado por Jowett (1993). 

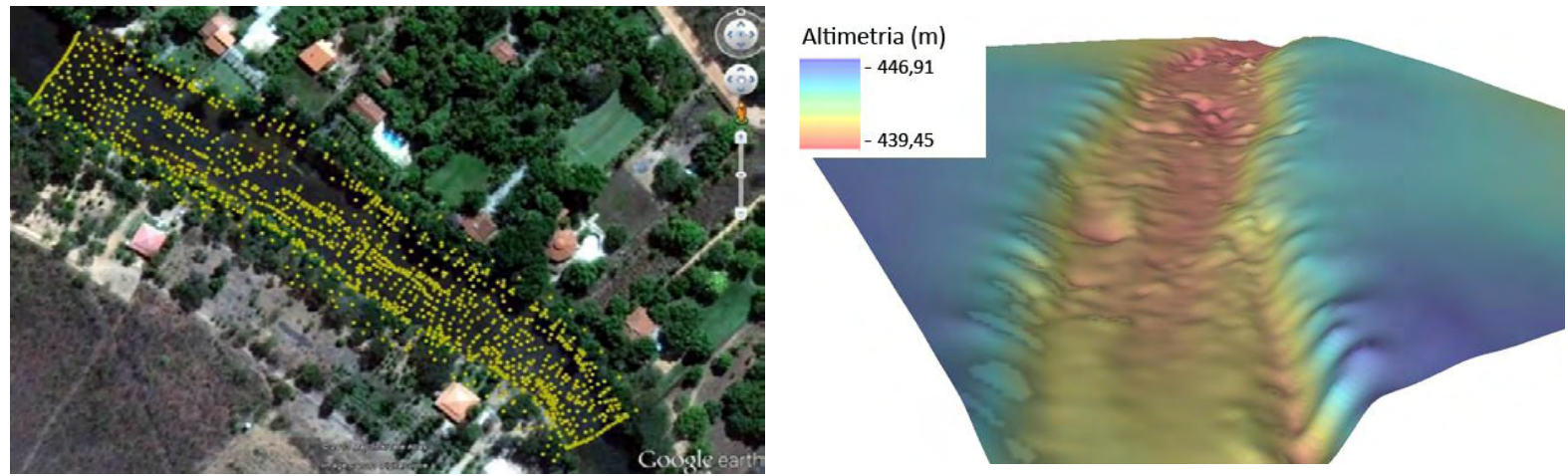

Figura 3. Distribuição dos pontos do levantamento batimétrico no trecho do baixo curso do rio de Ondas e vista em 3D da superfície batimétrica de montante para jusante.

Fonte: Adaptado de Google Earth (2013).

Os perfis batimétricos gerados para os dois trechos estudados no rio de Ondas permitiram a visualização da estrutura do leito e, principalmente, da distribuição dos diferentes ambientes, corredeiras e remansos no canal, confirmando as observações de Resck et al. (2007), que afirmam que os levantamentos batimétricos permitem a definição e visualização das características morfométricas do canal e melhor entendimento da estrutura e do funcionamento dos ecossistemas aquáticos.

Analisando os dois trechos de estudo do rio de Ondas, pode-se constatar que no trecho do alto curso o canal apresenta-se com margens vegetadas e predominância de leito arenoso, observando-se uma migração/erosão lateral na área, devido principalmente ao fato de o rio não possuir mais espaço e capacidade para erodir verticalmente o leito. No trecho do baixo curso, observa-se predominância de material grosseiro, elevada declividade, pouca vegetação em suas margens, e elevada energia que possibilita a erosão vertical do leito.

\subsection{Caracterização hidráulica}

Os parâmetros hidráulicos relacionados às características geométricas do trecho do alto curso estão descritos na Tabela 1.

Tabela 1. Dados médios dos parâmetros hidráulicos do trecho do alto curso do rio de Ondas durante os períodos sazonais.

\begin{tabular}{lcccc}
\hline Período & $\begin{array}{c}\text { Largura } \\
(\mathbf{m})\end{array}$ & $\begin{array}{c}\text { Profundidade } \\
\text { média }(\mathbf{m})\end{array}$ & $\begin{array}{c}\text { Velocidade } \\
\text { média }\left(\mathbf{m ~ s}^{-\mathbf{1}}\right)\end{array}$ & $\begin{array}{c}\text { Vazão } \\
\left(\mathbf{m}^{\mathbf{3}} \mathbf{s}^{-\mathbf{1}}\right)\end{array}$ \\
\hline Seco & 6,87 & 2,22 & 0,37 & 5,20 \\
Chuvoso & 19,92 & 1,50 & 0,32 & 9,07 \\
\hline
\end{tabular}

O fato do canal do trecho do alto curso possuir sinuosidades formando áreas de depressões e corredeiras, e de localizar-se em região com ambiente preservado, com amplo sistema radicular da vegetação das margens que proporciona maior estabilidade dos solos, pode ter auxiliado na estabilização da geometria hidráulica das seções durante o período de estudo. Isso pode ser constatado pelo decréscimo da velocidade média verificada a jusante, concluindo-se que a presença de galhos submersos no leito favoreceu o retardamento do escoamento superficial e do fluxo do canal, como evidenciado por Oliveira (2011) ao estudar a geometria hidráulica dos canais fluviais da bacia do rio Cascavel no Paraná.

Neste trecho, verificou-se que durante o período chuvoso há um elevado transbordamento do canal para a planície de inundação, mudando a configuração do leito. Nesses casos, as maiores profundidades ocorrem no talvegue, com pequenas variações da 
profundidade na planície de inundação, o que influencia os valores das profundidades médias das seções transversais, que são maiores durante o período seco em relação ao período chuvoso (Tabela 1).

Além disso, este transbordamento do canal provoca mudanças previsíveis no ambiente aquático interferindo na sua biota. Tal situação foi constatada por Tejerina-Garro e Mérona (2010) e Goldstein et al. (2007), que encontraram grandes diferenças nas assembleias de peixes relacionadas a oscilações da vazão, evidenciando que este é o principal fator que controla a comunidade ictiofaunística. Devido a este fato, os organismos aquáticos desse trecho podem encontrar-se também mais vulneráveis às atividades antrópicas de degradação dessas planícies.

No período chuvoso, as profundidades dentro do canal foram mais elevadas, possuindo profundidade máxima de $5,46 \mathrm{~m}$. No período seco, em que a vazão fícou concentrada no canal, sem transbordamento para a planície de inundação, a maior profundidade encontrada foi de $4,44 \mathrm{~m}$.

As características hidráulicas mensuradas no trecho do baixo curso do rio de Ondas estão apresentadas na Tabela 2.

Os resultados do estudo no trecho do baixo curso confirmaram a característica de um canal encaixado, com pouco transbordamento das margens e considerável elevação nas profundidades durante o período chuvoso. Neste trecho, durante o período chuvoso, a profundidade máxima observada foi de $1,86 \mathrm{~m}$, contrastando-se com o período seco, que esta profundidade apresentou valores de $1,52 \mathrm{~m}$.

Tabela 2. Dados médios dos parâmetros hidráulicos do trecho do baixo curso do rio de Ondas durante os períodos sazonais.

\begin{tabular}{lcccc}
\hline Período & $\begin{array}{c}\text { Largura } \\
(\mathbf{m})\end{array}$ & $\begin{array}{c}\text { Profundidade } \\
\text { média }(\mathbf{m})\end{array}$ & $\begin{array}{c}\text { Velocidade } \\
\text { média }\left(\mathbf{m ~ s}^{-1}\right)\end{array}$ & $\begin{array}{c}\text { Vazão } \\
\left(\mathbf{m}^{3} \mathbf{s}^{-1}\right)\end{array}$ \\
\hline Seco & 41,64 & 0,76 & 0,83 & 25,73 \\
Chuvoso & 46,47 & 1,06 & 1,39 & 66,44 \\
\hline
\end{tabular}

Pesquisas mostram que somente o estudo da velocidade e da profundidade não são significativos para elencar modificações nas comunidades aquáticas, sendo necessária a incorporação dos dados da vazão que influenciam decisivamente nessas comunidades (Cortes et al., 2008; Gorman e Kar, 1978; Kaufmann e Pinheiro, 2009; Tupinambás et al., 2011). Mesmo com tais afirmações, Marsh-Matthews e Matthews (2000) e Vlach et al. (2005) encontraram relação direta entre o aumento da profundidade e velocidade do canal com o aumento da diversidade de peixes.

A elevada flutuação sazonal na vazão, que se pode verificar nos dois trechos do rio de Ondas, resulta em alterações longitudinais e laterais do escoamento, que contribuem decisivamente para uma variada dinâmica das espécies aquáticas (Kaufmann e Pinheiro, 2009). Dentre as espécies da comunidade aquática, os peixes apresentam destaque pela necessidade desta variabilidade sazonal da vazão, que é fundamental na realização de suas diversas funções vitais (Lowe-McConnell, 1999).

Fantin-Cruz et al. (2008) afirmam que as variações espaciais e temporais das comunidades aquáticas respondem tanto à variação da vazão quanto às características morfométricas dos canais, refletindo nos diferentes comportamentos hidrodinâmicos dos trechos estudados no rio de Ondas, devido principalmente a descontinuidade serial resultante das variações litológicas ao longo do gradiente longitudinal deste rio. Dessa forma, considerando os aspectos estruturais do rio de Ondas, não é adequada a aplicação da Teoria 
do Rio Continuum, pois no alto curso do rio verifica-se uma energia hidráulica inferior à observada no baixo curso.

\subsection{Caracterização do leito}

As características ambientais diferenciadas dos trechos refletiram na composição granulométrica do leito do canal. Os resultados da análise granulométrica do trecho do alto curso mostraram predominância de textura arenosa $(88,94 \%)$, sendo ainda verificados seixos $(7,2 \%)$, argila $(3,5 \%)$ e silte $(0,35 \%)$.

As características granulométricas ocorrentes neste trecho estão associadas aos tipos de solos presentes na região, sendo eles o Neossolo Quartzarênico e o Gleissolo. O primeiro apresenta textura predominante de areia e o segundo tem essa textura principalmente nos horizontes superficiais (EMBRAPA, 2009), conforme constatado por Soares Neto (2005) e Castro et al. (2010) em estudos realizados no alto rio de Ondas.

No trecho do baixo curso, verificam-se a predominância de partículas com diâmetros grosseiros e com diferentes tamanhos e deposição de areia, com intercalações de materiais grosseiros próximos às margens.

Os resultados do método de medição do diâmetro médio das partículas mostraram a predominância de matacão $(76,9 \%)$ e bloco $(23,1 \%)$ neste trecho. Nos ambientes deposicionais marginais, nos quais foram verificadas associações entre areia e seixos, devido à impossibilidade de utilização do método do diâmetro médio das partículas, as amostras foram analisadas por pipetagem e peneiramento, obtendo como resultado os valores de $50,1 \%$ de areia, $46,7 \%$ de seixos, $2,5 \%$ de argila e $0,75 \%$ de silte.

Os resultados mostraram que a composição granulométrica desse trecho pode ser explicada principalmente pela litologia da região, associado à elevada velocidade do escoamento que auxilia no processo de erosão da rocha. Os solos nessa região são basicamente Neossolos Litólicos e Argissolos Vermelhos-Amarelos (Soares Neto e Souza, 2007). Os Neossolos Litólicos tem $90 \%$ ou mais de sua massa constituída por fragmentos de rocha com diâmetros maiores que $2 \mathrm{~mm}$, com predominância de cascalhos, calhaus e matacões (EMBRAPA, 2009).

A deposição de areia em alguns pontos das margens justifica-se pela diminuição da velocidade do escoamento, que propicia sua deposição nos bancos vegetados na área durante o período seco. Pode-se constatar, no entanto, que nessas áreas ocorrem intercalações entre areia e seixo, confirmando a elevada ocorrência de partículas com composição granulométrica grosseira.

Allan e Castillo (2007) apontam o material do leito como um componente crítico do ambiente aquático, principalmente por sofrer influência direta do ciclo hidrológico. Além disso, muitas espécies aquáticas possuem afinidade para determinada classe de diâmetro do material presente no leito (Souza e Barrella, 2009).

Dentre as preferências apresentadas pelos organismos aquáticos, há maior frequência para escolha de ambientes que possuem materiais com diâmetros elevados (Boëchat et al., 2013). Na maioria das vezes, eles se encontram com uma fauna mais diversificada, quando comparados com ambientes com materiais de granulometria fina (Barbour et al., 1999; Boëchat et al., 2013; Gordon et al., 2004; Minatti-Ferreira e Beaumord, 2006; Rodrigues et al., 2010).

Dessa forma, considerando a textura do material do leito, o trecho do baixo curso possui vantagens estruturais sobre o trecho do alto curso. Por sua vez, no alto curso predominam outras estruturas estratégicas do leito, como tocas, galhos submersos e variedade de plantas aquáticas, as quais, segundo Barbour et al. (1999) e Vlach et al. (2005), representam um ambiente com excelente complexidade do habitat, principalmente relacionada à alimentação e reprodução das espécies aquáticas. 
Silva (2010), estudando a influência da estrutura do habitat na assembleia de peixes do rio Cuiabá, constatou que o padrão de distribuição dessas assembleias dependeu totalmente da estrutura do leito. Além disso, o autor verificou que a composição das diferentes espécies foi totalmente distribuída entre os mais variados substratos, fato que pode ocorrer na bacia do rio de Ondas, localizada também no bioma Cerrado.

O leito é um componente multifatorial da análise estrutural do ambiente, que é influenciado por subparâmetros, como a textura, o grau de compactação, o tamanho das partículas presentes e a área superficial (Silveira, 2004), que podem, de maneira distinta, atuar na composição e abundância das espécies aquáticas, considerando suas adaptações.

Assim, considerando as diferentes preferências das espécies aquáticas relacionadas aos subparâmetros do leito e a diversidade particular de ambientes apresentada por cada trecho, não se pode afirmar com base nesse parâmetro que o trecho do baixo curso apresenta melhor disponibilidade de ambiente. Os dois trechos apresentaram diferentes características para ocorrência de uma fauna aquática diferenciada e diversificada.

\subsection{Disponibilidade de ambientes para os organismos aquáticos}

A complexidade da estrutura dos ambientes apresentada pelos dois trechos favorece a diversidade biológica da bacia do rio de Ondas, tendo em vista a disponibilidade de ambientes com diferentes características, que beneficiam as diversas aptidões das espécies aquáticas à estrutura física do canal.

Os trechos apresentaram diferenças nos parâmetros topobatimétricos, do leito e hidrodinâmicos, resultando em diferentes estruturas disponíveis, consequentemente, como previsto pela Teoria do Rio Continuum (Vannote et al., 1980), pode ocorrer também diferenças na diversidade de espécies, pelo fato do ambiente ser definidor dessa especificidade.

Condição semelhante à do rio de Ondas foi observada por Ferreira e Castro (2005), ao estudarem a estrutura dos ambientes de trechos do rio das Velhas, em Minas Gerais. Os autores confirmaram a influência das características físicas desses ambientes sobre a riqueza e abundância das comunidades aquáticas.

No trecho do alto curso, verificou-se como principais características formadoras do ambiente a predominância de leito arenoso, troncos submersos e macrófitas aquáticas. Além disso, é evidente a presença de margens escavadas, formando tocas que podem ser utilizadas para desova dos peixes. Pode-se constatar também, que há corredeiras e depressões, principalmente pela sinuosidade do canal, que favorecem a disponibilidade de ambiente (Fernandez, 2009).

Associada a tais fatores, e de maneira determinante e particular, o trecho do alto curso possui uma extensa mata de galeria inundável, que, com a elevação da vazão no período chuvoso, propicia o acúmulo da água proveniente do canal, disponibilizando ambiente diferenciado do verificado no período seco.

De maneira distinta, no trecho do baixo curso houve predominância de leito com material grosseiro, associado à elevada velocidade da corrente e baixa profundidade. Além disso, observa-se a disponibilidade de serapilheira para a comunidade aquática, e de diferentes ambientes, ocasionados pela variação nos diâmetros do material constituinte do leito, e deposições de areia em alguns pontos das margens, particularmente na margem esquerda.

No trecho do baixo curso, mesmo possuindo pouca oscilação quanto à inundação, por apresentar-se encaixado, ocorrem alterações na sua conformação física, pelo fato de vários matacões e pequenas ilhas vegetadas ficarem submersos durante o período de cheias, mudando temporariamente a configuração do canal. Mesmo com esta favorável heterogeneidade, pelo fato de estar localizado próximo ao centro urbano do município de Barreiras, esse trecho possui degradação em suas margens. 
Percebe-se, com as observações citadas, que o rio de Ondas apresenta características morfométricas diferenciadas entre os trechos do alto e baixo curso. No baixo curso há o predomínio de corredeiras e de materiais grosseiros, características esperadas de serem encontradas no alto curso, uma vez que os remansos e os materiais com diâmetros menores ocorrem predominantemente no baixo curso das bacias hidrográficas (Allan e Castillo, 2007; Rodrigues et al., 2010).

Conforme Riccomini et al. (2009), essas características estão associadas à estrutura geológica e geomorfológica das diversas regiões, sendo que a existência de rupturas nos declives causadas por falhas geológicas ou rochas ao longo dos rios podem formar ambientes de corredeiras e de cachoeiras.

\section{CONCLUSÕES}

As configurações físicas dos trechos do alto e baixo curso do rio de Ondas contribuem com diferentes ambientes disponíveis para a biota aquática, em particular os peixes, podendo resultar em distintas comunidades ícticas no alto e no baixo curso da bacia, mostrando a importância destes estudos no entendimento da estrutura dos ambientes e das comunidades aquáticas. Constatou-se ainda significante influência das características litológicas da região na geração da descontinuidade serial do rio, influenciando consequentemente nos processos ecológicos ocorrentes na bacia, relacionados principalmente à composição e distribuição da biota aquática.

\section{AGRADECIMENTOS}

À Fundação de Amparo à Pesquisa do Estado da Bahia - FAPESB (Termo de Outorga n $^{\circ}$ APP 0053/2011) e ao Conselho Nacional de Desenvolvimento Científico e Tecnológico CNPq (Processo n ${ }^{\circ}$ 472279/2013-0), pelo apoio financeiro.

\section{REFERÊNCIAS}

ALLAN, J. D.; CASTILLO, M. M. Stream ecology: structure and function of running waters. New York: Springer, 2007.

ASSOCIAÇÃO BRASILEIRA DE NORMAS TÉCNICAS - ABNT. Nbr 7181/84: solo análise granulométrica. Rio de Janeiro, 1984.

ASSOCIAÇÃO BRASILEIRA DE NORMAS TÉCNICAS - ABNT. Nbr 6457/86: amostra de solo - preparação para ensaios de compactação e ensaios de caracterização. Rio de Janeiro, 1986.

BARBOUR, M. T.; GERRISTSEN, J.; SNYDER, B. D.; STRIBLING J. B. Rapid bioassessment protocols for use in streams and wadeable rivers: periphyton, benthic macroinvertebrates and fish. EPA 841-B-99-002. $2^{\text {nd }}$ Edition. Washington: USEPA, 1999.

BESCHTA, R. L.; PLATTS, W. S. Morphological features of small streams: significance and function. Water Resources Bulletin, v. 22, n. 3, p. 369-379, 1986. http://dx.doi.org/10.1111/j.1752-1688.1986.tb01891.x

BEZERRA-NETO, J. F.; PINTO-COELHO, R. M. New morphometric study of Lake Dom Helvécio, Parque Estadual do Rio Doce (PERD), Minas Gerais: utilization of advanced methodology for bathymetric mapping. Acta Limonologica Brasiliensia, v. 20, n. 2, p. 117-130, 2008. 
BOËCHAT, I. G.; PAIVA, A. B. M.; HILLE, S.; GÜCKER, B. Land-use effects on river habitat quality and sediment granulometry along a 4th-order tropical river. Revista Ambient. Água, v. 8 n. 3, p. 54-64, 2013. http://dx.doi.org/10.4136/ambi-agua.1232

CASTRO, K. B.; MARTINS, E. S.; GOMES, M. P.; REATTO, A.; LOPES, C. A.; PASSO, D. P. et al. Caracterização geomorfológica do município de Luís Eduardo Magalhães. Planaltina: EMBRAPA Cerrados, 2010.

CORTES, R. M. V.; FERREIRA M. T.; OLIVEIRA S. V. D.; HUGHES, S. J. Combining habitat and biological characterization: ecological validation of the river habitat survey. Limnetica, v. 27, n. 1, p. 39-56, 2008. http://dx.doi.org/10400.5/5142

CUNHA, S. B.; GUERRA, A. J. T. Geomorfologia: exercícios técnicas e aplicações. Rio de Janeiro: Bertrand Brasil, 2011.

DAMASCENO, O. S. Geomorfologia fluvial da bacia hidrográfica do rio de Ondas. Caminhos da Geografia, v. 12, n. 39, p. 126-136, 2011.

EMPRESA BRASILEIRA DE PESQUISA AGROPECUÁRIA - EMBRAPA. Manual de métodos de análises de solos. Rio de Janeiro: EMBRAPA Solos, 1997.

EMPRESA BRASILEIRA DE PESQUISA AGROPECUÁRIA - EMBRAPA. Sistema brasileiro de classificação de solos. Rio de Janeiro: EMBRAPA-SPI, 2009.

FANTIN-CRUZ, I.; LOVERDE-OLIVEIRA, S.; GIRARD, P. Caracterização morfométrica e suas implicações na limnologia de lagoas do Pantanal Norte. Acta Scientiarum. Biological Sciences, v. 30, n. 2, p. 133-140, 2008.

FERNANDEZ, O. V. Q. Discriminação de habitats aquáticos no córrego Guavirá, Marechal Cândido Rondon (PR). Revista Geografias, v. 5, n. 1, p. 22-36, 2009.

FERREIRA H. L. M.; CASTRO P. T. A. Ecomorphological analysis of fluvial habitats of the upstream part of Rio das Velhas/MG, Brazil. Sociedade \& Natureza, v. 1, n. 1, p. $327-$ $336,2005$.

GOLDSTEIN, R. M.; MEADOR, M. R.; RUHL, K. E. Relative influence of streamflows in assessing temporal variability in stream habitat. Journal of the American Water Resources Association, v. 43, n. 3, p. 642-650, 2007. http://dx.doi.org/10.1111/j.17521688.2007.00051.x

GOOGLE. Google Earth. Programa computacional, versão 7.1.1.1888. [S.1.]: Google Inc., 2013.

GORDON, N. D.; MCMAHON, T. A.; FINLAYSON, B. L.; GIPPEL, C. J.; NATHAN, R. J. Stream hydrology: an introduction for ecologists. West Sussex: John Wiley \& Sons, 2004.

GORMAN, O. T.; KAR, J. R. Habitat structure and stream fish communities. Ecology, v. 59, n. 3, p. 507-515, 1978. http://dx.doi.org/10.2307/1936581

HAWKINS, C. P.; KERSHNER, J. L.; BISSON, P. A.; BRYANT, M. D.; DECKER, L. M.; GREGORY, S. V. et al. A hierarchical approach to classifying stream habitat features. Fisheries, v. 18, n. 6, p. 3-12, 1993. http://dx.doi.org/10.1577/1548-8446(1993)018<0003:AHATCS>2.0.CO;2

INSTITUTO BRASILEIRO DE GEOGRAFIA E ESTATÍSTICA - IBGE. Manual do Usuário: posicionamento por ponto preciso. Rio de Janeiro, 2009. 
JOWETT, I. G. Method for objectively identifying pool, run, and riffle habitats from physical measurements. New Zealand Journal of Marine and Freshwater Research, v. 27, n. 2, p. 241-248, 1993. http://dx.org.br/10.1080/00288330.1993.9516563

KAUFMANN, V.; PINHEIRO, A. Relações entre diversidade íctia e fatores hidrodinâmicos de um riacho na bacia do rio Uruguai. Biota Neotropica, v. 9, n. 1, p. 47-53, 2009. http://dx.doi.org/10.1590/S1676-06032009000100006

KRUEGER, C. P.; VEIGA, L. A. K.; FAGGION, P. L. Levantamento batimétrico no rio Uruguai. In: CONGRESSO BRASILEIRO DE CARTOGRAFIA, 21., 2003, Belo Horizonte. Anais... Belo Horizonte: IBGE, 2003.

LEANDRO, G. R. S.; SOUZA, C. A. Pantanal de Cáceres: composição granulométrica dos sedimentos de fundo no rio Paraguai entre a foz do rio Cabaçal e a cidade de Cáceres, Mato Grosso, Brasil. Revista Ambiente \& Água, v. 7, n. 2, p. 263-276, 2012. http://dx.doi.org/10.4136/ambi-agua.876

LOWE-McCONNELL, R. H. Estudos ecológicos de comunidades de peixes tropicais. São Paulo: EDUSP, 1999.

MARSH-MATTHEWS, E.; MATTHEWS, W. J. Geographic, terrestrial and aquatic factors: which most influence the structure of stream fish assemblages in the midwestern United States? Ecology of Freshwater Fish, v. 9, n. 1-2, p. 9-21, 2000. http://dx.doi.org/10.1034/j.1600-0633.2000.90103.x

MARTINS, M. E.; HONDA, E. A. Caracterização hidráulica dos canais da bacia da Água da Cachoeira e do ribeirão do Alegre. In: VILAS BÔAS, O.; DURIGAN, G. Pesquisas em conservação e recuperação ambiental no oeste Paulista. São Paulo: Páginas e Letras, 2004.

MINATTI-FERREIRA, D. D.; BEAUMORD, A. C. Adequação de um protocolo de avaliação rápida de integridade ambiental para ecossistemas de rios e riachos: aspectos físicos.

Revista Saúde e Ambiente, v. 7, n. 1, p. 39-47, 2006.

OLIVEIRA, E. D. Impactos da urbanização na geometria hidráulica de canais fluviais da bacia hidrográfica do rio Cascavel, Guarapuava/PR. 2011. 158 f. Dissertação (Mestrado em Geografia) - Universidade Estadual do Centro Oeste, Guarapuava, 2011.

QUEIROZ, F. L. L.; ROCHA, P. C. Avaliação de sedimentos do leito no canal Curutuba / planície fluvial do alto rio Paraná, Mato Grosso do Sul - Brasil. Revista AGB/TL, v. 7, n. 11, p. 147-164, 2010.

RESCK, R.; BEZERRA-NETO, J. F.; PINTO-COELHO, R. M. Nova batimetria e uma avaliação ecológica de parâmetros morfométricos da Lagoa da Pampulha (Belo Horizonte, Brasil). Revista Geografias, v. 3, n. 2, p. 13-23, 2007.

RICCOMINI, C.; ALMEIDA, R. P.; GIANNINI, P. C. F; MANCINI, F. Processos fluviais e lacustres e seus registros. In: TEIXEIRA, W. (Org.). Decifrando a terra. São Paulo: Companhia Editora Nacional, 2009.

RODRIGUES, A. S. L.; MALAFAIA, G.; CASTRO, P. T. A. A importância da avaliação do habitat no monitoramento da qualidade dos recursos hídricos: uma revisão. SaBios, v. 5, n. 1, p. 26-42, 2010. 
SILVA, S. E. Efeito do uso do solo e estrutura do habitat na assembleia de peixes de riachos da bacia do rio Cuiabá, MT. 2010. 105 f. Dissertação (Mestrado em Ecologia e Conservação da Biodiversidade) - Universidade Federal de Mato Grosso, Cuiabá, 2010.

SILVEIRA, M. P. Aplicação do biomonitoramento para avaliação da qualidade da água em rios. Jaguariúna: EMBRAPA Meio Ambiente, 2004.

SOARES NETO, J. P. Avaliação geoambiental da bacia do rio das Ondas no oeste da Bahia. 2005. 233 f. Tese (Doutorado em Geotecnia) - Universidade de Brasília, Brasília, 2005.

SOARES NETO, J. P.; SOUZA, N. M. Características fisiográficas da sub-bacia hidrográfica do rio das Ondas ao longo do tempo e suas relações ambientais. Magistra, Cruz das Almas - BA, v. 19, n. 2, p. 104-111, 2007.

SONTEK. Manual do sistema Riversurveyor S5/M9 versão 1.0. San Diego: YSI Incorporated, 2009.

SOUZA, C. E.; BARRELLA, W. Atributos ecomorfológicos de peixes do Sul do Estado de São Paulo. Revista Eletrônica de Biologia, v. 2, n. 1, p. 1-34, 2009.

TEJERINA-GARRO, F. L.; MÉRONA, B. Flow seasonality and fish assemblage in a tropical river, French Guiana, South America. Neotropical ichthyology, v. 8, n. 1, p. 145-154, 2010. http://dx.doi.org/10.1590/S1679-62252010005000005

TUPINAMBÁS, T. H.; CASTRO, D. M. P.; FRANÇA, J. S.; CALLISTO, M. Alterações de vazão em comunidades bentônicas: subsídio ecológico para parametrização de vazões ambientais. In: SIMPÓSIO BRASILEIRO DE RECURSOS HÍDRICOS, 19., 2011, Maceió. Anais... Maceió: ABRH, 2011. 1 CD-ROM.

VANNOTE, R. L.; MINSHALL, G. W.; CUMMINS, K. W.; SEDELL, J. R.; CUSHING, C. E. The river continuum concept. Canadian Journal of Fisheries and Aquatic Sciences, v. 37, n. 1, p. 130-137, 1980. http://dx.doi.org/10.1139/f80-017

VLACH, P.; DUŠEK, J.; ŠVÁTORA, M.; MORAVEC, P. Fish assemblage structure, habitat and microhabitat preference of five fish species in a small stream. Folia Zoologica, v. 54, n. 4, p. 421-431, 2005.

WARD, J. V.; STANFORD, J. A. The serial discontinuity concept in lotic ecosystems. In: FONTAINE, T. D.; BARTHELL, S. M. (Eds.). Dynamics of lotic ecosystems. Michigan: Ann Arbor Science, 1983.

WENTWORTH, C. K. A scale of grade and class terms for clastic sediments. The Journal of Geology, v. 30, n. 5, p. 377-392, 1922. 


Ambiente \& Água - An Interdisciplinary Journal of Applied Science
ISSN 1980-993X - doi:10.4136/1980-993X
www.ambi-agua.net
E-mail: ambi-agua@agro.unitau.br

\title{
Adubação com NPK e irrigação do girassol em Luvissolo: Comportamento vegetativo
}

\author{
doi: 10.4136/ambi-agua.1482
}

Received: 08 Aug. 2014; Accepted: 24 Sep. 2014

\section{Vinícius Batista Campos'; Lúcia Helena Garófalo Chaves²; Hugo Orlando Carvallo Guerra ${ }^{2 *}$}

\footnotetext{
${ }^{1}$ Instituto Federal de Educação, Ciência e Tecnologia do Amapá, Campus Laranjal do Jari, Amapá, Brasil

${ }^{2}$ Universidade Federal de Campina Grande (UFCG), Campina Grande, PB, Brasil Unidade Acadêmica de Engenharia Agrícola *Autor correspondente: e-mail: hugo_carvallo@hotmail.com, vinicius.campos@ifap.edu.br, lhgarofalo@hotmail.com
}

\section{RESUMO}

Entre as culturas utilizadas para a produção de biocombustíveis, o girassol é um das mais importantes. Apesar de existir informações na literatura, as necessidades hídricas e de nutrientes do girassol ainda não estão perfeitamente definidas. Com o objetivo de verificar os efeitos da adubação nitrogenada, fosfatada, potássica e o conteúdo de água disponível no solo (AD) sobre o comportamento vegetativo do girassol Embrapa 122 V2000, na Universidade Federal de Campina Grande, Campina Grande-PB, foi conduzido um experimento com quarenta e quatro tratamentos constituídos pela combinação de doses $\left(\mathrm{kg} \mathrm{ha}^{-1}\right)$ de $\mathrm{N}, \mathrm{P}$ e K de acordo com uma matriz baconiana, sendo 1: 0-0-0, 2: 0-80-80, 3: 80-80-80, 4: 100-80-80, 5: 60-0-80, 6: 60-100-80, 7: 60-120-80, 8: 60-80-0, 9: 60-80-80, 10: 60-80-100, 11: 60-80-120 e quatro conteúdos de água disponível (55, 70, 85 e 100\% da AD). O tratamento nove é o de referência, correspondendo às doses adotadas pelos produtores de girassol do Estado do Rio Grande do Norte. O delineamento foi inteiramente ao acaso em triplicata. Avaliou-se a altura de plantas, diâmetro do caule, número de folhas e área foliar, aos 40 e 60 dias após a semeadura. Os resultados indicaram que os efeitos da água disponível e adubação com NPK sobre a cultura do girassol ocorreram de forma independente, excetuando-se o número de folhas. Todas as variáveis apresentaram comportamento linear crescente em função da água disponível do solo, exceto a área foliar. Para as condições estudadas, as doses 100, 80 e $80 \mathrm{~kg} \mathrm{ha}^{-1}$ de $\mathrm{N}, \mathrm{P}_{2} \mathrm{O}_{5}$ e $\mathrm{K}_{2} \mathrm{O}$, respectivamente, proporcionaram os maiores índices de crescimento.

Palavras-chave: água disponível, Helianthus annuus L., nutrição mineral.

\section{NPK fertilization and irrigation of sunflowers in Luvisol: Vegetative behavior}

\section{ABSTRACT}

The sunflower is one of the most important crops used for the production of biofuels. While the literature does contain some information, the hydric and nutritional requirements of sunflowers are still not completely defined. In order to correct this deficiency, an experiment 
was conducted at the Federal University of Campina Grande, Campina Grande, Paraíba State, Brazil, with the aim of evaluating the effect of nitrogen, phosphate, and potassium fertilization and available soil water (AW) on the behavior of Sunflower cv. Embrapa 122 V2000. The experiment consisted of 44 treatments with compound fertilizers using a Baconian Matrix (1: 0-0-0, 2: 0-80-80, 3: 80-80-80, 4: 100-80-80, 5: 60-0-80, 6: 60-100-80, 7: 60-120-80, 8: 60-80-0, 9: 60-80-80, 10:60-80-100, 11:60-80-120 kg ha-1 of N, P and K, respectively, and four difference quantities of available soil water $(55,70,85$ and 100\%). The $9^{\text {th }}$ compound fertilizer treatment was considered the reference since its doses are those used by the region's sunflower growers in Rio Grande do Norte State. The treatments were arranged in a completely randomized design with three replications. The height, stem diameter, number of leaves and leaf area of the plants were evaluated at 40 and 60 days after sowing. The results showed that the effects of available soil water and NPK fertilization on sunflower crop occurred independently for all variables except for the number of leaves. All variables increased linearly as a function of available soil water, except for leaf area. For the conditions studied, the doses 100,80 and $80 \mathrm{~kg} \mathrm{ha}^{-1}$ of $\mathrm{N}, \mathrm{P}_{2} \mathrm{O}_{5}$ and $\mathrm{K}_{2} \mathrm{O}$ resulted in the highest growth rates, respectively.

Keywords: available soil water, Helianthus annuus L., mineral nutrition.

\section{INTRODUÇÃO}

O girassol (Helianthus annuus L.) é uma dicotiledônea anual da família Asteraceae, originária da América do Norte, mundialmente cultivada em todos os continentes, tendo como principais produtores a Rússia, Ucrânia, Argentina, entre outros (FAO, 2008). Essa planta se adapta bem a diversas condições edafoclimáticas, caracterizando-se pela tolerância a baixas temperaturas na fase inicial de desenvolvimento e pela relativa resistência a déficits hídricos. Pode ser cultivada em todas as regiões do país, pois o rendimento é pouco influenciado pelas latitudes e altitudes, assim como pelo fotoperíodo, o que facilita a expansão do cultivo no Brasil (Zobiole et al., 2010). É uma espécie extremamente versátil, tendo como principais produtos o óleo, produzido de seus aquênios (frutos), ração animal, além de ser utilizada na alimentação humana e como planta ornamental.

O girassol é uma planta que absorve uma quantidade muito grande dos principais macronutrientes, em comparação com a soja, milho e o trigo. Para que possa expressar todo o seu potencial produtivo, o suprimento de água e nutrientes deve ser adequado desde o início do seu desenvolvimento, principalmente a partir da emissão do botão floral, quando inicia o período de maior crescimento, acompanhado do aumento no consumo de água e da demanda nutricional.

De modo geral, as quantidades de nitrogênio, fósforo e de potássio recomendadas para o cultivo do girassol, variam de 40 a $60 \mathrm{~kg} \mathrm{ha}^{-1}$ de $\mathrm{N}, 40$ a $80 \mathrm{~kg} \mathrm{ha}^{-1}$ de $_{2} \mathrm{P}_{2} \mathrm{O}_{5} 40$ a $80 \mathrm{~kg} \mathrm{ha}^{-1}$ de $\mathrm{K}_{2} \mathrm{O}$. Entretanto, na literatura são encontradas recomendações com diferentes combinações destes elementos, em decorrência das condições edafoclimáticas e tipo de variedade que está sendo cultivada. Castro e Oliveira (2005), por exemplo, recomendam a adubação do girassol utilizando doses entre 40 a $60 \mathrm{~kg} \mathrm{ha}^{-1}$ de $\mathrm{N}$, devendo se utilizar em solos com médio teor de fósforo e potássio, 18 e $33 \mathrm{~kg} \mathrm{ha}^{-1}$, respectivamente. Quaggio e Ungaro (1997) indicam para o Estado de São Paulo, a aplicação de $50 \mathrm{~kg} \mathrm{ha}^{-1}$ de N, 20 a $70 \mathrm{~kg} \mathrm{ha}^{-1}$ de $\mathrm{P}_{2} \mathrm{O}_{5}$ e 20 a $60 \mathrm{~kg} \mathrm{ha}$ de $\mathrm{K}_{2} \mathrm{O}$.

A planta do girassol tem baixa eficiência no uso da água, uma vez que, cada litro de água consumido produz menos de dois gramas de matéria seca. Uma adequada disponibilidade de água durante o período da germinação à emergência é necessária para a obtenção de uma boa uniformidade na população de plantas. As fases do desenvolvimento da planta mais sensíveis ao déficit hídrico são do início da formação do capítulo ao começo da floração (afeta mais o 
rendimento de grãos) e da formação e enchimento de grãos, que é a fase de maior consumo de água pelo girassol (afeta mais a produção de óleo). De uma forma bastante prática, a fase mais crítica ao déficit hídrico é o período compreendido entre cerca de 10 a 15 dias antes do início do florescimento e 10 a 15 dias após o final da floração (Embrapa, 2000). Apesar dessas informações, e de outras disponibilizadas na literatura, as necessidades hídricas do girassol ainda não estão perfeitamente definidas, podendo variar de 200 a $900 \mathrm{~mm}$ ciclo $^{-1}$, o que constituiu o incentivo para novas pesquisas nesta área.

Neste sentido, objetivou-se avaliar o efeito da adubação mineral com NPK sobre o comportamento vegetativo do girassol variedade Embrapa 122 V2000, para diferentes níveis de água disponível no solo.

\section{MATERIAL E MÉTODOS}

\section{1. Área de estudo}

O experimento foi realizado em estufa agrícola, no período entre julho e outubro de 2010 na Unidade de Engenharia Agrícola do Centro de Tecnologia e Recursos Naturais da Universidade Federal de Campina Grande (UFCG) localizada no município de Campina Grande, PB, cujas coordenadas geográficas são: 654'10" S e 3550’02”O.

Segundo a classificação de Köppen, o clima da região é Csa (mesotérmico subúmido), temperatura média anual oscilando entre 19,2 e $27,5^{\circ} \mathrm{C}$ e umidade relativa do ar média de $83 \%$.

\subsection{Tratamentos e delineamento experimental}

O delineamento experimental foi inteiramente ao acaso, com três repetições, sendo testados 44 tratamentos de uma matriz baconiana formada por quatro níveis de água disponível no solo $(55,70,85$ e $100 \%)$, submetidos a quatro doses de nitrogênio $(0,60$, 80 e $100 \mathrm{~kg} \mathrm{ha}^{-1}$ de $\left.\mathrm{N}\right)$; fósforo $\left(0,80,100\right.$ e $120 \mathrm{~kg} \mathrm{ha}^{-1}$ de $\left.\mathrm{P}_{2} \mathrm{O}_{5}\right)$ e potássio $(0,80,100$ e $120 \mathrm{~kg} \mathrm{ha}^{-1} \mathrm{de}_{2} \mathrm{O}$ ), totalizando 132 parcelas (Tabela 1). A dose de adubação referencial foi $60,80,80 \mathrm{~kg} \mathrm{ha}^{-1}$ de $\mathrm{N}, \mathrm{P}$ e K, respectivamente.

\subsection{Condução do experimento}

O solo utilizado no experimento foi proveniente de um Luvissolo Crômico órtico coletado na camada superficial $(0-20 \mathrm{~cm})$, no município de Boqueirão, PB, o qual foi seco ao ar, destorroado, homogenizado, peneirado e caracterizado quanto os aspectos físicos e químicos, segundo metodologia descrita pela Embrapa (1997), tendo como características: $\mathrm{pH}$ $\left(\mathrm{H}_{2} \mathrm{O}\right)=5,7 ; \mathrm{Ca}^{2+}=3,40 \mathrm{cmol}_{\mathrm{c}} \mathrm{dm}^{-3} ; \mathrm{Mg}^{2+}=1,85 \mathrm{cmol}_{\mathrm{c}} \mathrm{dm}^{-3} ; \mathrm{Na}^{+}=0,18 \mathrm{cmol}_{\mathrm{c}} \mathrm{dm}^{-3}$; $\mathrm{K}^{+}=0,16 \mathrm{cmol}_{\mathrm{c}} \mathrm{dm}^{-3} ; \mathrm{H}^{+}+\mathrm{Al}^{3+}=2,15 \mathrm{cmol}_{\mathrm{c}} \mathrm{dm}^{-3}$; matéria orgânica $=6,03 \mathrm{~g} \mathrm{dm}^{-3} ; \mathrm{P}=14,63$ $\mathrm{mg} \mathrm{dm}$; areia $=644 \mathrm{~g} \mathrm{~kg}^{-1}$; silte $=137 \mathrm{~g} \mathrm{~kg}^{-1}$; argila $=219 \mathrm{~g} \mathrm{~kg}^{-1}$; densidade do solo $=1,31 \mathrm{~kg} \mathrm{dm}^{-3}$; densidade de partículas $=2,68 \mathrm{~kg} \mathrm{dm}^{-3}$ e porosidade total $=51 \%$.

Cada unidade experimental foi constituída por um vaso plástico com capacidade volumétrica de $35 \mathrm{~L}$ onde foram acondicionados $32 \mathrm{~kg}$ de solo. Um tubo de acesso de PVC para medição do conteúdo de água do solo com uma sonda segmentada FDR (Reflectometria no Domínio da Frequência) DIVINNER - 2000 foi inserido no solo dos tratamentos a ser mantidos a $100 \%$ da água disponível no solo para as plantas. O volume de água para $100 \%$ da água disponível no solo (AD) foi calculado determinando o conteúdo de água do solo na capacidade de campo (CC) e no ponto de murcha permanente (PMP) no laboratório $(\mathrm{AD}=\mathrm{CC}-\mathrm{PMP})$. Os volumes de reposição de água para os demais tratamentos foram calculados proporcionalmente a partir do volume de água para $100 \%$ da água disponível no solo. As irrigações foram conduzidas diariamente visando manter os respectivos níveis de água dos tratamentos. 
A adubação com $\mathrm{N}, \mathrm{P}_{2} \mathrm{O}_{5}$ e $\mathrm{K}_{2} \mathrm{O}$ foram realizadas na forma de uréia, superfosfato triplo e cloreto de potássio, respectivamente. Independente do tratamento utilizado, o fósforo foi aplicado integralmente na fundação e o potássio parcelado em três aplicações com mesmo quantitativo, em fundação, aos 28 e 52 dias após a semeadura (DAS). O nitrogênio também foi parcelado, sendo $50 \%$ da dose aos 28 DAS e os demais aos 52 DAS. Foi fornecido também a cada unidade experimental, aos 28 DAS, o correspondente a $2 \mathrm{~kg} \mathrm{ha}^{-1}$ de boro, proveniente de ácido bórico.

O semeio foi realizado no dia 16 de julho de 2010, sendo diretamente no vaso, utilizando 10 sementes de girassol, variedade Embrapa 122 V-2000, distribuídas e distanciadas de forma equidistante, a uma profundidade de $0,02 \mathrm{~m}$. Aos $20 \mathrm{DAS}$, realizou-se o desbaste, deixando apenas uma planta por unidade experimental.

Tabela 1. Doses de nitrogênio $(\mathrm{N})$, fósforo $(\mathrm{P})$ e potássio $(\mathrm{K})$ correspondentes aos tratamentos que compuseram a matriz baconiana.

\begin{tabular}{|c|c|c|c|c|c|c|c|c|c|}
\hline \multirow{2}{*}{ Tratamentos } & $\mathbf{N}$ & $\mathbf{P}_{2} \mathbf{O}_{5}$ & $\mathbf{K}_{2} \mathbf{O}$ & \multirow{2}{*}{$\frac{\text { Água }}{\%}$} & \multirow{2}{*}{ Tratamentos } & $\mathbf{N}$ & $\mathbf{P}_{2} \mathbf{O}_{5}$ & $\mathrm{~K}_{2} \mathrm{O}$ & \multirow{2}{*}{$\frac{\text { Água }}{\%}$} \\
\hline & \multicolumn{3}{|c|}{----- kg ha ${ }^{-1}$} & & & \multicolumn{3}{|c|}{----- kg ha"----- } & \\
\hline 1 & 0 & 0 & 0 & 55 & 23 & 0 & 0 & 0 & 85 \\
\hline 2 & 0 & 80 & 80 & 55 & 24 & 0 & 80 & 80 & 85 \\
\hline 3 & 80 & 80 & 80 & 55 & 25 & 80 & 80 & 80 & 85 \\
\hline 4 & 100 & 80 & 80 & 55 & 26 & 100 & 80 & 80 & 85 \\
\hline 5 & 60 & 0 & 80 & 55 & 27 & 60 & 0 & 80 & 85 \\
\hline 6 & 60 & 100 & 80 & 55 & 28 & 60 & 100 & 80 & 85 \\
\hline 7 & 60 & 120 & 80 & 55 & 29 & 60 & 120 & 80 & 85 \\
\hline 8 & 60 & 80 & 0 & 55 & 30 & 60 & 80 & 0 & 85 \\
\hline $9^{*}$ & 60 & 80 & 80 & 55 & $31^{*}$ & 60 & 80 & 80 & 85 \\
\hline 10 & 60 & 80 & 100 & 55 & 32 & 60 & 80 & 100 & 85 \\
\hline 11 & 60 & 80 & 120 & 55 & 33 & 60 & 80 & 120 & 85 \\
\hline 12 & 0 & 0 & 0 & 70 & 34 & 0 & 0 & 0 & 100 \\
\hline 13 & 0 & 80 & 80 & 70 & 35 & 0 & 80 & 80 & 100 \\
\hline 14 & 80 & 80 & 80 & 70 & 36 & 80 & 80 & 80 & 100 \\
\hline 15 & 100 & 80 & 80 & 70 & 37 & 100 & 80 & 80 & 100 \\
\hline 16 & 60 & 0 & 80 & 70 & 38 & 60 & 0 & 80 & 100 \\
\hline 17 & 60 & 100 & 80 & 70 & 39 & 60 & 100 & 80 & 100 \\
\hline 18 & 60 & 120 & 80 & 70 & 40 & 60 & 120 & 80 & 100 \\
\hline 19 & 60 & 80 & 0 & 70 & 41 & 60 & 80 & 0 & 100 \\
\hline $20^{*}$ & 60 & 80 & 80 & 70 & $42^{*}$ & 60 & 80 & 80 & 100 \\
\hline 21 & 60 & 80 & 100 & 70 & 43 & 60 & 80 & 100 & 100 \\
\hline 22 & 60 & 80 & 120 & 70 & 44 & 60 & 80 & 120 & 100 \\
\hline
\end{tabular}

*Tratamentos de referência, que corresponde às doses de adubação usadas para girassol no Estado do Rio Grande do Norte.

\subsection{Variáveis avaliadas e análise estatística}

Aos 40 e 60 dias após a semeadura (DAS) avaliou-se a altura de plantas (AP), diâmetro caulinar (DC), número de folhas (NF) e área foliar (AF). Na contagem das folhas foram consideradas as que apresentaram comprimento mínimo de $0,03 \mathrm{~m}$ e que estivessem sadias, 
ou seja, fotossinteticamente ativas; a medição do diâmetro do caule foi feito com o auxílio de um paquímetro, com as leituras sendo efetuadas na região do colo de cada planta a $0,05 \mathrm{~m}$ do solo; o comprimento do caule foi definido mensurando-se a distância entre o colo da planta e a inserção da folha mais nova no período da colheita das plantas; o cálculo da área foliar foi feita segundo metodologia proposta por Maldaner et al. (2009) cuja fórmula é:

$$
\mathrm{AF}=0,1328 \times \mathrm{C}^{2,5569}
$$

em que:

$\mathrm{C}=$ comprimento da nervura central de cada folha da planta sendo que o somatório final das áreas por folha fornecia o valor da área foliar da planta.

Os dados obtidos foram submetidos à análise de variância e de regressão, utilizando o software estatístico SAEG (Eyclydes, 1997).

\section{RESULTADOS E DISCUSSÃO}

A altura de plantas foi afetada significativamente pela adubação NPK e pela irrigação conforme a Tabela 2.

Tabela 2. Resumo das análises de variância para altura de plantas (AP) e diâmetro caulinar (DC) do girassol (variedade Embrapa 122-V2000) aos 40 e 60 dias após a semeadura (DAS) submetido à adubação com NPK e conteúdo de água disponível no solo.

\begin{tabular}{|c|c|c|c|c|c|}
\hline \multirow{3}{*}{ Fonte de variação } & \multirow{3}{*}{ GL } & \multicolumn{4}{|c|}{ Quadrado médio } \\
\hline & & \multicolumn{2}{|c|}{ Altura de plantas (cm) } & \multicolumn{2}{|c|}{ Diâmetro de caule (mm) } \\
\hline & & 40 DAS & 60 DAS & 40 DAS & 60 DAS \\
\hline Adubação (NPK) & 10 & $1217,566^{* *}$ & $284,644^{* *}$ & $6,373^{* *}$ & $1,621^{\mathrm{ns}}$ \\
\hline Irrigação (I) & 3 & $1074,617^{* *}$ & $4099,089^{* *}$ & $39,011^{* *}$ & $64,726^{* *}$ \\
\hline NPK x I & 30 & $131,25937^{\mathrm{ns}}$ & $73,729^{\text {ns }}$ & $2,009^{\mathrm{ns}}$ & $0,927^{\mathrm{ns}}$ \\
\hline Resíduo & 88 & 119,186 & 109,101 & 1.510 & 0,956 \\
\hline Total & 131 & - & - & - & - \\
\hline CV (\%) & - & 11,17 & 10,46 & 11,48 & 8,57 \\
\hline $\mathrm{N}$ - Regressão Linear & 1 & $80,504^{\text {ns }}$ & $123,697^{\text {ns }}$ & $0,165^{\mathrm{ns}}$ & $3,978^{*}$ \\
\hline N - Regressão Quadrática & 1 & $52,083^{\text {ns }}$ & $11,701^{\mathrm{ns}}$ & $2,851^{\mathrm{ns}}$ & $0,500^{\mathrm{ns}}$ \\
\hline P - Regressão Linear & 1 & $874,666^{\text {ns }}$ & $96,013^{\text {ns }}$ & $35,113^{* *}$ & $0,793^{\text {ns }}$ \\
\hline P - Regressão Quadrática & 1 & $3687,152^{* *}$ & $280,333^{\text {ns }}$ & $0,653^{\text {ns }}$ & $0,020^{\mathrm{ns}}$ \\
\hline K - Regressão Linear & 1 & $33,376^{\mathrm{ns}}$ & $960,800^{* *}$ & $0,0006^{\mathrm{ns}}$ & $0,126^{\mathrm{ns}}$ \\
\hline K - Regressão Quadrática & 1 & $453,255^{\mathrm{ns}}$ & $141,453^{\text {ns }}$ & $0,140^{\mathrm{ns}}$ & $0,725^{\mathrm{ns}}$ \\
\hline I - Regressão Linear & 1 & $2999,041^{* *}$ & $12188,784^{* *}$ & $112,592^{* *}$ & $189,765^{* *}$ \\
\hline I - Regressão Quadrática & 1 & $158,183^{\text {ns }}$ & $14,467^{\mathrm{ns}}$ & $1,401^{\mathrm{ns}}$ & $0,967^{\mathrm{ns}}$ \\
\hline N - Linear x I Linear & 1 & $0,300^{\mathrm{ns}}$ & $4,189^{\mathrm{ns}}$ & $2,493^{\mathrm{ns}}$ & $0,015^{\mathrm{ns}}$ \\
\hline P - Linear x I Linear & 1 & $7,824^{\mathrm{ns}}$ & $109,203^{\text {ns }}$ & $3,101^{\mathrm{ns}}$ & $1,333^{\mathrm{ns}}$ \\
\hline K - Linear x I Linear & 1 & $91,576^{\mathrm{ns}}$ & $190,403^{\text {ns }}$ & $0,141^{\mathrm{ns}}$ & $0,027^{\mathrm{ns}}$ \\
\hline
\end{tabular}

Nota: *, ${ }^{* *}$ significativo a 5 e $1 \%$, respectivamente, e ${ }^{\text {ns }}$ não significativo, pelo teste F. GL: Grau de liberdade. CV: coeficiente de variação. 
Os valores da altura da planta submetida às doses de $\mathrm{P}_{2} \mathrm{O}_{5}$ ajustaram, aos 40 DAS, ao modelo de regressão polinomial de segunda ordem (quadrática). Já, com as doses de $\mathrm{K}_{2} \mathrm{O}$, aos 60 DAS, os valores da altura de plantas ajustaram de forma linear, corroborando Ahmad (1989) que indica que a altura de plantas é significativamente afetada pela aplicação de $\mathrm{K}_{2} \mathrm{O}$ (Figura $1 \mathrm{~A}$ e B). Isto porque o potássio ocasiona $\mathrm{o}$ funcionamento dos estômatos, assimilando $\mathrm{CO}_{2}$ e aumentando a taxa fotossintética, influenciando, consequentemente, o crescimento das plantas. Os níveis de água utilizada para as plantas, tanto aos 40 como aos 60 DAS, influenciaram linearmente a altura das plantas (Figura 1C). Observou-se que as maiores alturas das plantas de girassol foram obtidas com as maiores doses de fósforo e potássio $\left(120 \mathrm{~kg} \mathrm{ha}{ }^{-1}\right)$. Segundo Taiz e Zeiger (2004), o fósforo é componente de muito fósforo-açúcares necessário na fotossíntese, respiração e participa na formação de nucleotídeos (DNA e RNA) e de fosfolipídios presentes nas membranas, por isso, é importante no metabolismo vegetal influenciando o crescimento das plantas.

$\mathrm{O}$ manejo da água disponível elevou linearmente a altura das plantas de girassol variedade Embrapa 122 V2000. Analisando as equações de regressão (Figura 1C), observou-se um acréscimo de 22,29 e $29,67 \%$ entre os tratamentos extremos de reposição de água aos 40 e 60 DAS, respectivamente.
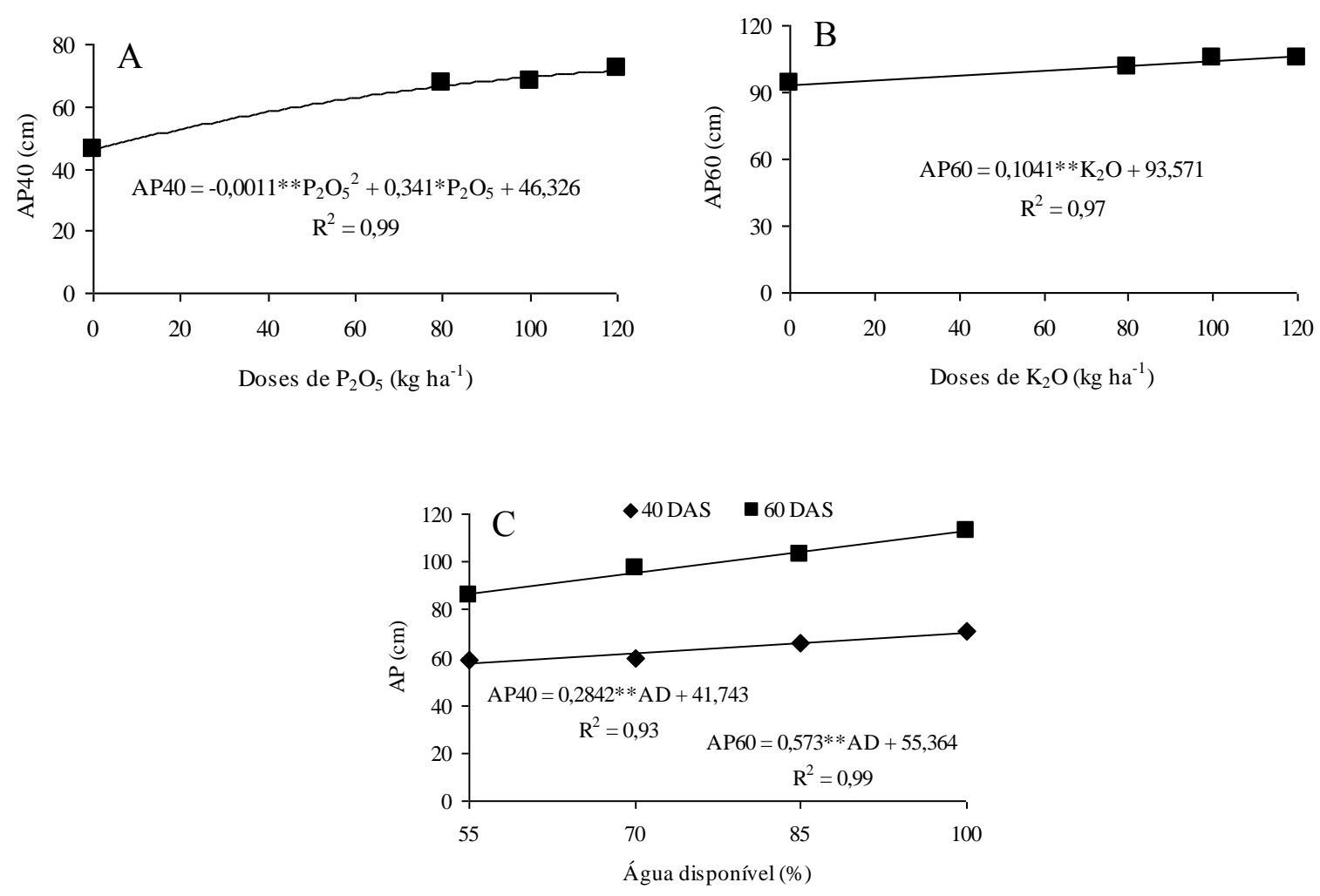

Figura 1. Altura de plantas do girassol Embrapa 122 V2000 aos 40 e 60 DAS em função das doses de $\mathrm{P}_{2} \mathrm{O}_{5}(\mathrm{~A}), \mathrm{K}_{2} \mathrm{O}(\mathrm{B})$ e do conteúdo de água disponível no solo (C).

Tan et al. (2000) relataram que a irrigação plena e limitada aplicada em diferentes fases de crescimento aumentou significativamente o crescimento vegetativo do girassol, particularmente a altura de plantas. Para Gomes et al. (2012), avaliando o desempenho de girassol sob diferentes lâminas de irrigação suplementar, a altura das plantas do girassol cultivar Araguá 4 respondeu de forma linear à irrigação, em todos os estádios analisados.

Muralidharudu et al. (2003) avaliando a resposta do girassol ao fornecimento de $\mathrm{P}$ em Vertissolos, concluíram que a aplicação de fósforo, em geral, resulta no crescimento em altura 
do girassol. Tendências semelhantes foram observadas por Mojiri e Arzani (2003) e Bakht et al. (2010a). Os resultados corroboram também com os obtidos por Soleimanzadeh et al. (2010) ao afirmarem que a altura final das plantas foi influenciada pelas doses de $\mathrm{K}_{2} \mathrm{O}$. Para Mobasser e Tavassoli (2013) os componentes de crescimento e rendimento foram afetados significativamente por diferentes níveis de água de irrigação.

Com exceçao da fertilização NPK aos 60 DAS, o diâmetro caulinar foi afetado estatisticamente pelas doses de fósforo (aos 40 DAS) e nitrogênio (60 DAS). Em relação ao regime hídrico, o diâmetro caulinar, nos períodos avaliados, aumentou significativamente com o incremento da água disponível no solo (Tabela 2).

De acordo com a literatura, o diâmetro do caule é uma característica muito importante no girassol, pois permite que ocorra menos acamamento da cultura e facilita seu manejo, tratos e colheita. Conforme os resultados obtidos, os valores de diâmetro caulinar aos 40 DAS aumentaram com as doses de fósforo, onde essa elevação resultou numa superioridade de $24,86 \%$ da maior dose de $\mathrm{P}_{2} \mathrm{O}_{5}\left(120 \mathrm{~kg} \mathrm{ha}^{-1}\right)$ em comparação ao tratamento controle (Figura 2A). Para os tratamentos fertilizados aos 60 DAS, foi detectado ajuste linear crescente com aumento com as doses de N, proporcionando, assim, uma superioridade de $24,69 \%$ do tratamento $100 \mathrm{~kg} \mathrm{ha}^{-1}$ em relação ao controle (Figura 2B).

Semelhantemente ao observado para a altura de plantas, a água disponível influenciou estatisticamente o diâmetro caulinar de forma linear, resultando em maiores valores da variável $(11,94$ e $13,02 \mathrm{~mm})$ nos tratamentos de $100 \%$ de água disponível, para avaliação aos 40 e 60 DAS, respectivamente (Figura 2C).
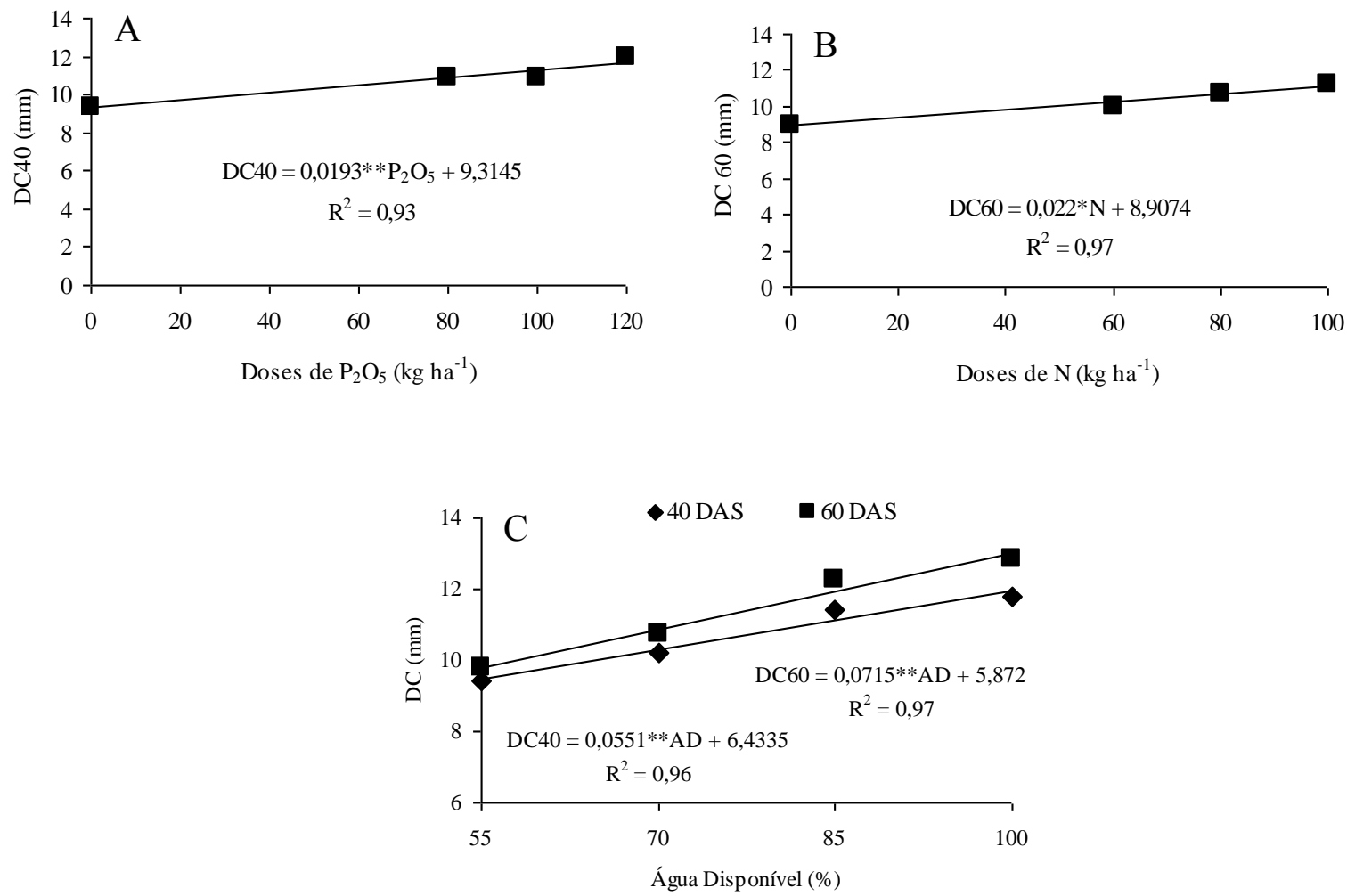

Figura 2. Diâmetro caulinar do girassol Embrapa 122 V2000 aos 28 e 52 DAS em função das doses de $\mathrm{P}_{2} \mathrm{O}_{5}(\mathrm{~A}), \mathrm{N}$ (B) e do conteúdo de água disponível no solo (C).

Avaliando a influência do manejo da irrigação e nitrogênio sobre características agronômicas e rendimento do girassol, Khaliq e Cheema (2005), obtiveram resultados significativos da adubação nitrogenada sobre o diâmetro do caule de girassol. Segundo os 
autores, não foi registrada interação entre irrigação e aplicação de nitrogênio. Esses resultados estão coerentes com os obtidos por Abdel-Motagally e Osman (2010), para adubação com nitrogênio. Sobre o efeito positivo da adubação fosfatada na cultura do girassol, Chaniara et al. (1989) afirmaram que o diâmetro do caule aumentou com o incremento da aplicação de fósforo.

Ayub et al. (1999), ao estudarem o efeito de diferentes fontes e doses de potássio sobre a produçao, as caracteristicas do crescimento e o teor de óleo do girassol, não observaram interferência significativa das doses de potássio no diâmetro do caule.

Os resultados do regime hídrico foram semelhantes aos obtidos por Nemazi et al. (2008), em estudo sobre o efeito do estresse hídrico e desfolha do girassol em condições controladas. Baseado na pesquisa, uma redução no teor de água no solo a 60 e $30 \%$ da capacidade de campo, resultou numa diminuição de 20 e $46 \%$ do diâmetro do caule em relação ao controle, respectivamente. Esses dados também corroboram com os obtidos por Guedes Filho et al. (2013) e Gomes et al. (2012).

O suprimento adequado de fósforo, uma vez que interferi diretamente nas taxas fotossintéticas, resulta no aumento de número de folhas e na área foliar. Por outro lado, a carência deste elemento reduz o número de folhas, porque promove a senescência precoce das folhas mais velhas. Nesta pesquisa o número de folhas do girassol Embrapa 122 V2000 foi influenciado significtivamente pela interação das doses de fósforo vs conteúdo de água disponível no solo aos 40 e 60 DAS (Tabela 3).

Tabela 3. Resumo das análises de variância para o número de folhas (NF) e para área foliar do girassol (variedade Embrapa 122-V2000) aos 40 e 60 dias após a semeadura (DAS) submetida à adubação com NPK e conteúdo de água disponível no solo.

\begin{tabular}{|c|c|c|c|c|c|}
\hline \multirow{3}{*}{ Fonte de variação } & \multirow{3}{*}{ GL } & \multicolumn{4}{|c|}{ Quadrado Médio } \\
\hline & & \multicolumn{2}{|c|}{ Número de folhas (NF) } & \multicolumn{2}{|c|}{ Área foliar $\left(\mathrm{cm}^{2}\right)$} \\
\hline & & 40 DAS & 60 DAS & 40 DAS & 60 DAS \\
\hline Adubação (NPK) & 10 & $17,739^{* *}$ & $39,096^{* *}$ & $185572,53^{* *}$ & $284202,6^{* *}$ \\
\hline Irrigação (I) & 3 & $103,292^{* *}$ & $32,573^{* *}$ & $164600,92^{* *}$ & $215584,0^{* *}$ \\
\hline NPK $x$ I & 30 & $5,004^{\mathrm{ns}}$ & $10,456^{\mathrm{ns}}$ & $152123,03^{\text {ns }}$ & $2071576,7^{\mathrm{ns}}$ \\
\hline Resíduo & 88 & 5,787 & 6,969 & 160614,01 & 22136157 \\
\hline Total & 131 & - & - & - & - \\
\hline CV (\%) & - & 13,78 & 14,47 & 17,10 & 18,70 \\
\hline N - Regressão Linear & 1 & $0,0375^{\mathrm{ns}}$ & $4,150^{\mathrm{ns}}$ & $20681,18^{\mathrm{ns}}$ & $10159,08^{\mathrm{ns}}$ \\
\hline N - Regressão Quadrática & 1 & $2,5208^{\mathrm{ns}}$ & $0,333^{\text {ns }}$ & $1620,37^{\mathrm{ns}}$ & $6138,93^{\text {ns }}$ \\
\hline P - Regressão Linear & 1 & $2,400^{\mathrm{ns}}$ & $9,600^{\text {ns }}$ & $21091,12^{\text {ns }}$ & $11163,99^{\mathrm{ns}}$ \\
\hline P - Regressão Quadrático & 1 & $16,333^{\mathrm{ns}}$ & $40,333^{*}$ & $8478,57^{\mathrm{ns}}$ & $118138,56^{\mathrm{ns}}$ \\
\hline K - Regressão Linear & 1 & $0,337^{\text {ns }}$ & $29,400^{*}$ & $138627,09^{\text {ns }}$ & $252336,47^{\mathrm{ns}}$ \\
\hline K - Regressão Quadrática & 1 & $2,520^{\mathrm{ns}}$ & $10,083^{\mathrm{ns}}$ & $613753,92^{* *}$ & $606012,27^{* *}$ \\
\hline I - Regressão Linear & 1 & $306,818^{* *}$ & $37,346^{*}$ & $137274,10^{\text {ns }}$ & $138340,10^{\mathrm{ns}}$ \\
\hline I - Regressão Quadrática & 1 & $2,454^{\mathrm{ns}}$ & $54,734^{* *}$ & $150103,58^{* *}$ & $861915,63^{* *}$ \\
\hline $\mathrm{N}$ - Linear $\mathrm{x}$ I Linear & 1 & $1,140^{\mathrm{ns}}$ & $4,083^{\mathrm{ns}}$ & $7824,48^{\mathrm{ns}}$ & $56876,65^{\mathrm{ns}}$ \\
\hline P - Linear x I Linear & 1 & $34,003^{*}$ & $36,750^{* *}$ & $15732,64^{\mathrm{ns}}$ & $178750,22^{\text {ns }}$ \\
\hline K - Linear x I Linear & 1 & $17,520^{\mathrm{ns}}$ & $27,603^{* *}$ & $99117,26^{\mathrm{ns}}$ & $263225,01^{\text {ns }}$ \\
\hline
\end{tabular}

Nota: ",** significativo a 5 e $1 \%$, respectivamente, e ${ }^{\text {ns }}$ não significativo, pelo teste F. GL: Grau de liberdade. CV: coeficiente de variação. 
De acordo com a Figura 3A, o número de folhas aos 40 DAS em função da adubação fosfatada aumentou de forma quadrática com os tratamentos de 55; 70 e 100\% AD e de forma linear com $85 \%$ AD sendo que o maior número de folhas (20,81 unidades) foi obtido na dose de $80 \mathrm{~kg} \mathrm{ha}^{-1}$ de $\mathrm{P}_{2} \mathrm{O}_{5}$ sob manejo de irrigação de $100 \% \mathrm{AD}$. Observou-se tambem que na dose de $120 \mathrm{~kg} \mathrm{ha}^{-1}$ de $\mathrm{P}_{2} \mathrm{O}_{5}$, quanto menor o volume de água no substrato, menor o número de folhas do girassol. Isto mostra que o aumento da adubação, entretanto, com deficit hidrico não influencia positivamente o desenvolvimento das plantas.

O número de folhas em função da adubação fosfatada, aos 60 DAS, não foi afetado nos níveis de de 85 e 100\% AD apresentando valores médios de 19,83 e 17,92, respectivamente. No entanto, o número máximo de folhas foi 20,40 unidades correspondente a estimativa de $83,88 \mathrm{~kg} \mathrm{ha}^{-1}$ de $\mathrm{P}_{2} \mathrm{O}_{5}$ com $70 \%$ AD (Figura 3B).

Da mesma forma, o número de folhas das plantas de girassol foi influenciado significtivamente pela interação das doses de potássio vs conteúdo de água disponível no solo aos 60 DAS (Tabela 3). Entretanto, os dados do número de folhas se ajustaram de forma quadrática somente com 55 e $70 \%$ AD cujo maior número de folhas (23,29 unidades) foi obtido na dose estimada de $51 \mathrm{~kg} \mathrm{ha}^{-1}$ de $\mathrm{K}_{2} \mathrm{O}$. Com os tratamentos de 85 e $100 \%$ AD obteve-se valores médios, em torno de 21 e 18 folhas, respectivamente (Figura 3C).
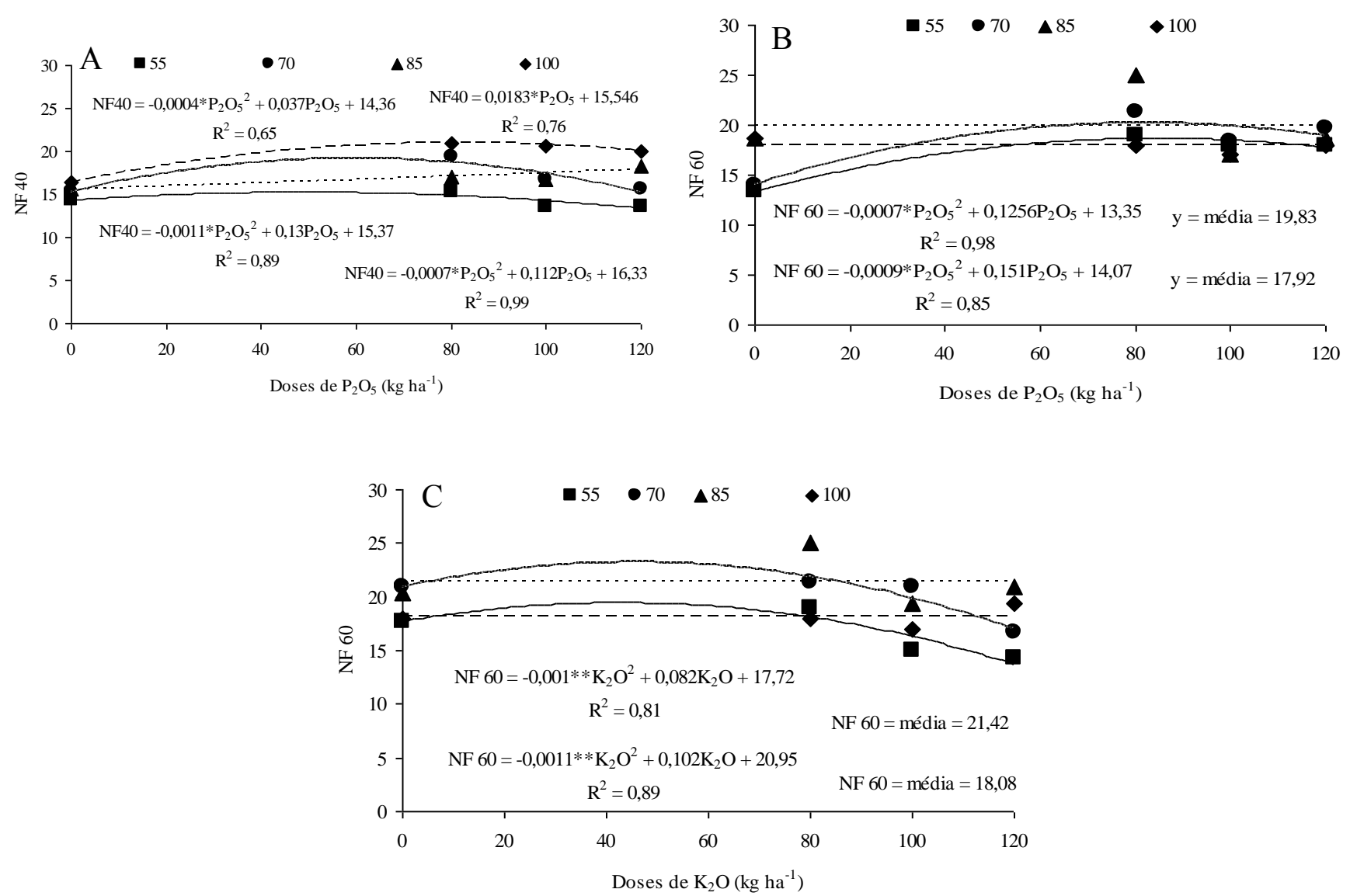

Figura 3. Número de folhas (NF) do girassol variedade Embrapa 122 V2000 em função da interação da adubação fosfatada e conteúdo de água disponível aos 40 (A) e 60 DAS (B) e adubação potássica e conteúdo de água disponível do solo aos 60 DAS (C).

O efeito negativo do estresse hídrico sobre a taxa de aparecimento de folhas de girassol foi registrado por Gholinezhad et al. (2011). Segundo os autores, o máximo e o mínimo de taxa de aparecimento foliar foi obtido com irrigação adequada e estresse hídrico severo, respectivamente. Para Taiz e Zieger (2004), o turgor celular estimula a expansão e ou 
alongamento das celulas; logo a restrição de água limita não só o tamanho, mas também o número de folhas; fatos constatados na presente pesquisa. Os dados do efeito depreciativo do estresse hídrico sobre o número de folhas do girassol, corroboram com os observados por Thakuria et al. (2004) e Bakht et al. (2010b).

De acordo com os resultados da análise de variância apresentada na Tabela 3, os dados da área foliar do girassol Embrapa 122 V2000 foram influenciados significativamente pela aplicação de potássio e conteúdo de água disponível no solo, aos 40 e 60 DAS, ajustados de forma quadrática.

A área foliar, aos 40 e 60 DAS, aumentou de forma quadratica com a aplicação de potássio nas plantas de girassol, obtendo-se os maiores valores com as doses de 70,75 e 72,62 $\mathrm{kg} \mathrm{K}_{2} \mathrm{O} \mathrm{ha}^{-1}$, respectivamente (Figura 4A). Os resultados dessa pesquisa corroboram com os apresentados por Ayub et al. (1999). Pela investigação destes autores, a área foliar máxima do girassol foi obtida na dose de $100 \mathrm{~kg} \mathrm{ha}^{-1}$ de cloreto de potássio.

As plantas com 55\% AD apresentaram uma menor área foliar, nas duas datas avaliadas. Contudo, os maiores valores da área foliar, foram encontrados 83,25 e 77,93\% AD, aos 40 e 60 DAS, respectivamente (Figura 4B). Vários trabalhos encontrados na literatura fazem referência ao efeito negativo do estresse hídrico sobre a área foliar do girassol a exemplo de Tan et al. (2000), Petcu et al. (2001), Thakuria et al. (2004) e Bakht et al. (2010b).
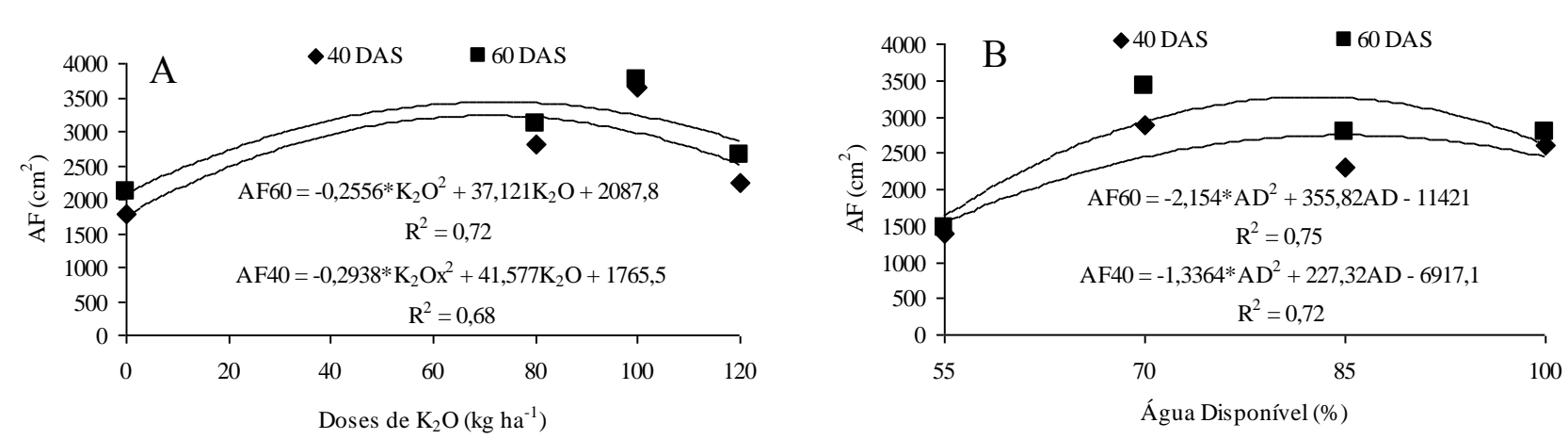

Figura 4. Área foliar (AF) do girassol variedade Embrapa 122 V2000 aos 40 e 60 DAS em função da adubação com $\mathrm{K}_{2} \mathrm{O}(\mathrm{A})$ e do conteúdo de água disponível no solo (B).

Em todas as pesquisas, quer seja sob condições de vaso, quer seja em cultivo de campo, a área foliar do girassol decresceu nos tratamentos com menor conteúdo de água disponível. Esses resultados estão compatíveis com os relatados por Thair et al. (2002), ao afirmarem que plantas de girassol sob estresse hídrico apresentaram redução de $25,56 \%$ da área foliar quando comparado aos tratamentos de $100 \%$ da água disponível. Naim e Ahmed (2010) apontaram que o estresse hídrico diminui a fotossíntese devido ao fechamento dos estômatos. A redução da taxa fotossintética diminuiu a translocação de carboidratos e reguladores de crescimento, o que resulta em redução da pressão de turgescência e da área foliar, além da diminuição do crescimento.

\section{CONCLUSÕES}

A altura da planta, o diâmetro do caule, o número de folhas e a área foliar do algodão foram afetados pela adubação NPK e pelo conteúdo de água disponível no solo para as plantas;

Com exceção da área foliar todas as variáveis apresentaram comportamento linear crescente em função da água disponível do solo; 
Para as condições estudadas, as maiores doses de $\mathrm{N}, \mathrm{P}_{2} \mathrm{O}_{5}$ e $\mathrm{K}_{2} \mathrm{O}$ e o maior conteúdo de água disponível no solo proporcionaram os maiores índices de crescimento.

\section{AGRADECIMENTOS}

Ao Conselho Nacional de Desenvolvimento Científico e Tecnológico (CNPq) pelo financiamento da pesquisa e pela concessão de bolsa de pós-graduação ao primeiro autor.

\section{REFERÊNCIAS}

ABDEL-MOTAGALLY, F. M. F.; OSMAN, E. A. Effect of nitrogen and potassium fertilization combinations on productivity of two sunflower cultivars under East of Elewinate Condictions. American-Eurasian Journal Agricultural and Environmental Sciences, Dubai, v. 8, n. 4, p. 397-401, 2010.

AHMAD, F. Effect of potassium application in different proportions with nitrogen and phosphorus on growth, yield and oil content of sunflower. 1989. 100f. Ph.D - Thesis - University Agricultural, Faisalabad, 1989.

AYUB, M.; TANVEER, A.; AMIN, M. Z.; SHARAR, M. S.; PERVAIZ, A. Effect of differents sources and levels of potash on yield and oil content of spring sunflower. Pakistan Journal of Biological Sciences, Singapore, v. 2, n. 3, p. 801-803, 1999.

BAKHT, J.; SHAFI, M.; YOUSAF, M.; SHAH, H. U. Physiology, phenology and yield of sunflower (autumn) as affected by NPK fertilizer and hybrids. Pakistan Journal Botany Sciences, Singapore, v. 42, n. 3, p. 1909-1922, 2010a.

BAKHT, J. J.; SHAFI, M.; YOUSAF, M.; KHAN, M. A. Effect of irrigation on physiology and yield of sunflower hybrids. Pakistan Journal Botany Sciences, Singapore, v. 42, n. 2, p. 1317-1326, 2010 b.

CASTRO, C; OLIVEIRA, F. A. Nutrição e adubação do girassol. In: LEITE, R. M. V.; BRIGHENTI, B. de C.; CASTRO, A. M. (Ed.). Girassol no Brasil. Londrina: Embrapa Soja, 2005. p. 317-373.

CHANIARA, N. J.; PATEL, J. C.; MALAVIA, D. D.; BALDHA, N. M. Effect of irrigation, nitrogen and phosphorus on the productivity of sunflower. Indian Journal of Agronomy, New Delhi, v. 34, n. 4, p. 399-401, 1989.

EMPRESA BRASILEIRA DE PESQUISA AGROPECUÁRIA - EMBRAPA. Centro Nacional de Pesquisa de Soja. Tecnologia de Produção do Girassol: sistema de produção. Londrina: Embrapa Soja, 2000. 158p.

EMPRESA BRASILEIRA DE PESQUISA AGROPECUÁRIA - EMBRAPA. Centro Nacional de Pesquisa de Solos. Manual de métodos de análise de solo. 2. ed. Rio de Janeiro: Embrapa Solos, 1997. 212 p.

EUCLYDES, R. F. Manual de utilização do programa SAEG (Sistema para análises estatísticas e genéticas). Viçosa: UFV, 1997. 59 p.

FOOD AND AGRICULTURAL ORGANIZATION - FAO. FAOSTAT - Statistics database 2008. Disponível em: <http://apps.fao.org/>. Acesso em: 10 out. 2011. 
GHOLINEZHAD, E.; AYNABAND, A.; GHORTHAPEH, A. H.; NOORNOHAMADI, G.; BERNOUSI, I. Effect of drought and nitrogen rates on grain yield, quality traits and physiological indices in sunflower hybrid Iroflor at different plant density. World Applied Sciences Journal, v. 14, n. 1, p. 131-139, 2011.

GOMES, E. P.; FREDI, G.; ÁvilA, M. R.; BISCARO, G. A.; REZENDE, R. K. S.; JORDAN, R. A. Produtividade de grãos, óleo e massa seca de girassol sob diferentes lâminas de irrigação suplementar. Revista Brasileira de Engenharia Agrícola e Ambiental, Campina Grande, v. 16, n. 3, p. 237-246, 2012. http://dx.doi.org/10.1590/S1415-43662012000300001

GUEDES FILHO, D. H.; SANTOS JÚNIOR, J. A.; CHAVES, L. H. G.; CAMPOS, V. B.; OLIVEIRA, J. T. L. Água disponível no solo e doses de nitrogênio no crescimento do girassol. Revista Brasileira de Agricultura Irrigada, Fortaleza, v. 7, p. 201-212, 2013. http://dx.doi.org/10.7127/RBAI.V7N300016

KHALIQ, A.; CHEEMA, Z. A. Influence of Irrigation and Nitrogen Management on Some Agronomic Traits and Yield of Hybrid Sunflower (Helianthus annuus L.). International Journal of Agriculture \& Biology, Faisalabad, v. 7, n. 6, p. 915-919, 2005 .

MALDANER, I. C.; HELDWEIN, A. B.; LOOSE, L. H.; LUCAS, D. P.; GUSE, F. I.; LUZZI, M. P. B. Modelos de determinação não-destrutivo da área foliar em girassol. Ciência Rural, Santa Maria, v. 39, n. 5, p. 1356-1361, 2009. http://dx.doi.org/10.1590/S0103-84782009000500008

MOJIRI, A.; ARZANI, A. Effects of nitrogen rate and plant density on yield and yield components of sunflower. Journal Science Technics Agricultural Nature Research, New Jersey, v. 7, p. 115-125, 2003.

MOBASSER, H. R.; TAVASSOLI, A. Effect of water stress on quantitative and qualitative characteristics of Yield in sunflower (Helianthus annuus L.). Journal of Novel Applied Sciences, Moorebank, v. 2, n. 9, p. 299-302, 2013.

MURALIDHARUDU, Y.; MURTHY, I. Y. L. N.; REDDY, K. P. C.; REDDY, B. N.; CHANDRANATH, H. T. Response of sunflower (Helianthus annuus L.) to phosphorus application in vertisols. Helia, Novi Sad, v. 26, n. 39, p. 147-154, 2003. http://dx.doi.org/10.2298/HEL1358101S

NAIM, A. M. E.; AHMED, M. F. Effect of Irrigation Intervals and Inter-row Spacing on vegetative growth characteristics in Sunflower (Helianthus annuus L) hybrids in Shambat soil. Journal of Applied Sciences Research, Faisalabad, v. 6, n. 9, p. 1440$1445,2010$.

NEZAMI, A.; KHAZAEIA, H. R.; BOROUMAND REZAZADEHB, Z.; HOSSEINIC, A. Effects of drought stress and defoliation on sunflower (Helianthus annuus) in controlled condition. Desert, Tehran, v. 13, n. 0, p. 99-104, 2008.

PETCU, E.; ARSINTESCU, A.; STANCIU, D. The effect of hydric stress on some characteristics of sunflower plants. Romanian Agricultural Research, Fundulea, v. 16, p. 15-22, 2001.

QUAGGIO, J. A.; UNGARO, M. R. G. Girassol. IN: RAIJ, B. VAN; CANTARELLA, H.; QUAGGIO, J. A.; FURLANI, A. M. C. (Eds.). Recomendações de adubação e calagem para o Estado de São Paulo. Campinas: IAC, 1997. 198p. 
SOLEIMANZADEH, H.; HABIBI, D.; ARDAKANI, M. R.; PAKNEJAD, F.; REJALI, F. Response of sunflower (Helianthus annuus L.) to drought stress under different potassium levels. World Applied Sciences Journal, Dubai, v. 8, n. 4, p. 443-448, 2010 .

TAHIR, M. H. N.; IMRAN, M.; HUSSAIN, M. K. Evaluation of sunflower (Helianthus annuus L.) inbred lines for drought tolerance. International Journal of Agriculture \& Biology, Faisalabad, v. 4, n. 3, p. 398-400, 2002.

TAIZ, L.; ZEIGER, E. Fisiologia vegetal. 3. ed. Porto Alegre: Artmed, 2004. 613 p.

TAN, S.; BEYAZGUL, M.; AVCIERI, Z.; Y. KAYAM, Y.; KAYA, H. G. Effect of irrigation at various growth stages on some economic characters of first crop sunflower. Journal Anadolu, Izmir, v. 10, n. 2, p. 1-34, 2000.

THAKURIA, R. K.; HARBIR, S.; TEJ, S. Effect of irrigation and antitranspirants on growth and yield of spring sunflower (Helianthus annuus L.). Indian Journal of Agronomy, New Delhi, v. 49, p. 121-123, 2004.

ZOBIOLE, L. H. S.; CASTRO, C.; OLIVEIRA, F. A.; OLIVEIRA JÚNIOR, A. Marcha de absorção de macronutrientes na cultura do girassol. Revista Brasileira de Ciência do Solo, Viçosa, v. 34, n. 2, p. 425-433, 2010. http://dx.doi.org/10.1590/S010006832010000200016 